


\section{THE UNIVERSITY}

\section{OF ILLINOIS}

\section{LIBRARY}

595.79

D/3h

v.2.

BIOLOGY

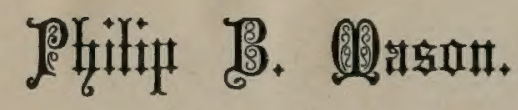




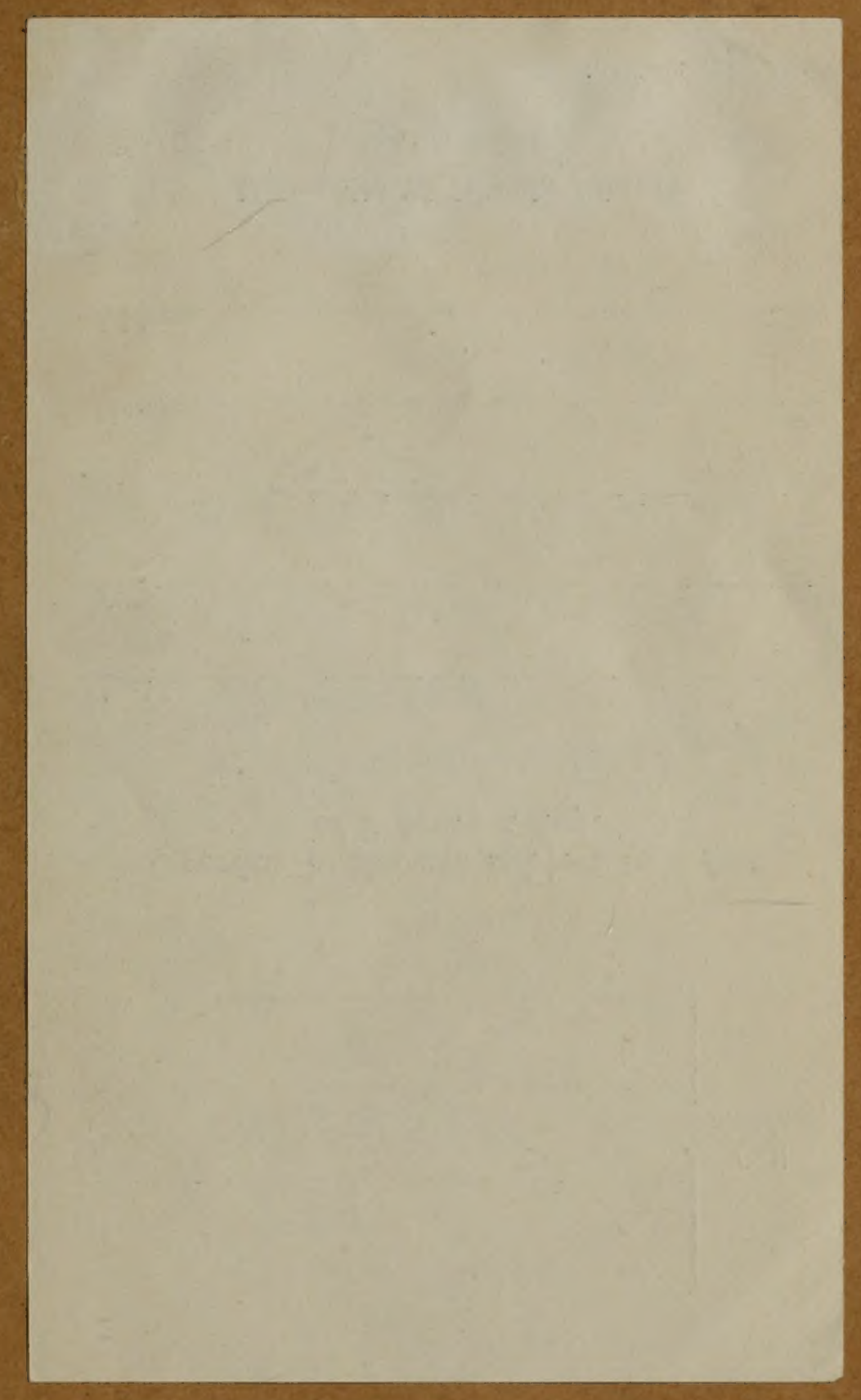





\title{
HYMENOPTERA EUROPAEA
}

\author{
PRAECIPUE \\ BOREALIA, \\ FORMIS TYPICIS NONNƯLLIS.
}

SPECIERUM GENERUMVE EXOTICORUM PROPTER NEXUM

SYSTEMATICUM ASSOCIATIS,

PER

FAMILIAS, GENERA, SPECIES ET VARIETATES DISPOSITA ATQUE DESCRIPTA

$A B$

ANDREA GUSTAVO DAHLBOM.

TOMUS SECUNDUS.

CHRYSIS in Sensu linnaeano.

ACCEDUNT XII TABULLAE ÁERI INCISAÉ.

B ER OL I N I

PROSTAT IN LIBRARIA FRIDERICI NICOLAI

MDCCCLIV. 


\section{A51140XVIH}

\subsection{0}

\section{z्याओ \\ -}

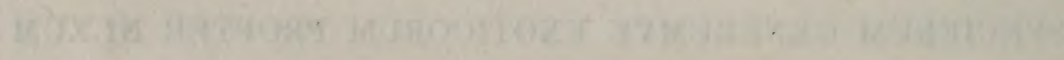

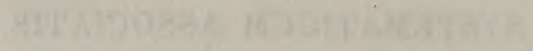

Denique caelesti sumus ommes semine oriundi.

- Lucret. II, 990.

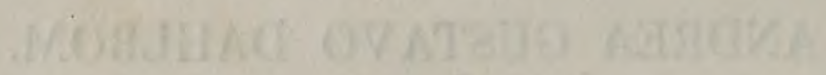

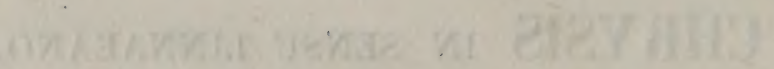




\section{Argumentum.}

1. Praefatio pag. $\mathbf{v}-\mathbf{x v r}$.

II. Catalogus Hymenopterorum Chrysidiformium, Collectionum De Sehestedii et Tönder Lundi (in Museo Regio Havniensi asservatorum ) pro Fabricii „Systemate Piezatorum" Typicorum pag. xvir et XVIII.

III. Catalogus Hymenopterorum Chrysidiformium, Collectionis Fabricii (Musei Universitatis Kieloniensis) pro „Systemate Piezatorum“ Typicorum pag. xıx.

IV. Revisio „Systematis Piezatorum“ Chrysidiformium pag. $\mathbf{x x}-\mathbf{x x I I}$.

V. Characteres Familiarum et Generum, atque Adumbrationes submonographicae Specierum Hymenopterorum Chrysidiformium nobis cognitarum pag. 1 388.

VI. Explicationes Iconum pag. 389 - 397.

VII. Index systematicus pag. 398- 401.

VIII. Index alphabeticus pag. $402-409$. 


\section{molagmogen}

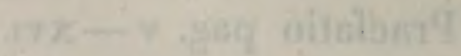

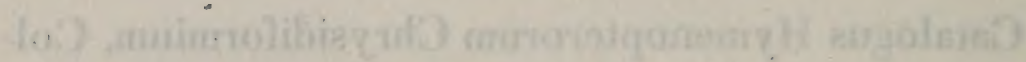
if

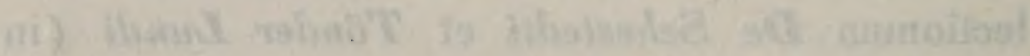

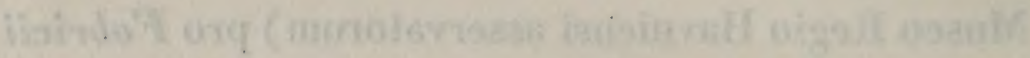

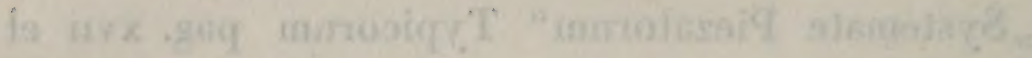

.Inxing

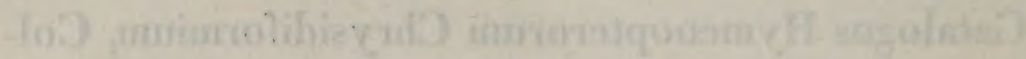

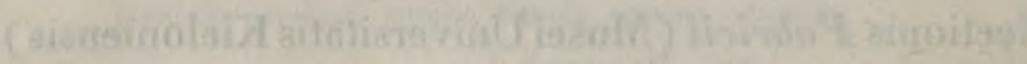

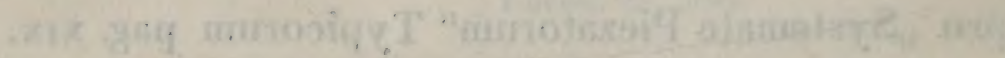

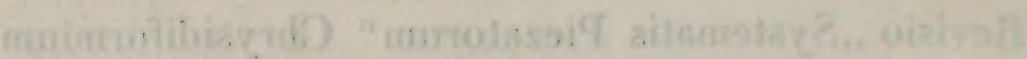

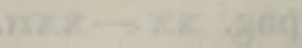

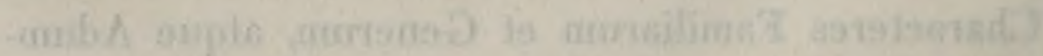

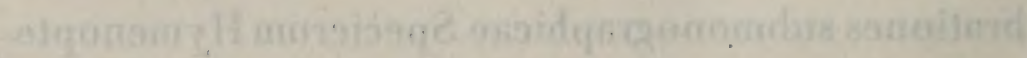

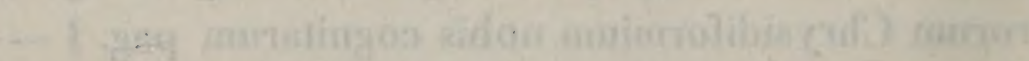

25:

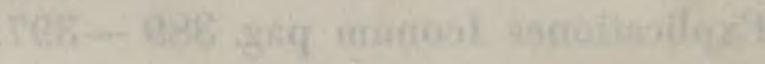

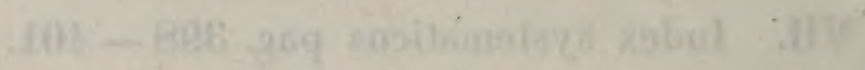

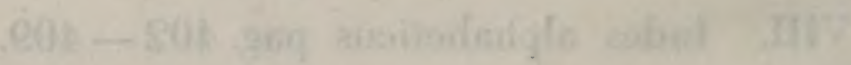




\section{PRAEFA TIO.}

Quam anno 1845 orbi erudito promiseram Chrysidarum descriptionem, eam nunc tandem amicis fautoribusque huius operis offero. Quod aliquanto tardius, quam in votis fuerat, volumen hoc in lucem prodeat, paucis ut excusem necesse est.

A compluribus entomologis rogatus, ut Fabriciani systematis Piezatorum accuratam ederem synonymiam, hoc in me delatum munus recusare nolui. Cum autem Fabricii definitiones, veterum scriptorum more, collective, ut ita dicam, factae, in incerto saepius nos relinquant, cuinam generi vel speciei tribuendae sint, cum praeterea apud eum multae designationes omissae sint, quibus nos, accuratioribus indagationibus consueti, carere non possumus, nullo alio modo mihi visus sum propositi mei finem assequi posse, nisi quae Havniae atque Kieloniae asservantur Fabricii specimina typica cum Fabricii synonymis sedulo compararem, et ditissimum museum Berolinense ipse inspicerem.

Ad hunc finem rex noster clementissimus 0scar, omnium bonarum artium fautor atque promotor, ineunte 
anno 1847 itineris impensas petenti mihi liberalissime concessit.

Die 5. mensis Iulii eiusdem anni 1847 itinere suscepto primum Havniam me contuli, et congregationi naturae studiosorum Scandinaviae adfui. Ibi a viris egregiis Christiano Drewsen, Schiödte doctore, qui regii musei sectioni entomologicae praeest, Staegero et Westermanno maxima benivolentia exceptus sum. Westermannus tanta fuit liberalitate, ut integram suam Chrysidarum collectionem, non solum in itinere mecum asportandam, sed etiam in patriam reduci maximo otio domi perlustrandam mihi concederet.

Musei Havniensis copiae entomologicae - quatenus Fabricii specimina typica continent - ex duabus collectionibus congestae sunt, quarum priorem vir beatae memoriae de Sehestedt, minister regius, alteram beatus Tönder Lund, vectigalis director, ante hos 14 fere annos museo donaverunt. Specierum numerus circa decem milium est. Exemplaria in scrinïs et cistis vitro coopertis more solito adservantur. Cistarum fundus non subere, sed cera tectus est.

Nonaginta et una species in museo Havniensi a me perlustratae, triginta hoc volumine descriptae sunt.

Havnia relicta Lubecam et Kieloniam me contuli. Lubecae Mildii collectionem et amoenissimas eius insectorum picturas inspexi. Kieloniae inde a die 30 . men- sis Iulii usque ad diem 7. mensis Augusti commoratus, benivolentia et opera virorum clarissimorum Boie, justiciarii, et Saxesen, doctoris, opportunitatem nactus sum, antiquam illam Fabricii collectionem, in museo universitatis Kieloniensis adservatam, perlustrandi. 
Cuius collectionis, a Fabricio universitati concessae, de natura et indole cum vir clarissimus Schaum doctor, in Ephemeridibus entomologicis Sedinensibus tum in universum, tum in specie de Scarabaeis abunde egerit, non est quod in ea describenda commorer.

Ibi viginti novem Fabricianae collectionis species a me indagatae, viginti sex hoc volumine descriptae sunt.

Inde a Kielonia viam ferream secutus Altonam Hamburgum et Berolinum adii. Altonae vir clarissimus Sommer divitem suam elegantemque Scarabaeorum et Papilionum collectionem mihi monstravit, et - quae eius est benignitas - insignia nonnulla Rhynchotorum et Hymenopterorum exemplaria dono mihi dedit.

In Hamburgi et Eppendorfii vicinis, anno 1838 una cum viris egregiis Thorey et de Winthem a me perlustratis, nunc eodem modo me moraturum fore speraveram, sed longe aliter res evenit. Thorejus in itinere absens erat, et carissimum Winthemium morbo phthisico letali correptum ac fere exhaustum inveni; qui quamvis a molestissima tussi paene loquela impediretur, tamen me per litterarum commercium sibi coniunctissimum, et tot excursionum olim comitem, benigniter excepit. Cuius viri praematuram quidem, sed diu expectatam mortem historiae naturalis amici mecum lugent.

Inde a die 7. mensis Augusti usque ad diem primum mensis Octobris Berolini commoratus sum. Vir clarissimus Klug, regi a consiliis intimis, museum entomologicun, curae suae creditum, quotidie perlustran- 
dum mihi permisit, et insectorum describendorum liberalissime copiam mihi fecit, imo venerabilis senex omnia ea, quorum ipse descriptionem antea inceperat Chrysidarum europaearum exemplaria mihi obtulit. (2ua eximia benignitate adeo adiutus sum, ut angusto bimestri spatio 116 species conferrem, 130 species integras describerem, $\mathbf{1 6}$ tabulas synopticas componerem et trecentarum Chrysidarum unguiculas microscopice pervestigarem. Hunc ultimum laborem minime exantlare potuissem, nisi vir clarissimus Stein, professor, egregii sui microscopii usum mihi concessisset.

Musei Berolinensis praestantiam, ab aliis merito laudatam, extollere non meum est. Cuilibet ingredienti - sitne historiae naturalis peritus necne maximam admirationem movet tot tantorumque animalium bene ordinata dispositio, doctorum hominum sagacissimis inquisitionibus constituta et servata. In colligendis et adservandis insectis vir clarissimus $K$ hug fere quinquaginta annorum operam impendit, a ministerio regis, cui harum rerum cura est, magna cum liberalitate adiutus.

Cum Berolini degerem, a viris summis, libero Barone Alexandro ab Humboldt, nec non libero Barone a D'Ohsson, studiorum meorum fautoribus praeclarissimis, incitatus sum, ut Viennam visitarem, ubi insectorum Europae meridionalis et imprimis dustriae, hucusque non satis cognitorum largissimam messem expectare possem. Absentiae autem venia cum nonnisi ad finem mensis Octobris mihi concessa esset, hoc itinere invitus abstimui. Attamen mihi Lundam reduci - quantum fuerit gaudium meum quisque la- 
cile intelliget - per commendationem Alexandri $a b$ Humboldt, omnia Viennensis musei Hymenoptera, ad Sphecum Chrysidarum et Vesparum genera pertinentia, a viro clarissimo Vincentio Kollàr, professore, per cursum publicum missa sunt.

Opere meo Berolini absoluto, Neostadium-Eberswaldam adii, ut dilectissimum amicum atque fautorem meum, virum clarissimum Ratzeburg, professorem, visitarem, et opus eius celeberrimum, cui titulus »die Forstinsekten“ denuo tractarem, eiusque scientia, omnibus numeris absoluta fruerer, qua insectorum indolem et mores tamdiu perscrutatus est. Qui cum fraterno amore me recepisset atque cupidissime de laboris mei statu inquisivisset, paullo post sollertia sua effecit, ut bonae notae libraria Friderici Nicolai Berolinensis libri mei edendi impensas faceret.

Praeter eas quas modo indicavi subsidias studiis meis entomologicis magnum auxilium attulerunt, quos honoris caussa nomino, viri clarissimi $C$. $I$. Boheman, professor Holmiensis; P. F. Bouché, hortulanus Berolinensis; Brischke, praeceptor Gedanensis; Cornelius, praeceptor Elberfeldensis; C. A. Dohrn, societatis entomologicae Sedinensis praeses; Hagen, doctor Regimontanus; Hoffmeister, pastor Nordhusiensis, Hassus-Casselanus; a Kiesenwetter, Saxo; Josephus Iriechbaumer, doctor Curiensis, Rhaetus; Loew, professor Posnaniensis; Luiben, scholae Ascherslebensis director; Tischbein, qui rei saltuariae praeest Herrsteinii in monte Hundsrück; P.F. Wahlberg, professor Holmiensis; I. Wahlberg, architectus militaris Holmiensis; Zeller, gymnasii Glogaviensis director. 
Iam anno 1846 vir clarissimus liber Baro Mlaximilianus Spinola, nobilis Genuensis, humanissime mihi permisit, ut locupletissimam suam Chrysidarum collectionem mecum asportatam usque ad opus meum completum apud me servarem.

Eadem liberalitate vir clarissimus I. W. Zetterstedt, professor Lundensis, lapponicorum insectorum collectionibus suis ut uterer mihi concessit, paternoque suo consilio studia mea promovit.

His instructus auxiliis ineunte anno 1848 Chrysidarum monographiam scribere incoepi, quo in opere perficiendo, ab officii mei curis detentus, paucis tantum horis subsecivis adhibitis, biennium consumpsi.

Hoc quod nunc prodit secundo volumine eundem quam in priore ordinem, a plerisque entomologis probatum, secutus sum. Quae mihi innotuerunt Chrysidarum 213 species, eas 12 generibus et 6 familiis distinctas descripsi.

Quarum familiarum signa diagnostica fere haec sunt: maxillae inferiores, labium inferius et lingua, aut breviora et minus procurrentia aut elongata et prominentia; venter convexus aut concavus; margo analis qui in ultimo abdominis segmento dorsalis reperitur, aut dentatus aut non dentatus aut serratus; pedum unguiculae planae aut dentatae aut serratae.

Ad generum characteres stabiliendos nonnisi immutabiles et satis patentes corporis partes, in speciebus definiendis sculpturam et armaturam adhibui. Colores nomnunquam constantes, plerumque autem variabiles inveni. 
De abdominis mirabili structura paucis hic dicendum est. Cum inter entomologos opinio invaluerit, abdominis dorsum in genuinis Chrysidibus (exceptis Clepte, Heterocoelia et masculis Parnopibus) e tribus segmentis dorsalibus esse compositum, ego in specierum descriptionibus ab hac opinione recedere nolui, sed ultimam vel tertiam dorsi partem, segmentum abdominis dorsale tertium a me nominatam, in duas sectiones distinxi; sectio prima semicircularis i. e. area basalis sive antica maximam segmenti partem complectitur, sectio altera i. e. area analis, area postica sive margo apicalis pone primam in extremo segmento sita est; cf. fig. $A$. et $B \cdot^{*}$ )

A.

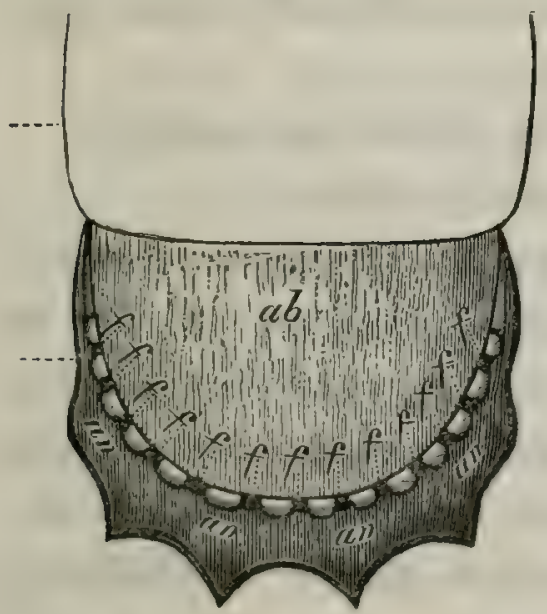

B.

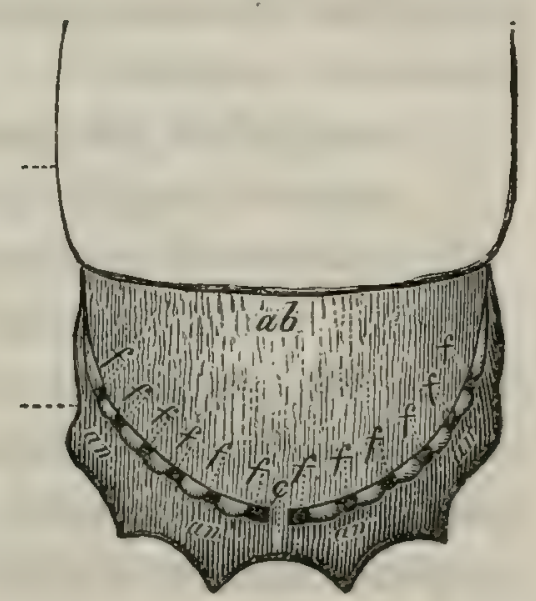

Quicunque autem hanc animalculi partem accuratius indagaverit, is facile viro clarissimo Spinolae as-

-) $2=\Lambda$ bdominis segmentum dorsale $2:$ dum; $3=$ abdominis se. gmentum dorsale 3 :tium; $a b=$ area basalis sive area antica; ffff $=$ foveolae $=$ series foveolarum ante-apicalis in Fig. $A$. continu, in Fig. B. per carinulam nedianam $c$ interrupta; $a n, a n, a n, a n=$ area analis, = area postica sive margo apicalis. 
sentietur, iam inde ab anno 1808 (in Ins. Ligur.) asseveranti, abdominis segmentum dorsale tertium duobus segmentis dorsalibus constare. Quam partium rationem in plerisque speciebus ego quoque inveni; tertium itaque segmentum dorsale pro supradicta area basali, quartum et ultimum pro area anali habenda sunt, et series foveolarum anteapicalis nihil aliud erit nisi margo catenatus duriusculus, ultima duo segmenta dividens. Quam sententiam abunde confirmant thoracis segmenta dorsalia et pectoralia, haud raro ita inter se coniuncta, imo concreta, ut nonnisi adhibitis suturis recte distingui possint.

Impressio basalis normalis segmenti dorsalis primi non solum a musculo erectore laterali, sed saepius ab aequalibus metanoti protuberantiis efficitur. Quae protuberantiae constant

1) marginibus vel asserculis rectis aut curvatis,

2) metanoti angulis postico-lateralibus,

3) postscutelli continuatione dentata aut aculeata.

Hanc continuationem rarius, margines autem et angulos semper inveni. Secundum protuberantias plus vel minus elevatas constitutae sunt impressiones, quas igitur eo tempore factas fuisse liquet, quo animalculi, nymphae statum adepti, omnes partes mollissimae erant.

Primi et secundi segmenti dorsalis margines laterales, nomnunquam ad perpendiculum decurvati, in utroque latere singularem efficiunt aream, tenui plus minusve elevato margine circumdatam, quam aream lateralem intramarginalem appellavi. Cuius areae sculptura et forma ad distingueudas arcte cognatas spe- 
cies nonnunquam usui mihi fuit. Conferantur delineationes specierum no. 186. 187. 185. 190. 191.

Corporis longitudinem in parte postica inde a verticis summo margine usque ad extremum ultimi segmenti dorsalis marginem analem commensuravi.

Ad minuendam in speciebus dignoscendis et comparandis molestiam, tabulas synopticas nonnullas composui, quas ad insectorum studium haud parvo auxilio fore spero.

Fabricianorum typorum accurata cognitio cum cuivis entomologo accepta esse debeat, triplicem composui catalogum

1) Fabricianorum typorum musei regii Havniensis,

2) eorundem musei Kieloniensis, et

3) Fabriciani systematis Piezatorum, quoad Chrysidem Linnaei continet, revisionem.

Ad illustrandas Chrysidarum sculpturam et armaturam maximi erat momenti, ut descriptionibus meis accuratas adderem delineationes. Specierum insigniores formas artifex egregius Milde depinxit, singulari regis nostri clementissimi munere adiutus. Nonnullarum partium adumbrationes, calamo scriptorio rudiuscule quidem, maxima tamen diligentia a me factas, typis ligneis expressas, suis locis textui insertas reperies.

Mirabuntur fortasse qui insectorum formas multis generibus distinguere consueverunt, me divites Hedychridarum et Chrysididarum familias tam paucis, illam tribus, hane uno genere circumscripsisse, species autem tot sectionibus et subsectionibus divisisse. Pluribus generibus adhibitis, dicent illi, sectionum atque 
phalangum paene inextricabilis multiturlo minuenda erat. Quibus respondisse sufficiet, distinctionum naturam et indolem ante omnia esse perpendendas; dummodo constantibus, certis, facilibus signis utamur, nec molesta nec inextricabilis erit divisio; quid quod indagatoris laborem minuet; si logicis, quae vocant, argumentis nititur systema, tantum abest ut in errorem immergat, ut potius recta via ad scopum ducat investigatorem. Quamnam confusionem nimia generum creandorum cupido pepererit, experientia docet. Nobis autem semper visum est, non alias in libro quam in natura adhibendas esse distinctiones. Quas si natura ipsa leves fecerit, in transitionibus autem plane deleverit, quonam iure eas in generibus constituendis adhibeamus? Vel rei entomologicae peritior - ut de tironibus taceam - nonnisi tentando synonymias nova ista genera discernere poterit.

Attamen ingenue confiteor, species nonnullas, v. c. Chrysidem lynceam et Chrysidem Gueudei (no. 191. 192), capitis et thoracis forma a reliquis diversas, novo generi adscribi potuisse, quod monente viro clarissimo Klug Pyriae nomine nuncupare licuisset.

Limnaeana, Fabriciana omniaque antiqua generum. nomina, prout in familiarum nomina abierint, delenda esse rati auctores nomulli, in scriptis suis hanc regulam secuti sunt. Tunc igitur nullum habebimus Scarabaeum, nullum Curculionem, mullam Blattam neque Cicadam; nova invenienda sunt nomina, antiquis generibus intrudendae Scarabaeidum, Curculionidum et id genus appellationes. Cui mutationi, a perpancis scriptoribus tentatae, assentiri non possum, quippe 
quam rei entomologicae ullam allaturam esse utilitatem nego. Antiqua genera ubi necessarium in plures sectiones dividere, ibi formam centralem, vetere nomine insignitam, quasi typum principalem, stabiliendam censeo.

Itaque in Chrysididis disponendis Chrysidis nomen ei indidi familiae, quae maximum specierum numerum complectitur, cuius in omnibus terrae regionibus formae quas vocant repraesentantes occurrunt, in quam denique reliquarum familiarum formae conveniunt. Hoc igitur Chrysidis genus quasi centrum omnium Chrysididarum Hymenopterorum habeo. Ab-

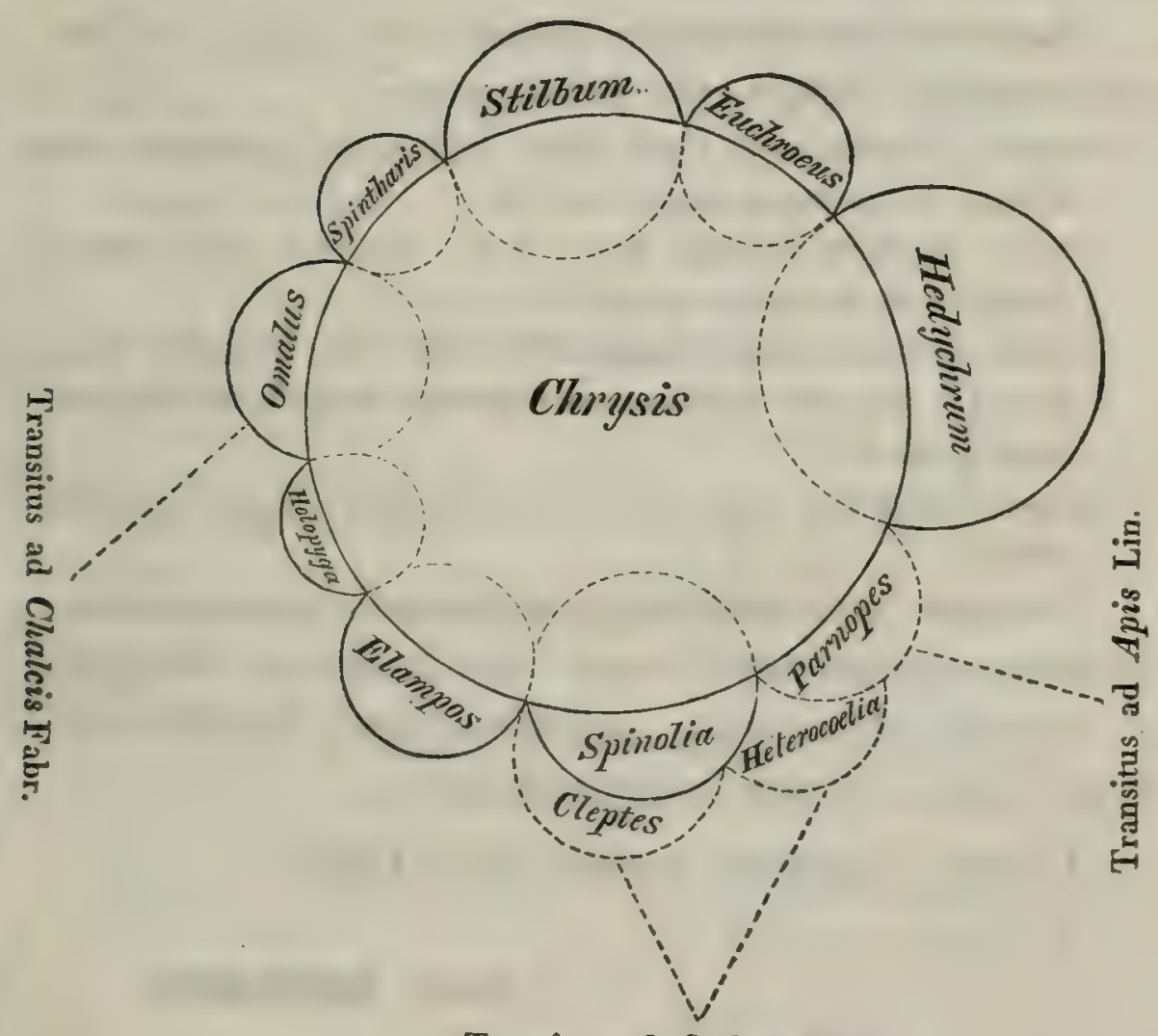

Transitus ad Codrus Fabr. 
solutissimam autem Chrysidarum formam non in hoc genere, sed in eo, quod Stilbum nuncupatur, quaerendam esse putaverim. Cuius generis species, quasi eodem modulo a natura formatae, tam arcte inter se cognatae sunt, ut nonnisi minutiis quibusdam differant.

Genus Spintharis, quod ante genus Chrysis ponendum erat, minus recte post hoc genus a me collocatum esse hic monendum censeo. Spintharis enim, quae transitionem facit ab Euchroeidibus ad Hedychridas, in systemate naturali aptissimum locum occupat inter Omalum, Hedychrum et Chrysidem, non autem inter arctissime coniuncta genera Chrysidem et Stilbum.

Superest ut sequentia nomina synonyma, in descriptionibus omissa, hic subiungamus:

Chrysis bidentata De Geer Mem. 2.2.837:2 nostrum esse videtur Hedychrum anale no. 40.

Chrysis nitidula Ahrens Faun. 4:12 nostrum esse videtur Hedychrum fervidum no. 53.

Chrysis maculata Fabr. Suppl. 258:12-13; Coqueb. Icon. Tab. 14. Fig. 10 nostrum esse videtur Hedychrum Zimmermanni, no. 32.

Chrysis integrella nob. Spec. no. 73 lege Chrysis neglecta Shuck.

Denique opus nostrum, pulcherrimorum insectorum familiam complectens, omni qua potuimus diligentia et sollertia elaboratum, entomologorum benivolentiae iterum atque iterum commendamus.

Lundae Gothorum mense Maio 1852.

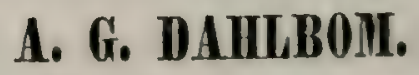


II. Catalogus Hymenopterorum Chrysidiformium, Collectionum de Seluestedti et Tönder-Lundi (in Museo Regio Havniensi asservatorum), pro Fabricii "Systemate Piezatorum" Typicorum.

1. "Diplolepis Chrysis e Porto Farino

-Vahlu . . . . . . . . . = Cleptes ignita nob. $\sigma^{\text {. }}$

2. "Cleptes semiaurata" . . . . = id. nob. $\sigma$.

3. "Cleptes ignita e Tunis Vall" . = id. nob. ㅇ.

4. "Chrysis splendida" . . . . . = Stilbum id. nob.

5. "Chrysis festiva e Grinea" . . . = Euchroeus id. nob.

6. "Chrysis calens ex Italia" . . . . = Stilbum id. nob.

7. "Clurysis scutellaris" . . . . = id. nob.

8. "Chrysis oculata" . . . . . . = id. nob.

9. "Chrysis lusca. Schlanbusclu . . = id, nob.

10. "Chrysis Iyncea e Guinea Thonning $=\mathrm{id}$. nob.

11. "Clerysis fulgida ex Austria . . . = id, nob.

12. "Chrysis purpurea" . . . . . = Euchroeus id. nob.

13. "Chrysis caerulipes e Tunis Vahl."

Forma aliena, non typica . . . . = Chrysis Vahli nob.

14. "Chrysis ignita". . . . . . = id. nob.

15. "Chrysis bidentata e Zawan Vahl";

forma aliena, non typica . . . . = Chrysis erythromelas nob.

16. "Chrysis integra". 2 Specinoina:

unum se Tanger Schousboe» . . = Chrysis Sehestedi nob.

alterum »e Tunis Valul"; forma typica $=$ Chrysis integra nob.

17. "Chrysis gloriosa e Tanger Schous-

boe", forma aliena, non typica . . = Chrysis Schousboei nob.

18 "Chrysis lucidula" . . . . . . . = Hedychrum id. nob. ㅇ.

19. "Chrysis fervida e Tanger Schousboe" .......... . = IIedychrum id. nob. 오. 
20. "Chrysis aurata" . . . = Omalus id. nob.

21. "Chrysis regia German. et Ital." = Hedychrum lucidulum nob. $\delta$.

22. "Clerysis fasciata ex Amer, mer.

Smidt." Forma typica. . . . = id. nob.

23. Chrysis fasciata "var. ex Amer.

mer." forma aliena, non typica . = Chrysis distinctissima nob.

24. "Chrysis cyanea". . . . = id. nob.

25. "Chrysis parvula ex Amer. mer.

Smidt." . . . . . . . = id. nob.

26. "Chrysis smaragdula ex Ind.

Or." Forma aliena, non typica = Chrysis Schiödtei nob.

27. "Chrysis nov, sp. ex Algier Stub." = Chrysis cyanopyga nob.

28 "Chrysis nov. sp. ex Ind. Or." . = Chrysis orientalis nob.

29. "Chrysis nov. sp. ex Amer. me-

rid. Smidt. affinis smaragdulae" $=$ Chrysis Smidti nob.

30. "Parnopes : carnea e Tanger

Schousboer" . . . . . . = id. nob. 
III. Catalogus Hymenopterorum Chrysidiformium, Collectionis Fabricii (Musei Universitatis Kiel.), pro „Systemate Piezatorum" typicorum.

1. "Chrysis splendida" . . . . = Stilbum id. nob.

2. "Chrysis smaragdula"; non typica = Chrysis Schiödtei nob.

3. "Chrysis oculata" . . . . . = id. nob.

4. "Clurysis scutellaris" . . . = id. nob.

5. "Clurysis carnea" . . . . . = Parnopes id. nob.

6. "Chrysis fulgida" . . . . . = Chrysis id. nob.

7. "Clirysis ignita". . . . . . = id. nob.

8. "Chrysis bidentata". . . . . = id. nob.

9. "Chrysis succincto". . . . . = id. nob.

10. "Chrysis lucidula" . . . . . = Hedychrum id. nob. 오.

11. "Chrysis fervida" . . . . . = Hedychrum id. nob.

12. "Chrysis aurata" . . . . = Omalus id.nob.

13. "Chrysis regia" . . . . . = Hedychrum lucidulum nob. $ð$.

14. "Chrysis cyanen" . . . . . = id. nob.

15. "Clerysis amethystina". . . . = id. noh.

16. "Chrysis 6-denlata" . . . = id. nob.

17. "Chrysis refulgens" . . . = Chrysis austriaca nob. ${ }^{\prime}$

18. "Chrysis fasciata". . . . = id. noh.

19. "Chrysis parvula" . . . . . = id. nob.

20. "Chrysis austriaca". . . . . = id. nob.

21. "Chrysis caerulans" . . . . = Chrysis id. nob. var.

22. "Chrysis gloriosa" . . . . . = Holopyga ovata nob. var.

23. "Chrysis pusilla" . . . . = Omalus id. nob.

24. "Clirysis aenea" . . . . . = Omalus id. nob.

25. "Chrysis purpurata ơㅇ․ . = Chrysis nitidula nob.

26. "Clrysis caerulipes" . . . . = id. nob. 
IV. Revisio "Systematis Piezatorum" Chrysidiformium.

Nomina systematica Fabricii: Nomina systematica hodierna:

1. Cleptes semi-aurata. Piez. $\mid=$ Cleptes semiaurata Hym. Europ. 154:1. - Dlbm. Catal. Mus.
Havn. II : 2.

2. Cleptes nitidula Piez. 154:2. = Cleptes nitidula H5m. Eur. 2. Spec. No. 2:

3. Cleptes splendens Piez, 155:3. = Cleptes semiaurata H. E. 2. Sp. No. 3. ठ․

4. Cleptes ignita Piez. $155: 4 .$, )

II : 3. - Diplolepis Chrysis $\}=$ Cleptes ignita H. E.2. Spec. No.4. Fabr. Piez. $150: 5$.

5. Cleptes stigma Piez. 155:5.

6. Cleptes fulgens Piez. 155:6. Neque ad Genus Cleptis, nec ad

7. Cleptes muscarum Piez.156:7. Familiam quidem Cleptidarum, sed

8. Cleptes Iarvarum Piez. 156:8. ad Familiam Chalcididarum Walk.

8. Cleptes larvarum Piez. 156:8. vel Pteromalinorum Daln. perti9. Cleptes coccorum Piez. 156:9. nent.

10. Cleptes minutus Piez. 156:10.

1. Chrysis splendida Piez.170:1. Dlbm. Catal. Mus. Havn. II:4, $\}=$ Stilbum splendidum Hym. Eur. 2. $\left.\begin{array}{l}\text { Dlbm. Catal. Mus. Havn. II :4, } \\ \text { Catal. Mus. Kiel. 111:1. }\end{array}\right\} \begin{aligned} & \text { Stilbum splendidum } 199 . \text { var. } 6 . \\ & \text { S. }\end{aligned}$

2. Chrysis smaragdula Piez.171:2. = Chrysis smaragdula H. E. 2. Sp. No. 190.

3. Chrysis festiva Piez. 171:3. = Euchroeus festivus H. E. 2. Sp. Dlbm. Catal. Mus, Havn. II: 5. No. 209.

4. Chrysis sculcllaris Piez. 171:5. $)=$ Chrysis scutellaris Hym. Europ.

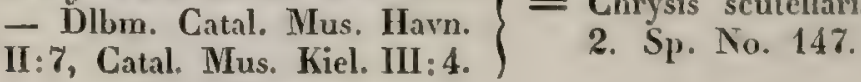




\section{Revisio Systematis Piezatorum Chrysidiforminm. xxı}

Nomina systematica Fabricii: Nomina systematica loodierna:

5. Chrysis oculata Piez, 171:6. $\mid=$ Chrysis oculata H. E. 2. Spec II:8., Catal. Mus. Kiel. III:3. No. 176.

$\left.\begin{array}{l}\text { 6. Chrysis lusca Piez. } 171: 7_{.} \\ \text {Dlbm. Catal. Mus. Havn. II:9. }\end{array}\right\}=$ Chrysis lusca H.E.2. Sp. No.166.

7. Chrysis lyncea Piez. 172:8. = Chrysis lyncea H, E. 2. Sp. No. Dlbm. Catal, Mus. Hav.II: 10. 191.

8. C'hrysis Panzeri Piez, 172:9. = Elampus Panzeri H. E. 2. Spec. No. 21 .

9. Chrysis caerulans Piez. $=$ Chrysis caerulans Hym. Europ. 2. 172:10. Dlbm. Catal. Mus.
Kiel. III: 21.

10. C'hrysis fulgida Piez. 172:11.

Dlbm. Catal. Mus.Havn.II: 11 $=$ Chrysis fulgida H. E. 2.Sp.No.136. et Catal. Mus. Kiel, III : 6 .

11. Chrysis purpurata. Piez. 172:12. Dlbm. Catal. Mus.
Kiel. III: 25.

12. Chr. purpurata Piez, 172:12. = Euchroeus purpuratus II.E.2. Sp. No. 204. Not. ben. exclusis $F$ abricii verbis "ano quadridentato", quae ad Chrysidem nitidulam pertinent.

13. Chr. caerulipes Piez. 173:13. $=$ Chrysis caerulipes H. E. 2. Spec. Dlbm. Catal. Mus. Kiel.III:26. $\quad$ No. 7\%.

14. Clurysis ignita Piez.173:14.)

Dlbm. Catal. Mus. Havn. II:14 = Chrysis ignita H. E. 2. Sp.No.165. et Catal. Mus. Kiel. III : 7.

15. Chrysis austriaca Piez. I= Chrysis austriaca Hym. Eur.2. 173:15. Dllom. Catal. Mus.
Kiel. III: 17 et 20.

16. Chrysis bidentata Piez. $\mid=$ Chrysis bidentata H. E. 2. Spec. Kiel. III : 8.

17. Chrysis integra Piez.174:17.

Dlbm. Catal. Mus. Hav. If:16. $=$ Chrysis integra H. E. 2. Sp. No.86. exemplar "e "Tunis Vall".

18. Chrysis maculataPiez.174:18 =? Hedychrum Zimmermanni H. E. 2. Sp. No. 32.

19. Chr. succincta Piez. 174:19. $=$ Chrysis succincta II. E. 2. Sp. Dlbm. Catal. Mus, Kicl. III:9. No. 149. 


\section{xxıI Revisio Systematis Piezatorum Chrysidiformium.}

Nomina systematica Fabricii: Nomina systematica hodierna:

20. Chr. gloriosa Piez. 174:20. $=$ Holopyga ovata H. E. 2. Spec. Dlbm. Catal.Mus. Kiel.III:22. No. 28. var. $i$.

21. Chr. luciduln Piez. 174:21. $)=$ Hedychrum lucidulum H.E.2. Sp. $\left.\begin{array}{l}\text { Dlbm. Catal.Mus. Havn. II : 18 } \\ \text { et Catal. Mus. Kiel. III : 10. }\end{array}\right\}=$ No. 45. ․․

22. Chr, dimidiata Piez, 174:22 = Chr, bidentata H.E.2.Sp. No.142.

23. Chrysis fervida Piez. 175:23. $)=$ Hedychrum fervidum H. E. 2. $S_{\mathrm{P}}$. Dlbm. Catal. Mus Havn.1I:19 $\}$ No. 53. et Catal. Mus. Kiel. III : 11.

24. Chrysis aenea Piez. 175:24. $=$ Omalus aeneus H. E. 2. Sp. Nío. Dlbm. Catal. Mus. Kiel. III:24. 13. var. $a$.

25. Chrysis aurata Piez.175:25. $\}=$ Omalus auratus H. E. 2. Sp. No.8. $\left.\begin{array}{l}\text { Dlbm. Catal. Mus. Havn. II:20 } \\ \text { et Catal. Mus. Kiel. III:12. }\end{array}\right\} \begin{aligned} & \text { Omalu. } \\ & \text { var. } \boldsymbol{a} \text {. }\end{aligned}$

26. Chrysis regia Piez. 175:26.) = Hedychrum lucidulum Hym. Eu-

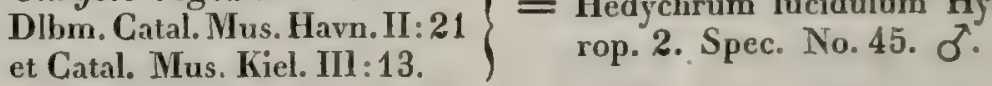

27. Chr. fasciata Piez. $175: 27$.)

Dlbm. Catal. Mus. Havn.II:22 $\}=$ Chr. fasciata H. E. 2. Sp. No.106. et Catal. Mus. Kiel. III : 18.

28. Chr. sexdentata Piez.175:28. $=$ Chrysis sexdentata H. E. 2. Sp. Dlbm. Catal. Mus. Kiel. III : 16.) No. 183.

29. Chrysis cyanea Piez.176:29. Dlbm. Catal. Mus. Havn.II:24 = Chr. cyanea H. E. 2. Sp. No. 102. et Catal. Mus. Kiel. III : 24.

30. Clir. parvula Piez. 176:30.

Dlbm. Catal. Mus. Havn. II:25 = Chr. parvula H. E. 2.Sp. No.103. et Catal. Mus. Kiel. III : 19.

31. Chrysis nitidula Piez. 176:31 = Chr. nitidula H. E. 2. Sp. No.117.

32. Chrysis amethystina Piez. $\mid=$ Chrysis amethystina H. E. 2. Sp. 176:31. Dlbm. Catal. Mus.
Kiel. 11 : 15.

33. Chrysis pusilla Piez, 176:33.) = Omalus pusillus H.E.2. Sp. No.10.

$\left.\begin{array}{l}\text { 1. Parnopes carnea Piez 177:1. } \\ \text { Dlbm. Catal. Nus. Havn. II:30 }\end{array}\right\}=$ Parnopes carnea H. L. 2. Spec.

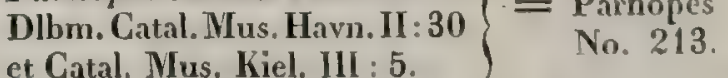


V. Characteres Familiarum et Generum, atque

Adumbrationes sub-monographicae

\section{Specierum}

Hymenopterorum Chrysidiformium nobis cognitarum. 
Species "ipsas non vero numerum earum auctum attendendum judicavi “.

Linné Flor. Srec. Praefat. 


\section{I R Y I I S}

in sensu Linnaeano

comprehendit:

Insecta Hymenoptera, a nobis Chrysidiformia appellata, magnitudine mediocria, parva aut minuta, coJoribus metallice splendidis elegantissime ornata, corpore semicylindrico, adtacto 1 . animalculo perterrito in globulum contractili vel convolubili; antennis geniculatis 13 -articulatis, ad orem insertis; oculis integerrimis; metanoto utrinque angulo postico-laterali l. dentiformi I. mucroniformi; pedibus cursoriis mediocribus; alis membranaceis 4 planis: anticis paupere venosis et cellulosis, cellulis completis tantum 3 vel ad maximum 4, - posticis pauperrime venosis et omnino absque cellulis; abdomine adhaerente: ano aut mutico, aut dentato, aut serrato, feminae plerumque tubulo retractili stylifero munito.

II. 


\section{CARACTERES NORMALES.}

\section{Hymenopterorum Chrysidiformium.}

Corpus cataphractum mediae, minoris aut minutae magnitudinis, ovato-oblongum, ovatum aut ovato-rotundum, glabriusculum aut pubescens, impresso-punctatum aut punctulatum; quoad convexitatem aliis semicylindricum, aliis subhemisphaericum, in Cleptidis subcylindricum.

Caput thorace latius aut ejusdem latitudine, superne transversum, totum rotundato-subcubico-triangulare; cavitas oris mediocris.

Mentum corneum semicylindricum, ad basin plerumque angustius quam ad apicem.

Ligula plurimis membranacea parra conica: tantum in Parnope magis dura longissima tenuis subfiliformis apiceque bifida.

Maxillae corneae, plurimis mediocres processubus duobus: interiori brevi acuto, exteriori majori rotundato-obtuso ciliato; in Parnope sunt maxillarum processus longissimi tenues acuminati, secundum longitudinem plicati, unacum ligula rostrum 1 , ut in Apibus promuscidem constituentes.

Palpi graciles: plurium maxillares 5 -articulati, labialibus $2-3$-articulatis longiores; in Parnope sunt maxil- 
lares et labiales biarticulati brevissimi, instar setularum brevium rigidarum obsoletissimi.

Mandibulae magnitudine et crassitie rariantes, plerumque trigonae, subtus nonnihil excavatae, apice inciso 1 2-3 - crenatae 1. denticulatae.

Labrum parvum: aliis subsemilunatum, aliis lineare et obtusum, plerumque absconditum, margine apicali plus minus cilintum.

Clypeus transversus: 1. brevissimus, 1. brevis 1. raro mediocris, disco plerumque longitudinaliter convexus et utrinque immersus.

Antennae geniculatae, filiformes, fere ad oris aperturam h. e. in basi l. juxta basin clypei insertae, approximatae, in vivis eximic vibratiles, in mortuis nonnihil curvae 1. plus minus involutae, articulis 13 compositae: scapo maximo conico, pedicello et articulo terminali plerumque brevissimis.

Oculi laterales integerrimi ovati plus minus exserti.

Facies inter orbitae et clypei basin pro scapis quiescentibus impressa I. immersa et ibidem subtilissime transverse strigosa; quae ampla impressio plerunque cavitas facialis nominatur.

Frons et Vertex depresso-convesa l. convexo-depressa.

Stemmata I. triangulariter I. arcuatim in vertice disposita. Margo occipitalis prothoracem versus aliis muticus, aliis angulatus, aliis subspinoideo productus.

T'horax subcylindricus, plus minus convexus, antice 1. arcuato-obtusus 1. truncatus, postice truncatus.

Dorsulum suturis longitudinalibus, quarum medianis 2 plerumque distinctioribus. 
Scutellum et Postscutellum aliis mutica, convexa, aliis conica, aliis mucronata.

Metanotum inaequale rugulosum, reticulatum aut clathratum, postice utrinque angulo laterali l. obliquo 1 . subhorizontali dentiformi 1. mucroniformi plus minus prominente armatum.

Pectus coriaceum; mesopleura utrinque in angulum l. lobun caracteristicum arcuatum aut triangularem efformata.

Pedes cursorii mediocres, pilosi et pubescentes; trochanteres inaequaliter conici; condyli subcylindrici; femora compresso-fusiformia; tibiae conico-cylindricae, aliis muticae, aliis rigide setulosae, aliis spinulosae; tibiarum anticarum singula calcare unico, tibiarum posteriorum singula calcaribus duobus armatae. Tarsorum articuli subfiliformes.

Unguiculi tarsorum valde caracteristici, infra aut mutici, aut unidenticulati, aut serrulati 1. pectinulati.*)

Alae quoad longitudinem et latitudinem mediocres, at venarum et cellularum pauperes: anticae plerumque solum 3 praebent cellulas completas scil. costalem, medialem et primam submedialem; cellula radialis quidem adest, at apice saepissime aperta nec nisi rarius rel rarissime ibidem clausa. Cellularum cubitalium et discoidalium tantummodo vestigia obsoleta 1 . obsoletissima per venulas rudimentarias vel $s$. $d$. coecas inchoata sub lente observantur. Venae in ala antica sunt: costalis, postcostalis, medialis, analis, venula transverso-medialis, venula

*) Notam maxime caracteristicam ab unguiculorum armatura sumtam debemus Cel. Wesmac̈l, qui eandem primus exploravit et adhibuit. 
transverso-submedialis, stigma el vena radialis, - omnes distinctae; rudimentariae sunt vena cubitalis et vena discoidalis.

In ala postica tantum unica adest cellula scil. coslalis angusta et obsoleta; venae ejusdem alae sunt: costalis, postcostalis, medialis, analis nec non setulae rigidae recurvae regionis stigmaticalis.

Abdomen thoraci adhaerens I. ovale 1. ovatum, I. rotundato - subquadratum, 1. subhemisphacricum, supra convexum; subtus planum et marginatum, post mortem propter viscera contracta et exsiccata concavum et subfornicatum; in Cleptidis et supra et subtus convexum; segmenta cataphracta conspicua 3-4-5: segmentum dorsale ultimum plurimis margine apicali valde caracteristicum scil. ibidem 1. integerrimum 1 . in centro excisum I. emarginato-dentatum 1. serratum aut serrulatum.

Differentia sexus: Mas plerumque brevior et angustior, ano mutico non tubulifero; segmenta abdominalia marium Cleptidarum et Parnopidarum etian plura quam feminarum earundem familiarum. - Femina plerumque major et robustior, rarius pictura diversa, ano munito tubulo stylifero annulato retractili et usque ad l. dimidiam I. tolam I. ultra corporis longitudinem extensili.

Habitatio. In collibus, fossis, muris locisque argillosis, sabulosis et arenosis nec non in parietibus, saepibus ligneis et caespiticiis truncisque arborum emortuarum vermisecalis soli expositis occurrunt et nidulantur Hymenoptera Chrysidiformia; plantarum praecipue umbelliferarum flores et folia in sylvis, lucis et nemoribus, pratis campis et hortis quoque frequentant. Adtactae vel terrefactac corpus in globulum ita convolvunt, at caput el 
pedes inter mesopleurarum lobos et ventris fornicem fere tota absconduntur; periculo autem praeterito celerrime aufugiunt. In copula rarissime deprehenduntur; cujus rei exempla tantummodo de Clepte semiaurata, Hedyclio lucidulo, Holopyga ovala et Chryside ignita afferre possum.

Metamorphosis completa, annua. Ova deponunt feminae pallida elliptica in nidis*) Hymenopterorum Spheciformium, Vespiformium vel Apiformium in quibus larvulae pseudoparasitae *) nutriuntur Aphidibus aut aliis Insectis larvisve mollioribus paralysatis, quae nidi mater genuina pro suis pullulis nutriendis diligenter reportavit.

Larvulae Hymenopterorum Chrysidiformium apodae subvermiformes.

Pupae aut nudae aut folliculatae.

Propter structuram trophorum, abdominis segmenti dorsalis ultimi nec non unguiculorum tarsorum diversam, haec Insecta in 6 familias subnaturales commode disponi possunt.

*) Exceptionem ab hac regula naturae praebent feminac Cleptis semiauratae et Cleptis nitidulae, que ova deponunt in larvis Nemati Grossulariae, intra quorum corpora larvulae Cleptium vitan vere parasiticam ferunt. Cl. Bouché anno 1847 Berolini milii narravit se accepisse Omalum auratum e larva Nemati Grossulariae exclusum. 
Tab. Syn. Famil. ex Ord. Hymenopt. Chrysidiform. 7

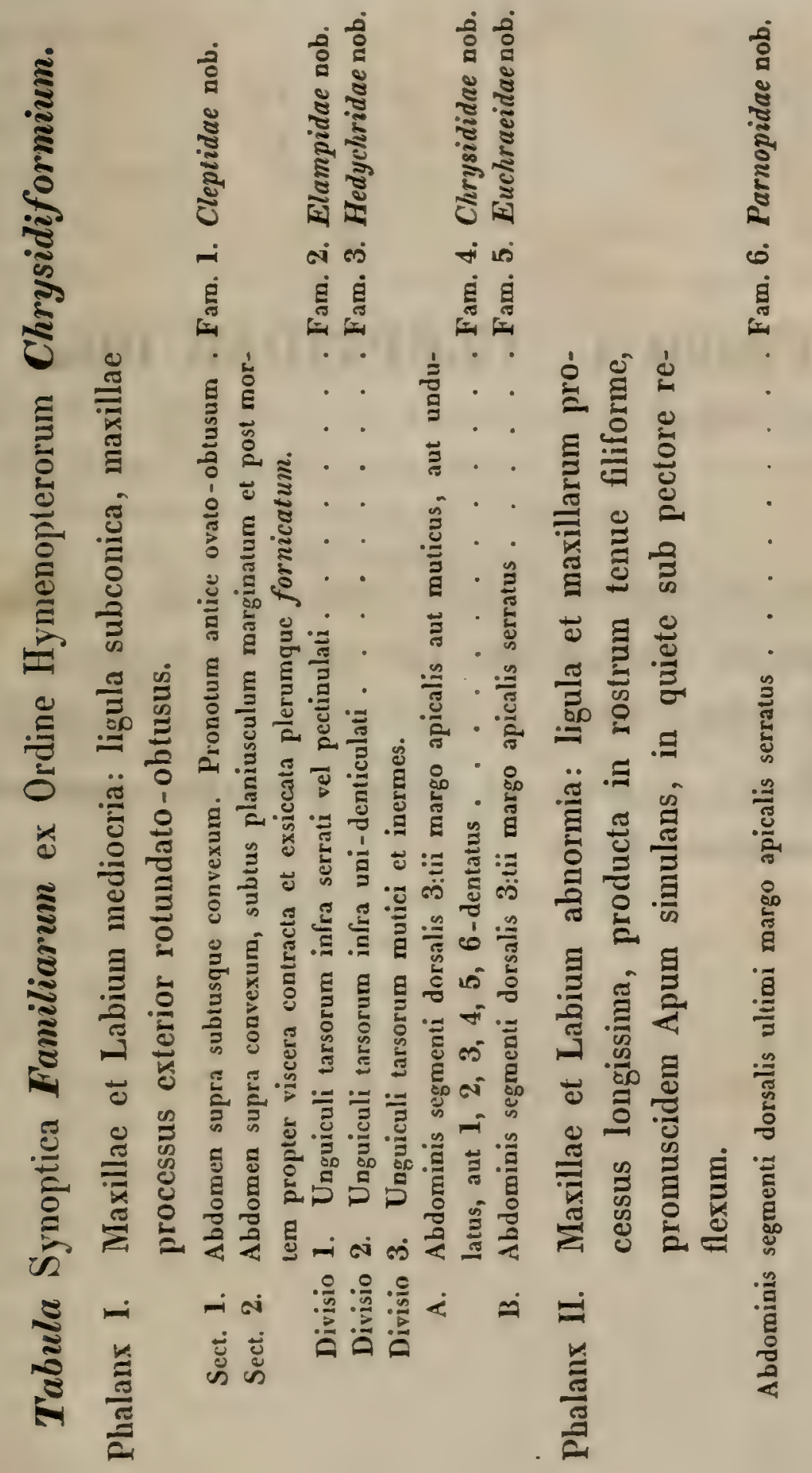




\section{Familia 1. CLEPTIDAE Dlbm.}

Pronotum angustum, antice ovato-l. arcuato-obtusum. Unguiculi tarsorum infra sub medio unidenticulati. Alae anticae cellula medialis apice oblique truncata. $\Lambda$ bdomen et supra et subtus convexum, segmentis conspicuis $4-5$. 


\section{T'ab. Synoptica Generum Faniliae Cleplidarum.}

\section{Tabula Synoptica Generum Familiae Cleptidarum.}

A. Abdominis segmenta invicem saagnitudine subaequalia scil, unum altero parumper majus; segmenti dorsalis ultimi margo apicalis muticus (non dentatus) . . . Genus 1. Cleples Auclorum.

B. Abdominis segmentum 2:dum maximum, I:num nediocre, reliqua abbreviata; segmenti dorsalis ultimi margo apicalis dentalus : . Genus 2. Heterocoelia nob. 


\section{Genus CLEPTES Latr.}

Gen $\mathrm{Cr}$. et Insect. Tom. 4., pag. 44, Gen. 473, et Auctorum recentiorum plurinorum.

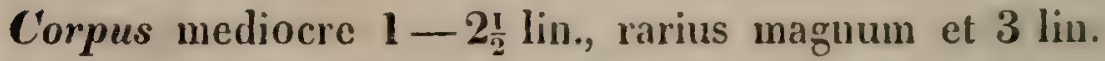
decimal. longun, ellipticum, proportionaliter angustum, raro crassum.

Caput transversum orbiculatum collo thoracis latius; frons convexa, raro depressa, nunquam (ut in Chryside pro scapis quiescentibus) foveata, tantum sulculo mediano tenuiter impressa. Ligula subrotundata integra. Maxillae processus externus et terminalis membranaceus late obtrigonus, ad apicem latior veluti truncatus aut obtusissimus; processus internus parvus dentiformis. Mandibulae breves robustae latae trigonae, subtus carae, ab origine ad mediun incrassatae, apice compressiusculae tridenticulatae: denticulo interno minori, apicalibus duobus invicem subaequalibus. Antennae longiusculae subrobustae filiformes aut subsetaceae, ad orem in clypeo insertae.

Theracis pronotum 1. transversale 1. sublongitudinale, colliforme, capite paullulum angustius, antice arcuato-obtusum. Dorsulum pronoto brevius, transversum, obtuse parallelogrammicum, convexo-depressum suturis longitudinalibus plerumque 4 impressum. Scutellum pronoto paullo brevius, disco convexo-depresso plano obtuse subrectangulo. Metanotum transversum subcubicum, fere ut in Ampulice*) constructum, supra planum et varie scul-

*) Cfr. Hym. Europ. 'Tom. 1, 'Tab. Exam. Synopt, No. 3 . 
ptis. pag. 10-11.

Sect. lidum ob-

dominis dor-

ulae laterales

mple - fu-

Sect. ic modice

$-2 \frac{1}{2} \operatorname{lin}$.

scum 1. cya-

a) Lanea, pectus,
postico laeve
2. Cleptes nitidula Fbr. Europa.

b) Stus et meta-

1. Cleptes fasciata Dlm. Brasilia. ipice fumeo-

Óae, disco et

ठ’ - . . 3. Cleptes semiaurata Fbr, Europa.

Sa dorsulum

; pedes basi irsale 3:tium macula objiceo - . 4. Cleptes ignita Fabr. Q Europa. Africa.

ditrinque maeurs. Pedes

eurs. Pedes

5. Cleptes ignita nob. $\sigma^{\top}(=$ Diplolepis Chrysis Fabr.)

punctulorum

a! . - 6. Cleptes aurata nob. Turcia.

b.-aurata. . 7. Cleptes orientalis nob. Turcia. 


\section{Tabula Synoptica Specierum Generis Cleptis. pag. 10-11.}

Sect. I. Metanoti anguli postico-laterales maximi, in mucronem validum obtusum procedentes.

Corpus magnum robustum $3 \frac{1}{2}$ lin. decimal. long. Caput, metanotum et abdominis dorsum piceo-nigra. Antennarum annulus, thorax, pedes, abdominis basis et maculae laterales testacea. Alae hyalinae: anticae basi obsolete - disco distinctissime et ample - fu-

Sect. II. Metanoti anguli postico-laterales mediocres aut parvi, hinc modice aut leviter prominuli. Corpus mediocre aut subparvum $1 \frac{1}{2}-2 \frac{1}{2} \mathrm{lin}$. decimal. long.

Divis. 1. Abdomen lacte brunneum 1, subcastaneum: apice 1. piceo-fuseum I. cyanescens 1. rarius aureo-maculatum.

A. Abdomen non aureo-maculatum

a) 우 Caput et dorsulum nigro- aut cuprco-aenea, prothorax et pedes fulva 1. subcastanea, pectus, scutellum et metathorax cyanea. Alae sordidae, non fasciatae. Pronotum margine postico laeve

b) 우 Caput, prothorax et mesothorax cupreo- ct aureo-viridia 1. cupren-aurata; pectus et metathorax viridi-cyanea. Pedes testacei aut ferruginei. Alae hyalinac: disco et spice fumeofasciatae.

$\delta$ Caput, thorax et basis pedurn viridi-cyanea, tibiae tarsique testacea, Mlae hyalinae, disco et apice sordidae nec rite fasciatae.

$\delta$ 오 Pronotum intra marginem posticum serie punctorem 1. punctulorum transversa

B. Abdomen aureo-maculatum

우 Pronotum margine postico laeve. Caput et prothorax viridi-aut cupreo-aurata; dorsulum cupreo-aeneum, postscutelli tuberculum interdum concolor; metathorax cyancus; pedes basi picei, apice testaceo-fusci; alae sordidae, non fasciatae. Abdominis segmentum dorsale 3;tium macula I. trapeziformi 1. triangulari amplissima nigro-picea, I. utrinque I. apice macula obsolela viridi- aut cupreo-aurea; segmentum 4:tum totum aureum ano summo piceo...

$\sigma^{7}$ Caput et thorax viridi-cyanea; abdominis segrocolum dorsale 3 :tium nigro-aeneum utrinque macula viridi-aurea, segrnentum 4 :tum cupreo-auratum, 5:tum vel anale caeruleum. Pedes basi viridi-cyanei tibiis fuscis, tarsis ferrugineis . . . . . .

Divis. 2. Abdomen tolum aureum. Pronotum margine postico serie punctulorum transversa.

a) Corpus parvum. Caput, thorax, pedes antennaeque viridi-cyanea

2. Cleptes nitidula Fbr. Europa.

1. Cleptes fasciala Dlm. Brasilia.

3. Cleptes semiaurata Fbr, Europa

4. Cleptes ignitr Fabr. Q Europa. Africa.

5. Cleptes ignita nob. $\delta$ ( = Diplalepis Chrysis Fabr.)

b) Corpus majusculum crassum, Caput, pronotum et mesonotum viridi- et cupreo-aurata. .

6. Cleptes aurata nob. Turcia.

7. Cleptes orientalis nob. Turcia. 
ptum, utrinque angulo postico-laterali supero prominulo interdum mucroniformi, postice truncatum.

Alae mediocres, plurimis apicem abdominis vix attingentes; anticae cellulis distinctis 4: costali, mediali et 1:mo submediali clausis adeoque completis, radiali lanceolalo - ovata apice 1 . aperta 1 . clausa; renis costali, postcostali, mediali, anali, stigmate et radiali firmibus, reliquis modo rudimentariis; vestigium cellulae submedialis 2:dae, discoidalis et quidem cubitalis sub lente observatur, hae autem cellulae nunquan explicatac semper rudimentariae 1. tantummodo inchoatae, unde notis Generis caracteristicis non adscribi possunt. Ala postica cellulis - praeter costalem praesentem - omnino caret, nec venas nisi 3 babet distinctas: costalem continuam, postcostalem obsoletam et analem basi firmiorem; reliquae venae, si occurrant, semper pallidae l. s. d. caecae sunt.

$\boldsymbol{P}$ edes pilosi; unguiculi tarsorum infra, inter apicem et centrum, unidenticulati.

Species Cleptis Generis 6 cognoscimus: 5 Europaeas et 1 Americanam. 
1. Cleptes fasciata Dalınan 오.

IIaxima $3 \frac{1}{2}$ lin. decimal. longa, robusta, cinereo-hirla, testacea amnulo antennarum abdominisque basi et maculis lateralibus concoloribus; capile, metanoto clathrato abdominisque dorso nigris; alis subhyalinis, anticis fumeo-bifasciatis.

Cleples fasciata Dalman Mus. Holmiens, Dlbm. Dispos. 1845. 1. 2.

Habitat. in Brasilia; specimina duo vidi, unum in Musco Holniensi asservatum a D. Schönherr donatum, alterum in Museo Berolinensi 1847.

Observ. Species sui Generis gigantea. Corpus sparse cinereo - et griseo-hirtum, quae hirsulies etiau in pedibus et in ala antica abundanter obvenit. Caput nigro-aeneum punctato-rugulosum; vertex et frons depressa, parumper immersa. Oculi magni prominuli. Antennarum scapus validus piceus uno latere testaceus, margine apicali infra prominulo; pedicellus et articulus proxime sequens testacei uno alterove latere infuscati; reliqua pars antennae nigra. Mandibulae ante apicem rufo-piceae. Palpi fusci. Thorax et pedes rufo-testacei. Pronotum et dorsulum crasse et subcrebre punctata, superficie subscabriuscula 1. subasperula, scutellum nitidum sparse punctatum. Metathorax et pleurarum vitta nigro-picea; postscutellum tubere discoidali transverso compressiusculo rectangulo abtusiusculo; metanotum irregulariter cubicum, supra clathratum: lineis elevatis transversis, obliquis et longitudinalibus invicem concurrentibus, areas nitidas angulatas majores et minores includentibus, postice in truncatura carinulis discoidalibus 3 aequaliter remolis, interstitiis oblique striatis; anguli postico-laterales maximi 


\section{Cleptes.}

mucronoidei inaequales prismatico-conici obtusi. Alae subhyalinae, colore testaceo non nisi parumper tinctae venis firmibus fuscis, stigmate crasso lanceolato - elliptico; vena radiali ab origine in medium crassa et dislinctissima, tum abrupte desinente et tantummodo vestigium tenue et obsoletum suae praesentiae offerente; fasciae fumeae 2 in ala antica adsunt: una infra basin alae perobseleta, - altera a stigmate in marginem posticum ducta lata distincla saturata; antice in hac fascia currit linea hyalina lenissime arcuata (a stigmate in discum), vestigium et riam renae cubitalis non explicatae indicans. Abdomen politum nitidissimum segmentis 1:mo et 2:do impunctatis, 3:tio et 4:to sparse punctatis; segmentum 1:mum pallide testaceum margine apicali nigro-piceo; 2:dum et 3:tium nigro-picea, basi utrinque macula laterali magna testacea; 4:tum totum nigro-piceum; tubulus analis robustus testaceus; venter parte basali testaceus, parte apicali nigro-piceus. Pedes lutei aut rufo-testacei, tarsis apice fuscis.

2. Cleptes nitidula Fabr. 오.

Parva $1 \frac{1}{2}-2$ lin. decimal. long. prothorace, abdumine, pedibusque ex parte, castaneis; capite dorsuloque nigro-aeneis, thorace postico viridi-cyaneo, ano nigropiceo, alis sordidis.

Ichncimon nitidulus Fabr. E. S. 2. 184. 211; - Coquebert Icon. 'Tab. IV. fig. 5.

Cleptes nitidula Fabr. Piez. 154. 2; - Panz. Fn. fascic. 106. Tab. 11; - Dlbm. Exercit. 11ym. 25. 2; - V'esmä̈l Note 3. 2; - Dlbm. Dispos. 1. 2.

Mabitat in plurimis Europac regionibus at rarius et solitarie; in borealibus plagis, ubi non ultra $60^{\circ}$ latilu- 
dinis boreal. transgreditur, inter species rarissinas habetur; in plantarum foliis et floribus obrenit. Ex Italia et Gallia D. Spinola, e montibus Austriae subalpinis D. Kollìr, e Silesia D. Dohrn et e Brandenburgia D. Bouché mihi specimina benerole communicarunt. In Belgia a D. Wesmaël et in Anglia a D. Westwood lecta. Intra Sveciam ad Hóberg Westro-Gothiae D. Gyllenhal et ad Kiviks-Esperỏd Scaniae D. D. Fallén et Zetterstedt hanc speciem in Umbellatarum floribus rarissime observarunt; individuum in terra ambulantem d. 7. Sept. 1838 in urbe Lund Scaniae in ricinitate Ribis Grossulariae ipse deprehendi. Comes Le Pelletier de SaintFargeau de hac specie ita scripsit: „j'ai vu le Cleptes nitidula allonger beaucoup son tuyau auprès d'une larre de Tenthrède, et le pousser vivement contre elle. Quoiqu'il lui eût fallu pour cela recourber son abdomen et diriger ce tuyau entre ses pattes en avant de la tète, l'opération entière fut l'affaire d'une seconde"; Referente Cel. Westwood in opere classico, Modern Classification of Insects" pag. 179.

Observ. Femina. Non solum colore et magnitudine minori sed esiam structura partium a Cl. semiaurata $q$ facile dignoscitur. Caput mediocre, at minus quam in Cl. semiaurata 오 et totum convexum nigro-acneum nitidum sparse punctulatum sulculo mediano distincta. Antennae fuscae, angustiores quam in $\mathbf{C l}$. semiaurata ${ }^{\circ}$, flagellum basi apiceque plus minus rubro- 1 . fusco-testaceum, scapus interdum ejusdem picturae. Pronotum fulvum aut laete brunncum, sparse punclulatum, nonnihil angustius et convexius quam in Clepte semiaurata. Dorsulum nigro-aeneum et scutellum cyaneum parcius punctata. Me- 
tathorax viridi-cyaneus, postscutellum disco planiusculum; metanotum utrinque inter discum et marginem laterilem sulculo oblique latiusculo ruguloso, ceterun carinulis pluribus 1. obliquis 1. subrectis tenuibus: interstitiis irregulariter et tenuiter rugulosis, interstitiis lateralibus plerumque transverse coriaceo-rugulosis, interstiliis centrali-basalibus fere longitudinaliter coriaceo-rugulosis: uterque angulus postico-lateralis spinoideus parvus et parum prominulus. Abdomen ovatum depresso-convexum nitidum sparse punctatum laete brunneum, segmentis 2:do et 3:tio magnitudine subaequalibus, hoc apice et segmento 4:to (s. anali) nigro-piceis. Tubulus analis robustus brunneo-testaceus, interduin apice fuscus. Venter dorso abdominali concolor at convexior. Pedes pictura rariant: a) plerumque trochanteres, genua, tibiae tarsique rufo-testacea, coxac fuscae viridi - et cyaneo-nitidae, femora l. tota coxis concolora l. uno latere testaceo-picta; b) rarius pedes toli rufo-testacei. Alae subbreves parum ultra medium ab. dominis extensae, sordidae, nunquam albo-fasciatae, sed subvena radiali punclum pallidum minus distinctum et a stigmate in discum linea albida leniter arcuatim ducta observantur. Venae, quae adsunt, firmae fusco-brumucac; rena radialis fere tota firma, tamen apice subtili marginem alae altingit cellulam radialeın concludens adeoque completam efformans.

\section{Cleptes semiaurala $\delta$ 오.}

Mediocris $2 \mathrm{lin}$. decimal. long. thorace postico viridicyaneo aut cyaneo-viridi, abdomine tibiis tarsisque laete castaneo-rufis, ano fusco plus minus cyanescente; ơ cnpile thoraceque viridi-ryaneis, antennis fuscis, alis sub- 
Iyyalinis disco leviter nebulosis; 오 capite et antico thorace viridi-cupreo-auratis splendidissimis, antennis basi rufo-testaceis, alis hyalinis disco et apice obsolete sordide fasciatis.

Spllex semiaurata Lin. Fn. Sv. 1661 et S. N. 2. 946. 35. $\delta$.

Ichneumon semiauratus Panz. Fn. Germ. 51. 2. ठో; Fabr. E. S. 2. 184. 210. ㅇ․

Chrysis semiaurata Oliv. Encycl. meth. Ins. V. 676. 21. $\sigma^{7}$. teste Wesinaël.

Cleptes semiaurata Fabr. $\sigma^{7}$ in Museo Havniensi, ubi exemplar 15 picum a Fabricio determinatum ipse anno 1847 exanninavi.

Cleptes semiaurata Fabr. Piez. 15! 1; - Latr. Gen. Crust. et Ins. IV. 44. \&; - Jur. Hym. 298. ㅇ; Dlbm. E. H. 23. 1. $\sigma^{7} q ;-$ Vesmaël Not. 2. 1. $\delta^{\top}$ 우; - Dlbm. Dispos. 2. 4. $\delta^{\top}$.

Iclneumon auratus Panz. Fn. Germ. 52. 1. 9 .

Cleptes auratus Megerl. in Mus. Vienn. 9 teste Kollàr.

Cleptes splendens Fabr. Piez. 155. 3. $\sigma^{7}$.

Cleptes pallipes Lepell. Annal, du Mus. VII. 119. 3. ㅇ.

Habitat mensibus Julio et Augusto per totam Europam vulgaris; regionibus septentrionalibus exceptis, in quibus adhuc non observata est. In foliis Ribis Grossulariae, ubi larvae Nemati Grossulariae copiosissime adfuerunt, utrumque sexum ludentem in horte Lundensi iterum iterumque observavi. De moribus ita scripsit $\boldsymbol{D} e$ Saint-Fargeau (in Encyclopédie Méthodique Tom X. pag. 9.): „J'ai vu une femelle du Clepte semidoré entrer successivement à reculons dans les trous qu'avoient formés en s'enfonçant en terre un grand nombre de larres d'une Tenthrédine qui aroient vecu sur un mème grosciller. L'année suivante je jouis à cette même place d'un spectacle fort brillant; une centaine de mâles et quelques femelles de celte espèce, couroient dans tous les sens sur le petit espace de terrain où les larres de Tenthrédine s'étoient cachées, et reflétoient toutes les coulcurs des 
pierre précieuse. Ce spectacle se renouvela pour moi plusieurs jours de suite de dix à onze heures de matin; ces. individus dispersoient après cette heure, et je pense que ceux que je voyois chaque jour étoient nouvellement éclos dans cet endroit."

Observ. Femina. Caput cupreo-auratum splendidum; frons minus convexa, punctura nomnihil crebior quam in $\boldsymbol{C l}$. nilidula, sulculus medianus antice abruptus. Antennae crassiores quam in $\boldsymbol{C l}$. nitidula, apice fuscae, scapus, pedicellus et flagelli tres l. quattuor articuli basales fulvi aut rufo-testacei. Prothorax, mesothorax et postscutellum cupreo-riridi aut viridi-cupreo aurata splendida. Pronotum paullo latius, paullo minus convexum atque distinctius punctatum quam in $\mathbf{C l}$. nitidula. Metanotum ut in $\boldsymbol{C l}$. nilidula simillime constructum sculptum et armatum; carinulae longitudinales tamen paullulum distinctiores. Abdomen late castaneo-brunneum, nitidum punctulatum griseo-pilosellum; segmentum 2:dum brevius quam 3:tium; hoc apice et anus nigro-picea, plus minus violaceo aut cyaneo-micantia; tubulus analis crassus testaceus; venter dorso convexior. Tantum in unico specimine abdominis segmenta 1:mum et 2:dum macula dorsali fusca insignia vidi. Pedes eandem picturam praebent, quam apud Cleplem nitidulam jam annotavimus. Alae fere ad anum extensae, aliis hyalinae disco apiceque sordidae 1. subfasciatae; aliis sordidae: basi latius at inter discum et limbum apicalem indeterminate hyalinae; venae firmae fuscae; cellula radialis non omnino completa, scil. venae radialis apex subtilis proxime ante marginem obliteratus.

Mas quoad structuram et sculpturam corporis, atque II. 
1. Cleptes.

picturam metathoracis abdominisque of simillimus, at nolis sequentibus dignoscitur: caput et thorax tota riridicyanea aut cyaneo-viridia, anus viridi-cyanescens. Alae nunquam fasciatae, sed subhyalinae et disco obsoletae vel nebulosae.

4. Cleptes ignita Fabr. $\sigma^{7}$ ㅇ.

Mediocris 2 lin. decimal. long. melathorace viridicyaneo, abdomine ferrugineo: postice aurato macula fusca, alis sordidis.

Mas: caput et thorax viridi-cyanea; abdominis segmentum 3:tium nigro-aeneum utrinque macula viridi-aurea, segmentum 4:tum cupreo-auratum, 5:tum rel anale caeruleum. Pedes basi viridi-cyanei tibiis fuscis, tarsis ferrugineis.

Ichneumon Chrysis Fabr. E. S. 2. 185. 213.

Diplolepis Chrysis Fabr. Piez. 150. 5. secundum exemplar typicum e Porto Farino a Prof. $\boldsymbol{V} a h l$ reportatum et in Museo Havniensi asservatum, quod ibidem aestate 1847 examinavi.

Femina : caput et prothorax viridi-l. cupreo-aurata; dorsulum cupreo-aeneum, postscutelli tuberculum 1. concolor I. aureo-viride; pedes basi picei, apice testaceo-fusci. Abdominis segmentum 3:tium disco macula amplissima 1. trapeziformi 1. triangulari nigro-picea, utrinque macula laterali obsoleta viridi-aurea: segmentum 4:tum aureum ano summo piceo.

Ichneumon ignitus Fabr. E. S. 2. 184. 212.

Cleptes ignita Fabr. Pier. 155. 4.

Diplolepis Chrysis Fabr. Piez. 150. 5. ubi Auctor disit: „Aculeus exsertus longitudine corporis", quae verba tantummodo feminae applicari possunt.

Habitat in Barbaria D. Vahl, in Italia D. Spinola, in Austria D. Kollàr. 
Observ. Femina. Ciaput et pronotum ita picta, punctata et latiuscula ut in Cleplis semiauralae $\$$; ita convexa et pronotum margine postico laeve (serie punctorum transversa carens) ut in Clepte nitidula. Antennae fuscae, flagellis basi et apice obsolete brunneo-testaceis. Mandibulae brunneae, basi apiceque fusci. Palpi fuscoet brunneo - varii. Mesonotum punctis nonnullis distinctioribus adspersum. Dorsulum violaceo-cupreum 1. cupreoaeneum, convexo-depresso planum, sulcis longitudinalibus 4 distinctioribus; scutellum magnum convexum 1. dorsulo concolor 1 . viridi-auratum. Postscutellum lateribus viridicyaneum, tuberculo discoidali convexo aeneo-cupreo aut viridi-aurato parcius punctato. Pectus et metathorax viridi-caerulea; metanotum disco irregulariter subtiliter coriaceo-rugulosum (nec ut in praecedentibus carinulatum), utrinque sulco normali profundo latiusculo obliquo, angulis postico-lateralibus mediocribus triangularibus non mucronatis. Abdomen normaliter constructum, confertim subtiliter punctulatum, segmento 3:tio paullulum majore quam 2:do; venter segmentis 2 basalibus rufo-testaceis, apicalibus 2 nigro-piceis, marginibus segmentorum apicalibus testaceo-scariosis; tubus sexualis fusco-testaceus. Alae mediocres, ultra $\frac{2}{3}$ abdominis longitud. extensae, sordidae, subhyalinae, non fasciatae, venis firmibus fuscis; cellula radialis completa scil. venae radialis apex, licet subtilis et difficile observandus, tamen alae marginem attingit.

Mas tantum notis 1. pro sexu normalibus 1 . in diagnosi specifica jam allatis dignoscitur. 
5. Cleptes aurata Dlbm. $\delta$.

Parva $1 \frac{1}{2}$ lin. decimal. long. viridi-cyanea, abdomine viridi-aureo, alis subhyalinis.

Dlbm. Dispos. 1845. 2. 6.

Habitat in Turcia; ad Bosphorum a I. Hedenborg detecta, Mus. Reg. Acad. Scient. Holm.

Observ. Caput, thorax, pedes antennaeque ut in Cleptis semianratae $\sigma^{7}$ picta, constructa et sculpta, sculptura tamen subtilior et caput antice paullo magis convexum. Postscutellum autem et metanotum eandem habent formam et sculpturam ac in $\mathbf{C l}$. ignita. Abdomen totum undique viridi-auratum, griseo-pubescens confertim punctulatum, ovatum, mediocre, rentre paullulum couvexius quam dorso, segmentis dorsalibus 1:mo apice indeterminate et 2:do macula discoidali-continua (h. e. a basi in apicem ducta) subrectangula aeneo-nigricantibus. Alae hyalinae, non sordidae licet propter pubescentiam ita apparent; venae mediocres fuscne; cellula radialis clongata ovato-lanceolata: alae dextrae completa scil. venae radialis apex licet obsoletus tamen marginem alae tangit, alae sinistrae aperta et igitur incompleta.

6. Cleptes orientalis Dlbm. ㅇ.

Robusta $2 \frac{2}{3}$ lin. decimal. long. capite, thorace antico abdomineque aureis, alis fuscis sordidis.

Ulabitat in Turcia, Mus. D. Drewsen.

Observ. Species eximia et elegans Clepli auratae affinis, at crassitie, magnitudine, pictura, structura et sculptura valde diversa. Corpus nitidissimum robustum et proportionaliter crassius quam in ulla alia specie hujus Generis. Caput crebre punctatum viridi-aureum, vertice 
et clypeo cupreo- 1. rubro-aureis, mandibulis maxillis palpis et pagina capitis infera nigro-piceis. Antennae nigrofuscae. Pronotum et mesonotum subcrasse et subcrebre punctata, viridi-aurea; pronotum postice subgibbo-convexum, linea intra marginem posticum impressa minus distincta. Dorsulum subplanum vel saltem levissime convexum suturis longitudinalibus 4: lateralibus obsoletis, medianis 2 distinctioribus crassis. Scutellum magnum convexo-depressum. Pectus punctatum et metanotum cyanea opaca area pleurarum supra pedes intermedios et infra alas posticas immersa nitidissima marginata. Metanotum antice longitudinaliter rugulosum, postice areis 2 marginatis angulatis nitidis punctatis; anguli postico-laterales leves, acute triangulares. Pedes fusci: femoribus cyanescentibus viridi-resplendentibus, tibiarum et tarsorum articulationibus ferruginantibus. Alarum tegulae magnae nigro-aeneae, viridi-aureo-splendentes. Alae sordidae fusco-brunnescentes venis firmibus piceis, cellula radiali subcompleta. Abdomen robustum, late ovatum, cupreoaureum, basi lateribusque viridi-resplendens, confertim punctulatum, punctura segmenti analis subcrassa; segmentum anale apice summo coarctatum pro meatu tubuli. Tubulus analis ferrugineo-testaceus, crassus (tibia crassior), longitudine $\frac{2}{3}$ abdominis; margines segmentorum ventralium piceo-scariosi.

\section{Genus HETEROCOELIA nob. ह̇regos (diversus) et rothice (venter).}

Abdominis segmenta dorsalia: 2:dum maximum, 1:mum mediocre, reliqua abbreviata, ultimum vel anale margine 
apicali dentatum. In ceteris a Genere Cleptis non diversum.

Species hujus Generis tantum unica (Africana) nobis cognita.

7. Heterocoelia nigriventris nob.

Mediocris aut subparva vix 2 lin. decimal. long. piceo-nigra thorace antico tarsisque rufis; alis posticis hyalinis, anticis fumeo-fuscis albo hyalino fasciatis.

Cleptes nigriventris Dlbm. Dispos. 1845. 1; 1 .

Habitat in Algieria D. Paykull, Mus. Holmiens.

Observ. Propter capitis formam, thoracis colorem, alas breves et abdominis pilositatem cineream Mutillis parvis masculinis haud dissimilis. Corpus cano-cinereoque pubescens et pilosum. Caput subparrum pendulum ovale, totum fronte et vertice convexum, confertius punctatum, vix nitidum subopacum, nigrum fusco-pubescens. Oculi parvi exserti globosi. Antennac ad tuberculum clypealem carinatum rufum insertae, approximatae, robustae, longitudine mediocres, fuscae, articulis 1:mo, 2:do, 3:tio et 4:to rufo-brunnescentibus; scapus prismatico-obconicus, leniter arcuatus, apice incrassatus. Mandibulae breves robustae subsanguineae. Maxillae et palpi brunnea. Prothorax et mesothorax sanguinei. Pronotum convexiusculum punctato-rugosum sulco mediano profundo continuo, antice angustius crasse marginatum, lateribus verticalibus concaviusculo-compressum. Mesonotum subplanum subtiliter coriaceum parce punctulatum: dorsuli sulci 2 mediani obliqui sat profundi crassiusculi; laterales 2 obsoleti. Postscutellum vix visibile, margine melanoti basali transverso-arcuato connatum. Metathorax irregulariter cubicus, supra latior quam infra, postice concaviusculotruncatus, totus nigro-piceus apice rufescens. Metanotum 
supra subhorizontale carinulis longitudinalibus 5 distinctis et $\mathbf{2}$ obsoletis (margine utroque laterali non computato), interstitiis transverse striato-rugulosis; anguli poslico-laterales crassi mucroniformes lineares inaequales obtusi; metanoti truncatura et latera coriaceo-rugulosa. Pedes fusci, articulationibus tarsisque rufis, femoribus anticis infra concoloribus. Alae breves, medium abdominis tantum attingentes; posticae albo-hyalinae subtiles: venis costali gracillima et mediali robusta abrupta testaceis, ceterum avenae. Alae anticae fumeo-fuscac subviolascentes fasciis albo-hyalinis 2 amplis et ab uno margine in alterum continuis: una s. basali minus determinata, altera cellulas medialem, submedialem, discum et cellulae radialis basin intercedit; in hac fascia nullas venas at sub cellula submediali ramulos duos obsoletos vidi; renae ceterum mediocres fuscae aut rufo-brumnescentes, stigma testaceum; vena radialis elongata modice armata circ. $\frac{1}{5}$ a margine remota cellulam ibidem apertam relinquens; in medio disci observatur linea longitudinalis subtilissima albissima (sine dubio restigium venae rudimentariae), apice triramosa _ ramulis: infero et intermedio simplicibus, supero iterum biramuloso. Abdomen crassum ovato-conicum obtusum, convexius quam in Clepte, per formam et griseam sparsamque pilositatem abdomen Mutillarum paullulum simulans, totum nigro-piceum nitidum sparse punctulatum marginibus segmentorum apicalibus brunneo-scariosis; segmentum 1:mum mediocre convexissimum; segmentum 2:dum maximum depresso-convexum 3:tium, 4:tum et 5:tum s. anale submediocra at simul sumta saltem $\frac{1}{4}$ breviora quam segmentum 2:dum. Segmentum anale dorsale ( $=$ Valvula analis dorsali) $\longrightarrow$ apice testaceum, triarcuato-emarginatum et hinc 4 -denticulatum. 


\section{Familia 2. ELAMPIDAE Dlbm.}

Thorax subtetraëdro cylindricus.

Pronotum transverso - subquadratum.

Unguiculi tarsorum infra serrati 1 . potius subtiliter pectinulati.

Alae anticae cellula medialis apice: introrsum angulato - emarginata, extrorsum - stigma versus - acuminata, scil. venula transverso-medialis profunde arcuato 1. subangulato inflexa.

Mandibulae apice inciso 3-dentatae.

Abdomen segmentis cataphractis conspicuis 3, supra convexum, subtus 1 . planiusculum marginatum 1 . (praesertim in emortuis et exsiccatis) concavum et subfornicatum. 


\section{Tabula Synoptica Generum Familiae Elampidarum.}

A. Abdominis segmentum dorsale 3:tium in centro narginis apicalis excisum: excisura libera nec unquam marginata . . . . . . . Genus 3. Omalus Panzer.

B. Abdominis segmentum dorsale 3:tium in centro marginis apicalis truncatoermarginatum: emarginatura triangularis aut semicircularis, libera aut marginata et plus minus repleta margine continuato depresso-plano; segnenti margo lateralis ante emarginaturam utrinque uni- aut bi-sinuatus. . Genus 4. Elampus Spinola.

C. Abdominis segmentum dorsale 3:tium margine apicali integerrimum . . Genus 5. Holopyga Dlbm. 


\section{Genus OMaluS Panzer ex parte.}

Corpus parv̀un 3 millimetr. -2 lin. decimal. long. rarius ultra, proportionaliter crassum, oratum, ovalo-rotundatum aut interdum quidem subhemisphaericum.

Mandibulae subbreves aut mediocres robustae, paullulum arcuatae, extus convexae; intus: a medio in apicem cavae, a basi in medium incrassatae, apice crenulato-tridenticulatae.

Abdomen breviter et late ovatum interdum subbemisphaericum, segmenti dorsalis 3 :tii margo apicalis in centro excisus 1. emarginatus: excisura l. emarginatura libera nec marginata.

Species Omali Generis 7 cognoscimus: 6 Europaeas et 1 Africanam.

Sect. I. Abdominis segmentum dorsale 3:tium in centro unarginis apicalis distinctissime et subtriangulariter excisum l. emarginatum.

8. Omalus auratus Dlbm.

Parvus 1-1 $1 \frac{3}{4}$ lin. decimal. long. viridi-cyaneus, raro violaceus aut aenescens, abdominis dorso l. rubro l. viridi-aurato l. viridi-nigroque aeneo, emarginaturne analis lobis mediocribus obtuse subtriangularibus, postscutello gibbo subhemisphaerico, alis apice sat fumosis.

Varietates. Omali aurati.

Divis. 1. Area pronoti postica et dorsuli antica nitida sparse punctulata. Caput et thorax plurimis viridi-cyanea, paucis pure cyanea aut violacea. 
?ag. $26-27$.

Sect. I. Abdou S- $_{\text {- }}$ tinctis:

A. ]

a) Postscutelly viridis. C . 8. Omalus auratus nob. Europa.

b) Postscutellu clarissime aे 9. Omalus parvulks Klug. Lusitania.

B. $I_{m}$

$e_{\text {te }}$

o.

the

a. 10. Omalus pusillus Fabr. Europa.

Sect. II. Abdoni-

ter em

A. 5 -

$\mathrm{P}_{13}$

P. 11. Omalus punctulatus nob. Europ. meridional.

B. $I_{3}$

C.

s1. 12. Omalus caeruleus De Geer, Europa.

C. $\mathrm{F}_{\mathrm{e}}$

$P_{s}$

II. 13. Omalus aeneus Panz. Europa.

D. $\mathrm{p}$

si

n. 14. Omalus coriaceus nob. Africa. 


\section{Tabula Synoptica Specierum Generis Omali. pag. 26-27.}

Sect. 1. Abdominis segmentum dorsale 3:tium in centro marginis apicalis distinctissime et subtriangulariter emarginatum.

A. Emarginaturae lobi nediocres, obtuse triangulares.

a) Postscutellum modice gibbum. Abdominis color dorsalis 1. aureus 1. viridi-âeneus 1. aeneaviridis, Corpus mediocre. Alae apice sat fumosae . . . . . . . . . . . . .

b) Postscutellum valde conicum. Abdomen auratum disco aeneo-fuscum, Corpus parvum. Alae

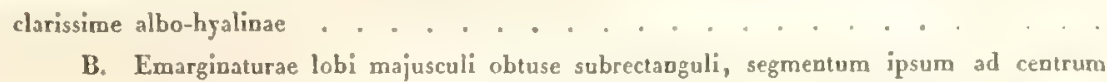
emarginaturae brevissime subcarinulatum: scil. ad certum luminis situm sub lente detegitur lineola supra emarginaturam elevata brevissima. Postscutellum valde conicum. Color corporis viridi-cyaneus aut cyaneo-viridis. Corpus parvum. Alae apice sat fumosae.

Sect. II. Abdominis segmentum dorsale 3:tium in centro marginis apicalis leniter emarginatum.

A. Pronotum et dorsulum punctis mediocribus adspersa. Alae subhyalinae 1. parumper sordidae. Abdominis dorsum aureum, confertim dislincte punctulatum. Corpus parvum . . . . . . . . . . . . . . 11

B. Pronotum et praecipue dorsulum punctis crassis adspersa; alae apice sat fumosae. Corpus caeruleurn aut cyaneum plus minus virescens et aenescens; abdominis dor-

sum sparse subtiliter punctulatum. Corpus submagnum . . . . . . . 12. Omalus caeruleus De Geer, Europa.

C. Pronotum et dorsulum politissima subimpunctata; abdominis dorsum subtilissime parcius punctulatum, margine summo apicali tenuissime testaceo scarioso. Corpus mediocre viridi-cyaneum. Antennae normales longiusculac . . . . . . 13. Omalus aeneus Panz. Europa.

D. Pronotum et dorsulum subopaca subtilissime coriacea; abdominis dorsum subtilissime punctulatum, margine apicali limbo lato albo-scarioso. Corpus parvum cyaneum. Antennae breves crassiusculae

10. Omalus pusillus Fabr. Europs.

8. Omalus auratus nob. Europa

9. Omalus parvulus Klug. Lusitanis

11. Omalus punctulatus nob. Europ. meridional. 
A. Abdominis dorsum rubro-aureum.

var. a. S. normalis: abdominis dorsum disco amplius aut arctius nigro-aenescens; venter viridis nigro-maculatus.

Clirysis aurata Lin. Fn. Sv. 1666; Fabr. Piez. 175. 25. secunduun exemplaria Typica, quae in Museis Havniensi et Kiliensi anno 1847 examinavi; Panz. Fn. Germ. 51. 8; Dlbm. Mon.' Chr. 16. 14.

Elampus auratus Wesmaël Not. 6. 1.

Omalus auratus Dlbm. Dispos. 4. 5. 1.

var. $b$. abdominis dorsum disco plus minus nigro-aenescens, venter auratus.

var. c. abdominis dorsum totum rubro-aureum immaculatum, venter viridis nigro-maculatus.

Hedychrum auratum Megerl. Mus. Vienn. teste Kollar in litteris.

Elampus scintillum Klug. Mus. Berolin. secundum exemplaria in Museo Berolinensi anno 1847 a me comparata.

var. d. abdominis dorsum totum rubro-aureum immaculatum, venter aureo-viridi-et nigro-maculatus.

B. Abdominis dorsum laete viridi-aureum, venter viridi-nigroque variegatus.

var. e. abdominis dorsum disco obsolete nigro-aenescens.

Omalus auratus Megerl. Mus. Wien. teste Kollàr qui specimen cummunicavit.

var. $f$. abdominis dorsum disco distinctissime et amplissime nigro-aeneum.

Hedychrum auratum Megerl. Mus. Vienn, secundum specimen ab Amico Kollar communicatum.

Divis. 2. Area pronoti postica polita sub-epunctulata, dorsulum subtilissime coriaceum subopacum.

var. g. caput et thorax cyanea; abdominis dorsum aureum, disco nigro-aenescens, margine viridi-resplendens. 
var. . caput et thorax cyanea; abdominis dorsum nigro-aeneum limbo viridi.

var. i. Caput, thorax et abdominis dorsum nigro-aenea, bujus margo viridis.

Habilat in muris, parietibus et truncis arborum, in foliis et floribus hortorum umbelliferis, immo quidem in gallis plantarum aestate plus minus frequenter. Varietates $\boldsymbol{a}, \boldsymbol{c}, \boldsymbol{e}$ et $\boldsymbol{f}$ amplissimam praebent distributionem geographicam, scil. ab Asia minori et insulis maris mediterranei per Turciam, Graeciam, Italiam, Hispaniam, Andalusiam ceterasque Europae regiones usque ad vicinitatem circuli polaris Lapponiae observatae et collectae sunt; varietates $b$, d et $g$ rariores sunt, scil. var. $b$ semel in Svecia mihi et semel in Finlandia D. Nylander obvia, var. $d$ in Graecia a D. Hoew et var. $g$ in Graecia a $D$. Loew et in Silesia a D. Zeller Iecta; rarictates $/ l$ et $i$ tantummodo in horto Berolinensi semel deprehendit I). Bouché.

De vivendi modo hujus speciei ita mihi scripsit oculatissimus Kollàr: "prope Viennam in Gallis Cynipedis lignicolae nidulatur, Aphidibus prolem nutriens." - Attamen haec magni momenti observatio, quam C.el. hollàr grati debemus, non ita explicari possit, ut Omalus auratus ipse Aphides raptaverit et in Gallam introduxerit. Mihi multo verisimilius est quandam Pemphredonidarum aut Crabronidarum speciem Gallas a Cynipede lignicola jan relictas pro nido adhibuisse, ibidem ova sua deposuisse, Aphides pro sua prole nutrienda collegisse et introduxisse. Constat enim copiam Hymenopterum Aculeatorum, praecipue e Crabronidarum et Pemphredonidarum familiis, pullulos suos $\Lambda$ phidibus nutrire; ex gr. 
Stigmus pendulus, Passaloecus borealis, Diodontus tristis et pallipes, Cemonus unicolor, Trypoxylon figulus, Rhopalum tibiale el clavipes, Lindenius albilabris et Panzeri, Crossocerus Aphidum, IVesmaëli, elongatulus etc. Hinc opinor Omalum auratum detegisse asylum, quod in galla Cynipedis lignicolne sibi paravit quaedam Pemphredonida aut Crabronida, in eodem ovula peperisse, ex ovulis provenisse Omali larvulas, quae in hoc Pemphredonidae aut Crabronidae domicilio vitam parasiticam aut pseudoparasiticam degerunt. Mentem suam de observatione a CI. Rudder facta, respectu habito ad vitam et metamorphosin Chrysidis cujusdam Angliae vulgaris (ignitae?), modo fere simillimo professus est Cel. Weslwood, ita dicens (in Modern Classificalion of Insects pag. 177): „I am indebted to Mr. E. Rudder for some particulars relative to the common species of Chrysis, which that gentleman reared from larvae found in cells filled with Aphides, formed in the pith of an elder twig stuck into the ground. In this case, I apprehend, the nests were those of a species of Pemphredon, or some allied genus, and the Chrysis was parasitical in the nest: the mode in which the larva fed upon the Aphides (of whic there were at least sixty in each cell) was observed by him; but it might certainly have been the larva of the maker of the nest which was thus occupied."

Cl. Bouché Professori Ratzeburg et mihi anno 1847 narravit se Omalum auratum e larva Nemati Grossulariae Berolini exclusum accepisse.

Observ. Var. a. Caput robustum transversum latiludine pronoti. Mandibulae robustae nitidae apice brunneae, basi I. virescentes 1. cyanescentes. Clypeus nitidus 
1. cyaneus 1. viridis 1. aureo resplendens. Cavitas facialis laevissima nitidissima viridis aut cyanea. Antennarum scapus et pedicellus viridi-cyanei, flagellum nigro-fuscum. Frons inter stemmata et oculos modice punctato-reticulata, viridis aut cyanea. Oculi exserti rotundate ovati fusco-brunnei. Regio stemmatum nitida sublaevis 1. parumper punctulata. Capilis margo et posticus et pone oculos lateralis distincte punctatus. Vertex cyaneus l. viridis. Thorax robustus gibbus latiusculus. Pronotum antice ad marginem distincte punctatum, postice area nitida semicirculari laevi parce punctulata. Dorsuli pars antica laeviuscula nitida parce punctulata; ejusdem pars postica nec non scutellum metanotum et pleurae crasse punctatoreticulata; sternum nitidum coriaceum. Pictura thoracis mirifice ludit; in plurimis tamen pronoti margo anticus, mesonoti et metanoti latera totumque pectus laete riridia, leviter cyaneo-imbuta, - pronoti area postica et mesonoti discus cyanea aut violacea, - metanoti gibbositas nigro- aut violascenti-aenea; interdum rero totus thorax cyaneus. Femora et tibiae uno latere cyanea, altero viridia videntur, omnino secundum luminis situm in quo observantur. Tibiarum calcaria pallide testacea. Tarsi basi tibiis concolores, apice brunnei. Unguiculi tarsorum testaceo brunnei subpellucidi. Alae basi clare albohyalinae, apice ample saturate fumosae, venae brunneae 1. testaceo brumneae; Cellula radialis tantum inchoata, scil. vena radialis mox infra stigma, instar ramuli stigmaticalis, abrupte desinit. Abdomen mediocre, nitidum, dorso subtiliter punctulatum, punctura marginali praesertim apicali distinctiori. Venter laevissimus vix punctulatus; segmentum ultimum disco confertius punctulatum. 
9. Omalus parvulus Klug.

$\frac{3}{4}$ lin. decimal. long. violaceo-cyaneus abdominis dorso rubro-aurato, disco aeneo-fusco, emarginaturae analis lobis mediocribus obtuse triangularibus, postscutello conico, alis clarissime albo-hyalinis.

Elampus parvulus Klug Mus. Berolin.

Habitat in Lusitania D. Moffmannsegg, Mus. Berolin.

Observ. Simillimus Omalo aurato minori, at alis clarissime albo-hyalinis mox distinguitur. Caput politum sparse punctatum nigro-violaceum. Pronoti pars antica cyanea modice punctata; pronoti pars postica et dorsulum polita nigro-violacea punctis majusculis paucioribus adspersa; scutellum convexum, postscutellum gibbum conico tuberculiforme obtusum, crasse punctato-reticulata cyanea; reliquus thorax cyaneus. Abdomen omnino ut in Omalo aurato. Alae pure albo-hyalina absque umbra, venis brunnescentibus.

10. Omalus pusillus Fabr.

Parvus $\frac{2}{3}-1 \frac{1}{8}$ lin. decimal. long. viridi-cyaneus abdominis emarginaturae analis lobis majusculis obluse subrectangulis, postscutello l. subconico l. subhemisphaerico, alis apice sat fumosis.

Chrysis pusilla Fabr. Piez. 176. 33. secundum Exemplar in Museo Kiel anno 1847 a me exarninatum.

Hedyclirum pusillum Megerl. Mus. Vienn. Quod specimen pro Descriptione Fabriciana typicum amice communicavit Cel. Kollàr.

Elampus violoceus Mus. Spinolae.

Habitat ,in Austria Mus. D. de Megerle" Fabr. I. c., in Turcia D. Drewsen, in Silesia D. Zeller, in Posnania D. Loew, in Brandenburgia prope Berolinum in campo are- 
noso Moabite in floribus Gnaphalii indiriduum unicum d. 18. Augusti 1847 legi.

Observ. Et sculptura et structura corporis Omalo aurato valde similis et affinis, at forma loborum emarginaturae analis, carinula supra emarginaturam, et abdomine cyaneo-viridi facile distinguitur. Mandidulac lacte brunneae basi virescentes. Clypeus nitidus subtiliter coriaceus, margo orbitarum punctatus cyaneo-viridia, reliqua pars capitis cyanea. Sculptura et color capitis thoracisque omnino ut in Omalo aurato. Scutellum nonnunquam aureo-resplendens. Metanoti gibbositas etiam ut Omalo aurato valida et variabilis, itaque in aliis ad forman conicam, in aliis ad formam subhemisphaericam magis adgreditur. Abdomen politum nitidissimum riridissimum aut cyaneo-viride, apice (quemadnodum in Omalo auralo) sparse griseo-pilosellum, totum dorso punctulatum: punctura disci adeo subtilissima ut interdum difficillime animadvertitur; segmentum 3:tium basi subtiliter punctulatum, apice nec non secundum totum marginem distinctius punctatum et ibidem plus minus aureo-nitens, margo summus testaceus et ante lobum emarginaturae utrinque unisinuatus; emarginaturae lobi majusculi obtusi, interdum aureo-splendentes; supra emarginaturam analem observatur lineola longitudinalis minuta elevata cum ipso angulo emarginaturae intimo continua. Venter violaceo, aeneo et viridi-pictus. Pedes cyanco-virides, tarsis apice lacte brunneis. Alac ut in Omalo aurato. 
Sect. II. Abdominis segmentum dorsale 3:tium in centro marginis apicalis leniter emarginatum.

11. Omalus punctulatus Dlbm.

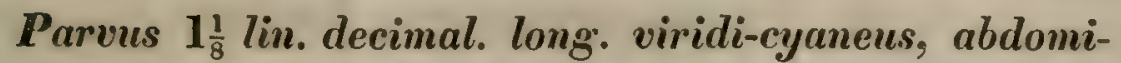
mis dorso aurato confertim distincle punctulato, pronoto et dorsulo punctis mediocribus adspersis, postscutello gibbe hemisphaerico vix conico, alis subhyalinis.

Hedyclerum minimum Dufour in litteris.

Habitat in Gallia meridionali D. Dufour, qui specimen in provincia Landes ad St. Sever delegit et mihi amice dedit, in Sicilia D. Schiödte.

Observ. Affinis Omalo aurato, at margine seginenti abdominalis dorsalis 3:tii apicali tantum leniter emarginato, abdominis dorso undique distincte punctulato, pronoto absque area illa nitidiori, quae apud $\mathbf{O m}$. auratum. constanter obvenit, alis subhyalinis etc. satis dignoscitur. Caput cyaneum aut violaceum concinne punctato-reticulatum. Palpi fusci. Mandibulae apice piceo-brunneae, basi virescentes. Clypeus viridi-aenescens. Cavitas facialis violascens. Antennae nigro-fuscae, basi aeneo aut violaceo virescentes. Thorax viridi-cyaneseens nitidus tegulis nigro-aut fusco-acueis; pronotum et dorsulum tota punctis majusculis non profundis adspersa; scutellum, metanotum et pleurae punctato-reticulata; gibbositas metanoti hemisphaerica, a latere veluti subconica obvenit. Pedes ut in Omalo aurato. Alae hyalinae, propter pubescentiam nonnihil sordidae, venis et cellulis omnino ut in Omalo aurato. Abdomen convexius et fere brevius quam in Om. aurato, nitidum virescenti-aureum, confertim distincte punctulatum, punctura apicalis distinctior II. 
quam discoidalis; segmenti 3:tii emarginatura lenis obtusa, segmenti margo apicalis et lateralis tenuis testaceo scariosus; venter aeneo-, cyaneo-, viridi- et aureopictus.

\section{Omalus coeruleus De Géer.}

Mediocris 2 lin. decimal. long. robustus, cyaneus aut violascens viridi-aut aeneo-resplendens, abdominis dorso sparse subtilissime punctulato, pronoto et praecipue dorsulo punctis crassis adsperso, postscutello gibbo subconico, alis apice sat fumosis.

Chrysis caerula De Géer Mem. 2. 837. 2; Dlbm. E. H. 33. 17. var. a. ㅇ.

Sphlex violacea Scop. Ent. Carn. 793.

Chrysis violacea Rossi Fn. Eir. II. 123. 848.

Omalus nitidus Panz. Fn. Germ. 97. 17.

Hedychrum nitidum Panz. Krit. Revis. 105.

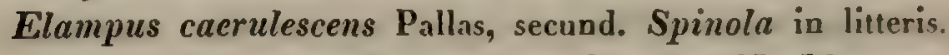

Chrysis fuscipennis Dlbm. Men. Clır. Sv. 15. 13.

Elampus gibbus Klug Mus. Berolin.

Chrysis chalybaea Ziegl. secund. Spinola in litteris.

Hedychrum imperiale Schuck. secund. Klug in Mus. Berolin.

Elampus-violaceus Wesmaël Not. 6. 2.

Omalus caeruleus Dlbm. Dispos. 1845. 5. 3.

Habilat mense Julio in variis Europae mediae et meridionalis regionibus raro, in borealibus rarius; intra Scandinaviam usque ad $65^{\circ}$ latitudinis borealis obserrata, scil. ad Stensele Lapponiae Umensis d. 3. Julii 1832 a me lecta.

Observ. Inter elegantiores sui Generis. Corpus robustum crasse rotundato-ovatum, viridi-cyaneum aut caeruleum, color cyaneus interdum et purpureo et aureo resplendens. Caput et vertex lata. Frons concinne punctato-reticulata, vertex laeviusculus parce punctulatus. Ca- 
vitas facialis laevissima polita splendida riolascens. Antennarum scapus robustus viridi-cyaneus, flagellum tenue nigro-fuscum. Clypeus viridis nitidus inaequalis, margine apicali undatus. Mandibulae robustae basi virides, apice brunneo-aenescentes. Palpi fusci. Thorax latus convexiusculus. Pronotum et dorsulum punctis majusculis adspersa. Pronoti margo anticus virescens punctis confertis, area postica subsemilunata magis nitida plerumque cyanca punctis paucioribus. Dorsuli discus purpureoviolaceus. Scutellum, metanotum et pleurae crasse punctato-subclathrata; metanoti gibbositas 1. subhemisphaerica l. obtuse subconica. Pedes viridi-cyanei aut cyaneovirides tarsis fuscis. Alae basi hyalinae: venis brunneis, cellulis ut in Omalo auralo, apice saturate fumosae. Abdomen late oratum, dorso viridi-cyaneum et instar chalybis politi splendidum, subtilissime sparse punctulatum, apice griseo-pilosellum punctis distinctioribus; segmenti dorsalis 3:tii margo apicalis leniter attamen acute emarginatus, utrinque arcuato - obtusissimus. Venter viridis, interdum aureo-resplendens: limbo violaceo aut aeneo nonnihil purpurascente.

13. Omalus aeneus Panzr.

Parvus aut mediocris 1-2 lin. decimal. long. cyaneus aut violascens plus minus viridi- aut aeneo-resplendens, abdominis dorso sparse subtilissime punctulato, pronoto et dorsulo politissimis sub-epunctalis, postscutello gibbo-convexo, alis apice sat fumosis.

var. $\boldsymbol{a}$. pronoti area et dorsulum violacea, abdominis dorsum viridi-cyaneum disco plus minus intensive violaceum. 
Clrysis aenea Fabr. E. S. 2. 242. 17; Piez. 175. 24; Panz. Fn. Germ. 51. tab. 17; Dlbm. Mon. Chr. Sr. 17. 6.

Omalus aeneus Panz. Fn. Germ. 85. t. 13; Dlbm. Dispos. 1845. 5. 2.

Chrysis caerulea Dlbm. E. H. 33. 17. var. b.

Elampus affinis Wesnaël Not. 6. 3.

Elampus violaceus Klug in Mus. Berolin. secundum exemplarinib.

Elampus excisus Klug in Mus. Berolin. $\}$ a.1847 a me examinata.

Hedychrum aeneum Megerl. Mus. Vienn. teste Kollar.

var. $b$. vertex, pronoti area, metanotum et discus abdominis subaspbaltico-1. gagathico nigro-aenea, margo abdominalis viridis.

Omalus flavipennis Megerl. Mus. Vienn. teste Kollìr.

var. c. Corpus viride leviter cyanescens, abdominis margo lateralis nonuihil viridi-auratus.

Omalus aeneus Megerl. Mus. Vienn. teste Kollar.

Habitat in foliis et floribus plantarum mensibus Junio, Julio et Augusto passim; Italiae: Spinola; Belgiac: Wesmaël; Austriae: Kollàr et Megerle von Miillfeld: Germaniae: Panzer, Klug, milhi; Daniac: Staeger. Var.a in Svecia a Scania usque in Lapponiam maxime meridionalem, Var. $b$ in monte Omberg Ostro-Gothiae d. 22. Julii 1834 mihi obviae. Var. $c$ adhuc tantum in Austria et Prussia observata. E Finnlandia varietatem a mihi transmisit Dom. Sallberg.

Observ. Var. a. Similis et affinis Omalo caeruleo; sed pronoto et dorsulo politissimis, metanoto tantum convexo, scutelli disco basali polito non punctato, cavitate faciali a stemmatibus remota etc. facile dignoscitur. Corpus eleganter viridi-cyaneum splendidum. Frous, pronotum antice arcuatim, dorsuli uterque margo lateralis, metanotum et pleurae punctato-reticulata, punctis melanoti et pleurarum crassioribus. Capitis margo poslicus subcoriaceus, minus distincte punctulatus. Vertex pone stem- 
mata subpolitus. Palpi, mandibulae, clypeus, antenuae et pedes ut in Omalo caeruleo. Flagella paullulum robustiora, caritas facialis latior brevior adeque a stemmate magis remota*), quam in Omalo caeruleo. Pronoti area postica magna semicircularis et dorsulum totum politissime punctulis perpaucis plus minus visibilibus. Scutellum punctis crassiusculis non reticulatum, basi area parva triangulari polita laevissima. Metanoli discus normaliter convexus. Alae ut in Omalo caeruleo. Abdomen viridi-cyaneum politum splendidissimum, paullulum angustius quam in Omalo caeruleo, subtiliter punctulatum; punctis disci minutis sparsissimis, limbi confertis distinctioribus; segmentum 3:tium omnino ut in Omalo caeruleo constructum; venter viridis ant viridi-aureus limbo piceo, interdum totus viridi-auratus, hoc tamen varius.

\section{Omalus coriaceus Dlbm.}

Parvus vix 1 lin. decimal. long. cyaneo-violascens abdominis dorso subtilissime punctulato, segmenti 3:lii margine apicali late albo-scarioso, pronoto et dorsulo subtilissime coriaceis subopacis, postscutello subhemisphaerico, antemnis brevibus crassiusculis, alis apice sat fumosis.

Habitat in Caffria Africae meridionali-orientalis, a Ciel. Joh. Wahlberg ibidem detectus.

Observ. Species eximia licet propter minutam corporis magnitudinem inconspicua. Habitu non dissimilis exemplaribus minutis Omali aenei. Corpus totum viola-

*) Hanc uotan caracteristicam primus observavit et 1. c. annolavit 1). Vresmaël. 
scens 1. potius chalybaeum. Caput crassiusculum orbiculatum, vertice convexo latiusculo subtilissime coriaceo subnitido. Stemmata parva arcuatim disposita. Oculi mediocres rotundato-subovati. Frons leniter punctatosubreticulata; cavitas facialis a stemınatibus longe remota mediocris, non ampla, nitida. Antennae breves crassiusculae basi nigro-aenescentes, flagellis fuscis apice brunnescentibus. Mandibularum apices castanei. Pronoti margines anticus et laterales nec non mesopleurae coriacea, confertim irregulariter punctata; pars pronoti postica et maxima atque totum dorsulum subtilissime coriacea subopaca 1. parum nitida punctulis minutis paucis et vix conspicuis adspersa. Scutellum convexum, disco subtilissime coriaceum subopacum, lateribus parce punctulatum. Metanoti gibbositas hemisphaerica, distincte punctato-reticulata; latera metanoti ample sed non profunde clathrata. Abdomen convexissimum politum chalybaeum splendidum punctulis minutis vix conspicuis adspersum; segmenti 2 :di margo apicalis summus leviter testaceo-scariosus; segmenti 3:tii margo apicalis late albo-scariosus pellucidus, ad centrum lenissime cmarginatus. Venter niger. Pedes nigri, parum viridi- et cyaneo-micantes tarsis brunneis. Alarum tegulae viridi-aenei. Alae ut in Omalo aurato constructae, hyalinae, anticae a medio in apicem saturate fumosae.

\section{Genus ELAMPUS Spinola.}

Corpus mediocre aut parvum $1-2 \frac{1}{2}$ lin. decimal. long. ellipticum.

Mandibulae Jongiusculae minus robustae falcatae com- 
. pag $38-39$.

Sect. I. Se mar-

giła ob-

$\mathbf{v e}$

Divis. 1. Anuatus.

A.

. 15. Elampus bidentulus Klug. Europa.

. . 16. Elampus ambiguus nob. Italia.

B. . . 17. Elampus Spina Spinola. Europa meridionali.

Divis. 2. Al, exaar-

giv trian-

gu|. . 18. Elampus truncatus Dlbm. Europa.

Sect. II. Sę̧ parte rarnante.

Divis. 1. Abisinua-

tus Post-

sct

A.

. . 19. Elampus chrysonotus nob. 'Turcia.

20. Elampus productus Spinola. Europa meridional.

3 aeque

. . 21. Elampus Panzeri Fabr. Europa.

B. 1. 22. Elampus caeruleus Klug. Europa.

Divis, 2. Able uni-

$\sin \}$ instar

opqediocre

robet pun-

ctul. . 23. Elampus Gnyi Spinola, Chili. 
Tabula Synoptica Specierum Generis Elampi. pag $38-39$.

Sect. 1. Segmenti abdominalis dorsalis 3:tii emarginatura caret plica illa marginali, quae apud Species Sectionis II:dae maxime caracteristica obrenit.

Divis. 1. Abdominis segmenti dorsalis 3 :tii margo lateralis ante emarginaturam utrinque bisinuatus.

A. Abdomen virescenti-aureum. Corpus mediocre

a) Postscutellum horizontaliter conicun, non mucronaturn

b) Postscutellum roucronatum: mucrone triangulari

B. Abdomen viride. Corpus majusculum. Postscutellum mucronatum

Diris. 2. Abdominis segmenti dorsalis $3:$ tii margo lateralis ante emarginaturam unisinuatus; emarginatura roediocris arcuata marginata margine punctato subsulcato; lobis lateralibus triangularibus. Corpus mediocre viridi-cyaneum . . . . . . . . . . . . . . .

Sect. II. Segmenti abdominalis dorsalis 3:tii emarginatura plerumque ex parte rarius tota repleta: plica marginali planum caracteristicum efformante.

Divis. 1. Abdominis segmenti dorsalis 3 :tii margo lateralis ante emarginaturam utrinque bisinuaus; (emarginaturae) plica marginalis entarginaturam ad $\frac{1}{2}-\frac{2}{3}$ excipit ct replet. Postscutelli mucro linearis.

A. Abdomen aureum, plus minus viridi-resplendens

a) Caput et thorax aurata .

b) Caput et thorax viridi-cyanea aut cyaneo-viridia

1. Postscutelli mucro triangularis

2. Postscutelli mucro sublinearis scil. apice transversus obtusus et fere aeque latus ac basi ..............

B. Abdomen purpureo-violaceum . . .

18. Elampus truncatus Dlbm. Europa.

15. Elampus bidentulus Klug. Europa

16. Elampus ambiguus nob. Italia.

17. Elampus Spina Spinola, Europa meridionali.

19. Elampus chrysonotus nob. "Turcia.

20. Elampus productus Spinola. Europa meridional.

21. Elampus Panzeri Fabr. Europa.

22. Elampus caerulens Kilug. Europa.

Divis, 2. Abdominis segmentis dorsalis $3:$ tii margo latcralis ante emarginaturam utrinque unisinuatus; (emarginaturae) plica marginalis senicircularis totum emarginaturam instar operculi replet. Postscutelli mucro brevis transversus semicircularis. Corpus mediocre robustum cyaneum. Thorax crasse punctatus Abdomen confertirn purctatum et pun-

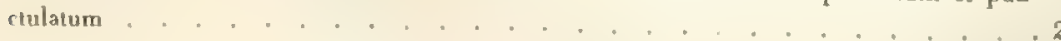




\section{Elampus.}

pressac, extus convexo-arcuatae, intus a medio versus apicem carae, apice tridentatae: dente apicali majore, interioribus marginalibus gradatim minoribus.

Clypeus mediocris transversus subsemilunatus, disco convexiusculus utrinque concaviusculo-depressus.

Antennae tenues, longitudine mediocres.

Postscutellum 1. conicum 1. processu mucronoideo armatum.

Alae et pedes fere ut in Omalo.

Abdomen segmentis cataphractis conspicuis 3: segmentum 3:tium ad centrum marginis apicalis subito coarctatum et ibidem revere truncato-emarginatum: emarginatura 1. libera l. aucta et partion repleta margine continuato depresso planum singulare verticale efformante; segmenti 3:tii margo lateralis utrinque ante emarginaturam aut uniaut bisinuatus.

Species Elampi Generis 9 cognoscimus: 8 Europaeas et 1 Americanam.

Sect. I. Segmenti abdominalis dorsalis 3:tii emarginatura libera, caret omnino plica illa marginali, quae apud Species Sectionis II:dae tam singularis et caracteristica obvenit.

15. Elampus bidentulus Pelletier.

Sulparvus 1 - $1 \frac{1}{2}$ lin. decimal. long. viridi-cyaneus abdomine viridi-aurato: segmenti 3:tii margine ante emarginaturam utrinque bisinuato, postscutello mucronato. var. $a$. Abdomen rubro-auratum. var. b. Abdomen viride aut viridi-aenescens.

Hedychrum bidentiulum Pelletier Encycl. Method. 
Elampus bidentulus Klug in Mus. Berolin.

Elampus pusillus Vesmaël Not. 7. 4. (non Chrysis pusilla Fabr. quae ad Omalim pusillum pertinet).

Habilat in Europa media et meridionali passim; Belgiae frequenter observata a D. Wesmaël; Galliae: I). Le Pelletier De Saint Fargeau; Helvetiae: D. Kriechbaumer; Germaniae: D. D. Klug et Lüben; Angliae D. Westwood. In Modern Classification pag. 178. refert Cel. Westwood: Anglicum quendam Entomologum observasse feminam hujus speciei ova deponentem in nido Psenis caliginosi.

Observ. Var. a. Habitu et colore similis Elampo $\boldsymbol{P a n z e r i}$, et cum hoc in Collectionibus commixta. Caput et thorax viridi-cyanea nitida. Frons confertim subpunctulata. Mandibulae apice late castaneae, basi viridi-, aureo- et aeneo-variegatae. Antennae basi viridi-cyaneae, flagellis nigro-fuscis. Pronotum et dorsulum nitidiora subpolita sparsius punctata. Scutellum, metanotum et pleurac confertim subcrasse punctato-reticulata. Metanoti gibbosilas conico-pyramidea, metanotum postice truncatum. Pedes virides aut cyaneo-virides tarsis fuscis aut brunneis. Alae hyalinae limbo apicali late fumosae. Abdomen dorso convexissimum splendidum aureum confertim punctulatum; segnentum 3:tium cupratum, ad centrum marginis apicalis anguste triangulariter emarginatum: emarginatura libera lobis obtusis subtriangularibus; margo (ejusdem segmenti) lateralis utrinque inter emarginaturam et basin segmenti ita bisinuatus, ut in medio marginis lateralis exstet angulus, sinu inferiore distinctiore quam superiore. Venter viridis nigro - et cyaneo-maculatus. 


\section{Elampus.}

16. Elampus ambiguus Dlbm.

Parvus $1 \frac{1}{4}$ lin. decimal. long. violaceus abdomine viridi-aeneo-aurato, segmento 3:tio ante emarginaturam utrinque bisinuato, postscutello mucronato.

Habitat in Europa meridionali rarius; specimen unicum, forte Italicum, in mea Collectione possideo, quod ut var. Elamp. Panzeri mihi transmissun est.

Observ. Individuis minoribus Elampi Panzeri similis; at dignoscitur: capite thoraceque tolis saturate violaceis, metanoti mucrone subtriangulari $h . e$. apice distincte angustior quam basi, segmenti 3:tii emarginatura libera absque plica. - Caput et thorax tota saturate violacea antennis fuscis, pedibus viridi-cyaneis, tarsis brunneotestaceis. Caput, pronotum, dorsulum et scutellum punctis majusculis parce adspersa. Metanotum punctato-reticulatum; mucro obtuse triangularis crasse sculptus. Pleurae coriaceae, confertim at minus regulariter punctata. Alae parte basali hyalinae venis brunneis, parte apicali brumneo-funosae. Abdomen nitidun viridi-aeneum nonnihil auratum, confertim punctulatum; segomenti 3:tii emarginatura subarcuata, submarginata lobis mediocribus triangularibus, margine laterali utrinque bisinuato, sinu inferiore distincto et superiore obsoleto.

\section{Elampus Spina Spinola.}

Submagnus $2 \frac{1}{4}$ lin. decimal. long. violascenti et purpurascenti-aeneus abdomine cyaneo-viridi, segmento 3:tio ante emarginaturam utrinque bisinuato postscutello mucronato.

Hedychrum Spina Lepelletier Annal, d. Mus, d'list, nat. cal. 38. pr. 121. fig. $2,3$.

Elampus Spina Mus. Spinolac. 
Habitat in Gallia, a D. Latreille detectus, e cujus Collectione Illustr. Spinola accepit individuum quod describendi caussa mihi benevole misit.

Observ. Inter majores et insigniores species hujus Generis. Caput et thorax subconfertim punctata nitida violascenti et purpurascenti-subaenea. Clypeus viridissimus epunctatus. Mandibulae, tibiarum calcaria tarsique testacea. Margo occipitalis utrinque, versus pronotum, acutum angulum emittit. Melanotum crasse punctato-reticulatum; mucro validus horizontalis sublinguaeformis I. potius triangularis scil. apice obtuso angustior quam basi. Pleurae confertim et modice punctato-reticulatae. Pedes - practer tarsos et calcaria - viridi-cyanescentes aut cyaneo-virescentes. Alae basi hyalinae, apice brunneo fumosae. Abdominis dorsum viridi-cyaneum nitidum confertim punctulatum; segmentum 3:tium magis viride, confertius punctulatum, mox ante summum marginem (quemadmodum in Chrysuris quibusdam ex gr. Chrysura sulcata) - nonnihil incrassatum; margo summus ipse tenuis, ad centrum arcuato-emarginatus, emarginatura libera lobis parvis subconicis; margo lateralis utrinque bisinuatus.

18. Elampus truncatus Dlbm.

Subparvus $1 \frac{1}{4}-1 \frac{3}{4}$ lin. decimal. long. viridi-cyaneus segmento 3:tio ante emarginaturam utrinque unisinuato, postscutello obtuso-conico.

Clerysis truncata Dlbm. E. H. -35. 18.

Elampus minutus Dlbm. Dispos 5. 2. An id. Wesmaël Not. 7. 5?

Elampus azureus Klug Mus. Berolin.

Habitat in plurimis Europae medine et meridionalis regionibus passim; ad Bosphorum Turciae a D. Heden- 
borg lectus; in Germania ad Glogaviam a D. Zeller, et ad Aschersleben a D. Liiben captus. Intra Sveciam primum a D. Gyllenhal obserratus; in Scaniae Blekingiaeque umbellatis plura specimina mense Julio deprehendi.

Obserr. Magnitudine, habitu et postscutelli structura similis et affinis Omalo bidentulo; sed colore corporis cyaneo, segmenti 3:tii emarginatura lata subarcuata marginata etc. mox distinguitur. Corpus nitidum viridicyaneum. Caput confertim modice coriaceo punctatum, fronte subreticulata. Mandibulae nigro-fuscae, apice piceo-brunneae. Antennae nigrae, basi cyaneo-virescentes. Pronotum et dorsulum sparse modice punctata; scutellum, metanotum et pleurae regulariter punctato-reticulata. Metanoti gibbositas obtuse conico-pyramidalis. Pedes viridicyanei aut cyaneo-virides, tarsis brunneis aut fusco-brunneis. Alae parte basali clare hyalinae venis brunneis, parte apicali saturate brunneo-fumosae; fumositas alarum posticarum minus ampla minusque intensiva. Abdomen politum viridi-cyaneum modice convexum, confertim subtiliter punctulatum; segmentum dorsale 3:tiun magis virescens et distinctius sparse punctatum, ad centrum marginis truncato-emarginatum lobis magnis triangularibus; emarginatura aut late et obtuse subtriangularis, marginata: margine concaviusculo opaco coriaceo punctis majoribus nonnullis; segmenti 3:tii margo lateralis 1. totus 1. saltem inferiore parte testaceus scariosus et nonnihil pellucidus, inter medium et emarginaturae lobum leniter arcuatoundatus 1 . sinuatus, inter medium et basin est hic margo (scil. lateralis) I. rectus 1 . leniter curvus I. lenissime sinuatulus; hoc in casu margo lateralis exstat bisinuatus 1. bisinuatulus angulo sinus intercedente. 
Sect. II. Segmenti abdominalis dorsalis 3:tii emarginatura plus minus repleta: plica marginali planum caracteristicum efformante.

19. Elampus chrysonotus Dlbm.

Parvus $1 \frac{1}{4}$ lin. decimal. long. viridi- et cupreo-auratus, abdominis segmento 3:tio ante emarginaturam bisinuato, postscutello mucronato mucrone obtuse subtriangulari.

Habitat in Turcia, Mus. D. Drewsen.

Observ. Corpore toto virescenti et cupreo-aurato ab omnibus hujus Generis speciebus facillime dignoscitur. Mandibulae apice testaceae. Antennarum flagella nigrofusca. Caput, pronotum et dorsulum punctis mediocribus confertim adspersa. Scutellum, metanotum et pleurae subcrasse punctato-reticulata. Postscutelli mucro validus cupreo-aeneus crassissime punctato-reticulatus triangularis apice obtusus. Pedes aureo-virides tarsis testaceis. Alae hasi hyalinae venis brunneis, apice sordidae vix fumosae. Abdomen cupreo-aureum confertim punctulatum; segmenti 3:tii emarginatura ad $\frac{3}{4}$ repleta: plica marginali nigro-picea semilunata nitida concaviuscula; segmenti 3 :lii margo lateralis utrinque bisinuatus angulo distincto sinus intercedente, ad sinum inferiorem observatur sulculus marginem sequens. Venter aureo-viridi et nigro-piceo pictus.

20. Elampus productus Klug.

Magnus robustus $2_{3}^{2}$ lin. decimal. long. virescenticyaneus abdomine aurato, segmento 3:lio utrinque bisimuato, postsculello mucrone obtuse subtriangulari.

Elampus productus Klug Mus. Berolin.

Habitat in Lusitania et Gallia rar. 
Observ. Maxime affinis et similis Elampo Panzeri; sed dignoscitur: corpore majore latiore, punctura distinctiori, postscutelli mucrone obtuse subtriangulari scil. apice distincte angustiore quam in medio. Mandibulae et tarsi brunnea.

21. Elampus Panzeri Fabr.

Mediocris $1 \frac{1}{4}-2 \frac{1}{2}$ lin. decimal. long. viridi-cyaneus, abdomine viridi-aurato, rarius unicolore viridi, segmento 3:tio ante emarginaturam ulrinque bisinuato, postscutello mucronalo: mucrone reclangulari apice transversa obtuso.

Chrysis scutellaris Panz. Fn. Germ. 51. 11.

Chrysis Panzeri Fabr. Piez. 172. 9; - Dlbm. Mon. Chr. 18. 17.

Elampus Panzeri Latr. Gen. Tom. IV. pag. 45; - Dlbm. Dispos. 5. 3. Elampus Ramburi Spinola, var. minor., Mus. Spinolae.

Habitat in foliis et floribus plantarum, praescrtim Unbellatarum, totius Europae mense Julio plus minus frequenter; intra plagas boreales tamen parce obvenit et quibusdam regionibus quidem rara fit species et ultra $65^{\circ}$ latitudinis borealis adhuc non observata.

Observ. Corpus elongatum. Caput et thorax viridi-cyanea, sparse modice punctata. Mandibularum apices et tarsi flavo-testacei. Scutellum, metanotum et pleurae punctato-reticulata. Metanoti mucro validus linearis subrectangularis apice obtusiusculo non angustior quam in medio; qua nota haec species ab affinibus certe et facillime distinguitur. Pectus 1. viridi-cyaneum 1. unicolore viride l. aurato-virescens. Interdum caput et thorax violacea, et metanoti mucro nigro-aeneus. Abdomen ple rumque viridi-auratum, nomnullis auten I. rubro-auratum 
1. aureo-viride 1. viridi-aeneum, omnibus nitidum et proportionaliter ovatum; segmenta dorsalia 1:mum et 2:dum confertim punctulata; segmentum 3:tium distinctius crebre punctatum; margo lateralis segmenti 3:tii utrinque distinctissime bisinuatus: sinu inferiori angusto profundo arcuato, sinu anteriori latissimo levi, inter ambos sinus adest lobus marginalis arcuatus flavo-testaceus laevissimus; emarginaturae plica planum nigro-piceum efficit 1. arcuatum l. semilunatum 1 . instar ferri equini formatum: aperturam plus minus angustam aut latam, arcuatam aut triangularem relinquens. Venter viridi-cyaneus aut viridi-auratus. Pedes, praeter tarsos, virides aut viridi-cyanei. Alae hyalinae, aliis tantum disco-aliis tota parte apicali brunneo sordidae et subfumosae.

\section{Elampus caeruleus Pallas.}

Subparvus vix $1 \frac{1}{2}$ lin. decimal. long. purpureo-violaceus abdominis segmento 3:tio utrinque bisinuato, postscutello mucronato: mucrone rectangulari apice obtuso.

Chrysis caerulea Pallas secund. Klug in Mus. Berolin.

Elampus caeruleus Klug Mus. Berolin.

IIabitat in Tauria, D. Pallas; in Prussia ad Berolinum, D. Erichson, Mus. Berolin.

Observ. Ad picturam similis Elampo truncato. Corpus totum purpurascenti-violaceum nitidum. Capnt et thorax sparse punctata. Postscutelli mucro proportionaliter robustior quam in Elampo Panzeri. Abdomen proportionaliter brevius et latius quam in Elampo Panzeri, purpureo-violaceum nitidum, segmentis 1:mo et 2:do confertius subtiliter punclulatis, segmento 3:tio sparse punctu- 
lato, apice marginulato et emarginato, emarginatura arcuata: plica nigro-fusca verticali nitida semilunata.

23. Elampus Gayi Spinola.

Robustus $1 \frac{1}{2}$ lin. decimal. long. cyaneus abdominis segmento 3:tio margine laterali utrinque unisinuato, postscutello mucronato: mucrone brevi semicirculari.

Elampus Gayi Mus. Spinolae.

Habitat in Chili, D. Gay, Mus. Spinolae.

Observ. Ad magnitudinem et colorem corporis Elampo truncato forsitan proximus. Corpus crassiusculum, intensive cyaneum nitidum. Frons et pleurae modice punctato-reticulata. Pronotum et dorsulum punctis majusculis rotundis adspersa. Scutellum et metanotum saturate nigro-violacea subpurpurascentia, crasse punctatoreticulata. Postscutelli processus depresso-planus semicircularis crasse punctato-reticulatus marginatus: margine tenui subconvexo laevissimo. Antennae et tarsi fusca; scápus et pedes viridi-cyanei. Abdomen subbreve ovatum: segmenta 1:mum et 2:dum cyanea confertim distincte punctulata; segmentum 3:tium viridi-cyaneum crebre punctatum, margine laterali tenui fusco laevi, mox ante emarginaturam leniter unisinuato; emarginatura repleta: lobo nigro-piceo totam emarginaturam instar operculi semicircularis excipiente.

\section{Genus HOLOPYGA Dlbm.}

Corpus robustum crassum rotundato- ovatum.

Mandibulae crassae trigonae apice inciso - 3dentatae: dentibus conicis approximatis, apicali majori. 
Abdomen segmentis cataphractis conspicuis 3: segmento 3:tio margine apicali integerrimo, tantum in unica specie undulato.

Species Holopygae Generis 6 cognoscimus: $2-3$ Europaeas, 2 Americanas et 1 Africanam.

Sect. I. Abdominis segmenti dorsalis 3:tii margo apicalis leniter undulatus.

\section{Holopyga Dohrni Dlbm.}

Robusta $2 \frac{1}{3}$ lin. decimal. long. viridi-cyanea punctatissima alis basi hyalinis apice brunneo fumosis, venis nigro-piceis.

Habitat in Cuba Cel. Dohrn, in New-York C.el. Kriechbaumer, qui mihi specimina amice donarunt.

Observ. Corpus robustum crassum. Caput thoraci arcte applicatum, pronotum et dorsuli pars antica irregulariter confertim punctata et punctulata, scil. punclis majoribus et minoribus mixtis; dorsuli pars postica, scutellum, metanetum et pleurae crasse punctato-reticulata. Dorsuli lobus intermedius basi area minuta transrersa subrectangulari l. subsemilunata minus determinata coriacea nec punctata. Mandibulae nigro-piceae basi rircscentes. Antennae nigro-fuscae, scapo pedicelloque riridi-cyaneis. Dorsuli lobus intermedius basi violascens 1. nigro-aencus, ceterum caput et thorax tota viridi-cyanescentia. Melanoti anguli postico-laterales validi triangulares depressi punctulato-coriacei. Pedes viridi-cranei tarsis brunneis. Abdomen late oratum, dorso I. unicolore cyaneum 1. viridi-cyaneum punctatissimum; segmenti 3:tii margo apicalis leniter undulatus, scil. ad centrum obsolete 
1gae. pa. $47-48$.

Sect. I. Alatus.

- . 24. Holopyga Duhrni nob. America.

Sect. II. A.

Divis. 1.

A. tis . 25. Holopyga luzulina Klug. America.

B. dominis

. . 26. Holopyga janthina Spin. Africa.

Divis. 2. A

bdomi-

. . 27. Holopyga punctatissima nob. Europ. meridionali.

A. ax aliis

uratum.

. . 28. Holopyga ovata pallas ex. p. Europa.

B. lureum,

x parte

. 29. Holopyga micans Klug. Andalusia. 


\section{Tabula Synoptica Speciemm Generis Holopygae. pag. 47-45}

Sect. I. Abdominis segmenti dorsalis 3:tii margo apicalis leniter undulatus.

Corpus robustum viridi-cyaneum punctatissimurn . . . . . . . . . 24. Holopyga Duhrni nob. America.

Sect. II. Abdominis segmenti dorsalis 3:tii margo apicalis integerrinus.

Divis. 1. Corpus viridi-cyaneum, caeruleum aut violascens.

A. Corpus caeruleum punctatissimum, abdominis segmentis basi nigro-subfascialis. 25. Holopyga luzulina Klug. America

B. Corpus viridi-cyaneum 1. violascens modice punctatum et punctulatum, abdominis segmentis concoloribus . . . . . . . . . . . . . . 26. Holopyga janthina Spin. Africa.

Divis. 2. Abdorninis dorsum 1. viridi-1, rubro-auratum.

Subdivis. 1. Corpus crasse punctatissimum. Caput et thorax cyanea; abdominis dorsum aureo-viride

27. Holopyga punctatissima nob. Europ. meridionali.

Subdivis. 2. Corpus modice punctatuma et punctulatum.

A. Metanotum modice converum. Abdominis dorsum unicolor. Caput et thorax aliis cyanca 1. viridi-cyanea, aliis thoracis dorsum aut antica aut moxima parte auratum. Corpus robustum

28. Holopyga ovala Pallas ex, p. Europa.

13. Metanotum gibbum obtuse-conicum. Abdominis dorsum hicolor: apice aureum, basi macula maxima subtriangulari nigro-aenea. Thoracis dorsum maxima ex parte auratum. Corpus minutum . . . . . . . . . . . . . . . .

29. Holopyga micans Klug. Andalusis 
emarginulatus et utrinque lenius sinuatus; in medio marginis lateralis utrinque angulus obtusus difficile animadvertitur. Venter viridi-cyaneus, segmentorum marginibus apicalibus fusco-scariosis; segmenta $\mathbf{1}$ et $\mathbf{2}$ nitidissima perparce punctulata, segmentum 3:tium confertissime punctulatum basi nigro-aeneum.

Sect. II. Abdominis segmenti dorsalis 3:tii margo apicalis integerrimus.

25. Holopyga luzulina Klug.

Rubusta 2 lin. decimal. long. laete caerulea capite thoraceque crasse punctatissimis, abdomine confertim modice punctato segmentis basi nigro subfasciatis, alis subhyalinis apice leviters umbrosis.

Hedychrum luzulinum Ḱlug Mus. Berolin. secundum Spinola.

Habitat in Brasilia, D. D. Milde et Spinola, qui specimina benevole communicarunt.

Observ. Valde similis et affinis Holopygae Dohrni, cum hac autem nullo modo confundenda; nam praeter alias notas praecipue segmenti 3:tii margine apicali integerrino certissime dignoscitur. Corpus laete caeruleun, scapis pectore pedibusque viridi-splendentibus. Caput confertim modice punctatum, pronotum et dorsulum confertim et crasse at irregulariter punctata. Pleurae, scutellum et metanotum crassius et regulariter punctato-reticulata. Margines suturales segmentorum thoracicorum paullulum virescunt. Pedes viridi - cyanei tarsis fuscotestaceis. Alarum tegulae et venae brunneo - aut nigropiceae. Abdominis dorsum confertim punctulatum, segmentis basi nigro-violaceis apice cyaneis, segmento 3:tio 
confertissime et distinctissime punctulato. Venter viridicyaneus.

26. Holopyga janthina Spinola.

Mediocris $1 \frac{1}{2}$ vix 2 lin. decimal. long. cyanea aut viridi-cyanea capite thoraceque modice punctatis et punctulatis, abdomine concolore confertim subtiliter punctulato, alis anticis basi hyalinis ceterum ample sordidis.

var, $a$. abdomine violascente.

Hedychrum janthinum Spinola (non Klug).

var. $b$. abdomine viridi-cyaneo.

Habitat in Africa, ad Promontorium Bonae spei a D. Westermann detecta et ibidem a D. Draege revisa. Museis D. D. Westermann et Spinola.

Observ. Paullo brevior et minus robusta quam Holop. luzulina, a qua ceterum facile dignoscitur punctura multo minus crassa (fere ut in Holop. ovata constructa), abdomine unicolore (nec segmentis basi nigricantibus), alis amplius sordidis e. s. p. Venter nigropiceus nitidissimus, parumper violascens.

27. Holopyga punctatissima Dlbm.

Robusta $2 \frac{1}{4}$ lin. decimal. long. viridis aut cyaneo-viridis punctatissima abdomine aurato-viridi, alis basi hyalinis apice ample fumosis.

Habitat in Insula Rhodo, a Cel. Loew mense Majo detecta et mihi benigne communicata.

O bserv. Species valde distincta; a subsequente (Holopyga ovata), cui simillima et maxime aftinis, per puncturam abdominalem primo intuitu dignoscitur. At habitu, sculptura capitis et thoracis nec non pictura pedum et 
alarum Holop. ovata nullo modo diversa. Scil. mandibulac viridi-cyaneae apice nigro-brunneae. Clypeus viridis. Cavitas facialis cyanea subtilissime coriacea; frons, vertex, genae et tempora viridia, leviter cyanescentia punclis majoribus et minoribus confertis. Antennarum scapi viridi-cyanei, flagella nigro-fusca. Oculi ovati mediocres. Stemmata majuscula nigro-brunnea. Pronotum viride et dorsulum viridi-cyaneum punctis majoribus et minoribus confertim et irregulariter punctata. Dorsuli lobus intermedius area basali transversa subrectangulari nigro-aenea subopaca coriacea subtilissime punctulata. Scutellum et metanotum viridi-cyanea, pleurae virides, crassius et regulariter punctato-reticulata. Pectus viride aut viridicyanescens coriaceum nitidum. Pedes virides parum cyanescentes, tarsis saltem apice brunneo-testaceis. Alae basi leriter hyalinae, ceterum ample sordidae, venis nigro-brunneis, tegulis nigro-aeneis. Abdomen breve et late ovatum, sat convexum, confertissime punctatum et punctulatum punctis majoribus et minoribus mixtis, aureoviride. Venter nitidus subpolitus nigerrimus, sparse et subtiliter punctulatus.

28. Holopyga ovata Pallas ex parte.

Robusı submagna, mediocris aut parva, $1 \frac{1}{2}-2 \frac{1}{2}$ lin. decimal. long. thorace l. viridi-cyaneo $l$. antica l. maxima parte dorsali aurato, pronoto et dorsulo modice at irregulariter punctatis, sculello toto punctato, abdomine auvato l. aureo-viridi conferlim punclulato, alis sordide umbrosis basi hyalinis.

Habitat per plurimas Europae regiones, ab insulis maris mediterranei usque in Sveciam mediam, atque pro- 
pter clima regionum tam diversarum valde diversum etiam variationes picturae et magnitudinis corporis locupletas ostendit; cujus exemplum optime hic testatur Conspectus:

Divis. 1. Caput et thorax aut viridi-cyanea aut unicolore cyanea.

var. a. 2 lin. decimal. long. abdomine aurato-riride; dorsuli lobus intermedius basi area transversa nigro-aenea subopaca subtiliter punctulata.

Unicum Exemplar in Insula Rhodo legit D.

Loew.

var. b. $1 \frac{1}{2}-2 \frac{1}{2}$ lin. decimal. long. abdomen auratum; dorsuli lobus intermedius basi area transversa nigro-aenea subopaca subtiliter punctulata.

Chrysis ovata Pallas Mscrpt. teste Klug in Mus. Berolin.

Elampus punctatissimus Klug in Mus. Berolin.

Hedychrum regium Megerl. Mus. Vienn. teste Kollar.

Hedychrum auratum Drewsen in litteris.

In Insulis Archipelagi: D. Loew; Italia: D. Spinola; Austria: D. Kollàr; Prussia: D. D. Dohrn et Luiben; Srecia: Scania (ad Esperöd d. 12. Augusti 1835), - Ostro-Gothia (in monte Omberg d. 22. Juli 1835 in copula cum var. $c$ !) et Gottlandia (in pratis Gothem d. 18. Juli 1841) mihi obria.

var. c. $1 \frac{1}{2}-2 \frac{1}{2}$ lin. decimal. long. abdomen auratum, dorsulum totum punctatum.

Chrysis regia Collect. Fallén.

Hedychrum lucidulum $\sigma^{\top}$ Nylander in litteris.

Helvetia: D. Kriechbaumer; Germania: D.D. Liiben et Zeller; Svecia: D. D. Fallén et 
Zettersledt qui hanc varietatem cum varietate $b$. copulatam deprehenderunt.

Divis. 2. Caput et thorax viridia. var. d. $1 \frac{1}{2}$ lin. decimal. long. abdomen auratum; dorsuli lobus intermedius basi area transversa nigro-aenea subtiliter punctulata.

Holopyga amoenula Dlbm. Dispos. 4. 1.

Duo specimina ex Insula Rhodo vidi, unum a D. Hedenborg alterum a D. Loew lecta.

Divis. 3. Pronotum aureo-maculatum.

var. e. $\mathbf{1}_{3}^{2}$ lin. decimal. long. Abdomen auratum, caput et thorax viridi-cyanea; pronotum utrinque macula discoidali et ad marginem anticum linea minus distincta viridi-aurcis. Dorsulum totum punctatum.

Specimen unicum e Sicilia mihi communicavit D. Schiödte.

var. f. $\mathbf{1}_{4}^{3}$ lin. decimal. long. Abdomen auratum, caput et thorax viridi - cyanea; pronotum aenescentiviride, ad marginem anticum macula centrali viridi-aurea; dorsulum totum punctatum aeneum viride irroratum.

Specimen unicum ad Aschersleben Germaniae legit D. Luiben.

Divis. 4. Pronotum et dorsulum, interdum quoque scutellum et postscutellum, aurata.

var. g. $\quad 1_{4}^{\frac{3}{4}-2}$ lin. decimal. long. Caput et thorax viridicyanea; pronotum, dorsulum et abdomen aurea; dorsulum totum punctatum.

Sicilia: D. Schiödte.

var. l. 1 $1 \frac{1}{2}-2$ lin. decimal. long. Caput et thorax cya- 
nea; pronotum, dorsulum et abdomen aurata, illa plus minus viridi-resplendentia; dorsuli lobus anticus basi area transversa 1 . concolore 1. viridi1. nigro-aenea subtiliter punctulata.

Hedychrum obtusicolle Megerl. Mus. Vienn. teste Kollar.

Elampus ignicollis Klug Hus. Berolin. I secundun speciruina ibid. Elampus cyaneocepherlus Klug MIus. Berolin. Sanno 1817 a me examinata.

Insula Rhodo: D. Loew; Austria: D. Megerle von Miihlfeld.

var. i. 2 lin. decimal. long. Pronotum, dorsulum, scutellum, postscutellum et abdomen 1. rubro- 1. viridi-aurata. Caput et ceterae thoracis partes viridi-cyanea; dorsulum 1. totum punctatum l. in basi lobi intermedii area transversa concolore aut aenescente subtiliter punctulata.

Chrysis gloriosa Fabr. Piez. 174. 20

Hedychrum lucidulum Pelletier. teste Spinola.

Hedychrum cyanocephalum Megerl. Mus. Vienn, teste Kollar.

Barbaria: D.Desfontaines secund. Fabr. 1.c.: insula Rhodo: D. Hedenborg; Sicilia: D. Schiödte; Austria: D. D. Megerle von Miihlfeld et Spinola; Prussia: D. D. Liiben et Zeller.

Observ. Illis nuper allatis novem varietatibus sequentes notae sunt communes: Mandibulae basi cyaneovirescentes, apice brunnescentes. Caput, pronotum et dorsulum confertim et irregulariter punctata et punctulata, scil. puncta majora et minora mixta; quod crassitiem puncturae attinet: dorsulum semper optimum I. primum, pronotum medium et caput ultimum tenet locum. Punclura frontis inter stemmata et cavitatem facialem subre- 
ticulata. Totum scutellum, metanotum et pleurae regulariter et plus minus crasse punctato-reticulata. Pedes et antennarum scapi viridi - cyanei, tarsis brunneo- et flagellis nigro-fuscis. Alae sordidae, basi hyalinae, venis brunneis. Alae posticae fere totae hyalinae, apice leviter sordidae. Abdominis dorsum nitidum, confertim subtiliter punctulatum; venter nigro-piceus nitidissimus.

\section{Holopyga micans Klug.}

Parva vix ultra 1 lin. decimal. long. thoracis abdominisque dorso aureis, capite violaceo, metanoto postico et pectore cyaneis, abdomine basi macula maxima triangulari nigro-aenea, alis hyalinis venis fuscis, postscutello conico obtuso protuberante.

Elampus micans Klug in Valtls Reise secundurn typurn in Museo Berolinensi anno 1847 a me examinatum.

Elampus fulgidicollis Mus. Spinolae.

Habitat in Andalusia: Dom. Waltl, Mus. Berolin. D. Chiliani Mus. Spinolae.

Observ. Corpus parvum omnino ut in Elampis elongato-oratum. Caput cyanescenti-violaceum modice subreticulato-punctatum. Antennae mediocres fuscae, scapus viridi-micans. Mandibulae apice brunnescentes. Pectus, mesopleurae et pars postica metanoti cyanea, leviter viridimicantia. Thorax gibbo-convexus; pronotum et dorsulum valde nitida sed non polita, punctis majusculis (non profundis) rotundatis modice adspersa, viridi-aurea; scutellum, postscutellum gibbum tuberculiforme subconicum obtusum, crasse punctato - reticulata viridi- et cupreoaurea, hoc in centro fuscum. Mesopleurae modice crasse punctata subreticulata; metanotum punctatum et rugulo- 
sum. Abdomen ut in Elampis formatum at ano integerrimo, cupreo-aureum nitidum confertim subtiliter punctulatum, basi macula triangulari aenea viridi-marginata maxima, scil. a basi segmenti 1:mi fere ad apicem segmenti 2:di extensa; segmenti 3:tii margo apicalis summus violascens. Venter nitidus nigro-piceus, segmentis 2:do et 3:tio basi utrinque leviter viridi-micantia. Pedes virescenti-cyanei, tibiarum apices et tarsi brunneo-fusci. 
Hedychridae.

\section{Familia 3. HED YCHATDAE DIbm.}

Mandibulae apice bidentatae: dente apicali angusto curvo longiore, dente interiore brevissimo latissimo nonnihil emarginato.

Thorax subtetraëdro-cylindricus.

Pronotum transverso subquadratum.

Unguiculi tarsorum infra unidenticulati.

Alae anticae cellula medialis latere apicali: oblique I. arcuato - I. angulato - emarginata propter venulam transverso medialem l. arcuato - I. angulato - inflexam. Abdomen segmentis cataphractis conspicuis 3, supra convexum, subtus planiusculum marginatum 1. (praesertim in emortuis et exsiccatis) concavum et subfornicatum; segmenti 3:tii margo apicalis plerisque integer. 


\section{Tabula Synoptica Specierum Generis Hedychri.}

pag. $58-59$.

Sect. I. Corpus violascens, cyanescens, coerulescens aut virescens.

Divis. 1. Abdominis segmenti dorsalis 3: tii margo apicalis trisinuatus et utrinque 1. unispinosus 1. uni-angulatus; sinus laterales centrali distinctiores.

Subdivis. 1. Abdominis segmenti $3:$ tii angulus apicali-lateralis obtusus sinu proximo levi (non profundo). Corpus magnum .. . . . . . . .

Subdivis. 2. Abdominis segmenti $3:$ tii angulus apicali-lateralis spinoideus sinu proximo profundo. Corpus mediocre

30. Hedychrum brasilianum Spin. Brasilia.

31. Hedychrum coelestinum Klug. Europa meridional., Asia minor et Africa.

Divis. 2. Abdominis segmenti dorsalis 3 :tii margo apicalis non sinuatus nec spinosus.

Subdivis. 1. Abdomen nigro-violaceum.

A. Corpus parvam; pronotum et dorsulum polita parce et sparse punctata; abdomen subtiliter modice punctulatum nitidum subpolitum: segmenti 3 : tii margo apicalis arcuatus concolor

B. Corpus mediocre; vertex, pronotum, dorsulum parum nitidum, crebre punctato-subreticulata; abdomen crebre punctulatum non politum; scgmenti $3:$ tii margo apicalis obtusangulus albidus angulis 3 :tio perobsoletis. .

Subdivis. 2. Abdomen (ut etian corpus) cyaneuts aut viride.

A. Corpus mediocre $1 \frac{1}{4}-2 \frac{1}{4}$ lin. decirnal. long.

a) Vertex et thoracis dorsum subcrasse punctato-rugulosa. Pronoti margo anticus fovea centrali longitudinali. Abdomen crebre punctulatum et punctatum. Corpus

b) Vertex, pronotum et dorsulum punctulato-reticulata. Pronoti margo anticus foveola centrali transversa. Abdomea crebre punctulatum. Corpus mediocre.

* Vertex et thoracis dorsum opaca. Abdominis punctura crebrior et distinctior. Corpus cyaneum viridi-pictum et viridi-resplendens. . . . . 35. Hedyclerum chalibaeum Klug. Europ.

** Vertex et thoracis dorsum nilida. Abdominis punctura parcior et subtilior.

Corpus totum viride. . . . . . . . .

c) Vertex, pronotum et mesonotum (nitidiona) punctis et punctulis irregulariter adspersa.

* Abdomen nitidum confertissime punctulatum. Alae apice fumosae . . 37. Hedychrum chloroideum Ziegl. Europa.

**Abdomen politurn parce et subtilissime punctulatum. Alae hyalinae. . . 38. Hedychrum metallicum nob. Fennia.

B. Corpus minuturn. Caput et thorax coriaceo-punctata et punctulata . . . . . 39. Hedychrum Zelleri nob. Europa.

Hedychrum Zimmermanni Dlbm. Americ. Boreal.

34. Hedychrum difficile Spin. Chili.

36. Hedychrum timidum nob. Bengal.

Sect. II. Corpus cyaneo-, viridi - et aureo-variegatum.

Divis. 1. Abdominis segmenta dorsalia $1: \mathrm{m}$ et $2: \mathrm{m}$ virescenti-cuprea, segmentum $3: \mathrm{m}$ cyaneum, Corpus submediocre $1 \frac{4}{5}$ lin, decimal. long. Vertex, pronotum, mesonotum et postscutellum cupreo-aeneo-viridia . . . . . . . . . .

Divis. 2. Abdominis omnia segmenta dorsalia aurata, a colore viridi parcius aut largius 40. Hedychrum anale Klug. Lusitania. imanixto dilutius aut intensius resplendentia. 
Subdivis. 1. Abdominis scgmentum dorsale 2:dum carinula rodiana distincta.

A. MIargo apicalis segmentorum dorsalium $2:$ di et $3:$ tii incrassatus. Corpus $1 \frac{2}{3}$ lin. decimal. long. Caput, pronotum et mesonotura aureo - viridia; abdomen concolor, disco subroseopurpurascente . . . . . . . . . . . . . . . • . . .

B. Margo apicalis segmentorum dorsalium 2 :di mulicus, 3:tii incrassatus. Corpus $1 \frac{1}{3}$ lin. decirnal. long. fere ut in Hedychro aheneo pictum . . . . . . . . .

Subdivis. 2. Abdominis segmenta dorsalia mutica, carinula mediana carent.

1. Pronotum et dorsulum irregnlariter coufertim punctata (non reticulata).

a) Corpus rasimum $3-3 \frac{1}{2}$ lin. decinal. long. valde robustum. Caput et thorax cyaneo-viridi-picta. Abdomen lacte viridi-auratura, confertissime punctatum . .

b) Corpus mediocre $2-2$ lin. decimal. long. modice robustum. Vertcx, pronotum, mesonotum et abdomen cuprea aut cupreo-viridia, hoc modice confertin punctaturm et punctulatum. . . . . .

B. Pronotum et mesonotum regulariter punctato-reticulata.

a) Abdomen punctatum et punctulatum.

* Caput et thorax cyanea plus minus viridi-picta. .

** pronotum aureum.

*** pronotum et dorsulum aurea

**** Vertes, pronotum et mesonotusn aurea.

b) Abdomen confertius subtilius punctulatum. Corpus parvum. Vertex, pronotum et mesonotum 1. viridia 1. aureo-viridia 1. viridi-cuprea. Abdomen viridi-au-

C. Pronotum coriaceurn (subopacum), mesonotum nitidum, sparse punctata. Corpus sub-
45. Hedychrum lucidulum nob. var, a. ) $\left.\begin{array}{ll}\text { var. } b . \\ \text { var. c. }\end{array}\right\}$

46. Hedychrum minutum Pellet. Europa. parsum.

a) Abdomen igueum.

* Vertex, pronotum, nesonotum et scutellum cuprata.

* Caput et thorax purpurascenti-violacea . . :

47. Hedychrum cupratum hlug. Europ.

b) Abdomen cupreum. Caput et pars antica thoracis viridia, nonniliil aureo - aut aenea - picta

I) Vertex, pronotum et mesonotum confertim punctato-coriacea, subopaca.

a) Vertex, pronotum, mesonolum et abdomen sordide aurea. Abdomen subtiliter confertissime punctulatum . . . . . . . . .

b) Vertex, pronotum et mesonotum aureo-rividia. Abdomen subtiliter sparse puactulatum roseo-testaceum, dorso a colore viridi-aeneo sat resplendens. . .

E Vertex, pronotum et mesonotum polita parce punctata. Corpus submediocre 2 lin. decimal. long. Caput, pronotum et mesonotum viridi-aurea - et aenco-picta. Abdomen viridi-aureum aut aureo-viride

48. Hedychrum purpurascens nob. Silesia.

49. Hedychrum inlegrum nob. Europa.

50. Hedychrum coriaceum nob. Europa.

51. Hedychrum femoralum Megerl. Austria.

52. Hedychrum fervidum Fabr. Luropa.

Sect. III. Corpus bicolor: Caput et thorax cyanea, nonnihil viridi-picta. Abdomen roseo-ferrugineum (non auratum). Corpus mediocre

53. Hedychrum roseum liossi. Europa. 
A. Ma13 .

lonsca-

pus 41. Bedychrum ahenenm Ḱlug. Luropa.

B. Malin.

dec . 42. Hedychrum incrassalum Spin. Sicilia

1. Pro

tax

. 43. Hcdychrum virens lílug. Europa.

no-

tim

13. Pre

14. Hedychrum rutilans Mcgenl. Luropa.

45. Hedychrum lucidulum nob.

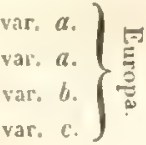

tunı

au-

46. Hedychrum minulum Pellet. Europa.

C. Proub-

par"

47. Hedychrum cupratum Klug. Europ.

-48. Hedychrum purpurascens nob. Silesia. aut

1). $V_{e 1}$

49. Hedychrum integrum nob. Europa.

liter

50. Hedychrum coriaceum nob. Europa. un-

E. Verlin.

51. Hedychrum femoratum Mcgerl. Austria.

decinen

viric, 52. Hedychrum fervidum Fabr. Luropa.

Sect. III.cta.

53. Hedychrum roseum Rossi, Luropa. 
consentaneum videfur fundamento tam instabili Sectiones 1. Sub-Genera construere.

Species Hedychri Generis 23 cognoscimus, scil. 18 Europaeas, 1 Africanam, 1 Asiaticam et 3 Americanas.

Sect. I. Corpus violascens, cyanescens, caerulescens aut virescens.

Divis. 1. Abdominis segmenti dorsalis 3:tii margo apicalis trisinuatus et utrinque aut angulatus aut subspinosus. Alae basi hyalinae, apice sat fumosae.

30. Hedychrum brasilianum Spinola.

Magnum robustum 3 lin. decimal. long. cyaneum parum virescens ventre nigro-piceo, confertim punctatum, pronoti margine antico foveola centrali, abdominis segmenti 3:tii angulo apicali-laterali obtuso sinubus levibus.

Habitat in Brasilia: D. Buquet, Mus. Spinolae.

Observ. Habilu et sculptura Hedychro lucidulo majori maxime affine. Corpus latum cyaneum parum viridiresplendens. Mandibulae apice piceae. Clypeus punctis majoribus adspersum. Caritas facialis mediocris, a stemmatibus sat remota, confertim transverse striata; ceterum totum caput confertim punctato-subreticulatum. Thorax latus omnino ut in Hedychr. lucidulo constructus, totus dorso pleurisque confertim punctato-subreticulatus, punctis pronoti et dorsuli modicis, - scutelli melanoti pleurarumque magnis et crassis. Pedes cyaneo-virides largiter cinereo-pilosi; tibiae posticae angulatac; calcaria tota tarsique apice lestaceo-brunnea. Alarum venae nigro-brun- 
neae; venula transverso-medialis intus lenissime arcuata. Abdominis dorsum confertim: basi punctulatum et apice punctatum; segmenti 3:tii margo apicalis brunneus leniter 3sinuatus: sinus uterque lateralis terminatur ad angulum obtusum. Venter nigro-piceus nitidissimus segmentis ultimo et penultimo disco punctatissimis.

31. Hedychrum caelestinum Klug.

Mediocre aut submagnum 2-3 lin. decimal. long. viride aut viridi-cyaneum ventre concolore, sparse modice punctatum, pronoti margine antico foveola centrali, abdominis segmenti 3:tii angulo apicali-laterali spinoideo sinubus distinctioribus; alis anticis totis brunneis violaceo-resplendentibus, posticis sordidis.

Hedychrum caelestinum King Mus. Berolin.

? Hedychrum amethystinum. Klug Mus. Berolin.

Cymura splendida Dlbm. Dispos. 4. 1.

Habitat in Russia meridionali D. Klug, in Turcia ad Bosphorum D. Hedenborg, in Aegypto D. Waltl, in Africa ad Cap. Bon. Sp. D. D. Drege et Westermann.

Observ. Magnitudine variat: naximum exemplar e regione Bosphori, exemplaria magna ex Africa, mediocria ex Aegypto vidi.

Corpus viridi-cyaneum, interdum dorso toto viride. Caput, et pronotum antice, nonnihil angustiora quam in ceteris speciebus; qua forma Hed. caelestimum Stilbos nonnihil simulat. Mandibulae longiusculae apice brunneae. Clypeus punctulatus, margine apicali truncatus nigro-piceus. Cavitas facialis mediocris, a stemmatibus longe remota, confertim transverse striata. Antennae breves flagellis nigro-fuscis. Frons et reliquae partes ca- 
pitis superficiei confertim modice punctato-subreticulatae. Pronotum et mesonotum nitida punctis modicis crebrius aut parcius adspersa. Metanotum et mesopleurarum pars supra crasse punctato-reticulata. Mesopleurarum pars inferior et metathoracis latera sub angulis spinoideis sita nitidissima confertim transverse striata. Pectus coriaceum. Pedes viridi-cyanei griseo-pilosi, tarsis testaceis aut ferrugineis. Alae anticae venula transverso-medialis intus distincte arcuata, hinc cellula medialis oblique arcuatoemarginata et versus stigma obtuse apiculata. Abdomen supra subtusque cyaneum aut viridi-cyaneum, marginibus segmentorum ventralium plus minus nigro-piceis; dorso depresso-convexum segmentis 1:mo et 2:do sparse punctulatis; segmentum 3:tium modice punctatum margine apicali distincte trisinuato et bispinoso, - sinubus: centrali levi, lateralibus profundis, supra hos angulus spinoideus procedit; segmenta ventralia 2:dum et 3:tium disco subtiliter et confertim punctulata.

Divis. 2. Abdominis segmenti dorsalis 3:tii margo apicalis muticus, nec sinuatus nec spinosus.

32. Hedychrum Zimmermanni Dlbm.

Parvum $1 \frac{1}{4}$ lin. decimal. long. salurate violaceum mesopleuris, scutello, metanoto pedibusque viridi-cyaneis, ventre nigro, pronoto et dorsulo politis perparce punctntis, abdominis margine apicali arcuato, alis hyalinis apice sat fumosis.

Dlbm. Dispos. 1845. 2. 1.

Habitat in New Iersey Americae borealis, D. Zimmermann; qui 3 specimina solita sua benevolentia nobis transmisit. 
Observ. Structura similis Omalo aurato. Corpus latum rotundato-ovatum. Frons et facies cyaneo-rirides confertim punctulatae. Mandibulae apice brunneae. Vertex violaceus subpolitus parce punctulatus. Antennae normaliter pictae. Pronotum et dorsulum polita parce punctulata violacea, dorsuli lobus intermedius nigro-aeneus. Scutellum, metanotum subhemisphaericun, mesopleurae lobus triangularis maximus, crasse punctato-reticulata. Pedes cyaneo-virides tarsis fuscis, horum anteriorum articulis tribus ultimis paullulum dilatatis. Alarum venae nigro-brunneac; venula transverso-medialis distincte arcuato-inflexa, hinc cellula medialis margine apicali distincte arcuato-emarginata. Alarum tegulae nigro-piceae. Abdomen breve latum ovato-rotundatum subhemisphaericum, basi transversum, dorso confertim subtiliter punctulatum subpolitum violaceum limbo interdum cyaneovirescente; venter niger fusco-pubescens.

33. Hedychrum janthinum Draege.

Mediocre 2 lin. decimal. long. capite thoraceque supra aeneo-virescentibus crebre modice punctato-reliculalis, sublus viridi-cyaneis, abdomine nigro-violaceo purpurascente, margine apicali oblusangulo scarioso-albido, alis hyalinis apice sat fumosis.

Inabitat in Africa ad Cap. bon. sp. D. Draege, Mus. D. Drewsen.

Observ. Structura et sculptura affinis et similis $\boldsymbol{H e -}$ dychro roseo. Mandibulac et antennae normales. Clypeus transverso-subsemilunatus. Facies et infera pagina capitis cyanea. Frons et vertex aeneo-virides. Pronotum et dorsulum viridi-aenea crebre modice punctato-reticulata. 
In pronoto fovea mediana perobsoleta. Scutellum dorsulo concolor at punctis majoribus sparsis. Postscutellum et mesopleurae lobus normalis crasse punctato-reticulata viridia; reliquae thoracis partes cyaneae. Pedes cyanci, parum virescentes, tarsis brunneo-testaceis. Alarum tegulae et venae fusco-brunneae; venula transverso-medialis angulato-inflexa, hinc cellula medialis latere apicali angulato-emarginata. Abdominis dorsum crebre punctulatum segmentis 1:mo viridi-cyaneo, 2:do et 3:tio nigro-violaceis purpureo-resplendentibus, 3:tii margine apicali tenuiter scarioso-albido obtusissime et latissime subtriangulari. Venter piceo-niger violaceo-micans, perparce pilosopunctatas.

34. Hedychrum difficile Spinola.

Mediocre vix 2 lin. decimal. long. caeruleum abdomine viridi-cyaneo, thoracis dorso subopaco crebre subcrasse punctato, abdominis segmenti 3:tii margine apicali arcuato-integerrimo, alis hyalinis.

Habitat in Chili D. Gry, Mus. D. Spinolae.

Observ. Corpus robustum sublineare. Caput et thorax caerulea confertim subcrasse punctato-rugulosa subopaca. Pronoti margo anticus foveola centrali longitudinali brevissima. Nandibulae et antennae normales. Thoracis dorsum nommihil convexum. Dorsulum suturis nullis, basi area media centrali semicirculari subtiliter punctulata subnitida et nonnihil virescente. Scutellum, metanotum et mesopleurae lobus normalis crassius punctatoreticulata. Pedes cyaneo-virides griseo-pubescentes tarsis brunneis. Alae albo-hyalinae venis nigris, cellula medialis latere apicali profunde obtuse angulato-emarginata. 
Abdomen supra subtusque viridi-cyaneum, dorso crebre punctulatum.

35. Hedychrum chalybaeum Klug.

Mediocre circa 2 lin. decimal. long. viridi-cyaneum vertice thoraceque opacis confertissime punctulato-reticulalis, abdominis segmenti 3:lii margine apicali utrinque angulo minuto obtuso obsoleto, alis basi hyalinis tum sordidis.

var. $a$. Corpus $2 \frac{1}{4}$ lin. decimal. long. violascenti-cyaneum subchalybaeum, vertice pronoto dorsulo et scutello transversim obsolete viridi-aeneo-pictis.

Chrysis chalybaea Pallas teste Klug.

Hedychrum chalybaeum Klug Mus. Berolin.

var. $b$. 2 lin. decimal. long. Corpus cyaneum, vertice transversaliter inter oculos, pronoti margine antico et postico nec non linea mediana, dorsulo maculis 3 indeterminatis et scutelli disco obsolete viridibus; abdominis discus viridi-resplendens.

Hedychrum caerulans Klug Mus. Berolin.

Hedychrum caerulescens Shuck. Ent. Mag. 17. p. 172. 3.

var. c. vix 2 lin. decimal. long. Vertex, pronotum et dorsulum tota viridia, scutelli discus levissime viridi-pictus. Abdomen viridi-cyaneum.

var. d. $1 \frac{1}{2}-1 \frac{1}{3}$ lin. decimal. long. Vertex, pronotum, dorsulum et scutellum viridia; postscutellum disco levissime viridi-pictum; abdomen cyaneovirescens.

Hedychrum viride Mus. Berolin.

Habitat in Europa media et meridionali mense Julio parce aut raro. In Russia meridionali a D. Pallas de- 
6. Hedychrum.

tectum; in Prussia ad Berolinum D. D. Bouché et Klug; in Silesia ad Glogaviam D. Zeller.

Observ. Structura et sculptura Hedychr. lucidulo ơ simillimum. Corpus fusco- et cinereo-pilosum et pubescens. Frons, vertex, pronotum et dorsulum confertissime punclulato-subreticulata 1. subgranulata opaca subarida. Scutellum postscutellum et mesopleurae lobus normalis modice crasse punctato-reticulata. Thoracis dorsum etiam ut in IIedychr. lucidulo magis depressum quam convexum. Mandibulae apice rufo-piceac. Clypei margo apicalis truncalus nigro-piceus. Cavitas facialis a stemmatibus sat remota, confertim transverse strigosa. Antennae breves normaliter pictne. Pronotum foveolis 2 obsoletis: una marginis antici centrali transversa, altera mediana longitudinali. Pectus totum subtiliter coriaceum. Pedes viridi-cyanei tarsis testaceo-brunneis. Alarum tegulae nigro-fuscae venis 1 . fuscis 1 . brunneis; venula transverso mediali leniter arcuato-inflexa. Abdomen late ovato-obtusum, basi transversum, margine apicali ad utrumque latus angulo minuto obsolelo obtusiusculo, dorso depresso-convexum viridi-cyaneum nitidum confertim punctulatum; scgmentum 3:tium inter discum et apicem ab uno margine laterali ad alterum linea transverso-arcuata minus determinata impressum. Venter nigro-piceus nitidus.

36. IIedychrum timidum Dlbm.

Subparvum $1 \frac{1}{2}$ lin. decimal. long. viride: verlice thoracisque dorso nitidis confertim modice punctulato-reticulatis, abdominis segmenti 3:tii margine apicali utrinque angulo minulissimo absoletissimo, alis basi hyalinis tum sordidis.

II. 
Habilat in Bengalia, D. Westermann, qui specimen unicum anno 1847 mihi amice dedit.

Observ. Valde similis et affinis IIed. chalybaeo, a quo tamen facile dignoscitur: corpore paullulum minore toto viridi, antennarum flagellis basi testaceis, capite thoraceque nonnihil nitidis, thorace minus depresso, punctura thoracis forte minus crassa nec tam arcte conferta quam in Hedychr. chalybaeo, ventre viridi-cyaneo, segmenti 3:tii margine apicali convexius arcuato etc. - Corpus parvum, totum punctulato-reticulatum. Mandibulae rufobrunneae, basi nigro - et viridi-pictae. Antennarum scapi cyanei, flagella basi rufo-testacea, tum fusca. Scutellum, metanotum et mesopleurae lobus normalis crasse punctalo-reticulata. Pedes virides, genubus, tibiarum apicibus tarsisque brunneo-testaceis; alarum tegulae et renae his concolores, tegularum basis virescens; venula transversomedialis modice arcuato-inflexa. Abdomen cyaneo-riride subtiliter sparse punctulatum, segmenti 3:tii uterque angulus apicali-lateralis minutissimus obsoletissimus rix nisi difficile detegendus.

37. Hedychrum chloroideum Ziegl.

Mediocre 2 lin. decimal. long. viridi-cyaneum verlice, pronoto et mesonoto nitidioribus sparse punclatis et punclulatis, abdomine confertissime punctulato margine apicali arcuato integerrimo tenuissime testaceo, alis basi hyalinis tum sordidis.

var. $a$. Corpus viridi-cyaneum colore 1. viridi 1. cyanco superante.

Hedychrum viride Zeller in litucris.

Hedycherum ririllissimum Megerl. Mus. Vienn. teste Köllir. 
var. b. Corporis dorsum cyaneum, abdomine viridicyaneo.

Chrysis chloroidea Ziegl. secundum Spinola in litteris.

Hedychrum clloroideum Mus. Spinolae.

Hedychrum caerulescens Pellet. secund. Klug Mus. Berolin. et secund. Kollar Mus. Vienn.

Elampus caerulescens Klug. Mus. Berolin.

Iabitat in Europa media et meridionali mensibus Majo - Julio passim; Turcia: D. Drewsen; Graecia: D. Loew; Austria: D. D. De Christophori, Kollùr et Megerle von Muillfeld; Silesia: D. Zeller.

Observ. Maxime affinis et similis Hedychr. chalybaeo; at capile latiore, vertice pronoto et dorsulo nitidioribus, punctura harum partiun irregulari et sparsa, venula transverso-mediali profunde arcuato-inflexa etc. distinguitur. - Caput breve, latum vertice amplo, pone oculos superne nomnihil ampliatum. Mandibulae breves robustae aeneae, apice 1 . ante apicem rufo-brunneae. Clypeus brevissimus. Antennae mediocres; scapus antice cyaneus postice virescens, flagella nigro-fusca. Cavilas facialis laevissima nitidissima cyanea subpolita, vix nisi subtilissime coriacea. Frons et vertex confertim punctata. Pronolum breve et mesonotum convexo-depressa nitida sparse punctata et punctulata; pronoti nargo anticus transverse-arcuatus. Dorsulum transverso-rectangulum suturis longitudinalibus subtilibus distinctissimis rectis. Metanotum et mesopleurae lobus normalis crasse punctato-reticulata. Alarum venae fusco-brunneae; cellula medialis margine apicali profunde arcuato-emarginata et versus stigma obtuse apiculata. Abdomen proportionaliter rotundato-ovatum, basi transversum, dorso nitidum et mais convexum quam in Hedychro chalybaeo, confer- 
tissime punctulatum; segmentum 3:tium aequaliter conrexum (nec ut in Medychr. chalybaeo ante apicem linea (ransverso - arcuata impressum), ${ }_{2}$ margo apicalis summus tenuissime testaceus arcuatus integerrimus; interdum vero segmentum 3:tium apice viridi-aureo resplendens. Venter piceo-nigerrimus nitidus. Pedes viridi-cyanei aut cyaneovirides; tibiarum apices, calcaria ct tarsi rufo-testacea.

38. Hedychrum metallicum Dlbm.

Parvum 1! lin. decimal. long. viride, nonnilit cyaneopiclum, verlice pronolo el dorsulo nitidioribus sparse pumctulatis, abdomine polito sparse subtilissime punclulalo, segmenlomum marginibus basalibus nigro-neneis, segmenti 3:lii margine apicali convexo-arcuato, alis hyralinis.

IIabital in Finlandia; unicum exemplar d. 27. Julii 1844 detegit Cel. Nylander.

Observ. Affinis Hedychr. chloroideo var. 6 ; sed corpore minore et magis metallice splendente, abdomine polito subtilissime sparse punctulato, segmentorum marginibus basalibus nigro-aeneis, alis hyalinis etc. mox distinguendum.

Corpus parvum robustum subtiliter cinereo-pubescens. Caput nitidum sparse punctulatum, quemadmodum ut in Hedychr. chloroideo formatum, cyaneum scapis concoloribus, fronte viridi. Antcnnarum flagella longiuscula nigro-fusca. Mandibulac apice brumescentes. Thorax modice convexus. Pronotum et dorsulum nitidiora sparse punclulata laete viridia; dorsuli suturae subtiles distincte $\mathbf{f}$, laterales antice abbreviatae rectae, medianae continuae basi arcuatae; lobus dorsuli intermedius basi area parra transversa subsemilumata nigro-aenea, sublilissime pun- 
6. Hedychrum.

ctulata. Scutellum, metanotum et mesopleurac lobus magnus modice punctato-reticulata viridi-cyanea. Sternum cyancum. Pedes cyaneo-virides, calcaribus tarsisque brunneo-testaceis. Alae hyalinae, ad costam cellulae radialis parumper sordidae, tegulis et venis fusco-brumeis; cellula medialis profunde angulato-emarginata, versus stigma obtuse apiculata propter venulam transverso-medialem profunde obtusangulo-inflexam. Abdomen proportionaliter rotundato-ovatum, basi transversum, margine apicali convexo-arcuatum, dorso modice convexum politum a colore metallico-viridi splendidum atque sparse subtilissime punctulatum, segmentis basi fasciola angusta subsemilunata nigro-aenea. Segmentum 3:tium totum aequaliter convexum; margo apicalis summus tenuissime nigro-brumeus opacus et ad utrumque latus non nisi quam obsoletissime minutiosissimeque angulatus. Venter nitidissimus piceoniger.

39. Hedychrum Zelleri Dlbm.

Minutum $\frac{3}{4}$ vix 1 lin. decimal. long. viride capite thoraceque subtiliter coriaceis conferlim punctulatis subopacis, abdomine subnilido conferlissime sublilissime punchulato, segmenti 3:tii margine apicali convexo-arcuato temissime testaceo, alis subhyalinis.

Dlbm. Dispos. 1845. 2. 2.

Habitat in Silesia, ad Glogaviam d. 28. Augusti 1841 detectum a Cel. Zeller.

Observ. Ad habitum valde affinis Hedychr. minulo; at corpore omnium Chrysidum miuntissimo toto dorso viridi, capite thoraceque subtiliter coriaceis alisque hyalinis facillime dignoscitur. 
Corpus aeruginoso-viride, ventre nigro-piceo politissimo et glaberrimo, tarsis pallide testaceis. Mandibulae et antennae normales, licet proportionaliter tenues. Ciaput et thorax subtiliter confertim punctulata: scutellum, postscutellum et mesopleurae lobus parvus subreticulata. Alae hyalinae, apice levissime umbrosae; tegulae viridi-fuscoaeneac; venae subtiles, teslaceo-brunnescentes; venula transverso-medialis levissime incurva. Abdomen nonnihil nitidum, confertissime et subtilissime punctulatum.

Sect. II. Corpus cyaneo-, viridi - et aureo-variegatum.

Divis. 1. Abdominis segmenta dorsalia I:mum et 2:dun virescenti-cuprea, segmentum 3:tium cyaneum.

40. Hedychrum anale Klug.

Submediocre $1 \frac{4}{5}$ lin. decimal. long. capite thoraceque infra cyaneis supra viridi-cupratis, abdominis segmentis 1:mo el 2:do cupreo- l. subroseo-auratis, 3:lio cyaneo: summo margine temuissimo pallido pellucido, pedibus basi cyaneo-viridibus apice caslaneis, alis anticis sordide hyalinis.

Elampus analis Klug Mus. Berolin.

Habilat in Lusitania, D. Hoffmannsegg Mus. Berolin.

Observ. Habitu non dissimilis speciebus quibusdam generis Spintharis, sed ano mulico mox dignotum; ab affinibus per segmentum 3:tium cyancum facillime distinctum.

Caput rotundato-triangulum angulis temporalibus magnis, infra cyancum, frons concolor. Vertex amplus punclato-rugosus, vix reticulatus, viridi-cuprafus. Stemmala 
exserta majuscula in arcum disposita. Oculi magni ovati exserti. Frons laevis plana, subtiliter coriacea, caerulea, apice nonnihil argenteo-sericen. Antenuae breviusculae sub-angustae, scapus cyaneo-virescens, flagellum nigrofuscum pubescens. Clypeus brevissimus inaequalis virescens, ad centrum marginis apicalis impresso-subtruncatus. Mandibulae nigrae, medio alque toti palpi lacte brunnescentes. Totum pectus cyancum nitidissimum fere absque sculptura. Dorsum thoracis crasse punctatum rugosum rotundato-subreticulatum viride; pronotum, dorsulum, scutellum et postscutellum viridi-cuprata; mesopleurae virides subclathratae. Abdomen confertim punctatum nitidum at non splendidum, cano puberulum; segmenta 1 :mum et 2:dum laete virescenti-cuprea 1 . potius roseo-aurata, illud basi et hoc apice lacte viridi-nitida; segmentum 3:tium cyaneum, ante marginem apicalem linea obsoleta et disco macula obsoleta viridibus; punctura segmentorum anticorum fortior quam segmenti 3:tii, hoc etiam minus nitidum; in segmento 2:do ad certum situm luminis lineola mediana plana subpolita observari potest. Coxac et femora viridi-cyanea; trochanteres nigro-brunnei; genua, tibiae tarsique testaceo-brunnea; tibiae macula viridi-fusca. Alac anticae sordide hyalinae, disco praesertim sordidae; posticae hyalinae. Venter valde fornicatus nigro-piceus.

Jivis. 2. $\Lambda$ bdominis omnia segmenta dorsalia aurata, a colore viridi parcius aut largius immixto dilutius aut intensius resplendentia.

Subdivis. 1. Abdominis segmentum dorsale 2:dum carinula mediana distincta. 
41. Hedychrum aheneum Pallas.

Submediocre $1_{3}^{2}$ lin. decimal. long. pectore el melanolo cyaneis, verlice pronoto et dorsulo aureo-viridibus; abdomine aeneo-viridi, disco leviter obsolete roseo-aurato, segmento 2:do carinula mediana, marginibus segmenti 2:di et 3:lii apicalibus incrassatis.

Chrysis ahenea Pallas teste Klug in Mus. Berolin.

Elampus ałeneus Klug Mus. Berolin.

Iabitat in Russia australi D. Pallas, Mus. Berolin.

Observ. Species propter sculpturam valde singularis et distinctissima. Caput cyaneum. Frons subtiliter coriacea, normaliter concaviuscula, utrinque albo-sericea. Vertex reticulato-punctatus viridis, in regione stemmatum aureo-aenescens. Antennac mediocres normales, scapus infra fuscus, supra cyaneo-virens; flagellum fuscum. Oculi et stemmata normalia. Mandibulae ante apicem palpique testaceo-bruunei. Thoracis dorsum modice crasse punctato-reticulatum; pronoti impressio mediana obsoleta, difficile observanda; mesopleurae subclathratae; peclus totum, pronoti margo anticus, scutelli latera et totus melathorax cyanea; pronotum et dorsulum viridi-aureo-cuprata; sculellum et mesopleurae disco subaurato-viridia. Abdominis dorsum totum confertim reticulato-punctatum; segmentum 1:mum viride, leviter aurco-micans; segmentum 2:dum viride, disco cupreo micans: carinula mediana basi obsoleta, disco apiceque distincta el cum margine apicali incrassato cohacrente figuram $\Perp_{-}$ancorac fere simulante; segmentum 3:tium basi purpureo-violascens, disco viride, apice leviler cupreo 1. aurato - virescens; margo apicalis undato-incrassatus, in centro rudimentum 
carinulae medianae inceptae abruptae (non explicatae) observari potest; margo apicalis summus tenuis pallido subpellucidus. Venter nitidissimus laevissimus nigro-piceus, disco utrinque viridi - maculatus. Pedes cyaneovirides, tibiarun apices et toti tarsi testacei l. sublutescentes. Alac hyalinac, apice leviter sordidac; venis brunneis, tegulis cyaneis.

42. Hedychrum incrassatum Spinola.

Robustum $1_{\frac{1}{2}}^{\frac{1}{2}}$ lin. decimal. long. supra viride leviter aureo-micans, abdominis segmento 2:do carinula mediana subcontinua, segmento 3:lio carinula mediana brevissima antice abrupta el obliterata, margine apicali: segmenti 2:di mutico, segmenti 3:tii incrassato.

Habitat in Sicilia: D. Chiliani, Mus. D. Spinolae.

Observ. Maxime affinis et similis Hedychro aheneo; at corpore magis viridi, tegulis testaceis; segmenti 2:di margine apicali mutico nec incrassato, seginento 3:tio apice carinula mediana distincta, ut diversa species certissime facillimeque dignoscitur.

Caput omnino ut in Hedychro aheneo sculptum et coloralum; at vertex fere totus viridis, nec nisi obsolelissime in regione stemmatum cupreo-nitidiusculum. Thorax cliam candem picturam et sculpturam habet, quam ad Hedycher. aheneum jam descripsimus; ea solum differentia, quod pictura aurata obsoletior et pronoli impressio mediana distinctior obveniunt. Abdomen quoque sculptura et pictura IIedychri ahenei simillima gaudet; at IIedychri incrassati segucutum 2:dum margine apicali mulicum (nec tat in $\boldsymbol{H}$. aheneo incrassatum); segmentum 3:tium apice fortius incrassatum, saturate viridi-cupreum: 
e centro ejusdem marginis excurrit carinula mediana in discum segmenti ducta. Alae hyalinae; tegulae et renae fusco-testaceae; venula transverso-medialis modice arcuato-inflexa; ceterum quod pedes et rentrem attinet, ulla differentia inter IIedychrum aheneum et Hedychrum incrassatum non existit.

Subdivis. 2. Abdominis segmenta dorsalia mutica, carinula mediana carent.

A. Pronotum et dorsulum confertim irregulariter punctata, non regulariter punctato-reticulata.

43. Hedychrum virens Klug.

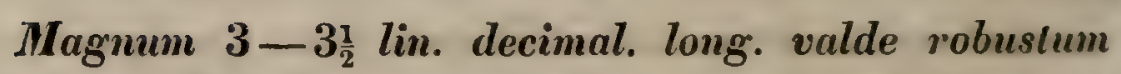
cyaneum abdomine punctatissimo viridi-aurato, alis basi breviter subhyalinis, ceterum totis fumato-fuscis.

Mas vix 3 lin. decimal. long. vertice et pronoto viridibus, abdomine aurato-viridi.

Hedyclirum virens Klug Mus. Berolin.

Femina $3 \frac{1}{2}$ lin. decimal. long. capite thoraceque cyaneis levissime viridi-pictis, abdomine viridi-aurato.

"Hedychrum nov. sp." Mus. Spinolae.

Habilat in Rossia meridionali et Lusitania forte rarissime.

Observ. Mas. Caput normale, subangustum, confertim crasse punctatum cyancum vertice viridi. Mandibulac rufo-brunneae, basi cyanescentes. Clypeus margine apicali gibbus, utrinque transverse concaviusculo-impressus. Palpi maxillaeque lestace-brumescentes. Thorax ronvexo-depressus. Pronolum viride, confertim crasse 
et irregulariter punctatum, antice nonnihil angustatum et hinc late subtrapezinum. Dorsulum minus confertim at paullo crassius irregulariter punctatum, scutellum punctis crassioribus 1. potius foveolis orbiculatis adspersum, postscutellum et mesopleurae rite punctato-reticulata, metanotum punctato-rugosum, - omnia una cum pectore cyanea, disco suturisque leviter viridi-picla. Pedes virescenti-cyanei; femora et tibiae posteriora uno latere, tibiarum omnium apices et omnes totique tarsi fuscobrunnea. Alarum tegulae nigro-fuscae, venae concolores: medialis et submedialis basi testaceae; venula transversomedialis leviter inflexo-arcuata. Abdomen robustum latum modice convexum, totum dorso punctis crassiusculis crebre et irregulariter obsertum 1. quasi cribratum: punctis obliquis haud raro confluentibus; margo apicalis segmenti 3:tii ad utrumque latus nonnihil emarginatus et hinc angulo dentiformi munitus, spatium inter hos dentes convexo-arcuatum et in centro immersum l. subimpressum; segmenta dorsalia viridia, disco aureo-nitida; macula basalis segmenti 1:mi subaenea; segmenti 2:di et 3:tii margo basalis laevis subpolitus tenuiter nigro-piceus. Venter inaequalis, planiusculo-fornicatus, nitidissimus punctis nonnullis adspersus, nigro-piceus, segmentis utrinque ante apicem macula parva viridi-lucida.

Femina quoad sculpturam et structuram $\sigma$ simillima, et solum pictura atque magnitudine dignoscitur; scil. caput et thorax tola cyanea: tantum in regione stemmatum, in pronoti et mesopleurarum disco, in dorsuli utraque parte laterali, nec non in suturis el rugulis metanoti l. maculac I. striolac indeterminatae virides observantur. Abdomen viridi-aurcum; segnenti 1:mi macula basalis, segmentique 
2:di margines basalis et apicalis tenuiter, cupreo-uitidissimi.

44. Hedychrum rutilans Megerl.

Mediocre $2-2 \frac{1}{2}$ lin. decimal. long. modice robustum verlice, pronoto, mesonolo abdomineque viridi-cupreis aut cuprato-viridibus; abdomine confertim modice punctalo et punctulato, alis sordide hyalinis.

var. $a$. circ. $2 \frac{1}{2}$ lin. decimal. long. vertex viridis: fascia ampla aeneo-cuprea; pronotum aeneo-cupreum, antice indeterminate virescens; dorsuli utraque area lateralis 1 . viridi - aurata I. viridi-cuprata, area media basi cuprea: ad centrum marginis basalis 1. viridi-cyanescens 1 . fusco-aenea, apice virescenti-cyanea. Scutellum macula discoidali aeneo-cuprea viridi-limbata. Abdomen viridicupreum.

Hedychrum rutilans Megerl. teste Kollàr in Mus. Vienn.

Hedyclerum fervidum Shuck. Ent. Mag. 17. p. 172; id. Pellet. teste Klug in Mus. Berolin.

Hedychrum speciosum Drewsen in litt.

var. b. 2 lin. decimal. long. vertex viridis, regione stemmatum leviter cupreo-aenescente; pronotum viride, postice obsolete 1 . indeterminate aeneocupratum; dorsuli utraque area lateralis viridis, area media cyanescens: basi utrinque aeneocuprea; scutelli macula discoidalis cupreo-viridis. Abdomen viridi-cupreum.

Hedyclerum aulicum Klug in Mus. Berolin.

var, c. $2 \frac{1}{3}$ lin. decimal. long. vertex viridis, regione stemmatum acnea; pronotum viride et aureo levissime nitens; dorsuli utraque area lateralis viridis 
limbo cyaneo, area media cyanea basi utrinque cupreo-aenea; scutelli discus subaureo-viridis, basi I. concolor l. cupreo-aenescens. Abdomen viridi-cupreum.

Hedychrum regium Pellet. secund. Spin. in litt.

Hedychrum intermedium Mus. Spinolae.

IIabitat in Anglia, Germania, Gallia, Hispania, passim.

Observ. Antea forsitan confusum cum Hedyclero lucidulo o, cui quodammodo simile, at pictura et sculptura constanter dignoscitur. Corpus robustum. Palpi brunnei. Ligula testacea. Mandibulae apice nigro-piceae, ante apicem rufo-testaceac, basi violaceo- et viridiaenescentes. Caput mediocre. Clypeus inaequalis nitidus virescens parce punctulatus, apice nigro-aeneus, margo apicalis ad centrum arcuatus. Antemnae mediocres, scapo cyanescente, flagello nigro-fusco. Cavitas facialis viridicyanea nitida, confertim transverse strigosa. Orbita oculorum, frons et vertex confertim punctata aut punctulata, hic subnitidus I. subopacus. Thorax longiusculus subrectangularis, dorso depresso-convexiusculus. Pronotum magnum, dorsulum illo $\frac{1}{3}$ nisi $\frac{1}{2}$ brevius, transverso - rectangularia, plus minus nitida, irregulariter confertim transverse subrugulosa et confertius irregulariter punctata punctis mediocribus; scutellum punctis majoribus adspersum. Pronotum in centro unarginis antici foveola transversa. Postscutellum, et mesopleurae lobus normalis, crasse punclato-reticulata 1 . potius subclathrata. Metanoti reliquae partes irregulariter rugulosae et punctatae. Pectus et pedes nitida, coriacea, punctulis paucis adspersa viridi-cyanea aut unicolore cyanea; tibiarum apices, calcaria tarsicjue teslaceo-brunnea. Alarum tegulac el renac nigro-fuscae; 
venula transverso-medialis modice arcuato-inflexa. Abdomen unediocre, dorso subdepresso-convexum nitidissimum modice punctatum et punctulatum; segmentum 3:tium ante apicem paullulum immersum, margo apicalis 3: sinuatus: sinus centralis lenissimus parum conspicuus, sinus laterales distinctiores, pone utrumque sinum lateralem procedit angulus parvus distinctus subacutus. Venter piceo-niger politissimus, segmentis disco ulrinque confertim pubescenti-punctulatis.

B. Pronotum et mesonotum regulariter punctato - reticulata.

a) Abdomen punctatum et punctulatum.

45. Hedychrum lucidulum Dlbm.

Robustum submagnum, medium aut parvum $3-1 \frac{1}{4}$ lin. decimal. long. facie, metathorace, pectore, pedibusque ex parte, viridi-cyaneis aut cyaneo-viridibus, tarsis l. fusco- l. rufo-testaceo-brunneis; thoracis pictura dorsali variante; abdominis dorso nitido rubro-aureo plus minus viridi-resplendente, modice punclato et punctulato.

var. a. o 2 lin. decimal. long. pronotum aureum.

var. $b$. \& $1 \frac{1}{4}-3$ lin. decimal. long. Pronotum et dorsulum aurea.

Chrysis lucidula Fabr. E. S. 2. 242. 15; Piez. 174. 24.

Hedychrum lucidulum Latr. Gen. Ins.; Panz. Fn. Germ. 51. 5; Wesmaël Not. 4. 3.

Hedychrum siculum Megerl. Mus. Vienn. teste Kollàr.

Hedychrum fervidum Drewsen in litteris.

var. c. 오 $1 \frac{1}{2}-2$ lin. decimal. long. Vertex, pronotum, dorsulum et scutelli macula basalis magna, aurea. 
Hedychrum lucidulum var. Zeller in litteris.

Hedychrum ardens Latr, teste Klug in Mus. Berolin., Vesmaël Not. 4. 2.

var. d. $\sigma^{7} 1 \frac{1}{4}-3$ lin. decimal. long. Caput et thorax viridi-cyanea aut cyaneo-viridia.

Chrysis regia Fabr. E. S. 2. 233. 19; Piez. 175. 26; Panz. Fn. Germ. 51. 9.

Hedlychrum regium Wesmaël Not. 5. 5.

Hedychrum aulicum Christophori Mus. Spinolse.

Hedychrum congestum Klug Mus. Berolin.

Hedychrum pedatum Klug Mus. Berolin.

Hedychrum viridicolle Klug Mus. Berolin.

Hedychrum alterum Dufour in litt.

Chrysis analis Zeller in litteris.

Mabitant varietates $b$ et $d$ per totam Europam a Turcia meridionali et insulis maris mediterranei usque in Sveciam mediam et meridionalem I. frequenter 1. parce; in Gottlandia has rarietates in copula observavimus. Varietas c raro obvenit Galliae: Latreille, Siciliae et Silesiae: Zeller, Brandenburgiae in horto Berolinensi: Bouché. Varictatem a tantum in insula Rhodo legerunt IIedenborg et Loew.

Cel. Walckenaer, in opere classico Mlémoires sur les IIalictes sequentem magni momenti observationem publicavit: „un des plus faibles de ces ennemis des Halictes „est la Chrysis lucidula, où la mouche dorée brillante; "elle se couche le plus souvent à côté du trou de nos „abeilles (Halictes), derrière le rempart qui l'entoure, et "qui est formé par les parcelles de terre qu'elles ont „retirées du trou. J'ai vu quelques-unes de ces Chrysis „se glisser avec adresse et promptitude dans le trou, mais "je ne les ai point vues sortir. Il arrive encore plus "frequeminent que ces Chrysis sont apperçues par un de 
„nos Halictes, dans la cachette où elles se tapissent; alors „celui-ci plane au-dessus de l'ennemi commun, ce qui „amène un second Halict, et bientôt un troisième, et enfin „un plus grand nombre; toutes alors planent au-dessus „de la Chrysis, qu'elles semblent redouter d'attaquer, „et qui se tient immobile: enfin, lorsque les abeilles „(Halicles) se trouvent suffisamment rassurées par leur "grand nombre, une d'entre elles fond sur la Chrysis, „qui s'enfuit, et que la troupe poursuit alors arec une „, sorte de fureur, et chasse de l'espace qui est le théatre "de son active industrie."

Cel. de Saint Fargeau contendit Hedychrum regium ova deponere in nido Megachilis murariac, quam observationem potius ad historiam Holopygae ovatae 오 referendam esse a sequentibus rationibus existimo: 1) quod Hedychrum regium ( I. Chrysis regia Fabr.) est mas ILedychri luciduli, ut nuperrime demonstravimus, 2) quod mares Chrysidum nunquam visitant nidos, in quibus illorum feminac ova parasitica deponunt, 3) quod Holopyga ovnta a multis Entomologis cum Hedychro regio confusa est.

Observ. var. $a$. ․ Non solum nola antea allata, sed etiam abdominis dorso crebrius et distinctius punctato a reliquis varietatibus dignoscitur. - An propria species?

Var. b. s. normalis. ․ Caput mediocre, confertim punctulato-reticulatum, interdum subgranulatum, viridicyaneum aut cyaneo-viride, fronte verticeque depressoconvexis. Palpi et mandibulac brumescentes, hae basi virescentes. Labrum ovato-obtusum nigro-aeneum pilosopunctatum, parum nitidum. Clypeus viridis nitidus, sub- 
tilissime coriaceus sparse punctatus, disco longitudinaliter convexus, margine apicali undato brunneo ad centrum utrinque sulculo transverso. Cavitas facialis mediocris nitida subtiliter transverse strigosa. Antennae nediocres normaliter pictae. Stemmata in triangulum subaequilateralem disposita; regio stemmatum interdum aureo-subirrorata. Pronotum, mesonotum, postscutellum et mesopleurae lobus normalis regulariter et confertim punctatoreticulata, eo tamen modo, ut sculptura postscutelli crassior quam scutelli et mesopleurae, hujus autem crassior quam pronoti et dorsuli. Color pronoti et dorsuli l. rubrol. viridi-aureus. Metanoti partes laterales oblique rugosae. Scutellum, metathorax et pectus viridi-cyanea, hoc coriaceum et sparse punctatum. Pedes 1 . viridi-cyanei l. cyaneo - virides subtiliter coriacei piloso - punctati, tarsis fusco aut testaceo-brunneis. Alae sordidae, basi et disco plus minus hyalinae, tegulis brunneis interdum basi virescentibus, venis fusco-brunneis, venula transverso-mediali leviter arcuato-inflexa. Abdomen robustum, rotundatoovatum, fusco-pubescens, dorso modice convexum splendidum subpolitum rubro - I. virescenti-aureum, mediocriter punctatum et punctulatum; segmentum 1:mum confertius punctulatum, hujus macula basalis nigro-aenea, interdum segmenti 2:di margo basalis indeterminate concolor; segmentum 3:tium ante apicem paullulum arcuato-immersum; hujus segmenti margo apicalis ad utrumque latus angulo parvo plus minus conspicuo obtuso 1 . acutiusculo munitus, spatium apicale inter ambos angulos situm arcuatum integerrimum. Venter piceo-niger nitidus, confertim fusco-piloso punctatus et punctulatus; segmentum penultimum apice arcuato-emarginatum, ex emarginaturae centro II. 
excurrit apiculus obtusus corneus robustus, minutus aut minutissimus. Valvula analis ventralis semilunata inaequalis: rima mediana subtilissima et obsoletissima.

var. $c$. Simillima var. $a$, nec magnitudine vere diversa; etenim licet individua var. $c$ non aeque magna sunt ac maxima individua rarietatis $b$, tamen permulta varietatis $b$ exemplaria possideo, quae exemplaribus rarietatis $c$ valde minora. Itaque re vera tantummodo per picturam haec varietas $\boldsymbol{c}$ a varietate $\boldsymbol{b}$ dignoscenda; scil. capitis pars suprema (a cavitate faciali in marginem occipitalem sumta), pronotum et dorsulun aurea, scutellum basi macula magna semicirculari aurea viridi-marginala, ceterum cyaneum.

var. d. $\sigma^{\pi}$ magnitudine, et proportionaliter etiam sculptura magis minusve crassa, conferta aut sparsa variat. Caput et thorax plerumque viridi-cyanea, vertice et thoracis dorso colore viridi aut interdum quidem colore aureo et viridi irroratis. Venter muticus absque apiculo (qui ad partes $q$ sexuales pertinet).

b) Abdomen confertius et subtilius punctulatum.

46. Hedychrum minutum Pellet. de St. Farg.

$1 \frac{1}{2}-1 \frac{3}{4}$ lin. decimal. long. thoracis dorso regulariter punctato-reticulato unacum vertice $l$. viridi-aeneove-aurato l. cuprato; abdomine subtiliter confertissime punctulato $l$. viridi-l. cupreo-aurato, segmenti 3:tii margine apicali convexissime arcuato-integervimo, alis sordidis basi hyalinis.

var. $a$. vertex atque discus pronoti et dorsuli viridi- 
aenei, scutellum riride-immaculatum; Abdomen viridi-auratum.

Chrysis integra Dlbm. Mon. Chr. 16. 15; E. H. 35. 20. var. b. Hedychrum integrum Dlbm, Dispos. 3. 5.

var. $b$. vertex atque discus pronoti et dorsuli nec non basis scutelli aeneo - aurata; Abdomen viridiauratum.

Chrysis integra Dlbm. E.H. I. c. var. a.

var. c. vertex, pronotum, dorsulum, scutellum et basis postscutelli cuprata; Abdomen viridi-auratum.

Hedyclırum minutum Pellet. secund. Spinola.

Hedyclirum Duponti Mus. Spinolae (specirnen e Mexico).

Chrysis caeruleo-cephala Megerl. Mus. Vienn. teste Kollàr.

Elampus fervidus Klug Mus. Berolin.

Habitat mensibus Junio - Augusto in locis arenosis nec non in foliis et floribus plantarum per plurimas Europae regiones. Exemplar varietatis $c$ e Mexico a $\mathbf{D}$. Dupont reportatum in Museo Excell. Spinolae ( ,Hedyclirum Duponti Spin." nominatum) examinavi.

Observ. Inter minores Chrysides haec species jure quidem numeranda, licet nullo modo minuta dici potest, quandoquidem longitudo corporis lineam decimalem superat. Mandibulae apice brunneae, basi virescentes. Clypeus et facies 1. virides I. cyanescentes et plus minus nitentes, haec inter clypeum et oculos concaviusculoplana subtilissime confertissime punctulato-coriacea. Frons et vertex confertim punctulata, aliis subreticulata, aliis coriaceo-subgranulosa. Antennae robustae, scapus viridis aut viridi-cyanescens breviusculus, flagellum fuscum. Thoracis dorsum et mesopleurae lobus parvus regulariter punctulato-reticulata, at punctis levibus nec profundis nec 
conferlis, unde reticulatis non in omnibus individuis facile observatur. Pectus et pedes cyanco-virides, tarsis testaceis. Alae ad costam in cellula radiali umbra fumosa: tegulae aeneae; venae tenues brunneo-testaceac, venula transverso-medialis fortiter arcuato-inflexa. $\mathrm{Ab}$ domen mediocre, dorso modice convexum cupreo-auratum limbo 1 amplius 1 . angustius viridi-resplendente, nitidum confertissime subtiliter punctulatum. Venter politissimus nigro-piceus, macula discoidali riridissima.

C. Pronotum coriaceum (sub-opacum), meso notum nitidum, sparse punctata.

47. Hedychrum cupratum Klug.

Subparvum vix 2 lin. decimal. long. supra totum cupreo-igneum: fronte, thoracis margine antico et poslico pectoreque viridibus; pronoto et dorsulo coriaceis opacis, illo crasse, hoc modice sparse punctatis, pedibus aureo-nitidus, alis subsordide hyalinis, in cellula radiali subcompleta fumosis.

Elampus cupratus Klug Mus. Berolin.

Habitat in Italia D. Rossi, in Helvetia D. Kriechbaumer.

Observ. 9. Corpus ut in Hedychro minuto constructum. Caput griseo-pilosellum. Vertex cupreus, aeneomaculatus, punctato-subrugulosus. Frons viridi-subaurata, nitida coriaceo subrugulosa. Antennae mediocres fuscae; scapus e cupreo resplendens. Clypeus 1. medio viridis et utrinque virescenti-aureus 1 . totus cupreus. Mandibulae nigro-piceae, ante apicem laete brumneae, basi cupreae. Palpi fusci. Thorax sparse griseo-pilosellus. 
Pronotum irregulariter coriaceo-rugulosum: pars autica humilior viridis, ad centrum marginis antici foreola profunda nigro-aenea, - pars postica paullulum elevatior aureo - cuprea punctis crassiusculis adspersa. Mesonotum punctis et punctulis adspersum: dorsulum coriaceo-subopacum, scutellum nitidum. Postscutellum conspicue punctato-reticulatum viridi-auratum; mesopleurac simillime sculptae et pictae; reliquae thoracis partes subaureo-virides. Abdomen igneo-cupreum nitidissimum sparse griseo-pilosellum subtiliter punctulatum. Venter inaequaliter concaviusculus nitidus parce pilosello-punctulatus nigro-piceus, segmento 2:do utrinque macula maxima viridi-aurata. Pedes griseo-pilosi, aureo-virides, femora et tibiae ad certum situm luminis a colore cupreo splendidissima observantur. Alarum tegulae cupreo-aeneae nitidae; venae firmae nigro-piceae; venula transversomedialis aurato-inflexa.

48. Hedychrum purpurascens Dlbm.

Robustum subparvum vix 2 lin. decimal. long. capite thoraceque aeneo-purpurascenti-violaceis, abdominis dorso igneo-aurato, pronoto coriaceo subopaco sparse crasse punctato, mesonoto nitido subcrasse punctato, alis hyalinis cellula radiali completa.

Habitat in Silesia; specimen unicum d. 16. Augusto 1840 ad Glogaviam detegit et mihi amice dedit Cel. Zeller.

Observ. Valde similis Hedychro cuprato; at facile dignoscitur: corpore crassiore, capite latiore, vertice non coriaceo sed nitido et distincte punctato, dorsulo nitido distinctius punctato, pedibus non aureis sed viridi-aeneis, 
alarum cellula radiali completa, colore capitis thoracisque aeneo-purpurascenti-violaceo etc.

Corpus sparse cano-pilosellum. Vertex et orbita pone oculos nitida distincte modice punctata 1. punctulata. Frontis punctura subreticulata. Facies subconcariusculo plana punctulato - coriacea, secundum longitudinem medianam subtiliter transverse strigosa. Antennae robustae nigrofuscae, scapus viridi-aeneus. Clypeus depresso-convexus politus semilunatus. Mandibulae aeneac, basi macula virescente, apice piceo-brunneae. Pronotum rite transverso-rectangulum antice sulco transverso, in sulci centro foveola rotundata; maxima pars pronoti punctis rotundatis majusculis adspersa. Mesonotum nitidum depresso-convexum crebre punctatum et punctulatum; dorsulum suturis longitudinalibus: lateralibus obsoletis antice abruptis, medianis profundis continuis nonnihil curvis. Postscutellum et mesopleurae lobus mediocris regulariter punctato-reticulata. Pedes aeneo-virides 1. viridi-aenei: tibiis apice, calcaribus tarsisque testaceis. Alae hyalinae tegulis aeneis, venis tenuibus fuscis, venula transversomediali fortiter arcuato-inflexa, cellula radiali completa. Abdomen mediocre, late ovatum, apice fortissime arcuatum integerrimum, dorso igneo-auratum et subtiliter confertim punctulatum. Venter politissimus sparse pilosellopunctulatus nigro-piceus, segmento 2:do macula centrali mediocri viridi-aurata.

49. Hedychrum integrum Dlbm.

Robustum parvum vix $1 \frac{1}{2}$ lin. decimal. long. capite thoraceque viridi-aenescentibus, abdominis dorso cupreoaurato, pronoto coriaceo parum nilido irregulariter parce 
punctato, mesonoto polito parce punctulato, alis hyalinis apice leviter sordidis, cellula radiali incompleta.

Clirysis integra Dlbm. E. H. 35. 20. var. $c$.

Hedychrum cupreum Dlbm. Dispos. 3. 4; quod nomen rejeci, ne propter denominationem consonantem haec species cum Hedychro cuprato confundetur.

Habitat in Europae borealis locis campestribus arenosis soli urenti expositis, a medio Julii ad finem Augusti passim. In Scania, Ostrogothia, Gottlandia, Helsingia, nec non in jugo alpino Norwegiae prope sinum Lewangerfjord mihi obvia. Ad Uleåborg Finlandiae a D. Nylander lecta.

Observ. Maxime affine Hedychro minuto; at certe dignoscitur: pronoto coriaceo sparse punctato (non reticulato), mesonoto polito parce et irregulariter punctulato, abdomine cupreo et multo subtilius - immo! microscopice (at non confertissime) punctulato.

Corpus sparse et longe cano pilosum. Caput nitidum aeneo-virens modice punctulatum; vertex cupreo-aeneus; stemmata magna in triangulum aequilateralem approximata; oculi mediocres; frons punctato-rugulosa; facies viridicyanescens planiuscula, subtiliter et confertim transverse strigosa. Antennae mediocres fuscae, scapus aeneus. Clypeus nitidus viridis, apice truncatus. Mandibulae robustae virescenti-aeneae, parte apicali late rufo-testacea. Palpi brunnescentes. Pronotum depresso-convexum parum nitidum punctulato-coriaceum, viride, disco l. postice plus minus aenescens, lateribus et parte postica punctis majusculis rotundatis adspersis; antice ante marginem sulculo transverso obsoletiori; in centro marginis antici foveola obsoleta rotundata. Mesonotum politum punctis 
et punctulis irregulariter adspersum, viridi- 1. cupreoaeneum. Dorsulum suturis longitudinalibus \& omnino ut in Hedychr. purpurascenti constructis. Postscutellum riride punctato-reticulatum, reliqua pars metanoti irregulariter subclathrata viridi-fusca. Mesopleurae lobus parvus, confertim coriaceus punctis nonnullis crassis, viridis. Pectus subtiliter coriaceum fusco-rirescens. Pedes aenei, nonnibil viridi-nitentes, longe et parce pallido-pilosi, tibiis apice, calcaribus tarsisque rufo-testaceis. Alae subhyalinae apice leviter sordidac, in cellula radiali incompletissima observatur umbra linearis nonnibil fumosa; tegulae fusco-brunneae subaenescentes; renae tenues fusco-testaceae, costa et stigmate fusco-brunneis subpiceis, venula transverso-mediali fortiter arcuato-inflexa. Abdomen mediocre ovato-rotundatum, limbo parce et longe albido-pilosum, apice integerrimum, dorso modice convexum politum cupreum confertim microscopice punclulatum; venter nigro-piceus nitidus sparse piloso-punclulatus, segmento 2:do macula centrali mediocri viridiaurata.

D. Vertex, pronotum et mesonotum confertim punctulato-coriacea subopaca.

50. Hedychrum coriaceum Dlbm.

Parvum $1 \frac{1}{2}$ lin. decimal. long. vertice pronoto mesonoto abdominisque dorso sordide viridi-auratis, hoc confertissime subtilissime punctulato, pedibus viridi-cyaneis. larsis testaceis, alis sordide hyalinis.

Habitat in Italia et Finlandia. Tantum 3 specimina vidi, 2 in Italia a I). Zeller et unicum ad Helsingfors Finlandiae a D. Nylander detecta. 
Observ. Magnitudine, habitu et pictura valde simile et affine Hedychri minuti varietati $c$; at primo intuitu dignoscitur sculptura capitis et thoracis, scil. caput et thoracis dorsum confertissime punctulato-coriaceo-opaca. Facies cyanea concaviusculo-plana, pone scapos subtilissime transverse strigosa, lineola mediana elevata. Antennae robustae: scapus nigro-aeneus, flagellum nigrofuscum. Clypeus nitidus viridis punctulatus, apice subtruncatus. Mandibulae robustae, viridi-nigroque aeneac, apice rufo-testaceae. Labium, palpi maxillaeque nigropicea. Pronotum et mesonotum confertissime subtiliter punctulato-coriacea subgranulosa opaca. Pronotum margine antico concaviusculo-depresso aeneo-viride, sulculo mediano longitudinali profundo postice abrupto; disco punctulis nonnullis rotundatis, non profundis, subnitidis hinc inde adspersum. Scutelli margo posticus viridis. Metathorax cyaneus, postscutellum punctato tenuiter reticulatum. Pectus viridi-cyaneum; mesopleurae lobus mediocris punctato tenuiter reticulatus. Pedes viridicyanei griseo-puberuli, tibiis apice, calcaribus tarsisque rufo-testaceis. Alarum tegulae aeneae; venac fuscobrunnescentes mediocres, venula transverso-medialis profunde arcuato-inflexa. Abdomen rotundato-ovatum, dorso parum nitidum, confertissime subtiliter punctulatum sordide aureum, limbo nonnihil viridi-resplendente; venter nigro-piceus politus nitidissimus, segmento 2:do 1 . toto concolore 1. macula parva centrali viridissima simplici aut gemina. 
51. Hedychrum femoratum Megerl.

Parvum $1 \frac{1}{2}$ lin. decimal. long. vertice pronoto el mesonoto aureo-viridibus, abdomine roseo-ferrugineo dorso conferlim subtiliter punctulato, segmentis limbo apicali ex aureo-viridi splendidis, pedibus rufo-testaceis basi viridi- et nigro-aeneis, alis sordide hyalinis.

Hedychrum femoratum Megerl. Mus. Vienn. teste Kollar.

Habitat in Austria; specimen unicum ad Viennam ab Illustr. Megerle von Miihlfeld olim detectum, amice communicavit Cel. Kollàr.

Observ. Locum systematicum habeat haec species optimum inter Hedychrum coriaceum et Hedychrum roseum, utrique affinis et ab utroque diversus. Vertex et thoracis dorsum confertissime punctulato-coriacea subgranulosa opaca aureo-viridia. Frons, facies et clypeus saturate cyanea 1 . violacea, ita ut in Hedychr. coriaceo constructa; mandibulae, antennae et trophi etiam hujus speciei. Pronotum antice concaviusculo truncatum, sulculo mediano longitudinali abrupto, utrinque ad sulcum lineola transversa elevata subtiliori. Postscutellum cyaneum et mesopleurae lobus parvus punctato-tenuiter reticulata. Metathorax et pectus viridi-cyanea coriacea. Alae subsordide hyalinae, venis fuscis subgracilibus, venula transverso-mediali profunde arcuato-inflexa, tegulis brunneis. Venter rufo- 1. roseo-testaceus politissimus.

E. Vertex, pronotum et mesonotum polita parce punctata.

52. Hedychrum fervidum Fabr.

Mediocre 2 lin. decimal. long. robustum politum modice punctatum $l$. rubro- $l$. viridi-auratum metathorace 
6. Hedychrum.

pectoreque cyanescentibus, ventre nigro-piceo, alis sordide hyalinis.

Chrysis férvida Fabr. Piez. 175. 23. secundum specimina 5 typica, quae in Museo Kiel. anno 1847 examinavi.

Hedychrum fervidum Megerl. Mus. Vienn. teste Kollàr; VVesmaël Not. 5. 4.

Hedychrum nitidum Pellet. Mus, Spinolae.

Hedychrum candens Klng sec. Zeller in litteris.

Hedyclerum laetum Zell. in litteris.

Elampus candens Klug Mus. Berolin.

Habitat in Italia et Silesia: D. Zeller; - Belgia prope Bruxellam: D. Hannon, teste D. Wesmaël I. c.; - Gallia: D. Latreille, Mus. D. Spinolae; - Austria: D. D. Drewsen, Kollìv et Megerle von Mühlfeld.

Observ. In serie Hedychrorum systematica certe infimum locum tenet baec species, etenim toto habitu nec non structura corporis partium speciebus Holopygarum nimis affinis; exceptis tarsorum unguiculis infra ita unidenticulatis ut apud Hedychra omnia.

Corpus totum ovatum, parce et breviter fusco-hirtellum. Caput breve robustum triangulariter orbiculatum, viridi-aut cupreo-aureum, politum, sparse punctulatum. Caritas facialis profunda violacea subtilissime transverse strigosa. Antennae longitudine mediocres, robustae, nigro-aeneae, basi nonnihil purpurascentes. Clypeus viridipurpureo- aut nigro-aeneus, ad centrum marginis apicalis emarginulato-arcuatus. Mandibulae robustae fuscoaeneae nitidae, basi macula viridi, apice rufo-testaceae 1. rufo-piceae. Maxillae, palpi et labium nigro-brunnea nitida. Thorax truncato-ovatus convexus, antice nonnihil declivis; pronotum et mesonotum polita, I. viridi- 1. cupreo-aurata, parce modice punctata; scutelli discus lac- 
vissimus (absque punctis). Metanotum et lobus mesopleurae submagnus crasse punctato-reticulata. Dorsulum suturis longitudinalibus quatuor, omnibus distinctis et subprofundis, at non crassis: lateralibus ut solito antice abruptis, medianis continuis antice - hamulorum instar arcuatis; lobus dorsuli intermedius basi area transrerso lineari parva, confertissime et subtilissime punctulata. Pectus cyaneum nitidum. Pedes 1. viridi-1. cyaneo-1. nigro-aenei, plus minus purpurascentes, calcaribus et unguiculis rufo-testaceis; femora parce pilosa; tibiae et tarsi confertissime cano-1. griseo-pubescentes, pube sericeonitida adpressa rigida. Alae sordidae, basi hyalinae, tegulis magnis convexis politis, venis mediocribus fuscobrunneis, renula transverso-mediali profunde subangulato inflexa. Abdomen longiusculum ovatum, basi transversum, ano deflexo, dorso nitidum punctatissimum l. viridi- 1. cupreo-aureum, rarius aureo-viride, segmentorum margines apicales semper fusco-brunnei. Venter nigro-piceus politissimus nitidissimus, parce pilosello-punctulatus.

Picturam ita variantem observavi, scil.

var. a. Caput, pronotum, mesonotum et abdomen cupreo-aurea plus minus viridi-resplendentia.

var. $b$. Caput, pronotum abdominisque dorsum aureoviridissima; dorsulum cupreo-aureum lobo intermedio basi viridi; scutellum violaceo-purpurascens. 
Sect. III. Corpus bicolor: caput et thorax cyanea plus minus viridi-picta; abdomen roseo-ferrugineum (non auratum).

53. Hedychrum roseum Rossi.

Mediocre 2 lin. decimal. long. viridi-cyaneum aut violascens confertim punctatum subopacum, abdomine roseo-testaceo l. roseo-ferrugineo confertim punctulato nitido.

Chrysis rosea Ross. Mant. Ins. 132. 290; Fn. Etr. 2. Tab. 8. fig. 7; Dlbm. E. H. 31. 14.

Clirysis rufa Panz. Fn. Germ. 79. 16; Jur. Hym. 297.

Hedychrum roseum Lepel, Ann. du MLs. VII, 123. 13; Wesmä̈l Not. 4. 1; Dlbm. Dispos. 2. 3.

Chrysis Rosae Dlbm. Mon. Chrys. 13. 11.

Habitat in Europae locis argillosis aridis optima aestate passim; intra Scandinaviam adbuc non altius quam inter $59^{\circ}$ et $60^{\circ}$ latitudinis borealis observata.

Observ. Corpus fusco- et griseo-pubescens, pilosum et hirtum. Caput et thorax ut in Chrysidibus genuinis constructa, confertim punctata subreticulata, I. viridi-cyanea l. cyanea 1. violacea. Mandibulae acneae, ante apicem rufo-testaceae. Clypeus depresso planus politus, margine apicali truncatus et marginulatus. Antennae mediocres nigro-fuscae. Facies pone scapos leviter concaviuscula subtiliter transverse strigosa, canalicula mediana subtilissima nitida. Pronotum fovea mediana antice profunda, postice versus marginem obliterata. Dorsulum suturis medianis 2 profundis continuis, ad utrunque latus lineola elevata subpolita continua, plus minus distincta. Pectus nitidum sparse punctatum. Pedes viridi-cyanci, tibiis apice, calcaribus 
6. Hedychrum.

tarsisque totis ferrugineis. Alae sordidae, basi hyalinae, tegulis et venis fusco-brunneis, venula transverso-mediali profunde arcuato-inflexa. Abdomen late rotundato-oratum, plus minus nitidum griseo-pubescens, roseo-testaceum aut roseo-ferrugineum, dorso confertim punctulatum. Venter politus nitidus parce piloso-punctulatus, segmentis 1:mo et 2:do apice, 3:tio ad maximam partem nigro-piceis. 


\section{Familia 4. CHRYSIDIDAE Dlbm.}

Mandibulae apice bi- aut tri-denticulatae, dente apicali angusto paullulum curvo longiore.

Thorax subtetraëdro-cylindricus; pronotum transverso subrectangulum, margine postico l. transverso l. emarginato-arcuato; postscutellum aliis muticum et l. planum 1 . convexum, aliis tuberculiforme, aliis conicum, aliis fortiter mucronatum.

Tarsorum unguiculi infra mutici (nec dentati, ut in Hedychridis; nec serrati, ut in Elampidis).

Alae anticae cellula medialis latere apicali l. oblique secta 1. oblique et leniter arcuato-emarginata propter venulam transverso-medialem l. oblique rectam l. oblique et leniter arcuato-inflexam.

Abdomen segmentis cataphractis conspicuis 3, supra convexum, subtus aut planiusculum et marginatum aut concavum et subfornicatum; segmenti dorsalis 3:tii margo apicalis varie formatus: 1. muticus, 1. undulatus, I. angulatus, I. dentatus et tunc dentibus obtusis aut acutis 1-6 armatus. 
Hanc Familiam, ex Ordine Hymenopterorum Chrysidiformium speciebus locupletissimam, in plura Genera divisam habere voluerunt Systematici, cujus rei`exempla sunt Chrysura, Cymura, Chrysis, Platycoelia, Pyrosoma, Poeciloechroa, Pyria, Nemophora, Spintharis, Stilbum, et forsitan adhuc plura. Sed caracteres, pro illis enumeratis Generibus condendis et stabiliendis, propositi 1. adeo absconditi fuerunt, 1. per formas partium luxuriose rariabiles adeo confluentes aut evanescentes, ut Geuera ejusmodi fundamentis enixa potius ad confusionem quam ad explicationem Systematis ducant. Igitur quaestio de copia Generum hujus familiae omnino nihil valere nobis videtur. Et re vera tantummodo duo 1. ad maximum tria Genera in hac familia ostendit ipsa natura, scil. Chrysidem Latr., Spintharem Kl. (sensu strictiori) et Stilbum Latr. 


\section{Tabula Synoptica Generum Chrysididarum.}

A. Postscutellum liberum, h. e. sub scutello non ulla parte absconditum.

a) Abdominis segmenti dorsalis $3:$ tii margo apicalis normalis, h. c. leviter I. (ad maximum) mediocriter prominulus et saepissime non aliter compositus quam ipsum segmentum.

b) Abdominis segmenti dorsalis 3 :tii 7. Genus Chrysis Lin., Fabr., Latr. p. margo apicalis eximie prominens, pellucidus submembranaceus aut subcoriaceus, non crustaceus adeoque aliter compositus quam ipsum segmentum.

8. Genus Spintharis Kl. p.

B. Postscutelli tota pars basalis sub scutello abscondita et solum pars apicalis instar mucronis validi exarati procedens

9. Genus Stilbum Fabr., p.

Latr.

II. 


\section{Adumbrationes in Chrysididarum Genera et Species.}

7. Genus CHPYSIS Lin. p., Fabr. p., Latr. p.

Corpus 1. parvum, 1. mediocre, I. magnum, 1. maximum, 3 millimetr. $-\frac{1}{2}$ pollic. decimal. long. et proportionaliter l. angustum 1 . robustum et crassum.

Caput plerisque mediocre, I. rotundato - triangulare 1. orbiculatum, rarius subquadratum et maximum.

Mandibulae mediocres, plerumque ut in specicbus Generum praecedentium formatae; tantum in unica (quantum cognoscimus, scil. Chr. Ehrenbergi) specie maximae elongatae et subampliatae, quemadmodum in Passaloecis Pemphredonidarum; apice bi- aut tridenticulatac.

Abdomen ovatum, ovale, ellipticum, lineare, subrectangulum aut denique subquadratum, angulis tumc rotundatoobtusis; segmenti dorsalis 3:tii margo apicalis aut muticus et inermis, ant undulato-angulatore inaequalis, aut 1-, 2-, 3-, 4-, 5-, 6-dentatus.

Alae anticae cellulis completis: costali, mediali, et discoidali 1:ma; cell. radiali et submediali 1:ma 1. completis 1. subcompletis 1 . apertis et incompletis; cell. cubitali 1:ma rarissime completa, plerumque incompleta, submedialis 2 :da incompleta.

Alae posticae tantummodo vena costali, postcostali et cellula costali completis gaudent; reliquarum renarum et cellularum solum vestigia obsoleta aut obsoletissima animadvertuntur.

Species Chrysidis Generis circ. $110-120$ cognoscimus, scil. 50-60 Europaeas, reliquas I. Africanas, I. Asia- 
ticas, I. Americanas; quae omnes secundum inermitatem aut armaturam abdominis analem ita commode in 8 Phalanges disponi possunt ut obveniunt in

Phal. I:ma. Chrysides ano integerrimae, ex. gr. Chr. incrassata, Chr.Humboldti, Chr.austriaca, Chr. integrella, Chr. coerulipes, etc.

Phal. II: da. Clhrysides ano inaequales, ex. gr. Chr. elegans, Chr. versicolor, Chr. crassimargo, Chr, unicolor, etc.

Phal. III:tia. Chrysides ano uni-dentatae, ex. gr. Chr. succinclula, Chr. Leachei.

Phal. IV:ta. Chrysides ano bi-dentatae, ex. gr. Chr. bihamata, Chr. prasina.

Phal. V:ta. Chrysides ano tri-dentatae, ex. Chr. Bohemanni, Chr. cyanea, Chr.parvula, etc.

Phal. VI:ta. Chrysides ano quadri-dentatae, ex. gr. Chr. fasciata, Chr. chiliensis, Chr. coevulans, Chr. nitidula, Chr. fulgida, Chr. bi-dentata, Chr. succincta, Chr. ignita, etc.

Phal. VII:ma. Chrysides ano quinque-dentatae, ex. gr. Chr. lusca.

Phal. VIII:va. Chrysides ano sex-dentatae, ex. gr. Chr. splendens, Chr. Wahlbergi, Chr. 6-dentata, etc.

Ut vero Lectores hac dispositione co facilius et commodius uti possint, propriam Tabulam Synopticam ante descriptionem specierum singulae cujusque talis Phalangis, qualis speciebus abundantis, offerre voluimus; Phalangibus autem 3:tiae, 4:tae et 7:mae, ut species modo perpaucas continentibus, proprias Tabulas Synopticas adjicere haud necessarium duximus. 
Phalanx I. Chrysides ano integerrimue. Scil. Abdominis segmenti 3:tii margo apicalis integerrimus.

Sectio I. Corpus cyaneo - et viridi-variegatum.

54. Chrysis capitalis Dlbm.

Mediocris $2 \frac{1}{2}$ lin. decimal. long. crasse punctata fuscopubescens viridis vertice, dorsuli area intermedia abdominisque segmentis 2:do parte basali 3:tioque saturate cyaneis, flagellis uno latere tarsisque totis testaceis, alis sordide hyalinis, segmenti 3 :tii serie ante apicali irregulari foveolis majoribus et minoribus alternis.

Habitat in Africa meridionali; ad Cap. B. Sp. a D. Westermann detecta.

Observ. Magnitudine et habitu nonnihil similis minoribus exemplaribus Chrysidis nitidulae. Corpus quemadmodum Chrys. ignitae oblongum et punctatum. Caput viride, supra crebre punctatum, macula verticis nigro-riolacea; infra stemmata et supra cavitatem facialem adest area transversa linearis nitida inaequalis aureo - viridis margine elevato fusco undique circumdata; cavitas facialis viridis nitida, lateribus coriacea punctulato-subgranulosa, disco longitudinali confertissime transverse strigosa. Antennae subbreves robustae; scapus et pedicellum subcyanescenti-viridia, flagellum rufo-testaceum postice nigrolineatum, disco nonnihil crassius quam basi et apice unde subfusiforme obvenit. Clypeus mediocris nitidus viridis sparse punctatus, ad centrum marginis apicalis truncatus et aureo-resplendens. Mandibulae piceo-brunneae, basi macula virescenti-aurea. Thorax longiusculus subcylin- 


\section{a sistens Synopsin}

rum Generis Chrysidis. pag. 100 et 101.

Si

majoribus rotundatis et

54. Chrysis capitalis nob. Africa.

diocre.

i separatis aequalibus sub. jugiformi . . . .55. Chrysis Jugum nob. Africa.

1 rotundis numerosis con-

.56. Clirysis hilaris nob. America.

rvum.

is profundiusculis. . 57. Chrysis tarsata K1. Germania.

Se.

. . . . . 58. Chrysis basalis nob. Africa.

ham transverse aureo - et

x. cyancus aureo-viridi-

saliter concaviusculo-im-

tata, saturate aurea; se-

. . . . . . . 59. Clirysis incrassata Kl. Europ. merid.

e aurata; segraenti 3 :tii

-60. Chrysis cyanura Pall. Rossia merid.

ec ante marginem callo-

btica saturate aurea . 61. Chrysis Humboldti Dlbru. Rhodus.

: tium concolor, nec nisi

pictura cyanea aut cya-

te basali paullulum con-

is tenuis transversus . 62. Chrysis transversa nob. Graecia.

ric punctorum paullulum

nter cyaneus; postscutel-

. . . . . . 63. Chrysis gastrica Kl. Lusitania.

nico-prominulum.

. . . . . . 64. Chrysis sulcata Dlbm. Rhodus.

a . . . . . 65. Chrysis varicornis Spin. Hispan. Aegyp 


\section{Tabula Synoptic I: ma sistens Synopsin \\ Phalangis I:mae Specierum nobis ognitarum Generis Chrysidis. pag. 100 et 101.}

\section{Sectio I. Corpus cyaneo - et viridi-variegatum.}

A. Corpus crasse punctatum. Alae sordidae. Corpus medic $\mathrm{e}$

Abdominis segmenti 3 :tii series ante-apicalis irreg ${ }^{4}{ }^{\text {ris }}$ e foveolis majoribus rotundatis et minoribus subalternantibus

B. Corpus modice punctatum. Alae hyalinac.

a) Abdominis segmenti 3 : tii margo apicalis immariatus. Corpus mediocre.

1. Abdominis segmenti 3 : tii series ante-apicis regularis foveolis separatis aequalibus sub-

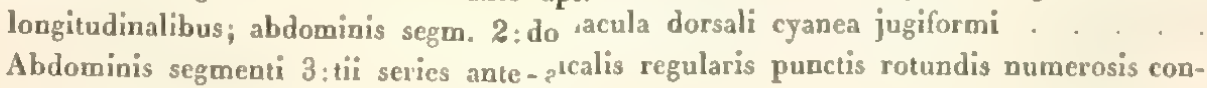

fertis. Corpus viridissimum immacularm

b) Abdominis segmenti 3 : tii margo apicalis igro-aeneus. Corpus parvum. Abdominis segmenti $3:$ tii series an-apicalis irregularis punctis profundiusculis .

54. Chrysis capitalis nob. Africa.

55. Chrysis Jugum nob. Africa.

56. Chrysis hilaris nob. America.

57. Chrysis tarsata Kl. Germania.

Sectio II. Corpus cyaneo-, viridi-, aene- et aureo-variegatum.

Divis. 1. Abdominis dorsura aureum, segmentu 1:mura viridi-cyaneum

Divis. 2. Abdominis dorsum aureum; segmenum 3 :tium cyaneum, nonnunquam transverse aurco- et viridi-striatum, ante seriem apicalm calloso-marginatum. Thorax cyancus aureo-viridimaculatus.

A. Abdominis segmentum 3 :tium, ante marginem callosum, transversaliter concaviusculo-immersum; segmenta duo antica punctato-coriacea.

a) Abdominis segmenta dio antica modice et confertion punctata, saturate aurea; se-

b) Abdominis segmenta duo antica crasse punctata, viridissime aurata; segmenti $3:$ ti pars basalis crasse panctata . . . . . . . . . . . .60. Clrysis cyanura Pall. Rossia merid.

B. Abdominis segrocntum 3:tum parte basali depresso-convexum nec ante marginem callosum, concavo-immersum, callus formose iricolor; segmenta duo antica saturate aurea . 61, Chrysis Humboldti Dlbm. Rhodus.

Divis. 3. Abdominis dorsum aureum, plus minus viridi-micans; segmentum $3:$ tium concolor, nec nisi summo margine apicali rarius aeneo- aut purpurascenti-fusco.

Subdivis. 1. Thorax nunquam aureus nec aureo-fasciatus, sed aut viridis pictura cyanea ant cyaneus pictura aurato-viridi,

Tribus 1. Venter cyaneus aut viridis plus minus nigro-pictus.

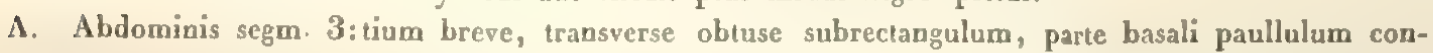
caviusculo-immersum, hinc ante seriem marginulo calloso, margo apicalis tenuis transversus . .62. Chrysis transversa nob. Graecia.

B. Abdominis segm. 3:tiurn mediocre, semilunatum convexum, apice sub serie punctorum paullulum incrassatum.

a) Abdominis dorsum viridissine auratum 1. potius aurato-viride; venter cyaneus; postscutel-

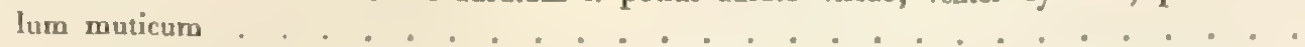

b) Abdominis dorsum igneo-aurcum; venter viridis; postscutellum conico-prominulum.

1. Antennarum flagella nigro-fusca ............... . 64. Chrysis sulcata Dlbm. Rhodus.

2. Antenuarum flagella medio alternatim albo- et gigro-annulata . . . . . . .65. Chrysis varicornis Spin. Hispan. Aegypt. 
Tribus 2. Veuter igneo-pictus.

Manipulus 1. Abdom. segm. 3:tii margo apicalis sub serie paullu m incrassatus, series anteapicalis irregularis foveolis parvis orbiculatis nigrisine inde confluentibus

Manipulus 2. Abdom. segmenti 3 : tii margo apicalis muticus, $\mathrm{h}_{1}$ incrassatus.

A. Abdom. segm. 3:tium apice parunper angustius quam basi, margo ap lis subtransversus.

a) Abdomen politum sparse punctatum, segmentum 3 :tium ad margine apicalem utrinque angulo obtuso, series ante-apicalis distincta, Corpus magnum. Caput bidi-cyaneum.

b) Abdomen confertissime punctulato-coriaceum subopacum, segmenti 3:tii mar apicalis utrinque arcuato-obtusus, series ante-apicalis perobsoleta. Corpus mediocre aut subparv $n$ fronte aurea.

B. Abdom. segmentum 3 :tium apice distincte et conspicue angustius quam basi.

a) Segmenti $3:$ tii margo apicalis ad medium transversus.

Segmentum abdom. 3: tium punctato-coriaceum, series ante-apicalis I. Igfundior et

66. Chrysis ruficentris nob. MIs. Pajkull

67. Chrysis atestriaca Fabr, Europa.

68. Chrysis aurifrons Kil. Graccia b) Segmenti $3:$ tii margo apicalis totus perfectissime arcuatus.

1. Series punctorum ante-apicalis (in abdom, segmento $3: t_{0}$ ) obsoleta aut obschissima.

* Abdomen confertim modice punctatum nitidum virescenti-aureura; postscutellum muticum.

** Abdomen confertissime punctulato-coriaceum subopacum coccineo-aureum; postscutellum obtu. . nicum I. potius tuberculiforme

2. Series punctorum ante-apicalis (in abdom, segmento $3:$ tio) distinctissima.

69. Chrysis bicolor Dlbru. Luropa

70. Chrysis simplex Kl. Europ. merid.

71. Chrysis aerala nob. Helvetia.

* Abdomen confertim crasse punctatum igneo-auratum. Corpus magnum. Abdominis segraentum 3:tilt. longiusrulum, apice subanguste 1. subelliptice arcuatum, margine apicali surno purpurascenti nigro acneo, Postscutellum conicum .

* Abdomen confertissime punctulato-coriaceum viridi-auratum. Corpus mediocre Abdomin tum 3 : tium semicirculariter arcuatum, margine apicoli

Subdivis. 2. Thorax aut aureo- fasciaus aut antica et

Tribus 1. Vertex, pronolum,

Tribus 2. Vertex, pronotum,

Tribus 3. Abdomen cupreo - aut igneo-aureum.

foveolis paucis segmenti abdom. 3:tii viridi-aeneus; series ante-apicalis anticus et

Manipulus 2. Margo apicalis segmenti abdom. 3:tii I. nigro-aeneus 1. cyaneus; scries ante-apicalis foveolis multis mediocribus. Frons, pronotum et dorsuli areac laterales aurca. Corpus parvum: . . . . pronotim et dorsuli

Manipulus 3. Margo apicalis segmenti abdon. 3:tii imanculatus, dorso abdon. concolor.

A. Dorsulum crasse punctatum.

a) Postscutellum acute conicum. (Caput, pronotum et mesonotum aurea).

b) Postscutellum muticum convexum. (Pronotum et mesonotum aurea.)

1. Abdom. segm, dorsale 3:tium totum modice cononotum aurea.)

* Segmenti abdom. 3:tii series ante-apicalis fotum modice convexum, nec immersum nec callosum.

* Segmenti abdom 2. Abdominis segmentum dorsale 3 : lium disco obsolctissime transversaliter immersum, tum
supra seriem lenissime vix conspicue callosum

B. Dorsulum coriaceum. (Postscutellum gibbum obtusum, Pronotum et scutcllum aurea).
72. Chrysis refulgens Spin. Europ. merid.

7, Chrysis integrella nob. Enropa.

74. Chrysis cuprata Hoffmga. Sicilia.

75. Chrysis viridana nob. Graecia.

76. Chrysis Germari nob. Europ. merid. ct Malta

77. Chrysis candens Germ. Europ. merid.

78. Chrysis caerulipes Fabr. Europ med, et merid.

79. Chrysis Gyllcnhali nob. Svccia.

80. Chrysis socia nob. Europ. macrid.

81. Chrysig dichroa Kl, Eur, merid, et $\Lambda$ sia anin. 82. Chrysis uniformis nob. Europ. maxime merid. 
Tribus 2. Veuter ig

Manipulus 1. At

Manipulus 2. $\Lambda 1$

apjsis rufeventris nob. Mus. Paykull.

A. Abdom. segm. 3:tium

a) Abdomen politum sp

angulo obtuso, series ysis anstriaca Fabr, Europa.

b) Abdomen confertissim

arcuato-obtusus, seriesysis aurifrons Ii]. Graccia.

B. Abdom. segmentum 3:

a) Segmenti 3 : tii margo

Segmentum abdo

distinctior 1. minysis bicolor Dlbn. Europa

b) Segmenti 3 : tii margo

1. Series punctorum

* Abdomen confertim modice puysis simplex Kl. Europ. mcrid.

* Abdomen confertissime punctu

nicum I. potius tuberculiforme ysis aerata nob. Ilelvetia.

2. Series punctorun

* Mbdornen confertim crasse pun longiusculur, apice subanguste anco. Postscutellum conicumysis refulgens Spin. Furop. meril

* Ibdomen confertissime punctu

tum 3:tium semicirculariter arrysis inlegrella nob. Europa.

Subdivis. 2. Thorax aut

Tribus 1. Vertex, rysis cuprate IIoffmgg. Sicilia.

Tribus 2. Vertex, Irysis viridana nob. Graccia.

Tribus 3. Abdomeı

Manipulus 1. M]

fo

Manipulus 2. M

ałrysis Germari nob. Europ. merid, et Malta

ar

Manipulus 3. M

${ }^{a r}$ rysis candens Germ. Europ. merid.

A. Dorsulum crasse punct:

a) Postscutellum acute crysis cacrulipes Fabr. Europ med. amerid

b) Postscutellum muticux

1. Abdom. segm, d

* Segmenti abdow. 3:tii series 4rysis Gyllenhali nob. Svecia.

** Segmenti abdom. 3:tii series arysis socia nob. Europ. merid.

2. Abdominis segra

supra seriem len rysis dichroa K’l. Eur, merid, et $\Lambda$ sia min.

B. Dorsulum coriaceurs. לrysis uniformis nob. Lurop. maxime meril. 
dricus basi apiceque truncatus, crebre subcrasse punctatus: punctura subreticulata viridis; pronoti truncatura antica nec non cavitas lateralis sterno proxima, dorsuli area intermedia et metanoti area postica cyanea. Pedes virides pubescentes ex uno alterove latere cyanescentes, calcaria pallida, tarsi testacei. Alae hyalinae, disco sordidae brunneo-subfumosae; venae firmae piceo-brunneae, cellula radialis elongato-lanceolata incompleta scil. summo apice anguste aperta; cellula discoidalis completa transversa rhombea. Abdomen longitudine capitis et thoracis simul sumtarum, longitudinale subrectangulare, basi truncatum et normaliter impresso-foveatum, apice obtusum 1. Ienissime arcuatum, dorso modice convexum et subcrebre punctatum, seginentum 1:mum viride: ante marginem apicalem linea transversa abbreviata cyanea; segmenta 2:dum et 3:tium cyanea: illud apice fascia viridi lateraliter ampliata, hoc ante seriem apicalem linea transversa viridi; venter basi viridis, apice viridi-cyaneus.

\section{Chrysis Jugum Dlbm.}

Mediocris $2 \frac{1}{4}$ lin. decimal. long. confertim modice punctata cinereo-pubescens laete viridis, abdominis segmentis 2:do macula utrinque el 3:lio fascia basali cyaneis, illa (segmenti 2:di) cum lineola basali angustissime cohaerente jugum formante; flagrellis tarsisque fuscis, alis hyalinis disco levissime sordidis, abdominis segmenti 3:lii serie ante-apicali regulari foveolis separalis aequalibus sublongitudinalibus.

IIabitat in Ciaffria, a Dom. J. Wahlberg detecta; Mus. Reg. Acad. Scient. Stockholm.

Observ. Corpus sublineare confertion modice pun- 
ctatum nitidum laete viride, pube cinerea adspersum. Caput orbiculatum superne convexum. Infra stemmata, inter oculos, cavitati faciali proxime, adest carinula laerissima nigro-fusca transverso-arcuata aream ex parte includens transversam nitidam subpolitam inaequalem vix punctatam. Cavitas facialis cyaneo-viridis confertissime transverse strigosa, lateribus albido-1. cano-sericeo-pilosa. Antennae basi cyaneo-virides nitidi, flagella robusta subbrevia cinereo-brunnea, propter pubem subtilissimam adpressam subopaca. Clypeus brevis politus sparse punctulatus, medio longitudinaliter carinato-convexus, ad centrum marginis apicalis subemarginato-truncatus, totus viridis: margine apicali nec non labro minuto apiculato nigro piceis. Mandibulae nitidae nigro-piceae, basi rirides. Pronotum transverso-rectangulum: disco lineola mediana obsoletissima difficillime conspicua, utrinque macula parra viridi-aurea, truncatura antica concaviuscula polita pulchre purpurascenti-violacea, foveolis 2 discoidalibus notata. Margo pronoti posticus, suturae dorsuli 2 longitudinales alarumque tegulae cyanea; sternum sublaere aut punctulis tantum patcis adspersum nitidissimum viride, in diverso situ luminis plus minus cyaneo-resplendens. Pedes cyaneo-virides nitidi largiter griseo-piloselli calcaribus pallidis, tarsis fuscis. Alae hyalinae venis brunneis; in anticarum cellulis radiali, cubitali et discoidali striae sordidae vix observantur; cellula radialis lanceolato-acuminata apice late aperta, vena cubitalis prius desinit quam vena radialis apici alae approximala. In alae anticae margine postico sub apice venulae Iransverso-medialis 2:dae adest punctum corneum piceo-brumeum. Abdomen normaliter constructum, confertim modice punctatum et pun: 
ctulatum nitidum laete viride; segmenti dorsalis 1 :mi impressio basalis triramosa cyaneo-nigricans; segmenti 2:di margo basalis nigro-aeneus, utrinque macula lateralis rotundata coerulea cum margine baseos nigro - acneo cohaerens formam jugi simulans; e centro marginis nigro-aenei excurrit in discum lineola subtilis coerulescens; segmentum 3:tium basi margineque apicali cyaneum, disco fascia viridissima, sub hac fascia (et ante summum marginem apicalem) nonnihil immersum propter seriem ante-apicalem; haec (series) regularis foveolis distinctis separatis aequalibus sublongitudinalibus 12; margo infra seriem convexiusculus sparse punctulatus, margo summus tenuissimus transverso-obtusus sub-arcuatus. Venter viridicyaneus apice fuscus.

56. Chrysis hilaris Dlbm.

Mediocris $2 \frac{1}{4}$ lin. decimal. long. modice punctata abdomine subtiliter punctulato, cinereo-pilosa laete cyaneoviridis, antennis tarsisque concoloribus apice nigro-fuscis, alis lyyalinis, anticis ad costam cellulae radialis fumosis, segmenti 3:tii serie ante-apicali irregulari foveolis numerosis ad latera evanescentibus.

Habitat in Americae New-York, Reverend. Dom. IIofmeister, qui specimen amice donavit.

Observ. Habitu quodammodo simulans Halictos minores virides. Caput confertim modice punctatum pendulum, vertice convexo latum, totum largiter cinereo-fusco-pilosum. Carinula inter oculos transverso-arcuata, quan in praecedentibus distincla obrenit, haec species omnino caret; facies depressa confertim punctata, cavitas 
facialis fere nulla. Antennae robustae elongatae filiformes cano-sericeo pubescentes; scapus totus, pedicellus et flagelli articuli tres basales supra virides, reliqua pars antennae nigro-fusca; flagelli articuli intermedii infra tumidiusculi. Clypeus magnus depresso-convexus nitidus punctatus viridis, margine apicali nigro-piceo truncatus. Mandibulae basi virides, apice piceae; oris partes inferiores brunneo-fuscae. Thorax convexo-depressus. Pronotum breve latum transverso-rectangulum depressum, subcrasse punctatum, antice leviter truncatum, truncatura concaviuscula confertim punctulata foveolis 2 centralibus politis; marginis postici anguli humerales magni versus tegulas deducti, unde ipse margo quasi rectangulariter emarginatus obvenit. Metanoti anguli postico-laterales magni conici. Pleurae confertim modice punctatae I. potius granulato-coriaceae; sternum subpolitum sparse punctulatum cyaneo-viride. Pedes concolores cinereo-pilosi, tarsis apice et toto margine infero nigro-fuscis. Abdomen longitudinale, sublineare scil. basi disco et apice fere aequaliter latum, basi apiceque obtusum, undique cyaneovirescens nitidum, dorso confertim et fere subtiliter punctulatum, segmenti 1:mi impressio basalis simplex et mediocris nec ramosa; segmentum 3:tium conrexum usque ad seriem ante-apicalem; haec irregularis, lacris (nec profunda) foveolis l. longitudinalibus 1. rotundatis numerosis, disco distinctis, ad latera cum reliqua punctura confluentibus et hinc quasi obliteratis; margo apicalis infra seriem depressus latiusculus (saltem non angustus) punctulatus oblusus leniter arcuatus; venter confertim et subtilissime pubescenti-punctulatus. 
57. Chrysis tarsata Klug.

Parva $1 \frac{1}{2}$ lin. decimal. long. subangusta punclalorugulosa griseo-pilosa cyaneo-viridis pedibus concoloribus, tarsis brunneo-testaceis, abdominis dorso aureoviridi, margine anali nigro-aeneo, alis hyalinis.

Chrysis tarsata Klug in Museo Berolinensi 1847.

Habitat in Germania; species rarissima ad Berolinum a Cel. Klug detecta.

Observ. Magnitudo et statura valde similis individuis minoribus Chrysidis cyaneae, at pictura et margine anali diversa. Totum dorsum corporis et mesopleurae punctato-rugulosa, superficies totius corporis sparse griseo-pilosa. Caput laete virens; facies pone antennas utrinque parce albido-subsericea. Antennae fuscae; scapus, pedicellus et flagelli articulus 1:mus viridi-micantes. Mandibulae apice laete et palpi brunnescentes. Pronotun cyancum macula transversa magna utrinque ad sulcum medianum laete viridi. Mesonotum totum et mesopleurae viridia. Metanotum cyaneum. Sternum viride suturis et maculis cyaneis. Abdominis dorsum punctulato-rugulosum parum nitidum, aureo-viride et pracsertim ad latera atque ad apicem segmentorum ex hoc colore micans; basis segmenti 1:mi cyanescens; margo apicalis segmenti 3:tii nigro-aeneus vix incrassatus, series ante-apicalis concolor e punctis profundiusculis irregularibus constituto; ceterum est segmentum 3:tium aequaliter depresso-convexum. Venter disco viridis, basi apiceque nigricans. Pedes virides micantes, tarsis brunneis I. brumnco-testaceis. Nlae subhyalinae venis fuscis; cellulis mediali et discoidali 1:ma (hoc exemplum est in Chrysidis Genere rarissimum) completis, submediali 1:ma el 2:da nec non radiali subcompletis. 
Sectio II. Corpus cyaneo-, viridi-, aeneo - et aureovariegatum.

Divis. 1. Abdominis dorsum aureum, segmentum 1:mum riridi-cyaneum aut cyaneo-viride.

58. Chrysis basalis Dlbm.

Parva $1 \frac{1}{2}$ lin. decimal. long. angusta sublinearis conferlim punctulato-coriacea parum nitida cyaneo-viridis, abdominis segmento 1:mo concolore, segmentis 2: do et 3:tio igneo-aureis, alis hyalinis cellula radiali incompleta.

Habitat in Algieria; I. Rambur, Mus. Spinolae.

Observ. Forte Mas. Affinis Chrysidi Leachei. Corpus largiter cinereo - et cano-puberulum. Caput et thorax confertim punctato - et punctulato-coriacea, punctis mediocribus et minutis. Frons et vertex conrexiuscula. Cavitas facialis mediocris, superne rix transverse marginulata, secundum longitudinem mediam transverse confertim strigosa. Antennae normales basi virides, flagellis nigro-fuscis. Clypeus brevis depresso-planiusculus sparse punctulatus viridis nitidus margine apicali leniter cmarginato-arcuato nigro-piceo, mandibulis et trophis concoloribus. Thorax convexus, dorsuli suturae 2 medianae hasi nonnihil curvae. Tegulae cyaneae. Pedes virides tarsis fuscis. Alae hyalinac; venac brunneae; cellula radialis angusta lanceolato-acuminata, apice modice aperta: venae radialis apex costae appropinquatus sed cum hac non conjunctus. Abdomen latitudine thoracis, capite thoraceque simul sumtis vix brevius, ovale, apice nonnihil angustiore arcuato-obtusum, dorso modice convexum confertissime punctulatum; segmentum l:mum viride impres- 
sione baseos normali margineque summo apicali cyaneis, lineola mediana subtilissima elevata parum conspicua; seguenta 2:dum et 3:tium igneo-aurea, seguentum 3:tium totum convexum seric ante-apicali subimmersa: foveolis minutis ovatis 1 . rotundatis numerosis, intermediis distinctis, lateralibus obsoletis et cum reliqua sculptura confluentibus. Venter viridis cyaneo-resplendens.

Divis. 2. Abdominis dorsum aureum 1. auratum; segmentum 3:tium cyaneum, nonnunquaın transverse nureo- et viridi-striatum, ante seriem apicalem - calloso-marginatum. Thorax cyaneus aureo-viridi maculatus.

59. Chrysis incrassata Spin. ㅇ.

Mediocris $2 \frac{1}{4}-2 \frac{1}{2}$ lin. decimal. long. modice punctata cyanea abdominis segmento 3:tio concolore, ante marginem callosum concaviusculo immerso, thorace aureoviridi-maculato, abdominis segmentis duobus anticis confertim modice punctato-coriaceis purpureo-aureis, alis sordidis basi et apice hyalinis.

var, $a$. Pronolum (exceptis sulcis 3 cyaneis et truncatura antica sub: violascente), dorsuli vitue 2 et scutcllum aurco-viridia; vertex inter oculos, ante stemmata 1. viridis 1. aureo-viridis.

Clrysis incrassata Spinola Annal. de la Soc. Entomol. d. Fr. 1838. 454. 5 .

var. b. Thorax cyaneus viridi-piclus; vertex concolor.

Chrysis integra Megerl. in Mus. Vienn. teste Kolliar.

IIabilat in Austria ad Viem. Jom. Megerle de Miihlfeld, teste Dom. Kollier in Mus. Vienn.; in insula Cintsica Dom. Chiesi, Mus. Dom. Spinola. 
Observ. in var. a. Ad habitum et sculpturam haud dissimilis Chrys. bicolori, sed structura abdominis segnenti 3:tii facillime distinguitur. Corpus robustum. Caput suborbiculatum. Vertex ubi stemmata jacent nigro-aeneus aut subviolascens, ad frontem et oculos laete subaureoviridis. Antennae breves subtenues fuscae apice brunnescentes. Clypeus majusculus subtrapezinus, basi cyaneus, lateribus virescens, apice nigro-aeneus, disco parce punctatus: versus antennas convexus et versus apicem declivis, margo apicalis prominulus depressus transversus. Mandibulae cyanescentes, macula media viridi, apice laete brunnescentes. Pronotum parte collari nigro-aeneum aut nigro - violaceum, parte dorsali subaureo-viride foreis 3 cyaneis: mediana subtriangulari, posticis (utrinque unica) dorsulo propius longis lineari-lanceolatis sulciformibus. Dorsuli vittac 2 ( 1 in singulo lobo laterali), tegulae, scutellum, postscutellum ad utrumque latus et metanoti anguli postico-laterales laete viridia ex aureo splendentia; mesopleurae macula concolore. Scutellum et postscutellum crassius punctata distincte reticulata. Pectus punctulatum subcoriaceum nitidum cyaneum. Pedes cyanei; tibiae ad certum situm luminis nonnihil riridi-nitent; tibiae anticae latere interiore tarsique omnes brumnescentes. Abdomen purpureo-aureum: segmentis 1:mo macula basali triangula, 2:do margine basali 3:tioque toto cyancis; segmentum 3:tium in centro: cavitatis, marginis callosi et marginis apicalis leviter viridi-micans, in disco hujus segmenti linea mediana subelevata polita. Venter cyaneus viride-irroratus. 
60. Chrysis cyanura Klug. 오.

Submagna robusta 3 lin. decimal. long. crasse punctata cyanescens abdominis segmento 3:tio concolore, ante marginem callosum concaviusculo-immerso, verlice thoracisque dorso aureo-viridi maculalis, abdominis segmentis duobus anticis crasse punctatis viridissime auralis, alis hyalinis, ad cellulae radialis marginem costalem sordidis.

Chrysis cyanure Klug Mus. Berolin. 1817.

Habitat in Rossia meridionali, a D. Pallas detecta; Mus. Berolin.

Observ. Praecedenti (Chr. incrassatae) major, robustior et sculptura crassiori, abdomine magis viride etc. facile dignota. - Caput longiusculum. Oculi ovati. Stemmata magna. Vertex latus et occiput irregulariter punctato-rugulosa cyanea; inter oculos stemmata et frontem plaga viridis; inter stemma anticun et frontis marginem superiorem lineolae longitudinales tres elevatae irregulares. Frons et clypeus cyaneo-virides. Antennae breviusculae, crassitie mediocres, scapus longus cyaneus, dorso leviter virescens; pedicellus et flagellum fusca, ille et hujus basis supra obsolete riridi-nitida; flagellum praeterea infra nec non apice testaceo-brumuescens. Clypeus ut in C\%r. incrassata simillimus. Thorax crasse punctato - reticulatus. Pronotum lateribus cyaneum, supra aureo-viride impressionibus 3 cyaneis: mediana distinctiori suborata, reliquis ad marginem posticum utrinque sitis lanceolatis transversis minus profundis et hinc obsoletis. Dorsuli area mediana cyanea, leviter viridi irrorata, utraque area lateralis saturate aureo-viridis margine laterali cyaneo; scutellum viridi-reticulatum punctis omni- 
bus cyaneis. Pectus et metanotum cyanea, hujus anguli postico-laterales et macula mesopleurarum discoidalis virides. Abdominis segmenta dorsalia duo antica simillime ita crasse punctata ut ipse thorax, a punctis et punctulis conferlis subcoriaceo-rugulosa, nitida, saturate viridi-aurata vel aureo-virescentia; in segmento 2:do lineola mediana plana subpolita adest; macula basalis segmenti $1: m i$, margo basalis segmenti 2:di tenuiter et totum 3:tiun seginentum cyanea; hoc basi depresso-convexum et sparse crasse punctatum, tum transverse arcuato-concaviusculoimmersum et pone hanc immersitatem iterum incrassatum in marginem paullulum callosum et nonnihil virescentem; sub hoc callo observatur series ante-apicalis distinctissima e foveolis profundis circiter $15-20$, quarum maxime lateralibus paullo minoribus orbiculatis, reliquis autem et plurimis majoribus subquadrangulis carinula intercedente separatis; margo apicalis proprius tenuis prominulus cyaneus, ad latera brunnescenti-subdiaphanus. Pedes cyanei, parum viridi-nitentes; tibiae apice tarsique toti brunneo-testaceae. Alae subhyalinae renis firmibus brunneis, cellulis: costali, mediali et discoidali 1: ma completis, submediali 2 : da et radiali distincte incompletis scil. cellula radiali apice latissime aperta propter venam radialem parum curvam apiceque a vena costali late remotam; ad marginem costalem cellulae radialis et in limbo apicali umbra sordida observanda.

61. Chrysis Humboldti Dlbm.

Submagna robusta 3 lin. decimal. long. crasse punctata capite thoraceque cyaneo-viridibus aureo-irroratis, abdominis segmentis duobus anticis confertim crasse pun- 
clatis purpureo-aureis, segmento 3:tio colore: cyaneo aureo et viridi transverse striato: lota parte basali depresso-convexo, alis hyalinis ad costam cellulae radialis sordidis.

Chrysura Humboldti Dlbm. Dispos. 1845. 6. 4.

Habitat in insula Rhodo, a D. Hedenborg detecta; Mus. Reg. Acad. Scient. Stockholm.

Observ. Quod magnitudinem Chrysidi austriacae proxima. Corpus robustum subcylindricum crasse punctatum, cano - et cinereo-pubescens. Caput orbiculatum confertim modice punctatum subrugulosum, ante et post stemmata l. viridi- l. viridi-aureo-irroratum. Vertex depresso-convexus. Cavitas facialis magna, superne per marginem elevatum stemmati approximatum limitata, infra marginem illum punctata, tum subtilissime transverse strigosa et punctulato-coriacea lateribus cano-sericeis. Antennae robustae cano-sericeo puberulae perbreves; scapus longissimus dimidiam flagelli longitudinem aequans, totus viridis; pedicellus, et articulus 3:tius elongatus, uno latere virides, altero fusci, reliqua pars antennae testacea. Frons et facies paullum angustiores quam in aliis speciebus, unde oculi nonnihil approximati obveniunt; oculi magni exserti orales. Clypeus magnus irregulariter subtrapezinus viridis nitidus sparse punctatus et punctulatus, convexo-depressus, ante apicem area semicirculari declivi aureo-splendida, margo apicalis transversus leniter emarginato-arcuatus, margo basalis ad centrum (propter antennas) bis arcuato-emarginatus, angulis laterali-basalibus latis arcuato-obtusis. Mandibulae basi virides plus minus aureosplendentes, apice brunnescentes; maxillae palpi labiumque brunnea. Thorax orato-subcylindricus, confertim 
crasse punctatus. Pronotum subgibbum supra viridi-cyaneum sulco mediano latiusculo, antice utrinque striola aut macula transversa 1. lactius viridi l. aurea, postice utrinque juxta marginem sulco transverso obsoleto; truncatura antica polita violascens. Dorsuli area media virescenti-cyanea, areae laterales 1 . virides 1 . viridi-aureae et tantum margine laterali externo cyaneae. Scutellum et mesopleurae viridia aureo-irrorata, metanotum cyaneum 1. viridi- 1. aureo-irroratum. Sternum viridi-cyaneum. Pedes virides tarsis testaceis. Tegulae ovales virides. Alae hyalinae, in cellulis radiali, cubitali et discoidali paullulum sordidae; venae brumeae; vena radialis ab apice alae longe remota, hinc cellula radialis laete aperta; haec ceterum sublanceolata; vena costalis mox post angulum venae radialis evanescit. Abdomen robustum, supra modice convexum, infra subplanum, ambitu subrectangulum, latitudine thoracis, longitudine capitis thoracisque simul sumtarum, segmenta dorsalia 1:mum et 2:dum purpureo - aurea, viridi-resplendentia, confertissime crasse punctato-rugulosa: punctis rotundis politis virescentibus, rugulis microscopice punctulatis aureo-purpurea; segmenti 1:mi impressio basalis et segmenti 2:di margo basalis violascentes limbo tenuissimo viridi; segmentum 2:dum duplo longius quain 1: mum, vix ultra, lineola mediana planiuscula antice distinctiore postice evanescente; segmentum 3:tium nonnihil majus quan 1: mum, parte basali depressoconvexum sparse punctatum subopacum saturate cyaneum: margo callosus mediam segmenti partem occupans et ab uno margine laterali in alterum extensus validus transverso-arcuatus, confertissime punctulatus nitidus viridiaureus; reliqua pars segmenti-viridis; series ante-apicalis, 
callum immediate sequens, regularis foveolis 20 profundis subaequalibus suboratis approximatis, particula apicalis (infra seriem) planiuscula sparse punctulata nitida, margo apicalis summus tenuissimus angustissimus fusco-brunneus transverso-obtusus, vix nisi obsoletissime arcuatus latitudine abdominis. Venter depresso-planus pube subtilissima adpressa cano-sericea adspersus, laete viridis ex aureo nonnihil resplendens.

Divis. 3. Abdominis dorsum aureum, plus minus viridi-micans; segmentum 3:tium concolor, nec nisi summo margine apicali rarius aeneo- aut purpurascenti-fusco, aut cyanescente.

Subdivis. 1. Thorax nunquam aureus nec aureofasciatus, sed aut viridis pictura cyanea aut cyaneus pictura aurato-viridi.

Tribus 1. Venter cyaneus aut viridis plus minus nigro-pictus.

62. Chrysis transversa Dlbm.

Latiuscula $2 \frac{2}{3}$ lin. decimal. long. crasse punctata cyaneo-viridis, abdomine obluse subrectangulo l. subquadrato basi viridi, lateribus et ante apicem virescenti-aureo, segmento 3:tio brevi transverse subrectangulo, ante seriem apicalem leviter calloso-marginato, margine apicali transverso tenui, alis albo-hyalinis ad costam cellulae radialis incompletae limboque apicali sordidis.

Habitat in Asia minori; specimen unicum a D. Loew detectum.

Observ. Ad habitum nomnihil affinis Chrys. foveaII. 
tae (No. 93.). Ciorpus robustum crassum, antice posticeque transverso-obtusum, totum cinereo- et cano-pubescens pilosellumque, dorso crasse punctatum. Caput viride, pone stemmata et occipite cyanescens. Vertex modice punctatus subreticulatus. Antennae modice robustac breviusculae fuscae, scapus et flagelli basis nonnihil viridi-micantes. Clypeus mediocris transversus medio convexiusculus. Mandibulae piceae, basi macula viridi, ante apicem laete brunnescentes. Thorax crasse punctato-reticulatus viridis; pronotum fovea mediana subcontinua, antice truncatum: truncatura medio polita concaviuscula subaenea, utrinque parce punctulata nitida viridis; dorsuli area media cyanea viridi-irrorata, areae laterales virides intus subaureo-irroratae; scutellum, mesopleurae, discus postscutelli et latera metanoti viridia; reliquae partes thoracis cyaneae, plus minus viridi-nitentes. Abdominis segmentum dorsale 1:mum crasse punctatum viride, basi macula cyanea, apice utrinque ex aureo-viridi resplendens; segmentum 2:dum confertim at mediocrius crasse punctatum viridi-aureum, praesertim ad latera et in apice, basi macula subtriangulari violacea; segmentum 3:tium breve, obtuse transverso-subrectangulum 1 . late et breviter subtrapezinum, inaequale: basi linea marginali violacea sparse punctatum, tum transverse concaviusculo-immersum punctato-coriaceum cupreo-aureum et deinde paullulum incrassatum in marginulum subcallosum viridi-auratum; series ante-apicalis sub hoc immediate sita e punctis et foveolis mediocribus orbiculatis numerosis constituitur; margo apicalis tenuis, paullulum prominulus, late transversus, vix nisi lenissime et inaequaliter currus, utrinque arcuato-oblusus. Venter viridis, pedes concolores tarsis 
fuscis. Alarum venae et cellulac omnino ut in Chr. cyanura, at vena radialis magis curva et apertura cellulae radialis minus Iata.

\section{Chrysis gastrica Klug. ㅇ.}

Oblonga 3 lin. decimal. long. et ultra, modice robusla, antice crasse punctato-reticulata cyaneo-viridi-irrorala, abdomine punclulato-coriaceo viridissime auralo, segmentis 2:do et 3:lio fasciola basali nigro-aenea, segmento 3:lio mediocri semilunato, ante seriem non calloso, margine apicali incrassato non sulcato, alis hyalinis disco sordidis, cellula radiali incomplela.

Spintharis gastrica Klug Mus. Berolin.

Habitat in Lusitania, D. Hoffmannsegg; Mus. Klug.

Observ. Habitu fere Chrysidi integrae similis, licet major, robustior et aliter sculpta pictaque. Caput cyaneum: vertice et occipite modice punctato-reticulatis viridi-irroratis. Antennae crassiusculae subbreves, basi virescentes, medio fuscae, apice brunnescentes. Clypeus subbrevis viridis, medio convexiusculus, margine apicali transverso. Mandibulae ut in Chr. transversa. Thorax longiusculus minus crassus, cyaneus, supra subaureo-viridi-irroratus; pronotum anlice truncatum: truncatura cyanea, parte colliformi aureo-viridi, spatio centrali politissimo aeneo. Abdomen ovato-oblongum confertim punctato-coriaceum, viridissime aureum, segmenta 2:dum et 3:tium in margine basali fasciola lanceolata nigro-aenea apice aureo-marginata; segmentum 3:tium mediocre semilunatum, modice depresso-convexum, disco vix nisi quam lenissime transwerse immersum, series ante-apicalis e foveolis parvis orbiculatis hinc inde subconfluentibus con- 
stituta; margo apicalis nonnihil incrassatus aureus. Venter cyaneus, lineola mediana et margines segmentorum virescentes. Alae hyalinae; in cellula radiali ad costam et stigma, nec non in disco cell. discoidalis, cubitalis et submedialis 2: dae umbra sordida observatur; venae alarum firmae brunneae; cellula radialis apice subanguste aperta. Pedes 1. cyaneo-virides 1. viridi-cyanei, tarsis testaceobrunneis.

64. Chrysis sulcata Dlbm.

Oblonga $2 \frac{1}{2}-3$ lin. decimal. long. subrobusta, antice punctato-reticulata cyaneo-viridi-variegata, abdomine ovali confertim punctato et punctulalo igneo-aureo, segmentorum 2:di et 3:tii marginibus basalibus nigroaeneis, segmento 3:lio magno semicirculari modice convexo, margine apicali infra seriem incrassato distincte transverse sulcato, alis hyalinis disco sordidis, cellula radiali incompleta, postscutello conice promimulo.

Chrysura sulcata Dlbm. Dispos. 1845. 7.6.

Habitat in Sicilia: D. Kollàr Mus. Vienn.; - in insula Rhodo, mense Majo, D. D. Hedenborg et Loew.

Observ. Caput, thorax et pedes largiter cano- et cinereo-fusco-pubescentia. Caput mediocre, rolundatosubcubico-triangulare viride, vertice convexo-depresso confertissime punctato - et punctulato reticulato; cavitas facialis obsoleta, superne cum fronte confluens: carinula illa limitativa omnino carens, tota confertissime punctulato-coriacea. Antennae mediocres; scapus, pedicellus et articulus 3:tius virides, articuli reliqui omnes nigro-fusci. Clypeus subbrevis transversus viridis, disco longitudinaliter convexiusculus, utrinque leviter immersus, margine 
apicali leniter emarginato-truncatus, toto margine apicali et laterali piceo-brunneus. Mandibulae et partes oris inferiores concolores. Thoracis dorsum et latera confertim modice punctato - reticulata parum nitida; pronotum transverso-rectangulum sulco mediano lanceolato postice abrupto, truncaturae anticae spatium centrale politum; dorsuli suturae 2 discoidales rectae nigro-violascentes; postscutellum crassissine punctatum, obtuse conicum, horizontaliter prominulum; sternum et pedes viridia, sparse punctulata nitida, tarsis nigro-fuscis. Alae hyalinae, in cellulis discoidali, cubitali et ad costaın cellulae radialis sordidae; tegulae convexae ovales virides nitidae sparse punctulatae; venae piceo-brunneae, vena radialis apici alae approximata sed cum eodem non connata cellulam radialem lanceolato-acuminatam apice apertam et incompletam linquens. Abdomen ovale longitudine capitis thoracisque simul sumtorum, dorso modice convexum igneoaureum, confertim et crasse et subtiliter punctatum scil. punclis magnis et minutis mixtis; segmenti 1:mi inpressio basalis normalis nigro-aenea postice lineola peripherica viridula; segınentorum 2:di margo basalis, 3:tii margo basalis et stummus apicalis etiam nigro-aenei; segmentum 3:tium modice convexum, totum punctis minoribus crebre obsertum, series ante-apicalis aliis distincte aliis obsoletius immersa: foveolis parvis nigris rotundatis, mediis plerumque distinctis, lateralibus interdum I. confluentibus 1. ommino obliteratis; paullo infra hanc seriem est segmenti particula apicalis veluti deflexa 1. inclinata, praestans superne: quasi carinulam transversam laevissimam; inter hanc obsoletam carinulan et summum marginem apicalem restat spatium transverso-lineare arcuatum 
concaviusculo-depressum confertissime punctulato-coriaceum, sulcum simulans; hoc tamen spatium sulciforme valde varians observavi; scil. in aliis distinctissimum, in aliis minus explicatum et hinc obsoletum. Venter viridis aut cyaneus nigro-maculatus.

65. Chrysis varicornis Spin.

Oblonga vix 3 lin. decimal. long. subrobusta, irregulariter conferlim et punctala et punctulata subcoriacea, cyaneo-viridi-variegata, abdomine ovali igneo-aureo, segmenti 2:di margine basali nigro-aeneo, segmento 3:tio magni semicirculari depresso-convexo, margine apicali infra seriem leviter incrassato, alis liyalinis disco sordidis cellula radiali completa, postscutello conico prominula, antennarum flagellis medio testaceis.

Chrysis varicornis Spinol. Annal. d. I. Soc. Entomol. d. Fr. 1838. 449. V.

Habitat in Hispania, D. Chiliami; - in Aegypto, D. D. Fischer et Walt: Mus. Spinolae.

Observ. Simillima Chrysidi sulcatac el forte ejus mas. Dignoscitur: antennarum articulis a 3:tio usque ad penultimum numeratis flaro-testaceis nigro fusco-marginatis, capite et thorace parum nitidis, sculptura thoracis et capitis subtiliori, confertiori et subcoriacea, alarum cellula radiali completa (itaque apice clausa nec aperta), abdomine confertissime subtiliter punctato: segmenti 3:tii margine apicali leviter incrassato, spatio antem illo sulciformi, quod in eodem margine Chrysidis sulcatae nuper antea descripsimus, I. obsoletissimo ef rudimentario I. omnino obliterato. 
Tribus 2. Venter igneo-pictus.

Manipulus 1. Abdominis segmenti dorsalis 3: tii margo apicalis infra seriem paullulum incrassatus.

66. Chrysis rufiventris Dlbm.

Mediocris $2 \frac{1}{2}$ lin. decimal. long. confertim irregulariter punctata et punctulata subcoriacea cyaneo-viridis, abdomine undique igneo-aureo, alis hyalinis disco sordidis, cellula radiali completa, postscutello conico.

Specimen unicum e Collectione Paykulliana Musei Reg. Acad. Scient. Stockholm. communicavit D. Boheman, patria non indicata.

Observ. Simillima Clrr. varicorni Spin., et forsitan hujus varietas? tamen dignoscitur corpore breviore et angustiore, antennarum flagellis nigris, sculptura thoracica minus crassa, abdominis segmenti 3 :tii serie ante-apicali irregulari foveolis profundioribus, ventre igneo, etc.

Caput, thorax, pedes et alae omnino ut in Chryside varicorni; antennae autem breviores, nigro-fuscae, scapo solo cyaneo-viridi; cavitas facialis superne carinula transversa caret, adeoque a fronte non separata. Oculi minores quam in Chr. varicorni et magis distantes. Abdomen eandem formam, sculpturam et picturam in dorso pracbet quam apud Cler. sulcatam; at segmenti 3:tii series ante-apicalis diversa et irregularis medio per carinulam brevissimain depresso-planam interrupta, utrinque foveolis 6 - $\$$ profundioribus nigris invicem fere alternation confluentibus, margo apicalis infra hanc seriem omnino ut in Chryside sulcala constructus; venter igneo-auratus splendidissimus marginibus segmentorum piceo-nigris. 


\section{Clarysis: Phalanx I.}

Manipulus 2. Abdominis segmenti dorsalis 3:tii margo apicalis laevis muticus, non incrassatus.

A. Abdominis segmentum dorsale 3:tium apice parumper angustius quam basi (margine apicali subtransversum).

67. Chrysis austriaca Fabr. $\sigma^{7}$ 오.

Magna 3-4 lin. decimal. long. robusta confertim irregulariter punctata subcoriacea viridi-cyanea fronte immaculata, abdomine subpolito sparse punctato: segmenti 3:lii serie ante-apicali distincta foveolis parvis orbiculatis numerosis, margine apicali transverso utrinque angulo obtuso, cellula radiali subcompleta.

Chrysis austriaca Fibr. Piez. 173. 15. secunduso exemplar typicum, quod e Collectione Megerlei Mus. Vienn. amice communicavit D. Kollàr.

Clerysis austriaca Fabr. 9 sccund. specimen, quod in Collectione F'abriciana Mus. Kiel, aestate 1847 examinavi.

Clurysis ignita Fabr. var. Ent. Syst. 2. 241. 10. in Descriptione.

Chrysis refulgens Fabr. of secundum specinen, quod in Collectionc Fabriciana Mus. Kicl. aestate 1847 examinavi.

Chrysis inermis Zett, in litteris.

Habitat per plurimas Europae regiones passim; Galliae: D. Latreille Mus. Spinolae, Helvetiae: D. Kriechbaumer, Austriae: D. Megerle von Miihlfeld; Germaniae: D. D. Loew, Sturm et Zeller; Daniae: D. Westermann; Sveciae: D. D. Fallén et Zetterstedt, Scaniae mihi obvia.

Observ. Affinis et similis Chrysidi bicolori; at dignoscitur: capite ad mandibulas subaeque lato ac ad verticem, abdominis segmento 3:tio apice solummodo parumper angustiori quam basi, serie anteapicali plerumque distincta, margine apicali utringue angulo obtuso, etc. 
Corpus robustum submagnum cano- et fusco-pubescens. Caput et thorax confertim irregulariter punctatorugosa coriacea parum nitida saturate cyanea. Caput subquadratum, scil. ad mandibularum basin vix angustius quam ad verticem, subtus utrinque angulo occipitali munitum; vertex convexiusculo-depresso-planus. Cavitas facialis laevis (non profunda), planiuscula, superne libera (nec per carinulam transversam a fronte separata). Oculi mediocres 1. potius subparvi. Antennae robustae, 오 longitudine mediocres, $\sigma^{\top}$ nonnihil longiores, utriusque sexus nigro-fuscae, scapo pedicelloque cyaneo-viridibus. Clypeus submagnus transversus subtrapeziformis sparse punctulatus, cyaneus plus minus viridi-pictus, convero-subcarinulatus, margine apicali truncato, laterali obliquo laevissimis nitidis subtilissime coriaceis nigro-piceis; mandibulae hujus coloris robustac nitidae, basi viridi-aut viridi-aeneo picta, apice bidentatae. Palpi et ligula nigro-fusci, maxillac et mentum viridi-aenescentes. Thorax subcylindricus, supra convexo-depressus I. vice versa; pronoti truncatura antica subperpendiculariter declivis non concaviuscula; pronoti margo anticus (mox post truncaturam) virescens sulco mediano postice abrupto; dorsuli suturae 2 medianae profundae crenulatae nonnihil curvac, areae laterales disco virides nitidiores. Metanoti anguli postico-laterales mediocres. Pedes viridi-cyanci, calcaribus subtestaceis tarsis fusco-brunneis. Alarum tegulae cyaneo-virescentes ovales. Alac hyalinae, in cellulis radiali, cubitali et discoidali plus minus sordidae; venae firmac piceo-brumneac; cellula radialis magna lanceolata subcompleta scil. vena radialis mox ante apicem alae subtiliter desinit cellulam linguens angustissime apertam. 
Abdomen robustum sublineare, longitudinem capitis thoracisque simul sumtorum vix aequans, depresso-convexum nitidum aut nitidissimum et tunc subpolitum, in aliis confertim, - in aliis et plurimis sparse modice punctatum et punctulatum igneo-aureum; segmenti 3:tii series anteapicalis rarius obsoleta plerumque distincta, foveolis parvis orbiculatis numerosis, margo apicalis late transversus, laevissime arcuatus utrinque angulo distincto obtuso, margo lateralis obliquus. Venter depresso-planus 1. totus unicolore igneus 1. nigro- et igneo-maculatus, sparse pilosopunctulatus.

68. Chrysis aurifrons Klug.

Mediocris $2-2 \frac{1}{2}$ lin. decimal. long. confertim irregulariter punctulato-coriacea; capite antice abdomineque virescenti-aureis, hoc confertim punctulato-coriaceo, segmenti 3:tii serie ante-apicali obsoleta foveolis minoribus haud paucis at sub-obliteratis, margine apicali latoobtusissime transverso-arcuato, cellula radiali incompleta.

Chrysis aurifrons Klug Mus. Berolin.

Clerysis integra var, chrysocephala Mus. Spinolae.

Habitat in Italia, Mus. Spinolae; in Asia minori teste D. Loew, qui specimen mense Aprilis 1841 lectum communicavit.

Observ. Habitu similis Chrysidi integrellae; al pictura capitis, sculptura et proportione segmenti 3:tii valde diversa. - Corpus cano-pilosum. Caput pone stemmata et in occipite cyaneum, antice a stemmatibus posticis usque ad clypei marginem apicalem auratus, at color aureus superne saturatior, inferne magis virescens. 
Vertex modice punctulato-subreticulatus. Antennae mediocres crassiusculae fuscae, basi virescentes. Clypeus submediocris aureo-viridis inaequalis, margine apicali summo nigro-aeneo sub-undulato transverso. Mandibulae piceae, basi virescentes. Palpi graciles fusci. Thorax modice punctatus et punctulatus, coriaceus subopacus violascenti-cyaneus; margines pronoti, dorsuli utraque vitta lateralis, scutelli discus, metanoti anguli laterales et pleurae viridia. Pronotum antice sulco mediano distincto. Abdomen mediocre, dorso modice punctato-coriaceum virescenti-aureum nitidum modice convexum; segmentum 3:tium mediocre, totum modice convexum, apice late transversum utrinque arcuato-obtusum; series ante-apicalis levis et obsoleta e punctis parvis rotundis constituta; venter igneus viridi- et nigro-maculatus. Pedes virides, tibiis latere exteriore aureo-nitidis, tarsis brunneis. Alarum cellula radialis subcompleta, triangulariter lanceolata; vena radialis in disco angulum subrectum format.

B. Abdominis segmentum dorsale 3:tium apice di stincte et conspicue angustius quam basi.

a) Abrom. segmenti 3:tii margo apicalis ad medium transversus.

69. Chrysis bicolor Dlbm. $ð$ 오.

Mediocris aut magna $2 \frac{1}{2}-4$ lin. decimal. long. fuscoet cano-pubescens et pilosella, confertim 1. modice 1. subcrasse punctata viridi-cyanea, abdomine igneo-aureo plus minus viridi-resplendente conferlim punctato aut punctulato nitido aut coriaceo-subopaco, segmenti 3:tii serie ante-apicali variante 1 . distincta 1 . obsoleta $l$. ob- 
soletissima l. omnino obliterata, alis hyalinis disco normaliter sordidis, cellula radiali $l$. completa $l$. subcompleta 1. incompleta.

Chr. bicolor Dlbm. Mon. Chr. 1829. 10. 6.

Chr. flammea Pallas in Manuscript. secund. Klug. Mus, Berolin.

Chr. mutica Klug Mus. Berolin.

Clir. austriaca Zett. Ins. Lapp. 466. 6. Nota.

IIabilat per maximam partem Europae, a regionibus littoralibus maris mediterranei usque in Lapponiam maxime borealem, parce aut raro. Intra Scandinaviain in floribus Umbellatarum montis Omberg Ostro-Gothiae d. 22. Julii 1835, in insula Furillen juxta Gottlandiam d. 21. Julii 1841, in Helsingia d. 23. Augusti 1840 in foliis Alni, in jugo alpino Norrwegiae juxta Lewangerfjord in foliis fruticum 2-3. Julii 1840. D. Zetterstedt et mibi obvia. Metamorphosin subit in Osmiae nigriventris nido, ubi larva vitam gerit parasiticam aut saltem pseudoparasiticam. Scil. in nota, quam Ins. Lapp. 466. 6. allegavit Cel. Zetterstedt, vidimus se, quum ad Kjellunge Gottlandiae d. 26. Julii nidum Osmiae nigriventris Nyland. (=Anthophorae nigriventris et inermis Zett. Ins. Lapp.) detegit, omnes cellulas ejusdem nidi folliculatas aperuisse et in singulo folliculo imaginem Osmiae memoratae completam invenisse, in unius autem cellulae folliculo, quum hunc aperuit, specimen vivum et completum Chrysidis bicoloris nob. (=Chrysidis austriacae Zetl., non Fabr.) deprehendisse. Eundem nidum atque specimen C.hrysidis ibidem deprehensum mihi dono dedit Illustr. detector. Folliculus, in quo Chrysis inclusa fuil, folliculis Osmiarum simillimus.

Observ. Maguitudo corporis et sculptura abdomi- 
nis, praecipue vero hujus series ante-apicalis in hac specie ita variant, ut variationes tamquam diversae species facile existimentur. Sed per examen scrupulosum multorum Chrysidis bicoloris individuorum, quae Amici et Cultores Scientiac e regionibus Europae diversissimis comparandi caussa miserunt, mihi contigit formas sculpturae unam in alteram ita sensim transgredientes observare, ut mihi persuasum sit illas pro speciebus diversis determinandis materiem plane nullam praebere. Nec a ratione cellulac radialis racillante, tali scopo attingendo notae constantes erui possunt. Tunc nihil aliud restat nisi magnitudo, quae, ceteris paribus, sola per se ad species unius ejusdemque Generis distinguendas nullo modo sufficit.

Chrysis bicolor valde affinis et similis est Chrysidi austriacae; at, praeter alias notas, mox dignoscitur: abdominis segmenti dorsalis 3 :tii margine apicali distincte et conspicue angustiore quam margine basali, capite ad mandibulas angustiore, cellula radiali et abdominis seric ante-apicali ad formam variantibus, metanoti angulis postico-lateralibus majoribus quam in Chryside austriaca, etc.

Corpus in exemplaribus nuper exclusis aut non detritis largiter cinereo-fusco et cano-pubescens. Caput rotundato-triangulum, confertim modice punctatum aut punctulatum, rugosum aut rugulosum subopacum 1. parumper nitidum, cyaneum et laeviter viridi-pictum aut virescens et cyaneo-pictum. Vertex el frons convexiusculo-depressa. Ad utrumque stemma supremum adest macula minuta nitida. Cavitas facialis concaviusculo-depressa punctulato-coriacea subgranulata opaca, superne 
libera (non marginata), medio sulculo Iongitudinali obsoleto nitidiori subpolito. Clypeus transverso-subtrapezinus punctatus, medio subcarinato convexus, cyaneus viridi-micans aut aeneo-viridis, marginibus laterali et apicali piceis, hoc subemarginato-truncato. Mandibulae robustae, basi virescentes, apice piceae. Antennae normales, apice nigro-fuscae, scapo pedicello et articulo 3:tio cyaneo-viridibus. Thorax subcylindricus capite non latior, confertim modice punctatus et punctulatus: punctura aliis magis regularis et tunc saltem in antica parte subreticulata, aliis minus regularis ad formam rugulosam plus minus appropinquans, aliis denique irregularis et in formam coriaceam abiens, hinc igitur fit thorax in aliis nitidus, in aliis vix nitidus, in aliis subopacus; pronoti sulcus medianus sublanceolatus postice abruptus, truncatura antica obliqua; dorsuli suturae 2 medianae profundae crenatae subrectae; postscutellum basi fovea centrali magna; metanoti anguli postico-laterales normales; color thoracis plerumque cyaneus; pronoti linea transversa indeterminata aut maculae 2 indeterminatae, utraque area lateralis in disco, scutelli discus, maculae et striae metanoti pleurarumque viridia; aliis vero thorax maxima ex parte virescens pictura cyanea. Pedes cyaneo-virides aut viridi-cyanei calcaribus testaceis, tarsis fuscis. Tegulae ovales viridi-cyaneae subnitidae sparse punctulatae. Alae hyalinae in cellulis: radiali ad costam, cubitali, discoidali et submediali plus minus sordidae; venae firmae piceae, radialis apex 1 . cum costa distinctissime conjuncta, 1. costam vix attingens, 1. a costa paullulum remota; hinc cellula radialis, quae lanceolato-acuminata apud omnes est, I. completa l. subcompleta 1. aperta et omnino in- 
completa. Abdomen ellipticum sublineare, dorso depressoconvexum modice punctatum et punctulatum, interstitia scil. punctulis minutis adspersa, unde superficies dorsalis magis l. minus confertim punctata, et propter sculpturam aut vagam aut confertam magis 1 . minus coriacea et magis 1. minus nitida obvenit, segmenta plerisque purpureoaurea, nonnullis igneo-aut coccineo-aurea, magis l. ıninus intensive viridi-splendentia, ad certum situm luminis lineola mediana subelevata inter basin et partem apicalem segmentorum observatur; segmenta 2:dum et 3:tium margine basali nigro-aenea; segmentum 3 :tium minus convexum quan in Chr. austriaca, confertius sculptum, punctato- et punctulato-coriaceum, series ante-apicalis ita varians: a) distincla foveolis numerosis circiter 20 parvis irregularibus hinc inde confluentibus, tunc margo apicalis infra seriem latiusculus depressus, b) obsoleta foveolis multis obliteratis at per lineam transversam immersam semper determinanda, c) omnino obliterala, quo in casu totum segmentum convexo-depressum et undique aequaliter punclato - aut punctulato-subcoriaceum, vestigio seriei ante-apicalis hujusque foveolarum carens; margo segmenti 3:tii summus in omnibus arcuatus et medio transversus, unde arcus plus minus depressus obvenit; venter confertim punctulato-coriaceus pubescens igneus nigro-maculatus.

b) Abdominis segmenti 3:tii margo apicalis perfectissime arcuatus.

70. Chrysis simplex Klug. 오.

Magna 3 lin. decimal. long. robusta pubescens et pilosella, capite ad mandibulas lato thoraceque confertim 
irregulariter punctatis subrugulosis viridi-cyaneis, postscutello convexo mutico, abdomine conferlim punctulatocoriaceo subruguloso plus minus nitido rubro-aut virescenti-aureo, segmenti 3 :tii serie ante-apicali obsoletissimo suboblilerato, cellula radiali incompleta apice anguste aperta.

Chrysis simplex Klug Mus. Berolin.

Chrysis integra var. major Spinol.

Wabitat in Europa meridionali rarius; in Graecia D. Friwaldsky, Mus. Spinolae.

Observ. Quoad capitis structuram Chrysidi austriacae affinis, sed abdominis sculptura et colore facillime distinguenda. - Corpus robustum cano-cinereoque pubescens et pilosellum. Caput magnum subquadratum, ad mandibulas vix minus latum quam ad rerticem, confertim punctulato - rugulosum subgranulosum, fusco- et cano-pilosum, vertex et frons lati convexo-depressi viride-cyanei facies concaviusculo-plana riridis. Antennae breves proportionales; scapus pedicellus et articulus 3:tius infra cyanei, supra virides, ceteri articuli nigro-fusci. Clypeus latus transversus subtrapezinus inaequalis depressus sparse punctatus nitidus subaurato-viridis, margine apicali piceo: ad latera oblique ad centrum recta truncato. Mandibulae robustae piceae, extus longitudinaliter sulcatae, quiescentes fere recta transrerse jacent et ex parte sub clypeo absconditac. Thorax robustus confertim punctatus et punctulatus coriaceus parum nitidus, cyaneus: pronoti margine et postico indeterminate, dorsuli areis lateralibus disco, scutelli et postscutelli disco, metanoti utraque parte postico-laterali mesopleurisque plus aut minus virescentibus. Pronotum transverso- 


\section{Chrysis: Phalanx $I$.}

rectangulum, punctato-subreticulatum sulco mediano lanccolato minus determinato abrupto; truncatura antica subperpendicularis: foveola centrali politissima nigro-picea. Dorsuli suturae 2 medianae subrectae crenulatae nigricantes. Punctura scutelli crassior quam ceterarum thoracis partium. Metanoti anguli postico-laterales robustae, magnitudine mediocres. Pedes virides, uno alterove latcre cyanescentes aut aenescentes, calcaribus testaceis, tarsis brunneis. Alarum tegulae ovales viridi-cyaneae nitidae sparse punctatae. Alae hyalinae in cellula radiali ad costan leviter sordidae; venae firmae piceae; cellula radialis elongata lanceolata apice anguste aperta. Abdomen robustum ovale, latitudine mesothoracis, at capite thoraceque simul sumtis longius, confertissime punctulatocoriaceum sericeo-nitens, viridi- aut purpureo-aureum, seginenti 1: mi impressio basalis semicircularis et segmenti 2:di margo basalis nigro-aenescentes; in disco segmentorum 1:mi et 2:di carinula mediana levissima planiuscula subpolita ad certum situm luminis observatur. Segmentum 3:tium totum aequaliter convexum, series ante-apicalis fere obliterata, tantummodo ut vestigium hujus considerari potest linea immersa obsoleta transverso-arcuata foveolas nonnullas obsoletissimas forens; margo apicalis summus nigro-piceus perfecte convexo-arcuatus. Venter planus confertissime et subtiliter punctulato-coriaceus, punctulis segmenti 2:di obliquis, segmento 1 :mo basi utrinque punctis nonnullis crassis adsperso, igneo-auratus marginibus segmentorum violascentibus.

71. Chrysis aerata Dlbm. .

Oblonga robusta $3 \frac{1}{2}$ lin. decimal. long. modice punII. 
ctata, antice fusco-Tirtella, aenescenti-viridis abdominis dorso confertissime punclulato-coriaceo subopaco aureococcineo, apice anguste arcuato; serie ante-apicali irregulari foveolis numerosis obsoletis sublinearibus, ventre concaviusculo igneo nigro-maculato, alis hyalinis disco el ad costam nornilit sordidis, cellula radiali subcompleta, postscutello obtuse conico subtuberculiformi.

Habetat in Helvetia, ad St. Luzi ab oculatissimo Doctore Kriechbaumer d. 13. Junii 1847 detecta et mihi benigne communicata.

Observ. Valde similis et affinis Chrysidi caerulipedi (No. 78), at sculptura et pictura facillime dignoscilur. Caput et thorax saturate viridia, rertice et thoracis dorso in colorem aeneum vergentibus. Caput rotundatotriangulum, prothorace distincte latius, sparse fusco- et cano-hirtellum, confertim modice punctato-rugulosum: vertex convexo-depressus; stemmata magna brumeo-testacea pellucida; cavitas facialis planiuscula confertius punctulato-coriacea, spatio mediano subtilius punctulato. Antennae mediocres nigro-fuscae: scapo toto, articulisque 3:bus proxime sequentibus supra, virescentibus. Clypeus et mandibulae ut in Chryside caerulipedi constructa et colorata; mandibulae tamen robustiores. Thorax nitidus parce fusco-hirtellus coriaceus modice punctatus et punctulatus, scil. puncta majora et punctula minuta confertim mixta; pronotum antice foveola centrali; dorsuli suturae 2 medianae lenissime curvae fuscae crenulatae; postscutellum obtuse conicum 1 . potius in centro tuberculiforme crasse punctato-reticulatum, basi forea centrali. Pedes aeneo-virides tarsis fuscis. Alarum tegulae aeneo - virides. Alae hyalinae, in alarum anticarum cellulis basalibus et 
medianis praecipue rero ad costam cellulae radialis adsunt umbrac brumneo - fumosae. Abdomen ut in Chryside caerulipedi constructum; segmenta dorsalia aureo-coccinea, confertissime punctulato-coriacea et subgranulosa, hinc minus nitida et fere opaca, lineola mediana subtilis et elevata ad certum situm luminis continua videtur per omnia segmenta; segmentum 3:tiun apice anguste arcuatum, in regione ubi series jacet nonnihil immersum, margo apicalis depressus nomnihil humilior quan reliqua pars segmenti, series ante-apicalis irregularis foveolis numerosis obsoletis leniter impressis linearibus. Venter eandem structuram et picturam praebet quam apud $\boldsymbol{C h}$ rysidem caerulipedem.

72. Chrysis refulgens Spinol. 9.

Maxima 4 lin. decimal. long. robusta pubescens, capile normali thoraceque confertim punctato-subreticulatis cyaneis viridi-pictis, raro totis viridibus, postscutello conico, abdomine confertim modice punctato purpureoaureo viridi splendente, segmento 3:lio mox supra seriem subcalloso-marginulato, serie ante-apicali distinctissima, cellula radiali incompleta apice anguste aperta.

var. $a$. Caput et thorax cyanea, leviter viridi-picta, abdomine purpureo-aureo.

Chrysis refulgens Spin. Ins. Lig. Tom. I. 8. No. 4. Tab. 1. fig. Ill. secundum specimina typica, quae Illustr. Spinola e Museo suo mihi benigne communicavit.

Chrysis scutellata Panz. teste Spinola in litteris.

var. b. Caput et thorax viridia, abdomine viridi-aureo.

Habitat in Europa meridionali et Asia minori parce aut raro; Dalmatiae: D. Dahl, Mus. Vienn.; Italiae ad Genuam a D. Spinola primum detecta; etiam in Rossia

$$
\text { 9* }
$$


meridionali observata, ut fertur; in Asia minori ad Patara mense Majo a D. Loew lecta.

Observ. var. $a$. Species eximia et elegans, forte maxima individua hujus Phalangis praestans. Caput rotundato-triangulare pendulum, cyaneum confertim punctulatum subrugulosum opacum; vertex et frons converodepressa; oculi subparvi at valde exserti; facies concaviusculo-plana, coriaceo-granulosa, sulculo mediano pone antennas subtili polito violascente aut virescente. AnIennae mediocres nigro-fuscae, articulis 3 : bus basalibus cyaneis plus minus virescentibus. Clypeus mediocris subquadratus longitudine paullo latior, depressus inaequalis punctulato - coriaceus, cyaneus 1 . plus minus virescens, margine apicali summo piceo. Mandibulae robustae piceae, basi macula viridi-cyanea. Thorax coriaceus, punctato-reticulatus saturate cyancus: pronoti striolae transversae indeterminatae, striola longitudinalis in dorsuli utraque area laterali, margines postici et laterales scutelli postscutellique, nec non maculae metanoti postico-laterales virescentes. Pronotum transverso-rectangulum; truncatura antica subperpendicularis leviter concaviuscula, fovea centrali politissima nigro-violacea, margo posticus emarginato-subarcuatus. Dorsuli suturae 2 medianae paullulum curvae crenulatae nigrae. Postscutellum obtuse conico-prominulum. Metanoti anguli postico-laterales robusti lati, obtuse triangulares, ad longitudinem mediocres. Pedes cyaneo-virides aut viridi-cyanei, calcaribus brunneo-testaceis, tarsis fuscis. Alae hyalinae; anticae disco ample et saturate brunneo-fumatae; tegulae ovales confertim punctulatae viridi-cyaneae aut vice versa subnitidac; venae firmae piceae; cellula radialis lanceolata 
incompleta apice modice aperta, vena radialis ab alae apice paullulum remota. Abdomen magnum, capite thoraceque simul sumtis $\frac{1}{4}$ nisi $\frac{1}{3}$ longius, robustum ovale dorso depresso-convexum, confertim punctatum nitidum purpureo-aureum, basi lateribusque plus minus viridiresplendens, impressio basalis segmenti 1 :mi parva semilunata nec non margines basales segmentorum $2:$ di et $3:$ tii nigro-aenescentes; carinula mediana subtilis in segmentis 1:mo et 3:tio distincta aut obsoleta et plerumque abrupta, in segmento 2:do distinctissima et subcontinua; segmenti 3:tii series ante-apicalis immersa, - hinc ipsum segmentum (mox supra seriem) quasi calloso-marginulatum, - medio per carinulam brevissimam intercedentem depresso-planiusculam interrupta, utrinque foveolis 9 profundiusculis approximatis subovatis; (segm. 3:tii) margo summus apicalis fusco-aenescens arcte 1. subcompressoarcuatus, unde segmenti ambitus fere formam obtusissime conicam accipit. Venter depresso-planiusculus, in exsiccatis plus minus concaviusculus, confertissime punctulatus, nitidus igneus nigro-maculatus.

73. Chrysis integrella Dlbm. ơ

Mediocris aut subparva 2-3 lin. decimal. long. subrobusta sublinearis pubescens capite normali thoraceque confertim punctulato-coriaceis subopacis viridicyaneis, postscutello convexo mutico, abdomine confertissime subtiliter punctulato - coriaceo opaco, segmento 3:tio mox supra seriem subcalloso-marginulato, serie ante-apicali distinctissima, cellula radiali incomplela apice late aperta.

Chrysis integra Panz. var. minor, secundurn Museum Spinolae. 
Chrysis austriaca Dlbm. Mon. Chr. 14. 12, Excercit. Hym. 31. 15. et Dispos. 7, 7, exclusis synonymis Fabricii.

Habitat in locis argillosis ab Italia maxime meridionali usque in Fenniam meridionalem Junio - Julio passim. Tauriae: D. Parreis, Mus. Vienn. teste D. Kollàr; Italiae: D. Spinola ; Helvetiae: D. Kriechbaumer; Galliae: D. D. Dufour et Latreille; Saxoniae: D. Tischbein; Silesiae: D. Zeller; Brandenburgiae: D. Ratzeburg; Sreciae: Scania D. D. Fallén et Zetterstedt; ad Lund Scaniae in pariete argillaceo d. 15-18. Julii 1830 utrumque sexum in nido Odyneri parietum legi - Westrogothia D. D. Gyllenhal et Schönherr - Ostrogothia prope urbem Wadstena d. 11. Julii in sepimento ligneo mihi quoque obvia. Finlandiae ad Helsingfors: D. Nylander.

Observ. Corpus lineare subellipticum fusco- et canopubescens pilosellumque. Caput et thorax confertim punctulata subcoriacea parum nitida cyanea, punctura frontis et pronoti interdum subreticulata. Caput normale rotundato-triangulum; frons, facies et clypeus plerisque virides. Cavitas facialis mediocris superne subtilissime et obsolete limitata, medio subtilissime transverse strigoso-coriacea, carinula mediana subtilissima. Antennae longitudive mediocres, tenues nigro-fuscae, scapo et pedicello virescentibus. Clypeus mediocris vix magnus, transversus subtrapezinus inaequalis punctulatus nitidus, margine apicali piceo, ad centrum arcuato-emarginato subtruncato. Mandibulae piceae, basi aeneo-virescentes. Pronotum nargine antico el humerali plus minus virescens, foveis tribus: mediana lanceolata postice obliterata, reliquis duabus ad marginem posticum utrinque sitis linearibus. Tegulae plerumque virides. Dorsuli suturae 2 medianae crenulatae 
paullulum curvae. Scutellum, metanotum et pectus interdum viridi-picta. Metanoti anguli postico-laterales mediocres. Pedes viridi-cyanei' tarsis colore variantibus: 1. fuscis 1. brunneis 1. testaceis. Alae hyalinae disco leviter sordidae; venae mediocres piceae; cellula radialis lanceolata incompleta, apice late aperta; vena radialis parum curva, ab apice alae longe remota. Abdomen capiti thoracique simul sumtis ad longitudinem aequale, thorace fere latius, lineari-ellipticum, dorso depresso-convexum confertissime subtiliter punctulato-coriaceum, segmento 1: mo etiam punctis crassioribus adsperso, igneo-aureum basi et limbo plus minus viridi-micans, ad certum situm luminis lineola mediana subelevata subtilissima in segmento 2:do vix conspicitur; segmenti 1:mi impressio basalis nigro-aenea, segmeutorum 2 :di et 3:tii margines basales interdum concolores; segmentum 3:tium in duas regiones divisum: regio antica $s$. basalis maxima semilunata convexo-depressa, - regio postica s. apicalis triplo brevior linearis arcuata basi seriem fovens; series ante-apicalis distinctissima, sub margine regionis basalis immediate sita, regularis foreolis mediocribus rotundatis approximatis numerosis circ. 16-20; venter depresso-planus, plus minus concaviusculus, confertissime subtilius punctulato-coriaceus, nitidus igneus nigro-maculatus, interdum viridi-resplendens.

Subdivis. 2. Thorax aut aureo-fasciatus aut antica et majori parte aureus.

74. Chrysis cuprata Klug. ㅇ.

Subparva via 2 lin. decimal. long. robustula crasse punctata cyaneo-viridis, vertice, thoracis abdominisque 
dorso viridi-cupreis, ventre maxime fornicato nigroaeneo levissime viridi-picto, serie ante-apicali foveolis rotundis profundis subpellucidis numerosis distinctissimis, alis subhyalinis venis fuscis.

Clerysis cuprata Hoffmannsegg secund. Mus. Berolin.

Spintharis cuprata Klug Mus. Berolin.

Habitat in Sicilia, D. Zeller Mus. Berolin.

Observ. Habitus fere Chrysidis integrellae (No. 73). Corpus cinereo-pubescens et pilosellum. Occiput modice punctatum et frons punctulata aenescenti-viridia. Vertex crasse punctato-reticulatus aeneo-cupreus. Frontis sulcus medianus politus impunctatus. Antennae mediocres robustae basi virescentes, medio fuscae, apice brunneae. Clypeus ut in Chryside crassimargo constructus at brevior, disco aeneo-cupreus; mandibulae quoque ut in Chr. crassimargo pictae. Thoracis dorsum et mesopleurae crasse punctato-reticulata. Pronoti pars colliformis violascens, macula centrali viridi, utringue fovea profunda orbiculata; pars posterior pronoti, dorsulum, scutellum et mesopleurarum discus viridi-aeneo-cuprea; metanotum et pleurae viridia. Tegulae virides nitidae testaceo-marginatae. Pronoti pars posterior antice impressione centrali irregulari. Sternum nitidum parce punctulatum cyaneum limbo viridi. Abdomen mediocre suboratum, dorso modice convexum, viridi-aut subaene-cupreum, parum nitidum; segmenta 1:mum, 2:dum et 3:tium modice confertim punctata; margo apicalis humilis prominulus arcuatus. Pedes virides tarsis brumeo-testaceis. Cellula radialis ovato-lanceolata subcompleta, scil. venae radialis apex costae intactae approximatus: apertura igitur valde angusta. 
75. Chrysis viridana Dlbm. 오.

Parva gracilis $1 \frac{1}{3}$ lin. decimal. long. modice punctaln viridis, vertice pronoto mesonoto abdominisque dorso viridissime auratis, hoc punctulato-coriaceo margine apicali purpureo-aureo, serie ante-apicali levi (non immersa) punctis parvis rotundis distinctis, ventre modice fornicato nigro-, viridi- et igneo-maculato, alis hyalinis venis fuscis, cellula radiali completa.

\section{Habitat in Asia minori, D. Loew.}

Observ. Corpus fere ut in Chryside succincta constructum, sparse cinereo- et cano-pilosellum. Caput viride latiusculum, vertice et occipite punctalo-subreticulatum; oculi parvi valde exserti; antennae longiusculae subangustae fuscae, a basi fere in medium viridi-nitidae; clypeus parvus, apice subtransversus; mandibulae nitidae piceo-brunneae; palpi tenues fusci. Thoracis dorsum et mesopleurae subtiliter punctato-reticulata; pronotum et mesonotum viridissime aurea, dorsuli area media basi coriacea; metanotum et pectus viridi-cyanea aut cyaneoviridia. Pedes virides tarsis fuscis. Alae hyalinae, ad costam cellulae radialis nonnihil sordide subfumosae; cellula radialis triangulariter lanceolata completa, scil. venae radialis apex tenuis costam tangit. Abdomen ovato-oblongum confertim punctulatum fere coriaceum, viridi-aureun, modice depresso-convexum; segmenti 3 :tii series ante-apicalis centro interrupta 1. subobliterata, margo apicalis summus arcuatus tenuis (non incrassatus) paullulum oblique prominulus rufo-aureus.

\section{Chrysis Germari Wesmaël.}

Parva vix 2 lin. decimal. long. oblonga subrobushula 
crasse et modice punctata cyanea, pronoti margine anlico et mesonoto viridi-aureis, abdomine cupreo-aureo punctato et punctulato, segmenti 3:tii margine apicali viridi-aeneo, serie ante-apicali foveolis paucis crassioribus, ventre plano viridi-cyaneo-picto, alis hyalinis: venis fuscis, cellula radiali completa.

Chrysis nitidula Germar. It. Dalmat. 260. 43; Faun. Ins. Europ. IV. fig. 12. (Non Fabricii Chr. nitidula, quae in nostra Phalange VI:ta occurrit.)

Clirysis Germari Wesmaël Notice etc. pağ. 11.

Habitat in Europa meridionali rar.; in Croatia a D. Germar detecta; in insula Melita maris mediterranei a D. Antonio Schembri inventa, teste D. Zetterstedt qui specimen Melitense mihi dono dedit.

Observ. Chrysidi succinctae valde similis, at corpore minore, ano integerrimo etc. facile dignoscitur. Corpus parvum subrobustum fusco- et griseo-pubescens pilosellumque nitidum. Caput orbiculatum conrexum modice punctatum et punclulatum, pronoto paullulum latius cyaneum, maculis parvis irregularibus ante et post virescentibus; cavitas facialis confertim punctulato-coriacea, laete viridis utrinque argenteo-pilosella. Clypeus brevissimus inaequalis punctulatus viridis nitidus. Antennae robustae nigro-fuscac cano-puberulae, scapo viridi, pedicello et articulo 3:tio supra ex parte concoloribus. Mandibulae breves robustae, basi virescentes, medio rufo- et apice nigro-piceae. Pronotum breve transversum subrectangulum cyaneum, pars antica viridi-aurea punctatosubreticulata: fovea mediana obsoleta, truncatura antica declivis aenescenti-viridis: antice fovea centrali gemina polita violacea; pars postica modice punctata 1. punctu- 


\section{Chrysis: Phalanx $I$.}

lata subcoriacea; margo posticus summus vix nisi lenissime emarginato - arcuatus, potius subrecta transversus. Mesonotum et postscutellum convexa; illud subcrasse punctato - reticulatum; dorsulum viridi - cupreum suturis 2 medianis angustissimis subparallelis, basi tantum levissime curvis, scutellun viridi-aureum. Metanotum et pectus punctulato - coriacea cyanea, mesopleuris nonnihil virescentibus; postscutellum confertim punctulato - subreticulatum; metanoti anguli postico-laterales mediocres triangulares. Pedes cyaneo-virides cano-pilosi femoribus robustis, tarsis testaceo-brunneis. Alae hyalinae venis fuscis, cellula radiali elongata triangulariter lanceolata completa. Abdomen subellipticum, thorace nonnihil angustius, dorso modice convexum 1. depresso-convexum nitidum, confertim modice punctatum et punctulatum: carinula mediana subtili et obsoleta tantum ad certum situm luminis visibili, segmentum 1:mun viride maculis 2 maximis lateralibus indeterminatis medio conniventibus viridi-auratis: impressione basali magna triangulari cyanea; segmenta 2:dum et 3:tium cuprea ad margines et ad latera viridi-resplendentia; segmenti 3 :tii margo apicalis subanguste arcuatus viridi-aeneus subtiliter punctulatus coriaceus; series ante-apicalis foreolis paucis irregularibus profundis nigris circ. 7: extimis 1 . basali-lateralibus 1:ma et 2:da minoribus, 3:tia majore, centrali maxima gemina. Venter planus nitidus pilosello-punctulatus, segmentis: 1:mo cyaneo, 2:do viridi marginibus cyaneis, 3:tio viridi basi leviter aureo-micante, margine apicali testaceo-scarioso. 
77. Chrysis candens Germar. $q \sigma$.

Subparva vix 2 lin. decimal. long. robustula modice punctata cyanea, pronoto, dorsuli areis lateralibus, abdominisque dorso confertius punctulato-coriaceo, aureis; segmenti 3:tii margine apicali cyaneo, serie ante-apicali foveolis mediocribus numerosis, ventre viridi-nigroque picto modice fornicato, alis hyalinis ad costam cellulne radialis nonnilit sordidis, venis fuscis.

Mas fronte immaculata, cellula radiali completa.

Chrysis ephippium Ziegl, teste Kollàr Mus. Vienn.

Femina fronte aurea, cellula radiali incompleta.

Chrysis candens Germar. Fn. Ins. Europ. IV. 13; Klug in Mus. Berolio.

Habitat in Germania media et meridionali rar., D. D. Germar, Klug, Kollàr et Ziegler. In Croatia a D. Germar primum detecta.

Obs e rv. Magnitudine et statura Clrysidi succinctae affinis, at ano integerrimo et ㅇ fronte aureo-maculata dignoscitur. Summa vero affinitas Chrysidis Germari, ab hac autem pictura thoracis et $q$ frontis, praesertimque sculptura abdominis distinguitur. - Caput cyaneum punctulato-rugosum et subreticulatum, $q$ macula frontali magna (supra antennas, inter orbitas sita) aurea: supra et infra arcuato - emarginata margine virescente, $\sigma$ frons immaculata. Clypeus transversus magnus latus subtrapezinus, medio continue carinulatus, utrinque subconcaviusculo-depressus, margine apicali subangulato - (at non profunde) emarginatus. Cavitas facialis medio subpolita, parcius subtiliter punctulata, lateribus punctulato-subcoriacea et cinereo - puberula. Antennae mediocres fuscae, scapus cyanescens 1 . virescens. Thorax cyaneus dorso punctulato-reticulatus coriaceus, pronoto et dorsuli areis 
lateralibus 1. rubro-1. subriridi-aureis; pronoti sulcus medianus abruptus, truncatura antica 1 . cyanea l. violacea, dorsuli area media 1 . viridi-cyanea I. violacea; scutelli discus, metanoti suturae et anguli postico-laterales plus minus virescunt. Pedes aut virides aut pure cyanei tarsis brunneis. Alae anticae cellula radialis triangulariter lanceolata, in $\sigma^{\top}$ completa, in $q$ subcompleta: scil. venae radialis apex mox ante costam obliterata spatium angustum apertum relinquens. Abdomen ovale, dorso modice convexum subviridi-aureum confertim punctulatum subcoriaceum, non politun licet micans; segmentum 3:tium disco lenissime transverse immersum, interdum ad ipsam seriem tenuiter viridi-marginatum; series ante-apicalis obsoleta e punctis numerosis irregularibus constituta, margo apicalis tenuis (non crassus) nigro-aeneus l. violascens 1. plerumque cyaneus. Apud \& sunt segmenta ventralia: 1:mum et 3:tium nigra viridi-maculata, 2:dum totum viridissimum leviter aureo-micans; $\sigma^{\nearrow}$ venter violaceus aut viridi-cyaneus, marginibus segmentorum apicalibus teslaceo-scariosis.

78. Chrysis coerulipes Fabr. $\sigma^{7}$ 오.

Oblonga robusta $3 \frac{1}{2}-4$ lin. decimal. long. modice punctata purpureo-aurea, metanoto pectore pedibusque coeruleis, abdominis dorso confertim punctato-coriaceo nitido, serie ante-apicali regulari foveolis numerosis (majoribus et minoribus simul sumtis circ. 14), ventre igneo nigro-maculato concaviusculo-plano, alis hyalinis disco et ad costam sordidis, venis fuscis, cellula radiali subcompleta, postscutello acute conico.

Chrysis coerulescens Fabr. Ent. Syst. Suppl. 257. 9. 
Chrysis cocrulipes Fabr. Piez. 173. 13; Megerl. Mus. Vienn. Specimen idem typicum, quod Fabricius in Syst. Piez. 1. c. descripsit, e Musen Imper. Viennensi coroparationis caussa benigne transmisit D. Kolliar.

Habitat in arenosis Europae mediae et meridionalis passim; in Brandenburgia ad Neustadt-Eberswalde semel lecta a D. Ratzeburg, in Silesia: D. Schneider secundum D. Dolern in litteris, in Austria a D. Megerle de Miihlfeld primum detecta test. Fabricio 1. c. et D. Kolliar, in Italia: ad Genuam D. Spinola et ad Buda D. Zeller; in Turcia Europaea D. Drewsen.

Observ. Corpus oblongum ellipticum robustum. Caput rotundato-triangulare pendulum aureum viridi-resplendens, coriaccum confertim punctulatum, vertice convexo-depresso subreticulato, stemmatibus magnis testaceo-pellucidis; cavitas facialis perpendiculariter declivis planiuscula subtiliter confertim punctulata: spatio mediano nitidiori subigneo sublaevi aut parcissime punctulato, canalicula mediana subtili. Antennae breves robustae, brevissime fusco- et cano-puberulae nigro-fuscae: scapo I. coeruleo I. violaceo, duobus proxime sequentibus articulis supra virescentibus. Clypeus magnus gibbus crasse punctatus aurato -aeneus, margine apicali prominulo nigropiceo nitido, ad latera obliquo subemarginato, ad centrum late truncato. Mandibulae basi punctulato-rugosae viridi-cyaneae, ceterum nitidae politae piceae, ante apicem nonnihil rufescentes; pagina inferior capitis coerulea aut cyanea, margine aureo-viridi. Thorax subcylindricus supra depresso-convexus; pronotum et mesonotum I. virescenti- 1. purpureo-aurea confertim punctato-rugosa, illud anticae sulco mediano abrupto; dorsuli suturae 2 
medianae subprofundae continuae crenulatae fuscescentes. Scutellum sat convexum. Metanotum et pectus coerulea punctulato - coriacea minus nitida. Postscutellum acute conicum. Sternum nitidissimum sparse et subtiliter punctulatum viridi-cyaneum. Pedes coerulei aut cyanei viridi-resplendentes, tarsis fuscis. Alae subliyalinae, disco obsolete et ad costam cellulae radialis saturatius brunneo-fumosae l. sordidae, venis fuscis. Tegulac alarum violaceae nitidae sparse subtiliter punctulatae. Abdomen elongatum ovale, capite thoraceque simul sumtis conspicue longius, apice anguste arcuatum; segmenta 1:mum et 2:dum viridi-aurea nitidiora, illud confertim punctato-rugosum, hoc confertius punctulatum; segm. 3:tiun plerisque intensius coccineo-aureum minus nitidum subopacum confertissime punctulato-coriaceum; in segmentis 2:do et 3:tio lineola mediana elevata ad certum situm luminis animadrertitur; segmenti 3:tii series ante-apicalis foveolis approximatis mediocribus rotundatis ant ovatis circ. 14; in quibusdam speciminibus est segmentum (3:tium) supra seriem subcallosum et supra callum deinde subimmersum, in aliis autem omnino muticum; in quibusdam sunt seriei foveolae distinctae et majores, in aliis minores et obsoletae, in aliis obsoletae et confluentes, quarum nonnullac interdum quidem eranescunt. Venter 1 . planiusculus 1. concariusculus, semper carinula mediana subtilissima instructus, igneus ex aureo- et viridi-resplendens: segmenta 1:mum et 2:dum maculis basalibus binis longitudinalibus 3:tiunque macula basali transversa atris.

79. Chrysis Gyllenhali Dlbm.

Súbmediocris $2 \frac{1}{2}$ lin. decimal. long. oblonga suban- 
gusta modice punctata coerulea, antennis fuscis, pronoto mesonoto abdominisque dorso viridi-aureis, hoc modice subconfertim punctulato nitido; segmento 3:tio toto modice convexo, serie ante-apicali regulari foveolis parvis rotundatis separatis 12, margine apicali late outuse arcuato, ventre viridissime aurato nigro-maculato concaviusculo plano, alis hyalinis venis brunneis, cellula radiali completa, postscutello mutico depresso-convexo.

IIabitat in Westrogothia Sveciae, a D. Gyllenleal olim detecta et sub nomine ,Chr. nitidula Grmr." mihi donata.

Observ. Maxime affinis Chrysidi dichroae (No. 81), a qua tamen facile dignoscitur corpore paullulum angustiore, thorace proportionaliter longiore et minus convexo, abdominis dorso nitido, nec ullo modo coriaceo, minus confertim punctato; segmento abdominis 3 : tio toto modice convexo, nec immerso nec calloso, margine apicali paullo latius et obtusius arcuato, alis totis hyalinis. Ceterum, quoad reliquam sculpturam et picturam corporis, ulteriorem diversitatem detegere nequeo.

Caput rotundato-triangulare totum coeruleum, modice punctato-rugulosum; vertex convexo-depressus; cavitas facialis punctulata: spatio mediano subtiliter coriaceo, distincle canaliculato. Antennae breves robustae fuscae scapo cyaneo. Clypeus mediocris carinato-convexus punclulatus viridi-cyaneus, margine apicali nigro-piceo polito truncato. Mandibulae breves robustae, basi viridi-cyaneae, apice rufo-piceac. Thorax convexiusculo-depressus; pronotum et mesonotum confertim modice punctatorugosa nitida viridissime aurea; pronotum sulco mediano subcontinuo paullo ante marginem posticum desinente, Iruncatura antica sparse punctata viridi-aenea foveolis 
2 centralibus politis. Metanotum coeruleum concinne (nec crasse) punctato-reticulatum forea basali centrali. Pleurae concolores parum viridi-nitentes punctato-rugulosae. Sternum et pedes nitida, parce piloso-punctulata, viridi-cyanea tarsis fuscis. Abdomen ovali-ellipticum, dorso modice convexum nitidum splendidum viridi-aureum confertim punctulatum, in segmentis 2 : do et 3 : tio lineola mediana subtilis elevata ad certum situm luminis animadrertitur; segmentum 3 :tium non minus convexum quam 2:dum 1. 3:tium, margo apicalis obtuse arcuatus et parum brevior quam margo basalis; series ante-apicalis regularis levis (non profunda) foveolis parvis rotundatis separatis circ. 12: mediis distinctioribus, extimis 1. lateralibus obsoletis. Ventris segmentum 1:mum viridi-aeneum apice nigro-piceum, 2:dum viridissime aureum: macula basali maxima reniformi atra (segm. 3:tium in nostro unico individuo mutilatum).

80. Chrysis socia Dlbm.

Subparva 2 lin. decimal. long. oblonga angusta modice punctata coerulea antennis apice laete brunneis, pronoto mesonoto abdominisque dorso viridi-nureis, lhoc modice punctulato nitido, segmento 3 :tio toto modice convexo, serie ante-apicali regulari foveolis minutis orbiculatis approximatis numerosis circ. 16, margine apicali late obtuse arcuato, ventre viridissime aurato nigromaculato, concaviusculo-plano, alis hyalinis ad costam cellulae radialis sordidis, cellula radiali subcompleta, scutello mutico depresso-convexo.

Habitat in Sicilia, D. Groltmann; Mus. Vienn.

Observ. Clerysidi Gyllenhali affinis et simillima;

II. 
at dignoscitur corpore angustiore et breviore, abdominis segmenti 3:tii serie ante-apicali foveolis minutis approximatis numerosis 16 et ultra. - Caput ut in Chryside Gyllenhali formatum, totum punctulato-coriaceum opacum cyaneum, vertice circa stemmata et genis leviter virescentibus. Facies sparse cano-pilosella. Cavitas facialis confertim subtiliter punctulato-coriacea subgranulosa. Antennae breves robustae: scapo cyaneo, articulis tribus proxime sequentibus supra virescentibus, reliquis laete brunneis aut subferrugineis. Thoracis forma, sculptura et pictura omnino ut in Chryside Gyllenhali, ea solum differentia quod: punctura paullo minus crassa, pronoti sulcus medianus brevior, truncatura antica viridi-cyanea. Abdomen quoque eandem formam, picturam et puncturam, quam Chr. Gyllenhali, habet; at segmentorum dorsaliun punctura paullulum subtilior et - comparata paullo minus conferta, segmenti 3 :tii series ante-apicalis paullulum impressa (nec ita levis ut in $\boldsymbol{C h r}$. Gyllenhali) foveolis minutis approximatis numerosioribus circ. 16 et ultra, margo apicalis infra seriem conspicue brevior quam in Chr. Gyllenhali. Ventris segmenta: 1:mum nigrum, disco utrinque virescens; 2 :dum basi macula maxima reniformi aeneo-nigra, apice macula tridentata viridissime aurea; 3:tium basi virescenti-aureum, apice nigro-piceum, marginibus omnium segmentorum apicalibus testaceo-brunneo-scariosis. Alae hyalinae venis piceis, ad costam cellulae radialis umbra linearis fumosa, cellula radialis subcompleta,

81. Chrysis dichroa Klug.: $\sigma^{7}$ ㅇ․

Oblonga robusta $2 \frac{1}{3}-3$ lin. decimal. long. subcrasse 
punclata viridi-cyanea; pronoto, mesonoto, abdominisque dorso aureis, hoc confertim punctulato l. nitido l. coriaceosubopaco, segmento 3:tio infra discum transversaliter obsoletissime immerso et tum ante seriem lenissime calloso, serie ante-apicali l. regulari l. irvegulari foveolis parvis numerosis aut distinctis aut confluentibus, ventre l. concaviusculo-plano 1. modice fornicato igneo nitidissimo nigro-maculato; alis subhyalinis, venis fuscis, celluli radiali completa, postscutello mutico convexo.

Spintharis dichroa Klug Mus. Berolin.

Chrysis coerulipes var. coeruleo-cephala Mus. Spinolae.

Chrysis bicolor. Megerl, Mus. Vienn. teste Kollàr.

Habilat in Austria D. D. Klug, Kollàr, Parreys et Spinola; in Italia ad Buda, D. Zeller; in Asia minori ad Ephesum mense Aprili 1812, D. Loew.

Observ. Sculptura et pictura maxime affinis duabus proxime praecedentibus (Chr. Gyllenhali et Chr. sociae); at certe distinguitur corpore majori et robustiori, punctura thoracica crassiori, abdominis segmento 3 : tio infra discum obsoletissime immerso et tum mox ante seriem apicalem lenissime calloso. Etiam Chrysidi coerulipedi valde similis, sed capite coeruleo et postscutello mutico convexo certe dignoscitur.

Caput mediocre rotundato-triangulare, pronoto paullulum latius, confertim modice punctatum subrugulosum coeruleum aut cyaneum parum nitidum subopacum, orbita viridi. Vertex convexo-depressus. Cavitas facialis mediocris subtilissime sparse punctulata, canalicula mediana subtili abrupta. Antennae breves robustae nigrofuscae cano et fusco-puberulae, scapo infra cyaneo, sequentibus duobus articulis supra virescentibus. Clypeus 
mediocris cyaneo-viridis punctulatus, medio carinato-convexus, utrinque concavus, margine apicali polito piceotruncato. Mandibulae mediocres basi virides, tum piceae et ante apicem rufescentes. Pronotum et mesonotum coriaceo-rugulosa crasse punctata virescenti-aurea; pronoti sulcus medianus propter rugositatem irregularis, truncatura antica concaviuscula subcrasse punctata viridi-aenea: areola centrali polita utroque sulco arcuato; dorsuli suturae 2 medianae subprofundae subrectae crenulatae basi parumper curvae. Postscutellum convexo-obtusum modice punctato-reticulatum. Metanoti anguli postico-laterales mediocres triangulares. Metanotum cyaneum, ad latera nonnihil virescens. Pleurae rugosae punctatae subopacae, sternum nitidum subtiliter punctulatum viridi-cyanea aut cyaneo-viridia, pedes concolores tarsis fuscis. Alae hyalinae, interdum disco sordidae, tegulis l. viridiaeneis 1 . viridi-cyaneis, venis piceis, cellula radiali completa triangulariter lanceolata: angulo disci recto, in cellula radiali vitta brunneo-fumosa. Abdomen capite thoraceque simul sumtis longius, latitudine thoracis, oraliellipticum, dorso convexum purpureo-, coccineo- aut virescenti-aureum, confertissime punctulatum $\mathrm{I}$. coriaceosubopacum I. nitidum, in disco segmentorum 2:di et 3:tii lineola mediana subtilis elevata animadvertitur; segmentum 3:tium disco (1. paullulum inferius) obsolete aut obsoletissime (scil. varie in variis individuis) transverse subarcuato-immersum, tum (secundum immersitatem) lenissime callosum 1. convexo-subelevatum, series ante-apicalis infra hanc lenissimam convexitatem sita regularis aut irregularis scil. foveolis parvis ovalibus numerosis distinctis aut confluentibus constituta; margo apicalis fortiter 
convexo - arcuatus, angustior quam margo basalis, convexodepressus subtiliter punctulatus, sumnus brumneus; segmenta ventralia basi nigra, apice ignea marginibus summis apicalibus piceo-scariosis, segmentum anale piceum.

82. Chrysis uniformis Dlbm.

Oblonga modice robusta vix 3 lin. decimal. long. cyanea, pronoto scutello et postscutello viridi-aureis, abdomine coccineo - aureo, pronoto et dorsulo coriaceis, postscutello gibbo obtuso, abdomine confertissime punctulato - coriaceo, segmento 3:tio disco immerso, tum ante seriem apicalem perpaullulum incrassato, serie ante-apicali sub marginulo illo semiabscondita continua foveolis mediocribus numerosis approximatis, alis hyalinis venis fuscis, cellula radiali incompleta, apice late aperta, vena radiali ab alae apice longe remota.

Habitat in Asia minori, mense Aprili, D. Loew.

Observ. Valde affinis Chrysidi dichroae et Clrysidi coerulipedi; at dorsulo coriaceo et segmento abdominis 3:tio aliter constructo certe distincta. - Caput confertim modice punctato - coriaceum fusco - cyaneum, frons utrinque ad oculos linea 1 . macula orbitali viridi. Singulum quodque stemma in singula sua fovea situm. Antennae modice robustae, subbreves fuscae, basi cyaneae leviter virescentes. Clypeus magnus transversus latus depressus inaequalis, parce punctulatus viridis, apice nigro-aeneus, ad centrum marginis apicalis leniter enarginulatus. Mandibulae nigro-piceae ante apicem brunnescentes. Pronotum robustum viridi-sordide auratum coriaceum punctis et punctulis adspersum, sulcus medianus longitudinalis profundus cun sulculo ante marginem 
posticum transverse ducto obsoleto subcohaerens. Dorsulum totum cyaneum coriaceum. Scutellum convexum, postscutellum gibbum obtusum crasse, punctato-reticulata viridi-sordide aurea. Metanotum et pectus cyanea viridi-picta. Pedes uno latere virides, altero cyanei, tarsi fusci. Alae hyalinae, ad marginem cellulae radialis costalem sordidae subfumosae; cellula radialis valde incompleta, apice hiante aperta propter venam radialem lenissime curvam et fere rectam, apice a costa late remolo. Abdomen coccineo-aureum confertim punctulato-coriaceum, ad latera et ad apices 1:mi et 2: di viridi-aureoresplendens, segmentum 2:dum margine basali violascente; segmentum 3:tium disco leniter concaviusculo-subimmersum, tum prominulum in marginem subcallosum triangulariter arcuatum, qui seriem ante-apicalem ex parte abscondit; haec (series ante-apicalis) e punctis mediocribus approximatis hinc inde confluentibus constituta continua; margo apicalis tenuis arcuatus. Venter totus igneus.

Phalanx II. Chrysides ano inaequales. Scil. Abdominis segmenti 3:tii margo apicalis inaequalis, lenissime aut undulatus aut angulatus; quae tamen inaequalitas plerumque nonnisi oculo acute armato detegi potest.

Sectio I. Inaequalitas marginis apicalis (in abdominis segmento dorsali $3:$ tio) obsoleta aut obsoletissima.

83. Chrysis Dalmanni Dlbm. 오

Mediocris oblonga circ. 3 lin. decimal. long. crasse 
Phalangis II: dae Spec .

Sectio I. Inaequalitas marginis apicalis (se aut obsoletissima.

A. Abdomen violaceum: fascia segmentorum d et venter cyanea: pronoto, tegulis, scutello lenissime inaequalis, scil. utrinque inter basi)lbm. Mus. Paykull.

B. Abdomen aureum segmento 3 : tio toto cyane

a) Vertex, pronotum, dorsuli areae lateral duo antica aurea; segmentum 2:dum fa supra seriem macula centrali viridi, seri noribus 8-12, margo apicalis lenissimnn. Cap. b. sp.

b) Thoracis dorsum abdominisque segment. aeneo, segmentum 3:tium ante seriem a sulcis numerosis profundis parallelis bre lateribus et apice ita leniter sectus ut at cyanescentia . . . . . . . s Dlbm, Mus. Payk.

c) Caput et thorax cyaneo- et viridi-varię 3:tium basi nigro-aeneum, disco subat veolis linearibus subellipticis non conflur. Tunis.

C. Abdomen aureum: segmenti 3 : tii margo ap nissime inaequalis, series ante-apicalis foreol

a) Pronoti margo anticus et dorsulum aurt. var. c. Europa

b) Pronotum aureum ........ var. d. $\}$ media et

c) Dorsulum viridi-aureum ..... var. e. meridional

Sectio II. Inaequalitas marginis apicalis 1 stincta.

Divis. 1. Centrum marginis apicalis (abdominis se!

Subdivis. 1. Abdominis segmentum 3 :tium ant apicali excipienda nullo modo pro

A. Abdominis dorsum parte basali virescens, $p$ aeneo-purpurascens leniter undulatus; series cribus. Caput, thorax et pedes cyaneo-viridt. var. $g$.

B. Abdominis dorsum parte basali cyaneo-vire: segmenti 3 :tii series ante-apicalis. Caput, *

C. Abdominis dorsum totum 1. viridi-aeneum ]

a) Abdominis segmenti 3 :tii margo apicali put, thorax et pedes cyanco-viridia al aureo-viridi; venter igneus; scries ante-.

$$
\text { var. h. }\left\{\begin{array}{c}
\text { Europa } \\
\text { media et } \\
\text { var. } f .
\end{array}\right.
$$




\section{Tabula Synoptica II: da sistens Symopsin}

\section{Ihalangis II: daw Speciermm nol,is cognitarum (ieneris Chrysidis. pag 150 et 151.}

Sectio I. Inaequalitas marginis apicalis (segmenti abdominis 3:tii dorsalis) obsoleta aut obsoletissima.

A. Abdomen violaceum: fascia segmentorum dorsalium 1:mi et 2:di apicali viridi; caput, thoras et venter cyanea: pronoto, tegulis, scutello et postscutello aureis; segmenti 3 :tii margo apicalis lenissime inaequalis, stil. utrinque inter basin et apicem obsoletissine quasi angulatus

B. Abdomen aureun segrento 3 : tio toto cyaneo.

a) Vertex, pronotum, dorsuli areae laterales, scutellum, postscutellum et abdominis segmenta duo antica aurea; segmentum 2:dum fascia basali ampla arcuata cyanea; segmentum 3:tium supra seriem macula centrali viridi, series ante-apicalis irregularis foveolis majoribus et mi-

boribus $8-12$, margo apicalis lenissime undulatus. Venter cyaneus . . . . " " .

84. Chrysis simeosa Dlma. Cap. b. sp. aeneo, segmentum 3 :tium ante seriem macula centrali viridi, series ante-apicalis inmersa: sulcis numerosis profundis parallelis brevibus sublinearibus, margo apicalis subarcuatus, at lateribus et apice ita leniter sectus ut anguli 4 perobsoleti exstent. Pectus, pedes et venter cyanescentia.

3:tium basi nigro-acneum, disco subaureo-viride, apice cyaneum, series ante-apicalis foveolis linearibus subellipticis uon confluentibus circ. 14, margo apicalis subundulatus. .

C. Abdomen aureum: segmenti 3 : tii margo apicalis aeneus aut violascenti-purpureus, arcuatus lenissime inaequalis, series ante-apicalis foveolis numerosis rotundatis mediocribus.
a) Pronoti margo anticus et dorsulum aurea
b) Pronotum aureum. . .
c) Dorsulum viridi-aureum

Sectio II. Inaequalitas marginis apicalis (segmenti abdominis 3:tii dorsalis) distincta.

Divis, 1. Centrum marginis apicalis (abdominis segmenti $3:$ tii) emarginulatum

Subdivis. 1. Abdominis segmentum 3 :tium anle marginem apicalem sub-leve h. e. pro serie anteapicali excipienda nullo modo profunde sulcatum.

A. Abdominis dorsum parte basali virescens, parte apicali auratum; segmenti 3 : tii margo apicalis aeneo-purpurascens Ieniter undulatus; serics ante-apicalis foveolis numerosis rolundatis mediocribus. Caput, thorax et pedes cyaneo-viridia. Venter cyaneo-viridi-et aurco-pictus

B. Abdominis dorsum parte basali cyaneo-virescens, parte apicali viridi-auratum. Margo apicalis segmenti 3 : lii series ante-apicalis, Caput, thorax, pedes ct veater ut in $\mathbf{\Lambda}$. . . . . . .

C. Mbdominis dorsum totum 1, viridi-aencum 1. aeneo-auratum 1. aureum.

a) Ahdominis segmenti $3: t i$ margo apicalis discolor scil. acneus (totus leniter undulatus). Caput, thorax et pedes cyanco-viridia aut viridi-cyanea; pronolum antice utrinque macula aureo-viridi; venter igneus; scries ante-apicalis foveolis rotundatis mediocribus . 
b) Abdominis scgmenti 3:tii margo apicalis dorso concolor.

a. Margo apicalis sub initio seriei, h, c. inter basin lateralem et centrum emarginulatum, angulo obsoleto munitus.

1. Corpus subparvum punctulatum subcoriaceum parum nitidum, 1. viridi-aeneum l. aeneoauratum, tarsis 1. fuscis 1. testaceis, alis albis . . . . . . . . . 94. Clerysis versicolor Spinol. Italia.

2. Corpus mediocre aut submagnum modice punctatum nitidissimum cyaneum, pronoto, mesonoto abdominisque durso viridi-aureis, tarsis fuscis, alis soldide lyalinis. . .

B. Margo apicalis inter basin laterale
et thorax cyanea plus viridi-picta.

1. Corpus majus crasse punctatum; pedes virides, tibiae extrorsum aureo-splendentes, tarsi albo-testacei; abdom, segm. 3: tii series ante-apicalis foveolis profundis pacis .

2. Corpus brevius, minus crasse punctatum. Pedes cyaneo-virides tarsis fuscis; abdorn. segmenti 3 :tii series ante-apicalis foveolis confluentibus minus distinctis $\cdot . \cdot$.

D. Corpus totum cyaneum viridi-picturn, magnitudine mediocre, confertim punctatum subnitidum;
segmenti 3 :tii series ante-apicalis foveolis majusculis profundis subnumerosis; margo apicalis

utrinque angulo laterali distincto
Subdivis. 2. Abdominis segmentum dorsale 3 : tium pro serie ante-apicali excipienda utrinque profunde transverse sulcatum; series ante-apicalis per carinulam medianam interrupta foveolis sulciformibus; margo apicalis fortiter incrassato-callosus, sumrus pone emarginaturam centralem utrinque integerrimus. Corpus magnum robustum confertim modice punctatum cyaneura; vertex et thoracis dorsum viridi-irrorata. Abdominis dorsur cocrinen-aureum . . . . . . . . . . . . . .

Divis. 2. Centrum marginis apicalis (abdominis segmenti $3 ;$ tii) integerrimum.

Subdivis. 1. Abdominis segmenti $3:$ tii margo utrinque sub initio seriei ante-apicalis angulis duobus distinctis munitus.

Pronotum, dorsuli areae laterales abdominisque dorsum aurea; abdom. segmenti 3 : tii margo apicalis purpurascenti-acneus . . . . . . . . . .

Subdivis. 2. Abdominis segmenti 3 :tii margo utrinque sub initio seriei ante-apicalis angulo unico plus minus distincto munitus.

A. Abdominis segmenti dorsalis 3 :tii series ante-apicalis foveolis longitudinalibus sulciformibus linearibus paucis.

Corpus mediocre, robustum nitidum punctatum viridi-aeneum, alis albis

88. Chrysis mediocris nob. Austria.

89. C'hrysis emarginatula Spinol. Italia. 90. Chrysis mixta nob. Liguria.

91. Chrysis carinata Spinol. Cap. b. sp.

92. C'hrysis crassimargo Spinol Hispan.

87. Chrysis elegans Pellet. var, a. Europ.

B. Abdominis segmenti dorsalis 3 : tii series ante -apicalis foveolis rotundatis numerosis.

a) Abdomen aureurn: margo apicalis convexus punctatus (aeneus aut purpurascens). Alac sordide hyalinae.

1. Pronotum et dorsuli areac laterales aurea.

2. Pronotum et mesonotum aurea.

87. Clirysis elegans Pellet. var. a $b$.

orpus aut violaceum aut coeruleum. Alae albissimae.

1. Abdominis segmenti 3 :tii margo apicalis paullulum angustior et prominulus, inter

2. Abdominis segmenti 3 : tii margo apicalis latus brevissinus, inter angulos depressoarcuatus, Corpus lacte cocrulcum.

95. Chrysis albipennis Klug. Austria. 96. Chrysis unicolor Dlbm, Svecia. 
b) Abdoninis segmenti 3 :tii nargo apicalis dorso co

c. Margo apicalis sub initio seriei, h. c. inter basir gulo obsoleto munitus.

1. Corpus subparvum punctulatum subcoriaceu anratum, tarsis 1 . fuscis 1 . testaceis, alis alb lia

2. Corpus mediocre aut submagnum modicc mesonoto abdominisque dorso viridi-aureipia

B. Margo apicalis inter basin lateralem et centrum ct thoras cyanca plus viridi-picta.

1. Corpus majus crasse punctaturo; pedes vi tarsi albo-testacei; abdom. segm. 3 :tii seri

2. Corpus brevius, minus crasse punctatum. segmenti 3 :tii series ante-apicalis foveolis

D. Corpus totum cyaneum viridi-picturn, magnitudine m segracnti 3 :tii series ante-apicalis foveolis majusculis utrinque angulo laterali distincto . . . . . . b. b. sp.

Subdivis. 2. Abdominis segmentum dorsale 3 : tium pro funde transverse sulcatum; series ante-apica veolis sulciformibus; margo apicalis fortiter naturaw centralem utrinque integerrimus. C punctatum cyaneura; vertex et thoracis do coccinen-aureum . . . . . . Llispan.

Divis. 2. Centrum marginis apicalis (abdominis segmenti 3: Ialia.

Suldivis. 1. Ahdominis segmenti 3 :tii margo utrinque bus distinctis munitus.

Pronotum, dorsuli areae laterales abdol 3 : tii margo apicalis purpurascenti-ac, Europ.

Subdivis, 2. Abdominis segmenti 3 :tii margo utrinques plus minus distincto munitus.

A. Aldominis segmenti dorsalis 3 :tii scries ante-apicalis nearibus paucis.

Corpus mediocre, robusturn nitidum punctatum vpt.

B. Abdorninis segmenti dorsalis $3:$ tii series ante-apicalis

a) Abdomen aureum: margo apicalis convexus punct dide hyalinae.

1. Pronotura et dorsuli areac laterales aurea $f b$.

2. Pronotum et mesonotum aurea . . . .

b) Corpus aut volaccum aut coeruleum. Alae albis!

1. Mbdominis segmenti $3: t i i$ margo apicalis angulos converissime arcuatus. Corpus vitria.

2. Abdominis segmenti 3 : tii margo apicalis 1 arcuatus. Corpus lacte coeruleum . . .ia. 
punctata cyanen, pronolo tegulis scutello et postscutello aureis, abdomine violaceo segmentis 1:mo 2:doque fascia apicali viridissima, segmenti 3:tii margine apicali lenissime inaequali scil. utrinque inter basin et apicem obsoletissime quasi angulato, alis sordidis brunneo-liyalinis, cellula radiali subcompleta.

Chrysis torquata Dalman Mus. Paykull.

Chrysis Dalmanni Dlbm. Dispos, 1845. 12. 9.

Specimen unicum, e Collectione Paykulliana Musei R. Acad. Scient. Stockholm., communicavit D. Boheman, patria non indicata.

Observ. Statura fere Chrysidis bidentatae. Corpus oblongum subsemicylindrico-lineare confertim punctatum. Caput mediocre suborbiculatum, confertim modice punctatum subreticulatum subviolaceum; vertex amplus convexo-depressus, ante stemma anticum leviter viridipictum; stemmata magna testaceo-pellucida; oculi mediocres ovati parum exserti et parum convexi ; cavitas facialis magna subprofunda, superne per carinulam transversam subrectangulo-arcuatam inter oculos ductam limitata, viridi-cyanea nitida, confertissime subtiliter transverse strigosa: canalicula mediana superne profundiore, inferue subtili. Antenuae perbreves robustae, scapo viridi-cyaneo, pedicello flagellisque fuscis, hoc apice infra fuscotestaceo. Clypeus mediocris, disco carinato - convexus subpolitus viridi-aureo-resplendens, utrinque immersus sparse punctatus, cyaneo-viridis, margine apicali nigropiceus truncatus. Mandibulac parvac piceae, basi aeneovirescentes. Palpi fusci; horum articuli terminales, maxillarum processus el ligula brumeo-testacei. Thorax cylindricus, antice vix angustior quam caput, dorso et 
mesopleuris confertim et crasse punctato-subreticulatis, dorsuli punctura paullo minus profunda et paullo magis conferta quam in partibus jam memoratis. Pronotum convexo-depressum subplanum transverso-rectangulum aureum lateribus cyaneis; truncatura antica nitida subriolacea parce punctata foveolis binis centralibus; margo posticus leniter arcuato-emarginatus subtruncatus. Dorsuli areae laterales saturate cyaneae leviter viridi-irroratae, area media nigro-aenea. Scutellum et postscutellum aurea. Metanotum, pleurae et sternum cyanea viridipicta; metanotum sub postscutello crassissime exaratum sulco transverso-arcuato lato et profundo, per carinulas nonnullas in foveas $3-5$ diviso; anguli postico laterales nediocres. Pedes viridi-cyanei, tarsis brunneo-testaceis. Alae sordidae brunneo-hyalinae venis brunneis; cellula radialis lanceolato-acuminata, venula transverso-medialis basi brevissime et obtuse angulato-inflexa. Abdomen sublineare, ovato - ellipticum, dorso modice conrexum confertim punctatum parum nitidum saturate cyancum 1. potius violaceum. Abdominis segmenti 1:mi impressio basalis triramosa scil. e sulcis tribus profundis concentricis constituta: sulco medio longitudinali externis obliquis subtransversis; fascia apicalis mediocris linearis nitida viridissima. Abdominis segmenti 2: di fascia apicalis latior et concolor, ad latera perparum aureo-nitens, antice utrinque arcuato-emarginata et hinc in segmenti discum angulum acutum promittens. Abdominis segmentum 3:tium carinula mediana subtilissima vix conspicua, paullulum infra basin macula discoidali parra obsoleta aeneo-virescens; ante seriem non vere callosum nec marginatum, sed potius brevissime conveso-declive; series ante-api- 
calis post hanc declivitatem posita, igitur paullulum humilior quam pars anterior segmenti, medio interrupta, foveolis profundis majusculis circ. 14, scil. 7 utrinque; margo apicalis convexo-depressus mediocris subcoufertim punctulatus, summus arcuatus, attamen ad utrumque latus obtusissime quasi bi-angulatus. Venter fornicatus viridicyaneus nitidus subtiliter punctulato-coriacens.

84. Chrysis sinuosa Dalman. $ठ 7$.

Mediocris oblonga robusta 3 lin. decimal. long. crasse punctata viridi-cyanea: pronoto, dorsuli areis lateralibus tegulis scutello postscutello abdominisque segmentis 1:mo 2:doque aureis, loc fascia basali ampla arcuata subreniformi segmentoque 3:tio cyaneis; hujus serie anteapicali irregulari foveolis paucis majoribus et minoribus, margine apicali leniter undulato, alis sordidis.

Clirysis sinuosa Dalman Collect. Paykull.

Chrysis sinuata Dlbm. Dispos, 1845. 12. 18.

Habitat in Africa ad Cap. bon. sp. D. Paykull, Mus. Reg. Acad. Scient. Stockholm.

Observ. Caput robustum oblongum sublineari-ellipticum, totum dorso pleurisque confertim crasse punctatum. Caput mediocre rotundato-triangulare, thorace antico paullulum latius, pone tempora utrinque in margine occipitali angulo spinoideo munitum, confertim punctato-subreticulatum, cano pubescens et pilosellum, cyaneum; vertex convexo-depressus antice aureo-virescens; stemmata magna subimmersa clara. Cavitas facialis magna subprofunda cyaneo-viridis nitida, subtilissime confertim transverse strigosa, medio canaliculata, lateribus et stlperne omnino ut in Chr. Dalmami marginata, margine 
supero transverso ad centrum nonnihil arcuatim insinuato. Oculi mediocres ovati exserti. Antennae breves robustae: scapo cyaneo-viridi, pedicello fusco, flagello rufo-testaceo basi et infra fuscescente. Clypeus mediocris viridis nitidus, disco convexus et politus, utrinque immersus et punctulatus, margine apicali nigro-piceo ad centrum truncato. Thorax depresso-cylindricus confertim crasse punctato-reticulatus. Pronotum transverso-rectangulum submagnum aureum: plicis lateralibus deflexis et truncatura antica cyaneis, hac punctulata foveis medianis 2 lanceolatis profundis. Mesonotum et postscutellum aurea, dorsuli area intermedia cyanea. Metanotum viride: sulco transverso - arcuato crenato cyaneo, angulis postico-lateralibus brevibus crassis obtuse triangularibus apice auratis. Pleurae virides, pectus et pedes cyaneo-viridia, tarsis fusco-brunneis. Alae sordidae venis brumneis, tegulis aureis. Abdomen robustum mediocre, capite thoraceque simul sumtis vix brevius, sublineare, dorso modice convexum, confertim crasse punctatum; segmentum 1:mum aureum: impressione basali magna cyanea postice viridilimbata obsolete subtrisulcata scil. sulcis multo minus distinctis quam in Chr. Dalmanni; segmentum 2:dum altreum fascia basali magna arcuata postice emarginata el hinc subreniformi cyanea: limbo postico viridi; segmentum 3:tium cyaneum, ad seriem macula centrali indeterminata viridi, totum modice convexum nec pro serie quidem excipienda immersum, carinula mediana subtilis tantum disco et ante seriem visibilis; series ante-apicalis irregularis medio interrupta utrinque foreolis $4-5$ majoribus et minoribus, harum externis subconfluentibus, margo apicalis mediocris punctulatus convexo-depressus, 
summus late arcuatus et lenissime obtuso-undulatus; venter concaviusculo-planus, a segmentorum plicis lateralibus sat marginatus et hinc quasi fornicatus videtur, viridicyaneus.

\section{Chrysis erythromelas Dlmn. 우?}

Mediocris oblonga subangusta 3 lin. decimal. et ultra long. modice punctata cyanea, macula verticis, dorso thoracis, abdominisque segmentis 1:mo et 2:do aureis, hoc fascia basali angusta nigro-aenea, segmento 3:tio basi nigro-aeneo apice cyaneo, disco lineola $l$. macula viridissima, serie ante-apicali regulari foveolis longinsculis linearibus sulciformibus mumerosis, margine apicali leniter obtuse angulato, alis hyalinis venis piceis.

Chrysis crythromelas Dalman Mus. Paykull., Dlbm. Dispos. 1845. 11. 15.

Specimen e Collectione Paykulliana Musei Reg. Acad. Scient. Stockholm. communicavit D. Boheman, patria non indicata.

Observ. Chrysidi simuosae ad picturam similis; at corpore angustiori, sculptura minus crassa et margine apicali abdominis segmenti $3:$ tii leniter angulato facillime distincta. - Corpus quoad magnitudinem mediocre, elongatum lineari-subcylindricum subangustum. Caput orbiculatum confertim punctulatum saturate cyaneum: macula magna transversa inter oculos et stemmata virescenti-aurea; cavitas facialis magna cyanea nitida parum virescens, medio distincte canaliculata, utrinque lạevissima (nec transverse strigosa, ut in praecentibus speciebus), lateribus confertim punctulato-coriacea, superne partem apicalem maculae aureae excipiens et ibidem per lineolam trans- 
versam obsolete elevatam subarcuatam submarginata. Antennae breves robustae fuscae: scapo cyaneo, pedicello extus virescente. Clypeus brevissimus viridi-cyaneus sparse punctulatus, disco convexiusculus nitidus, margine apicali piceo truncato. Mandibulae robustae nigro-piceac, basi cyaneo-virescentes. Thorax depressiusculocylindricus, antice capite paullulum angustior, dorso modice punctato-reticulatus (et pronoto, mesonoto atque postscutello) aureus nonnihil viridi-resplendens; metanoto postico, pronoti plicis inferioribus lateralibus, pectore pedibusque cyaneis, tarsis fuscis. Pronotum transverso-reclangulum, margine postico leniter arcuato-emarginatum, antice impressione mediana suborata pone discum obliterata, truncatura antica violacea 1. potius nigro-aenea sparse punctulata: disco subpolita foreolis 2 centralibus. Metanotum posticum ad formam normaliter licet obsolete sculptum; anguli postico-laterales perbreves triangulares nec non pleurae punctato-rugulosi. Pectus vitidum subtiliter punctulatum. Alarum anticarum cellula radialis elongata ovato-lanceolata apice sat aperta; venula transrersomedialis non recta, sed basi leniter arcuato-inflexa. Abdomen lineari-ellipticum capite thoraceque simul sumtis paullulum longius, dorso convexum confertim modice punctatum; segmenta dorsalia 1:mum et 2:dum igneo-aurea limbo parumper viridi-nitentia, segmenti 1:mi impressio basalis magna subtriramosa cyanea aut subviolascens, segmenti 2:di fascia basalis angusta sublanceolata nigroaenea; segmentum 3:tium magnum, basi conrexum nitidum modice punctatum nigro-aeneum, in ceteris totum cyaneum macula centrali aurco-viridissima, disco transversaliter obsoletissime immersum confertissime punctatum 
subrugulosum et subopacum, tum - ante seriem - brevissime et lenissime conrexo-declive 1 . submarginatum, series ante-apicalis immersa medio interrupta, foveolis numerosis circ. 14 longitudinalibus sulciformibus linearibus, margo apicalis mediocris convexo-depressus punctulatus: summus quodammodo arcuatus, at apice utrinque ita sectus ut anguli 4 obsoleti et latera 6 exstant. Venter fornicatus nitidus cyaneus confertim punctulato - coriaceus.

86. Chrysis integra Fabr.

Mediocris circ. $2 \frac{1}{2}$ lin. decimal. long. modice punctata cyaneo - et viridi-variegata, segmentis abdominis 1:mo 2:doque aureis, 3:lio basi nigro-aeneo, disco subaureoviridi, apice cyaneo, margine subundulato, serie anteapicali foveolis linearibus subellipticis non confluentibus circ. 14, medio per interstitium viride interrupta, alis subsordide hyalinis venis piceis.

Clerysis integra Fabr. E. S. 2. 241. 12. et Piez. 174. 17. certissime! secundum specimen typicum, e Tunis Africae a D. Vahl reportatum, quod in Museo Havniensi aestate 1847 examinavi.

Mabitat in Hispania et Africa D. Vahl.

Observ. Caput cyaneum: macula ante stemmata viridi. Thorax cyaneus; pronotum, dorsuli areae laterales, scutellum et postscutellum viridia. Pedes cyanei viridi resplendentes tarsis fuscis.

o Chrysidis elegantis varietates $c, d$ et $c$ in Sect. 2. descriptas (Spec. 87) quaeras. 
Sectio II. Inaequalitas marginis apicalis (in abdominis segmento dorsali $3:$ tio) distincta.

87. Chrysis elegans Le Pell. $ð 70$.

Mediocris oblonga $1 \frac{3}{4}-2 \frac{3}{4}$ vix 3 lin. decimal. long. confertim modice punctata parum nitida viridis aut cyanen, pedibus concoloribus, tarsis $l$. fuscis l. Urunneo-testaceis, thoracis dorso plemimque aureo-fasciato, rarius viridi l. viridi-cyaneo, abdominis dorso 1. toto aureo l. basi virescente et apice aurato, lineola mediana subelevata laevissima (segmento 3:tio non sulcato), serie ante-apicali foveolis numerosis rotundatis mediocribus, margine apicali brevi semper discolore et l. angulato l. undulato.

Varietalurn Dispositio.

A. Thoracis dorsum aureo-pictum.

var. a. $\delta^{7}$. $2 \frac{3}{4}-3$ lin. decimal. long. Pronotum, dorsuli areae laterales abdominisque dorsum aurea; hujus segmenti 3 :tii margo apicalis purpurascenti-aeneus, ad centrum integerrimus, utrinque sub initio seriei ante-apicalis angulis duobus lateralibus distinctis rounitus; venter nigro-piceus maculis maxinois viridi-aureis aut igneis.

Chrysis elegans Le Pellet. Encyclop. Méthod. secund. Klug MIus.

Berolin.

Spintharis elegans Klug Mus. Berolin.

Chrysis sicula Mus. Spinolae.

Chrysis rufiventris Dalman Mus. Paykull.

Chrysis candens Dlbm. Dispos. 1845. 10. 13.

var. ab. ㅇ. vix 3 lin. decimal. long. Pronotum, dorsuli areac laterales abdominisque dorsum aurea; hujus segmenti 3 :lii margo apicalis aenescenti-purpureus, ad centrum integerrimus, utrinque sub initio seriei ante-ápicalis angulo unico laterali distincto munilus; venter nigro-piceus igneo-maculatus.

Chrysis sicula Mus. Spinolae.

var. $b$. vix $2 \frac{1}{4}$ lin. decimal. long. Pronotum, rnesonotum abdominisque dorsum aurea; hujus segmenti $3:$ tii margo apicalis aene- 


\section{Chrysis: Phalanx II.}

scenti-purpurcus, ad centrum integerrimus, utrinque sub initio serici ante-apicalis angulo unico laterali distincto munitus; venter nigro-piceus viridi- et aureo-maculatus.

var. $e$. ㅇ. $2 \frac{3}{4}$ lin. deciraal. long. pronoti margo anticus, dorsulum abdoninisque dorsum aurea; hujus segmenti 3:tii margo apicalis nigro-aeneus lenissime et obsoletissime inaequalis; renter nigro-piceus igneo-maculatus.

var. d. 오 $2 \frac{1}{3}$ lin. decimal. long. pronotum abdominisque dorsum aurea; hujus segmenti 3: tii margo apicalis aeneo-purpurascens lenissime et :obsoletissine inaequalis; venter nigro-piceus viridi-et aureo-maculatus.

var. c. ․ $2 \frac{1}{2}$ lin. decimal. long. dorsulum viridi-auratum; abdominis dorsum aureum, segmenti 3 : tii margo apicalis purpurascentiaeneus leniter sed non obsolete inaequalis; venter nigro-piceo et igneo-aureo pictus.

Chrysis separata Dlbm. in litteris 1847.

B. Thoracis dorsum aut cyancum pictura viridi, aut viride pictura cyanea.

var. $f$. $\sigma^{\top} \cdot 2_{4}^{3}$ lin. decimal. long. abdominis dorsum aurcum, segmenti 3:tii margo apicalis nigro-aeneus leniter undulatus; venter igneus nigro-piceo-pictus.

Chrysis sicula var. $\beta$. Mus. Spinolae.

var. g. $\sigma^{7} .3$ lin. decimal. long. abdominis dorsum parte basali cyaneo-virescens, parte apicali viridi-aureum, segmenti 3:tii margo apicalis nigro-aeneus purpureo-irroratus leniter undulatus, venter aureo-viridis nigro-pictus.

Chrysura confluens var, Dlbm. Dispos. 1845. 6. 2.

var. h. J. $2 \frac{3}{4}$ lin. decimal. long. abdominis dorsun basi viridi-cyaneum apice lateribusque aureo-viridissimum, margo apicalis viridi- et purpureo-aeneus leniter undulatus, venter viridis.

Chrysura confluens Dlbm. Dispos. I. c.

Habitat mense Majo in Europa meridionali et Asia minori passim.

Observ. var. $a$. Corpus oblongum proportionaliter angustum lineare subcylindricum confertim modice punctatum cano- et cinereo-pilosum. Caput robustum ovatosubcubicum punctato-reticulatum viride. Stemmata mediocria. Oculi ovales depresso-convexi parum exserti. 
Cavilas facialis planiuscula libera (superne non marginata) tota confertin punctulata medio distincte canaliculata, utrinque anguste polita et impunctata. Antennae perbreves robustae fuscae, scapus infra et pedicellus supra aureo-splendentes. Clypeus breris inaequalis punctatus et punctulatus nitidus viridis aureo-resplendens, apice piceus truncatus. Mandibulae robustae piceae basi riridimaculatae. Thorax cylindricus viridis aut viridi-cyaneus, dorso pleurisque modice punctato-reticulatus; pronotum transverso-rectangulum aureum, postice leniter arcuatoemarginatum, antice sulco mediano profundo angusto paullo ante marginem posticum desinente; plicae laterales deflexae coriaceae virides; truncatura antica concariuscula viridis parum aureo-nitens sparse punctata: spatio centrali polito piceo foveolis binis. Dorsuli areae laterales aureae, area media violascens aut nigro-aenea basi violacea, suturis duabus normalibus longitudinalibus paullulum curvis. Scutellum magnum depresso-convexum. Postscutellum mediocre convexum rarius subgibbum et tunc a latere visum quasi obtuse conicum; metanoti anguli posticolaterales breves latiusculi acutanguli coriacei. Sternum cyaneo-virescens aut viridi-cyaneum punctulatum nitidum. Pedes cyanescenti-virides nitidi, coxis tibiisque secundum diversum situm luminis aureo-resplendentes; tarsi l. fusci 1. brunnei 1. brunneo-testacei. Alae sordide hyalinae, ad costam cellulae radialis stria funea, tegulis nigro-aeneis, venis piceis, venula transverso-mediali basi distincte arcuato-inflexa. Abdomen longitudine capitis thoracisque simul sumtorum, latitudine thoracis, sublineare, dorso modice convexum, confertim mediocriter punctatum subcoccineo-aureum; segmenti 1:mi impressio basalis submagna 
basi fusca aut cyanescens limbo viridi, margo apicalis summus piceus; segmenti 2:di margo basalis nigro-aeneus, in disco seggmentorum 2:di et 3:tii lineola mediana abrupta subelevata obsoletissima tantummodo ad certum situm luminis animadvertitur; segmentum 3:tium ut praecedentia conrexum, at punctura interdum paullulum crassior et confertior, unde hoc segmentum tunc minus nitidum atque nonnunquam subopacum obvenit; series ante-apicalis continua (medio non interrupta) foveolis numerosis mediocribus rotundatis; margo apicalis brevis convexiusculus aut depresso-convexus robustus aeneus purpureo-punctulatus, summus humilis tenuis subtilissime coriaceus impunctatus, parte centrali subtransversus, lateribus utrinque oblique late et leniter bis emarginulatus, hinc ad utrumque latus marginis apicalis duo anguli exstant bene distincti. Venter concaviusculo-planus, itaque modice fornicatus; quod tamen variat in variis individuis, secundum modum quo animalculum in agone mortis viscera leniter aut fortiter contraxit; pictura ventralis etiam variationibus subjecta, plerisque tamen obvenit venter nigro-piceus aut nigro-aeneus: plicis lateralibus nec non segmentorum 1:mi et 2: di macula discoidali maxima obcordata viridibus, aureis aut igneis, segmentum 3:tium 1. lineola maculave mediana $\mathrm{l}$. maculis duabus magnis subapicalibus concoloribus $\mathrm{l}$. totum nigerrimum immaculatum:

Reliquae nobis cognitae varietates hujus speciei, scil. var. $a b, b, c, d, e, f, g$ et $h$, tantummodo notis jam antea sub caractere specifico demonstratis a varietate nuperrime descripta differunt. 
88. Chrysis mediocris Dlbm. $ð$.

Oblonga $2 \frac{1}{2}$ lin. decimal. long. modice punctata nitidissima cyanea, metanoto pedibusque concoloribus tarsis fuscis, pronoto mesonoto abdominisque dorso subpolito viridi-aureis (hoc lineola mediana carente), segmenti 3:tii (non sulcati) serie ante-apicali foveolis numerosis rotundatis mediocribus, margine apicali mediocri concolore, ad centrum emarginulato, ulrinque sub initio seriei angulo laterali obsoleto.

Chrysis bicolor Megerl. Mus. Vienn. var.

Habitat in Austria rarissime; ad Vienn. a D. Megerle de Miihlfeld olim detecta, teste $\mathrm{D}$. Kollàr, qui specimen e Museo Viennensi amice communicavit.

Observ. Similis et affinis Chrysidi eleganti; at corpore nitidissimo, punctura minus crassa minusque profunda, capite paullulum angustiore, metanoto pectore pedibusque purissime cyaneis, abdomine apice angustiore, margine apicali ejusdem coloris ac ipso abdomine etc. certe facillimeque dignoscitur.

Corpus oblongum lineari-ellipticum fusco- et canopubescens pilosellumque nitidissimum. Caput oratum latitudine thoracis, crebre (non confertim) punctulatum cyaneum, vertice subruguloso viride-irrorato. Stemmata mediocria 1. subparva. Oculi ovales depresso-conrexi non exserti. Cavitas facialis mediocris libera (superne non marginata), disco laevissima impunctata politissima, canalicula mediana subtilissima, lateribus punctulato-coriacea 1. subrugulosa. Antennae breves robustae fuscac cano-puberulae, scapo nonnihil viridi-micante. Clypeus brevis politus nitidus viridis, disco carinatus, lateribus concavo-immersus, apice truncatus aurato-aeneus. Man- 
dibulae piceae ante apicem rufo-brunnescentes, basi cyanescentes. Thorax mediocris ovato-subcylindricus, paullulum brevior et paullulum robustior quam in Clr. eleganti, pure cyaneus pronoto et mesonoto viridi-aureis, totus punctis minoribus levibus (non profundis) crebre (non confertim) obsertus, hinc sculpturae superficies minus regularis, adeoque non reticulata sed potius subrugulosa, tantummodo in scutello et postscutello reticulatis quaedam obvenit, puncta scutelli majora sunt quam reliquarum partium. Pronotum transverso-rectangulare, sulco mediano distincto sublanceolato (paullulum latiore et minus profundo quam in Chr. eleganti) ante marginem posticum desinente, margine postico arcuato-emarginatum; truncatura antica cyanea punctulata subcoriacea minus nitida subopaca: spatio centrali parvo polito piceo foveolis binis, antice posticeque aureo-aeneo irrorato; pronoti plicae laterales deflexae concavo-impressae cyaneae. Dorsuli suturae 2 medianae non rectae sed basi nonnihil curvae. Scutelli cavitates laterales cyaneae viridi-marginatae; in scutelli disco basali adest areola minuta subtriangularis laevissima polita (qua nota omnino caret $\boldsymbol{C h}$. elegans, cujus totum scutellum est punctato-reticulatum). Metanotum convexo-gibbum punctulato-coriaceum subrugulosum; postscutellum confertim modice punctato-subreticulatum margine postico virescens; area sub postscutello sita semicircularis foveis $4-6$ magnis profundis in arcum dispositis limitata, anguli postico-laterales mediocres triangulares. Pleurae modice punctatac (non reticulatae) nitidae. Sternum nitidissimum sparse punctulatum. Pedes nitidi viridi-cyanei tarsis nigro-fuscis. Alae sordide hyalinae venis piceis; cellula radialis subcompleta 
scil. apice peranguste aperta, venula transrerso-medialis basi leniter arcuato-inflexa. Abdomen mediocre, capite thoraceque simul sumtis paullulum brevius, suborali-ellipticum apice paullulum angustius, dorso modice convexum, confertim modice punctatum nitidissimum viridiaureum; segmenti l:mi impressio basalis mediocris cyanea Irisulcata, margo apicalis ejusdem segmenti summus cupreo-splendens; segmentum 2:dum disco concolor, margine basali anguste nigro-aeneum; segmentum 3:tium apice angustius quam in Chr. eleganti, series ante-apicalis continua (medio non interrupta) foreolis numerosis mediocribus rotundatis profundis approximatis nigris, margo apicalis mediocris aut submagnus convexiusculus sparse subtiliter punctulatus nitidissimus abdomini concolor, summus centro lenissime emarginulatus, utrinque conrexo-arcuatus et sub initio seriei ante-apicalis obsoletissime angulalus. Venter concaviusculo-planus nitidus subtilissime coriaceus nigro-piceus, plicis lateralibus nec non segmentorum 1:mi 2:dique macula discoidali maxima obcordata et 3 : tii lineola mediana viridi-aureis.

89. Chrysis emarginatula Spinol. $\delta$.

Magna oblonga robusta $3 \frac{1}{2}$ lin. decimal. long. punctatissima subnitida viridi-cyanea pedibus cyaneo-viridibus. tibiis extus aureo-splendentibus, tarsis luteis, abdominis dorso viridi-aureo lineola mediana subelevata subdistincla, segmenti 3:tii (non sulcati) serie ante-apicali foveolis remolis rotundatis subprofundis majusculis, margine apicali majore concolore ad centrum leniter emarginalo ulrinque arcualo-integerrimo.

Chrysis emarginalula Spinol. Ins. Lig. Fascic, 4:tus 239. LXXXIV. 
secundum specinen typicum e IIuseo Spinolae nili benigne communicatum.

IIabitat in Italia rarissime; olim ad Genuam ab Illustr. Spinola detecta.

Observ. Quoad habitum maxima individua Chrysidis ignitae simulans. - Corpus robustum elongatum lineare subsemicylindricum, cano- et fusco-pubescens pilosellumque, punctatissimum punctis mediocribus, subcoriaceum parum nitidum. Caput ovato-triangulare confertim modice punctato-rugulosum cyaneum; vertex latus depresso-convexus; stemmata magna; oculi mediocres ovati exserti; frons viridis; cavitas facialis concolor mediocris confertim punctulato-coriacea, disco longitudinaliter anguste polita distincte canaliculata, lateribus largiter canosericeo-pilosella. Antennae uediocres robustae, scapus viridis aureo-nitens, pedicellus supra igneus, articulus 3:tius infra virescens, reliqua pars antennae per articulos alternatim rufo-brunneos et nigro-fuscos annulata. Clypeus mediocris viridissime aureus nitidus sparse punctulatus, disco carinato-convexus, apice transversus nigro-piceus. Mandibulae robustae nigro-piceae, basi viridi-maculatae, ante apicem rufo-piceae, apice fortiter bidentatae. Palpi brunneo-testacei. Thorax ovato-cylindricus; pronotum et dorsulum subcoriacea parum nitida punctatissima punctis modicis; pronotum capiti arcte approximatum cyaneum supra ad maximam partem virescens, truncaturae anticae spatium centrale politum piccum; dorsuli areae laterales cyaneae macula discoidali viridi, area media nigro-aenea; scutellum viride leviter aureo-irroratum, postscutellum caeruleum, modice convexa crasse et regulariter puuclato-reliculata; area sub postscutello brevis semicir- 
cularis punctato-rugulosa foveata violacea; metanoti anguli postico-laterales perbreves triangulares virides coriacei remote punctati; pro-pleurae et meta-pleurae coriaceac viridi-cyaneae, meso-pleurae rugulosae remote punctatae virides; sternum viridissimum nitidum parum aureo-micans. Pedes viridissimi sparse punctulati nitidissimi, femoribus crassis fusiformibus, tibiis extus aureo-splendentibus, tarsis albo-testaceis 1 . potius subluteis impunctatis cano-puberulis. Alae subhyalinae, in disco singulae cujusque cellularum umbra obsoleta laete brunnescens aut sublutescens; cellula radialis incompleta late aperta, brevior quam in praccedentibus et ab apice alae sat remota; tegulae viridi-aeneae, venae laete brunneae, venula transverso-medialis basi leniter incurva. Abdomen submagnum elongatum capite thoraceque simul sumtis conspicue longius, latitudine thoracis, sublineare, dorso modice convexum viridi-aureum confertissime punctatum: punctis minoribus quam thoracis, subcoriaceum, lineola mediana subelevata subtili distincta et continua; segmenti 2:di margo basalis anguste nigro-aeneus; segmentum 3:tium subcupreo-aureum, limbo lateribusque viridi-nitens, punctis plurimis viridibus, margo basalis praesertim disco nigro-acneus; series ante-apicalis medio interrupta, foveolis numerosis profundis, majoribus et minoribus simul sumtis 16, sed uno latere foveolis 6 omnibus majoribus et altero latere foveolis $\mathbf{1 0}$ majoribus et minoribus alternis; segmenti 3:tii margo apicalis (post seriem) mediocris 1. majusculus depresso-convexus sparse punctulatus segmento concolor, summus angustissimus piceus centro leniter emarginatus, pone emarginaturam arcuato-integerrimus. Venter non fornicatus sed planus et secundum 
longitudinem medio subtiliter continue carinulatus, igneoaureus: segmentis 1:mo 2: doque basi nigro-aeneis.

\section{Chrysis mixta Dlbm.}

Mediocris oblonga 2 lin. decimal. long. confertim modice punctata subnitida virescenti-cyanea pedibus concoloribus tarsis fuscis, abdominis dorso salurate aureo, segmenti 3:tii (non sulcati) serie ante-apicali foveolis subconfluentibus minus distinctis, margine apicali majori ad centrum distinctius emarginato, pone lobulos arcuatointegerrimo.

Spintharis emarginatula Klug (non Spinolae), secundum specimen quod in MIuseo Berolinensi aeslate 1847 examinavi.

Habilat in Liguria, teste D. Klug in Museo Berolin.

Observ. Simillima Chrysidi emarginatulae Spin.; at dignoscitur: corpore breviore, minus crasse punctato, vertice viridi-aeneo, clypeo viridi-cyaneo non aureopicto, pedibus cyaneo-viridibus non aureo-pictis, tarsis brunneis nec albo-testaceis, ventre basi nigro apice igneo, abdominis dorso saturatius aureo, serie ante-apicali foveolis subconfluentibus minus distinclis, emarginaturae lobulis triangularibus subdentiformibus. Alae subhyalinae venis brunneis; cellula radialis lanceolata incompleta, apice modice aperta.

91. Chrysis carinata Spinol. $\sigma^{7}$ ?

Mediocris oblonga robusia 3 lin. decimal. long. confertim punctata subnitida tota cyanea aut violascens viridi-picta tarsis brumneis, segmenti 3:tii (non sulcali) serie ante-apicali foveolis submumerosis rolundatis majusculis profundioribus, margine apicali mediocri, ad 
centrum subacute emarginalo, sub initio seriei utrinque angulo laterali distincto.

Chrysis carinata Spinola in litteris.

Nemophora carinata Draege. Mus. Spinolae.

Habitat in Africa, ad Cap. bon. sp. a D. Draege detecta; Mus. Spinolae.

Observ. Ad habitum et picturam Chrysidi nitidulae quodammodo similis. Corpus robustum semicylindricum lineare punctatissinum punctis mediocribus, saturate cyaneum I. potius violaceun. Caput rotundato-triangulum thoraci arcte applicatum, pronoto paullulum latius; vertex amplus depresso-convexus fusco-hirtus, ante stemmata virescens coriaceo-rugulosus, inter stemma anticum et cavitatem facialem carinula transversa brevissima arcuata obsoleta; oculi mediocres ovati exserti; cavitas facialis lata planiuscula viridi-cyanea nitida, confertissime punctulato-coriacea, disco anguste subtilissime transverse strigosa et distincte canaliculata. Antennae elongatae tenues: scapo, pedicello et parte basali flagelli fusco-virescentibus, parte apicali flagelli subferrugineo. Clypeus submaguus latus viridissimus nitidus sparse punctatus, carinato - convexus, margine apicali transverso, anguste aureo-micante et leniter arcuato - enarginato. Mandibulae robustae piceae basi virescentes. Thorax robustus cylindricus convexus confertim crasse punctatus violaceus; pronotum supra, dorsuli areae laterales disco et area media basi scutellumque viride-irrorata. Pronotum subbreve transverso-rectangulum margine postico leniter arcuato-emarginatum, supra non sulcatum, truncaturae anticae spatium centrale semicirculare politum viride utrinque forcolis 2 : quarum proxima sulciformi et altera sive submarginali ro- 
tundata. Dorsuli suturae medianae profundae crenulatae, basi parum curvae. Scutellum et postscutellun convexiora, illud crassius et remote punctatum, hoc basi fovea centrali. Metanoti anguli postico-laterales mediocres angusti acutius triangulares. Pleurae punctulato-rugulosae cyaneae. Sternum nitidum viridi-cyaneum sparse et subtiliter punctulatum. Pedes cyaneo-virides nitidi griseopilosi tarsis brunneis. Alae brunneae subhyalinae venis firmibus piceis, venula transrerso-mediali basi subangulato-inflexa. Abdomen mediocre aut subbreve longitudine et latitudine thoracis, obtuse subrectangulum, dorso confertim modice punctatum et modice convexum violaceum, segmentorum 1:mi et 2:di margo apicalis virescens, lineola mediana subtilis subelevata in disco segmentorum 2 : di et 3:tii observanda; segmentum 3:tium confertius punctulatum coriaceum, series ante-apicalis medio interrupta, foveolis numerosis circ. 18 profundis rotundatis subpellucidis, mediis majoribus et in fundo purpurascentiviolaceis, extimis minoribus; margo analis mediocris depressus punctulatus coriaceus, centro leniter acutanguloemarginatus, pone emarginaturam utrinque late depressoarcuatus usque in angulum apicali-lateralem sub initio seriei situm; venter concaviusculo-planus nitidus viridicyaneus confertim pubescenti-punctulatus.

92. Chrysis crassimargo Spinol. ㅇ.

Submagna oblonga robusta $3 \frac{1}{3}$ lin. decimal. long. confertim modice punctata subritida saturate cyanea aut violascens, pedibus concoloribus, verlice pronolo el mesonoto parum aureo-viridi-irroralis, abdominis dorso coccineo-aureo, segmento 3:tio (pro serie excipienda) 
extra ordinem profunde transverse sulcato, serie anteapicali (in sulco sita) per carinulam medianam interrupta, foveolis longitudinalibus linearibus, margine apicali mediocri fortiter calloso-incrassato, summo ad centrum lenissime emarginulato, utrinque arcuato-integerrimo.

Chrysis crassimargo Spinol. Annal. Entomol. d. France 1843. p. 127.

Habitat in Hispania, D. Chiliani; Mus. Spinolae.

Observ. Ad habitum Chrysidi austriacae assimilis.

Corpus robustum ovato-oblongum sparse fusco- et cano-pilosellum. Caput, thorax et pedes cyanea; vertex ad frontem et oculos, pronotum utrinque ad sulcum medianum, striola in utraque dorsuli area laterali, macula mesopleurae et scutelli, viridia nonnihil aureo-irrorata; occiput et pronoti margo collaris, dorsuli area media et metanotum posticum, nigro-aenea. Coxae anticae et omnes tibiae ex uno latere virescunt; tarsi brunneo-testacei. Caput crassiusculum breve subovatum 1. ovato-subrectangulum, vertice et occipite modice punctato-reticulatis violascentibus aut nigro-acneis, antice cyaneum, inter oculos et stemmata 1. abrupte viridi-maculatum 1. transverse et continue aureo-viride; cavitas facialis mediocris punctulato-coriacea, medio canaliculata. Antennae robustae perbreves, scapus longiusculus 1. nigro-1. viridi-aeneus, flagellum fuscum crassiusculum et brevius quam in affinibus speciebus. Clypeus submagnus late transversus inaequalis parce punctatus cyaneus, macula ccutrali viridi, margo apicalis prominulus politus nigro-piceus lenissime depresso-arcuatus, margines laterales obliqui prominuli albotestacei. Mandibulae robustae punclatae nigro-piceae, ante apicem laete brunnescentes, basi levissime viridi-pictac. 
Palpi mediocres brunnei. Thorax ovato-cylindricus punctato-subreticulatus; pronotum transverso subrectangulum, sulco mediano crasso lanceolato, truncaturae anticae spatio centrali polito nigro-piceo utrinque foveola sulciformi. Dorsuli suturae 2 medianae crenulatae subrectae. Scutellum depresso-convexum. Postscutellum convexius; area sub postscutello semicircularis foveis 6 magnis in arcum dispositis limitata; metanoti anguli postico-laterales mediocres acute triangulares. Pectus sparse punctulatum. Alae subhyalinae tegulis aeneis, venis piceis, venula transverso-mediali parum arcuata, cellula radiali subcompleta scil. apice angustissime aperta. Abdomen ovali-ellipticum, thorace paullulum angustius, capite thoraceque simul sumtis nonnihil longius, dorso normaliter convexum confertim modice punctatum subcoriaceum coccineo-1. igneo-aureum viridi-nitens; segmenti $1: m i$ impressio basalis submagna tri-foveata et segmenti 2:di margo basalis angustus, nigro-acnei; segmenti 3 :tii series ante-apicalis foveolis circ. 12, h. e. 6 in utroque sulco. Venter nigro-aeneus: plicis lateralibus et segmentorum 2:di 3:tiique maculis ante-apicalibus maximis, igneis.

\section{Chrysis foreata Dlbm. 오}

Mediocris oblonga robusta $2 \frac{1}{3}$ lin. decimal. long. modice punctata nitida viridi-aenea pectore, ventre pedibusque cyaneo-viridibus, tarsis alarumque venis laete brunneis, alis albis, abdominis segmenti 3:tii serie anteapicali foveolis remotis longiusculis profundis linearibus sulciformibus, margine apicali majusculo humili depresso arcuato ad centrum integerrimo, sub initio seriei utrinque angulo laterali obsoleto. 
Chrysis foveala Dlbm. Dispos. 1845. 6. 2.

Habital in Aegypto, D. Hedenborg; Mus. R. Acad. Scient. Stockholm.

Observ. Pictura et magnitudine Clrysidi rutilanti nomnihil assimilis. Corpus robustum oblongum latiusculum subrectangulum crasse punctatum. Caput orbiculatum 1. potius rotundato-subtriangulum non crassum, punctulato-reticulatum viridi-cyaneum; frons et vertex virides. Stemmata mediocria elevata; oculi exserti orati; cavitas facialis mediocris superne transverse-marginata, tota riridis nitida, disco subpolita abrupte et subtiliter canaliculata, lateribus subtiliter punctulato-coriacea cano-sericeo pubescens. Antennae mediocres fuscae scapo viridi. Clypeus mediocris transversus latus nitidissimus viridis rix punclatus, medio carinato-convexus, utrinque foreatoinmersus, margine laterali obliquo testaceo, margine apicali nigro-piceo arcuato-emarginato. Mandibulae breves piceo-brunneae basi virescentes. Thorax robustus subcylindricus dorso pleurisque crasse et subremote 1. saltem non confertim punctato-reticulatus conrexo-depressus riridi-aeneus, qui color in mesonoto fere in cupreum abit; pronotum antice sulco mediano brevi suborato obsoleto; metanotum gibbo-convexum, area sub postscutello norınalis, anguli postico-laterales validi acute triangulares virides punctulato-coriacei. Sternum nitidissimum sparse punctulatum cyanco-viride; pedes concolores tarsis brunneo-testaceis. Alae albae, tegulis aenescentibus, renis laete brunneis, venula transverso-mediali basi distiucte arcuato-inflexa; cellula radialis valde incompleta, scil. apice hiante aperta, costa paullo post stigma desinit, venae radialis apex ab alae apice longe remolus. Abdomen 
robustum ovato-subrectangulum latitudine mesonoti, capite thoraceque simul sumtis paullulum brevius, dorso depresso-convexum nitidissimum politum viridi-aeneum punctis subcrassis rotundis crebre obsertum, lineola mediana subelevata subtili in segmentis $1:$ mo et 2 :do continua; segmenti 1:mi impressio basalis magna profunda semicircularis; segmentum 3:tium ante marginem apicalem leniter transverse arcuato-sulcatum, series ante-apicalis in hoc sulco (non profundo sed latiusculo) sita continua foveolis longitudinalibus linearibus sulciformibus profundis remotis circ. 14, margo apicalis brevis punctulatus depressus: summus depresso-arcuatus 1. potius rectangulo-arcuatus, utrinque sub initio seriei angulo laterali obsoleto; venter fortiter fornicatus viridis cyaneo-maculatus.

94. Chrysis versicolor Spinol. $\sigma^{7}$ ㅇ․

Subparva oblonga vix $1_{4}^{3}$ lin. decimal. long. confertim modice punctata et punchulata subcoriacea l. aeneoaurata l. viridi-aenea, pectore pedibusque cyaneo-viridibus, tibiis interdum aureo-splendentibus, tarsis $l$. fuscis 1. testaceis, alis albis venis brumneis, abdominis segmenti 3:lii serie ante-apicali foveolis numerosis parvis rolundatis, margine apicali convexo-arcuato ad centrum lenissime emargimulato, utrinque sub initio seriei angulo Iaterali retracto distincto obtuso.

Chrysis versicolor Spinola. Ins. Lig. Fascic. 4. pag. 241. LXXXV; secundum specimina typica c Museo Spinolac benevole conmunicata.

Ilabitat passim in Liguria, haud infrequens; D. Spinola. In Gallia meridionali, D. Latreille. 
var. $\boldsymbol{u}$. 오. Pronotum, mesonotum abdominisque dorsuro aeneo-aurata; tarsi brunnei articulationibus testaceis.

var. b. ㅇ. Pronotum abdominisque dorsum aeneo-aurata, mesonotum viridi-aeneum; tarsi brunnei articulationibus testaceis.

var. c. $\sigma$. Thoracis abdominisque dorsum viridi-aeneum; tarsi toti testacci.

Observ. var. $\boldsymbol{a}$. Hedychro minuto quodammodo assimilis, at multo major multoque crassius punctata, ceterum ano unguiculisque aliter constructis generice diversa. - Corpus ovato-oblongum, cum corpore specierum plurimarum hujus Generis comparatum subparvum scil. vix $1 \frac{3}{4}$ lin. decimal. long, totum dorso modice at confertius punctatum subreticulatum. Caput robustum rotundato-triangulum punctulato-subgranulosum viride, supra erecte cinereo-pilosellum, antice lateribusque largiter cano-sericeum, pone stemmata violascens. Stemmata et oculi mediocria, hi valde exserti. Cavitas facialis longa superne transverse marginata, margine angulato-arcuato stemmati approximato, tota crebre et subtiliter punctulatocoriacea, canalicula mediana distincta. Antennae robustae longiusculac fuscae cano-puberulae, scapo viridi-cyaneo. Clypeus brevis nitidus sparse punctulatus, disco convexocarinatus, margine laterali obliquo anguste testaceo, margine apicali arcuato-emarginato parum aeneo-aurato. Thorax subovatus; pronotum, dorsuli areae laterales et scutellum aeneo-aurata; dorsuli area media, metanotum et pleurae viridi-aenescentia 1 . aeneo-viridia; pronoti truncatura antica levis et metanoti area sub postscutello semicircularis, violascentes; pronotum antice sulco mediano obsoletissimo; dorsuli suturae medianae arcuatae; sternum subtiliter punctulato-coriaceum viridi-cyaneum l. cyaneoviride. Pedes cyaneo-virides tarsis brunneis, articulatio- 
nibus testaceis. Alae albo-hyalinae, ad costam cellulac radialis vitta fumea; venae brunneac, venula transversomedialis basi leniter arcuato-inflexa; cellula radialis incompleta, apice anguste 1 . angustissime aperta; alarum tegulae aeneo-virides. Abdomen mediocre ovale modice convexum aeneo-auratum, segmentis 2:do et 3:tio margine basali anguste nigro-aeneis; segmenta 1:mum et 2:dum confertim modice punctata, punclis segmenti 2:di minoribus quam punctis segmenti $1:$ mi; segmentum 3:tium confertissime punctulatum coriaceum; series ante-apicalis continua foveolis numerosis mediocribus rotundatis hic illic confluentibus; margo apicalis mediocris depressus tenuis subtilissime coriaceus, summus ad centrum lenissime et obsoletissime emarginulatus, haud procul a basi et sub initio seriei angulo laterali distinctiori; venter fornicatus aeneus, parum viridi-et igneo-pictus.

95. Chrysis albipennis Klug?. 오.

Subparva ovalis robusta $1 \frac{3}{4}$ lin. decimal. long. capite thoraceque confertissime punctulato-coriaceis opacis, abdomine confertim subtiliter punctulato subnitido, tota violacea alis albis, tarsis alarumque venis piceis, abdominis segmenti 3:lii serie ante-apicali foveolis numerosis parvis rolundatis, margine apicali anguste prominulo fortissime arcuato ad centrum integerrimo, utrinque sub initio seriei angulo laterali distincto subrecto.

? Elampus albipennis Klug. Mus. Berolin.

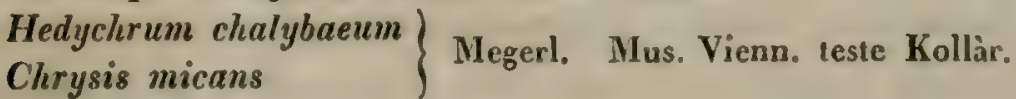

Habitat in Hungaria, Mus. Vienn.

Observ. Subsequenti, Chrysidi unicolori, maxime 
affinis; sed corpore nonnihil longiore, colore saturate violaceo, tarsis fuscis, abdominis segmenti 3:tii margine apicali magis prominulo anguste convexissime arcuato etc. facillime dignoscitur.

Corpus quoad magnitudinem robustum ovale. Caput et thorax confertissime punctulato-coriacen opaca. Caput robustum latum triangulariter rotundatum 1 . unicolore violaceum 1. viridi-aeneo-irroratum; stemmata mediocria; oculi breviter ovati maxime exserti; mox ante stemma anticum inter oculos lineola elevata transverso-arcuata obsoletissima; cavitas facialis levis planiuscula indeterminata conferlissime subtiliter coriaceo-granulosa, non canaliculata. Antennae breves subangustae fuscae scapo violaceo; os infra oculos subanguste prominulus. Clypeus proportionaliter submagnus subtrapezinus depressus sparse punctulatus margine apicali piceo lenissime arcuatoemarginato subtruncato. Mandibulae robustae fusco-piceae aut subaeneae, apice rufo-piceae; palpi fusco-brunnei. Thorax subbrevis robustus quadrato-subglobosus fere ut in Hedychris constructus, violaceus nonnihil viridi-aeneo irroratus; pronotum transversaliter subangustum sublineare, margine postico late emarginato-arcuatum, disco utrinque sulco transverso perobsoleto. Dorsuli suturae medianae subtiles obsoletae, basi arcuatae; postscutelli sculptura distincte reticulata; metanoti anguli postico-laterales mediocres robusti prismatice conici; mesopleurae apice utrinque acutangulae. Alac albo-hyalinae venis brunneis, venula transverso-mediali subrecta; cellula radialis incompletissima, scil. costa mox post stigma et rena radialis mox post angulum centralem desinunt. Abdomen ovatum, latitudine thoracis, longitudine rerticis 
thoracisque simul sumtorum, dorso convexum crebre subtiliter punctulatum violaceum subnitidum; segmenti 1:mi impressio basalis mediocris sulcis tribus radiantibus, margo apicalis summus virescens; segmentum 3:tium basi et disco leviter viridi-aenescens, utringue ante seriem lineola transversa obsoleta impressum, series ante-apicalis continua foveolis numerosis parvis rotundatis distinctis, non confluentibus; margo apicalis mediocris humilis depressus, anguste et fortissime arcuato-prominulus, summus brunneo-testaceus et centro integerrimus, utrinque sub initio seriei angulo laterali remoto distincto subrecto. Venter concaviusculo-planus violaceus marginibus segmentorum brunneo-testaceo - scariosis.

96. Chrysis unicolor Dlbm. $\sigma^{7}$.

Subparva breviter obtuse ovata robusta $1 \frac{1}{2}$ lin. decimal. long. capite thoraceque confertim punctulato-coriaceis, abdomine confertissime subtilissime punctulato tota subnitida coerulea tarsis brunneo-testaceis, alis albissimis venis piceis, abdominis segmenti 3:tii serie ante-apicali foveolis numerosis minulis rotundatis, margine apicali brevi late depresso-arcuato ad centrum $l$. integro l. integerrimo, sub initio seriei utrinque angulo laterali remoto distincto acuto.

Chrysis unicolor Dlbm. Exercit. Hymenopterol, 1831. 32.16.

Clirysura unicolor Dlbm. Dispos. 1815. 6. 1.

Ilabilat in Scania Sveciae rarius; captu valde difficilis; in campis arenosis: ad Lomma juxta mare balticum d. 19. Julii, ad Kjeflinge d. 30 . Julii 1830 (circ. 1 mil. a Lund) nec non in Sandhammar d. 14. Augusti 1838 milhi obvia.

II. 
Observ. Praecedenti (Chrysidi albipenni) simillima; at certe et facillime dignoscitur: corpore breviore laete coeruleo, abdomine subtilissime confertissime punctulato, segmenti 3:tii margine apicali brevi late depresse transverso-arcuato, etc. - Caput rotundato-triangulum, non crassum, vertice latum, totum coeruleum plus minus viridi-micans, punctulato-rugulosum; oculi orati ralde exserti; cavitas facialis nulla, facies inter oculos et antennas planiuscula confertissime subtiliter punctulato-coriacea. Antennae breves tenues, scapo pedicelloque viridi-cyanescentibus, flagello fusco-ferrugineo. Clypeus magnus subquadratus 1. subrectangularis, inaequalis depressus nitidus, parte basali punctulato-coriacea viridi-cyanea, parte apicali subdeclivi remote punctulata aenea, margine apicali summo leniter arcuato-emarginato. Mandibulae robustae aeneae, apice rufo-piceae bi-dentatae. Palpi maxillaeque brunnescentes. Thorax quadrato-subglobosus coriaceus crebre punctulatus coeruleus, parum viride irroratus; scutelli et postscutelli sculptura reticulata. Pronotum brevius et transversaliter angustius quam in Chryside albipenni; metanoti anguli postico-laterales mediocres depressi robusti triangulares; mesopleurae apice acutangulae. Sternum et pedes nitida coerulea sparse punctulata tarsis brunneotestaceis. Alae albissimae venis brunneo-testaceis, venula transverso-mediali et cellula radiali omnino ut in Chryside albipenni. Abdomen ovato-subsemiglobosum, latitudine mesothoracis, longitudine verticis thoracisque simul sumtorum, cocruleum parum viridi-micans, dorso confertissime subtilissime punctulatum; segmenti dorsalis $1: m i$ impressio basalis mediocris inaequalis, vix nisi leniter et obsolete sulcata; segmentum 3:tium semicirculare, series 


\section{Chrysis: Plhalanx III.}

ante-apicalis continua foveolis numerosis minutis orbiculatis; margo apicalis brevis depressus semicirculariter arcuatus, summus tenuissime albo - testaceus, ad centrum 1. lenissime et obsoletissime inaequalis l. integerrimus, sub initio seriei utrinque angulo laterali remoto distincto acuto, sub hoc angulo arcuato-emarginatus; venter concaviusculoplanus dorso concolor, marginibus segmentorum 1 . fusco1. piceo - scariosis.

Not. In Museo Berolinensi Hymenopteron quoddam "Elampus albipennis $K l$ l." inscriptum aestate 1847 vidi; quum vero structuram marginis apicalis segmenti abdominalis 3:tii et sculpturam corporis in specimine Klugiano accuratius mihi annotare neglexerim, pro certo affimare nequeo utrum Chrysidi albipenni an Chrysidi umicolori nunc descriptis referendum sit insectum illud Berolinense.

Phalanx III. Chrysides ano uni-dentatae. Scil. Abdominis segmenti dorsalis 3:tii margo apicalis apiculo centrali terminatus et hine uni-denticulatus, utroque latere arcuatoobtusus.

Hujus Phalangis tantum 2 Species nobis cognitae sunt, scil.

Chrysis succinctula. Spinol. et

Chrysis Leachei Shuck.

Chrysidis cyaneae varjetas, quae propter structuram analem in liac Phalange forsitan quaeritur, in Plialange V:ta spec. 102 invenitur.

97. Chrysis succinctula Spinol. ㅇ.

Oblonga mediocris $2-2 \frac{1}{2}$ lin. decimal. long. subcrasse punctata cyanea, pronoto antice, mesonoto aut toto aut 
dorsulo, abdominisque dorso auratis, segmenti 3 : tii margine apicali aeneo, ventre igneo-maculato, alis subsordide hyalinis, cellula radiali 1 . incompleta $l$. subcompleta.

Chrysis succinctula Spinola.

Spintharis succinctula Klug Mus, Berolin.

Clirysis assimilis Cluristophori in litteris ad Spinol.

Habitat in Europa media et meridionali; ad Berolinum, D. Klug; ad Genuam, D. Spinola; ad Milanum, D. Christophori.

Observ. Chrysidi succinctae quodammodo similis; at corpore minore, abdominis margine anali aliter constructo etc. mox distincta. - Corpus sparse griseo-pilosum. Caput modice punctato-subreticulatum cyaneun aut violascens, frons plus minus virescens. Caritas facialis disco profunda politissima. Clypeus viridis, disco gibboso-aeneus aut cupratus, in centro marginis apicalis leniter arcuato-emarginatus. Antennae mediocres robustac fuscac, scapus 1. virescens l. cyanescens. Palpi brunnei. Mandibulae pictura normali. Thorax subcrasse punctato-reticulatus. Pronotum 1. cyaneum 1. violascens, antice fascia aut fasciola viridi-aurea; dorsulum saturatius aureum; scutellum 1. concolor 1 . viride 1. cyancum; mesopleurac 1 . cyaneae 1 . macula aureo-viridi; reliquae thoracis partes cyaneae; metanoti anguli postico-laterales mediocres acute triangulares. Abdomen capite thoraceque simul sumtis longius, subcrasse punctatum viridi- et cupreo-aureum; segmenta 2:dum et 3:tium interdum margine basali nigra; segmenti 3:tii scries ante-apicalis continua foveolis numerosis profundis, margo apicalis ejusdem segmenti fusco- aut nigro-aeneus. Venter niger maculis 
viridibus et igneis. Pedes virescentes et cyanescentes, tibiae subaenescentes, tarsi testaceo-fusci. Alarum anticarum cellula radialis lanceolata 1 . incompleta 1 . subcompleta, venae radialis apex mox ante costam obliterata.

98. Chrysis Leachei Shuck. $\sigma^{7}$.

Parva subangusta $1 \frac{1}{4}-1 \frac{1}{2}$ lin. decimal. long. modice punctata cyanea pronoto antice, mesonoto abdominisque dorso viridi-aut viridissime aureis, abdominis segmentis apice coeruleo-marginalis, alis lyalinis cellula radiali completa.

Chrysis Leachei Shuckard. Entomol. Magaz. No. 17. pag. 168. 10.

Chrysis hybrida Pellet. secund. Mus. Berolin.

Spintharis nitidula Klug Mus. Berolin. (non Cler. nitidula Grmr. Cfr. nostram speciem No. 76).

Mabitat in Anglia, D. Shuckard; in Hungaria et Italia, D. Spinola; in Asia minori, D. Loew.

Observ. Inter elegantiores species hujus Generis. Praecedenti (Chrysidi succinclulae) affinis quidem et similis; at practer picturam abdominis notabiliorem, etiam corpore breviore et minus robusto; sculptura minus crassa: verlicis thoracisque confertiori et subcoriacea; apiculo centrali (marginis analis in abdominis segmento dorsali 3:tio) distinctiori, alarumque cellula radiali completissina, satis superque dignoscitur. 
Phalanx IV. Chrysides ano bi-dentatae. Scil. Abdominis segmenti dorsalis 3:tii margo apicalis utrinque dente unico laterali armatus.

Hujus Phalangis tantum 2 Species nobis cognitae sunt, scil.

Chrysis lihamata Spinol. et

Chrysis prasina Klug.

Chrysis assimilis Spinol., cujus o propter structuram analem forsitan in hac Phalange quaeritur, in Phal. VI. occurrit.

99. Chrysis bihamata Spinol. 오

Mediocris robusta $2 \frac{1}{2}$ lin. decimal. long. modice punctata nitida griseo-pilosa viridis, thoracis disco dorsali viridi-aenescente, abdominis dorso cupreo, margine apicali concolore: spinis robuslis, spatio inter spinas transverso lenissime arcuato.

Chrysis bihamata Spinol. Annal. Entomol. d. Fr. 183S. 450. VIII, secundum specimen tynicum e Museo Spinolae nobis communicatum.

Habitat in Aegypto, a D. Fischer detecta; Museis Spinolae et Westermanni.

Observ. Species propter armaturam analem singularis. Corpus robustum oblongo-ovatum nitidum. Caput mediocre, rotundato-triangulum, antice viridissimum, postice et infra cyaneum; occiput nitidum modice punctatum; vertex confertius punctulatum subcoriaceum; caritas facialis latiuscula subprofunda confertim punctulata nitida 1. cyanea 1 . cyaneo-viridis largiter cano-sericea praesertim ad latera; supra hanc cavitatem et infra stemma anticum adest carinula transversa levissima utrinque biramulosa. Antennae mediocres, subbreves fuscae scapo 
pedicelloque cyaneo - virescentibus. Clypeus mediocris transverso-rectangularis sparse punctatus, disco subconvexus, margine apicali subpiceo, laterali subtestaceo. Mandibulae nigro-piceae, basi viridi-maculatae, ad apicem rufo-piceae. Thorax mediocrís subquadrato-ovatus, dorso convexo-depressum nitidum subcrasse at non confertim sed potius subremote punctatum; pronotum mediocre transverso-rectangulum, disco viridi-auratum non sulcatum, antice paullulum angustius quam postice; truncatura levis subconvexo-declivis, macula centrali cuprea: foveola utrinque; dorsuli pictura variat: aut areae laterales virides leviter aureo-resplendentes et area media unicolore viridis, aut areae laterales viridi-cupreae et area media cupreo-aenea; scutellum I. Lotum cupreum, I. viride basi cuprea; metanotum viride: postscutellum subgibbum, anguli postico-laterales robusti triangulares punctulato-coriacei aureo-virides; mesopleurae superne viridi-aureae; sternum et pedes longius et sparsius pilosa nitidissima sparse punctulata viridia, uno alterove latere secundum situm luminis cyanescentia; pedes tamen extus plerunque subaureo-splendentes, tarsis brunneo-testaceis. Alae albohyalinae tegulis aenescentibus, venis 1 . brunneo-testaceis I. piceis, venula transverso-mediali basi leniter arcuatoinflexa; cellula radialis valde incompleta, scil. vena costalis paullo post stigma abrupte desinit et vena radialis desinit longe ante apicem alae. Abdomen robustum late subquadrato - ovatum, thorace parumper longius at non latius, dorso subcrebre punctulatum nitidum subpolitum cupreum limbo plus minus viridi-resplendens; segmenti 1:mi impressio basalis magna semicircularis sulco mediano distinctiori; seguentum 3:tium serie foveolarum ante-api- 
cali fere caret, quandoquidem puncta totius segmenti invicem simillima et aequaliter adspersa obreniunt, sed per initium marginis apicalis (paullulum humilioris) locus et vestigium talis seriei significata videntur; margo apicalis ipsi segmento concolor, brevis arcuatus, spinis 2 lateralibus robustis acute conicis fere subulatis remotis bi-dentatus, hinc inter spinas quasi late emarginatus videtur; sed spatium, quod inter spinas exstat, re rera late transversum et lenissime subarcuatum obvenit; lateraliter inter spinam et segmenti basin est margo leniter et obsolete angulatus; venter 1 . planus 1 . concariusculus viridi-cyaneus nitidus.

100. Chrysis prasina Klug. ð’

Mediocris robusta $2 \frac{2}{3}$ lin. decimal. long. modice at confertim punctata, vertice thoracisque dorso coriaceis subrugulosis, subtus cyanea supra cyaneo-viridis, abdominis dorso viridi-aureo, disco subcupreo, margine apicali cyaneo-viridi spinulis minutis subtilibus, spatio inter subrectangulari l. subtrapezino.

Chrysis prasina Klug Symb. Phys. Berolin. 1845. spec. 10. tab. XLV. fig, 10. (Specimen Klugi typicum non vidi.)

Clirysis billamata Westermann in litteris.

Habitat in Aegypto, Mus. D. Westermann; qui exemplar unicum amice communicavit.

Observ. Praecedenti (Chysidi bilhamatae) maxime affinis et similis, atque forsitan ejusdem sola rarietas mascula. Quum vero sculptura thoracica dorsalis nec non structura analis abdominis longe aliae sunt in Chryside prasina quam in Chryside bihamata, atque tantummodo unicum Chrysidis prasinae specimen adhuc examinare 
mihi contigit, hanc ut propriam speciem pro tempore potius habui, usque dum experientia pluribus individuis utriusque comparatis aliter doceat.

Corpus quoad magnitudinem et habitum omnino ut in Chryside bihamata; caput hujus. Antennae robustiores et longiores, sed scapus brevior quam in Clr. bihamata. Mandibulae nigro-piceae basi viridi-maculatae, ante apicem late rufo-testaceae; secundum Klugi descriptionem sint mandibulae „basi testaceae", qua pictura mandibulae nostri exemplaris carent. Thoracis dorsum confertim punctatum coriaceum 1 . subrugulosum laete cyanescenti-riride. Sternum riridi-cyaneum. Pedes et alae ut in Chryside bihamata, sed tegulae viridi-cyaneae. $\Lambda \mathrm{b}$ dominis segmentum dorsale 1:mum (in Westermanni exemplare, quod ante oculos habui) totum viride, parumper aureo-nitens; segmenta 2:dum et 3:tium cuprea, lateribus viridi-aureo-splendentia, segmenti 3:tii ınargo apicalis cyaneo - viridis nitidissimus depressus, spinis minutis subtilibus subulatis; spatium inter spinulas ita ter sectum ut latera trapezii tria exstent. In ceteris a Chryside bihamata non differt. 
Tab. Synoptica Gen. Chrysidis. Phalanx $V$.

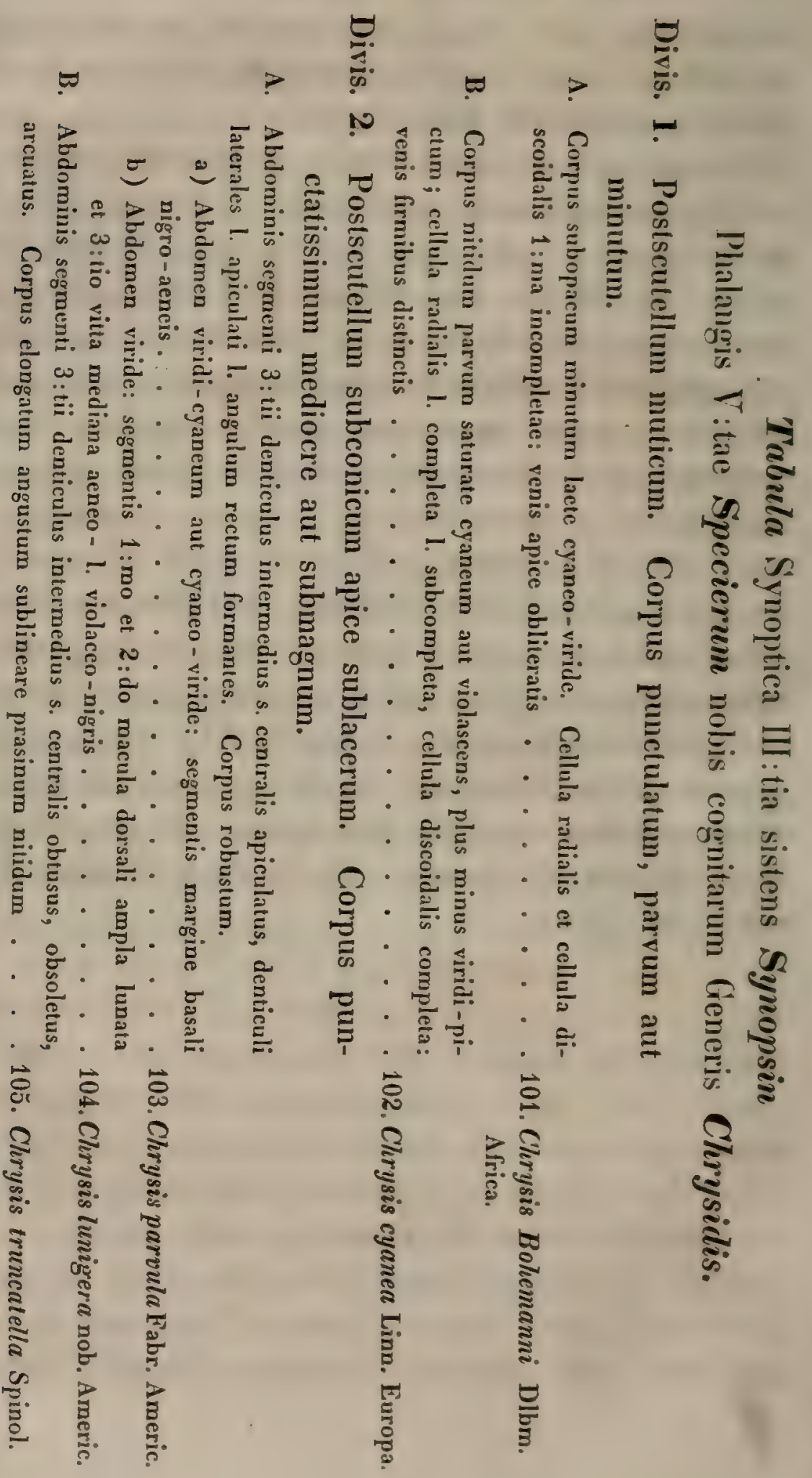


P'halanx V. Chrysides ano tri-dentatae. Scil. Abdominis segmenti dorsalis 3:tii margo apicalis denticulis unico centrali et duobus lateralibus armatus.

Divis. 1. Postscutellum muticum.

101. Chrysis Bohemanni Dlbm. $\sigma^{7}$.

Minuta gracilis linearis $1 \frac{1}{4}$ lin. decimal. long. punctulato-coriacen opaca coerulea tarsis nigro-fuscis, alis sublyyalinis ad costam cellulae radialis brunneo-fumosis, venis piceis, cellulis discoidali 1:ma et radiali incomplelis: venis apice obliteratis.

Clrysis Bohemanni Dlbm. Dispos.. 1845. 12. 20.

Habitat in Caffria; ad Port. Natal. a D. J. Waltberg detecta. Mus. R. Acad. Scient. Stochholm.

Observ. Clrysidi cyaneae similis et affinis; at corpore angustiore, abdominis dorso non nitido sed confertissime punctulato-coriaceo, armatura anali constante nec variante, cellulis radiali et discoidali incompletis etc. facillime dignoscitur.

Corpus parvum angustum lineare, totum dorso confertim punctulato-coriaceum subopacum, viridi-coeruleum, breviter cinereo-puberulum, pleuris, pedibus, metanoto postico ventreque nitidis. Caput proportionaliter robustum crassiusculum, superne amplum modice punctatoreticulatum convexum et quasi subglobosum obvenit; oculi ovato-rotundati exserti; cavitas facialis subprofunda, magnitudine medíocris semicircularis, tota viridissima et subtiliter transverse strigosa. Antennae longitudine et crassitic mediocres, nigro-fuscae puberulae scapo pedicelloque 
cyaneo - virescentibus. Clypeus brevissimus transrersus virescens, disco carinato-convexus. Mandibulae piceae. Thorax cylindricus dorso coeruleus, punctulato-subreticulatus, postice lateribusque virescens; postscutellum convexius; metanoti anguli postico-laterales parri triangulares. Abdomen lineare capite thoraceque simul sumtis Iongius, dorso depresso-convexum confertissime et subtiliter punclulato-coriaceum lacte cocruleum, lateribus et margine segmentorum apicali nonnihil viridi-nitens, in disco segmentorum lineola mediana subelevata; segmenti $\mathbf{1}$ :mi impressio basalis brevis levis et obsoleta; segmentum 3:tium ante seriem lenissime subimmersum, series ante-apicalis non in arcum sed in angulum rectum $\cdots . . . .$. ducta foveolis numerosis minutis approximatis; segmenti 3:tii margo apicalis subbrevis subtilissime coriaccus subtriangularis tridentatus: denticulis parvis subminutis conicis invicem aequaliter distantibus, spatio inter denticulos centralem et lateralem obliquo subrecto l. rix nisi lenissime arcuato; venter modice fornicatus nitidus cyaneo-viridis.

102. Chrysis cyanea Linn. $\sigma$ ?

Parva aut mediocris $1 \frac{1}{4}-2 \frac{1}{2}$ lin. decimal. long. modice punctata et punctulata nitida cyanea aut vialascens viridi-picta tarsis fuscis, alis sublyalinis, cellulis discoidali completa, radiali $l$. completa $l$. subcompleta: venis firmibus distinctis.

Chrysis cyanea Lin. S. N. 2. 948. 5. et Fn. Sr. 1667; - Fabr. E. S. 2. 243. 20 et Piez. 176. 29, secundum specimina Pabricii typica in Muscis lieg. Hanniensi et Kíliensi aestate 1847 a roc cxaminata. Panz. Fn. Germ. 51. 10 et Auctorum plurimorum. 
var. a. Abdominis segmenti dorsalis 3:tii margo apicalis 3-denticulatus denticulis acutis, margo lateralis angulatus.

$\left.\begin{array}{l}\text { Chrysis cyanea } \\ \text { Chrysis chalybaea }\end{array}\right\}$ Megerl. Mus. Vienn. teste Kollìr.

var. b. Abdominis segmenti dorsalis 3:tii margo apicalis 3-denticulatus denticulis I. omnibus subacutis 1. lateralibus obtusis, margo lateralis muticus (nec angulatus).

Chrysis discoidea Megerl. Mus. Vienn. teste Kollàr.

var. c. Abdominis segmenti dorsalis 3 :tii margo apicalis uni-denticulatus denticulo minuto centrali.

Chrysis chalybaeata K'lug. Mus. Berolin., secundum speciraen ibidern aestate 1847 a me examinatum.

Habitat in parietibus ligneis, truncis arborum, sacpibus et muris nec non in foliis floribusque plantarum soli urente expositis, a tempore verno usque in medium auctumnum per totam Europam vulgaris aut vulgatissima; ad domos et fenestras etiam frequenter adest, et extra Europam in Asia minori quidem observata.

Observ. Species tam vulgaris, quam omnibus sine dubio Entomologis cognita, descriptione completa non opus habere forsitan videatur; ne autem cum affinibus suis ex. gr. Chr. Bohemanni, Chr. assimili, Chr. nitidula, Clir. delicatula etc. confundenda, enumeratio notarum insigniorum structuram et sculpturam respicientium hujus speciei quoque debita sit.

Corpus magnitudine et pictura maxime varians, $a b$ una linea usque ad $2_{4}^{3}$ lin. decimal. longum, cinereo- et griseo-pubescens pilosellumque, nitidum, in aliis cyaneum aut violascens viridi-pictum, in aliis viride cyaneo-pictum, aliis rarissime totum viride aut totum cyaneum. Caput mediocre rotundato-triangulum subcubicum modice punctatum, vertice amplo convexo-depresso punctato-reliculato; margo occipitalis utrinque spinula bre- 
vissima obtusa transversa terminatus: cavitas facialis latiuscula profunda nitidissima confertim punctulato-coriacea, medio distinctius canaliculata. Antennac mediocres subbreves fuscae, scapo pedicelloque 1. cyanescentibus 1. virescentibus; clypeus mediocris aut subbrevis transversus remote modice punctatus nitidus, plerumque viridis, interdum aureo-splendens, margine apicali profunde et late arcuato-emarginatus, hinc totus ad ambitum quasi subreniformis. Mandibulac mediocres piceae, basi rirides aut cyaneae, ante apicem rufo-piceae; palpi maxillaeque fusca. Thorax ovato-subcylindricus, dorso confertim modice punctato-reticulatus; pronotum mediocre transversorectangulum convexo-depressum, sulco mediano profundo continuo, truncatura antica punctulato-coriacea: spatio centrali polito utrinque sulcato; dorsulum depresso-convexum suturis medianis rectis subcrassis subcrenulatis; scutellum et postscutellum convexiora; metanoti anguli postico-laterales mediocres robusti triangulares punctatorugulosi; mesopleurae punctato-reticulatae suturis crenatis 2: una perpendiculari apice elliptice inflexa, altera transversa; sternum, pedes et venter nitidissima sparse punctulata 1. viridi - cyanea 1. cyaneo- 1. aureo - viridia, tarsis fuscis. Alae subhyalinae ad costam cellulae radialis vix adumbratae, venis firmibus piceis, renula transverso-mediali basi parumper arcuato-inflexa; cellula discoidalis completissima, radialis 1 , rarius completa 1 . apice anguste aperta. Abdomen sublineare subrectangulum, latitudine fere thoracis, longitudine verticis thoracisque simul sumtorum, dorso modice convexum nitidum sparse punctulatum; segmentum 1:mum basi trisulcatum: sulco mediano longitudinali crasso profundo, sulcis lateralibus submargina- 
libus subtransversis obsoletis aut irregularibus; segmentum 3:tium 1. confertim I. confertius 1 . confertissime punctulatum, hinc: aliis nitidum, aliis subnitidum, aliis coriaceum et subopacum, disco omnibus transverse leniter aut lenissime immersum, mox supra seriem brevissime convexodeclive 1. submarginulatum, series ante-apicalis medio anguste interrupta: foveis crassis circ. 12 subconfluentibus, margo apicalis mediocris aut majusculus depressus subtilissime coriaceus tri-denticulatus: denticuli breves aut brerissimi, centralis semper distinctus, laterales ex utroque angulo apicali-laterali constituti l. acuti l. obtusi l. rarissime obliterati, quo in casu margo pone dentem centralem utrinque arcuato-obtusus obrenit; spatiun inter dentem centralem et dentes laterales 1 . arcuato-emarginatus I. convexo-arcuatus 1. undulatus 1. oblique subrectus, margo proprie lateralis (pone utrumque dentem laterali-apicalem situs) 1. muticus et tunc aut oblique rectus aut lenissine arcuatus, 1. superne lenissime arcuatus et inferne leniter lateque emarginatus indeque angulo centrali plus minus distincto munitus. Venter in rivis planus, in emortuis et exsiccatis concaviusculus.

Divis. 2. Postscutellum subconicum, apice sublacerum.

103. Chrysis parvula Fabr. $ð 79$.

Submagna elongata robusta $2 \frac{1}{2}-3 \frac{1}{2}$ lin. decimal. long. punclatissima nitida viridi-cyanea ant cyaneo-viridis abdominis segmentis margine basali nigro-aeneis, ano trumcato 3-denticulato: denticulo centrali apiculato, alis sordide hyalinis.

Chrysis parvula Fabr. Piez. 176. 30, secundum specimina typica in Museis Havniensi et Kiliensi aestate 1847 a me examinata. 
Chrysis 3-dentata Dlbm. Dispos. 1845. 13. 22.

Chrysis coerulans Dalman. Mus. Paykull.

Habitat in America; e Brasilia et Mexico a D. Liebmann reportata, Mus. D. Drewsen; e Carolina meridionali a D. Zimmermann amice communicata.

Observ. Chrysidi nitidulae individuis majoribus habitu et colore assimilis; at corpore robustiore et crasse punctatissimo, ano 3 -dentato etc. facile dignoscitur. -

Corpus elongatum viridi-coeruleum aut coeruleo-riride crasse punctatissimum nitidum cinereo-pubescens. Caput thoraci arcte applicatum robustum rotundato-triangulum, superne subcubicum, ore subangusto prominulo; vertex, dorsum thoracis et mesopleurae crasse punctatissima, conspicue reticulata; margo occipitalis utrinque sub temporibus spinula munitus; stemmata et oculi mediocria; illa parum elevata, in triangulum approximata, hi exserti rotundato-ovati; cavitas facialis submagna subprofunda subtrapezina viridissima nitida punctato-coriacea et transverse strigosa secundum medium subcarinulata, lateribus largiter cano-sericeis modice punctata, superne transrerse leniter marginata: margine sinuoso utrinque biramuloso parallelis sursum ductis, interdum obsoletis. Antennae robustae crassiusculae, magnitudine mediocres, fuscae cinereo-puberulac, scapo crasso cylindraceo-conico cyaneo-viridi, infra ad apicem leniter emarginato, pedicello articulisque flagelli duobus proximis supra concoloribus; clypeus mediocris transversus reniformis cyaneo-viridis, remote punctatus pubescens disco centrali conrexus, utrinque normaliter immersus, margine apicali summo nigropiceo. Labrum exsertum semicirculare gibbum punctulato-pilosellum. Mandibulae mediocres, basi virides, disco 
piceae, apice rufo-piceae, parce fulvo-1. luteo-pilosellae. Palpi maxillaeque fuscescentes. Thorax robustus cylindraceo-rectangularis antice posticerque truncatus, dorso depresso-convexus; pronotum ita modice ut truncatura antica fere obliterata, hujus spatium centrale triangulare politum, medio subtilissime canaliculatum utrinque foveola rotundata; dorsuli suturae medianae crassae nigrae crenulatae, antice angustiores et nonnihil curvae; scutelli margo basalis politus violascens; postscutellum conicum, basi fovea centrali rotundata, apice prominulo obtuso propter sculpturam crassissimam sublacerum; metanoti anguli postico-laterales robusti, magnitudine mediocres triangulares; mesopleurae marginibus duobus obliquis inferne angulum conjunctim efformantes; sternum et metapectus nitida punctulafa. Pedes cyaneo-rirides nitidi punctulati pubescentes et piloselli; tibiae posticae fortiter curvae nec non tarsi postici in toto latere inferiori pube sericea cinerea subrigida (scopulae instar) largissime et confertissime vestiti. Alae sordide hyalinae, ad costam cellulae radialis brunneo-fusco-fumosae, tegulis viridi-cyaneis, venis firmibus nigro-piceis, venula transverso-mediali basi modice arcuato-inflexa, cellula radiali elongata lanceolato-acuminata et cellula discoidali completis. Abdomen robustum elongatum capite thoraceque simul sumtis conspicue longius, orale basi apiceque subtruncatum, dorso nitidum sat convexum; segmentum 1:mum subcyancum crassius punctatum punctis viridibus, impressio basalis ampla profunda irregulariter triramosa, disco polita cyanea; segmenta 2:dum et 3:tium confertim modice punctata 1. viridia I. cyanea I. coerulescentia, marginibus basalibus: segmenti 2:di nomnihil strangulato et segmenti 3:tii conII. 
vexiore nigro-aeneis; segmenti 3 :tii series ante-apicalis per carinulam interrupta, foveolis circ. 12 crassis profundis 1. separatis 1. approximatis et interdum confluentibus; margo apicalis submagnus prominulus depressus tenuis non crassus nitidissimus I. viridis 1. purpureo-riolaceus, medio carinulatus, summus quasi truncatus, sed re rera tri-denticulatus: denticulo centrali subulato e continuatione carinulae constituto, utroque denticulo laterali (ex angulo apicali-laterali formato) acute triangulari, spatio inter denticulum centralem et utrumque denticulum lateralem transverse 1 . oblique recto 1 . arcuato 1 . arcuatoemarginato 1. subundulato; margo lateralis (a dente laterali ad basin segmenti sumtus) oblique rectus longius muticus; venter nitidissimus, I. planiusculus I. modice fornicatus, nitidus subtilissime coriaceus pubescens cyaneoviridis aut viridi-cyaneus.

104. Chrysis lunigera Dlbm. 오.

Submagna elongata robusta circ. 3 lin. decimal. long. punctatissima nilida viridis abdominis segmentis dorsalibus 1 :mo et 2:do macula ampla lunata et 3:tio vitta mediana aeneis $l$. violascentibus, ano summo violaceo truncato 3-denticulato: denticulo centrali apiculato, alis hyalinis.

"Chrysis nova species ex Amer. merid. Smidt." In Collectione Domini Tönder-Lund Musei Havniensis unicum specimen asservatur, quod ibidem aestate 1847 examinavi.

Habitat in America meridionali, a D. Smidt oliu detecta; Mus. Reg. Havnieus.

Observ. Magnitudo et summa affinitas Chrysidis parvulae, a qua tamen facile dignoscitur: colore riridi 
magis superante atque intensiore, pronoti lineola mediaua nigra, parte abdominis (ubi maculae lumatae adsunt) polita, margine summo anali polito pulchre violaceo per carinulam medianam viridem denticulo centrali terminatam abrupto.

105. Chrysis truncatella Spinol. ㅇ.

Elongata angusta sublinearis 3 lin. decimal. long. punctatissima viridissima suturis thoracis et incisuris abdominis nigricantibus aut violascentibus, ano truncato subtridenticulato: denticulo centrali obsoleto obtuso subarcuato, alis sordide hyalinis.

Chrysis truncatella Spinol. inedit.

Habitat in Brasilia, Mus. Spinolae; qui specimen e Collectione Latreillei acceptum benigne communicavit.

Observ. Chrysidi parvulae valde similis et affinis; at corpore gracili multoque angustiore, viridissimo, pronoto longitudinaliter sulcato, ani denticulo centrali obsoletissimo obtuso arcuato etc. facilline distinguenda.

Caput rotundato-triangulum modice punctato-reticulatum, supra convexum totum viride; stemmata submagna elevata in triangulum arcte approximata; cavitas facialis oblonga subrectangularis nitida punctulato-coriacea, medio subtiliter canaliculata, lateribus cano-sericea, superne transverse marginulata: marginulo utrinque ad oculos subarcuatim deflexo; cum hoc marginulo cohaerent marginuli duo frontales paralleli, superne (inter stemmata) in arcum transversum conjuncti et stemma anticum eo modo includentes. Antennae mediocres robustae fuscae, scapo toto, pedicello et articulo proxime sequente supra, virescentibus. Partes oris ut in Chryside parvula. Thorax 
cylindricus viridis crasse punctato-reticulatus, dorso sat convexus, et conspicue convexior quam in Chryside parvula; pronotum sulco mediano longitudinali brevi subovali; truncaturae anticae spatium centrale politum orbiculatum (non canaliculatum) foveolis 2 rotundissimis; dorsuli suturae 2 medianae angustae profundae rectae nigrae; postscutellum brevius quam in Chryside parvula. at apice magis lacerum. Sternum viridissimum nitidum punctulatum pubescens, pedes toti concolores; tibiae tarsique pedum posticorum omnino ut in Chryside parvula constructi. Alae etiam ut in Chryside parvula; sed cellula discoidalis minus bene explicata propter renam cubitalem et venulam transverso-discoidalem obsoletas tenuissimas et fere obliteratas. Abdomen elongatum angustum lineare capite thoraceque simul sumtis conspicue longius, totum viridissimum, dorso modice conrexum crebre punctatum; segmenti 1:mi impressio basalis mediocris triramosa nigro-violascens; segmenti 2 :di margo basalis substrangulatus, segmenti 3 :tii margo basalis convexus et anus, violacei; series ante-apicalis irregularis fere discoidalis h. e. disco distincta lateribus autem omnimo evanescens, medio interrupta per carinulam medianam viridem subacutam, quae ante summum marginem desinit (nec ut in Chryside parvula in denticulum centralem continuatur); foveolae paucae profundae confluentes; margo apicalis depressus truncatus, subtridenticulatus, denticuli laterales ex utroque angulo apicali-laterali formati acuti triangulares, denticulus centralis obtusus obsoletus arcuatus; venter nitidus viridissimus planiusculus, parumper concarus, punctulato-coriaceus pubescens.

Typis exscripsit A. W. Schade Berolinensis. 


\section{ysidis.}

\section{Sect. I. C}

\section{Divis.}

Divis.

Krysis fasciala Fabr. America.

Sulhrysis episcopalis $S_{\text {pin. }}$ Megypt.

Sultrysis Reichei Spiu.

$\mathrm{Su}$

Tribus 1. A

A. C

d

fí

a'lerysis assimilis Spin. Sicilia et Aegypt.

b"thrysis dissimilis nob. Bengalia.

B. $C$

dYhrysis Gayi Spin. Chili.

Tribus 2. A

Turb

M

A. Corpus i stinctissin sato-proflerysis chiliensis Spin. Chili.

B. Corpus du

a) Capui

disco

culo-1

juscullhrysis Nisseri nob. America.

b) Capur

dum .

sum,

foveol

recti Clrysis indigolea Dufour?

Manipulte

Mrysis clelorosoma nob. Java. Gernania. Gallia. Turba

MI

A.

Chrysis distinctissima nob. America merilion. 


\section{Phalangis VI:tae Specienum nobis cognitarum Generis Chrysidis.}

Sect. 1. Corpus violaceum, cyancum aut viride (rarissime aeneo-1, aureo-pictum).

Divis. 1. Abdominis segmentum dorsalc 3:tium seric ante-apicali omnino caret.

Corpus cyaneum viridi-pictum submagnum, totum punctatissimum . . . . . . 106. Chrysis fasciata Fabr, Arucrica.

Divis. 2. Abdominis segmentum dorsale 3 :tium serie ante-apicali senper instructum.

Subdivis. 1. Corpus purpurascenti-violaceum, vertex et dorsulum aurata. Corpus submagnum . 107. Clhrysis episcopalis Spin. Aegypt.

Subdivis. 2. Abdomen purpurascenti-violaceum, caput et thorax cyanea. Corpus mediocre - 119. Chrysis Reichei Spin.

Subdivis. 3. Corpus cyaneum aut niride: pictura viridi (rarissine acnea l. aurata).

Tribus 1. $\Lambda$ bdominis seomenti dorsalis 3 : tii dentes apicales intermedii obtusi.

A. Corpus modice punctatum et punctulatum, viride: pictura 1. cyanea 1. aurata; aldominis segmenti dorsalis $3:$ tii dentes apicales duo intermedii perobsoleti, per emarginaturam leven aut levissinam formati.

a) Scutellum immaculatum. Corpus parvum $1 \frac{1}{2}$ lin, deciral. long. . . . . . . . . 108

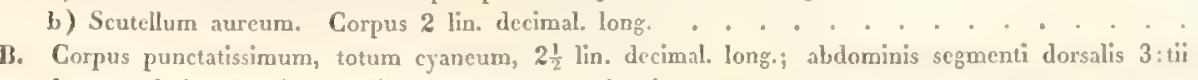
dentes apicales duo intermedii rolusti arcuato-obtusi

Clrysis assimilis Spin. Sicilin et Aegypt

109. Chrysis dissimilis nob. Bernalia.

Tribus 2. Abdominis segmenti dorsalis 3 : tii dentes apicales omes acuti.

Manipulus 1. Abdominis segmentum dorsale 3 :tium, supra seriem ante-apicalem margine convexiusculo plus minus incrassatum.

A. Corpus maximum punctatissimum; abdominis segmenta dorsalia 2 : dum et 3 : tium carinula mediana distinctissima; hoc utrinque transverse concavum, postice - supra seriem ante-apicalem - fortiter incras-

sato-prominulun, series ante-apicalis fovcolis. majusculis rotundis separatis . . . . . . . .

111. Chrysis chiliensis Spint. Chili.

B. Corpus mediocre.

a) Caput et thorax punctatissima. Abdomen dorso sparse punctatum, non carinulatum; segmentum 3 :tium disco leniter subconcariusculo-immersum, postice - supra scriem ante-apicalen - roodice convexiusculo-ruarginatum; series ante-apicalis continua foveolis minutis rotundis separatis, dentes apicales majusculi: intermedii subcurvi

b) Caput et thorax modice punctata. Abdomen dorso sparse punctulatum, segmentum 2:dum (et interdum quoque 3 :tium) carinula mediana subtili; segmentum 3 :tium disco Ieniter concaviusculo-immersum, postice - supra seriem - modice convexiusculo-raarginatum; series ante-apicalis interrupla foveolis mediocribus profundis non bene separatis, hinc inde confluentibus; dentes apicales mediocres recti . . . .

Manipulus 2. Abdominis segrnent, dorsale 3:tium - supra seriem anteapicalern - margine ullo non incrassatum . . . . . . . . . . . . . . . .

Turba 2. Abdominis segmentum dorsale 3 : tium modice depresso-convexum, disco non concaviusculum. Manipulus 1. Alae liyalinae aut sordide hyalinae.

A. Series ante-apicalis foveolis profundissirnis.

a) Corpus crasse punctatissimum cyaneum, abdom. segment. dorsal, 3 :tium foveolis ante-marginalibus maximis scparatis haud numerosis . . . . . . . . . . . .

110. Clhysis Gayi Spin. Cliti.

115. Chrysis distinctissima nob. America merilion. 
b) Corpus confertim punctatum coeruleum aut virescens, abdom. segm. dors. 3 : tum forcolis ante-marginalibus conlluentibus numerosis . . . . . . . . . . . . . . . .

B. Serics ante-apicalis foreolis mediocribus.

a) Corpus nediucre.

1. Ablominis segmenti dorsalis $3:$ tii dentes apicales robusti, majores aut mediocres.

$\boldsymbol{a}$. Corpus varii magnitudinis cyaneum aut vinlaceum virjdi-pictum, caput et thorax punctatissima; caput mediocre triangulare, thorax et abdomen subelongata subsemicylindrica.

$\times$ Abdominis dorsum cyanescens viridi-pictum.

+ Capitis cavitas facialis superae per carinulam transversam limitata (; abulom. segnenti dorsalis 3 : tii margo lateralis flexuosus) . . . . . . . . . . . . . . . . . . 11

ff Capitis cavitas facialis superne libera, scil. carinula transversa limitante onanino caret . . .

XX Abdominis dorsum violaceo-purpurascens (; segmenti 3:tii margo lateralis, ut in Chryside soluta no. 118, oblique rectus) . . . . . . . . . . . . . . . . . . . . .

B. Corpus mediocre, subtus cyaneum, supra viride acnco-1. aurato-micans; caput et thorax sparse modice punctata; caput 1. magnum 1. maximum cubicum; thorax brevis; abdomen ovatum.

X Corpus supra viride parumper aurco-nitens, caput magnum, alae albo-hyalinae venis tenuibus testaceis; segmenti 3 :tii series ante-apicalis distincta, dentes apicales subparvi . . . . . .

$\times \times$ Corpus supra viride distinctius acneo-aurato-nitens, caput maximum, alac sordide hyalinae venis frrmibus piceis; segmenti 3 :tii series ante-apicalis oblitterata, dentes apicales majusculi . . . .

2. Abdominis segmenti dorsalis 3 : tii dentes apicales parvi.

a. Caput et thoras crasse et regulariter punctata. Corpus viride. . . . . . . . . . . . . .

B. Caput et thorax modice et irregulariter et confertim xuguloso-punctata.

$X$ Corpus viride tarsis alluis

$\times \times$ Corpus viride tarsis fuscis

$X X X$ Corpus cyaneum tarsis fuscis

b) Corpus minutum tracile, totur confertim punctulatum subopacum viridi-cyancum . . . 126. Chrysis delicalula nob. Africa.

Manipulus 2. Mlac violascentes.

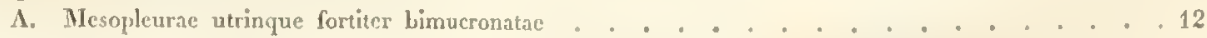

1. Mesopleurae margine et apicali et postico-laterali crenulatae et line obsolete denticulatac . .

Sect. II. Abdomen cyaneum aut viride: fasciis aut viridibus aut aureis distinctissimis,

Divis. 1. Metanotum centro postice mucronulatum.

Corpus submagnum punctalissimum purpurascenti-violaccum, segmenta abdominis dorsalia

fasciis mediocribus seladonico- viridibus formose picta . . . . . . . . . .

Wivis. 2. Metanotum inerme (non mucronatura).

Subdivis. 1. Abdominis segmenti dorsalis $3:$ tii dentes apicales omnes distincti, licet perbreves.

A. Corpus cyaneum; abdominis segmenta dorsalia aut duo aut tria aurco-fasciata; vertex, pronotum et scutcllum l, viridia l. aurata . . . . . . . . . . . 130

B. Corpưs coeruleum; aldominis segmenturn 2:dum aureum . . . . . . . . . 13

C. Corpus viride.

a) Abdominis scgnedum dorsale 2:dum utrinque macula laterali et $3:$ tium vitti mediann viridi-aureis . . . . . . . . . . . . .

b) $\Lambda$ bdom. segment. dorsale 2 :dum fascia apicali ampla et $3:$ tium utrinque nuacula laterali siridi-aurcis . . . . . . . . . . . . . .

Subdivis. 2. Mbdominis segmenti dorsalis 3 : tii dentes apicales internacdii obsoletissimi brevissimi, - externi breves distincti.

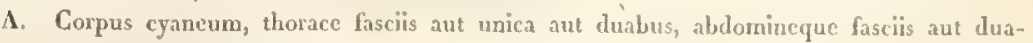
bus aut tribus aureis

B. Corpus viride, segmento abdominis dorsali 2: do toto aureo
16. Chrysis coerulans Tabr. America.
129. Chrysis spinigera $S_{\text {pin. }}$ Cayeun

130. Clrysis allernans Klug. Africa.

31. Chrysis Schesledli not. 'Tanger.

32. Chrysis aurcomaculala nob. Guinea.

133. Chrysis soror nol. lihodus.
7. Chrysis nitidula Fabr. Europa, America boreal. 18. Chrysis soluta nob. Srecia.

19. Chrysis Reichei Spin. Coromandel.
120. Chrysis Ehrenbergi Dlbm. Aegypt.

121. Chrysis megacephala Bohemn. Megypt.

22. Chrysis laeta Dracge, Africa,

23. Chrysis palliditarsis Spin. Acgypt.

124. Chrysis orientalis nob. India oriental.

Chrysis diversa nob. Acgypt.

27. Chrysis amelhystina Fabr. India oriental.

128. Chrysis I'cstermani nob. Gunea. 
b foveolis

116. Chrysis coerulans Fabr. America.

na; caput

ui dorsalis

117. Chrysis nitidula Fabr. Europa, Anscrica boreal.

. . 118. Chrysis soluta nob. Svecia.

ide soluta

. . 119. Chrysis Reichei Spin. Coromandel.

parse no-

; tenuibus

120. Chrysis Ehrenbergi Dlbm. Acgypt.

inae renis

121. Chrysis megacephala Bohemn. Aegypt.

122. Chrysis laeta Dracge. Africa.

. 123. Chrysis palliditarsis Spin. Aegypt.

. . 124. Chrysis orientalis nob. India oriental.

125. Chrysis diversa nob. Acgypt.

. . 126. Clerysis delicatula nob. Africa.

. . 127. Chrysis amethystina Fabr. India oriental.

1e. . 128. Chrysis Westermanni nob. Guinea.

simis.

is dorsalia

129. Chrysis spinigera Spin. Cayenn.

perbreves.

:iata; ver-

130. Chrysis alternans Klug. Africa.

131. Chrysis Sehestedti nob. Tangex.

tium vitta

132. Chrysis aureomaculala nob. Guinea.

ue macula

issimi bre-

aut dua-

134. Chrysis semicincta Lepel. Gallia. Hispania.

135. Chrysis zonata nob. Asia minor. 
Sect. III. AI

dii

A. 1

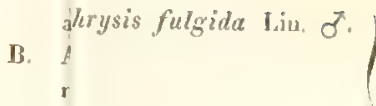

Europa

Krysis fulgida Liu. 오.

I

Thrysis exsulans uob. Bengalia.

Sect. IV. A

ur

Divis.

'Hrysis Ramburi Spin. Hispania.

Trysis simuata Dalnan. Africa

'hrysis cyanopyga nob. Algie! Divis.

'Hrysis splendidula Rossi. Europa.

'hrysis bidentata Lin. Europa.

'Hrysis pyrrhina Dlma. Mus. Paykull.

Sect. V. $\Delta b$

ap
A. C
nilhysis rutilans Olivier: Europa ncridional.
B. C
rithrysis terminala Megetle. Austria.

Sect. VI. A
A. A
Wirysis analis Spin. Italia, Ilispania, Malla, Rhodus.
B. N'trysis scutellaris Fabr. Europa.
C. $\lambda$
T'/hysis aeruginosa kilug. Berolin 
Sect. III. Abdominis dorsum aureum; segmentum 1 :mum violaceum aut cyaneum, inter dum quoque segmentum 2:dum macula concolore.

A. Abdominis segmentum dorsale 1 : num totura et 2:dum basi macula maxina senicirculari, violacea

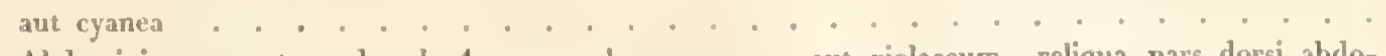

B. Abdominis segmentum dorsale 1 :mum solum cyaneum aut violaceum, reliqua pars dorsi abdominalis aurea.

a) Abdom. segment. dorsale 3:tium depresso-convexurn, apice distincte angustius quam basi, series ante-apicalis foveolis numerosis distinctis circ. 16 , dentes apicales robusti acute triangulares; alarum cel!ula radialis completa

b) Abdominis srgment. dorsale 3 :tium valde convexum, apice parum angustius guam basi, series ante-apicalis foveolis paucis circ. 8-10 quarum latcralibus obsoletis, dentes apicales angusti spinoidei; alarum cellula radialis incompleta

136.

Chrysis fulgida lin. $\sigma$.)

136.

Chrysis fulgida Liu. 오.

Europa.

Chrysis exsulans nob. Bengalia.

Sect. IV. Abdominis dorsum aureum: segmentum 3:tiun cyaneum: aut unicolor, aut maculis viridibus 1 , aureis.

Divis. 1. Abdominis segmenti dorsalis 3 : tii dentes apicales distincti.

A. Segmenti 3 : tii margo lateralis angulatus.

a) Segmentuna (abdominis dorsale) $2:$ dum immaculatum, segmentum $3:$ tium utrinque macula laterali-marginali aurea; dentes apicales breves latiusculi triangulares . . . . .

b) Segment. 2:dum basi linca arcuata cyanea, segnent. 3 : tium macula centrali viridi-aurea; dentes apicales mediocres spinoidei . . . . . . . .

B. Segmenti 3 : tii margo lateralis muticus (ann angulatus,) oblique rectus.

a) Corpus robustum; abdomen punctatum, seginenta $1:$ mum et $2:$ dum purpurea 1 . ignea, hoc lineola mediana elevata polita; segnenti $3: t i i$ series ante-apicalis foreis profundis mediocribus rotundis separatis . . . . . . . . . . . . . . . .

b) Corpus gracile; abdomen punctulatum, segraenta 1:mum et $2 \cdot d u n$ viridi-aurea, lineola mediana caret; segmenti $3:$ tii series ante-apicalis foveis profundioribus majoribus coëuntibus suhpellucidis . . . . . . . . . .

Divis. 2. Abdominis segmenti dorsalis 3 : tii dentes apicales obsoleti.

A. Thoracis dorsum aureurn

B. Thorax viridis, dorsuli area media cyanen

138. Chrysis Ramburi Spin. Hispania.

139. Chrysis simuata Dalman. Africa.

140. Chrysis cyanopyga nob. Algier

141. Chrysis splendidula Rossi. Europa

142. Chrysis bidentata Lin. Europa.

143. Clorysis pyrrhina Dlra. Mus. Paykull.

Sect. V. Abdominis dorsum aureum aut viridi-aureum, segmentum 3 : tium viride (: margo apicalis 1. concolor 1 . cyaneus, series ante-apicalis semper cyanea).

A. Corpus robustum. Abdomen aurato-viride 1. viridi-aureum confertius punctatum; segmenti $3:$ tii margo lateralis angulatus, dentes apicales breviores

B. Corpus angustum. Abdomen aeneo-auratum sparse punctatun; segmenti 3 : tii margo lateralis muticus, dentes apicales rajores

C'hrysis rutilans Olivier. Europa meridional.

Chrysis terminata Megerle. Austria.

Sect. VI. Abdominis dorsum aureum, segmenti 3:tii margo apicalis cyaneus.

A. Abdominis segmenti dorsalis $3:$ tii dentes apicales omnes triangulares. Thorax cyaneus nonnilíl

B. Abdom. segmenti dors. 3 :tii dentes apicales intermedii obtusi brevissimi. Scutellum aureum . . . 146. Chrysis analis Spin. Italia, Hispania, Malta, Rhodus.

C. Abdom. segmenti dors. 3 : tii dentes apicales intermedii obtusi, externi triangulares, omnes distincti. Thorax et ahdomen viridi-cuprea 
Sect. VII. Abdominis dorsum aureum, segmenti 3: tii margo apicalis aut acneus, aut rarissime viresecns.

1. Dorsulum (mesonoti) I. totum 1. saltem areis lateralibus auratum.

a) Abdom. segmenti dors. 3 :tii margo lateralis leniter aut lenissime angulatus, inter angulum et denten externum emarginatus, dentes apicales aut triangulares aut obtusi . . . . . 119. C'hrysis succincta Lin. Europa.

b) Abdom. segrnenti dors. 3 : tii margo lateralis arcuatus, dentes apicales robusti spinoidei subapproxinati 150. Chrysis Grahmami Spin. Sicilia. Ib. Dorsum (Lloracis) anreum.

a) Abdom. segnenti dors. 3:tii margo apicalis 4-angulatus . . . . . . . . . 151. CKrysis Sckousbuei uolb. 'Tanger.

b) Abulominis segmenti dors. 3 :tii margo apicalis viridissimus distincte 4 -dentatus, dentibus cxternis triangularibus, internediis arcuato-obtusissimis - . . " "

52. Clerysis Dretoseni nob. Africa.

Sect. VIII. Abdominis dorsum aurcum unicolor.

Divise 1. Tarsi albo-testacei.

A. Abdomen breviusculum, dorsulum (mesonoti) viridi-surcum . . . . 153 .

B. Ablomen longiusculum, thorax cyaneo-viridis . . . . . . . . . . . 151

Clerysis armena Spin. Armenia.

Divis. 2. Tarsi fusci brunnescentes.

Subdivis. 1. Abdominis segmenti dorsalis $3:$ tii dentes: laterales, h. e. margo proprius apicalis convexo-arcuatus, utrinque dente triangulari munitus, supra liunc dentern adest - in ccntro marginis lateralis - anoulus dentiformis. Pronotum et mesonotum viridicuprata . . . . . . . . . . . . . . . . . . . .

Subdivis. 2. Abdominis segmenti dorsalis 3 : tii dentes: apicales.

Turba 1. Abdominis segment. dors. 3:tium carinula mediana distinctiori, ntrinque concaviusculo-depres-

sum, totum confertim crasse punctatum, supra seriem marginulo incrassatum . . . . . . 156

Turia 2. Abdominis segmento dors. 3 : tium modice depresso-convexum.

Manipulus 1. Segmenti 3:tii margo apicalis monnilil angustatus, dentes intermedii prominuli valde approximati, externi remoti. Pronotum et dorsulum viridi-aurea, venter niger maculis igneis 157

Manipulus 2. Segnenti 3:tii margo apicalis latitudine mediocris, non angustatus, dentes omnes in unam plus minus curvas dispositi.

A. Scgmenti 3:tii dentes externi ab angulo laterali remoti, hoc angulo arcuato-obtuso .

B. Segmenti 3 : tii dentes externi ipsum angulun lateralem efficiunt.

$X$ Segmenti 3:tii dentes breves, aut omnes aut saltem intermedii obtusi.

1. Abdominis dorsum clipreum (segmenti 3 :tii serics ante-apicalis forcolis rotundis distinctis) . . . . . . . . . . . . 1

2. Abdominis dorsum aureum

Segmenti 3 :tii series ante-apicalis foveolis transversis confluentibus, dentes intermedii obtusi, externi triangulares . . . . . . . . . . .

Segmenti $3:$ tii series ante-apicalis foveolis rotundis distinctis, dentes internedii triangulares brevissimi, externi obtusi ............ 161. Chrysis aestiva nob. Mhodus.

3. Aldominis dorsum viridissirac aureum. Alarum cellula radialis completa . . 162. Chrysis impar nols. Rhodus

XX (Abdominis) segmenti (dorsalis) 3: vii dentes omnes robusti acuti: aut triangulares, aut spinoidei.

1. Venter nigro-, viridi-et aureo-maculatus. Pedes viridi- et cyanco-variegati.

a) Segmenti 3 :tii dentes apicales intermedii arcuati, apice nonnithil incurvi . . 163. Clerysis curvidens nob. Foropa meridional.

b) Segmenti 3:tii dentes apicales omnes triangulares recti ......165. Clirysis ignita Lin. Europa. Asia minor.

2. Venter igneus. Pedes aure-splendentes . . . . . . . . 164. Chrysis auripes Wesmacl. LEuropa. 
aut ra-

ngulum et

149. Chrysis succincta Lin. Europa.

proxinati 150. Chrysis Grohmanni Spin. Sicilia.

151. Chrysis Scloousbuei nob. 'Ianger.

is externis

152. Chrysis Drewseni nob. Ifrica.

153. Chrysis armena Spin. Armenia.

154. Chrysis manicala nob. $\Lambda$ sia mimor.

us apicalis

west - in

um viridi-

. 155. Chrysis angulata nob. Asia minor.

lo-depres-

156. Chrysis inaequalis nob. Europa meridional, et Asia minor.

valde apculis igneis 157. Chrysis pyrophena hlug. Gallia meridional. omnes in

. 158. Chrysis distinguenda Spin. Europa meridional.

tundis di-

- 159. Clerysis comparata Lepelet. Gallia.

is interme-

. . 160. Clirysis verna nob. Rhodus.

intermedii

- . 161. Chrysis aestiva nob. Rhodus.

- . 162. Chrysis impar nob. Rhodus.

slares, aut

ii.

. . 163. Chrysis curvidens nob. Europa meridional,

165. Chrysis ignila Lin. Europa. Asia minor.

164. Chrysis auripes IVesmacil. Europa. 
Phalanx VI. Chrysides ano quadri-dentatue scil. abdominis segmenti dorsalis 3 : tii margo apicalis denticulis quatuor armatus.

Sect. I. Corpus violaceum, cyaneuin aut viride (rarissime aeneo- 1. aureo-pictum).

Divis. 1. Abdominis segmentum dorsale 3:tium serie ante-apicali omnino caret.

106. Chrysis fasciata Fabr. $\sigma^{7}$.

Submagna elongala robusta sublinearis $3 \frac{1}{2}$ lin. decimal. long. punctalissima nilida viridi-cyanea abdominis segmentis margine basali nigro-aeneis, segmenli 3:tii dentibus parvis aut submediocribus aculis angustis distanlibus, alis sordidis subliyalinis, venis piceis.

Chrysis fasciatu Fabr. Piez. 175:27. secundum specimina 2 typica in Museo Havniensi asservala, anno 1847 a me examinata, ctiain in Collectione Fabricii in Museo Kiel. exemplar cjusdem nominis lustravi.

Chrys. punctatissima Spin. Annal. Ent. 1840. 200:48.

"Ifabitat in America meridionali Dom. Smidt, Mus. Dom. de Seliestedt" Fabr. I. c. Specimina a Dom. Spinola mihi describendi caussa benigne communicata, in Cayenne collegit Dom. Leprieur anmo 1539.

Observ. Individuis majoribus Clirysides milidulae valde similis, at corpore punctatissimo et praecipue struclura segmenti abdominis dorsalis 3:tii (, quod omnino II. 
caret serie foveolarum anteapicali, apud plurimas Chrysides normali,) ab illa et ab omnibus affinibus facillime dignoseitur.

Corpus robustum elongatum sublineare punctatissimum cyaneum aut viridi-cyaneum plus minus nitidum. Caput subbreve rotundato-triangnlum. Vertex amplus depressoconvexus. Stemmata in triangulum approximata, in macula nigricante plerumque sita. Oculi obtuse ovati. Caritas facialis elongata trapezina 1 . subrectangularis, riridissima aut viridi-cyanea confertim coriaceo-punctulata el albo-sericeo-pilosella. Clypeus huic concolor nitidus depressus coriace-punctulatus brevis, margine apicali late arcuato-emarginatus; margo occipitalis maris pone oculos utrinque angulo spinoideo armatus; mandibulae breres robustae, basi viridi-cyaneae, ceterum rufo-piceae. Antennae breves robustae: scapo pedicelloque rel viridibus 1. viridi-cyaneis, flagello fusco. Thorax subcylindricus, dorso convexus lateribusque punctatissimus, l. totus çaneus 1. pronoti et mesonoti marginibus viridi pictus; postscutellum gibbum sculptura crassissima saltem medio rugosum. Sternum et pedes nitida viridia aut viridi-cyanescentia punctulata cinereo-pubescentia articulis tarsorum ultimis fuscis. Alae posticae hyalinae; anticae subhralinae, disco plerumque sordidae, cellula radiali elongata apice late aperta. Abdominis dorsum cyaneum; segmenti 1:mi impressio s. regio basalis interdum nigro-violacea ant nigro-aenea, regio postica antice posticeque obsolete riridimarginata; segmenta 2:dum et 3:tium margine basali fasciolaque minus determinata ante marginem apicalem saepe virescentem nigro-acneis; abdominis dorsum, pracsertim in segmento 2: do ubi lineola mediana subelerata obser- 
vatur, sat convexum; sculplura 1. punclura segmenti 1 :mi aeque crassa ac dorsuli at minus conferta, punctura sequentium segmentorum minus crassa quam segmenti I:mi sed nonnihil crebrior; segmentum 3:tium totum depresso-conrexum, nec vestigium quidem seriei illius anteapicalis plurimarum specierum normalis praebens: dentes apicales 4 breves acuti spinoidei distantes emarginaturis spatiosis, intermedia triangulari, utraque laterali arcuata, margo lateralis inter dentem externum et basin non rectus sed non nihil flexuosus. Venter viridi-cyaneus aut vice versa, praesertim ad margines segmentorum dense cinereo-pubescens.

Divis. 2. Abdominis segmentum dorsale 3:tium serie forcolarum I. punctorum ante-apicali semper instructum.

Subdivis. 1. Corpus purpurascenti-violaceum, vertex et dorsulum aurata.

107. Chrysis episcopalis Spin. 오.

Submagna robusta elongata $2 \frac{1}{2}$ lin. decimal. long. conferlim modice punclata nilida purpurascenti-violacea fronte dorsuloque cupreo-auratis, serie anterpicali obsolela foveolis nonnullis (circ. 9) irregularibus, alis hyalinis disco fumeo-sordidis, venis piceis.

Chrysis episcopalis Spin. Annal. Entomol. 1838. 449:4.

Ilabilat in Aegypto, a D. Fischer detecta; Mus. de Spinola.

Observ. Individuis majoribus Chrysidis nitidulae quoad habitum assimilis. Corpus sparse griseo-pilosum. 
Caput modice confertim punctato-subreticulatum; verlex subcoriaceus, sub lente instar rubini rufo-splendens. Cavitas facialis planiuscula immarginata punctulato coriacea saturate violascens, largiter albo-sericeo pilosella, sulco mediano continuo aureo. Antennae mediocres fuscae, scapus violaceo purpurascens. Clypeus mediocris transversus inaequalis, purpureo-violaccus, subtrapezinus: marginc apicali transverso subtruncato nigro-aeneo. Labrum majusculum convexum subsemilunatum et mandibulae nigroaenea, hae apice testaceo-brunneae. Palpi tenues mediocres fusci testaceo-annulati. Caput infra riridi-cyaneum. circa foramen magnum purpureo-aureo-irroratum. Thorax dorso crasse punctatus convexiusculus; dorsulum rel totum vel ex parte cupreo-purpureum suturis 2 medianis crenulatis. Metanotum postice in foreolis aureo-resplendens. Sternum sparse punctulatum coriaceum, sulcis suturisque viridi aureis. Abdomen nitidum laevigatum, non politum, confertim subcrasse punclatum; impressio basalis aenea coriacea sparse punctulata, postice aureo-purpureo submarginata; segmentum 3:tium totum depresso-conrexum, dentes apicales mediocres acute triangulares, emarginaturae mediocres arcuatae: utraque lateralis latior quam centralis, segmenti margo latecalis inter basin et scriem subrectus 1. lenissime arcuatus. Venter confertim punctulatus nitidus purpureo-violascens. Tarsi fusci; horum unguiculi et tibiarum calcaria brunneo-testacea. Alac hyalinae, disco sordidae, venis firmibus piccis; cellula radialis triangulariter lanceolata apice sat aperta. 
Famil. C'lrysididae. Chrysis: Phalenx VI. 201

Subdivis. 2. Corpus cyaneum aut viride: pictura I. viridi 1 . rarissime aenea aut aurata.

Tribus 1. Abdominis segmenti dorsalis $3:$ tii dentes apicales intermedii obtusi.

108. Chrysis assimilis Spin. 079 .

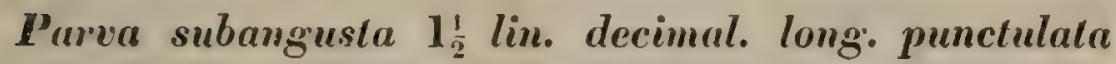
viridis, cyaneo-viridis ant viridicyanea, segmentis basi 3:lioque margine apicali cyaneis, hoc (margine) subliliter coriaceo, serie anteapicali per carimulam medianam minulam interrupto, alis sublyyalinis cellula radiali lanceolata subcompleta, apice angustius aperta.

Chrysis assimilis Mus. Spinolae.

Chrysis pumila Klug sec. Spinola in litteris.

IIabitat in Sicilia et Aegypto. Tria specimina lustravi: unum a D. Grolmann in Sicilia lectum, Mus. Vienn. teste D. Kollàr; alterum e Stansnio a D. Loew communicatum, tertium ex Aegypto D. Walll, Mus. D. Spinolae.

Observ. Corpus subangustum, quemadmodum Chrysydlis cyaneae formatum, cano- et griseo-pilosellum, confertim modice punctatum et punctulatum, minus nitidum subcoriaceum, virescens aut cyaneo-virescens, segmenta abdominis dorsalia: 2: dum margine basali et disco indeterminate, - 3:tium margine basali et apicali cyanca. Pectus, pedes et venter virides, hinc nonnihil nigro-maculatus, calcaria et tarsi testacea, horum apices fusci. Anteunae longiusculae, robustac fuscac scapo virescente. Abdominis segmenta dorsalia: 2: dum et 3:tium lineola mediana subtili subelevata continua, interdum obsoleta atut oblitterata; segmentum 3:tium disco utrinque subcon- 
caviusculo-depressum, series anteapicalis cyanea forcis profundis irregularibus, margo apicalis tenuis 1. rirescens 1. cyanescens subtilissime coriaceus opacus: utrinque denticulo triangulari acuto; spatium inter hos denticulos, per emarginaturam centralem levissimam, leniter arcuato-undatum, hinc duo quasi-dentes L brevissimi late obtusi exstant.

109. Chrysis dissimilis Dlbm. ㅇ.

Submediocris 2 lin. decinal. long. punctata viridis, pectore pedibusque concoloribus, tarsis fuscis, scutcllo aureo, segmentis basi cyaneis; segmenti 3 : tii serie anteapicali continuo, margine apicali prominulo depressoconvexo sparse punchulato, alis subhyalinis, ad marginem cellulae radialis costalem sordidis, cellula radiali lanceolata incomplela apice distincte aperta.

Ilabilat in Bengalia, a D. Westermann detecta; specimen unicum in Museo Westermanni asserratum lustravi.

Observ. Habitus Chrysidis succinctne majoris. Corpus griseo-pilosellum nitidum laete viride, dorso modice punctatum. Vertex, pronoti particula antica colliformis, dorsuli area media, et basis segmentorum abdominis dorsalium cyanea. Antennae breviusculae, scapus virescens, flagellum fuscum. Mandibulac normaliter pictac. Scutellum viridi-aureum, postscutellum aurato-viride. Abdominis segmentum dorsale 3 :tium totum modice convexum, series anteapicalis continua nec interrupla, forcolis mediocribus rotundis, quarum nonnullis confluentibus: margo apicalis, ut ipsum segmentum, convero-depressus promi- 
F'amil. Chrysydidae. Chrysis. Phalunx VI. 203

nulus sparse punctatus: dentibus lateralibus triangularibus distinctis, centralibus (quemadmodum in Chrys. assimili) brevissimis latis subarcuato-obtusis. Venter cyaneo-viridis nigro-naculatus.

\section{Chrysis Gayi Spin. $\delta$.}

Mediocris aul submagna robusta $2 \frac{1}{2}$ lin. decimal. long. punctalissima cyanea ventre pedibusque concoloribus, tarsis apice fuscis, alis hyalinis venis piceis, cellula radiali incompleta.

Clrysis Gayi Mus. Spinolae.

\section{Ifabitat in Chili, D. Gay; Mus. D. Spinola.}

Observ. Dentibus ani centralibus robustis latis arcuato-obtusis ab affinibus speciebus mox dignoscitur. Corpus sparse fusco- ct cano pilosellum, dorso confertim crasse punctatum, undique unicolore cyaneum. Caput longiusculum ovato-triangulare modice punctato-coriaceum. Frons nonnihil viridi-micans. Antennae breves, scapus cyaneus, Hlagellum fuscum. Clypeus depressus, paullulum longior quam in plurimis aliis speciebus, subtrapezinus, apice subarcuato-obtusiusculus. Mandibulae basi cyaneae, medio acneae, apice rufo-piceae. Palpi minuti fusci. Abdomen dorso tolo modice convexum, confertim crasse punctatum; segmenti 3:tii series anteapicalis foveolis inaequalibus; margo apicalis, ut reliquum abdominis dorsum, depresso-convexus crasse sparse punctalus, dentibus non longis at robustis et latiusculis: centralibus conspicue arcuato-obtusis, lateralibus remotis paullulum brerioribus triangularibus vix aculis sed potius subobtusiusculis. Venter nitidus sparse punctulatus. $A$ lac ad marginem costa- 
lem cellulae radialis nonnihil sordidac; cellula radialis triangulariter lanceolata imcompleta, apice late aperta.

Tribus 2. Abdominis segmenti dorsalis 3:tii dentes apicales omnes acuti.

Turba 1. Abdominis segment. dorsale 3 :tium disco transverse plus minus concaviusculo-immersum.

Manipulus 1. Abdominis segment. dorsale 3:tium, supra seriem anteapicalem, margine convexiusculo plus minus incrassatum.

111. Chrysis chiliensis Spin. ठ오.

IHagna aut submaxima punctatissima robusía elongata 4 lin. decimal. long. cyanea, scutello postscutelloque gibbo-convexis inermibns, abdominis segmentis dorsalibus 2:do et 3:tio carimula mediana distinclissima, hoc (segmento) utrinque transverse concavo, postice supra seriem anteapicalem forliler incrassato-prominulo, alis albo-hyalinis disco leviter sordidis, cellula radiali incompleta.

IRabitat in Chili, D. Gray; Mus. D. Spinola, qui specimina 2 milhi dono dedit.

Observ. Inter distinctiores et maxime eximias formas hujus generis. Corpus fusco et cano sparse pilosum et pubescens. Caput robustum rotundato-quadratum subcubicum confertim modice punctatum. Oculi breves ovati. Cavitas facialis magna subtrapezina profundiuscula confertim punctato-coriacea viride ant viridi-cyanco nitida, superne transverso-marginata. Antennac subbreres 
crassiusculac, basi cyaneo-virescentes, apice fuscae. Clypeus mediocris transversus punctulatus cavitati faciali concolor, disco convexus utrinque concaviusculo-depressus, ad centrum marginis apicalis late at leniter arcuato-emarginatus. Caput ad latera clypei mandibularumque basin utrinque angulato protuberans. Mandibulae robustae piceae; basi cyaneo- et viridi-pictac, interdum quoque ibidem nonnihil aureo-splendentes. Labrum breve linguaeforme convexum cyaneo-viride ciliatum. Palpi fusci pubescentes. Caput ad latera, inter verticem et basin mandibularum marginatum. Thorax crassus cylindricus punctatissimus dorso convexus. Pronotum latitudine occipitis, aut cyaneum aut viride punctis cyancis; truncatura antica foreis 2 centralibus sulciformibus longitudinalibus politis; pronoti regio postica convexo-depressa transversosubrectangula antice ad centrum Ieniter et obsolete cmarginata. Dorsuli suturae medianae crenatae continuae. Metanoti area postico-centralis subperpendicularis, basi planiuscula coriacea punctata, foveis 5 maximis in limbo excavata; anguli postico-laterales validi, lati compressi nomnihil curri obtuse triangulares coriacei punctis crassis adspersae. Mesopleurac crassissime punctatae, utrinque ad apicem sterno proprius arcuato-emarginatae et hinc utrinque crasse at breviter bidentatae, fere totae virides; pictura viridis etiam in scutello et metanoto obvenit. Sternum et pedes viridi-cyanei aut cyaneo-virides nitidi cinereo-pilosi punctulati; illud coriaceum; tarsorum articuli terminales fusci. Alae albo-hyalinae venis firmibus piceis, cellula radiali elongata lanceolata apice modice aperta. Abdomen robustum latitudine metanoti, capite thoraceque simul sumtis longius, obtuse subreclangulum, dorso 
modice convexum punctatissimum cyaneum marginibus segmentorum apicalibus plus minus rirescentibus; segmenta I:mum et 2:dum utrinque angulo laterali-apicali valido spinoideo munita; segmenti 3:tii series anteapicalis foveolis numerosis majusculis rotundis separatis, dentes apicales robusti acuti triangulares emarginaturis arcuatis, centrali breviori quam utraque laterali; margo lateralis proprius segmenti 3:tii obliquus subrectus nec arcuatus. Venter valde fornicatus viridi-cyaneus aut rice versa, punctatus et punctulatus, pubescens, pilosellus, nitidus.

112. Chrysis Nisseri Dlbm. ð゙.

Mediocris elongala subrobusta sublinearis $2 \frac{3}{4}$ lin. decimal. long. capile thoraceque confertim subcrasse punctalis, abdomine modice punctato viridi-cyanea nitida, scutello depresso-plano, posts cut ello sub u ucron at o. abdominis dor so mutico nec carimulata. segmento 3:lio disco leniter subconcaviusculo-immerso, postice supra seriem modice convexiusculo-marginato, margine Inlerali elongato obliquo subrecto, alis sordide ligalinis, cellula radiali subcompleta.

Chrysis Nisseri Dlbm. Dispos. 1845. 14:27.

IIabitat in Remedios Americae, D. Nisser: Mus. R. Acad. Scient. Stockholm., teste D. Boheman.

Observ. Chrysidi nilidulae ad habitum quodammodo similis.

Corpus cinereo-pubescens. Vertex, thoracis dorsum et latera abdominisque segmentum dorsale $1:$ mum modice punctatissima. Caput transversum triangulariter suborbi. culatum, lateraliter utrinque infra oculos marginatum. 
Vertex amplus convexo-depressus. Oculi mediocres ovatorotundati. Cavitas facialis mediocris subquadrata nitida virescens punctulato-coriacea, ad latera albo-sericeo-pubescens, superne per marginem verticinum transversum biarcuatum limitata. Antennac breres robustae fuscae, basi viridi-cyanescentes. Clypeus brevis transversus punctulatus viridis nitens, medio convexiusculus ad centrum marginis apicalis subemarginatus. Mandibulae normales. Palpi fusci breves tenues pubescentes. Pagina capitis inferior nitida riridis sublaevis, utrinque postice angulo valido obtuse spinoideo armata. Postscutelli margo posticus ad centrum obtusangulo prominulus et hinc quasi mucronatus. Metanoti anguli postico-laterales mediocres acute triangulares. Pectus viride nitens; mesopleurae crassissime punctato subreticulatae; sternum et pedes punctulati cyaneo-virides cinereo-pubescentes, tarsis apice fuscis. Alarum venae firmae piceae; cellula radialis angusta lanceolato-acuminata, apice angustissime aperta et hinc subcompleta. Abdomen ovale longitudine capitis thoracisque simul sumtorum, dorso modice convexum et modice punctatum segmento 1 :mo crasse punctatissimo; segmenta 1 :mum et 2:dun utrinque angulo laterali-apicali spinoideo minuto munita; segmenti $3:$ tii series anteapicalis foveolis numerosis rotundis minutis separatis, margo apicalis prominulus convexo-depressus punctulatus nitidus, dentes inaequales: externi breviores triangulares, centrales majores spinoidei nomnihil decurvi, emarginaturis arcuatis subaequalibus, centrali reliqquis forte paullulum latiori profundiori. Venter viridi-cyaneus punctulato-coriaceus pubescens. 
113. Chrysis indigatea Dufour? ð’ㅇ.

Mediocris elongata sublinearis $2 \frac{1}{2}$ lin. decimal. long. conferlim modice punclata cyanea aul coerulescens, scutello convexo-depresso postsculelloque convexiori inermibus, abdominis segmento dorsali 2:do lineola mediana subelevala, segmento 3:tio disco leniter concaviusculoimmerso, postice supra seriem modice convexiusculomarginato, margine (segmenti 3 :lii) laterali tolo flexuoso: basi convexo-arcualo, tum oblique arcualo-inflexo I. leniler emarginalo, alis hyalinis, cellula radiali completa aut subcompletr.

Chrysis indigotea Dufour Annal. Ent. 1840. 38:18.

Clerysis violacea Mus. Spinolae.

Habitat in Svecia, Grermania et forsitan quoque in Europa meridionali, rarius; duo specimina in nostra collectione adsunt, unum e Germania, alterum in Ostrogothia Sveciae a me olim deprehensum. Exemplar unicum etiam in Germania lectum sub nomine „Chrysis violacea Panz." amice communicavit D. Spinola. De sua Chryside indigotea ita scripsit D. Dufour 1. c.: „,cocrulea viridi-nitens abdomine apice quadridentato, antennis tarsisque nigris. Long. 3 lin. Nidificat in ramis exsiccatis Rubi fruticosi, parasita Odyneri rubicolae. D'un gros bleu, changeant parfois en vert, surtout aux flancs du corselet et sur les cotés des premiers segments de l'abdomen. Pattes et premier article des antennes vertes ou rarement bleus. Dernier segment de l'abdomen tronqué, arec quatre dents triangulaires aiguës". Quae pauca verba ad nostrum insectum, respectu scil. habito ad structuram et picturam corporis, omnino accommodari possunt. 
Famil. Clmysididce. Clerysis: Phalanx VT. 209

Observ. Corpus cano - et cinereo-pubescens et pilosellum, cyaneum aut cocruleum; margo occipitalis pone oculos, cavitas facialis et clypeus, pleurae, sternum, pedes et latera segmentorum abdominis dorsalium, interdum quoque margines horum segmentorum apicales e colore viridi plus minus nitida. Corpus omnino ut in Cleryside milidula constructum et punctatum, unde $\mathrm{C} / \mathrm{r}$. indigolen ut varietas Chrysidis mitidulae facile existimari posset; sed segmento abdominis 3:tio longe aliter constructo certissime dignoscitur. Cavilas facialis mediocris punctulatocoriacea, superne per carinulam transversam utrinque angulato-deflexum limitata. Clypeus transversus mediocris punctulatus, disco convexus utrinque concaviusculus, ad centrum marginis apicalis lenissime arcuato subemarginatus. Antennae mediocres robustae tarsique nigro-fusci, illae basi virescenti-cyaneae. Mandibulae basi parum rirescentes, medio nigro-apice rufo-piceac. Palpi fusci. Vertex, thoracis dorsum et latera, abdominisque segmentum dorsale 1:mum confertim modice punctata, seğmenta 2:dum et 3:tium punctulata. Metanoti anguli posticolaterales mediocres late triangulares. Abdominis segmenti 3:tii series ante-apicalis per interstitium minutum planiusculum interrupta, foreolis utrinque numerosis mediocribus subprofundis non bene separatis, hine inde confluentibus; margo apicalis nomihil prominulus, convexodepressus punclulatus, dentibus mediocribus triangularibus rectis, emarginaturis arcuatis invicem subacqualibus; margo lateralis obliquus flexuosus: parte basali convexoarcuatus, parte apicali leniter arcuato - emarginatus. V'enter confertissime punctulato - coriaceus pubescens nitidus cyaneus maculis quibusdam viridibus. Alae ad costam 
ccllulac radialis fumeo-sordidae, venis mediocribus brunneis.

Manipulus 2. Abdominis segmentum dorsale 3:tium - supra seriem ante-apicalem - margine ullo non incrassatum.

114. Chrysis chlorosoma Dlbm.

Mediocris oblongata angusta sublinearis $2 \frac{1}{4} \mathrm{lin}$. decimal. long. modice punctata viridis, dorsuli lobo centrali abdominisque segmentis dorsalibus basi cyaneis, segmenti 3:tii serie anterpicali interrupta foveolis utrinque distinctioribus rotundis, dentibus apicalibus mediocribus triangularibus, alis sordide hyalinis cellula radiali completa lanceolata.

Ifabilat in Java, Mus. D. Westermann. Tantummodo unicum exemplar vidi.

Observ. Corpus gracile cinereo-pubescens. Caput dorsum et latera thoracis abdominisque segmentum dorsale 1:mum confertim punctata subreticulata, cetera segmenta abdominis dorsalia modice punctata rel potius punctulata. Color corporis primarius laete riridis; macula verticis, truncatura pronoti antica, lobus centralis dorsuli et margines basales segmentorum abdominis dorsalium cyanescentes 1 . coerulescentes. Caput rotundatotriangulum pronoto paullulum latius; vertex amplus de- presso-convexus; oculi ovali; cavilas facialis mediocris punctulato-coriacea, superne per carinulam transrersam rectam limitata; antennae el mandibulae mediocres normaliter pictae; clypeus brevis inaequalis parce punctula- 
tus ad centrum marginis apicalis subtruncalus. Pronoti regio postica sulco mediano brevi. Alarum venae piceac. Tarsi apice fusci. Abdominis segmenti $3:$ tii regio antica Iransversaliter et leniter per totum discum concaviusculoimmersa, Venter viridis nigro-maculatus.

Turba 2. Abdominis segmentum dorsale 3:tium modice depresso-convexum, disco non concaviusculum.

Manipulus 1. Alae hyalinae aut sordide hyalinae.

A. Series ante-apicalis foreolis profundissimis.

115. Chrysis distinctissima Dlbm. $\sigma^{7}$.

Mediocris subrobusta elongata sublinearis $2 \frac{1}{2}-3 \frac{1}{2} l i m$. decimal. long. punctatissima cyanea aul viridi-ryanea, abdominis segmenti 3 : lii serie anteapicali profundissima per carinam medianam distincliorem interrupla foveolis maximis separatis linearibus, dentibus majoribus spinoideis, alarum cellula radiali incompleta.

Chrysis fasciata Spinol. Annal. Ent. 1840. 202:50.

Clerys. fusciata „var. ex Amer, merid.“ Hus. Havn. ubi Excmplar anno 1847 examinavi.

Clerysis intricans Mus. Spinolae.

Inabitat in America meridionali.

Observ. Corpus punctatissimum cinereo-pubescens. Capitis cavitas facialis longiuscula subtrapezina confertim punctulato-coriacca nitida virescens medio utrinque subtiliter transverse-strigosa, superne per carinulam subverticinam transversam subarcuatam limitata. Clypeus brevis nitidus viridis sparse punctulatus, medio convesus, utrin- 
que subconcaviusculo-depressus, ad centrum marginis apicalis truncatus. Antennac breves robustae et mandibulac mediocres, normaliter pictae. Mesopleurae apice utrinque tuberculato-tridentatae. Abdomen cyaneum; segmenta dorsalia margine basali nigro-riolacea; segmenta 1:mum et 2:dum margine apicali et 3:tium ante seriem, interilum virescentia; segmenta I et 2 angulo laterali-apicali spinoideo utrinque munita; segmenta 2:dum et $3:$ tium lincola mediana planiuscula 1. subconvexiuscula elevata, quae lineola in regione apicali segmenti 3 :tii formam carinulae distinctioris accipit. Segmentum 3:tium longiusculum subtrapezinum; serics anteapicalis: in parte dorsali profundissima, medio per carinulam intercedentem abrupta, foveolis utrinque $3-5$ maximis sublinearibus subpellucidis, - in parte laterali h. e. secundum marginem lateralem non profunda foveolis ibidem mediocribus rotundatis plus minus confluentibus; margo apicalis prominulus punctulatus subtenuis depressiusculus dentibus mediocribus spinoides: centralibus approximatis, lateralibus dislantibus, emarginatura centrali profundius arcuata modice lata, emarginaturis lateralibus non profundis at latis et obliquis; margo lateralis proprius longus obliquus subrectus. Venter nitidus pubescens punctulatus viridi-cyaneus nigro-maculatus. Alarum cellula radialis triangulariter lanceolata incompleta apice modice aperta.

116. Chrysis coeruleans Fabr. ơㅇ.

Mediocris subrobusla clongala sublinearis rive. 3 lin. decimal. long. conferlim modice punciala cosmlen aul vividis, abdominis segmenti 3 : lii serie anteapicali medio- 
Famil. Chrysididae. Clmysis: Phalanx VI. 213 cri per carinulam medianam brevissimam depressam interrupla, foveolis modice profundis numerosis plus minus confluentibus, alarum cellula radiali completa.

Chrysis coerulans Fabr. Piez. 172:20. secundum specimen typicum in Collectione Falriciana Mus. Universitat. Kiel. asservatum, anno 1847 a me examinalum.

"Habitat in Carolina" Fabr. l. c. Specimina 5 e Carolina meridionali prope Georgetown lecta benevole nobis transmisit amicus D. Zimmermann, qui hanc speciem ibidem frequenter observavit. E Cayenne, a D. Buquet lecta, sub nomine "Clrys. intricans" a D. Spinola communicata.

Observ. Corpus nitens cinereo pubescens l. viride 1. coeruleum I. viridi-cyaneum. Vertex, thoracis dorsum et pleurae abdominisque segmentum dorsale 1:mum confertim subreticulato-punctata. Caput robustum normale; Vertex amplus depresso-convexus; cavitas facialis longiuscula virescens confertim punctulato-coriacea, superne per carinulam transversam utroque apice arcuato-deflexam limitata. Clypeus brevis transversus punctulatus nitidus plerumque viridis, disco convexus et interdum aureo-resplendens, utrinque concaviusculus, ad centrum marginis apicalis truncatus. Antennae longiusculae, modice robustac, fuscae, basi l. virides et tunc interdum leviter aureopictae I. viridi-cyaneae. Labrum nigro piceum semilunatum subciliatum. Mandibulae basi virides, medio nigroet apice rufo-piceae. Abdominis dorsum modice convexum, margo basalis segmentorum 2 : di et 3 :tii nigro-aeneus; segrmentum 2:dum confertim modice punctatum, lineola mediana in aliis omnino obliterata, in aliis subelevata planiuscula et subpolita, in aliis subcarinulata; segmenII. 
tum 3:tium confertius punctulatum subcoriaceum disco plerumque viridi-cyaneum; series anteapicalis violacea; margo apicalis unediocris convexiusculus punctulatus: dentibus mediocribus, lateralibus triangularibus, centralibus spinoideis paullulum decurris, emarginaturis arcuatis, lateralibus latioribus el minus profundis, quum centrali. Pedes aliis virides et tunc interdum basi aureo-splendentes, aliis viridi-cyanei; omnibus sunt calcaria brunneotestacea et tarsorum apices fusci. Alae subhyalinae, ad costam (praecipue cellulae radialis) fumeo-sordidae, renis firmibus piceis, cellula radiali triangulariter lanceolata completa.

B. Series ante-apicalis foveolis mediocribus.

a. Corpus mediocre.

1. Abdominis segmenti dorsalis 3 :tii dentes apicales robusti, majores aut mediocres.

117. Chrysis nitidula Fabr. ơ오.

Mediocris aut submagna elongata robusla $3-4$ lin. decimal. long. confertim punclata nitida viridi-cyanea, pectore, lateribus abdominis, marginibus segmenlorum apicalibus pedibusque plus minus viride micaulibus, capitis cavitate faciali superne per carinulam transversam limitata, occipite utrinque unidenticulato, segmenli (abdominis ) 3:lii margine Ialerali 1. flexuoso 1. angulalo l. oblique subrecto, alarum cellulis discoidali et radiali completis.

var. a. $\overparen{O}$. Abdominis segmenti 3 :tii margo lateralis I. flexuosus 1. angulatus. 
Chrysis nitidula Fabr. E. S. 2. 243:21, - Piez. 176:31. - Dlbm. Mon. Chr. 11:7, E. H. 30:9, Dispos. 13:24.

Clurysis purpurata Fabr. E. S. 2. 210:9

Chrysis Iris Christ. ". et Piez. 172:12. $\}$ secundum Klug, in Musco

Chrysis cocrulans Meg. Latr. et Spin. Museis Viena. et Spinolae. Chrysis 4-dentata Meg. Mus. Vienn. teste D. Kollàr.

var. b. ㅇ. Abdominis segmenti 3:tii margo lateralis nec flexnosus nec' angulatus, sed oblique subrectus. Mus: D. Drewsen.

Habitat per plurimas Europae regiones, nec non in Amcrica septentrionali, passim. Intra Europam borealem usque in Fenniam meridionalem adscendit, teste D. William Nylander in litteris. In Sveciae mediae et meridionalis sepibus, truncis arborum et parietibus ligneis mense Julio a nobis observata. Ex America boreali a DD. Drewsen, Spinola et Westermann communicata.

Observ. Corpus robustum elongatum subcylindricum cano-cinereoque pubescens et pilosum. Caput, thorax abdominisque segmentum dorsale $1:$ mum confertim et subcrasse punctata subreticulata; segmentum 2 :dum confertim modice punctatum: punctis nediocribus et punctulis obsertum, segm. 3:tium punctulatum. Caput rotundato-triangulare cyaneum aut violascens ad singulum quodque stemma macula parva virescente. Caritas facialis viridis confertius punctulato-coriacea, lata subquadrata superne transverse-marginata, margine aliis distinctissimo aliis obsoleto, paullulum arcuato. Antennac robustac longiusculae nigro-fuscae, basi l. cyaneae 1 . viridi-cyaneae. Clypeus mediocris transversus, disco et lateribus normalis, ad centrum marginis apicalis truncatus, superficie nitidus punctulatus, 1. Lotus cyaneus 1. viridis et disco interdum aut violascens aut purpurascens. Labrum semi- 


\section{Famil. Chrysididae. Clirysis: Phalan. VI.}

lunatum nigro-piceum convexum ciliatum. Mandibulac mediocres robustae nitidae nigro-piceae, apice rufescentes, basi 1. virescentes 1. cyanescentes l. purpurascentes; capitis pagina inferior superne utrinque, prosternum rersus, angulo spinoideo armata, quae tamen armatura capite ex. gr. thoraci arcte adpresso non animadvertitur. Thorax dorso saturate cyaneus; pronoti regio postica transverse rectangula, I. concolor I. marginibus antico posticoque aut viridibus aut viridi-coerulescentibus, sulco mediano elliptico plus minus distincto. Mesonotum et metanotum 1. unicolore saturate cyanea l. plerisque eo modo viridiirrorata ut puncta cyanea et interstitia nonnihil virescenlia; haec pictura etiam in pleuris saepe occurrit, quibusdam aut individuis totum pectus viride; dorsuli suturae medianae rectae parallelae crenulatae; interdum quoque hae suturae et pronoti sulcus medianus purpurascunt, hinc itaque Fabricii verba (de Chr. purpurata 1. c.) ,thorax viridis lineolis in medio obscure purpurascentibus". Metanoti anguli postico-laterales mediocres triangulares coriaceae et punctatac. Pedes viridi-cyanei tarsis apice fuscis. Alae sordide hyalinae venis firmibus piceis aut fusco-brunneis, cellula radialis lanceolato-acuminata completa aut subcompleta. Abdominis dorsum modice convexum saturate cyaneum aut subviolascens; segmenta 1:mum et 2:dum lateribus margineque apicali l. laete coerulescentia 1. virescentia; segmentum 2:dum carinula mediana aliis obsoleta aut obsoletissima, aliis distincta aut distinclissima; segmenti $3:$ tii series ante-apicalis irregularis, medio brevissime interrupta, utrinque foveolis numerosis majusculis: harum nonnullis separatis rotundatis, aliis confluentibus et subtransversis; margo lateralis 
Famil. Chrysididae. Clorysis: Phalanx VI. 217

prominulus convexo-depressus punctulatus, dentibus robustis ad longitudinem mediocribus triangularibus subaequalibus, emarginaturis modice arcuatis, centrali plerumque lationi.

\section{Chrysis soluta Dlbm. ơ}

Angusta linearis vix 2 lin. decimal. long. confertim punctato et punctulato subcoriacea parum nitida, violacea, verticis lineola transversa, marginibus segmentorum abdominis 1:mi el 2: di apicalibus, femoribus tibiisque uno alterove latere virescentibus, pedibus ceterum ad colorem primarium brumeis, capitis cavitate faciali superne non marginala, occipite inermi, segmenti 3:tii margine laterali leniler flexuoso, alarum cellulis discoidali et radiali incompletis.

Chrysis nitidula Dlbm. Mon. Chrys. 1829. 11:7. var. 6.

IIfabitat in Svecia rarissime; exemplar unicum, a D. Professore Zettersledt detectum, in nostra collectione adest.

Observ. Chrysidis nilidulae maxime affinis et similis; at corpore multo breviore et angustiore, violaceo nec nisi locis in diagnosi nuperrime memoratis coeruleo-virescente, capitis cavitate faciali superne non marginata, occipite inermi, alarum cellulis discoidali et radiali incompletis, capite thoraceque modice punctato 1 . fere punctulato-coriaceis facillime distinguitur.

Vertex pone stemmata transversaliter virescens; etiam in pronoti dorsulo, metanoto el pectore pictura viridis obvenit, sed color harum partium pririus violaceus. $\mathrm{Ca}$ vilas facialis punctulato-coriacea nitida, superne cyanca 
apice virescens; clypeus mediocris transversus nitidus viridis punctis paucis adspersus, disco conrexiusculus, ad centrum marginis apicalis lenissime arcuato-emarginatus. Antennae ut in Cleryside nitidula. Labrum semilunatum mediocre subpulvinatum pubescens testaceo-brunneum. Mandibulae parte basali virides, parte apicali testaceobrunneae. Thorax cylindricus, metanoti anguli posticolaterales parvi coriacei punctali subacute triangulares. Alae hyalinae venis brunneis, renula transverso-medialis tantum dimidiata h. e. in medio inter renas radialem firmiorem et cubitalem subtilem inchoatam desinit, hinc cellula discoidalis facta est incompleta; Cellula radialis lanceolato-acuminata, apice modice aperta. Abdomen lineare, thorace paullulum longius at ejusdem latitudinis, dorso convexum, segmenta 1 :mum et 2: dum confertim punclulata, marginibus apicalibus nitidis coeruleo-virescentibus, illud (segm. 1 :mum) ceterum violaceum, hoc (segm. 2:dum) nigro-aeneum et nomnibil purpurascens, lineola mediana subtiliter carinulata; segmentum 3 :tium confertius subtiliter punctulatum, basi nigro-aeneum, apice nitidum cyaneum, series antenpicalis, dentes et margo lateralis ut in Chrys. nitidulae var. $\boldsymbol{\alpha}$. Venter viridi-cyaneus.

119. Chrysis Reichei Spinol. ㅇ․

Mediocris elongala robusta 3 lin. decimal. long. punctatissima punctis modicis, vix nilida, saturate cyanea abdominis dorso violaceo-purpureo, capilis cavilate faciali superne marginata, occipile inermi, abdominis segmenti 3:tii margine laterali recto, alarum cellulis discoidali completa, radiali subcompleta. 
F'amil. Clryssididae. C'mrysis: Plualum.x VI. 219

Clurysis Reichei Mus. Spin.

Pyria Reichei Spin. Annal. Enı. 1838. 448:3.

Illabitul in Africa meridionali ad Caput bonae spei, Mus. D. Spinolae:

Observ. Similis et affinis Chr. mitidulae, at pictura et punctura abdominis certe dignoscitur. Corpus robustum cinereo-fusco pubescens et pilosum. Caput et thorax confertissime punctata subreticulata parum nitida subopaca punctis mediocribus, pure cyanea; guttula ad singulum quodque stemma, orbitae oculorum, pronoti margo posticus et centrum dorsuli leviter virescentia; cavitas facialis, basis antennarum, clypeus, pectus et pedes coerulescenti-viridia aut viridi-cyanescentia. Caput robustum; vertex amplus transverso-rectangularis convexodepressus. Cavilas facialis quadrata confertim punctulatocoriacea, superne transrerse marginata, margine leniter bis arcuato; clypeus transversus brevis subsemilunatus crebre modice punctalus, disco gibbo-convexus, ad centrum marginis apicalis subtruncatus 1. potius lenissime arcuato-emarginalus. Antennae mediocres - 1. respectu wagnitudinis corporis subbreves, apice fuscae, scapus cyaneo-virescens, articuli 2 :dus et $3:$ tius cyanei. Labrum mediocre scmilunatum conycxum ciliatum mandibulaeque nigro - picea aenescentia, hae macula basali viridi-cyanea. Palpi fusci. Thorax crassus cylindricus. Pronotum maculis 2 nigro-acneis, una polita et immersa in centro partis anticac, altera opaca in centro partis posticae; scutelltum convexo-depressum et postscutellum convexum basi nomnihil nigro-aenescentia, sulurae dorsuli concolores crenulatae. Metanoti anguli postico-laterales magni robusti lati triangulares coriacei sparse crasse punctati. 
Pedes viridi-cyanei tarsis fuscis. Alae sordide hyalinae venis firmibus piceis; cellula discoidalis completa; cellula radialis elongata triangulariter lanceulata, apice anguste aperta. Abdomen subellipticum, basi truncatum, thorace paullulum angustius, longitudine capitis thoracisque simul sumtorum; dorso modice convexum, creberrime modice punctatum et punctulatum, vix nitidum subopacum pulchre violaceo-purpurascens, lineola mediana planiuscula subelevata nitida fusco-viridiaenea, in segmento 2: do continua, in $1:$ mo autem et $3:$ tio tantum apicem versus distincta; segmenta: 2:dum marginibus basali et apicali, 3:tium margine basali quoque aenescentia; segmenti 3:tii series anteapicalis medio breviter interrupta, utrinque foveolis numerosis majusculis profundis rotundatis scparatis nec confluentibus; dentes apicales mediocres triangulares, emarginaturis modice arcuatis invicem subacqualibus; margo lateralis (segm. $3:$ tii) obliquus subrectus et vix nisi obsoletissime flexuosus.

120. Chrysis Ehrenbergi Dlbm. 오.

Mediocris ovalis robusta $2 \frac{1}{2}$ lin. decimal. long. sparse modice punctata subtus cynnea supra virescens parumper aurato-nitida, tarsis lestaceis capite magno cubico, cavilate faciali superne non marginata, occipile inermi, abdomine ovato, segmenti 3:tii margine laterali paullulum flexuoso, alarum cellulis discoidali completa: radiali incompleta, venis tenuibus testaceis.

Platycelia Ehrentergi Dlbm. Dispos. 1845. 8:1.

Habitat in Aegypto, a D. IIedenborg detecta. Unicum specimen vidi, e Museo Reg. Acad. Scient. Stochholu. a D. Boheman communicatum. 
Famil. Chrysididae. Chrysis: Phalanx VI. 221

Observ. Species eximia per structuran capitis praccipue singularis. Corpus ovale nitidum cano-pubescens et pilosellum punctis sat mediocribus aut subparvis adspersum. Caput magnum rotundato-cubicum viride; vertex latus pronoto latior transverso-rectangularis, depressoconvexiusculus pone stemmata nomnihil aureo-nitens, margine occipitali leniter arcuato-emarginatus; tempora recta concaviuscula polita non punctata margine postico emarginata; oculi subparvi ovato-rotundati; caritas facialis quadrata mediocris confertim punctulata medio leniter et abrupte canaliculata. Antennae breves non crassae, fitscae scapo viridi; genae brevissimae, ad apicem temporun angulato-subprotuberantes; clypeus brevis transversus depressus parce punctulatus, ad centrum unarginis apicalis lenissime arcuato-emarginatus. Mandibulae elongalae acuminatae sublineares parumper arcuatae, margine infero-externo a basi usque ad $\frac{3}{4}$ totius longitudinis leniter arcuato-emarginatae, supra 1 . extus longitudinaliter sulcatae, totae piceae, ante apicem dilutiores, macula basali viridi; labrum parvum semilunatum. Thorax rectangularis capite angustior, dorso parum convexus; pronolum breve transverso-rectangulum sulculo mediano brevi, margine antico humili cyanescens, reliqua parte viridis parum aureo-nitens, lateribus concavo-emarginatis; dorsulum breve transverso-ovale et scutellum mediocre viridia parumper aureo-micantia; metanoti anguli posticolaterales subparvi triangulares. Pedes virides calcaribus tarsisque testaceis. Alae albo-hyalinae venis praeter costalem robustan tenuibus brunneo-testaceis, cellula radiali lanceolata apice aperta. Abdomen subbreve, thoracis longitudine, ovatum, basi transversum normaliter at leniter 
impressum, dorso modice convexum crebre punclulatum Jaete viride nomnihil aureo-splendens; segmentum dorsale 3:tium subsemilunatum, totun modice convexum nec in regione seriei quidem immersum, series anteapicalis continua: foveolis numerosis perparvis rotundis cum reliqua segmenti punctura facile confundendis, margo apicalis brevis subdepressus viridi-coerulescens: dentibus apicalibus mediocribus aut subparvis spinoideis, emarginaturis arcuatis, lateralibus levibus (nec profundis) subangustis (nec latis), centrali profunda et duplo nisi triplo latiore quam singula quaque lateralium; hinc utrinque dentes duo approximati, nec ulli mere centrales, existunt; renter cyaneus nigro- et viridi-maculatus, totus depresso-planus nec ullo modo fornicatus.

121. Chrysis megacephala Bohem. ㅇ․

Bradiocris ovalis robusta $2 \frac{1}{2}$ lin. decimal. long. sparse modice punclata, sublus cyanea, supra virescens a eneoauralo-nilida tarsis lestaceis, capile maximo cubico, cavitate fraciali superne non marginaln, occipile inermi, ablomime oxalo, segmenti 3:tii margine laterali flexuso, alarum cellutis discoidali completr, radiali incompleta, venis firmibus piceis.

Chrysis megacephula Boheman in litteris ad D. Westermann.

Habitat in Aegypto, Mus. D. Westermann.

Observ. Nimis affinis et similis Chrysili Elurenbergi atque ut hujus varielas primo intuitu facile existimanda. Attamen Chrysis megacephala sequentibus notis praccipue characteristicis certe dignoscitur, scil. vertice, pronoto, mesonoto abdominisque dorso conspicue acneo- 
aurato-nitidis; capite majore multoque crassiore; fronte latiore et oculis igitur magis distantibus; clypeo majore; thorace crassiore; femoribus et tibiis viridi-cyaneis; alis sordide hyalinis venis firmibus piceis; abdominis dorso crebrius punctato, segmenti 2 :di margine basali nigroaeneo; segmenti 3 :tii margo-apicalis prominulus et reliqua parte segmenti distinctius humilior, serie anteapicali fere obliterata 1 . a reliquis segmenti punctis vix discernenda, dentibus apicalibus majoribus. Cieterum inter has species non ulterior adest differentia.

Not. Cloysides Eltrenbergi et megacephala forsitan proprium Genus efficere possint, cui jam anno 1845 nomen "Platycelia" proposuimus. Quum vero notae Generis ejusmodi caracteristicae e structura trophorum ut opinamur praecipue sumi debent, nos las partes post mortem, ut constat, retractas et perfragiles (in exemplaribus tantum descriptionis caussa et bona fide traditis) dissecare non ausi sumus, praesertim quod hoc vix nisi detrimento animalculi fieri posset. Propter hanc rationem itaque necesse fuit, ut species memoratas in Genere Chrysidum saltem pro tempore collocaverimus.

2. Abdominis segmenti dorsalis 3 :lii dentes apicales parvi.

122. Chrysis lacta Draege. ${ }^{7}+$.

IITediocris $2 \frac{1}{3}-2 \frac{1}{2}$ lin. decimal. long. subcrasse punclata lacte viridis larsis fuscis, abdomine coeruleo- $l$. cyaneo-picto, segmenti 3:tii serie anteapicali immersa interrupta foveolis numerosis inaequalibus, dentibus bre- 
vibus robustis triangularibus, emarginaluris modice arcuntis subaequalibus.

Clurysis lneta Dracige. Mus. Spinolae.

Clurysis malachitica Mus. Berolin. secund. D. Drewsen in litferis.

IIabilat in promontorio bonae spei, D. Draege, Mlus. DD. Drewsen et Spinoltr; in Guinea, D. Weslermam.

Observ. ㅇ․ Corpus cinereo-pilosellum. Caput et thorax crasse aut subcrasse punctato-subreticulata. Caput laete viride, verticis macula centralis aenea. Antennae, clypeus et mandibulae normales; cavitas facialis subrectangularis subprofunda confertim punctulato-coriacea, superne transverse marginata: margine leniter et inaequaliter arcuato. Dorsuli area media disco nomnihil violacco-irrorata. Abdominis segment. dorsal. 1:mum laete viride subcrasse punctatum, 2:dum subcoerulescenti-riride modice punctatum, 3 :tium confertim punctulato-coriaceum cyaneum apice virens, margo sive regio apicalis regione basali nomnihil humilior, hinc series apicalis quasi immersa, foveolis numerosis inaequalibus scil. nonnullis mediocribus et nonnullis parvis, margo lateralis pone dentem exteriorem leniter arcuatus. Venter viridi-cyaneus aut vice versa, pedes concolores tarsis fuscis. Alae aut hyalinae aut sordide hyalinae: cellula radiali lanceolata aliis completa, aliis subcompleta, aliis distincte aperta. IMas aut feminae simillimus, aut dignoscitur: corpore paullulum breviore, abdominis dorso interdum toto subcrasse punctato, segmentis 2:do el 3:tio dorsalium totis cocruleis nec nisi leviter viridi-irroratis. 
123. Chrysis palliditarsis Spin.

Mediocris 2! lin. decimal. long. crapite thoraceque irregulariler modice punctatis subrugulosis coeruleo-viridis tarsis albo-testaceis, abdominis segmenti 3:lii serie anteapicali lenissime immersa continua foveolis circ. 10 rotundatis majusculis, dentibus brevissimis late triangularibus, emarginaluris leniter arcuatis: laleralibus latioribus.

Chrysis palliditarsis Spinola. Annal. Entomol. 1838. 449:6.

IIabilat in Aegypto, a D. MI. Fischer detecta; Mus. D. Spinolne.

Observ. Affinis et similis Clrysidi laelae. Corpus clongatum lacte viride, totum dorso Iateribusque confertim et modice at irregulariter h. e. subrugulo-punctatum. Pronoti margo anticus, dorsuli area centralis, tegulae, abdominisque segmenta 2 :dum et 3 :tium basi, coerulea. Antennarum scapus et pedicellus virides, flagellum ferrugineum aut lacte brumneum. Cavitas facialis normalis, superne marginata: margine transverso, utrinque ramulo deflexo longitudinali brevissimo. Alae albo-hyalinae venis brunneis, cellula radiali triangulariter lanceolata, apice late aperta. Abdominis segnenti 3:tii series ante-apicalis cyanea; margo apicalis mediocris prominulus convexodepressus punctulatus, summus tenuissimus testaceus; margo lateralis obliquus, leniter flexuosus.

124. Chrysis orientalis Dlbm. ㅇ․

Mediocris $2 \frac{1}{2}$ lin. decimal. long. conferlim modice punctata viridissima dorsulo abdomineque cyaneo-pictis, tarsis fuscis, abdominis segmento 3: lio tolo modice con- 
vexo, serie ante-apicali subobliterata: foveolis taniummodo perpaucis, dentibus mediocribus trinngularibus, emarginaturis modice arcuatis subaequalibus.

„Chrysis nov. sp. Ex Ind. orient." Mus. Havn.

Habitat in India orientali; specimen in Museo Havniensi anno 1847 vidi.

Observ. Habitus omnino proxime praecelentium. Corpus viridissimum dorsulo abdominisque segmentis 2 : do et 3:tio basi cyaneis. Alae hyalinae, disco sordidac. Venter viridis nigro-maculatus.

125. Chrysis diversa Dlbm. 오.

Mediocris $2 \frac{1}{2}$ lin. decimal. long. conferlim modice punctata cyanea viridi-picta tarsis fuscis, abdominis segmento 3:tio tolo modice convero, serie ante-rpicali distincta continu: foveolis numerosis mediocribus rotundatis, dentibus brevibus triangularibus, emarginaturis modice arcuatis exterioribus aequalibus.

Habilat in Aegypto, a D. Hedenborg detecta. Specimina duo e Museo R. Acad. Scieut. Stockholm. communicavit D. Boheman.

Observ. Simillima Chrysidi orientali, sed colore corporis magis cyaneo et praecipue segmenti 3:tii serie anteapicali conspicua certe distinguitur. Corpus lineare, breviter cano- et fusco-pubescens, cyaneum aut coerulescens; vertex, pronoti regio postica, dorsuli areae laterales et abdominis segmentum 3 :tium supra seriem plus minus viridia aut viridi-irrorata; scutellum, pectus et margines apicales interdum quoque laterales segmentorum 1:mi et 2 :di lacte viridia. Caput mediocre rotundato- 
subcubicum; rertex convexus; cavitas facialis rectangularis planiuscula confertim punctulato-coriacea, medio subtiliter canaliculata, superne leviter transverse marginata, marginulo bis arcuato. Antennae breves subrobustae fuscae scapo viridi-cyaneo. Clypeus mediocris transversus subtrapezinus punctulatus viridis nitidus marginibus: latcralibus testaceis, apicali piceo, disco lenissime et subtilissime carinulatus, utrinque leviter impressus, ad centrum marginis subtruncatus 1 . potius late et brevissime triangulariter cmarginatus. Thorax subcylindricus, dorso depresso-convexus; metanoti anguli postico-laterales parvi acute triangulares. Abdomen obtuse subrectangulum thorace paullulum longior at ejusdem latitudine, dorso modice convexum et modice confertim punctatum; segmenti $3:$ tii dentes apicales in una eademque linea transversa positi, omnes breves triangulares, at exteriores robustiores, centrales breviores obtusiusculi, emarginatura centralis paullo minus profunda paulloque minus lata quam singula quaque exteriorum; margo lateralis obliquus lenissime et obsoletissine flexuosus I. subrectus. Pedes cyaneo-virides aut viridi-cyanei tarsis fuscis; calcaria, margines apicales tibiarum articulorumque tarsorum brunneotestacea. Alae hyalinae venis mediocribus piceis, cellula radiali triangulariter lanceola apice aperta.

b) Corpus minutum gracile, totum confertim punctulatum subopacum viridi-cyaneum.

126. Chrysis delicatula Dlbm.

Parva gracilis linearis $1 \frac{1}{2}$ lin. decimal. long. conferlissime subreliculalo-punclata viridi-cyanea parumper 


\section{8}

Famil. Clrysididae. Chrysis: Phalanx VI.

nilida tarsis brumneis, abdominis segmentis $2:$ do et $3:$ lio margine basali nigro-neneis, segmento 3 :tio disco utrinque subdepresso supra seriem ante-apicalem conveviusculo, serie intervupta foveolis numerosis submediocribus rotundalis subpellucidis, dentibus apicalibus parvis triangularibus, emarginaluris: centrali modice arcuala, exlernis latis obliquis subtransversis l. lenissime arcualis.

Habilat in Caffria, a D. J. Wallberg detecta; specimen unicum e Museo R. Acad. Scient. Stockholm communicavit D. Boheman.

Observ. Quoad habitum Chrysidi Bohemanni similis. Corpus angustum lineare parumper cinereo- et canopubescens, pulchre laete cyaneum aut subcoeruleum; orbitae oculorum, cavitas facialis, clypeus, pronotum, scutellum, pectus, pedes, abdominisque segmenta $1:$ mum et 2:dum margine apicali et $3:$ tium ante seriem, a colore virescente plus minus micant. Caput rotundato-subcubicum sublongiusculum, regione supera verticem valde convexum, oculos et partem occipitis superiorem comprehendente subglobosum, regione infera inter verticem, oculos et mandibulas sita subquadratum; cavitas facialis planiuscula normaliter sculpta, medio subtiliter canaliculata, superne non marginata. Antennae mediocres fuscae, basi viridi-cyaneae. Clypeus subbrevis transversus punctulatus, disco parum convexus, ad centrum marginis apicalis leviter arcuato-emarginatus. Thorax cylindricus dorso depresso-convexus; in pronoti regione antica et humili adest area centralis polita nigro-aenea utrinque foveolata; regio pronoti postica et maxima transverso-rectangula antice truncata postice leviter arcuato-emarginata. Dorsuli area media violaceo-purpurascens. Postscutellum 
Famil. Chrysididae. Chrysis: Plealanx VI. 229

gibbo-convexum. Metanoti anguli postico-laterales minuti acute triangulares. Abdomen elongatum capite thoraceque simul sumtis fere longius, angustum lineare rectangulum, dorso modice convexum; segmenti 3 :tii regio maxima et basalis semicircularis regione apicali paullulum altior, disco utrinque paullum depressa, supra seriem lenissime convexo-emersa; margo (s. regio) apicalis (segmenti $3:$ tii) prominulus subdepressus subtilissime punctulatocoriaceus: emarginatura centralis mediocris et modice arcuata, emarginaturac exteriores ralde obliquae latae levissimie arcuatae et usque ad angulum laterali-apicalem ductae, hinc dentes centrales nonnihil approximati, dentes autem externi valde distantes remoti 1 . quasi retracti et ex ipsis angulis laterali-apicalibus efficiuntur. Alae hyalinae venis tenuibus brunneo-testaceis, cellula radiali lanceolata subcompleta scil. apice angustissime aperta.

\section{Manipulus 2. Alae violascentes.}

127. Chrysis amethystina Fabr. $\delta 7$.

Submagna robusta circ. 3 lin. decimal. long. modice confertim punctata viridis ant viridi-cyanea nitida alis violascentibus, inferdum basi apiceque albo-hyalinis, abdominis segmenti 3:tii serie ante-apicali interrupta foveolis magnis mumerosis inaequalibus, dentibus brevibus spinoideis, emarginaturis inaequalibus: externis latioribus, fronte inter oculos subangista, mesopleuris utrinque fortiter bi-mucronatis.

Chrysis amethystina Fabr. E. S. 2. 243:22. et Piez. 176:32. secundum specimen in Collectione Fabriciana Mus. Universit. Kil. asservatum, anno 1847 a me exaninatum.

Clrysis Westermanni Mus. Spinolne.

II. 
"Irabitat in nova Hollandia" Fabr. 1. c.; in India orientali frequenter, DD. Spinola et Westuood; e Bengalia et Java a D. Westermann communicata.

Observ. Chrysidi lynceae Fabr. ad habitum similis, at postscutello mutico et abdominis segmento 3:tio tantum 4-dentato mox dignoscitur.

Corpus sub-stilbiforme robustum elongatum sublineare fusco et cano pilosellum et pubescens. Caput subparvum pendulum rotundato-subquadratum, thoraci arcte applicatum, modice punctatum nitidum viride rarius cyanescens; vertex et sat convexus inter oculos angustius quan in plurimis speciebus, antice insignis area semicirculari stemma anticum excipiente et per carinulam arcuataın determinata f haec carinula cohaeret cum cavilatis facialis margine supero transverso utrinque ramulum longitudinalem oblique demittente; cavitas facialis elongata subrectangula, ad verticem tamen angustior quam ad mandibulas, confertim punctulato-coriacea, supra medium forea centrali, superne transverse-marginata: margine inaequali leniter et irregulariter arcuato, ex utroque hujus apice descendit ramulus obliquus longitudinalis secundum latera cavitatis, ubi tandem evanescit. Antennae robustae, 오 longitudine mediocres, $\delta$ breves crassae; scapus nec non articuli 2 : do et 3 :tio 1 . virides 1 . viridi-cyanei rarius toti cyanescentes, flagellum fuscum. Clypeus brevis transversus sparse punctulatus, disco modice convexus utrinque depressus, ad centrum marginis apicalis late et leniter arcuato-emarginatus. Mandibulae nigro-piceae, macula basali viridi et noununquam aeneo-aurato-nitida. Occiput utrinque pone cavitatem angulo spinoideo munitum. Tho- 


\section{Famil. Chrysididae. Chrysis: Phalanx VI. 231}

rax ovali-cylindricus confertim crasse et subreticulatopunctatus dorso sat convexus, antice truncatus et paullulum angustius quam postice, dorsulum subgibbum suturis medianis basi arcuatis, scutellum et postscutellum convexiora; metanotum sub postscutello foreato-sculptum, unde etiam Fabricius 1. c. „scutellum prominulum concavum " habet; metanoti anguli postico-laterales magni late triangulares; mesopleurae praecipue crasse sculptae, harum uterque lobus apice emarginatus et hinc fortiter crasse obtuseque bidentatus 1. quasi bi-mucronatus. Quoad picturam est thorax: aliis totus viridissimus unde etiam a Fabricio "thorax viridis" describitur, - aliis viridis tegulis, dorsuli area media ex parte et metanoto apice cyaneus, - aliis cyaneus viridi-irroratus, - aliis undique pure cyaneus. Pedes viridi-cyanei aut cyaneo-virides tarsis fuscis. Alae anticae saturate violaceae at nihilominus pellucidae, ima basis et summus margo apicalis plerumque albo-hyalini, quae hyalinitas basalis et apicalis rarissime ita ampliatur ut tantumınodo discus alae restet violaceus; venae omnes firmae piceae; alae posticae sordidae, in recentissimis individuis etiam nitorem praebent violascentem quo autem individua detrita omnino carent, margo harum alarum posticus semper late hyalinus; rarissime obveniunt alae posticae totae subhyalinae. Abdomen robustum elongatum capite thoraceque simul sumtis $\frac{1}{4}$ nisi $\frac{1}{3}$ longius subrectangulum, dorso valde convexum crebre modice punctatum, aut pure cyaneum lateribus obsolete virescens, aut coeruleum: segmentis 1 :mo utrinque macula subaurato-viridi, segm. 2: do lateribus et margine apicali ample laete viridi-nitido, segm. cyaneo et tantum disco plus minus virescente; hoc segmentum tra- 
pezinum et in $\sigma^{\pi}$ brevius quam in $\&$, regio antica $s$. basalis semicircularis aut tota depresso-convexa aut disco leviter subimmersa at apice supra seriem convexiusculoterminata, area postica s. (= margo) apicalis humilis depressa prominula; series ante-apicalis per carinulam plus minus distinctam interrupta utrinque foveolis magnis profundis inaequalibus scil. aliis (praecipue discoidalibus) longitudinaliter sulciformibus, aliis (praecipue lateralibus) orbiculatis; dentes apicales breves robusti spinoidei, exteriores valde remoti ipsum angulum laterali-apicalem efficientes; cmarginatura centralis mediocris arcuata, singula quaque exteriormm duplo latior obliqua subtransversa lenissime arcuata 1. potius subrectangularis; margo lateralis proprius oblique rectus. Venter fortiter fornicatus viridi-cyaneus, interdum nigro-maculatus.

\section{Chrysis Westermanni Dlbm. ㅇ.}

Submagna robusta $3 \frac{1}{4}$ lin. decimal. long. viridis nilidn abdominis segmento 3:tio limbo cyaneo, alis violascenlibus, (abdominis) segmenti 3 :tii serie ante-apicali interrupta foveolis mediocribus mumerosis aequalibus rotundatis, dentibus brevibus triangularibus, emarginaturis modice arcualis subaequalibus, fronte inter oculos nonnihil latiore, mesopleuris utrinque apice et postice cremulato-denticulatis.

Habitat in Guinea, D. Weslermann; qui specimen unicum communicavit.

Observ. Praecedenti (Chrys. amethyslinae Fabr.) simillima et maxime affinis, at per nolas sequentes praecipue certissimeque distinguitur, scil.: Corpus totum vi- 
Famil. C'hrysididae. Chrysis: Phalan.e VI. 233

ride, macula rerticis centrali nigro-aeneo purpurascente opaca, mesonoti disco leviter cyanco-irrorato, abdominis segmenti 2:di latera et segmenti $3:$ tii totus ambitus cyanea; caput et thorax crassius punctata, minus nitida; vertex minus convexus potius conrexo-depressus et frons inter oculos magis latus; cavitas facialis latior brevior quadrata; thorax cylindricus dorso depresso-convexus, postscutello convexo-prominulo crassissime punclato-reticulato; mesopleurae utrinque in margine et apicali et postico-laterali crenulatae el hinc obsolete multidenticulatae; abdominis segmentum 3:tium mediocre subsemilunatum: regio antica tola modice convexa, regio postica (= margo apicalis) subconvexo-depressa humilis promimula parce subtilissime punctulata; series anteapicalis tenuissime interrupta foveolis ommibus mediocribus profundis et rotundatis; dentes apicales breves triangulares; emarginaturae modice arcuatac subaequales, scil. exteriores centrali parumper latiores. In reliquis omnibus nolis cum individuis normalibus Clerysidis amethystinue exacte congruit.

Sect. II. Abdomen aut cyaneum aut viride: fasciis I. viridibus 1. aureis distinclissimis.

Divis. 1. Metanotum centro postice mucronulatum.

129. Chrysis spinigera Spin. 오.

Submagna robusta punclatissima 3 lin. decimal. long., nitida subtus viridis supra violaceo-purpurascens maculis et fasciis viridissimis ornata, segmenti (abdominis) 3:tii margine laterali ad basin albo-pellucido, dentibus apicalibus robustis acute triungularibus. 


\section{Famil. Chrysididae. Chrysis: Plialanx VI.}

Chrysis spinigera Spinola. Annal. Entomol. 1840. 201:49.

Habitat in Cayenne, a. D. M. Leprieur detecta; Mus. D. Spinola.

Observ. Inter elegantissimas hujus Generis species jure meritoque numeranda.

Corpus robustum crasse punctatissimum cano- et fuscopubescens et pilosellum. Caput late rotundato-triangulare thoraci arcte applicatum viride; vertex amplus medio violaceus foveolis omnibus in fundo viridibus; stemmata magna testacea; oculi maxime exserti breviter ovati; antice in vertice adest area obcordata per lineolam elevatam determinata, intra cujus apicem ( $=$ angulum superum) stemma anticum excipitur; basis ejusdem areae arcuato-emarginata ramulum longitudinalem utrinque demittens efficit limitem cavitatis facialis superum. Cavitas facialis subrectangularis subprofunda subtiliter punctulata albo - sericea cyaneo-viridis; clypeus concolor transversus subtrapezinus, disco carinulatus, utrinque concaviusculus, margine apicali truncatus. Labrum parvum semilunatum viride. Mandibulae subbreves robustae viridi-cyanescentes apice piceae. Thorax crassus subcylindricus dorsulo praecipue convexus; pronotum viride maculis 3 violaceo-purpurascentibus, dorsulum purpurascenti-violaceum marginibus lateralibus et arcu centrali viridibus, suturae medianae propter sculpturam obliteratae tantumque basi et apice distinctae, scutellum viride; postscutellum et metanotum viridia macula centrali violaceo-purpurascente; in centro metanoti (mox sub postscutello) adest mucro teres subbrevis oblique horizontalis subtruncato-oblusus. Pectus, sternum et pedes viridi-cyanea aut cyaneo-viridia, 
tarsis testaceo-brunneis, horum articulo basali saltem extus virescente; mesopleurarun uterque margo posticus crenato-undulatus et hinc obtuse et obsolete quasi multidenticulatus. Abdomen ovale elongatum capite thoraceque simul sumtis longius, latitudine thoracis, basi truncatum, dorso modice convexum crasse.punctatum, fasciis purpureo-violaceis et viridissimis alternantibus ita ornatum ut in singulo quoque segmentorum fasciolae $4 \mathrm{ob}-$ veniunt, scil. 1) fascia basalis purpureo-violacea, 2) $f$. subbasalis viridis, 3) f. medialis purpureo-violacea et 4) marginali-apicalis viridis; segmentum 1:mum basi triramoso foveatum I. exaratum, utroque angulo basali-laterali prominulo obtuso 1. potius oblique truncato; segmentum 2:dum lineola mediana leniter elevata planiuscula polita; segmentum 3:tium apice angustius quam basi: regio antica subsemicircularis basi et disco polita, basi parce punctata, disco lenissime subimmersa et non punctata, limbo toto crasse-punctata et convexo-terminata, regio postica (= margo apicalis) humilis convexo-depressa valde prominula tota polita; series anteapicalis per carinulam medianam viridem interrupta, utrinque ad carinulam fovea magna sulciformi longitudinali et lineari, reliquis foveolis numerosis parvis rotundis; dentes apicales robusti acute triangulares subaequales, emarginaturac mediocres: exteriores arcuatae at centralis subtriangularis; segmenti 3:tii margo lateralis valde obliquus, elongatus arcuato-flexuosus, basi albo-testaceo-scariosus, segmenti 2: di margo lateralis summus totus concolor. Venter modice fornicatus viridissimus, confertim subtiliter punctulato-coriaceus. Alac subliyalinae aut Ievissime sordide hyalinac, secundum costam cellulac radialis nonnihil fu- 
matae, venis firmibus brunneis, cellula radialis lanceolatosublinearis apice sat aperta.

Divis. 2. Metanotum inerme, non mucronatum.

Subdivis. 1. Abdominis segmenti dorsalis $3:$ tii dentes apicales omnes distincti.

130. Chrysis alternans Klug $\delta$.

Mediocris $2-3$ lin. decimal. long. conferlim modice punctata cyanea vertice pronoto et scutello l. viridibus 1. auratis, abdominis segmentis dorsalibus aut omnibus aut 1 : mo 2:doque, viridi-aureo-fasciatis.

var. $\boldsymbol{a} . \delta^{\nearrow}$. V. Vertex, pronotum, scutellum et postscutellum tota 1. viridi-aurea 1 . aureo-viridia, abdominis segmenta 1 : mum et 2: dum regione apicali amplissime concolora, segnentum 3:tium macula mediana cuneala viridi-aurata.

Chrysis (Poeciloechroa) alternans Klug, secundum Mus. Spinolne Poeciloechroa bifasciata Klug, secund. Mus. Drewseni.

var. b. . Vertex cyaneus antice lateribusque riridi-irroratus; pronotum, scutellum et postscutellum viridi-aurea, illud (pronotum) interdum maculis 2 1. 3 nigro-aeneis; abdominis omnia segmenta dorsalia apice fascia ampla viridi-aurea,

var. c. $\sigma^{\prime}$. Vertex et thoracis dorsum viridi-irrorata nec ullo modo aureo-picta, abdominis segmenta 1 : roum et 2 :dun apice fascia ampla viridi-aurea, segmentum 3 :tium ante apicen leviter viridi-irroratum.

Clirysis elegantula Mus. Spinolae.

Habitat in Aegypto et in Promontorio bonae spei, Museis DD. Drewsen, Spinola et Westermann.

Observ. Corpus confertius punctatum punctis sat mediocribus, fusco- et cano-pubescens robustum sublineare. Caput normale. Vertex subamplus depresso-convexus. 
Famil. Chrysididae. Clurysis: Plualunx VI. 237

Cavitas facialis subprofunda confertim punctulato-coriacea, medio canaliculata, superne marginala: marginulo transverso inaequali, utrinque ramulum longitudinalem obliquun perbrevem demittente. Antennac breves fuscae, crassitie mediocres, fuscae, basi viridi-cyaneac. Clypeus mediocris transversus trapezinus parce punctatus depresso subplanus, disco nonnihil convexus, margine apicali summo picco truncato. Mandibulae piceae, macula basali viridi. Maxillae et palpi l. fusco l. laete brumescentes. Thorax rectangularis, dorso depresso-convexus; dorsuli sulurae medianae lenissime curvae, crenulatae; metanoti anguli postico-laterales mediocres depresso-planiusculae coriaceae, non triangulares at versus abdomen curvae et hinc quasi 7 mucroniformes. Sternum et pedes punctulatocoriacei nitidi viridi-cyanei, tarsis brunneis. Alae aut subhyalinae aut sordide hyalinae, venis firmibus piceis, cellula radiali triangulariter lanceolata apice anguste aperta. Abdomen ellipticum aut potius obtuse subrectangulum, longitudine capitis thoracisque simul sumtorum, vix longius, latitudine thoracis, dorso modice convexum et confertissime modice punctatum, in colore cyaneo subopacum in colore viridi l. aureo nitidum; fascia segmenti rarius recte et aequaliter transversa: quo in casu maximam segmenti partem obtegit, plerisque autem mediocris et antice utrinque late profundeque arcuato-emarginata angulum medianum acutum elongatum sursum emittens, atque tunc pictura segmenti a duabus fasciis re vera constituitur, scil. antica saturate cyanea reniformi, poslica aut viridi-aurea aut rubro-aurea, antice bis-arcuato-emarginata angulo centrali aculissimo sursum ducto; pictura seguenti 3:tii eandem quoque formam interdum praebel, 
quum scil. ita bene explicata obvenit ut in var. b.; segmentum dorsale 3:tium totum modice aut subdepressoconvexum; series anteapicalis leniter immersa, medio interrupta foveolis utrinque distinctis numerosis mediocribus profundis, interdum vero et praesertim apud mares est superficies totius segmenti punctis ita aequaliter adspersa ut series plane evanescel; margo apicalis modice prominulus dentibus brevibus latis triangularibus acqualibus, emarginaturis modice arcuatis invicem quoque subaequalibus; margo lateralis (segmenti $3:$ tii) obliquus, aliis subrectus, aliis leniter arcuatus, aliis infra centrum plus minus distincte angulatus. Venter fornicatus viridi-cyaneus nitidus pubescens subtiliter punctulato-coriaceus.

\section{Chrysis Seheștedti Dlbm.}

Mediocris circ. $2 \frac{1}{2}$ lin. decimal. long. conferlim modice punctata coemulea abdominis segmento 2: do aureo.

Habital in Tanger, a D. Schousbo olim detecta; teste Collectione Schestedti Musei Regii Harniensis, ubi unicum exemplar hujus speciei, juxta specimen typicum Chrysidis integrae Fabr. asservatum, anno 1847 vidi et examinavi.

Observ. Magnitudo et statura fere Chrysidis igni1ae. Corpus coeruleum; abdominis segmentum dorsale 2:dum aureum; segmentum (abdominis) dorsale 3:tium totum convexo-depressum nec supra seriem incrassatum, margo apicalis prominulus planus, series anteapicalis distincta foveolis mediocribus non confluentibus, dentes apicales breves obtusi unde margo summus quasi undulatus obvenit. Antennae basi aeneo-virides, apice lutescentes, postice fusco-maculatae. 
132. Chrysis aureo-maculata Dlbm. $\delta$.

Subparva 2 lin. decimal. long. confertim modice punclata viridis, verlicis macula dorsulique area centralibus nigro-aeneis, abdominis segmenlis 2:do utrinque macula laterali 3 :tioque villa mediana viridi-aureis.

Habitat in Guinea, Mus. D. Westermann.

Observ. Statura fere Chrysidis succinctae. Corpus subangustum sublineare, confertissime modice punclatum, breviter cano- et cinereo-pubescens. Caput normale; cavitas facialis subrectangularis fortiter excavata, subtiliter transverse strigosa, canalicula mediana interrupta desinente in foveola centrali, lateribus punctulatocoriacea albo-sericea, superne transverse marginata: marginulo arcuato, utrinque sursum in verticem ramulum obliquum pone stemma desineutem emittente, hinc in rertice exstat area subtriangularis aut subsemicircularis. Antennae breves fuscae, basi virides. Clypeus brevis transversus parce punctulatus, disco parum convexus, ceterum depresso-planiusculus, margine apicali summo piceo truncato. Mandibulae breves robustae nigro-piceae, ante apicem rufo-piceae, basi macula laete viridi. Palpi maxillaeque nigro-fuscescentes; margo occipitalis utrinque angulo brevi dentiformi. Thorax cyliudricus, antice posticeque truncatus et hinc subrectangularis, dorso depresso-convexus, dorsuli suturae medianae rectae parallelae, postscutellum gibbo-convexum, metanoti anguli postico-laterales robusti punclato-coriacei convexo-depressi acute triangulares. Sternum et pedes magis nitida quam ceterum corpus at ejusdem coloris scil. lacte viridia et punctulata, tarsis fusco-brunneis. Alae hyalinae, tegulis 
cyancis, venis mediocribus brunneis, cellula radiali elongata subangusta lanceolato-acuminata completa. Abdomen sublineare, obtuse subrectangulum, latitudine thoracis, hoc paullulum longius, dorso modice convexum, confertissime modice et regulariter punctatum; segmentum 1 :mum lacte viride, fovea centrali triramosa cyanea; segmentum 2:dum luxuriose pictum, scil. 1) margo lateralis summus et macula disci maxima rotundata virides, 2) marginis basalis linea transversa angusta nigro-aenea utrinque cum macula laterali obliqua cyanea cohaerens, 3) margo summus apicalis et macula utrinque lateralis rotundata viridi-aurati; segmentum 3:tium cyaneum: regio anticas. basalis depresso-convexa, convexo-terminata et heic in limite ornata fasciola tenui aureo-viridi antice bis arcuatoemarginata et ramulum centralem (instar vittae) in discum sursum emittente, - regio postica (= margo apicalis) modice prominula nitida parce punctulata humilior (quam regio antica); series ante-apicalis brevissime interrupta foveolis profundis inaequalibus, scil. quibusdam magnis subquadratis, aliis 1 . mediocribus 1. parsis rolundatis; dentes apicales breves triangulares aequales, emarginaturae modice arcuatae invicem subaequales at exteriores nonnihil obliquae; margo lateralis proprius oblique rectus. Venter fornicatus nitidus punctulatus pubescens viridis, margine anali testaceo.

\section{Chrysis soror Dlbm. $\sigma^{7}$.}

Mediocris $2 \frac{1}{2}$ lin. decimal. long. confertim modice punctala viridis abdominis segmentis 2 :do fascia rpicali ampla et 3:tio ad basin utringue macula lnterali viridi-aureis. 
Famil. Chrysidedae. Chrysis: Phalanx VI. 241

IIabitat in insula Rhodo, a D. Hedenborg detecta; Mus. D. Loew.

Obserr. Similis et nimis affinis Chrysidi alternanti et forsitan ut hujus rarietas considerata; at dignoscitur: colore corporis primario viridi, abdominis segmento 1 : mo non fasciato, segmenti 3 :tii emarginaturis apicalibus levioribus, dentibus intermediis subobtusis, tarsis pallide testaceis, cavitatis facialis margine supero 5 -ramuloso, etc. - Caput viride; vertex amplus depresso-planus; cavitas facialis profunda, superne leniter transverse marginata: margine 9 quinque-ramuloso ramulis brevissimis, 3 sursum ductis, intermedio stemma tangente, 2 deorsum versis. Antennae breves robustae fuscae, basi virides. Clypeus brevis transversus punctatus, disco carinato-convexus, ad centrum marginis apicalis subtruncatus. Mandibulae et palpi nec non angulus marginis occipitalis utrinque prominulus omnino ut in Chryside alternante. Thorax et pedes virides, tegulae cyaneae margine externo viridi; dorsuli area media cyanea nonnihil viridi-irrorata. Tarsi eximie albo-testacei. Abdominis segmentum 1 :mum viride; seginentum 2:dum margine basali tenuiter nigro-aeneum, tum laetius viride et demum in parte apicali maximaque laete viridi-aureum; segmentum 3 :tium confertim punctato-coriaceum laete viride, utrinque macula laterali marginali indeterminata viridi-aurea, margo àpicalis sparse punctulatus viridi-coeruleus; series ante-apicalis foveolis mediocribus circ. 10; dentes apicales breves triangulares marginem leniter undulatum efformantes, margo lateralis proprius obliquus leviter arcuato-flexuosus. Alae hyalinae, disco nec non ad costam cellulae radialis nomnihil sordidae, renis firmis piceis, cellula radiali triangulariter 
lanceolata apice late aperta. Venter modice fornicatus lacte viridis nitidissimus, subtilissime punctulatus pubescens.

Subdivis. 2. Abdominis segmenti dorsalis 3:tii dentes apicales: intermedii brevissimi obsoletissimi, externi breves distincti.

134. Chrysis semicincta Lepel. ðㅇ․

Mediocris elongala robusta $2 \frac{1}{2}$ lin. decimal. long. confertim modice punctata cyanea: fasciis thoracis ant unica aut duabus, abdominisque aut ductus aut tribus aureis.

Chrysis semicincta Lepel. Mus. Spinolae.

Chrysis imperialis Dalman. Dibrn. Dispos. 11:17.

Chrysis bidentata Mus. Drewseni.

Mabitat in Hungaria, D. Drewsen; in Gallia et Hispania, D. Spinola; in Algieria, Mus. Paykull. teste D. Boheman.

Observ. Propter picturam corporis luxuriosam Chrysidi alternanti Lepel. valde similis et affinis, at corpore breviore, punctura minus crassa, capitis caritate faciali superne non transverse marginata, abdominisque segmenti 3:tii dentibus apicalibus intermediis brevissimis obtusis obsoletissimis etc. praecipue dignoscitur. - Corpus robustum sublineare, confertissime permodice punctatum, saturate cyaneum aut potius violaceum. Caput triangulariter rotundatum 1. suborbiculatum; vertex mediocris modice convexus, antice aut linea transverso-arcuata unica aut maculis 3 viridibus; cavitas facialis subquadrata pro- 
Famil. Chrysididae. Clrysis: Phalanx VI. 243

funda cyanea confertim punctulato-coriacea, medio subtiliter canaliculata, superne non transrerse marginata; antennae subbreves fuscae, basi viridi-cyaneae, flagello interdum subtus ante apicem nonnihil testaceo; clypeus mediocris transversus subtrapezinus sparse punctatus, disco nonnihil convexus margine apicali truncatus, totus aut cyaneus aut viridi-cyaneus. Mandibulae piceae basi viridi-maculatae. Thorax subbrevis rectangularis dorso depresso-convexus pronoto, scutello et postscutello 1. laete viridibus 1 . aureis et plus minus e viride resplendentibus; pronoti truncatura antica, dorsulo, metanoti segmento postico levissime viridi-irrorato pectoreque aut cyaneis aut violaceis, mesopleuris viridibus. Pedes viridi-cyanei tarsis fusco-brunneis. Alae subhyalinae venis firmibus brunneis, cellula radiali triangulariter lanceolata apice sat aperta. Abdomen sublinearc obtuse subrectangulum, in masculis breve, in feminis subelongatum et thorace fere duplo longius, omnibus latitudine thoracis, dorso modice convexum crebre punctatum punctis parvis; segmentum 1:mum aut laete viride aut viridi-aureum, fascia basali subangusta violacea; segm. 2:dum bifasciatum: fascia basali violacea mediocri subreniformi, fascia apicali coccineo-aurea amplissima antice leniter bis arcuato-emarginata angulo centrali brevissimo acuto sursum emittente; segmentum 3:tium violaceum: fasciola discoidali aut viridi aut viridi-aurea indeterminata scil. aliis lanceolata et aliis tenuissina et lineari atque interdum striolam medianan sursum emittente; segmenti 3 : tii regio (= margo) apicalis humilior prominula transversa subtrapezina, series anteapicalis continua foveolis non numerosis, mediocribus rotundatis separatis; dentes apicales 


\section{Famil. Chrysididae. Chrysis: Phalanx VI.}

externi breves triangulares distincti, dentes apicales intermedii brevissimi obtusi obsoletissimi, emarginatura centralis lenissima, emarginaturae externae leniter arcuatae latiores, hinc summus et proprius margo apicalis leniter undulatus im efformatur. Venter modice fornicatus cyaneus viridi-, nigro - et purpurascenti-pictus, superficie normaliter constructa.

135. Chrysis zonata Dlbm.

Subparva $1 \frac{1}{2}-1 \frac{3}{4}$ lin. decimal. long. confertim modice punctata subcoriacea viridis, dorsuli area media cyanea, abdominis segmento 2:do aureo.

Habitat in Asia minori, a D. Loew detecta; Mus.

\section{Loewi.}

Observ. Statura fere Clrysidis ignitae parvae. Pictura Chysidi sorori valde similis et affinis, at corpore multo minore et angustiore, punctura multo minus crassa, abdominis segmenti 3:tii dentibus apicalibus obtusis brevissimis obsoletissimis facile dignoscitur. - Corpus confertim punctatum subcoriaceum punctis permediocribus. Caput viride; cavitas facialis subangusta rectangularis profunda, medio subtiliter canaliculata, lateribus et superne punctulato-coriacea, superne transverse marginata: margine utrinque ramulum brevem longitudinalem deorsum versum emittenté; antennae mediocres basi virides, flagellum laete brunnescens. Clypeus transversus mediocris subtrapezinus disco convexus margine apicali truncatus. Mandibulae et instrumenta cibaria normalia. Thorax viridis: pronoti truncatura antica, dorsuli area media et metanoto segmento postico leviter viridi-irrorato, cyaneis. Pedes virides tarsis brunneis. Alae subhyalinae 
Famil. Chrysididae. Chrysis: Platanx VI. 245

venis subtenuibus brunneis; cellula radialis subtriangulariter lanceolata, apice anguste aperta. Abdomen ut in Chryside semicincta simillime constructum, at segmenti 3:tii margo proprius apicalis brevior, dentes autem et cmarginaturae simillimam praebent formam; segmentum 1 :mum cyaneo-viride: margine apicali aurato; segmentum 2: dum igneo-aureum: margine basali tenuiter nigro-aeneo; segmentum 3:tium viride: fascia basali violacea; series ante-apicalis interrupta: foreolis utrinque $4-5$ coeruleis majusculis profundis rotundatis. Venter planiusculus, viridi-cyaneo- et nigro-pictus, superficie normali.

Sect. III. Abdominis dorsum aureum; segmentum 1 :mum violaceum aut cyaneum, interdum quoque segmentum 2: dum macula basali concolore.

136. Chrysis fulgida Lin. $\sigma 7$ 오.

Mediocris aut magna robusta $2 \frac{1}{2}-4$ lin. decimal. long. nilida modice punctata cyanea plus mimus viridipicta, abdominis segmento 1:mo concolore, segmentis 2:do et 3:tio aureis, illo (2:do) ot macula basali maxima cyanea, segmento 3:tio depresso-convexo, serie anleapicali foveolis numerosis, margine apicali promimulo, dentibus apicalibus robustis triangularibus, alarum cellula radiali completa.

Chrysis fulgida Zett. Ins. Lapp. 433:1; Dlbm. Dispos. 1815. 9:6.

Mlas. Abdominis segmentum 2:dum cyaneum fascia apicali mediocri aurea, antice late profundeque arcuatoemarginata.

II. 
Chrysis fulgida Lin. Fn. Sv: Descr.

Cltrysis stondera Jur. Hym. 296. 1. XII. fig. 9.; - Vesnact Not. $9: 3$.

Chrysis stondera Panz. Fn, Germ, 107:12.

Chrysis haemorrhoea Meg. Mus. Vicnn. teste Kollitr in lilt.

Femina. Abdominis segmentum 2:dum totum aurcum immaculatum.

Chrysis fulgida Lin. S. N. 2. 948:7, Fn. S». 1669. Dingnos.;Fabr. E. S. 2. 240:8, Piez. 172:11.; - Panz. In. Germ. 79:15.

Irabitat in locis argillaceis et truncis rermisecatis per totam Europam, optima aestate passim; in sylva Alten prope Nordcap d. 4-7. Augusti 1821 a 1). Zetlerstedl observata.

Observ. Statura fere Chrysidis ignitae. Corpus robustum elongatum cinereo-pubescens. Caput transversum suborbiculatum confertius modice punctatum subrugulosum, plerisque supra cyaneum et antice rirescens: cavitas facialis lata subquadrata, levis nec profunda, confertim punctulato-coriacea, supra discum forea centrali, superne marginata: margine inaequali transverso, utroque apice breviter longitudinaliter deflexo; antemnae robustac longinsculae fuscae, basi cyaneae plus minus viridi micantes; clypeus mediocris transversus trapezinus sparse modice punctatus, disco convexus, utrinque concariusculodepressus, margine apicali nigro-piceo late et leniter arcuato-emarginatus, totus nitidus viridis, viridi-cyaneus aut rarius unicolore cyaneus. Labrum semilunatum convexum punctatum ciliatum parvum, mandibulae, maxillac palpique picea, macula mandibularum basali viridi. Margo occipitalis utrinque angulo parvo prominulo. Thorax dorso lateribusque crasse punctatus, aut cyancus aut purpurascenti-violaceus, aliis unicolor, aliis pronolo, dorsuli 
areis lateralibus, scutello et postscutello viridi-maculatis; metanoti anguli postico-laterales magni latiusculi triangulares; pectus et perles nitidissimi l. virides l. viridi-cyanei tarsis fuscis. Abdomen elongatum sublineare, latitudine thoracis, obtuse subrectangulum, capite thoraceque simul suntis longius, dorso modice convexum lineola mediana elevata subtili fere in omnibus segmentis visibili sed non continua, et rarissime tota explanata I. oblitterata; segmentum 1:mum raro unicolore cyaneum, saepissime utrinque macula laterali-apicali virescente; punctura segmentorum: 1:mi crassa, 2:di mediocris, 3:tii conferstissima e punctis parvis congestis constituta, unde superficies segmenti 3:tii subcoriacea apparet, at hoc segmentum non winus intensive fuhminans quam 2:dum; segmentum 3:tium saepe in $q$ disco utrinque nonnihil immersum; series anteapicalis interrupta foveolis vel mediocribus vel parris, rotundis numerosis; margo apicalis prominulus, dentes robusti triangulares; emarginaturae late arcuatae, centralis in $q$ saepe latior; venter nitidus viridi-, cyaneo- et nigro-pictus. Alae sordide hyalinae venis firmibus piceis; cellula radialis elongata, subtriangulariter lanceolata acuminata, plerumque completa, rarius subcompleta apice angustissime aperta.

137. Chrysis exsulans Dlbm.

Mediocris robusta $2 \frac{1}{2}$ lin. decimal. long. modice punclala cyanea, abdominis segmenlis: 1:mo concolore, 2: do el 3:lio igneo-aureis eximie convexis, dentibus apicalibus angustis fortiter spinoideis, alarum cellula radiali incompleta. 


\section{Famil. Chrysididae. Chrysis: Plealanx VI.}

Patria ignota, forte Bengalia; specimen unicum ut. „Chr. fulgidae varietas" amice communicavit D. Spinola.

Observ. Pictura Chrysidi fulgidae quidem simillima et maxime affinis, attamen notae sequentes Chrysidem exsulentem ut propriam speciem certe distinguunt. Corpus quidem brevius quam in speciminibus normalibus Chrysidis fulgidlae sed quoad longitudinem crassius; caput et thorax mediocrius punctata subcoriaceo-rugosa late virescenti-cyanea. Cavitas facialis brevis lata quadrata profunda subtiliter punctulato-coriacea, supra discum fovea centrali, superne distinctius marginata: margine transverso-arcuato. Thorax subbrevis crassus dorsulo gibbo-convexo, suturis medianis tenuibus nigro-aeneis basi fortiter arcuatis. Sternum et pedes riridissime cyanei nitidi. Alae hyalinae, ad costam cellulae radialis nec in basi cellulae cubitalis leviter sordidae; venae mediocres brunneac; cellula radialis brevior, non lanceolata sed latissime triangularis, apice anguste et distincte aperta. Abdomen subrectangulare I. potius elongato-subquadratum, quoad magnitudinem latius quam in Chrys. fulgida; punctura segmentorum 1:mi et 2:di paullulum crassior et magis regularis quam punctura thoracica; segmenta 2: dum et 3:tium convexiora (quam in Clrr. fulgida); segmenti 2:di lineola mediana subelerata polita planiuscula; segmentum 3:tium subbreve decurrum, series anteapicalis foveolis perpaucis circ. 8 magnis profundioribus rotundatis, margo apicalis proprius perbreris et humilis, dentes angusti cylindraceo-conici fortiter spinoidei, emarginaturae profunde arcuatae: utraque externa latior et paullulum profundior quam centralis. Omnes reliquas notas, in hac descriptione omissas, habet Clrrysis extsulans cum Chryside fulgida communes. 
Famil. Chrysididae. Clorysis: Plualanx VI. 249

Sect. IV. Abdominis dorsum aureum; segmentum 3:tium cyaneum: aut unicolor, aut maculis 1 . viridibus 1. aureis pictum.

Jivis. 1. Abdominis segmenti dorsalis 3:tii dentes apicales distincti.

A. Abdominis segmenti 3:tii margo lateralis angulatus.

138. Chrysis Ramburi Spin.

Mediocris $2 \frac{1}{2}$ lin. decimal. long. confertim modice punctata cyanea, dorso thoracis in ơ concolore, in 9 ad maximam partem aurato, abdominis segmentis dorsalibus 1:mo 2:doque totis et 3:lio utrinque macula laterali aureis.

Mas. Thorax cyaneus lateribus pectoreque nomnihil viridi pictus.

Chrysis Ramburi Mus, Spinolae.

Femina. Pectus et metanotum cyanea, pronotum et mesonotum aurea.

Clerysis bidentata Mus. Spinolae.

IIabitat in Europa meridionali rarius; marem unicum in Hispania a D. Rermbur detectum et feminam unicam in Lombardia a D. C'lerislopleori lectam communicavit D. Spinola.

Observ. Statura Chrysidis bidentatae, a qua tamen mox dignoscitur armatura anali distinctiori, segmenti 3:tii macula laterali aurea etc.

Mas. Caput el thorax confertius modice punctatocoriacea cyanea griseo - et cino-pilosella. Frons, facies el clypeus virides. Cavitas facialis mediocris profun- 


\section{Famil. Chrysididae. Clrysis: Phalanx VI.}

diuscula punctulato-coriacea superne levissime transverse marginulata: marginulo obsoleto bis arcuato. Antennae robustae breviusculae fuscae, basi viridi-cyaneae. $\mathrm{Cl}_{5}$ peus majusculus transversus subtrapezinus, disco convexiusculus utrinque depressus, margo apicalis nigro-acneus transversus. Mandibulae et palpi normales. Pronoti margo supra truncaturam transversus et uterque margo lateralis, scutelli macula basalis parra, melanoti suturae et anguli posticolaterales virides. Pectus cyaneum suturis et maculis viridibus. Pedes cyaneo-virides tarsis brunneis. Alae hyalinae, disco nomnilil sordidae, renis mediocribus brunneis, cellula radiali lanceolata, apice modice aperta. Abdominis dorsum confertim modice punctatum subcoriaceum lineola mediana subtilissime carinulata in segmento 2:do; segmenta $1:$ mum et 2:dum aurea, illud macula basali triangulari viridi, hoc margine basali tenuiter nigro-aeneo; segmentum 3:tium confertius modice punctato-coriaceum cyaneum, in utroque latere a basi usque in seriem macula marginali magna orata obliqua aurea viridi-circumscripta; totum hoc (3:tium) segmentum depresso-convexum nec in regione apicali quidem humile; series anteapicalis foveolis mediocribus numerosis rotundatis; margo apicalis mediocris, dentes breves triangulares latiusculi, emarginatura centralis modice arcuata, proxima-utraque leniter arcuata et paullulum latior (quam centrali); margo lateralis proprius centro angulatus. Venter niger: segmentis 2: do et 3 :tio ante apicem aureo-viridi - maculatis.

F'emina a mare suo notis sequentibus praecipue dignoscitur: capite toto cyaneo, lineola sub stemmate transversa curva viridi; clypei disco virescente; thoracis dorso 
Famil. Chrysididae. Clhyssis: Phalanx VI. 251

punctato-reticulato; pronoto el mesonoto viridi-aureis, illius truncatura antica et sulco mediano cyaneis; metanoto et pectore cyancis: suturis viridibus; pedibus cyancis: tarsis apice fuscis; ventre nigro-aeneo: segmentis apice cyaneo-maculatis.

139. Chrysis sinuata Dalman. + .

Ilediocris $2 \frac{l}{\mathrm{i}}$ lin. decimal. long. conferlim crasse punctala viridi- et ryaneo-picta, pronoto, striolis dorsuli duabus, srutello, postscutello, abdominis segmentis dorsalibus 1:mo el 2: do maculaque centrali segmenti $3: 1 i i$ aureis, segmento 2:do linea basali transverso-arcuala cyanea.

Chrysis simuatu Dalman. Mus. Paykulli; Dlbm. Dispos. 1845. 12:18.

IInbilat in promontorio bonae spei; teste D. Bohemen, qui specimen unicum e Museo R. Acad. Scient. Stockholm. communicavit.

Observ. Statura fere C'lrysidis bidentatne. Corpus subrobustum lineare, fusco- et griseo-pubescens. Caput rotundato-triangulum confertim modice punctato-subreticulatum viride; vertex amplus transverso-rectangularis parumper consexus macula centrali saturate cyanea; carilas facialis longiuscula rectangularis, disco profunda sublilissime coriacea subcyanescens, canalicula mediana distincla, limbo toto viridis et punctulata, superne distincte transverse marginata: margine utrinque longitudinaliter breviter deflexo; antennae mediocres fuscac, basi virides; clypeus viridis nitidus punctulatus transversus subtrapezinus, disco subcarinato-convexus, utrinque immersus, margine apicali piceo late lenissime arcuato-emarginatus. La- 


\section{Famil. Chrysididae. Chrysis: Plualanx VI.}

brum, mandibulae et reliquae oris partes normales. Thorax longiusculus subcylindricus crasse punctato-reticulatus; pronotum viridi-aureum, truncatura cyanea foveis medianis 3 parallelis approximatis; tegulae et dorsulum violaceae, hujus areae laterales introrsum viridi-auratae; scutellum rubra et postscutellum viridi-aurea; metanolum subaurato-viride foveis nonnullis cyaneis, angulis posticolateralibus robustis triangularibus; pectus viride; pedes viridi-cyanei, calcaribus albo-testaceis, tarsis fuscis; alae hyalinae, disco nonnihil sordidae, venis mediocribus brunneis, cellula radiali elongata subtriangulariter lánceolata, subcompleta propter venulam radialem mox ante apicem alae ita oblitteratam, ut inter costam et venam radialem apertura perangusta obvenit. Abdomen thorace nonnihil longius at latitudine thoracem aequans, obtuse subrectangulum, dorso subdepresso convexum confertim punctatosubreticulatum aureum: segmentum 2:dum basi fascia transverso-arcuata cyanea, medio angusta, utrinque ampliata, in hoc segmento carinula quoque mediana subtilis adest; segmentum 3 : tium cyaneum macula centrali riridiaurea lata triangulari, regio antica hujus segmenti convexo-terminata; regio postica (= margo apicalis) igitur paullulum humilior, ceterum modice prominula planinscula opaca subtiliter punctulato-coriacea; series antcapicalis continua (nec interrupta), foreolis numerosis profundis subsulciformibus; dentes apicales robusti acute triangulares, externi forsitan paullulum breviores; emarginaturae subprofunde arcuatae, externae obliquae et centrali nonnihil profundiores; margo lateralis proprius obliquus elongatus, ad centrum leniter angulatus, inter basin et angulum convexo- et inter angulum et apicem concaro-arcua- 
Famil. Chrysididae. Chrysis: Plualanx VI. 253

tus. Venter modice fornicatus nitidus subtiliter punctulatus pubescens cyaneo-viridis nigro-maculatus.

B. Abdominis segmenti 3:tii margo lateralis muticus (non angulatus), oblique rectus.

140. Chrysis cyanopyga Dlbm. $\sigma^{7}$.

Mediocris robusta 2 lin. decimal. long. confertim modice punctuta viridi-cyanea, abdominis segmentis dorsalibus 1:mo 2:doque aureis, hoc margine basali nigroaeneo, segmenti 3:tii serie ante-apicali foveolis separalis.

Chrysis analis Megerl, Mus. Vienn. teste Kollar in litt. (non Chrysis analis Spinola. Ins. Lig.).

Clrysis cyanopygo Dlbrn. in Mus. Havniensi anno 1847.

II abitat in Algieria; tantum duo specimina vidi, unum auno 1847 in collectione Seliestedli Musei R. Havniensis ita inscriptum „Chr. n. sp. Algier. Stub", alterum e Museo Imp. Viennensi a D. Kollar benevole transmissum.

Observ. Habitus fere Chrysidis ignitae. Corpus robustum confertim modice punctatum fusco- et canopubescens et pilosellum. Caput cyaneum, antice inter stemma, oculos et mandibulas viride; cavitas facialis mediocris subquadrata punctulato-coriacea, superne marginata: margine transverso $\sim$ bis arcuato; antennae mediocres robustae fuscae, basi virescentes; clypeus transversus mediocris trapezinus punctulatus nitidus, disco convexus et leniter carinulatus, utrinque immersus, margine apicali subtruncatus; mandibulac normales. Thorax mediocris; pronotum viride, truncalura violacca: areola 


\section{Famil. Clerysididac. Clerysis: Pluctanx VI.}

centrali semilunata immersa polita nigro-aenea; dorsuli area media basi nigro-acnea, apice violacea, areae laterales cyaneae introrsum virescentes; scutellum, metanoti anguli postico-laterales robusti triangulares et mesopleurae viridia, metanotum et pectus cyanea; pedes antice virides, postice viridi-cyanei, tarsis fuscis, calcaribus testaceis; alae subsordide hyalinae, venis mediocribus brunneis, cellula radiali elongata lanceolata completa. Abdomen robustum ovali-subrectangulum, capite thoraceque simul sumtis paullulum longius at latitudine thoracis, dorso modice convexum aureum; segmentum 2:dum basi nigroacneo-marginatum, lineola mediana subtiliter carinulata praesertim in disco distincta; segmentum 3 : tium cyaneum, in exemplare Havniensi unicolor, in ex. Viemnensi apice laete viride, regio hujus segmenti antica convexo-terminata, regio (= margo) apicalis paullo humilior depressoconvexa punctulata; series anteapicalis interrupta, utrinque foveolis paucis magnis separatis orbiculatis; dentes apicales mediocres aut subbreves triangulares fere in unam lineam transversam dispositi, emarginaturne modice arcuatae, centralis paullulum angustior quam singula quaeque externarum. Venter normaliter constructus, viridicyaneus nigro-maculatus.

141. Chrysis splendidula Rossi. ठั우

Mediocris ang usta linearis $2-2 \frac{1}{2}$ lin. decimal. lung. conferlius punchulato subcoriacea, aut viridi-cyanea. ant cyanen aul violacea, abdominis segmentis dorsalibus $1:$ mo 2:doque aureis immaculalis, segmenli 3 :lii serie anleapicali foveolis plus minus coufluentibus. 
Famil. Chrysididae. Clryssis: Plualanx VI. 255

Chrysis splendidula Rossi Fu. Etr. Edit. Illig. 1. 125:850 - Dlbm. Dispos. 1845. 9: 7 .

Clirysis cyanura Dalman Mus. Paykulli.

Ilabitat praescrtim in Europa meridionali et Asia minori, passim aut raro; sed in plagis quoque borealibus obvenit, licet rarissime, sic ad Glosbo in Helsingia Sveciae unicum specimen in locis arenosis d. 25. Augusti 1810 deprehendi.

Observ. Individuis parvis Clrysidis ignitae quodammodo similis, at corpore multo angustiore, punctura non crassa, abdominis segmento 3:tio toto cyaneo etc. facile distinguitur. Quoad picturam Chrysidi cyanopygae proxima; sed corpore angustiori gracili punctulato, abdominis segmento dorsuli 3:tio disco quidem leniter attamen distincte immerso, serie ante-apicali foveolis profundioribus, harum nonnullis in majores subrectangulas confluentibus, dentibus apicalibus robustioribus etc. satis superque dignoscitur.

Corpus elongatum gracile cinereo-pubescens punctis parvis crebre obsertum, capite thoraceque subcoriaceum. Caput robustum rotundato-subcubicum, aliis totum viride aliis cyancun; cavitas facialis subquadrata, disco profunda et subtilissime transverse strigosa, in vertice supra cavitatem facialem marginulus transversus utrinque deflexus aut distinctus aut obsoletus adest aut abest. Clypeus mediocris transversus nitidus punctulatus subsemilunatus, ad centrum marginis apicalis aut lenissime arcuato-emarginatus aut subtruncatus, ceterum superficic normali. Margo occipitalis utrincue angulo parro subacuto prominulo. Thorax aut pure cyancus, aut pronoto, dorsuli areis lateralibus et scutello viridi-irroratis 
pectoreque viridi-cyaneo, aut totus viridis. Metanoti anguli postico-laterales robusti triangulares.

Pedes aut viridi-cyanei aut cyaneo-virides tarsis fuscis. Antennae breves fuscae basi cyaneo- et viridi-nitentes. Alae subhyalinae, disco parumper sordidae, renis brunneis, costali, subcostali et radiali mediocribus, reliquis tenuissimis, cellula radiali mediocri ad summum apicem alae non extensa, lanceolata plérisque completa, rarius subcompleta. Abdomen elongatum capite thoraceque simul sumtis nonnihil longius, elliptico-rectangulum, dorso modice convexum punctulatum nitidum; segmenta 1 :mum et 2 :dum 1. viridi - 1. igneo-1. coccineo-aurea; segmentum 3:tium 1. cyaneum 1. violaceum, aliis unicolor, aliis apice virescens; quoad formam est hoc segmentum trapezinum, basi valde latius quam apice, in $\delta$ brevius quam in 9 ; regio antica $s$. basalis (segmenti $3:$ tii) semicircularis, disco leniter aut lenissime at nihilominus distincte immersa, supra seriem convexiusculo-terminata; regio postica (margo apicalis) humilior, depressiuscula subtilissime punctulato-coriacea utrinque paullulum declivis; series anteapicalis interrupta, foveolac profuudae majusculae inaequales scil. nonnullae separatae et orbiculatae, nomnullae confluentes et subquadrangulac; dentes robusti acuti triangulares subaequales scil. intermedii vix nisi levissime majores, emarginaturae mediocres modice arcuatac subaequales; margo proprius lateralis elongatus valde obliquus at nibilominus per se rectam lineam currens. Venter fornicatus, niggro-, cyaneo-, viridi-el aureo-pictus. 
Famil. Clerysididae. Chrysis: Phalanx VI. 257

Divis. 2. - Abdominis segmenti dorsalis 3:tii dentes apicales obsoleti.

142. Chrysis bidentata Lin. $\sigma^{\top}$.

Mediocris aut submagna $2 \frac{1}{2}-3 \frac{1}{2}$ lin. decimat. long. confertim crasse punctala viridi-cyanea, thorace abdominisque segmentis 1:mo et 2:do dorso aureis.

Chrysis bidentata Liu. S. N. 2.947:2. Q; - Fabr. E. S. 2.241:11 ct Piez. 173:16 (exclus. forte Synonym. De Geeri). ㅇ; Panz. Fn. Germ. 77:15. ठ7; - Dlbm Mon. Chr. 8:3 et E. H. 29:4. ठౌㅇ; - Wesmael Not. 10:4. ठㅇ․

Clerysis viridula Lin. Fn. Sv. 1668. ठౌ; - Dlbm. Dispos. 1845. 11:14. б우.

Chrysis dimidiata Oliv. secundum Wesmael I. c. nam opus Olivieri ipse non vidi; - Coqueb. Illustr. dec, 2:58. tab. XIV. fig. $2 \delta^{7}$ et fig. 3 q; - Fabr. E. S. S. 258. 15-16. $\delta^{\top}-$, Piez. 174:22. ఠ"; - Lepel. Annal. d. Mus. VII. 127:20. бㅇ․

Habitat mensibus Junio, Julio et Augusto in floribus et foliis plantarum, in locis argillosis plurimarum Europae regionum, nec non in Asia minori, plus minus frequenter. Specimen typicum Chrysidis bidentatae Fabr. in Collectione Fabriciana Musei Universitatis Kiel. anno 1847 ita accurate examinavi, ut dubium de Synonymia Fabricii non ulterius habeo.

Observ. Femina. Corpus robustum elongatum sublineare confertim crasse punctatum cinereo-fusco- et canopubescens. Caput mediocrius et confertius punctatum subrugulosum suborbiculatum cyaneum, antice plus minus viridi-nitidum; cavitas facialis lata subquadrata aut subtrapezina subtilissime transverse-strigosa limbo punctulatocoriacea, medio interdum subtiliter carinulata, superne aut obsoletius aut distincte transverse marginata: margine inaequali utrinque breviter longitudinaliter deflexo; cly- 
peus mediocris transversus nitidus punctatus et punctulatus, disco convexus et interdum subcarinulatus, utrinque modice depressus, margine apicali nigro-piceo ad centrum late truncato aut subtruncato; antennae breves robustac fuscae, basi aut cyanci aut viridi-cyanci interdum purpurascentes; labrum parvum semilunatum, mandibulac mediocres, piceae; hae basi viridi-maculatae; maxillae palpique normales; margo occipitalis utrinque angulo parro subacuto prominulo. Thorax mediocris cylindricus confertim crasse punctatus subreticulatus, parum nitidus et interdum subopacus, dorso modice convexus aureus; pronoti truncalura antica, metanoti segmentum posticum et pectus cyanea pleuris plus minus viridi-pictis; pronoti: truncatura antica foveis 2 centralibus nigro-aencis, -partis posticae sulcus medianus ellipticus plus minus profundus non continuus; metanoti anguli postico-laterales mediocres triangulares; pedes aut cyanci aut viridi-cyanei una cum sterno nitidissimi, tarsis brunneis calcaribus testaceis; alae sordide hyalinae tegulis cyaneis, aeneis aut nigro-piceis, nervis firmibus piceis, cellula radiali mediocri lanccolata, aut incompleta aut subcompleta scil. apice I. modice 1. angustius aperta. Abdomen robustum oralisubrectangulum capite thoraceque simul sumtis aut longitudine aequale aut paullulum longius, dorso confertim punctatum modice convexum; segmenta $1:$ mum et $2:$ dum 1. viridi- 1. igneo-aurea, illum basi fascia mediocri cyanca, - hoc I. muticum 1. lineola mediana subtilissime carinulata, margine basali tenuiter nigro-aeneum; segmentum 3:tium cyancum, disco aut macula centrali aut fasciola transrersoarcuata minus bene determinata virescenti; series anteapicalis interrupta, foveolac numerosac profundae inac- 


\section{Famil. Chrysididae. Chrysis: Plualanx VI. 259}

quales scil. et rotundatae et ellipticae et subrectangulares adsunt; regio (= margo) apicalis nonnihil humilior (quam regio antica) convexo-depressa punctulata prominula; dentes apicales: externi breves triangulares, intermedii brevissimi latissimi lenissime arcuato-obtusi; emarginatura centralis lenissima subangulata, emarginaturae externae late leniter arcuatac. Venter aut planus aut modice fornicatus, nitidus subtilissime punctulatus pubescens viridi-cyaneus, basi nigro-maculatus.

MIas feminae simillimus, at practer notas sexuales saepius quoque dignoscitur: capite, pectore, pedibus anoque ad maximam partem viridibus, leviter cyaneo-pictis; antennarum flagellis aut supra fuscis et subtus testaceis, aut totis testaceis fusco-annulatis.

143. Chrysis pyrinina Dalman. ठð.

Mediocris $2 \frac{1}{2}$ lin. decimal. long. conferlim punctulata viridis, dorsuli area media abdominisque segmento 3 :tio ryaneis, hoc fascin apicali viridi, segmenlis 1: mo el 2 :do aureis.

Chrysis pyrrhina Dalman Mus. Paykulli; teste D. Boheman, qui specimen unicum, patria non notata, e Museo R. Acad. Scient. Stockholm. amice communicavit; - Dlbm. Dispos. 1845. 9:8.

Observ. Praecedentis (Clrysidis bidenlati) mari simillimus; at notae characteristicae, quibus praecipue dignoscitur, sunt sequentes: caput et thorax confertim punclulato-rugulosa, nec crasse punctata, tota viridia: verticis macula centrali, pronoti truncatura antica dorsulique area media cynneis; abdomen crebre punctulatum; segmenti 3:tii margo apicalis brevior, cmarginatura centralis obsoletissima, unde margo summus potius 
fere 3 -dentatus quam 4-dentatus videtur; antennarum flagello toto fusco.

Sect. V. Abdominis dorsum aureum aut riridi-aureum, segmentum 3 : tium viride (margo apicalis 1 . concolor l. cyaneus, series ante-apicalis cyanea).

144. Chrysis rutilans Oliv. $\sigma^{7}$.

Mediocris aut magna robusta $2 \frac{1}{2}-4$ lin. decimal. long. subcrasse punclata viridis, abdominis dorso l. auralo-viride 1. viridi-aureo conferlius punctato, segmenli 3:tii dentibus apicalibus mediocribus aut brevibus, margine laterali angulato.

II as. $2 \frac{1}{2}-3$ lin. decimal. long. abdominis segmentis dorsalibus 1 : mo et 2:do viridi-aureis.

Chrysis rutilans Olivier, teste Latreille olim in litteris ad Spinola. . . . . . . . . MIus.

Chrysis Friwaldskyi Spinola . . . . . . . . Spinolae.

Femina vix 4 lin. decimal. long. abdominis segmentis dorsalibus 1:mo et 2:do saturate aureis.

Habitat in Lusitania, Italia, Gallia, Turcia ad Constantinopol. Feminam, in Tirolia a Cl. Prof. Apez detectam, in Museo Berolinensi anno 1817 vidi.

Observ. Species et pictura et ani sculptura facile dignota. Femina inter formosissimas Europac Chrysides jure numeranda: Corpus robustum sparse cinereo- et cano-pilosellum, dorso crasse subconfertim punctatum nitidum. Caput lacte viride, infra cyanescens; frontis sulcus medianus abruptus cyaneus. Clypeus mediocris aut submajusculus, transversus subtrapezinus, inaequalis, sparse punctatus viridis, disco convexiusculo linea transversa ar- 
Famil. Chrysididae. Chrysis: Plıalanx VI. 261

cuata 1. subrepanda cuprea, margine apicali nigro piceo politus et nonnihil arcuato-emarginatus. Mandibulae piceae nitidae parce punctatae, basi macula parva viridi, ante apicem rufo-piceae. Palpi brunnei. Pronotum laete viride, parum aureo-micans, truncatura et lateribus cyaneum. Mesonotum, mesopleurae et postscutellum etiam laete viridia; tegulac areaque dorsuli media cyaneae; metanotum et pectus concolora suturis et angulis viridibus. Pedes cyaneo-virides, calcaribus tarsisque brunneis. $\mathbf{A b}$ dominis dorsum subcrasse confertim punctatum nitidum; seginentum 1:mum aureum, basi viridi-cyaneum; segmentum 2:dun formose aureum margine basali tenuiter cyanescens; segmentum 3:tium viride, margine basali tenuiter cocrulescens, centro nonnihil aureo-micans; series ante-apicalis cyanea, per carinulam medianam viridem interrupta, foveolis profundis politis confluentibus fere sulcum transverso-linearem efformantibus; dentes apicales robusti, intermedii spinoidei, exteriores triangulares illis paullulum breviores. Venter niger cyaneo- et viridimaculatus.

Mas feminae suae notis characteristicis simillimus, nec nisi sequentibus diversus: Corpus brevius et magis viride, clypeus in exemplaribus a me examinatis non cupratus, abdominis segmenta dorsalia duo antica magis viridi- quam rubro-aurata, segmentum 3:tium disco non aureo-micans.

\section{Chrysis terminata Megerl.}

Mediocris $2 \frac{1}{2}$ lin. decimal. long. subcrasse punctata viridi-aeneo-purpurascens, abdominis dorso aeneo-aurato, segmenti 3:tii dentibus apicalibus majoribus, margine laterali concavo-arcuato.

II. 
Chrysis terminata Megerle von Mühlfeld, Mus. Vienn. Ieste Kollar.

Uabitat in Austria rarissime; specimen unicum e Museo Imp. Viennensi benevole transmisit Amicus D. Kollar.

Observ. Statura Chrysidis ignilae. Caput mediocre rotundato-triangulum confertim punctulato-rugulosum cinereo - et cano-pubescens aeneun opacum; vertex depresso-planiusculus; cavitas facialis quadrata planiuscula nitida cyanea punctulato-coriacea: foreola centrali, superne transverse marginata: margine $\sim m$ crenulato et hinc quadri-denticulato. Antennae mediocres fuscae, basi cyanescenti-virides. Clypeus transversus mediocris subtrapezinus punctulatus viridi-cyaneus, disco carinato-convexus, margine apicali ad centrum modice arcuato-emarginatus. Labrum parvum semilunatum convexum, mandibulae, maxillae palpique nigro-fuscae, vix aut parumper nitida. Margo occipitalis utrinque angulo prominulo parro subacuto. Thorax rectangularis confertim subcrasse punctatus pubescens et parum nitidum; pronotum, scutelli et postscutelli margines postici et mesopleurae fusco-viridiaenea; pronoti truncatura antica, dorsulum et metanotum fusco-aeneo purpurascentia; sternum nitidum punctulatum purpurascenti-violaceum; pedes nitidi cyanei, tarsis fuscis, calcaribus tibiarum brunneo-testaceis; alae hyalinae, tegulis violaceis, venis mediocribus brunneis, cellula radiali lanceolata subcompleta scil. apice peranguste aperta. Abdomen mediocre, thorace longius, capite thoraceque simul sumtis brevius, dorso sat convexum; segmentum 1:mum nitidum confertim punctatum subaeneo-cupreum limbo aurato-virescente; segmentum 2:dum nitidum non confertim at magis sparse punctatum, amplius acneo-cupreum, lateribus subaurato-viridi splendens, ad basin macula parva 
centrali opaca rotundata nigro-aenea; segmentum 3:tium confertissime punctulato-rugulosum seladonicum 1 . potius aeruginoso-viride parum nitidum, basi teuuiter et quasi sordide aureo-micans; series ante-apicalis interrupta, utrinque foveolae 5 profundae, harum 2 anticae subquadrangulae majores et 3 laterales rotundatae; margo apicalis brevis convexo-depressus subaurato-viridis, dentes robusti acuti, externi subtriangulares, intermedii nonnihil majores spinoidei, emarginaturae mediocres arcuatae sub-

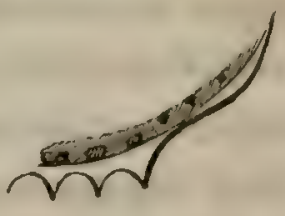
aequales, margo proprius lateralis obliquus concavo-arcuatus I. si velis late arcuato-emarginatus. Venter viridicyaneus.

Sect. VI. Abdominis dorsum aureum, segmenti 3:tii margo apicalis cyaneus.

146. Chrysis analis Spinola. $\sigma^{7}$.

Mediocris robusta $2-3 \mathrm{lin}$. decimal. long. confertim punctata viridi-cyanea abdominis dorso aureo, segmenti 3:lii margine apicali cyaneo, dentibus brevibus triangularibus.

Chrysis analis Spin. Ins. Lig. 2.26: XXVI. secundum specimina typica ab III. Auctore mihi communicata.

IIabitat in Europa meridionali ex. gr. Italia et Hispania nec non in insulis maris mediterranei. In Liguria a D. Spinola jam ad initium hujus saeculi detecta. In insula Rhodo mense Majo a D. Loew semel observala. Ex Insula Malta quoque communicata.

Observ. Chrysidi ignitae habitu valde similis ut jam Ill. Spinola l. c. notavit; sed structura, armatura et 
pictura segmenti abdominalis dorsalis 3 :tii facillime et certissime dignoscitur.

Corpus robustum elongatum pubescens. Caput confertim modice punctatum subcoriaceum cyaneum; cavitas facialis subquadrata subprofunda nitida punctulato-coriacea medio canaliculata, supra caritatem adest lineola transversa elevata inaequales plus minus distincta, aliis transverso-arcuata, aliis transversa et utrinque deflexa, virescens aut cyanea. Antennae breves, non crassae, fuscae, basi virides aut viridi-cyaneae. Clypeus mediocris aut subbrevis transversus punctulatus inaequalis nitidus plus minus viridi-pictus, ad centrum marginis apicalis late et lenissime arcuato-emarginatus. Mandibulac et reliquae oris partes normales. Thorax confertim punctatus subcoriaceus, aut unicolore cyaneus aut stria transversa pronoti, stria longitudinalis in utraque dorsuli area laterali, scutellum et postscutellum, virescentia, qui color rarissime in aureo-viridem abit, in exemplaribus ita pictis etiam vertex aureo-viridis macula centrali cyanea; pronoti sulcus medianus profundus non continuus; thoracis dorsum parum convexum, dorsuli suturae medianae mediocres subrectae 1. parumper curvae; metanoti anguli postico-laterales robusti latiusculi triangulares; pectus et pedes 1. unicolore cyanei 1. viridi-cyanei; tarsi fusci, subtus subtestacei; alae aut hyalinae aut subhyalinae scil. disco et ad costam cellulae radialis interdum plus minus fumcosordidae; venae firmae in majoribus individuis, in minoribus mediocres, semper piceae; cellula radialis lanceolata subcompleta. Abdomen subellipticum elongatum, capite thoraceque simul sumtis longius, dorso modice convexum plus minus nitidum confertissime punctatum, I. viridi- I. 
rubro-aureum margine basali segmentorum 2:di et 3:tii nigro-aeneo, margine apicali segmenti 3 : tii cyaneo; segmenti 3:tii area antica abrupte convexiusculo-terminata; series ante-apicalis interrupta sub limite $I$. termino areae anticae ex parte abscondita, foveolae numerosae profundae subovatae approximatae; margo apicalis mediocris prominulus parun convexus punctulato-coriaceus irregulariter subtrapezinus; dentes apicales breves subacute triangulares; emarginaturae mediocres leniter arcuatae, centralis recte transversa, externac I. conformes 1 . obliquae, hoc in casu dentes externi paullum postpositi h.e. tunc non in una et eadem linea siti ac dentes intermedii; margo proprius lateralis obliquus, aliis subrectus aliis lenissime flexuosus. Venter viridi-cyaneus fornicatus, interdum nigro - maculatus.

147. Chrysis scutellaris Fabr. $\sigma^{7}+$.

Mediocris circ. $2 \frac{1}{2}$ lin. decimal. long. confertim modice punctata viridi-cyanea scutello abdominisque dorso aureis, segmenti 3:tii margine apicali cynneo, dentibus: maris omnibus brevissimis obtusis, feminae externis brevibus triangularibus et intermediis oblusis brevioribus.

Chrysis scutellaris Fabr. E. S. S. 257:4-5, et Piez, 171:5, secundum specimina typica et in Museo R. Havniensi et in Collectione Fabriciana Musei Universitatis Kiel. anno 1847 a me lustrata; - Dlbm. Dispos, 1845. $10: 12$.

Cltrysis segmentata Dlbm. Mon. Chr. 1829. $9: 4$.

Chrysis Germari Ziegl, secundum Mus. Spinolae.

Habitat in variis Europae regionibus rarius; Italiae, D. Spinola; Austriae ad Viennam, D. Kollar; Daniae ad Havniam, D. Westermann; Sveciae in Scania tantum bis lecta, scil. a D. Eber\%. Munck af Rosenschöld 1829 
ad Kåseberga et a ne in Sandhammar d. 14. Aug. 1838 in floribus.

Observ. Chrysidi bidentatae affinis et similis; at corpore magis nitido et minus crasse punctato, scutello abdominisque dorso (praeter marginem hujus apicalem cyaneum) aureis facillime dignoscitur.

Corpus elongatum subangustum at nihilominus robustum fusco- et cano-pubescens confertim modice punclatum. Caput mediocre rotundato-triangulum aut potius suborbiculatum cyaneum, ante stenma anticum inter oculos 1. maculis tribus 1. fasciola unica transverso-arcuata viridissimis; cavitas facialis mediocris subquadrata aut subtrapezina nitida medio canaliculata ceterum normalis, superne non nisi obsoletissime marginulata; antemnae breves fuscac, basi viridi-cyaneae; clypeus mediocris transversus subtrapezinus punctulatus viridi-cyaneus, disco nonnihil convexus, margine apicali proprio depresso prominulo polito nigro-piceo subtransverso aut lenissime late arcuato-emarginato; mandibulae, maxillae et palpi fuscopiceae. Thorax cyaneus dorso parum convexus; pronotum antice utrinque striola transversa, dorsuli areae laterales interdum striola longitudinali viridibus; in pronoti truncatura antica foveolae 2 centrales interstitio polito nigro-aeneo; sulcus pronoti medianus 1. profundus 1 . obsoletus, non continuus; dorsuli suturae medianae crenulatae subrectae aut parumper curvae; scutellum 1. rubro1. viridi-aureum; postscutellum viridissimum, interdum aureo-resplendens; mesopleurae viridi-maculatae; sternum \& cyaneum, $\sigma^{\top}$ viridi-cyaneum; pedes \& cyanei, $\delta$ cyaneo-virides, tarsis utriusque sexus brumeis tamen dilutioribus in $\sigma^{7}$; metanoti anguli postico-laterales mediocres 
triangulares; alae subhyalinae, disco paullulum sordidae, venis firmibus piceis, cellula radiali lanceolata incompleta. Abdomen elongatum subovale capiti thoracique simul sumtis ad longitudinem aequale, dorso modice convexum et confertim modice punctatum I. viridi-1. rubro-aureum, margo basalis segmenti 2 :di et interdum quoque segmenti 3:tii tenuiter nigro-aeneus; segmentum 3:tium similiter ut in Chrysidi anali constructum; margo apicalis cyaneus, in $\delta$ multo brevior quan in 9 , dentes apicales brevissimi obtusi et emarginaturae lenissime arcuatae, unde totus hic margo apud $\delta$ leniter undulato-flexuosus obvenit; $f$ margo apicalis (segmenti $3:$ tii) mediocris prominulus $\mathrm{hr}$ subtrapezinus, dentes externi breves distincte triangulares, intermedii breviores obtusi, emarginaturae mediocres leniter arcuatae; series ante-apicalis apud utrumque sexum interrupta, foveolae numerosae profundae magnitudine mediocres ovatae aut subtriangulares. Venter modice fornicatus viridi-cyaneus.

\section{Chrysis aeruginosa Klug. ㅇ.}

Mediveris 2 lin. decimal. long. confertin punctulatosubcoriacea sublus nigro-cyanea supra viridi-cuprea, abdominis segmenti 3:tii margine apicali cyaneo, dentibus brevibus distinctis, externis triangularibus, intermediis oblusis.

Clerysis aeruginosa Klug., secundum specimen in Museo Berolinensi asservatum et ibidem anno 1847 a me descriptum.

Habitat in Prussia rarius, ad Berolinum a Cel. Klug detecta.

Observ. Habitus omnino Cluysidls succinctue. Ca- 


\section{Famil. Chrysididae. Chrysis: Phalanx VI.}

put viride fronte cyanea, vertex confertim subtiliter punctato-rugulosus aut subreticulatus. Clypeus mediocris aut subparvus inaequalis transversus viridis, margine apicali nigro-fusco transverso vix emarginulato. Mandibulae basi nigro- apice rufo piceae. Thoracis dorsum convesiusculum modice punctato-subreticulatum viridi-cupratum, metanoto pectore pedibusque cyaneis, illo (metanoto) suturis et angulis virescentibus. Abdominis dorsum modice convexum, confertim modice punctatum viridi-cupratum; segimentorum 1:mi et 2:di margo basalis nigro-aeneus; segmentum 3:tium medio leniter et transverse concaviusculo-immersum, at iterum - ante seriem - modice convexiusculum; series ante-apicalis foreolis parris rotundis circ. 12 distinctis, per carinulam medianam intercedentem brevissimam interrupta; margo apicalis prominulus sparse punctulatus viridi-cyaneus. Venter niger, cyaneo- et riridi-maculatus.

Sect. VII. Abdominis dorsum aureum, segmenti 3:tii margo apicalis aeneus (rarissime rirescens, hoc tantummodo in varietatibus masculis Chrysidis succinctae).

149. Chrysis succincta Linn. $\delta$ ㅇ․

Parva aut mediocris $1 \frac{1}{2}-2 \frac{1}{2}$ lin. decimal. long. confertim modice punctata viridi-cyanea, dorsulo l. tolo $l$. saltem areis lateralibus abdominisque dorso aureis. segmenti 3:tii margine apicali $l$. aeneo $l$. virescente, distinctius aut obsoletius 4-denticulato, dentibus l. triungularibus $l$. oblusis, margine laterali infra medium $l$. 
leniter 1. lenissime angulato, inter angulum et dentem apicalem plus minus distincte emarginato.

Chrysis succincta Lin. S. N. 2. 947:3; Fabr. E. S. 2. $241: 13,-$ Piez. 174: 19; Panz. Fn. Germ. 77: 16; Dlbm. Mon. Chr. 10:5 (exclus. Synonym. Chrys. nitidulae Ahrens. Fn. 4:12, quae Hedychrum fervidum $=$ Chrysis fervida Fabr. esse videtur), - Dispos. 1845. 10 : 10.

Chrysis cellaris De Crist. Mus, Spinolae.

Chrysis succinctula Spinol. Mus. Vienn. teste D. Kollar in litt.

Irabilat in locis sabulosis nec non in floribus umbellatarum totius Europae, Lapponia excepta; in regionibus meridionalibus parce, in Europa boreali autem ex. gr. Dania, et Svecia et Norwegia mensibus Junio, Julio et Augusto subfrequenter obveniens.

Observ. Corpus angustum at nihilominus proportionaliter robustum, elongatum pubescens confertim modice punctatum. Caput potius quadrato- quam triangulariter rotundatum, punctulato-subreticulatum, aliis cyaneum et antice aut viride aut cyaneo-virescens, aliis laetius viride et tantuminodo postice sublusque cyanescens, color viridis interdum ex aureo splendet; cavitas facialis mediocris quadrata profunda, 1. disco polita nitidissima et limbo punctulato-coriacea 1. tota punctulato-coriacea; in frontis convexitate, antequam haec in caritatem facialem abit, apud quaedam specimina adest carinula transversa 1. tantum in vestigio 1 . plus minus explicata, hoc in casu tamen inaequalis et irregularis; antennae breves fuscae, basi virescentes et nomnunquam aureo-nitidac, flagellum robustum subcrassiusculum; clypeus transversus brevis latus punctulatus viridi-cyanco- et nigro-aeneo-pictus, disco carinalus, ad centrun marginis apicalis plus minus distincte angulato-emarginatus, marginibus lateralibus obli- 
quis; mandibulae et reliquae oris partes normales. Thorax cylindricus confertim modice punctatus, dorso depresso-convexus, aliis viridis, aliis viridi-cyaneus, aliis pure cyaneus; omnibus autem (saltem 50 a me examinatis) pronotum antice fasciola raro continua, plerumque interrupta l. viridissima l. viridi-aurata; dorsulum plerisque aut viridi-aut rubro-aureum nitidum, paucissimis area media viridi-aenea; scutellum et metanotum $\mathrm{l}$. cyanea $\mathrm{l}$. viridi-cyanea 1. viridia; metanotum plerumque gibbo-convexum, angulis postico-lateralibus longiusculis angustis acute triangularibus; pectus raro totum cyaneum aut totum viride, plerumque viridi-cyaneum; pedes pictura valde variantes scil. 1. viridissimi tarsis albo-testaceis, 1. aureovirides tarsis brunneo-testaceis, 1. cyaneo-virides, tarsis brumneo-testaceis, 1. aeneo-purpurascentes femoribus infra ex parte viridi-nitidis, tarsis laete brunneis; alae hyalinne, ad costam cellulae radialis plus minus distincte fumatosordidae, venis brunneis et secundum proportionem atque magnitudinem reliquarum corporis partium aut mediocribus aut tenuibus, cellula radiali triangulariter lanceolata aliis subcompleta aliis et plerisque distincte aperta ideoque incompleta. Abdomen subovale aut potius subellipticum capite thoraceque vix I. parumper longius, dorso modice convexum et modice punctatum nitidum 1. viridi1. cupreo-1. igneo-1. coccineo-aureum margine basali segmentorum 2:di et 3:tii nigro-aeneo, margineque apicali segmenti 3:tii 1. viridi- 1. brunneo- 1. nigro-1. purpurascenti-1. cupreo-aeneo; in segmentis $2:$ do et $3:$ tio lineola mediana rarius omnino oblitterata, plerumque subtilissime carinulata, aut segmentis concolor aut viridi-aut nigro-aenescens; segmenti 3 :tii series ante-apicalis in ipso 
areae anticae termino declivi sita, interrupta; foveolae: $\mathbf{I}$. separatac numerosae profundae ovales, -1 . hinc inde confluentes et tunc inaequales pauciores et majores et minores; margo apicalis paullulum humilior quam segmenti regio basalis et maxima, depresso-convexus nitidus parce punctulatus, in variis individuis plus minus prominulus; dentes apicales eximie variantes ex. gr. in aliis (optima forna ) distincti, externi breviores triangulares, intermedii magis prominuli et obtusi, emarginaturae mediocres: em. centralis minus profunda et paullulum angustior, emarginaturae laterales obliquae et latiores, - in aliis dentes intermedii propter emarginaturam leviorem obsoleti, in aliis omnes dentes propter emarginaturas aut leves aut levissimas ita abbreviati obtusati et obsoleti ut margo non ulterius dentatus sed re vera leniter undulatus restat; margo lateralis aliis (forma oplima) infra centrum distincte angulatus et inter angulum et dentem lateraliapicalem oblique modice arcuato-emarginatus, quae tamen forma angulata per varios ut ita dicam transgressus sensim explanatur, quo facto etiam emarginatura illa memorata fit minus evidens. Venter modice fornicatus nitidissimus, raro totus viridis aut viridi-cyaneus, sacpissime aut igneus et nigro-aeneo-maculatus aut nigro-aeneus et splendidissime complissimeque igneo-aureo-pictus.

150. Chrysis Grohmanni Spinola. ㅇ.

Mediocris robusta $2_{4}^{\mathrm{L}}-2_{3}^{2}$ lin. decimal. long. confertim punclata cyanea, dorsuli areis lateralibus abdominisque dorso aureis, segmenti 3:tii margine apicali aeneo, dentibus robustis spinoideis subapproximatis, margine laterali arcuato. 
Chrysis Grohmanni Mus. Spinolae.

Chrysis gloriosa Dalman. Mus. Paykulli; Dlbm. Dispos. 10: 11.

Habitat in Sicilia, a D. Grolımam olim detecta, Mus. 1. Spinola; in eadem insula nuper a D. Scliodte revisa.

Observ. Chrysidi succinctae maxime affinis et similis, at facillime certissimeque dignoscitur: corpore crassiore, fortius punctato, capite thoraceque cyaneis (vertice et pronoto antice viridi aut aureo-viridi-pictis), dorsuli areis lateralibus aureis: area media ad suturas virescente, pedibus viridi-cyaneis tarsis fuscis, alis sordide hyalinis: venis firmibus piceis, cellula radiali elongata perfecte lanceolata subcompleta, abdomine robusto ovato, segmenti 3:tii dentibus apicalibus robustis triangularibus subspinoideis, emarginaturis latitudine mediocribus at profunde arcuatis, externis obliquis et paullulum latioribus quam centrali, hinc dentibus externis postpositis b. e. non in una eademque linea ac dentibus intermediis; margine laterali (segmenti 3:tii) elongato obliquo leniter arcuato; ventre igneo nigro-aeneo-maculato. In ceteris a Chryside succincta non differt.

151. Chrysis Schousboci Dlbm.

Mediocris circ. 2 lin. decimal. long. confertim modice punctala viridi-cyanea thoraceque abdomine dorso aureis, segmenti 3:lii margine apicali acneo 4-angulalo.

"Chrysis gloriosa e 'Tanger D. Schousboe" Cullect Seȟestedti Musei R. IIavniensis, secundum unicum specimen guod ibiden anuo 1847 examinavi.

IIabitat in Tanger, a D. Schousboe olim detecha, Mus. R. Havniensi. 
Famil. Clrysididae. Clerysis: Phalanx VI. 273

Observ. Magnitudo et habitus omnino Chrysidis succinctae formae optimae; ab hac rero dignoscitur: dorso thoracis toto aureo, abdominis segmenti 3:tii margine apicali $\bigsqcup$ per truncaturas laterales obliquas et media recta trapezii instar quadri angulato; serie anteapicali foveolis irregularibus, aliis distinctis, aliis confusis, medio per interstitium brevissimum interrupta. Ceterum Chrysidi succinctae simillima. Caput cyaneum viridi - maculatum. Venter nigro-aeneus, viridi- et cyaneomaculatus.

\section{Chrysis Drewseni Dlbm. $\sigma^{7}$ ?}

Magna robusta 3 lin. decimal. long. conferlim crasse punctatn subfus viridi-cyanen supra aurea, segmenti $3: t i i$ margine apicali viridissimo dislincte 4-dentato dentibus brevibus, externis acute triangularibus, intermediis latis obfusissime arcuatis.

Habitat in Africa meridionali, ad Promontorium bonae spei a D. Drrege detecta, Mus. D. Drewsen.

Observ. Habitus fere Clirysidis bidentatae. Corpus robustum elongatum sublineare obtusum pubescens. Caput latiusculum orbiculatum confertim modice punctatun subreticulatum viridi-aureum partibus faciali ovali et occipitali viridi-cyaneis; cavitas facialis quadrata subtiliter punctulata planiuscula lateribus albo-sericea; antennac breves fuscae, basi viridi-cyaneae; clypeus mediocris transversus trapezinus punctulatus nitidus, disco parum convexus, margine apicali piceo transwerso. Mandibulae (praeter maculam baseos viridem), maxillac palpique fusco-piceae. Thorax rectangulariter subcylindricus, confertim crasse punctato-reticulatus, subtus viridi-cyaneus, 


\section{Famil. Clarysididae. Chrysis: Phalanx VI.}

dorso parum convexo aureus; pronoti truncatura antica declivis coriaceo-rugulosa, cyanea, viridis, et aureo irrorata foveis centralibus 2 parallelis ovalibus planis politis viridibus; metanoti anguli postico-laterales robusti acute triangulares; pedes viridi-cyanei, tarsis basi fuscis, apice totoque latere infero brunneo-testaceis; alae sordidae, venis firmibus piceis, cellula radiali triangulariter lanceolata incompleta, apice modice aperta; tegulae alarum viridi-cyaneae. Abdomen ovale aut potius obtuse subrectangulum, longitudine capitis thoracisque simul sumtorum, dorso modice convexum et confertim punctatun nitidum rubro-aureum, limbo nonnihil viridi-micans; segmentum 3:tium totum depresso-convexum, series ante-apicalis irregularis et obsoleta scil. foveolis tantum ad latera detegendis, medio autem cum reliqua punctura commixtis; margo apicalis viridissimus, non nisi obsoletissime humilior quam regio antica et hujus puncturam praebens, dentes externi breves acute triangulares valde remoti, intermedii breves lati obtusissime arcuati; emarginatura centralis lata lenissima, emarginaturae externae breves obliquae modice arcuatac, unde ipse margo fere hanc formam ostendit; margo lateralis leniter curvus.

Sect. VIII. Abdominis dorsum unicolor: aut aureo-viride, aut viridi-aureum, aut pure aureum.

Divis. 1. Tarsi albo-testacei.

153. Chrysis armena Spinola. $\sigma^{7}$.

Mediocris 2 lin. decimal. long. et ultra, crassa obtusa suberasse punctata laete viridis, verlice dorsulo abdominisque breviusculi dorso laete viridi-auratis, pedibus 
Famil. Clrysididae. C'hrysis: Plutanx VI. 275

aureo-nilidis tarsis albo-teslaceis, segmenti 3:lii serie ante-apicali oblitterata, margine laterali: angulo subacuto.

Chrysis armena Mus. Spinolae.

Mabilat in Armenia, a D. Osculati detecta, Mus. D. Spinola.

Observ. Valde similis Chrysidi manicatae nob., quae forsitan ut varietas a quibusdam Entomologis existimetur; at Chrysis armena differt: corpore breviore et proportionaliter crassiore, abdomine breviore, hujus segmenti 3:tii margine laterali ad centrum angulo acutiusculo ctc. - Caput laete viride, pone stemmata aureovirescens; facies planiuscula subquadrata, pube albo-sericea largiter obducta, superne transverse marginata: margine inaequali. Antennae elongatae robustae, basi aeneovirides, flagellum supra fuscum, subtus luteum. Clypeus magnus longiusculus inaequaliter depresso-convexus viridis nitidus, punctulis paucis adspersus, margine apicali arcuato fusco. Mandibulae testaceae, margine basali virescentes, apice nigricantes. Palpi fusco-brunnei. Thoracis dorsum crasse punctato-subreticulatum. Pronotum obtusum, scutellum et postscutellum viridia, aureo-nitida ; mesopleurae, et metanoti anguli postico-laterales, auratovirides; metanotum viride. Dorsulum, pectus, pedes et venter viridi-aurea. Tegulae majusculae viridi-cyaneae. Tibiarum apices, calcarin tarsique albo-testacea. Alae subhyalinac, venis firmibus fuscis, cellula radiali lanceolata incompleta h. e. apice distincte aperta. Abdomen subbreve latiusculum, dorso convexum confertim subcrasse punclatum aureo-riride aut viridissime aureum; segmenti 1:mi impressio basalis amplior (quum in Clryside mamicala) et margine postico inaerualis; segmentum 3:tium 
aureo-viride, confertius modice punctatum; series anteapicalis oblitterata, per lineolam obsoletam tantum inchoata, foveolae inter reliqua segmenti puncta solummodo perpaucae et quidem difficile observari possunt; dentes apicales robusti, magnitudine mediocres, aequales acuti a latere paullulum remoti, interstitiis perfecte arcuatis; margo lateralis ad centrum angulo parro subacuto.

154. Chrysis manicata Dlbm. ð’ㅇ.

Submagna $3 \frac{1}{3}-3 \frac{1}{2}$ lin. decimal. long. robusta ovatooblonga crasse punctata cyaneo-viridis, abdomine longiusculo viridi-aureo, ventre nigro-, viridi-et igneopicto, pedibus viridibus, tarsis ( $\sigma^{\top}$ ) albo-testaceis (아 brunneis), segmenti 3 :tii serie ante-apicali distincta, margine laterali mutico (non acutangulo).

Habitat in insula Rhodo, mense Majo a DD. Hedenborg et Loew detecta. Mus. D. Loew.

Observ. Valde similis Chrysidi armenae Spin.; sed corpore longiore, thoracis dorso cyaneo-riridi nec aureo-maculato, serie ante-apicali distincta, segm. 3:tii margine laterali non angulato sed lenissime arcuato, rentre variegato, pedibus viridibus etc. ut propria species certe distinguitur. Cilypeus margine apicali nigro-aeneo polito prominulo non arcuatus sed transversus. Thorax viridis, dorsuli area media omnibusque suturis et dorsalibus et pectoralibus cyaneis. Antennac fuscae, basi virescentes, $\sigma^{\top}$ longiusculae, + breves. Alae byalinae, disco et in cellula radiali nomnihil sordidae, venis firmibus fuscis, cellula radiali triangulariter lanceolata incompleta, apice modice aperta. Abdominis segmenti 3 :tii series ante-apicalis distincta, foveolis irregularibus, majoribus 
Famil. Chrysididae. Chrysis: Platanx VI. 277

el minoribus simul sumlis circ. 12; dentes apicales robusti triangulares acuti, externi non a latere remoti sed angulum laterali-apicalem simul efficientes.

Divis. 2. Tarsi fusci.

Subdivis. 1. Abdominis segmenti dorsalis 3 :tii dentes: laterales, h. c. margo proprius apicalis convexo-arcuatus, utrinque dente triangulari munitus, supra hunc dentem adest in centro marginis lateralis - angulus dentiformis.

155. Chrysis angulata Dlbm. ㅇ.

Mediocris $2 \frac{1}{3}$ lin. decimal. long. subangusta punctala cyaneo-viridis, pronoto, mesonoto abdominisque dorso cupreis, pedibus viridibus tarsis fuscis.

IIabitat in Asia minori, a D. Loew detecta; Mus. Loewi.

Observ. Ad habitum simillima Chrysidi succinctae, et cum hac facile confundenda, nisi armaturam ani singularem respicias. - Caput suborbiculatum, confertim punctulatum viride, vertice cupreo-aeneo irrorato; cavitas facialis subquadrata profunda nitida subtiliter punctulata, superne non marginata; antennae mediocres fuscae, basi virescentes; clypeus mediocris margine apicali nigroaeneo transverso; mandibulae basi virides, medio et apice nigro-piceae, ante apicem brunneae; palpi fusci. Thoracis dorsum crassissime punctato-reticulatum; pronotum cupreum: sulco mediano profundo postice abrupto, truncatura antica lateribusque cyaneo-viridibus; dorsulum cupreum: suturis 2 medianis cyancis, area media basi vi-

$1 \mathrm{I}$. 
rescente; scutellum cupreum viridi-marginatum; postscutellum disco lacte viride, lateribus cyaneum; metanotum cyancum suturis et angulis subaureo-nitido viridibus; pectus cyaneum viridi-resplendens; pedes cyaneo-rirides tarsis fusco-brunnescentibus; alae subhyalinae, cellula radiali sublanceolata incompleta, apice modice aperta, venis firmibus piceis. Abdominis dorsum modice convexum cupreum; segmentum 1 :mum aureo-rirescens, basi crassius punctatum, apice punctulatum; segmentum 2:dum subconfertim punctulatum, margine basali tenuiter nigroaeneo; segmenti 3:tii margo proprius apicalis transrersus, leniter arcuato-obtusus, utrinque dente laterali robusto triangulari; margo (segmenti 3:tii) proprius lateralis superne lenissime arcuatus, inferne - inter centrum et dentem laterali-apicalem - distincte late arcuato-emarginatus, unde ad centrum angulus exstat mediocris dentiformis; linc margo lateralis utrinque bidentatus et margo proprius apicalis revera muticus restat. Venter pedibus concolor.

Subdivis. 2. Abdominis segmenti dorsalis 3:lii dentes: apicales.

Turba 1. Abdominis segmentum dorsale 3:tium carinula mediana distinctiori, utrinque concaviusculodepressum.

156. Chrysis inaequalis Dlbm. $\sigma^{\top} q$.

Mediocris $2 \frac{1}{2}$ lin. decimal. long. robusta confertim punctata cyanea abdomine virescenti-aureo, segmenti 
Famil. Chrysydidae. Chrysis. Phalanx VI. 279

3:tii serie ante-apicali foveolis profundis rotundatis magnitudine mediocribus, dentibus mediocribus approximatis l. triangularibus $l$. subspinoideis.

Chrysis inaequalis Dlbm. Dispos. 1845. 8:4. ㅇ․

Chrysis ignita var. Mus. Spinolae.

Habitat in Gallia meridionali, D. Latreille, Mus. D. Spinolae; in Italia, D. Zeller; in Bosphoria, D. Medenborg.

Observ. Affinis et similis Chrysidi ignitae, at ab hac aliisque affinibus structura segmenti abdominalis dorsalis 3:tii mox distinguitur.

Corpus robustum crassum obtusum largiter cinereofusco pubescens. Caput et thorax cyanea, confertim modice punctata parum nitida. Caput rotundato-triangulum robustum; cavitas facialis rectangularis planiuscula, non profunda, subtilissime transverse strigosa et punctulatocoriacea, medio canaliculata, superne fortiter marginata: margine acuto leniter transverse arcuato, ceterum tota nitida viridi-cyanea; antennae mediocres fuscae, basi viridi-cyaneae; clypeus concolor transversus brevis, punclulatus nitidus, medio nonnihil gibbo-convexus, utrinque leniter concaviusculus, ad centrum marginis apicalis subtransversus l. potius lenissime arcuato-emarginatus; labrum minutum semilunatum gibbum ciliatum, mandibulae palpique nigro-picea, macula mandibularum basali cyanea plus minus virescente; margo occipitalis utrinque prope basin angulo spinoideo munitus. Thorax robustus rectangularis convexus; mesopleurarum uterque apex emarginato-bidentatus; metanoti anguli postico-laterales magni lati acuminati, margine externo l. antico convexo-, postico concavo-arcuali; stcrnum et pedes viridi-cyanei 
tarsis fuscis; alae sordide hyalinae, venis firmibus piceis, cellula radiali triangulariter lanceolata incompleta apice anguste aperta. Abdomen robustum subovale, dorso sat convexum confertim crasse punctatum 1. viridi- 1. rubroaureum, margine basali segmentorum 2:di et 3:tii tenuiter nigro-aeneo; carinula mediana in segmentis 2 : do et 3: tio continua eximie distincta; segmenti 3 : tii regio basalis et maxima semicircularis, disco - utrinque ad carinulam - concaviusculo-depressa, tum mox ante seriem convexo-terminata; series ante-apicalis propter carinulam continuatam interrupta, foveolae profundae rotundatae magnitudine mediocres, aliis separatae et tunc pauciores, aliis approximatae et numerosae; regio (segmenti $3:$ tii) postica (= margo proprius) mediocris humilior nitidus punctulatus, dentes subapproximati robusti acute triangulares subspinoidei, emarginatura centralis anguste et profunde arcuata, emarginaturae externae nonnibil latiores et minus profundae, hinc dentes intermedii majores quam externi; margo lateralis valde obliquus, basi breviter convexo-, tum longe et lenissime concaro-arcuatus. Venter viridi-cyaneus.

Turba 2. Abdominis segmentum dorsale 3 : tium modice depresso - convexum.

Manipulus 1. Abdom. segmenti 3:tii margo apicalis nonnihil anguslatus, dentes intermedii prominuli valde approximati.

157. Chrysis pyrophana Klug. ㅇ.

Submagna ovato-oblonga vix $3 \frac{1}{2}$ lin. decimal. long. 
Famil. C'rysididae. Chrysis: Plualanx VI. 281

subcrasse punclata cyanea, fronte, pronoto, dorsulo, abdomineque supra, aureis; ventre nigro igneo-maculato.

Chrysis pyrrophana Klug. in Museo Berolinensi, ubi specimen anno 1817 descripsi.

Habital in Gallia meridionali, teste D. Klug I. c.

Observ. Habitus fere Chrysidis ignilae majoris, at per picturam thoracicam et armaturam analem species distinctissima. - Caput suborbiculatum purpureo-aeneum; cavitas facialis profunda igneo-aurea, lateribus confertim punctata, disco laevissimo polito punctis perpaucis adsperso; antennae breviusculae fuscae, scapus aeneus; clypeus huic concolor brevissimus, apice emarginato-truncatus 1. potius leniter angulato-emarginatus; mandibulae nigro-piceae, ante apicem subtestacea; palpi brunnei. Thorax cyaneus, suturis metanoti pectorisque nonnihil virescentibus; dorsum thoracis confertim modice punctatoreticulatum; pronotum et dorsulum viridi-aurea, illius truncatura antica violacea; pedes viridi-aenei tarsis brunneis; alae sordide hyalinae venis brunneis, cellula radiali lanceolata incompleta, apice modice aperta. Abdominis dorsum confertius modice punctatum, punctis multo minus crassis quam in thorace; segmenta $1:$ mum et 2:dum viridi aurea, hujus margo basalis tenuissime niger: segmentum 3:tium cupreum modice punctato-subreticulatum convexo-depressum, lineola mediana subelevata subplaniuscula in hoc - ut etiam in segmento 2:do - observanda; series ante-apicalis foveolis mediocribus rotundatis distinctis circ. 12, per carinulam medianam interrupta; segmenti 3:tii regio postica s. apicalis proportionaliter distincte angustior quam in aliis hujus Subdivisionis speciebus, unde etiam dentes magis approximati magisque 
prominuli obveniunt; margo apicalis prominulus convexodepressus sparse punctatus; dentes intermedii prominuli robusti obtusiusculi approximati, emarginatura inter hos dentes anguste arcuata; dentes externi pone illos - nec in illorum linea - siti, breviores latiores triangulares, utraque emarginatura inter dentem externum et medium obliqua late arcuata; marginis apicalis summus finis nigro-aeneus.

Manipulus 2. Abdominis segmenti dorsalis 3: tii margo apicalis latitudine mediocris, non angustatus, dentes omnes in unam lineam plus minus curvam dispositi.

A. Segmenti 3:tii dentes externi ab angulo laterali remoti, hoc angulo arcuato-obtuso.

158. Chrysis distinguenda Spinola. $\sigma ㅇ$.

Mediocris aut submagna $2 \frac{1}{2}-3$ lin. decimal. long. robusta confertim punctata 1 . viridi-cyanea l. cyanescentiviridis, abdominis dorso viridi-anreo, segmenti $3:$ tii serie ante-apicali foveolis numerosis profundis rotundatis mediocribus, dentibus plerumque brevibus triangularibus, emarginaturis modice arcuatis aequalibus.

Chrysis distinguenda Mus. Spinolae (non Annales Entomol. 18.38. 450:VII. quac species est toto corpore cyanco-viridis).

Habilat passim: in Gallia meridionali, D. Dufour: - in Austria, D. Kollar; - Italia, D. Spinola et Zeller; - in Sicilia, D. Drewsen et Kollar; - in Insula Rhodo, D. Loew.

Observ. Valde similis et aftinis Clurysidi ignilae 
Famil. Clhrysididae. Chrysis: Plualanx VI. 283

et Chrysidi austriacae; unde ab aliis ut Chrysidis ignitae et ab aliis ut Chrysidis austriacae varietas existimata fuit, ab ambabus autem structura segmenti abdominalis dorsalis 3 : tii valde diversa.

Corpus robustum obtusum crassum valde fusco- $\mathrm{e}^{t}$ cano - pubescens. Caput rotundato - triangulum confertim aut punctato- aut punctulato-rugulosum subreticulatum, rarius viride, plerumque cyaneum; cavitas facialis quadrata non profunda, punctulato-coriacea nitida $l$. viridis 1. viridi-cyanea, medio canaliculata, lateribus sericea, superne distincte marginata: margine transverso subrectangulari ad formam variabili 1 . recto $\square, l$. antice crenulato mry, l. antice concavo-arcuato $\sim$, I. subtra-

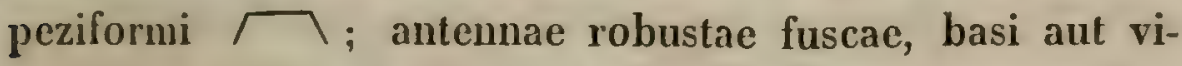
ridi-cyaneae aut rarius virides, maris elongatae, feminae mediocres aut subbreves; clypeus mediocris aut submagnus transversus subtrapezinus, depresso-planiusculus punctulatus nitidus cavitati faciali concolor, ad centrum marginis apicalis modice arcuato-emarginatus; labrum, mandibulae, maxillae palpique normalia, nigro-picea; margo occipitalis prope basin utrinque angulo prominulo spinoideo. Thorax crassus oblongo-quadratus confertim punctato-reticulatus, aut violaceus, aut cyaneus, aut viridi cyaneus, aut rarissime cyaneo-viridis, dorso depressoconvexus, postscutellun subgibbun aut saltem saepe margine postico-prominulum; melanoti anguli postico-laterales robusti latiusculi acute triangulares; pectus et pedes saepissime aut viridi-cyanei aut cyaneo-virides, tarsis fuscis; alae sordide hyalinae venis firmibus piceis, cellula radiali aut lanceolata aut subtriangulariter lanceolata incompleta apice anguste aperta. Abdomen obtuse sub- 
rectangulum plerumque curvum, thorace longius, capite thoraceque simul sumtis brevius, dorso modice convexum confertim punctatum aut viridi-aut rubro-aureum nitidum, margine basali segmentorum 2: di el 3:tii saepius nigro-aeneo; lineola mediana subelevata planiuscula in segmentis 2:do et $3:$ tio plus minus visibilis; segmenti 3: tii margo apicalis mediocris vix nisi rarius humilior quam regio antica, punctulatus, dentes ginaturae jam in diagnosi descriptae lateralis bis arcuatus: arcu supero brevi

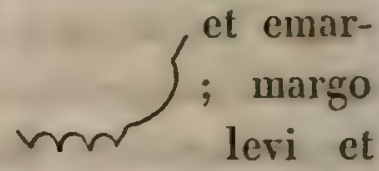
subperpendiculi, arcu infero maximo valde convexo obliquo. Venter aut viridi-cyaneus aut viridis et tunc nonnihil aureo-nitens, segmentorum marginibus nigricantibus.

B. (Abdominis) segmenti (dorsalis) 3 : tii dentes externi ipsum angulum lateralem efficiunt.

$\times$ segmenti $3:$ tii dentes breves, aut omnes aut saltem intermedii obtusi.

159. Chrysis comparata Lepel.

Submediocris 2 lin. decimal. long. viridi-cyanea, $a b$ dominis dorso cupreo basi viridi-aureo, segmenti 3 :lii serie ante-apicali foveolis parvis rolundis circ. 10, denlibus apicalibus perparvis triangularibus, intermediis oblusis, externis acutiusculis.

Clerys. comparata Lepel. secundum specimen typicum a D. Spinola communicatum.

Habitat in Gallia, Mus. Spinolae.

Observ. Descriptionem sufficientem dare nequeo, quandoquidem specimen typicum et mutilatum el mucore 
Famil. Chrysididae. Chrysis: Phalanx VI. 285

obductum est. Corpus submediocre toto dorso confertim modice punctatum. Venter riridi-aureus nigro-maculatus. Sternum cyanescens. Pedes virides tarsis brunneis. Alae subhyalinae venis fuscis, cellula radiali lanccolata incompleta, apice anguste aperta.

160. Chrysis verna Dlbm. 우.

Mediocris $2 \frac{1}{2}$ lin. decimal. long. cyaneo-viridis abdomine aureo, segmenti 3:tii serie ante-apicali foveolis profundis transversis confluentibus, dentibus apicalibus brevibus, intermediis obtusis, externis triangularibus, alis sordide hyalinis, venis firmibus fuscis, cellula radiali triangulariter incompleta apice anguste aperta.

IIabitat in insula Rhodo, mense Aprili a D. Loew detecta.

Observ. Praecedenti (Chrysidi comparatae) valde affinis, at ut hujus varietas difficile existimanda; etenim dignoscitur: corpore majore et robustiore, alis sordide hyalinis, cellula radiali triangulariter lanceolata, serie anteapicali (segmenti $3:$ tii) foveolis profundis transversis confluentibus e. s. p.

Corpus confertim modice punctatum. Caput viride, antice viridi- nec non occipite pure cyaneum, longiusculum rotundato-subrectangulum; cavitas facialis rectangularis non profunda, medio canaliculata, superne non marginata sed cum convexitate frontali confusa; antennae mediocres aut subbreves robustac fuscae, scapus aeneus; Clypeus mediocris transversus subtrapezinus punctulatus nitidus, disco leniter convexus, margine apicali piceo transverso. Pronotum, dorsuli areae laterales, tegulae, mesopleurae, postscutellum et metanoti anguli postico- 
laterales viridia; pronoti truncatura antica, dorsuli area media, metanotum et pectus cyanea. Pedes cyaneo-rirides tarsis fuscis. Abdomen dorso confertim punctatum modice convexum rubro-aureum, segmenti 1 :mi impressio basalis cyanea, postice viridi-marginata; segmenti margo apicalis mediocris prominulus, regione antica vix humilior et eandem atque haec puncturam - licet parciorem unltoque minus crassum praebens; dentes breves: externi latiusculi triangulares, intermedii lati arcuato-obtusi; emarginaturae ad latitudinem subaequales, at centralis Ienis et externac obliquae modice arcuatae, unde in hoc margine fere eandem formam videas quam apud Chrysidem Drewseni (no. 152) monstravimus.

\section{Chrysis aestiva Dlbm.}

Mediocris $2 \frac{1}{2}$ lin. decimal. long. conferlim modice punclata viridi-cyanea, abdominis dorso aureo, segmenti 3:tii serie ante-apicali foveolis mediocribus dislinctis numerosis, dentibus apicalibus brevissimis latis, extemis obtusiusculis, intermediis triangularibus, alis hyalinis venis fuscis, cellula radiali lanceolata subcompleta.

Habitat in insula Rhodo, mense Majo a D. Loew detecta. Mus. Loewi.

Observ. Valde similis Chrysidi vernae (proxime praecedenti), et forsitan ut varietas ejusdem existimatur; cui tamen opinioni abdominis segmenti 3 : tii series anteapicalis et armatura contradicunt. - Caput confertim punctulato-reticulatum, supra viride, infra cyaneum regione stemmatum subconcolore; cavitas facialis disco profunda politissima cyanea; antennac breves, apice fuscae, basi virescentes; clypeus brevissimus margine apicali angulato- 


\section{Famil. Chrysididae. Clerysis: Phalanx VI. 287}

emarginatus; mandibulae normales, basi parum viridipictae; palpi fusci. Thorax virescenti-cyaneus, dorso modice punctato-subreticulatus; pronoti regio postica utrinque, dorsuli areae laterales in disco, scutelli et postscutelli latera et metanoti anguli postico-laterales laete viridia; mesopleurae viridi-irroratae, tegulae et omnes reliquae thoracis partes virescenti-cyaneae; pedes secundum diversum lucem 1 . cyaneo-virides 1 . viridi-cyanei tarsis fuscis. Venter nigro-aeneus, segmentis apice maculis viridibus et igneis. Abdominis dorsum confertim modice punctatum et convexum saturate aureum; segmenti 1:mi impressio basalis normalis et segmenti 2: di margo basalis tenuis nigro-aenescentes; in segmentis 2:do et 3:tio ad certum situm luminis lineola mediana subelevata conspici potest; segmentum 3:tium medio, utrinque ad lineolam medianam subelevatam, lenissime transversaliter immersum, ante seriem convexiusculo-terminatum; margo apicalis mediocris parce punctatus regione antica distincte humilior; dentes apicales brevissimi lati angulos minutos efficientes; emarginatura centralis leniter transverse arcuata, emarginaturae externae latiores obliquae lenissime arcuatae, unde haec forma ${ }^{2}$ marginis apicalis exstat; margo lateralis obliquus.

\section{Chrysis impar Dlbm. $\sigma$.}

Subparva $1 \frac{2}{3}$ lin. decimal. long. cyaneo-viridis, abdomine viridissime aureo, segmenti 3:tii serie anteapicali foveolis parvis rotundis circ. 10, dentibus apicalibus parvis triangularibus, intermedie apice oblusis, alis hyalinis, cellula radiali completa. 
IIabilat in insula Rhodo, a D. Loew mense Aprili detecta; Mus. Loewi.

Observ. Valde similis et affinis Clerysidi comparalae (no. 159), quam mutilatam et mucore obductam rite examinare non potui. Structura et sculptura omnino Clerysidis comparatae. Caput et thorax laete viridia. Antennae robustae nigro-fuscae, basi virides. Clypeus apice leniter arcuato-emarginatus. Pronotum utrinque supra coxas fovea distinctissima ovata. Cavitas facialis subquadrata planiuscula superne distincte transverse arcuato-marginata. Pronoti truncatura antica et dorsuli area media cyaneac. Sternum et metanoti discus quoque cyanescunt. Pedes virides tarsis fuscis. Alarum venae brunneae, tantum costa et vena radialis firmae, reliquae tenues. Abdominis segmentum 1:mum aureo-viride, venter concolor, illius basis cyanescens; segmenta 2 :dum et 3:tium viridi-aurea; segmentum 3:tium totum modice subdepresso-convexum; series ante-apicalis interrupta, foveolac parvae profundae rotundatae circ. 10 distinclae; margo apicalis brevis punctulatus; dentes apicales parvi, omnes triangulares at intermedii summo apice obtusi, externi acuti; emarginaturae leniter arcuatae, centralis mediocres recte transversa, externae nonnihil obliquae et paullulum latiores; margo lateralis obliquus.

$\times x$ (Abdominis) segmenti (dorsalis) 3:tii dentes omnes robusti acuti: aut triangulares aut spinoidei:

163. Chrysis curvidens Dlbm. $\sigma^{7}$. Angusta subparva $1_{4}^{3}$ lin. decimal. long. conferlim 
Famil. Chrysididae. C'llrysis: Phalan.x VI. 289

modice punctata el punctulata viridi-cyanea pedibus ventreque concoloribus, abdominis dorso cupreo, segmenti 3:tii dentibus apicalibus: externis triangularibus, intermediis spinoideis arcuatis.

IIabitat in Europa meridionali; specimen unicum in nostra collectione adest.

Observ. Individuis parvis Chrysidis ignitae simillima et forsitan ut hujus varietas in Collectionibus determinata; sed quum inter 300 specimina Chrysidis ignitae a me examinata dentes segmenti 3:tii apicales semper rectos, nec in ullo alio quam in nostrae Chr.curvidentis unico specimine dentes intermedios leniter arcuatoincurvos observavi, hanc formam potiori jure ut propriam speciem proponere potuissem. Structura et sculptura corporis omnino Chrysidis ignilae. Abdominis dorsum cupreum confertim punctulatum, segmenti 2: di margo basalis nigro-acneus; segmenti 3 :tii series anteapicalis foveolis parvis rotundatis circ. 10 distinctis; margo apicalis mediocris, regione antica non humilior, dentes: hn externi recti triangulares latiusculi, intermedii spinoidei apice nomnihil incurvi, emarginaturae intermedia suborbiculata, externae leniter arcuatae obliquae et intermedia paullulum latiores.

164. Chrysis auripes Wasmaël. ర゚

Mediocris aut magna $2 \frac{1}{2}-4$ lin. decimal. long. confertim modice punctata viridi-cyanea, abdominis dorso creberrime punctulato aut viridi- aul rubro aureo: pedibus posterioribus antice, ventreque concoloribus (; segmenti 3:tii dentibus apicalibus triangularibus rectis). 
Chrysis auripes Wesmacil Not. 9:2.

Chrysis ignita Dlbm. Ex. Hym, 28:2. var. c.

IIabilat forsitan in plurimis Europae regionibus, licet adhuc (quantum constat) tantummodo in Belgia a D. Wesmrël juxta Bruxellam, in Gallia a D. Loew, et in Svecia a nobis lecta. Intra Sveciam solum 6 exemplaria observavimus scil. in trunco exsiccato horti Academici Lundensis d. 24. Mai 1831, in Blekingia ad Skillinge in loco arenoso inter plantas Solani tuberosi d. 22. Julii 1841, in floribus ad Wamblingbo Gottlandiae d. 5. Julii 1841 nec non in Jemtlandia ad Berge d. 6. Augusti 1810 in ligno sicco vermisecato.

Observ. Individuis majoribus et magis pictis Chrysidis ignitae nimis affinis et simillima; quae similitudo adeo fallax est, ut ambas species certe determinare non possis, nisi pedum ventrisque picturam accurate respicias.

Corpus robustum elongatum obtusum sublineare largiter fusco- et cano-pubescens. Caput robustum latum rotundato-quadratum confertim punctulato-coriaceum cyaneum; vertex amplus convexo-depressus transverso-rectangularis 1. unicolor 1 . ad singulum quodque stemma macula parva aut viridissima aut aureo-viridi, rarius (saltem in nostris exemplaribus) hae maculae ita ampliantur ut antice efformant fasciolam transversau tunc (CO) triramosam; cavitas facialis lata quadrata planiuscula nec profunda, medio canaliculata, superne transverse marginata: margine acuto-inaequali aut irregulariter transverso $\longrightarrow$, aut uni-arcuato $\frown$, aut bi-arcuato $\frown$, superficies totius caritatis aut viridis aut viridi-cyanea; antennac robustae fuscae basi virescentes, $\sigma^{7}$ longitudiue 
mediocres, + subbreves; clypeus mediocris transversus subtrapezinus parce punctulatus nitidus viridis, disco convexus et interdum aut aureo - aut aeneo-nitens, margine apicali nigro-piceo ad centrum modice arcuato-emarginato; labrum angustum elongatum acuminatum, parum convexum, ciliatum, mandibulae, maxillae et palpi nigropiceae; mandibularum basis aut viridi- aut aureo-aut cupreo-picta; margo occipitalis prope basin utrinque angulo prominulo parvo munitus. Thorax ad magnitudinem mediocris, crassus, antice tamen capite distincte angustior, totus confertim modice punctatus cyaneus: pictura aut viridi aut aureo-viridi splendente ita plerumque distributa, scil. in pronoti margine pone truncaturam anticam lineola transversa 1 . continua 1 . ad sulcum medianum brevem interrupta, tegulae alarum, macula centralis in scutelli margine basali, nec non vitta ampla obliqua mesopleurarum; in dorsuli areis aut omnibus aut lateralibus etiam color virescens aut in forma macularum aut quasi irroratus obvenit; metanotum et pectus saepius viridi-cyanea; rarissime totum thoracem cyaneum vidi; metanoti anguli postico-laterales robusti, magnitudine mediocres, latiusculi triangulares; pedes aut omnes aut posteriores ( $=$ inter medii et postici) antice $\mathbf{l}$. viridi- $\mathbf{l}$. rubro-igneove aurei, postice aut viridissimi aut viridicyanei, tarsis brunneis, calcaribus spinis spinulisque omnibus brumneo-testaceis; alae hyalinae, disco et ad costam cellulae radialis plus minus sordidae, venis piceis aut brunneis, mediocribus, externis robustis; cellula radialis 1. completa 1 . subcompleta I. incompleta, scil. nonnullis apice aut anguste aut angustissime aperta, fortiter triangulariter lanceolata. Abdomen robustum, capitis tho- 
racisque simul sumtorum longitudine, ovato-subrectangulum, dorso modice convexum aut viridi-aut rubro-aureum, creberrime modice punctatum et punctulatum, qua punctura creberrima superficies abdominis dorsalis subcoriacea atque - licet quodammodo nitens tamen - non vere splendida sit; segmentum 1:mum subcrasse punctatum, impressione baseos normali viridi-cyanea; lineola mediana elevata aut subelevata planiuscula polita impunctata in segmento 2:do atque etiam in segmento 3:tio (licet in hoc plerumque obsoletior) adest; segmenti 3:tii area maxima disco utrinque depressa, postice supra seriem convexiusculo-terminata; series ante-apicalis interrupta foveolis utrinque numerosis mediocribus rotundatis; regio (segmenti 3:tii) postica s. marginalis mediocris subtiliter punctulato-coriacea; dentes omnes robusti aut saltem mediocres triangulares recti; emarginaturae leniter arcuatae, latitudine subaequales, centralis recte transversa, externae obliquae; margo proprius lateralis obliquus, leniter aut curvus aut subflexuosus. Venter aut totus igneus aut plerumque ad basin segmentorum nigro-maculatus.

165. Chrysis ignita Linn. ठํ․

Parva, mediocris, aut magna 2 - 4 lin. decimal. long. confertim aut modice aut crasse punctata viridi-cyanea pedibus ventreque concoloribus, abdominis dorso aut 1 . viridi-aut igneo-aut cupreo-aureo (; segmenli 3:lii dentibus triangularibus rectis).

Chrysis ignita Linn. Syst. Nat. 2.947: 1., Fnn. Sv. 1665; - De Geer Ins. 2. 2. $832: 1$. Tab. 28, fig. $17-21$; - Fabr. E. S. 2. 241:10, Piez. 173:14; - Latreille Gen. Ins. 3:317; - Spi- 
Famil. Chrysididae. Chrysis: Phalanx VI. 293

nola Ins. Lig. 1:10; - Panz. Fn. 5:22; - Dlbm. Mon. Chr. 7:2, Ex. Hym, 28:2 var. $a$. 'et $b$., Dispos. 8:2; - Zett. Ins.

Lapp. 433:2; - Vesmaël Not. 9:1.

Chrysis obsoleta Dlbm. Dispos, 8:1. $\sigma^{7}$ var. Mus. Paykulli.

Chrysis Kollari Ziegl. secundum Mus. Spinolae.

Chrysis pyritosa Klug. secundum Mus. Berolinens.

? CHrysis cyanochrysa Forster. Nov. sp. ins. 88.

"Irabitat in parietibus et muris, praesertim argillaceis" (Lin. I. c.) nec non in foliis et floribus plantartun truncisque arborum soli urenti expositis per totam Europam Asiamque minorem vulgaris aut vulgatissima, ab ineunte vere usque in ultimum auctumnum vigens. In terris arcticis per totam Lapponiam cultam nec non inferalpinis maritimis Nordlandiae Finmarkiaeque usque ad Alten (prope Nord-Cap) procedit, observante Cel. Zetterstedt l. c. De moribus et vita pseudo-parasitica hujus optime cognitae speciei Cel. Latreille, Walckenaer et Westwood observationes ad delectandum aptissimas publicarunt. Scil. in "IHistoire naturelle des Fourmis" (Paris. 1802) pag. 320 ita scripsit Latreille: „J'ai trouvé „aussi très-fréquemment les chrysis doré femelle ( $=\boldsymbol{C} h r y$ „, sis ignii(a P), guettant avec patience l'instant où le phi"lanthe" (forte Cerceris ornata?) „sortiroit de son trou, „afin de s'y introduire, d'y déposer ses oeufs, et de dé„truire les espérances de ce dernier. Le philanthe s'ap„percevoit souvent du dessein du chrysis, et venoit lui "donner la chasse."

In Illustr. Wallencteri ,Mémoires des Abeilles Solitaires" (Paris. 1817) pag. 42 haec verba occurrunt: „J'ai „souvent vu la Chrysis dorée (=Chr. ignita) pénétrer „dans le trou du Cerceris ormala lorsqu'il s'y trouvait; "la Chrysis dorée commence par jeter du sable dans ce

II. 
„trou, probablement pour étourdir son ennemi: elle en„tre ensuite dans ce trou, y reste quelque temps, et en „ressort. J'ignore entièrement les motifs de ces fails; „un naturaliste plus heureux et plus habile les expliquera "peut-être un jour."

In Cel. Westwoodi "modern Classificution of Insects" (Londin. 1839. pag. 176) haec leguntur: „I bave con„stantly found our largest British species*) in company „with Odynerus antilope (see my article on the habits „of this insect, in the Trans. Entom. Soc. rol. 1.**), „of which it is doubtless the parasite, but which does "not appear to offer the slightest molestation, deterred, „possibly, as Latreille suggests, by the splendour of its "coat of mail."

Ipse Chrysidem ignitam in nido Odyneri murarii Fabr. prope Iund Scaniae pluries obserravi, et semel duo hujus Chrysidis individua e nidis duobus Odyneri memorati apud me exclusa sunt.

Observ. Pro hac specie, fere ubique rulgaris et omnibus naturae scrutatoribus sine dubio perbene cognita, diffusam descriptionem haud necessariam duxi; pracsertim quum plurima, de Chryside auripedi jam scripta, ad Chysidem ignitam applicanda sunt. Igitur tantummodo sequentes paucas notiones addere rolui. 1) Nagnitudo corporis e diverso climate minus dependere ridetur; etenim individua maxima ultra 4 lineas decimales longa in regionibus Sveciac borealibus legi, mediocria autem aut parva ex Europa meridionali accepi, - et rice

*) an Chrysis ignita?

**) "Transactions of the Entomological Society" non vidimus. 
versa; 2 ) capitis ejusque partium forma, structura, magnitudo et sculptura et pictura omnino ut in Chrysidi auripedi; 3 ) Labrum (saltem in exemplaribus vivis a me examinatis) mediocre gibbum semilunatum et rarissime orbiculatum, post mortem vero paullo retractum ut difficile conspicitur; 4) fasciolan, quam in antico vertice, apud Chrysidem auripedem, nonnunquam triramosam et aureo-viridem annotavimus, in hac specie non vidimus; 5) thorax plerumque cyaneus pictura viridi ut in Chrysidi auripedi; apud quaedam specimina thorax aut laete riridis, aut laete coerulescens, aut pure et saturate cyaneus, qui color tunc facile in purpureum vergit; 6) pedes in omnibus a me examinatis viridi-cyanei, tarsis 1 . nigro-fuscis l. laetius aut saturatius brunneis; 7) Abdomen plerumque longius quam in Clrysidi anripedi, ad crassitiem, picturam et sculpturam dorsalem quam maxime varians; scil. quoad colorem aut viridissime aureum, aut fulvo-, aut igneo-, aut cupreo-, aut cupreo-aeneo-aureum, aliis splendidissimum, aliis propter puncturan confertissimam modice nitidum; quoad puncturam aut in segmentis 1:mo et 2: do crasse creberrime punctatum, quo in casu carinula mediana non solum in segmento 2: do sed etiam in 1:mo conspicue evadit, - aut totum modice aut denique subtiliter punctatum; 8) abdominis segmenti dorsalis 3: tii series ante-apicalis ut in Chryside auripedi constructa, sed foreolae propter magnitudinem corporis variant scil. 1. majores 1. mediocres 1. parvae. 9) variationes coloris I. picturae ventralis jam a Cel. Wesmaël ita describitur: ,var. a. ventre rubro-aurato, var. b. ventre viridi-aurato, var. c. ventre viridi-cyaneo, segmento terlio interdum nigro"; quae descriptio etiam cum no- 
stra experientia omnino congruit, ea sola exceptione quod in nostris exemplaribus omnium varietatum rentrem semper licet plus minus ample, nigro-maculatum observavimus.

Phalanx VII. Chrysides ano quinque-dentatae; scil. abdominis segmenti dorsalis $3:$ tii margo apicalis dentibus quinque armatus.

166. Chrysis Iusca Fabr.

Mediocris aut submagna 3 lin. decimal. long. coeruleo-viridis dorsulo cyaneo, abdominis segmento dorsali 2:do utrinque macula aurea, segmenli 3:tii dentibus apicalibus mediocribus triangularibus.

Chrysis lusca Fabr. Piez. 171:7 secundum specimen anno 1847 in Museo R. Havniensi a me descriptum et exarainatum.

„Habitat in Italia Dom. de Sehlanbusch. Mus. D. Lund." Fabr. 1. c.

Observ. „Statura et magnitudo Chrysidis oculatae" Fabr. 1. c. Corpus robustum modice punctatum nitidum coeruleo-viride, dorsulo cyaneo. Antennae fuscae scapo subtus viridi. Abdominis segmentum dorsale 2:dum utrinque juxta marginem apicalem macula magna viridi-aurea; segmentum dorsale 3:tium basi convexiusculum, medio transverse concaviusculum, supra seriem iterum convexiusculum; series ante-apicalis foveolis rotundatis distinctis non confluentibus, medio per carinulam continuam interrupta, margo apicalis depressus nomnihil prominulus 
dentibus 5 mediocribus triangularibus subobtusis. Alae fusco-hyalinae.

\section{Chrysis Megerlei Dlbm.}

Mediocris $2 \frac{1}{2}$ lin. decimal. long. viridi-cyanea, abdominis segmentis dorsalibus 1:mo 2:doque aureis, 3:tio cyaneo dentibus apicalibus robustis spinoideis.

Clerysis analis Megerl. secundum Typum ab III. Spinola comrounicatum (; non Clirysis analis Spin. Ins. Lig. quam sub no. 146 descripsimus).

Ilabilat ad Veronam, a D. Conti detecta; Mus. Spinolae.

Observ. Statura et magnitudo fere Chrysidis analis Spin., ab hac vero mox dignoscitur segmenti abdominis 3 :tii margine apicali distincte 5 -dentato.

Corpus robustum obtusum pubescens confertim punctatum. Caput rotundato - triangulum cyaneum antice virescens. Cavitas facialis mediocris subrectangularis non profunda, superne transverse distincte marginata: margine utrinque decurvo (; antennae in specimine nobis communicato mutilatae); clypeus brevissimus transversus, ad centrum marginis apicalis breviter et modice arcuatocmarginatus, disco nonnihil gibbus; reliquae partes oris nigro-piceae. Thorax subbrevis crassus subquadratus confertim crasse punctatus cyaneus; pronoti pars postica, dorsuli areae laterales, scutellum, metanoti nargines et latera atque mesopleurae viridi-irrorata; dorsuli area media purpurascenti-violacea; metanoti anguli postico-laterales robusti lati triangulares; sternum et pedes viridiryanei tarsis fuscis; alae sordide hyalinae, venis firmibus brumneis, cellula radiali lanceolata apice auguste aperta. 
Abdomen robustum subovatum, basi transversum, capite thoraceque simul sumtis brevius, dorso sat convexum crebre modice punctatum nitidum; segmenta 1:mum et 2:dum aurea, hujus margo basalis nigro-aeneus; segmentum 3 : tium valde convexo-declive totum cyaneum et confertissime punctatum punctis mediocribus; series ante-apicalis profunda sub margine convexiusculo regionis anticae partim abscondita, foveolis profundis paucis nigroaeneis, hinc inde confluentibus et tunc transversis linearibus; margo apicalis brevissimus punctulatus, dentibus 5

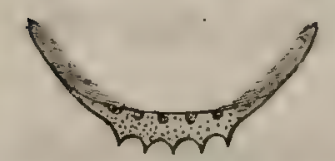
spinoideis aequalibus in lineam lenissime curvam sitis, emarginaturis mediocribus modice arcuatis, invicem latidudine et profunditate subaequalibus. Venter nitidissimus subtilissime punctulatus, pubescens, planus (nec fornicatus), viridi-cyaneus, segmentorum marginibus et basalibus et lateralibus et apicalibus aut nigricantibus aut nigro-brunneis.

Phalanx VIII. Chrysides ano sex-dentatae; scil. abdominis segmenti dorsalis 3:tii margo apicalis dentibus sex armatus.

Sect. I. Abdominis dorsum aureum.

Divis. 1. Abdominis segmenti 3:tii dentes: 4 apicales obsoleti obtusi, tantummodo ex ipsius marginis undulatione orti, 2 laterales distincti, scil. margo proprius lateralis ad centrum suum (infra initium seriei) angulo robusto munitus. 


\section{- 'hrysidis.}

Sect. I. Abd

Divis.

Chrysis pulchella Spin. Sicilia.

Chrysis dives Klug. Sicilia.

Clerysis Valeli nob. 'Tunis.

Divis.

Chrysis micans Rossi. Sicilia. Corsica. Rhodus.

Sect. II. Abi

ma

Cor7

Chrysis Scliönherri Dlbm. Africa.

Sect. III. A

pi

A.

i

Chrysis Zellerstedli Dlbm. Svecia et Nurwegin.

B. $A$

p

10

d) Clirysis equestris Dalman. Mus. Paykull 


\section{Tabula Synoptica $Y$ : ta sistens Synopsin}

\section{l'halangis VIll:vae Specierum nobis cognitarum. Generis Chrysidis.}

Secl. I. Abdominis dorsum aureum.

Divis. 1. Abdominis segmenti dorsalis $3:$ tii dentes: 4 apicales obsoleti obtusi, tantummodo ex ipsius marginis undulationc orti, - 2 laterales distincti, scil. margo proprius lateralis ad centrun sum (infra initium seriei) angulo robusto munitus.

A. Segmenti 3 :tii margo apicalis (infra sericm) immaculatus et ipsi segmento concolot.

a) Caput, pectus et metanotum cyanea, leviter viridi-picta; pronotum et naesonotum aurea,

abdominis segracnti dorsalis $3:$ tii series ante-apicalis concolor . . . . . . . . . .

b) Caput,

13. Segmenti 3 : tii margo-apicalis violaceo-cupreus; caput, pectus et metanotum coeruleo-aurea, pronotum et postsculcllum viridi-aurea, mesonotun cupreo-aureum . . . . . . . 160. Chrysis Jahli reb. 'lunis.

Divis, 2. Ablominis segmenti dorsalis 3 :tii dentes ornes 6: apicales, robusti spinoidei. Corpus robustum, caput et thorax cyanea plus minus viridi-picta; abdominis dorsum aureum, plus minus viridi- aut cupreo-splendidum, segmenta 2 :dum et 3 :tium basi fasciola sublineari nigro-2cnea

68. Chrysis pulchella Spin. Sicilia.

69. Clurysis dives hlıg. Sicilia.

171. Chrysis micans liossi. Sicilia. Corsica, Hilıdus.

Sect. II. Abdominis dorsum viridi-aureum, segmento 3:tio viridi-cyaneo; segmento 2 : do margine apicali et macula utrinque lateralis cyaneis.

Corpus robustum submagnum. Caput ct thorax punctatissima viridia, cyanco-et acneo-purpurascenti-picta, Mlae nigro-brunneac . . . . . . . . . . . . .

Sect. III. Abdominis dorsum violaceum fasciis aureis (. Caput et thorax cyanea viridipicta confertim punctata).

1. Abdominis segmenti dorsalis $3: t i i$ margo apicalis brevis, series ante-apicalis foveolis rotundatis mediocribus distinctis; dentes 2 extcrni obtusi arcuati, religui 4 triangulares breves; emanginatura inter dentes 2 centrales angustion et profundior, unde hi dentes nonnilil robustiores et paullo magis allumimati

13. Abdominis segmenti dorsalis 3 : thi margo apicalis amplus, basi utrinque depressus, in lace depressione jacet scries ante-apicalis forcolis Iongitudinalibus sulciformilus irregularibus; dentes 2 externi angulati, reliqui 4 triangulares robusti; cmarginatura inter dentes 2 centrales latior, unde hi lentes non approximati 
Sect. IV. Abdominis dorsum viridi-cyaneum, scgmento 2:do utrinque macula rotundata aurea. Corpus magnum viridi-cyancum. Alae brumnescentes.

A. Corpus mediocre. Abdomen creberime punctulatum, segmenti 2: di utraque macula ocellaris mi-

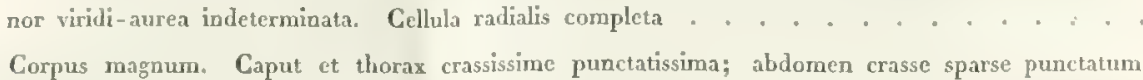
segmenti 2 : di utraque macula ocellaris major purpureo-aurea víridi-circumscripta. Cellula radialis incoropleta . . . . . . . . . . . . . . . .

176. Chrysis oculate Fabr. India oricntalis.

Sect. V. Abdominis dorsum aut viride, aut cyaneum, aut coeruleum, aut violascens, nunquam aureo-pictum.

Divis. 1. Postscutellum inerme (, non mucronaturn, licet interduna crasse sculptum).

Subdivis. 1. Abdominis segmenti 3 ; tii dentes: 4 apicales et 2 laterales; uterque dens lateralis prope basin marginis lateralis et juxta initium seriei ante $\sim$ apicalis situs.

A. Corpus mediocre obtuse subrectangulun punctatissimum viridi-cyaneum vertice pronoto et dorsulo aureo-maculatis; abdomen depresso-convexum, segmentum 2:dum carinula mediana distincta, segmentum 3:tium breve, basi immersurn, medio ad seriem transverse convexo-elevatum, scries foveolis parvis numerosis rotundatis subpellucidis, dentes apicales mediocres spinoidei; alac luya-

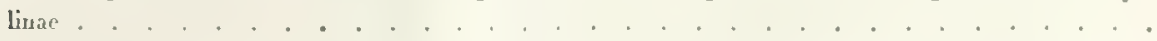
(17)

B. Corpus subragnum ovale capite thoraceque punctatissimis viride cyaneo-pictum ; abdomen sat con-
vexum modice confertim punctatum nitidissimum, segmentum 2 :dum carinula mediana caret, segracntum 3:tium mediocre (longius quam in Chr. splendente) depresso-convexum, ad seriem vix marginulatum, series foveolis minoribus numerosis rotundatis opacis, dentes apicales robusti triangulares; alae brunnescentes, basi hyalinue . . . . . . . . . . . . . 178 Subdivis. 2. Abdominis segmenti 3 :tii dentes: omnes 6 apicales.

Tribus 1. Dentes 2 externi ohtusi, ex apice marginis lateralis arcuato formati. Corpus mediocre sublineare, cyancum aut violaceum leviter viridi-pictum

Tribus 2. Dentes omnes 6 acuti: 1. triangulares 1. spinoidei.

Manipulus 1. Abdominis dorsura distincte fasciaturn: fasciis aut violaccis aut nigroviolaceis.

1. Abdominis segmentura dorsale 3 :tium totum convexo-depressum; margo analis 1. apicalis itarne non humilior quar maxima pars segmenti ante seriem, sed totum segmentum undique unam candesnque convexo-depressitatem praebet. Corpus viridi-cyaneum 3 lin. decimal. long., segmenti 3:lii series ante-apicalis foveolis rotundatis per lineam medianam elevatam convexo-planam interrupta, margo apicalis prominulus dentibus mediocribus robustis acute triangularibus, Alae fusco-lya-

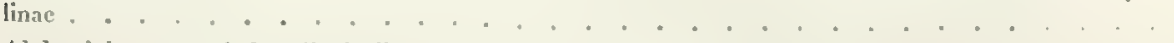

13. Abdoninis segmenti dorsalis 3 : tii margo apicalis 1 . analis (= area postica) semper humilior quam area antica, quac maximam segmenti partem efficit et supra scrien marginulo depresso-couvexo limitata est.

a) Segmenti 3 :tii area postica (= margo analis 1. apicalis) utroque latere albo-vittata. Corpus angustum sublineare $2 \frac{2}{3}-2 \frac{1}{3}$ lin. decimal. long.

1. Caput, thorax et abdominis segmentum dorsale 1 : mum crasse punctatissina, hujus reliqua segmenta confertim punctulata; segmenti 3 :tii area postica depresso-convexa sulbilissime co-

(5). Clarysis violacea Pauzr. Luropa sucdia el meri. dional, sfricat horeal. 
otundata

- cllaris mi-

175. Chrysis Schiödtei nob. India orientalis.

sunclatum,

Iula radia-

176. Chrysis oculata Fabr. India orientalis.

Jlascens,

ns lateraliv

et dorsulo

tincta, se-

$\mathrm{m}$, scries

alae liya-

. . 177. Chrysis splendens Klug, Africa.

a sat con-

caret, se-

seriero vix

ti triangu-

. . 178, Chrysis Wahlbergi Dlbm. Aliica.

orpus nue-

- 179. Chrysis violacea Panzr. Europa media et menidional, Africa boreal.

nut nigro-

lis itaque

Inam can-

uenti 3 : lii

interrupta,

usco-hya-

lior quam

180. Chrysis Smidli nob. Imesica meridional

o-conveso

Corpus

Ius reliqua

issime co- 
Chrysis Genbergi nold. Busilia.

Chrysis Klugi not. IBrisili.i.

1. Chrysis sexdendata Fabr. Anuica

$\Lambda$.

$$
\text { I }
$$

a

Chrysis modica noth. Afirat l.

Clerysis prominula Dllun. Mus, Paghull.

B. $A$

c

$\rightarrow$

Chrysis aequinoclialis Spiu. Bmsilia.

Chrysis insularis Gucrin. Cuba.

b

Chrysis inaequidens nob. Now-York. $4 \cdot$

Chrysis malachitica Dracge, Africa.

Chrysis smaragdula Fabr. Imerica horent.

Div

11

. Chrysis lyncea Fals. Wrica. 
riacca subvirescenti-çanea utroque latere toto alla pellucida, series foveolis parvis rotunda is, dentes mediocres acute triangulares, onnes invicem acyuales.

2. Caput, thorax et totum abdoninis dorsum crasse munctatissina; abdominis segmenti dorsaliv 3:tii area postica magis promiuula depressa nitidissima vage punctulata purpureo-violacea ad basin utriusque lateris vitta parva cuneiformi albo-peltucida, series foveis crassis longitudinalibus sulcifornibus circ. 14 in parte violacea per carinulam medianam intersupta, den. les innequales spinoidei: centrales duo brevissimi, externi duo majores. . . . . . . . 18

h) Segmenti 3 :tif area postica (= margo analis 1. apicalis) unicolor, cyanea aut virescens . . . 183.

Manipulus 2. Mlelominis dorsum non fasciatum.

A. Aldominis segmenti dorsalis $1: \mathrm{mi}$ utraque area lateralis intra-marginalis punctulato-coriacea. Corpus viride plus minus cyanescens.

a) Corpus mediocre; abdominis segmenti 3 : tii area antica vix nisi lenissime nedio jmmersa, areac posticae enarginatura centralis parum angustior yuam cxterna; alarum ccllula radialis con-

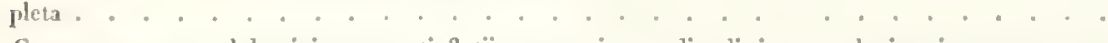

b) Corpus magnum; abdominis segnenti $3:$ tii area antica medio distincte at leniter immersa, areae posticae eruarginatura centralis multo angustior quan externa; alarum cellula radialis inconpleta, apice late aperta . . . . . . . . . . . . . . . . . . . . . . . . . . . . . . . . . . . . . .

B. Abdominis segmenti dorsalis 1 : mi area latcralis intramarginalis crasse punctata: punctis 1. numerosis et confertis 1. paucis et vagis.

a) Segmenit 3:tii emarginaturac (apicales): ormes aequales aut subaequales.

1. Corpus subparvum sublincare confertim modice punctatum subopacum coerulcum alis albis; scgmentum 3:tium totum depresso-convexum, scries foveolis parvis numerosis, dentes parvi . . . . . . . . . . . . . . . . . . gmenti 3:tii area antica medio transverse immersa, ante sericm subgibbo-convexa, series fovcolis multis distinctioribus, dentes robusti . . . . . . . . . . . . . . . .

b) Segmenti 3:tii cmarginaturac (apicales): utraque externa distincte angustior quam cmarginatura centralis. Corpus mediocre, modice punctatum viridi-cyaneum; segnentum abdominis 3 : tium depresso-convexum, scries foveolis parvis numerosis, cmarginaturae inaequales: centralis magna profunda triangularis, intermedia et externa arcuatac, illa mediocris, hace parva; dentes 2 centrales magni, uterque proximus raediocris, externi 2 minores, at onnes trangulares . . . . . 18

c) Segmenti 3:tii emarginaturae (apicales): utraque externa distincte latior quam emarginatura centralis. Slae sordidac, violascentes.

1. Corpus mediocre, modice punctatum viridi-cyanescens; abdominis segmenti 3 : tii area antica convexo-depressa, series levis (non profunde inmersa) fovcolis mediocribus orbiculatis . . 189.

2. Corpus maximum robustissimum punctatissinum viride aut viridi-cyaneun; segmenti 3 : tii area antica medio leniter immersa, series profunde immersa foveolis magnis profundis subquadrangulis . . . . . . . . . . . . . . . . . 190

189. Chrysis malachilica Dracge, Mfrim

Chrysis smaragdula Faln: Amcriva boreal.

Divis. 2. Postscutellum mucronatum. Corpus magnum.

1. Aldominis segnenti dorsalis $3:$ tii dentes: 4 apicales et 2 latcrales.

a) Postscutellum carinatum; segmenti 3 :tii emarginalura centralis recte transversa subrectangula.

191. Chrysis lyncen Falı. Mrica. 
b) Postscuellum plamm crassissine punctatissimum I. scrobiculaturn; scgmenti 3:tii marginatura rentralis 1. arcuata 1. triangularis.

1. Postsculellum acute conicum apice politum; segmenti 3 :tii emarginatura centralis arcuata parumper angustior quam proxima; dens lateralis parvus, ad centrum marginis lateralis situs, unde spatium iater dentes apicalem extermum et lateralen breve ant mediocre obvenit. .

2. Mostscutellum triangulare opacum; segmenti $3:$ lii emarginatura centralis triangularis, distincte angustior quam proxima; dens lateralis validus mox prope basin marginis situs, spatium - inter dentes lateralem et externum apicalem - longissimum . . . . . . . . .

I) Abdominis segmenti dorsalis 3:tii dentes: omnes 6 apicales (. Postscutellum longitudinaliter excavatun, totum crassissime punctatissinoum).

a) Abdominis segmentum dorsale 3:tium totum depresso-convexum, area antica postice 1. suprá seriem laud convexo-marginata, ambae igitur areac non nisi serie ipsa inconopleta distinAhdominis scgmentum dorsale 3:tium medio totum transverse concaviusculo-immersum, pu Dtice supra seriem convexiusculum, series ante-apicalis foveolis distizctis numerosis rotundatis
s.

194. Chrysis incerta nob. Cayenue.

195. Chrysis nobilis lilug. Acgypt. 
ginatura

arcuata

lis situs,

int - 192. Chrysis Gueudei Spin Madagascat.

distincte

spatium

193. Chrysis mucronala nol. Mrica.

$\mathrm{x}^{2} \mathrm{Y} \cdot \mathrm{Mca}$

1. suprat

distin-

194. Chrysis incerta nob. Cayeme.

$\mathrm{m}, \mathrm{po}-$

latis 195. Chrysis nobilis lilug. Acgypt. 
Famil. C'lerysididae. Clryssis: Phalamx VIII. 299

A. Abdominis segmenti 3:tii margo apicalis (infra seriem) immaculatus et ipsi segmento concolor.

168. Chrysis pulchella Spinola. 9.

Submediocris $2 \frac{1}{2}$ lin. decimal. long. confertim modice punctata viridi-cyanea, thorace ex parte abdomineque dorso viridi-aureis, segmenti 3:tii serie ante-apicali concolore.

var. a. pronotum et dorsulum viridi-aurea.

Chrysis pulchella Spin. secundum Klug in Museo Berolinensi 1847. var. b. pronotum et mesonotum viridi-aurea.

Clerysis dives Klug, secundum Muscum Spinolae.

IItabilat in Liguria, D. Spinola; - in Sicilia, DD. Grohmann, Kiesenvetter, Kollar et Schiödte.

Observ. Var. a. Habitu non dissimilis Hedychero lucidulo minori, sed abdomine angustiori et magis Chrysiformi, ano dentato etc. facillime dignoscitur. - Caput et thorax cyanea confertim modice punctato-subreticulata, interdum sub stemmate fasciola viridis; cavitas facialis rectangularis planiuscula non profunda, subtilissime coriacea, viridis aut viridi-cyanea, medio canaliculata, superne transverse marginata: margine $\mathcal{L}^{\circ}$ sursum biangulato, e singulo quoque angulo ramulum rectum versus stemmata superiora currentem emittente; antennae mediocres, scapus et pedicellus viridi-cyanea, flagellum fuscum; clyperus mediocris inaequalis, medio subcarinulatus, in disco apicali instructus area declivi triangulari polita nigro-picen, margo apicalis leniter arcuatoemarginatus. Mandibulae cum palpis normaliter pictac. Occiput utrinque angulo spinoideo obsoleto. Pronotum 
viridi-aureum: foveolis medianis antica cuprea, postica cyanea, truncatura utrinque et latera cyanea. Dorsulum viridi-aureum. Scutellum et mesopleurae leviter viridiirrorata; haec apice obsolete bituberculatae; reliquus thorax unacum pedibus cyaneus, hi l. uno l. altero latere virescentes tarsis aut brunneo-testaceis aut fuscis. Abdominis dorsum confertim modice punctatum viridi-aureum, lineola mediana subelevata polita ad certum situm luminis visibili; segmenta 2 :dum et 3 :tium margine basali tenuiter nigro-aenea; hoc modice convexum, series anteapicalis $8-10$ valde profundis magnis suboralibus; margo apicalis convexiusculus, sparse irregulariter punctulatus, modice undulato - 4-denticulatus, denticulis brevibus obtusis, aut brevibus aut brevissimis, externis subtriangu(a. $d l$ *) laribus, intermediis latis arcuato-obtusis, summus finis hujus marginis scarioso-testaceus et subpellucidus; dens uterque lateralis triangularis subacutus, per emarginaturam obliquam modice arcuatam a denticulo apicali proximo remotus, margo lateralis a basi usque in dentem suum leniter oblique flexuosus. Venter parumper fornicatus, segmenta basi nigra, apice lateribusque 1 . viridia 1 . cyanea 1 . ambobus coloribus mixtis variegata. Alae hyalinae, disco et ad marginem cellulae radialis costalem sordidae, venis fuscis; cellula radialis triangulariter lanceolata incompleta: apice modice aperta.

var. b. non nisi corpore paullulum breviore, scutello viridi-aureo a var. $a$. differt.

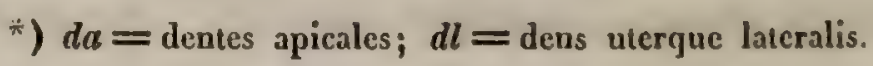


Famil. Chrysididae. Chrysis: Phalanx VIII. 301

169. Chrysis dives Lin. + .

Parva vix 2 lin. decimal. confertim punchulata, subtus viridi-cyanea supra aurea, abdominis segmenti 3:lii serie ante-apicali nigro-aenea.

Clirysis dives Klug, secundum Museum Spinolae.

IIabilat in Sicilia, D. Grolemann; Mus. D. Spinola.

Observ. Praecedenti simillima, at nullo ejusdem varietas; etenim certe dignoscitur: corpore minore et proportionaliter minus robusto, capite thoraceque punctulatoreticulatis, abdomine subtiliter punctato, vertice inter stemma anticum, oculos et faciem viridi-aurato, pronoto et dorsulo cupreis, scutello aureo, postscutello viridi-aureo, mesopleuris apice muticis (inermibus, nec tuberculatodenticulatis), abdominis segmenti $3:$ tii serie ante-apicali nigro-aenea, dentibus apicalibus internediis brevissimis obsoletissimis. In ceteris autem omnibus cum Chryside pulchella congruit.

B. Abdominis segmenti (dorsalis) 3:tii margo apicalis violaceo-cupreus.

170. Chrysis Vahli. Dlbm.

Mediocris 2 lin. decimal. et ultra long. crasse punclala, capile, pectore et melanoto coeruleo-aureis; pronoto, postscutello abdominisque segmento dorsali 1:mo viridi-aureis, mesonoto abdominisque segmentis dorsalibus 2: do et 3:tio cupreo-auris.

„Chrysis cocrulcipes, e Tunis Valll"; in Collectione Sellestedli Musei Reg. Havnicus. unicum specimen anno 1847 vidi.

IIabitat in Africa; ad Tunis a D. Vahl olim detecta. Observ. Valde assimilis Chrysidi candenti Germar. 
302 Famil. Clirysididae. C'lrysis: Phalanx VIII.

et nob. - Corpus crassum et crasse punctatum. Abdominis segmenta 2:dum et 3:tium margine basali nigroaenea, segmenti 3 :tii margo apicalis mediocris violaceocupreus, fere ut in Chryside pulchella constructus. Antennae tarsique nigro-fuscescentes, scapus illorum et pedes viridi-coerulei. Alae hyalinae venis fuscis. Venter purpureo- I. aeneo-violascens, marginibus segmentorum viridi-coerulescentibus.

Divis. 2. Abdominis segmenti dorsalis 3:tii dentes omnes 6: apicales, robusti spinoidei.

171. Chrysis micans Rossi. $\sigma^{\prime} q$.

Mediocris aut submagna $2 \frac{1}{2}-3$ lin. decimal. long. robusta confertim crasse punctala viridi-cyanea plus minus purpurascens, abdominis dorso viridi-cupreo-aurato, segmentis 2:do el 3:tio basi nigro-aeneis.

Clerysis micans Rossi Fn. secundum Museum Berolinense.

Chrysis similis Lepel. secundum Mus. Spinolae.

Ilabitat in insulis Corsica et Sicilia, D. Spinola; in insula Rhodo, mense Majo, D. Loew.

Observ. Statura fere Cllrysidis rulilantis. - Corpus elongatum crassum crasse punctatum pubescens; caput et thorax punctalo-reticulata viridi-cyanea, punctis plurimis nisi omnibus in fundo violaceo-purpurascentinitidis. Caput rotundato-triangulum aut suborbiculatum; cavitas facialis quadrata, modice profunda, punctulatocoriacen, superne transverse-marginata: margine subacuto inaequali; antennae breves fuscae, basi viridi-cyaneae; clypeus transversus mediocris subtrapezinus, disco con- 
Famil. Chrysididae. Chrysis: Phalane VIII. 303

vexus, ad centrum marginis apicalis leniter arcuato-emarginatus; labrum semilunatum convexum ciliatum nigropiceum; mandibulae et reliquae partes oris concolores; illae basi macula viridi-cyanea. Mesopleurae margine postico ad apicem crenulatae et quasi denticulatae; metanoti anguli postico-laterales lati robusti acute triangulares; sternum viridi-cyaneum, pedes concolores tarsis nigris; alae aut hyalinae aut plus minus sordidae, venis firmibus fusco-piceis, cellula radiali elongata lanceolata subcompleta: apice anguste aperta. Abdomen robustum sublineare, decurvum, basi transversum, apice arcuatum, dorso valde convexum viridi-cupreo-aureum; segmenti 1: $m i$ impressio basalis violascens, segmentorum 2 :di et 3:tii margo basalis aut ample aut tenuiter nigro-aeneus; segmenti 3:tii series ante-apicalis foveolis mediocribus numerosis rotundatis, margo apicalis mediocris regione antica humilior punctulatus, omnes 6 dentes in unam lineam leniter dispositi robusti, acute triangulares subspinoidei magnitudine subaequales, emarginaturae modice arcuatae, 3 intermediae aequales, 2 externae obliquac, margo lateralis obliquus lineam rectam currens nec nisi obsoletissime flexuosus. Venter fornicatus niger, paullulum violaceo-micans, segmentis viridi-cyaneo-maculatis.

Sect. II. Abdominis dorsum viridi-aureum, segmento 3 :tio viridi-cyaneo.

172. Chrysis Schönherri Dlbm.

Magna $3 \frac{1}{2}$ lin. decimal. long. confertim punctala robusta virescens aeneo-purpureoque picta, abdominis dorso viridi-aureo, segmentis: 2:do utrinque macula laterali 
3:tioque toto viridi-cyaneis, dentibus apicalibus brevibus: intermediis 4 acule-, externis 2 obluse-triangularibus, alis nigro-brunneis.

Chrysis Schönherri Dlbm. Dispos. 1845. 10:9.

Ilabilat in Africa meridionali-orientali, ad Port Natal a D. J. Walulberg detecta; Mus. R. Acad. Scient. Stockholm.

Observ. Species eximia et elegans. - Corpus robustum elongatum pubescens. Caput re vera rotundatosubquadratum, sed propter oculos exsertos subtriangulum apparet, confertim modice punctatum, antice virescens postice viridi-cyaneum, vertice subruguloso macula centrali purpurascenti aenea; cavitas facialis rectangularis parum profunda, punctulato-coriacea nitida, lateribus albo-sericea, supcrne marginata: margine transverso inaequali, utrinque decurvo; antennae magnitudine mediocres, robustae fuscae, basi viridi-cyaneae; clypeus mediocris nitidissimus transversus, disco convexus; mandibulae una cum reliquis oris partibus nigro-piceae, macula mandibularum basalis intensive aureo-viridis; margo occipitalis utrinque angulo minuto spinoideo. Thorax subbreris crassus sat convexus, crasse punctatissimus subreticulatus viridis, lateribus sternoque nitidus, dorso subopacus; pronoti margo posticus, dorsuli scutellique discus purpurascenti-aenea, metanoti discus coeruleus; postscutellum propter crassam sculpturam quasi crenulatus apparet; metanoti anguli postico-laterales robusti lati triangulares; pedes virides nitidissimi, tarsis nigro-fuscis, calcaribus spinulisque testaceo-brunneis; alae nigro-brumneac, venis concoloribus firmibus, cellula radiali lanceolata incompleta, tegulis cyaneis. Abdomen robustum lineari sub- 
Famil. Chrysididae. Clurysis: Phalanx VIII. 305

ellipticum, dorso sat convexum; seğmentum 1:mum confertim modice punctatum viridi-aureum, margine summo apicali tenuiter nigro-piceo; segmentum 2:dum confertim punctulatum viridi-aureum, utrinque macula laterali magna rotundata nec non centro marginis apicalis cyaneis; margo lateralis (segmenti 2:di) apice angulo acuto subspinoideo; segmentum 3:tium remote punctulatum viridi-cyaneum, disco vix nisi levissime et obsoletissime immersum, ante seriem convexiusculo terminatum, series ante-apicalis foveolis numerosis rotundatis, centralibus duabus distinctioribus, reliquis aut parvis aut mediocribus; margo apicalis brevis subtiliter punctulatus subcoriaceus, dentes apicales breves triangulares, intermedii 4 acuti, externi 2 obtusi; emarginaturae arcuatae: centralis paullulum angustior quam proxima, reliquae omnes invicem latitudine subaequales; margo proprius lateralis parum obliquus, leniter curvus. Venter fortiter fornicatus, viridi-cyaneus.

Sect. III. Abdominis dorsum violaceum fasciis aureis.

173. Chrysis Zetterstedti Dlbm. $\sigma$.

Submediocris $2 \frac{1}{2}$ lin. decimal. long. conferlim modice punctata violacea viridi picta, abdominis dorso subtiliter punctulato fasciis 3 arcuatis viridi-aureis, segmenti 3:tii serie ante-apicali foveolis mumerosis subparvis rolundalis, margine dentibusque apicalibus brevibus.

Clrysis Zetterstedti Dlbm. Dispos. 1845. 11:16. $\sigma^{\text {t }}$.

Ilabilat in Svecia et Norwegia rarius; duo tantum specimina vidi: unum in trunco Belulae albae exsiccato 


\section{Famil. Clirysididae. Chrysis: Plealanx VIII.}

soli exposito d. 7. Septembris 1840 ad Laxbro Nericiae et alterum ad Christianiam Norwegiae mense Julio 1814.

Observ. Species pulchra et bene distincta. - Corpus mediocre elongatum pubescens. Caput rotundatotriangulum confertim permodice punctatum cyaneo-virescens vertice violaceo; cavitas facialis lata transverse quadrata punctulato-rugulosa, parum profunda subplaniuscula, supra discun fovea centrali rotundata, superne irregulariter et obsolete transverse marginata; antennae breves robustae fuscae, apice dilute brunneae, basi viridicyaneae; clypeus transversus perbrevis nitidissimus, disco convexus, unargine apicali cupreo-aeneo ad centrum arcuato-emarginato; labrum subtriangulare convexum unacum mandibulis, maxillis palpisque nigro-piceum, basis mandibularum viridi-aureo picta; margo occipitalis utrinque angulo spinoideo minuto obsoleto. Thoras modice punctato-subreticulatus viridis aut cyaneo-viridis, pedibus concoloribus, tarsis fuscis; pronoti truncatura antica tota et partis posticae maculae 3 interdum coëuntes in fasciolum, dorsulum et scutelli discus violacea; metanoti anguli postico-laterales mediocres triangulares; alac sordide hyalinae venis mediocribus brunneis, cellula radiali lanceolata incompleta: apice late aperta, tegulis cyaneis. Abdomen obtuse rectangulun, basi non latius quam apice, longitudine capitis thoracisque simul sumtorum, dorso saturate violaceun: segmenta $1:$ mum et 2 :dum margine apicali 3: liumque ante seriem fasciola pulcherrima viridiaurea, medio angusta, utrinque ampliata totumque marginem lateralem obtegente; segmentum 1:mum modice punclatum, 2:dum subtiliter et fere remote punctulatum, 3: tium subtilius confertissime punctulatum; series ante- 
apicalis per carinulam brerem planam viridi-auream interrupta, foveolis profundis rotundatis numerosis violaceis, aut subparvis aut mediocribus; margo apicalis brevis aut totus viridis aut disco riridis et utrinque violascens; dentes 2 centrales robusti triangulares propter cmarginaturam centralem profunde et anguste arcuatam approximati, dentes 2 proximi breves triangulares, dentes 2 externi angulum laterali-apicalem efficientes lati arcuato-obtusi, emarginaturae externae latae obliquae arcualae, emarginatura inter dentem utrumque centralem et dentem proximum l. secundarium mediocris recte arcuata at reliquis lenior. Veuter aureo-viridis segmentis maculis marginibusque nigricantibus.

174. Chrysis equestris Dalman. 오.

IIIagna $3 \frac{1}{2}$ lin. decimal. long. confertim crasse punclata violacea viridi-picta, abdomine confertissime subtiliter punctulato fasciis 3 arcuatis viridi-aureis, segmenti 3:tii serie ante-apicali profunde immersa foveolis sulciformibus irregularibus, margine apicali valde prominulo, dentibus robustioribus.

Clirysis Zetterstedti Dlbm. Dispos, 1845. 11:16. ㅇ․

Patria ignota. Specimen unicum e Collectione Payliulli Mus. R. Acad. Scient. Stockholm. communicavit D. Bolieman.

Observ. Valde similis Chrysidi Zettersiedti(no.173), at corpore majore et robustiore, capite thoraceque crasse punctatis, abdominis dorso confertissime punctulato, segmenti 3:tii serie ante-apicali et margine longe aliter constructis, dentibus apicalibus majoribus et robustioribus certissime et facilline distinguitur. 
Corpus robustum elongatum pubescens. Caput ut in Chryside Zetlerstedti constructum, sculptum et pictum, at cavitas facialis superne distinctius marginata: margine transverso inaequali subacuto. Thorax unacum vertice crasse punctato-reticulatus, subtus lateribusque aut viridis aut cyaneo-viridis; pronoti margines, alarum tegulae, scutelli et postscutelli latera, metanoti anguli posticolaterales subbreves crassi triangulares et pedes concolora, ceterum toto dorso violaceus; alae sordide hyalinae venis firmibus piceis, cellula radiali lanceolata incompleta. Abdomen elongatum capite thoraceque simul sumtis longius, ovali-subrectangulum scil. apice paullulum angustius quam basi, dorso sat convexum saturate violaceum et ad certum situm Iuminis nonnihil purpurascens, segmentorum fasciae apicali-marginales viridi-aureae omnino ut in Chryside Zetterstedli constructae; segmentum 1:mum medio punctulatum, lateribus crasse punctatum; segment. 2: dum confertissime punctulatum; segm. 3:tium et confertissime et subtilissime punctulatum, series anteapicalis per carinulam viridem planiusculam interrupta, utrinque immersa; violaceae, irregula ovatae 1. triangula

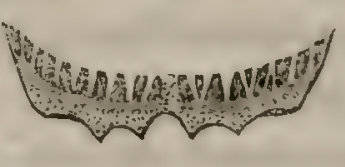
foveolae profundac res longitudinales 1 . res l. subsulciformes; dentes robusti, 4 intermedii triangulares, externi 2 angulum validum obtusum efformantes, emarginaturac 3 mediac recte arcuatac, centralis profundior quam proxima, externae duae latae oblique arcuatae. Venter riridicyaneus nigro-maculatus. 
Famil. Clrysididae. Clurysis: Plaalanx VIII. 309

Sect. IV. Abdominis dorsum viridi-cyaneum segmento 2: do utrinque macula rotundata aurea.

175. Chrysis Schiödtei Dlbm. 오.

Magna $3 \frac{1}{4}$ lin. decimal. long. crasse punctato-reticuIata virescens, abdomine creberrime punctulato viridicyaneo, segmento 2:do ulrinque macula parva viridianrata, alis brumnescentibus cellula radiali completa.

Chrysis smaragdula Westermann in litteris (; non Chr. smaragdula Fabr. quae, Americae borealis incola, in nostra Sect. 5. descripta est).

Chrysis smaragdula Collect. Selcestedti Mus. Reg. Havniens., ubi 2 specimina, "Ex Ind. Or." inscripta, anno 1847 examinavi et descripsi.

Habitat in India orientali. Specimen unicum in Tranquebaria lectum communicavit D.Westermann.

Observ. Propter maculas abdominis 2 viridi-aureas Chrysidi oculatae Fabr. assimilis, at longitudine et sculptura valde diversa.

Corpus magnum robustum elongatum sublineare obtusum. Caput et thorax confertim crasse punctata subreticulata viridia, vertice, dorsulo, scutellique disco fuscoaeneis. Caput suborbiculatum oculis exsertis; cavitas facialis quadrata subprofunda punctulato-coriacea viridissima, medio inaequaliter canaliculata, lateribus albo-sericea, superne marginata: margine $\sim \sim$ transverso inaequali flexuoso subacuto; antennae subbreves fuscae robustae, basi viridi-cyaneae; clypeus brevis transversus punctulatus nitidus viridis, disco nonnihil convexus, ad centrum marginis apicalis cupreo-aureo-micans et leniter arcuato-emarginatus; labrum semilunatum convexum piceum ciliatum; mandibulae breves, basi aureo-virides,

II.

21 
apice brunneae; mesopleurarum margo posticus ad apicem crenulatus et hinc ad certum situm luminis quasi denticulatus apparet; metanoti anguli postico-laterales magni triangulares; pedes virides tarsis fuscis, femora anlica antice subaenea, tibiae anticae exlus cyanescentes; alae brunneae venis concoloribus firmibus, cellula radiali elongata lanceolata, aut completa aut subcompleta et tunc apice angustissime aperta. Abdomen elongatum sublineare, capite thoraceque simul suntis longius, dorso sat convexum viridi-cyaneum nitidum; segmenta 1 :mum et 2:dum creberrine punctulata, hoc ad marginem apicalem utrinque macula subparva splendidissima viridi-aurea; segmentum 3:tium subremote punctulatum, disco leniter transverse inmersum, ante seriem convexiusculo-terminatum; series ante-apicalis immersa per carinulam medianam parvam convexiusculam interrupta, foveolae numerosae profundae majusculae arcuatae aut subquadratae, margo apicalis punctu tus den stis acute naturae 3

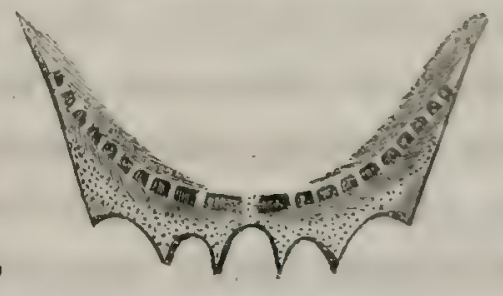
prominulus subtiliter latus fortiter 6 -dentatibus omnibus robutriangularibus, emargimediae arcuatae, centralis profundior, emarginaturae externae valde obliquae et latiores. Venter viridi-cyaneus nigro-maculatus.

176. Chrysis oculata Fabr. $\sigma^{7}+$.

Magna robusta 4 lin. decimal. long. crassissime punctalo-reticulata viridis, abdomine modice aut subcrasse punclato viridi-cyaneo, segmento 2:do utrinque macula ocellari purpureo - aurea viridi circumscripta, alis brunnescentibus cellula radiali incompleta. 
Clrysis oculata Fabr. Ent. Syst. 2. 239:5, Piez. 171:6; - Dlbm. Dispos. 1845. 15:34.

\section{Mabilat Tranquebariae, D. Westermann.}

Obserr. Valde similis Chrysidi Schiödtei; sed corpore majore et robustiore, capite thoraceque crassissime punctatissimis, abdomine modice punctato, segmenti 2: di maculis lateralibus majoribus rubro-aureis, mesopleuris apice utrinque fortiter mucronatis etc. certe dignoscitur.

Caput ut in Chrysidi Schiödtei constructum, maris paullulum angustius quam feminae; cavitatis facialis margo superus forma varians, scil. aut in Chryside Schiödlei, nut ita formatus. Thorax subbrevis, crassus, gib L L bus subovali-quadratus viridis; dorsuli su turae medianae et scutelli discus fusco-aenescentes, interdum totum mesonotum aeneo-purpurascens; postscutelli centrum crasse foveatum, submarginatum margine inaequali subcrenulato; metanoti anguli postico-laterales magni robusti triangulares; mesopleurae apice utrinque fortiter les tarsis apice fuscis; nis concoloribus robu mucronatae; pedes virialae brumnescentes, vestis, cellula radiali subtriangulariter lanceolata incompleta: vena radiali plerisque longe ante apicem alae oblitterata. Abdomen robustum elongatum capite thoraceque simul sumtis perspicue longius, ovali-subrectangulum scil. basi paullulum latius quam apice, dorso crasse punctatum valde convexum viridi-cyaneum, ceterum uti Chrysidis Schiödlei constructum, excepta segmenti 2 :di utraque macula laterali, quae major et distinctior - ut jam diximus - exstat. Venter viridi-cyaneus nigro-maculatus. 
Sect. V. Abdominis dorsum aut viride, aut cyaneum, aut cocruleum, aut violascens, nunquam aureopictum.

Divis. 1. Postscutellum inerme (non inucronatum, licet interdum crasse rugosum).

Subdivis. 1. Abdominis segmenti dorsalis 3:tii dentes: 4 apicales et 2 laterales; uterque dens lateralis prope basin marginis lateralis, et juxta initium seriei ante-apicalis, situs.

177. Chrysis splendens Klug.

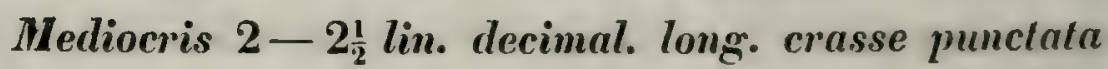
viridi-cyanea vertice thoraceque aureo-maculatis, abdominis dorso viridi-maculato, segmento 2:do carinula mediana distincta, alis hyalinis.

Chrysis splendens Klug secundum Mus. Spinolae.

Habitat in Africa meridionali ad promontorium bonae spei, Mus. D. Spinola.

Observ. Species formosa, Euchraeo purpuralo ad habitum paullulum similis. - Corpus ovale sparse cinereo-pilosum obtusum. Caput et thorax fortiter crasse punctata subreticulata. Caput rolundato-triangulum viride, vertice disco violaceo; oculi valde exserti, caritas facialis mediocris rectangularis subtiliter punctulato-coriacea, medio canaliculata, lateribus albo-sericea, superne transverse marginata margine sursum ter angulato h et emittente in verticem duos ramulos, supra stemma an- 
ticum concurrentes, aream subreniformem efformantes; antennae mediocres fuscae, basi cyaneo virescentes; clypeus viridis splendidus mediocris transversus inaequalis, margine apicali toto leniter arcuato-emarginato; mandibulae normaliter pictae, basi viridi-irroratae; labrum convexo-depressum semilunatum nigro-piceum; palpi maxillae et tubulus labialis brunnescentes; occiput utrinque angulo prominulo e carinula transversa orto. Pronotum violaceum aut cyaneum utrinque macula transversa viridiaurea; dorsuli areae laterales concolores, - area media I. cyanea I. purpureo-violacea, antice utrinque leviter viridi-irrorata; scutellum et postscutellum cyaneo-viridia aut viridi-cyanea. Metanotum cyaneum lateribus virescens. Mesopleurae aureo-virides, apice crenulatae et hinc irregulariter quasi denticulatae. Pectus viridi-cyanescens. Pedes cyaneo-virescentes tarsis fuscis. Alae hyalinae venis mediocribus piceis, cellula radiali subtriangulariter lanceolata subcompleta, apice anguste aperta. Abdomen fere ut in Hedychris formatum, dorso modice convexum confertim crasse punctatum, segmentum 2: dum carinula mediana distincta; segmentum 3:tium breve: sulco mediano brevi, totum - secundum basin - sulcatoimmersum, tum modice convexo-incrassatum ante seriem; series ante-apicalis foveolis mediocribus inaequalibus numerosis; margo apicalis mediocris parce punctulatus; dentes mediocres spinoidei a latere remoti, intermedii approximati; emarginaturae arcuatae, centralis angustior quam proxima; margo lateralis ad basin dente triangulari valido spinoideo a dentibus apicalibus longe remoto, interstitium - iuter hunc dentem et dentem apicalem externum - leniter convexo-arcuatum. Venter fornica- 


\section{Famil. Chrysididae. Chrysis: Phalanx VIII.}

tus violascens aut cyanescens, parum nigro - et viridipictus. Hanc speciem ita variantem vidi:

var. $a$. abdominis dorsum violaceum, segmentum 1 :mum utroque latere aureo-viride; 2 :dum disco macula utrinque et apice fascia, -3 :tium disco fasciola laete viridibus.

var. $b$. abdominis dorsum cyaneum; segmentum 1:mum disco transversaliter viride, 2 :dum utrinque et 3:tium disco indeterminate viridia.

178. Chrysis Wahlbergi Dlbm. $\delta 7$.

Magna $3-3 \frac{1}{2}$ lin. decimal. long. conferlim modice punctala cyaneo-viridis, alis fumalis apice hyalinis.

Chrysis.Wahllergi Dlbm. Dispos, 14:28.

Habitat in Africa meridionali-orientali, ad Port Natal a D. J. Wallberg detecta; Mus. Reg. Acad. Scient. Stockholm.

Observ. Staturae fere Chrysidis amethystinae Fabr.

Corpus robustum virescens pubescens confertim modice punctatum, punctura capitis et thoracis multo crassior quam abdominis. Caput rotundato - triangulum riridissimum macula verticis centrali cyanea; cavitas facialis rectangularis profunda, disco subtiliter transverse strigosa, lateribus punctulato-coriacea albo-sericeo subrillosa, superne transverse marginata: margine flexuoso, sursum ad centrum angulato et ex angulo o ramulum unicum versus stemma ductum emittente; $\sim$ in antennae mediocres apice brunneae, articulis basalibus 1 : mo viridi-cyanescenti, 2: do et 3:tio supra viridibus; clypeus subbrevis transversus irregulariter trapezinus, punctatus disco convexus, margine apicali late angulato-emarginatus 
Famil. Chrysididae. C'hrysis: Phalam.x VIII. 315

$m l$ margine utroque laterali oblique arcuatoma $u^{\circ} l^{\circ}$ ) emarginatus; labrum parvum semilunatum maxillaeque piceae, palpi testaceo-brumnei. Thorax crassus quadrato-subcylindricus, ad abdomen latius quam ad caput, viridis dorsuli area media basi concolore, ceterum aut mesonoto et postscutello aut tantummodo dorsuli area media (praeter basin) cyaneus; alarum tegulae aut virides aut viridi-cyancae; postscutellum propter sculpturam crassam margine postico manm leniter subangulato-prominulum; metanoti anguli postico-laterales maximi oblique transversi, irregulariter subtrapezinac; mesopleurae apice obtuse crenulato-denticulatae; pedes virides splendidi, tarsis nigro-brunneis. Alae fumatae, ad certum situm luminis nonnihil violascentes, apice albo-hyalinae, venis omnibus robustis piceis, celIula radiali elongata lanceolato-acuminata subcompleta: apice angustissime aperta. Abdomen robustum elongatum ovali-subrectangulum, capite thoraceque simul sumtis distincte longius, dorso sat convexum splendidum modice aut subcrasse punctatum, aut viridissimum aut viridicyaneum; segmenti 3:tii margo et apicalis et lateralis aut cyaneus aut violaceus, punctulatus; series ante-apicalis per carinulam brevissimam interrupta, foveolae mediocres profundac numerosae rotundatae; dentes: apicales triangulares, intermedii paullo sobustiores quam secundarii; emarginatura centralis subtriangularis, utraque

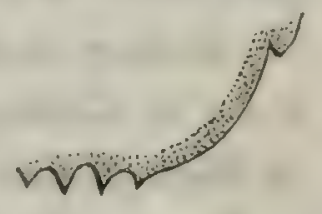

*) $m l=$ margo uterque latcralis, $m a=$ margo apicalis. 
proxima oblique arcuata; uterque dens lateralis ad basin marginis lateralis situs validus acutangulus, a dente apicali externe longe remotus, margo inter hunc et dentem apicalem proximum obliquus arcuatus. Venter fornicatus viridi-cyaneus.

Subdivis. 2. Abdominis segmenti dorsalis $3:$ tii dentes: omnes 6 apicales.

Tribus 1. Dentes 2 externi obtusi, ex apice marginis lateralis arcuato formati.

179. Chrysis violacea Panzer. $\delta$ 오.

Mediocris $2 \frac{1}{2}-3$ lin. decimal. long. angusta confertim modice punctato-reticulata violacea, capite antice pedibusque virescentibus, abdomine punctulato, lateribus apiceque viridi-cyanescente, segmenti 3 :tii dentibus apicalibus 4 mediocribus acute triangularibus, 2 externis majoribus obtusis, alis hyalinis.

Chrysis violacea Panz. Krit. Revis. 2. 103.

Chrysis sexdentata Panzer Fn. Germ. 51:12. nota bene: iconem pictam et "Habitat Norimbergae" (; exclusis: figura segrnenti abdominis ultimi aucti, diagnosi et descriptione, quae omnia pertinent ad Clirysidem sexdentatam Fabr. Confer no. 183 in hoc nostro libello):

Chrysis indigotea Dufour Annal. Entomol. 1840. 38 : 18. ठౌ. sccundum speeimen typicura a Cel: Dufour nobis donatur.

Habitat in Germania non raro D. Panzer Krit. Reris. 1. c.; in Grallia, ad St. Sever, "nidificat in ramis exsiccatis Rubi fruticosi, parasita Odyneri rubicolae" Duf. l. c. ; in Bavaria, D. Spinola; in Austria ad Viennam, D. Kollar; in America septentrionali, D. Latreille, Mus. Spinolae. 
Famil. Chrysididae. Chrysis: Phalanx VIII. 317

Observ. Quae de Chryside indigotea (no. 113) jam scripsimus, etiam ad hanc speciem maxima ex parte applicanda sunt; ea tamen certissima differentia, quod: apud Chrysidem violaceam thorax est totus violaceus, nec nisi sterno et metanoti lateribus virescens; - abdominis segmenti dorsalis 3 : tii dentes apicales 6 , quorum: ex 2 externae ab illis nonnihil remoti fortiter angulati obtusiusculi; emarginaturae 3 intermediae mediocres rectà arcuatae, centralis forsitan paullulum profundior, externac duae latae et obliquae; - venter viridis. In reliquis Chrysidi indigoteae simillima.

Tribus 2. (Abdominis segmenti dorsalis 3: tii) dentes apicales omnes 6 acuti: aut triangulares aut spinoidei.

Manipulus 1. Abdominis dorsum distincte fasciatum: fasciis aut violaceis aut nigricantibus.

A. Abdominis segmentum dorsale 3:tium totum convexo-depressum; area postica ( $=$ margo apicalis) igitur non humilior quam area antica $s$. basalis et maxima, sed totum segmentum undique unam eandenque convexo-depressitatem aut depressoconvexitatem praebet.

180. Chrysis Smidti. Dlbm.

Submagna 3 lin. decimal. long. viridi-cyanea, marginibus segmentorum abdominis dorsalium basalibus violascentibus, apicalibus virescentibus, segmenti 3:tii serie 
318 Famil. Clhrysididue. Chrysis: Phalamx VIII.

ante-apicali foveolis majoribus rolundatis, denlibus robuslis triangularibus, alis fusco-hyalinis.

„Chr. nov. sp. ex Amer. mer. Smidt affinis smaragdulac"; Specimen unicum in Collectione Lundio-Sehestedti Mus. Reg. Hasniens. ita, ut jam notavimus, inscriptum anno 1847 examinavi et descripsi.

IIabitat in America meridionali, a D. Smidt olim detecta; Mus. Reg. Havniens.

Observ. Caput, thorax, et abdominis dorsum basi, crasse - reliqua segmenta abdominis dorsalia modicepunctata. Caput cano-pubescens vertice fusco. Pectus et metanotum viridia suturis coerulescentibus. Pronotum nigro-aeneum limbo viridi. Mesonotum aut nigro-aeneum aut fusco-violascens maculis viridibus indeterminatis sparsis. Scutellum et postscutellum nullo modo gibba, sed in uno plano cum reliquo thoracis tergo. Metanoti anguli postico-laterales validi acuti conico-spinoidei. Pedes virides nonnilil coerulescentes, femoribus posticis postice nigro-aenescentibus, tarsis omnibus fuscis. Alae fusco-hyalinae. Abdominis dorsum depresso-consexum: linea mediana continua violacea laevissima, non conrexa nec impressa sed plana; segmentum 1 :mum viride, medio cyanescens; segm. 2:dum basi violaceo-fuscum, tum cyaneum et utrinque macula viridi minus distincta, margine apicali virescens; segm, 3:tium margine basali tenuiter violascens, tum viride, iterum violascens et ante seriem virescens, in hoc loco (scil. ante seriem) non convexosubelevatum nec convexo-quasi terminatum sed eandem convexo-depressitatem undique praebens; series anteapicalis mediocris per carinulam medianam brevissimam convexo-planam interrupta; margo apicalis modice pro- 
Famil. Chrysydidae. Chrysis. Phalanx VIII. 319 minulus convexiusculus, dentibus 6 mediocribus triangularibus acutis in arcum dispositis.

B. Abdominis segmenti dorsalis 3 :tii area postica (= margo apicalis et lateralis) humilior quam area antica, quae - maximam segmenti partem efficiens - supra seriem ante-apicalem marginulo depresso - convexo limitatur.

a) (Abdominis segmentis dorsalis 3:tii) area postica utroque latere albo-vittata.

181. Chrysis Genbergi Dlbm. $\delta$.

Mediocris sublinearis $2 \frac{2}{3}$ lin. decimal. long. viridicyanea, capite thorace abdominisque segmento dorsali 1:mo crasse punctata, reliquis abdominis segmentis punclulata, segmenti 3:tii area postica depresso-convexa sublilissime coriacea cyanea utroque latere toto albopellucida, serie ante-apicali foveolis parvis rotundatis, dentibus omnibus 6 mediocribus acule triangularibus, invicem subaequalibus.

Clrysis fasciata var. Westermann in litteris.

Ilabitat in Brasilia, Mus. D. Westermann. In memoriam amici, viri perillustris Dom. Pauli Genberg, Regis Regnique Sveciae Senatoris et Ministri, jam anno 1829 in studio Historiae naturalis Chrysidum unacum me versati, denominata.

Observ. Clerysidi fascialae (no. 106) quodammodo similis, praeserim si colorem corporis respicias; sed corpore angustiore et breviore, abdominis segmento 3:tio 
aliter constructo et $\mathbf{6}$-dentato nec non utrinque albo-vittato facillime dignoscitur.

Corpus subgracile sublineare subglabrum, nec nisi levissime pubescens. Caput, thorax et abdominis segmentum dorsale crasse punctata, illa subreticulata, hoc subremote punctatum. Caput rotundato-triangulum viride, macula verticis centrali parva cyanea, oculis exsertis; cavitas facialis subangusta rectangularis profunda, subtilissime punctulato-coriacea, nitida viridi-cyanea, medio profunde canaliculata, utrinque albo-sericeo-pilosella, superne non transverse marginata sed cum convexitate frontis confusa; supra cavitatem facialem inter stemmata et oculos adsunt circ. 6 lineae curvae elevatae hanc figuram Th fere referentes; antennac breves, crassitie mediocres, nigro-fuscae, basi viridi-cyaneae; clypeus brevis transversus subreniformis punctulatus, disco subcarinulatus, ad centrum marginis apicalis emarginatus; mandibulac unacum ceteris oris partibus normales. Thorax rectangulariter subcylindricus, dorso sat convexus, totus laete viridis: pronoti truncatura antica utrinque, dorsuli margo basali et suturis medianis, postscutelli disco et metanoti margine maxime postico cyaneis; pedes cyaneo-virides, tarsis basi fuscis apice brunneo-testaceis; pronotum utroque latere acute et subtiliter marginatum, hinc ejus angulus lateralis anticus uterque acutus; metanoti anguli postico-laterales mediocres triangulares; mesopleurarum margo lateribus et apice inaequalis minus distincte denticulatus; alac albo-hyalinac, venis mediocribus piceis, cellula radiali clongata lanceolata incompleta apice 
Famil. Clrysididae. Chrysis: Plaalanx VIII. 321

modice aperta. Abdomen elongatum capite thoraceque simul sumtis longius, sublineare, basi nonnihil latius quam apice, angulo utroque laterali - basali obtuse prominulo, dorso sat convexum, lineola mediana subelevata in segmento 2: do ad certum situm luminis observanda; segmentum 1: Inum crasse et subremote punctatum basi trifoveatum, fasciis 3: 1:ma s. basali et 3:tia s. apicali viridibus, media s. 2: da cyanea; segmentum 2: dum fasciis 4 : 1: ma s. basali nigro-aenea, 2:da cyaneo-viridi, 3:tia violacea, 4:ta s. apicali viridi; segmentum 3:tium cyaneum, basi tenuiter nigro-aeneum, ante seriem virescens, margine laterali albo-pellucidum, qui color paullo ante dentem apicalem externum desinit; series ante-apicalis foreolis parvis rotundatis numerosis; dentes omnes 6 apicales mediocres acute triangulares invicem subaequales in arcum dispositi, emarginaturae mediocres modice arcuatae etiam invicem aequales; margo lateralis leviter obliquus et prope dentem arcuatus.

182. Chrysis Klugi Dlbm. ㅇ. .

Mediocris sublinearis $2 \frac{1}{3}$ lin. decimal. long: punctatissima viridi-cyanea, abdominis segmenti dorsalis 3 :tii area postica prominula depressa nitidissima purpureoviolacea, ad basin utriusque lateris villula cuneala albopellucida, serie ante-apicali per carinulam medianam continuam interrupla, foveis crassis longiludinalibus sulciformibus circ. 14 in parte violacea, dentibus apicalibus spinoideis incequalibus: intermediis 2 brevissimis, secundariis 2 mediocribus, externis 2 majoribus.

IIabitat in Brasilia, Mus. D. Drewsen.

Observ. Valde similis proxime praecedenti (Chry- 


\section{Famil. Chrysididae. Chrysis: Platanx VIII.}

sidi Genbergi); sed corpore punctatissimo, postscutelli margine postico angulato-prominulo, mesopleuris apice distincte emarginato-bidentalis, praecipue vero constructione et armatura areae posticae segmenti abdominalis dorsalis 3:tii eminentissime dignoscitur haec eximia species.

Corpus elongatum angustum sublineare sparse fuscoet cano-pubescens, punctatissimum. Caput suborbiculatum viride, vertice pone stemmata cyaneo-irrorato; oculi valde exserti; cavitas facialis subangusta subrectangularis, profunda subtiliter punctulato-coriacea non canaliculata, lateribus albo-sericeo villosa, supra discum forea centrali, superne non marginata; proxime supra hanc cavitatem adest in vertice linea elevata subarcuata fere hanc<smiles>C1CCCC1</smiles>

figuram referens; antennae breves robustae fuscae, basi cyaneo-virides; clypeus transversus submediocris omnino uti Chrysidis Wesmaëli constructus; labrum parvum semilunatum punctulatum viride, ciliatum; mandibulae breves rufo-piceac, basi subaurato-virides; palpi maxillaeque fuscescentes. Thorax robustus quadrato-subcylindricus cyaneo-viridis, pronoti maculis 2 transrersis dorsulo intra marginem nigro-aeneis, pronoti truncatura antica et metanoti fovea postica maxima purpureo-riolaceis; pronotum utroque latere tenuiter subacute marginatum angulo utroque laterali-antico obtuso; postscutellum cyaneum, margine postico propter sculpturam crassam subcrenulatum et in centro ejusdem marginis leniter angulato-prominulum et subapiculatum, quemadmodum fere ut in descriptione Chrysidis Wallbergi (no. 178) jam demonstravimus; metanoti anguli postico-laterales 
robusti ralidi triangulares; mesopleurae ad apicem marginis postici leniter late arcuato-emarginatae et hinc

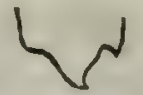

distincte bidentatae; pedes largiter cinereo-piloselli, secundum diversum situm luminis aut cyaneo-virides aut viridi-cyanei; tarsis brunneis, articulo basali extus pedibus concolore; alae hyalinae, secundum costam cellulae radialis linea fumea insignes, venis firmibus piceis, cellula radiali mediocri lanceolata incompleta apice sat aperta, rena radiali longe ante apicem alae oblitterata. Abdomen elongatum capite thoraceque simul sumtis distincte longius, subovale, basi transversum thorace fere latius, angulis laterali-basalibus validis obtusis, apice arcuato-angustatum, dorso toto crasse punctatum, punctis segmenti 1 :mi crassissimis; segmentum 1 :mum fasciis 4 : 1:ma purpureo-violacea, 2 : da viridi, 3 : tia violacea, $4: t a$ s. apicali-marginali tenui viridi; segmentum 2: dum fasciis quoque 4 scil. 1 :ma s. basali et 3 :tia nigro-aeneis subpurpurascentibus, 2: da et $4:$ ta virescenti-cyaneis, in hoc segmento lineola mediana polita planiuscula impunctata nigro-aenea obserranda; segmentum 3:tium elongatum 2 : do vix nisi parumper brevius, angustato-arcuatum: area antica s. basalis ad basin convexa nigro-aenea, disco et apice convexo-depressa virescenti-coerulea; - area postica (margines et apicalem et lateralem efficiens) valde prominula depressa polita parce subtiliter punctulata formose et saturate purpureo-violascens, carinula mediana acuta continua, utrinque juxta basin areae anticae est margo areae posticae - instar lineac cunciformis - albopellucidis; seriem foveolarum et dentes apicales jam in diagnosi satis notavimus; emarginaturae valde inaequales: 


\section{Famil. Chrysididae. Chrysis: Phalanx VIII.}

centralis (inter dentes intermedios) lata lenissime transverse arcuata, emarginaturae secundariae mediocres oblique arcuatae, emarginaturae externae latiores maxime oblique arcuatae; margo lateralis inter basin segmenti et dentem externum elongatus obliquus leniter curvus. Venter nitidissimus, leviter fornicatus, subtiliter punctulatus; viridissime cyaneus, basi nigro-maculatus.

b) Abdominis segmenti dorsalis $3:$ tii area postica unicolor, aut cyanea aut virescens.

183. Chrysis sexdentata Fałr. $\sigma^{\top}+$.

Mediocris aut magna $2 \frac{1}{2}-3 \frac{1}{2}$ lin. decimal. long. punctatissima, aut viridissima aut viridi-cyanea, mesopleuris apice obtuse tridentatis, abdominis segmenti dorsalis 3:lii serico ante-apicali foveolis mediocribus rotundatis numerosis, denlibus acute triangularibus unicum emarginaturis aequalibus.

Chrysis sexdentata Fabr. Ent. Syst. Suppl. 258:20-1, Piez. 175:28 certissime! secundum exemplar typicum, quod in Collectione Fabriciana Musei Universitat. Kicl. anno 1847 lustravi et descripsi.

Chrysis sexdentata Panzer Fn. Germ. 51:12. nota bene: figuram "segmenti abdominalis ultimi, diagnosin et descriptionem; sed nullo modo "Habitat Norimbergae", quae verba unacum icone picta ad Chrysidem violaceam (nob. no. 179) pertinent.

"Habitat Cajennae Dom. Richard" Fabr. 1. c. E Brasilia a D. Drewsen, e Cajenna a D. Spinola communicata. Etiam unicum specimen, in Senegal lectum, e Collectione Latreillei nobis transmisit D. Spinola.

Observ. Chrysidi violaceae tantummodo pictura quodammodo similis; sed in ceteris omnibus longe diversa. 
Corpus elongatum robustum punctatissimum, capite thorace abdominisque segmento dorsali 1:mo punctatoreticulatis, plus minus fusco - et cano-pubescens, aut raro totum viridissimum, aut raro cyaneum, plerumque viridicyaneum I. viridi-coeruleum fasciis et maculis nigricantibus et violascentibus pictum. Caput suborbiculatum viridicyaneum; cavitas facialis longitudinalis subtrapezina parum profunda punctulato - coriacea, lateribus cano-villosa, superne: margine transverso inaequali utrinque deflexo et hinc $\neg$ subrectangulari; antennae robustae, longitudine mediocres fuscae basi viridi-cyaneae, clypeus transversus mediocris viridis punctulatus, disco convexiusculus ad centrum marginis apicalis modice emarginatus utrinque arcuato-obtusus, hinc totus fere ita formatus; labrum subsemilunatum punctulatum parum convexum, aeneo-virescens, fulvo-ciliatum; mandibulae basi virescentes, ceterum unacum maxillis palpisque piceae. Thorax crassus ovato-subquadratus virescenti-coeruleus, pronoti fasciola aut continua aut interrupta, dorsulo 1. toto I. macula in singula quaque area nigro-aeneis; pronoti truncatura antica centro, scutellum et metanoti fovea maxime postica purpureo-violascentia; postscutellum cyaneum gibbum, margine postico ad centrum obtuse prominulo; metanoli anguli postico laterales robusti prismatico triangulares; mesopleurae utroque apice fortiter tridentatae; sternum et pedes viridi-coerulescunt, tarsis basi concoloribus, apice fuscis; alae aut subhyalinae aut sordidae, secundum costam cellulae radialis linea fumea, venis omnibus firmibus nigro-piceis, cellula radiali elongata lanceolata subcompleta, apice peranguste aperta. Abdomen robuII. 
slum elongatum capite thoraceque simul sumtis distincle longius, subovale, basi latius, apice angustato-arcuatum, dorso sat convexum crasse confertim punctatum, aut riride aut coerulescens, segmentis 2 : do et 3 :tio basi fascia nigro-violacea purpurascente, in his segmentis carinula mediana convexiuscula polita impunctata observanda; segmenti 3:tii series ante-apicalis per carinulam brerem robustam convexam interrupta, foveolae profundae mediocres rotundatac numerosac; dentes omnes 6 apicales mediocres at nihilominus robusti, acute triangulares, A quoad magnitudinem subaequales, in arcum dispositi; emarginaturae tres intermediae mediocres modice arcuatae, externae duae latiores et ralde oblique arcuatae. Venter viridi-cyaneus nigro-maculatus.

Manipulus 2. Abdominis dorsum non fasciatum.

A. Abdominis segmenti dorsalis $\mathbf{1}$ :mi area lateralis intramarginalis punctulato-coriacea.

184. Chrysis modica Dlbm. ㅇ․

Submediocris vix 3 lin. decinal. long., capite thoraceque modice punctato-reticulata abdomine punclulato. coeruleo-viridis, abdominis segmento dorsali 3:tio disco non nisi obsoletissime immerso, emarginaturis apicalibus tribus intermediis invicem subaequalibus, alis brunnescentibus, cellula radiali compleia.

Chrysis mediocris Dlbm. Dispos. 14:31.

Habitat in Africa; 3 specimina vidimus; unum in Guinea lectum, a D. Westermann nobis olim donatum; alte- 
rum e promontorio bonae spei a D. Spinola ut variet. Chrysidis malachiticae communicatum; tertium e Port Natal a D. J.Wahlberg reportatum, Mus. R. Acad. Scient. Stockholm.

Observ. Chrysidi sexdentatae Fabr. assimilis, at corpore vulgo breviore, capite thoraceque modice punctatis, abdomine confertim punctulato unicolore nec fasciato, mesopleuris non apice dentatis, alis brunnescentibus etc. satis superque dignoscitur.

Corpus mediocre elongatum pubescens; caput et thorax confertim modice punctato-reticulata. Caput rotundato-triangulum viride, verticis macula centralis violascens; cavitas facialis mediocris subrectangularis parum profunda, punctulata, disco aut subtiliter canaliculata aut foveola mediana, lateribus albo-sericeo villosa, superne leniter marginata: margine transverso inaequali; antennae robustae, longitudine mediocres, nigro-fuscae, basi viridicyaneae; clypeus transversus mediocris punctulatus, disco convexus, margine apicali toto leniter arcuato-emarginato; mandibulae ceteraeque oris partes normales. Thorax robustus rectangulariter subcylindricus, dorso modice. convexus, undique aut coerulescenti-riridis aut viridissimus, exceptis pronoti truncatura antica maculisque 3, dorsuli suturis medianis et interdum scutelli disco nigroaeneis; postscutellum cyanescens, margine postico obtuse subprominulum et propter sculpturam subcrenulatum apparet: metanoti anguli postico-laterales mediocres triangulares; pedes thoraci concolores, tarsis fuscis; alae brunnescentes, ad certum situm luminis subriolaceo-nitidae, pellucidae, venis firmibus piceis, cellula radiali subtriangulariter lanceolata completa. Abdomen elongatum ovali- 
sublineare, basi transversa latius quam apice arcuato, capite thoraceque simul sumtis longius, dorso modice convexum aut viridi-coeruleum aut coeruleo-viridissimum, aliis confertim aliis confertissime punctulatun; segmenti 3:tii area antica ad seriem convexo-decliri terminata, area postica mediocris depressiuscula subtilissime punctulato-coriacea; series ante-apicalis, dentes et emarginaturae apicales fere similline ut in Chryside sexdentata (no. 183). Venter viridis nigro-maculatus.

185. Chrysis prominula Dlbm. $\sigma^{7}$.

Maxima $4 \frac{1}{2}$ lin. decimal. long. robusta, capite thoruceque subcrassissime punctato-reliculata, abdomine punctulata viridis, abdominis segmento dorsali 3:tio disco distincte immerso, emarginatura apicali centrali multo angustiore quam reliquis, alis brumnescentibus cellula radiali incompleta.

Chrysis prominula Dlbm. Dispos. 1845. 14:32.

Patria nobis ignota; specimen unicum e Collectione Paylsulli Mus. R. Acad. Scient. Stockholm. communicavit D. Boheman.

Observ. Statura et pictura nec non abdominis punctura Chrysidi modicae affinis et similis; at corpore crassiori et fere duplo majori, capite thoraceque multo crassius punctatis, mesopleuris dentatis, segmento abdominis 3:tio disco distincte licet leniter immerso, emarginatura centrali angusta etc. facillime distinguenda. - Etiam C'hrysidem oculatam (no. 176) simulat, sed ab hac mox dignoscitur abdominis dorso immaculato, toto punctulato, serie ante-apicali foveolis omnibus rotundatis et - cum Chr. oculatae comparatis - parvis. 
Corpus crassum elongatum. Caput transverse suborbiculatum punctato-reticulatum, parte orali transverse subrectangulari, totum viridissimum, macula verticis centrali nigro-aenea opaca; cavitas facialis quadrata confertissime punctulato-coriacea, cano-pilosella, planiuscula, superne omnino ita marginata ut in Chryside oculata (cfr. figuram primam in descriptione no. 176); antennae breves fuscae basi viridi-cyaneae; clypeus omnino ita constructus ut in Chryside Wallbergi (no. 178, cfr. figuran ibidem); labrum mediocre semilunatum convexum virescenti-acneum; mandibulae brevissime subprismatice conicae acutae piceae basi virescentes; palpi fusco-brunnei. Thorax crassus oblonge quadrato-cylindricus, postice propter metanoti angulos conspicue latior quam antice, crassissime punctato-reticulatus viridis; pronoti truncatura antica, alarum tegulae et metanotum disco cyanea; pronoti fasciola postica, dorsuli vittae medianae $e^{t}$ discus scutelli opaco nigro-aenescentes; postscutelli centrum fere ut in Cleryside oculata obtuse prominulum et crasse foveatum nec non submarginatum: margine inaequali subcrenulato; metanoti anguli postico-laterales maximi fere ut in C'lrysidi Wallbergi (no. 178, cfr. figuram ibidem) constructi, at margo transversus posticus non ut in Chr. Wahlbergi arcuatus sed sic $\sim$ flexuosus; mesopleurae margine fere hoc modo

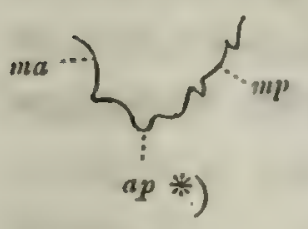

crenato-dentatae; pedes cyaneo-virides, tarsis fuscis, femori intermediis latere postico nigro-aenescentibus; alae brun-

*) $m a=$ margo anticus; $m p=$ margo posticus; $a p=$ apex marginis. 
nescentes venis piceis firmibus, cellula radiali lanceolata, apice late aperta. Abdomen ut in Chryside modica constructum; segmenta dorsalia 1:mum et 2:dum viridia, 3:tium cyaneum nec nisi apice leviter virescens; segmentum 3:tium disco leniter at distincte concaviusculo-immersum, tum ante seriem convexiusculo marginulatum; series ante-apicalis foveolis mediocribus aut potius subparvis rotundatis numerosis; regio segmenti postica prominula subdepressa subtiliter punctulato-coriacea; dentes apicales robusti, magnitudine mediocres et invicem subaequales, emarginaturae arcuatae inaequales, centralis angustior, secundariae mediocres obliquae, externae latiores obliquae. Venter cyaneo-vi-

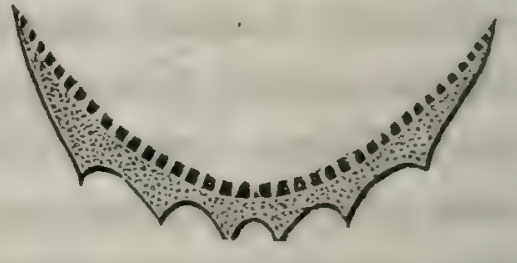
ridis nigro-maculatus fornicatus.

B. Abdominis segmenti dorsalis 1:mi utraque area lateralis intra-marginalis crasse punctata: punctis aut numerosis et confertis aut paucis et remotis.

a) Segmenti 3:tii emarginaturae apicales invicem magnitudine aequales aut subaequales.

186. Chrysis aequinoctialis Spinola. $\delta$ ㅇ.

Mediocris aut subparva 2-3 lin. decimal. long. sublinearis confertim modice punctata subopaca coerulea alis albis, abdominis segmento dorsali 3:tio toto depresso-convexo, serie ante-apicali foveolis dentibusque apicalibus parvis.

Chrysis aequinoctialis Mus. Spinolae.

Habitat in Brasilia, Dom. Reiche; Mus. Dom. Spinola. 
Observ. Corpus sublineare, totum coeruleum subopacum, confertim modice punctatum, ore pedibusque paullo viridi-nitidis. Caput transversum suborbiculatum; cavitas facialis subquadrata subtiliter punctulata planiuscula, non profunda superne transverse et subacute marginata (; antemnae in speciminibus missis mutilatae); clypeus transversus brevis parce punctulatus, disco parumper convexus, margine apicali late et leniter subarcuatoemarginatus; labrum parvum semilunatum nigro-aeneum; mandibulae ceteraeque oris partes piceac, illae basi virescentes; margo occipitalis utrinque angulo parvo dentiformi. Thorax subrectangulariter cylindricus, dorso depresso-convexus; metanoti anguli postico-laterales robusti acute triangulares; meso-pleurae margine obtuse 4-denticulatae; pedes corpori concolores, extus unacum tarsorum articulo basali virescenti nitidae, tarsis fuscis; alae albo-hyalinae, venis firmibus nigropiceis, cellula radiali lanceolata subcompleta: apice angustissime aperta. Abdomen subovale, basi transversum et conspicue latius quam apice arcuato, dorso modice conrexum et crebre punctatum; in utroque latere segmenti dorsalis $\mathbf{1}$ :mi intra marginem deflexum adest area cuneata concaviusculo-plana marginulata parce crasse punctata; segmenti 3: tii series ante-apicalis foveolis parvis aut minulis rotundatis numerosis; dentes apicales parvi acute triangulares in arcum lenem dispositi, invicem aequales; emarginaturae mediocres, modice arcuatae, 3 intermediae subaequales, externae 2 paullulum latiores et obliquae; venter viridi-cyaneus nigro-maculatus. 
332 Famil. Chrysididae. Chrysis: Phalanx VIII.

187. Chrysis insularis Guerin. ㅇ.

Magna $3 \frac{1}{2}$ lin. decimal. long. robusta confertim crasse punctata virescens, abdominis dorso purpurascenti-violaceo, alis hyalinis, disco violascentibus, segmento abdominis 3:lio ante seriem convexo submarginato, serie ante-apicali foveolis distinctioribus, dentibus apicalibus robustis.

Chrysis insularis Guerin, secundum Spinola.

Habilat in insula Cuba, Dom. Poey; Mus. Dom. Spinola.

Observ. Corpus robustum elongatum parum nitidum, subopacum, confertim crasse punctatum. Caput rotundato-triangulum lacte viride; cavitas facialis rectangularis parum profunda, subplaniuscula, crebre punctulata, medio canaliculata, superne marginata: margine omnino ut in Chryside oculata (no. 176, cfr. figuram ibidem primam) formato; antennae longiusculae, non crassae, fuscae, basi cyaneo-virides; clypeus mediocris transversus irregulariter subtrapezinus $น$, punctulatus, disco modice convexus, ad centrum marginis apicalis leniter arcuato-emarginatus; labrum parvum semilunatum aenescens; mandibulae castaneae, basi viridi-pictae. Thorax crassus quadrato subcylindricus viridis, dorso valde convexus; dorsulum et scutellum basi, metanotum maxima ex parte, cyanea; metanoti anguli postico-laterales validi irregulariter prismatico-triangulares acuminati

marginibus flexuosis; mesopleurarum margo fere ut in Chryside aequinoctiali (no. 186, cfr. figuram ibidem) 
crenulato-denticulatus; pedes viridi-cyanci tarsis fuscis; alae basi apiceque albo-hyalinae, disco fumatac et violascentes, venis omnibus nigro-piceis firmibus, cellula radiali subtriangulariter lanceolata incompleta, apice anguste aperta. Abdomen robustum subovale basi transversun, capite thoraceque simul sumtis conspicue longius, dorso crebre crasse punctatum interstitiis subtilissime punctulatis, pulchre violaceum purpurascens ambitu viridi-coerulescente, margine basali segmentorum 2 : di et 3:tii nigro-aeneo; segmenti dorsalis $\mathbf{1}$ : mi utraque area lateralis intramarginalis: sublinearis apice angustata, tota modice concava punctis crassis parce adspersa, superficie ceterum subtilissime coriacea, utrinque marginulata; in segmento 2:do lincola mediana elevata subtilis obsoletissima; segmenti 3:tii area antica s. basalis medio leniter at distincte transverse immersa, ante seriem convexo-terminata; series ante-apicalis per carinulam medianam brevissimam convexam obsoletam interrupla, foveolae numerosae rotundatae mediocres, distinctiores quam in Chryside aequinoctiali; dentes apicales robusti acute triangulares, centrales 2 paullulum majores quam secundarii 2 mediocres, externi 2 breviores et latiores, omnes 6 leniter arcuatim dispositi; emarginaturae mediocres, centralis obtuse subtriangularis, reliquae scil. et secundariae et externae invicem aequales atque nonnibil obliquae. 
334 Famil. C'hrysididae. C'hrysis: Phalanx VIII.

b) (Abdominis) segmenti (dorsalis) 3:tii utraque emarginatura externa angustior quam emarginatura centralis.

188. Chrysis inaequidens Dllom. $\sigma^{7}$.

Mediocris $2 \frac{2}{3}$ lin. decimal. confertim modice punctata abdomine punctulato, viridi-cyanea, alis sordide hyalinis. Habilat in New-York; specinen unicum ab Amico Kriechbaumer nobis donatum.

Observ. Statura fere Chrysidis nitidulae $\sigma^{\top}$ (no. 117).

- Corpus quoad crassitiem mediocre, supra viridi-cyaneum; capite antice, pectore pedibusque viridibus, rentre fortiter fornicato ad maximam partem concolore, tarsis pedum fuscis. Caput et thorax confertim modice punctata; cavitas facialis (capitis) rectangularis punctulatocoriacea, mediocris, superne marginata: margine transverso inaequali subarcuato; antennae mediocres fuscae, basi virides; clypeus brevis transversus punctulatus, disco convexus, ad centrum marginis apicalis leniter arcuatoemarginatus; mandibulae piceae, basi virescentes. Thorax crassus subquadrato-cylindricus, dorso sat convexus; metanoti anguli postico-laterales robusti triangulares; meso-pleurarum margines obsolete crenulatae. Abdomen mediocre, longitudine capitis thoracisque simul sumtorum, obtuse rectangulum, dorso sat convexum et creberrime punctulatum; segmenti dorsalis 1:mi utraque area lateralis intramarginalis cuneiformis punctis crassis fere alternatim dispositis adspersa, marginulata leniter concava; segmentum dorsale 3 :tium breve, totum depresso-convexum; series ante-apicalis foveolis rotundatis 
Famil. Chrysididae. Chrysis: Plealanx VIII. 335

numerosis inaequalibus scil. et majoribus et mediocribus et parvis; dentes apicales acute triangulares, magnitudine inaequales: bustiores, secundarii 2 mediocres, externi 2 breviores et paullo latiores; emarginaturae etiam inaequales: centralis magua triangularis profunda, secundariae 2 mediocres modice et nonnihil oblique arcuatae, externae 2 parvae etiam oblique arcuatae.

c) (Abdominis) segmenti (dorsalis) 3:tii utraque emarginatura externa latior quam emarginatura centralis.

189. Chrysis malachitica Draege. ㅇ.

Mediocris 3 lin. decinal. long. confertim modice punctata viridi-cyanea, abdominis segmenti dorsalis $3:$ tii serie ante-apicali foveolis mediocribus orbiculalis mumerosis, alis hyalinis, disco brunneis et violaceo-micanlibus.

Chrysis malachitica Draege, secundum Museum Spinolae.

Chrysis laeta Mus. Berolin., secundum Drewsen in litteris.

IIabitat in Africa meridionali, ad promontorium bonac spei a Dom. Draege detecta. Specimina tantum 2 vidi, unum a Dom. Spinola et alterun a Dom. Drewsen communicata.

Observ. Statura fere Chrysidis modicae (no. 184). - Corpus mediocre subovale viridi-cyaneum, confertim modice punctatum, punctura verticis thoracisque subreticulata et nomihil crassiore quam punctura abdominis, thorace abdomineque dorso valde convexis. Caput ro- 


\section{Famil. Clorysididae. Clhrysis: Plaalanx VIII.}

tundato-triangulum oculis valde exsertis; cavitas facialis rectangularis subprofunda, disco subtilissime transverse strigosa, ambitu punctulato-coriacea, lateribus cano-sericeo-villosa, superne marginata: margine fere hujus figurac; antennac perbreves robustae fuscac, basi viridicyaneae; clypeus transversus mediocris trapezinus parce punctulatus planiusculus, disco parumper convexus, ante marginem apicalem linea transversa impressus, ad centrum marginis apicalis lenissime arcuato-cmarginatus; mandibulae piceae, basi virescentes; labrum mediocre semilunatum gibbum cyaneo-virescens. Tíorax crassus, ad ambitum quadrato-subcylindricus, dorso valde convexus; metanoti anguli postico-laterales omnino ut in Clrr. alternanti (no. 130, cfr. figuram ibidem) constructi; mesopleurae utroque apice distincte $h / 3$-dentatae; pedes cyanco-virescentes tarsis fuscis; alae basi apiceque hyalinae, disco saturate brumneac et ad certum silum luminis violascentes, venis firmibus fuscis, cellula radiali triangulariter lanceolata incompleta, apice modice aperta. Abdomen ovatum, capite thoraceque simul sumtis longius, dorso valde convexum et confertim modice punctatum, in segmentis 2:do et 3:tio lineola mediana subelerata planiuscula polita impunctata viridi observanda; segmenti 3:tii series ante-apicalis per carinulam brevissimam convexam medianam interrupta, foveolae profundae, magnitudine mediocres, orbiculatac numerosac; dentes apicales acute-triangulares inaequales: 2 intermedii paullulum majores, 2 secundarii mediocres, 2 externi perbreves; emarginaturae quoque inaequales: centralis mediocris perfecte 
Famil. Chrysidedae. Chrysis: Platanx VIII. 337

transverse arcuata, secundariae paullum latiores et oblique arcuatae, externae lenius, latius et (quam secundariae) magis oblique arcuatae. Venter fornicatus viridi-cyaneus.

190. Chrysis smaragdula Fabr. 오.

Maxima subsemipollicaris robustissima et punctatissima, aut viridissima aut viridi-cyanea, abdominis segmenti dorsalis 3:tii serie ante-apicali foveis magnis profundis quadratis numerosis, alis violascentibus, basi hyalinis.

Chrysis smaragdula Fabr. Ent. Syst. 2. 239:2, Piez. 171:2; Dlbm. Dispos. 1845. 15:33.

„Habilat in America boreali "Fabr. 1. c. Specimina duo e Carolina meridionali Americae borealis, prope Georgetown lecta, nobis transmisit et amice donavit Dom. Zimmermann.

Observ. Inter maximas et maxime eximias species Generis, unde juxta Stilbum splendidum a Fabricio etiam collocata fuit.

Corpus robustissimum maximum $4-5$ et ultra lin. decimal. longum adeoque distincte punctatissimum ut punctura oculo quidem inermi bene observatur. Caput transversum suborbiculatum, antice viridi-splendidissimum; cavitas facialis lata subquadrata confertim irregulariter punctulata modice profunda, disco sublaevigata et abrupte canaliculata, superne marginata: margine transverso bi-arcuato $\sim$ polito inpunctato; antennae robustae, magnitudine mediocres fuscae, basi virides 1 . viridi-cyaneae; clypeus magnus transversus latus politus splendidus parce punctulatus, disco subgibbus, ad centrum marginis apicalis interdum cupreo-aenei leniter et late ar- 


\section{Famil. Clrysididae. Chrysis: Plalanx VIII.}

cuato-emarginatus, angulis lateralibus late arcuato-obtusis, hinc ambitus clypei subreniformis apparet; mandibulae valde robustae, licet magnitudine mediocres falcatae acuminatae, margine interno leniter inciso obsolete unidenticulatae, piceo-brunneae, macula basali aut riridicyanea aut viridissima et apice aureo-cuprea; labrum maximum perfecte semicirculare convexum politum fulrociliatum, basi testaceum, disco viride, margine apicali cupreo (; reliquae partes oris in speciminibus nostris mutilatae); margo occipitalis utrinque angulo ralido subobtuso munitus. Thorax crassus, crassissime punctatoreticulatus, oblongo-subquadratus, postice tamen latius quam antice, dorso modice convexus scutello et postscutello subgibbis, totus aut viridissimus aut riridi-cyaneus; pronoti area utraque lateralis perpendiculariter deflexa irregulariter subtrapezina superne crassissime punctata, postice fortissime foreata: forea postica supera saltem triplo minore quam fovea postica infera; mesopleurae apice utroque fortiter obtuse bispinosae et ceterum marginibus irregulariter et varie crenulatae; metanoti anguli postico-laterales

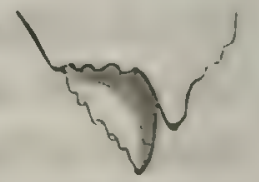
maximi, omnino ut in Chryside prominula (no. 185, cfr. figuram ibidem) constructi; pedes aut viridi-cyanci, aut viridissimi trochanteribus posterioribus apice cupreo-marginatis, tarsi fusci latere externo plus minus ample pedibus concolore; sternum punctulato-coriaceum, mesosternum latissimum modice concavum. Abdomen magnum ovali-subrectangulum, basi transversum et latius quam apice modice arcuato, dorso modice convexum politum 
splendidum et crasse punctatum, aut viridi-cyaneum, aut viridissimum margine basali segmentorum $1:$ mi et $2: \mathrm{di}$ cyaneo; segmenti dorsalis 1 :mi utraque area lateralis intramarginalis subcuneiformis prasse punctata interstitiis punctorum subtilissime punctulatis, leniter concaviuscula; segmenti 2 : di uterque antelaterali-apicalis brevissime spinoideus; segmenti 3 : tii series ante-apicalis per carinulam medianam brevem convexam interrupta, foveae magnae profundae subquadratae politae purpureo-violaceae numerosae; margo apicalis mediocris subdepressus parce modice punctatus; dentes apicales robusti acute triangulares, 4 intermedii subaequales, 2 externi breviores; emarginaturae arcuatae, centralis et duae secundariae mediocres latitudine subaequales, at illa his paullulum profundior, duae externae obliquae latiores et leniores. Venter modice fornicatus viridi-cyaneus segmentis basi nigris. Alae brunneae violascentes basi hyalinae, interdum etiam apice subhyalinae, venis omnibus firmibus piceis, cellula radiali elongata lanceolata incompleta: apice modice aperta.

Divis. 2. Postscutellum mucronatum (. Corpus in nostris magnum).

Subdivis. 1. Abdominis segmenti dorsalis 3:tii dentes: 4 apicales et 2 laterales.

191. Chrysis lyncea Fabr. $0^{7} 9$.

Magna aul maxima cire. 4 lin. decimal. long. conferlin modice punctata viridis, cyaneo-viridis aut viridicyanea, abdominis segmento dorsali 2:do ",utrinque 


\section{Famil. Chrysydidae. Clerysis: Phalanx VIII.}

ocello coerulescente", segmenti 3:lii emarginalura centrali recte transversa subrectangulari, postscutelli mucrone plano anguste triangulari supra carinato.

Chrysis lyncea Fabr. Ent. Syst. 2. 240:6, Piez. 172:8 secundum 2 specimina in Museo Havniensi 1847 a me examinata.

Stilbum lynceum Dlbm. Dispos. 1845, 15:1.

Habitat in Africa subfrequenter; in Sierra Leona primum detecta; e Guinea, - Senegal, — Promontorio bonae spei et Port Natal nobis communicata.

Observ. Species elegans, propter dorsum thoracis abdominisque valde convexum, postscutellum mucronatum, etc. Stilbos simulans.

Corpus elongatum, proportionaliter angustum, at nihilominus robustum, confertim modice punctatum parum pubescens, aut cyaneo-viride, aut viridi-cyaneum, aut viridissimum. Caput rotundato - subtriangulum, of nonnihil latius et suborbiculatum, $\sigma^{\pi}$ paullo angustius paulloque longius; cavitas facialis trapezino-rectangularis profunda confertissime punctulato - coriacea, disco abrupte canaliculata, superne obsolete marginata: scil. margine transverso brevi saepius a sculptura verticis oblitterato; antemnae mediocres fuscae, basi aut virides aut viridi-cyaneae; clypeus transversus submagnus aut saltem plus quam mediocris, reniformis punctulatus disco convexus; labrum mediocre semilunatum modice convexum, subfilissime punctulatum subopacum fuscum fulvo-ciliatum; mandibulae aut piceac aut rufo-piceae, basi virides et interdum ibidem cupreo-pictae; maxillae nigro-piceae, palpi l. concolores 1. testaceo-brunnei; margo occipitalis utrinque angulo spinoideo plus minus curvo. Thorax orato-subcylindricus antice posticeque transversus, dorso valde 


\section{Famil. Chrysididae. Chrysis: Phalanx VIII. 341}

convexus; pronotum antice posticeque arcuato-emarginatum, utraque area lateralis perpendiculariter deflexa e foveis aut duabus aut tribus excavata

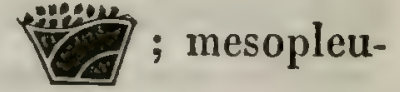
rae utroque apice mucrone validissimo perpendiculari subprismatice l. trigono-conico matae; supra hunc mucronem est pleura per sculpturam in 4 areas rum 3 anticae areae sublineares

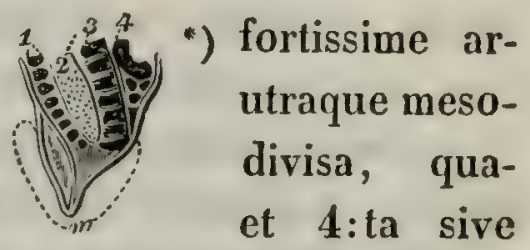
postica subtriangularis; ceterum observatur: in area antica (1) series foveolarum rotundarum, - in area 3:tia (3) series fovearum subquadratarum carinulis interjacentibus separalarum, - in area postica (4) series foveolarum inaequalium, area 2:da (2) est convexo-depressa sublaevis nec nisi parcissime subtilissime punctulata; metanoti anguli postico-lateralis magni, apparent triangulares, at re vera ut in Chryside Wahlbergi (no. 178, cfr. figuram ibidem) efformati; postscutellum disco elevatum et postice unispinosum: spina subborizontalis ultra finem metanoti posticam extensa, anguste triangularis apice obtusiusculo, supra carinula mediana polita planiuscula impunctata, utrinque ad hanc carinulam est spina crassissime punctata; pedes aut viridi-cyanei aut viridissimi parce piloselli; tibiae posticae longiores lenissime curvae, toto latere postico linea cinereosericea insignes, quae pubescentia etiam latus inferum tarsorum totum soleae instar obtegit; tarsi pedibus concolores, apice nigro-fusci; alae sordidae subbrunnescenti-

*) 1. = mesopleurae area antica -2 . dito $2: \mathrm{da},-3$. dito $3: t i a$, 4. area postica; $m=$ mucro apicalis mesopleurae.

II. 


\section{Famil. Clerysididae. Chryssis: Plealanx VIII.}

hyalinae, ad certum situm luminis subviolascentes, renis firmibus nigro-piceis, cellula radiali elongata triangulariter lanceolata aut incompleta aut subcompleta, scil. apice angustissime aperta. Abdomen conico-ovale capite thoraceque simul sumtis $\frac{1}{4}$ longius splendidum, dorso valde convexum et modice punctatum; segmenti dorsalis 1 : mi utraque area lateralis intramarginalis perpendiculariter deflexa, irregulariter subrectangularis sublinearis

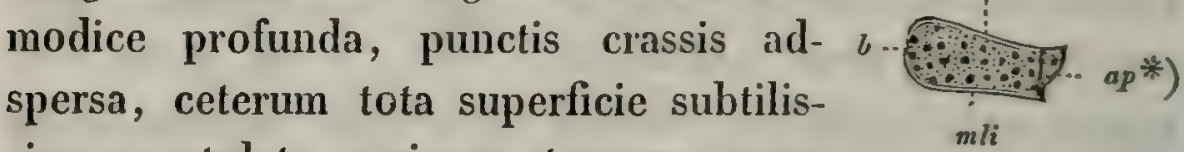
sime punctulato - coriacea et opaca; areae consimiles etiam in lateribus segmenti 2 :di adsunt, sed hae basi truncatae (nec obtusae); margo lateralis scgmentorum 2:di et $1:$ mi apice angulo spinoideo terminatus; in utroque disco laterali segmenti dorsalis 2 : di observatur macula mediocris ocellaris aut cyanea aut violaceo-purpurascens, rarius „pupilla fulva“ ut scripsit $\boldsymbol{F} \boldsymbol{a}$ - bricins l.c.; segmentum dorsale 3:tium subtrapezinum, plerisque cyaneum, basi nec non ante seriem rirescens: area basalis sublonge 1 . alte arcuata, disco tota transverse concaviuscula immersa, ante seriem convexiusculo terminata; area postica mediocris modice prominula subdepressa parce punctulata; series ante-apicalis continua, foveolae mediocres rotundatae numerosae; dentes apicales 4 robusti triangulares, magnitudine subaequales, uterque dens lateralis brevis angustus acutissime spinoideus, a dente apicali longe remotus, centro marginis lateralis propior quam basi; emarginaturae 3: omnes apicales in-

*) $m l s=$ margo lateralis superus; $m l i=$ margo lateralis inferus; $b=$ basis; $a p=$ apex. 
Famil. Chrysididae. Chrysis: Phalanx VIII. 343

aequales: gularis, in proximae

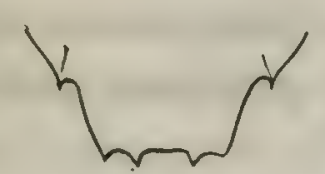

centralis transrersa subrectanaliis latior, aliis emarginaturae et latitudine et profunditate aequalis; ambae emarginaturae externae modice arcuatae nonnihil obliquae. Venter planiusculus subtilissime coriaceus, aut viridis aut viridi-cyaneus, nonnihil nigromaculatus, interdum ex aureo resplendens.

192. Chrysis Gueudei Guerin. $\delta$.

Magne $3 \frac{1}{2}$ lin. decimal. long. crasse punctata viridis, abclominis segmento dorsali 2: do immaculato, segmenti 3 :tii emarginatura centrali arcuata, postscutelli mucrone plano anguste triangulari, toto supra crassissime punctato, apice polito.

Chrysis Gueidei Guerin, secundum Museum Spinolae.

Habilat in Madagascar, a Dom. Gueude detecta; Mus. Dom. Spinola.

Observ. Valde similis et affinis praecedenti (Chrysidi lynceae Fabr.); at praecipue notis sequentibus certe dignoscitur: capitis cavitate faciali superne non transverse marginata, nec vestigium quidem talis marginis praebente; - posiscutelli toto mucrone crassissime punctato I. foveolis crassis rotundis sculpto, summo apice laevissime polito; - metanoti angulis postico-lateralibus ut in Chryside insulari (no. 187, cfr, figuram ibidem) constructis, excepto tamen margine postico transverse arcuato; - abdominis segmento dorsali multo breviore, disco obsoletius immerso et ante seriem lenissime convexo, - seriei foveolis paullulum minoribus et minus numerosis, foveola centrali ceteris majore, - emarginatura apicali centrali ad magnitudinem mediocri at perfecte et sub- 


\section{Famil. Clhrysididae. Chrysis: Phalanx VIII.}

profunde transverse arcuata, - emarginaturis duabus apicalibus externis latioribus minus profundis, leniter et oblique arcuatis.

193. Chrysis mucronata Dlbm. 오.

Maxima 4 lin. decimal. long. robusta sublinearis vertice thoraceque crassissime punctatissimo-reticulatis, abdomine modice punctato et punctulato viridis, abdominis segmento dorsali 2:do immaculato, segmenti 3:tii emarginatura centrali triangulari, poslscutelli mucrone plano brevi obtuse et late triangulari toto scrobiculato.

Habitat in Africa meridionali, ad promontorium bonae spei a Dom. Draege detecta; unicum specimen nobis communicavit Dom. Spinola.

Observ. Nullo modo varietas Chrysidis lynceae nec Gueudei, a quibus longe discrepat.

Corpus elongatum lineare. Caput transrersum suborbiculatum, occipite et vertice crasse punctato-reticulatum; cavitas facialis magna subquadrata modice profunda, cinereo et cano-pubescens, confertissime punctulato-coriacea, disco transverse arcuato-strigosa, superne conspicue marginata: margine fere hujus 2 il S formac (; antennae speciminis communicati mutilatae); clypeus mediocris transversus subtrapezinus punctulatus depressus, disco parum convexus, margine apicali leniter arcuato-emarginatus; labrum mediocre semilunatum punctulatum convexum virescens fulvo-ciliatum; mandibulae breves piceae, basi virides, ceterae partes oris brunneofuscae; capitis pagina infera ad marginem baseos leniter 
transverse arcuato-marginulata: marginulo utrinque angulo valido spinoideo terminato. Thorax rectangulariter cylindricus crassissime punctatissimo 1. potius foveolatoreticulatus, viridis, dorso magis depressus quam convexus; pronoti maculae transversae maximaque pars mesonoti nigro-aenea purpurascentia opaca; pronotum antice arcuatum, postice arcuato-emarginatum, utroque margine laterali supero acuto flexuoso; pronoti utraque area lateralis intramarginalis deflexa ita sculpta ut reliquus thorax; mesopleurae margine postico et apicali crenulatae et hinc obtuse denticulatae, adeoque nullo modo ut in Chr. Iyncea et Gueidei constructae; postscutelli mucro mediocris (, sed - cum Chrysidis lynceae comparatus subbrevis), obtuse triangularis atque ita crasse sculptus ut reliqua superficies thoracica; metanoti anguli posticolaterales robusti acute triangularis; pedes virides tarsis fuscis; alae sordidae hyalinae venis robustis piceis; cellula radiali falcato-lanceolata completa. Abdomen ovali subrectangulum capite thoraceque simul sumtis subbrevius, dorso modice convexum; segmentum 1:mum viride crasse punctatum, segm. 2: dum concolor modice et confertim punctatum, segm. 3:tium viridi-cyaneum confertissime punctulatum; segmenti dorsalis $1:$ mi et 2 : di areac laterales intramarginales ejusdem formae, quam nuper apud Chr. lynceam (no. 191) descripsimus; segmentum dorsale 3:tium fere duplo brevius quam segmentum 2:dun, convexo-depressum; series ante-apicalis continua nec per carinulam ullam medianam interrupta, foveolae rotundatae numerosae inaequales: majores et minores fere alternantes; dentes 4 apicales (cfr. hanc figuram $d a$ ) mediocres acute triangulares, a latere paullulum remoti, 


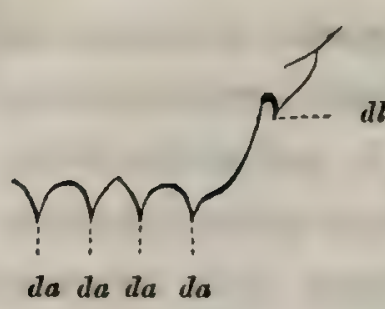

dentes centrales vix nisi obsoletissime robustiores quam externi; dens uterque lateralis (cfr. hanc figuram dl) acute triangularis robustus, a dente apicali externo longissime remotus et juxta basin marginis lateralis collocatus, spatium - inter dentem lateralem et dentem apicalem externum - oblique arcuatum; emarginaturae ad latitudinem et profunditatem mediocres, externae duae arcuatae, centralis triangularis. Venter viridicyaneus.

Subdivis. 2. Abdominis segmenti dorsalis 3:tii dentes: omnes 6 apicales (. Postscutelli mucro in nostris longitudinaliter excavatus, at simul crassissime punctatus).

194. Chrysis incerta Dlbm. $\delta$.

Magna robusta $3 \frac{1}{2}$ lin. decimal. long. crasse punctata cyanea alis violascentibus, abdominis segmento dorsali 3:tio tolo depresso-convexo, serie ante-apicali incompleta foveolis paucis rotundalis hinc inde confluentibus.

Chrysis smaragdula Encyclopéd. methodiq. teste Spinola in litteris.

Habitat Cajennae, Dom. Buquet; Mus. Dom. Spinola.

Observ. Cum Chrysidi nobili KI. (no. 195), cui plurimis notis simillima, facile confundenda; nec nisi colore pure cyaneo (exceptis pedibus et capite antico virescentibus), abdominis segmentis dorsalibus 2:do et 3 :tio punctulatis (non crasse punctatis), segmenti (abdominis) dorsalis 3:tii tota superficie depresso-convesa, 
Famil. C'rysididae. CHrysis: Phalanx VIII. 347

serie ante-apicali incompleta: foveolis paucis, et mediocribus et parvis hinc inde confluentibus, dignoscitur.

195. Chrysis nobilis Klug. ㅇ.

Mrgna rolusta $3 \frac{1}{2}-4$ lin. decimal. long. crasse punctata cyaneo-viridis alis violascentibus, abdominis segmento dorsali 3:tio medio transverse concavinsculoimmerso, ante seriem convexiusculo, serie ante-apicali completa foveolis numerosis rotundatis mediocribus dislincte separatis.

Clirysis notilis Klug. Symb. Pliys. Dec. V. 1845. Tab. XLV. fig. 2. Pyria stilboides $S_{\text {pin. Annal. Ent. 1838. } 446: 3 .}$

Stilbum sexdendatum Guérin Revue zool. 1842. 145:2 secundum Klug I. c.

Stilbum Hedenborgi Dlbm. Dispos. 16:2.

C'llrysis producta Mus. Spinolae.

Habilat in Aegypto; "ad Ambukol mense Augusto" detecta, teste Dom. Klug 1. c. Deinde etiam in Aegypto iterum lecta a Dom. Walt, Mus. Dom. Spinola, - et a Dom. Hedenborg ad Bahrel Abiad, Mus. Reg. Acad. Scient. Stockholm.

Observ. Statura fere Clirysidis oculatae (no. 176), sed abdomine convexiore, crassius punctato et aliter constructo armatoque.

Caput rotundato-triangulun viride, confertim punctato-reticulatum; caritas facialis magna subquadrata modice profunda punctulato-coriacea superne transverse marginata: margine fere lujus 9 formae; antennae robustae, magnitudine submediocres nisi longiusculae nigro-fuscae, basi cyaneo-virides aut vice versa; clypeus transversus mediocris subtrapezinus parce punctulatus, 
disco modice convexus, margine apicali aut transverso aut leniter arcuato-emarginato; labrum submagnum semilunatum convexum, parce subtiliter punctulatum, subfulvociliatum, viride aut cyanescens; mandibulae robustae piceae, basi virescentes; palpi fusci; margo occipitalis utrinque angulo valido spinoideo munitus. Thorax crassus oblonge quadrato - cylindricus, sat convexus, crasse punctatus, punctis pronoti et dorsuli non confertis sed remotis aut vagis, ut jam Cel. Klug et Spinola l. l.c.c. docent, punctis aut scutelli postscutelli confertis unde harum partium superficies reticulata exstat; dorsulum margine antico nitidius et sublaere atque subtilissime punctulatum; pronoti utraque area lateralis intramarginalis fere ut in Chryside lyncea (no. 191, cfr. figuram ibidem) constructa, ea tamen exceptione quod majores foveae sunt plures; mesopleurae fere hoc Lo modo apice dentatae et crenulatae; postscutelli mucro mediocris linguaeformis, supra totus sulco mediano excaratus, cavitate scrobiculata; metanoti anguli postico-laterales omnino ut in Cler. prominula (no. 185, cfr. figuram ibidem) constructi; pedes eandem habent picturam et constructionem, quam apud Chrysidem lynceam (no. 191) jam descripsimus; alae brunneae violascentes, venis firmibus piceis, cellula radiali lanceolata incompleta: apice modice aperta. Abdomen robustum conico-ovale, basi transversum et - propter mucronem metanoti ut docet Cel. Spincla 1. c. - triangulariter impressum, ceterum dorso valde convexum splendidum aut viridissimum aut cyaneo-virescens, segmento 3 :tio plerumque cyaneo et margine anali virescente; segmentum dorsale 1 : mum crasse 
vage punctatum interstitiis subtiliter punctulatis, utraque area lateralis intramarginalis quoad ambitum et profunditatem omnino ut in Clryside lyncea (no. 191, cfr. figuram ibidem ) formata, non vero ita crasse punctata, sed tota punctulato-coriacea et tantummodo punctis perpaucis crassis; segmentum dorsale 2: dum crasse vage punctatum punctis discoidalis magis remotis quam lateralibus, interstitiis omnibus politis impunctatis; segmentorum 1:mi et 2:di uterque margo lateralis apice brevissime acute spinosus; segmentum dorsale 3:tium mediocre: area basalis polita, disco modice 1 . leniter concavoimmersa et remote modice punctata, toto ambitu confertim modice punctata et ante seriem leniter convexo terminata; series ante-apicalis continua (non interrupta), foveolae mediocres rotundatae distinctae numerosae; margo analis mediocris modice punctatus et punctulatus subcoriaceus nonnihil depressus; dentes omnes 6 apicales, mediocres, spinoidei, invicem magnitudine subaequales; emarginatura centralis mediocris perfecte transverse arcuata, emarginaturae ambae secundariae (utraque per se) nonnihil latiores et forsitan - rite observatae - etiam paullulum profundiores quam illa atque oblique arcuatae; utraque emarginatura externa conspicue latior quam singula quaeque secundariarum, atque obliqua et leniter arcuata. Venter aut planiusculus aut modice fornicatus, viridi-cyaneus, nigro-maculatus. 


\section{Genus SPINTHARIS Klug.}

Corpus aut parvum aut submediocre $2-2 \frac{1}{2}$ lin. decimal. long. et proportionaliter robustum, elliptico-sublineare obtusum, punctatum.

Caput mediocre, aut transversum suborbiculatum aut longitudinale et rotundato-triangulum; cavitas facialis subrectangularis, superne marginata; antennae breves; mandibulae mediocres falcatae, margine interiore ante apicem acute unidenticulatae.

Thorax aut ovato-subquadratus aut quadrato-cylindrico-subcubicus; alarum anticarum cellulae: costalis, medialis et $\mathbf{1}$ :ma discoidalium completae, - submedialis 1: ma et submedialis 2: da aut completae aut subcompletae, - radialis incompleta; alae posticne ut in genere Chrysidis; pedes etiam hujus generis, tibiae aut saltem basi tarsique toti brumeo-testacea. Abdomen fere ut in Hedychro constructum, subbreve obtusum rotundatosubquadratum modice convexum; segmentum dorsale 1:mum mediocre; segmentum dors. 2: dum maximum, illo duplo longius; segmentum dorsale 3:tium brevissimum, scil. duplo brevius quam 1:mum et fere quadruplo brevius quam 2:dum, subperpendiculariter conrexo-declire, margo (hujus segmenti) apicalis eximius et propter consistentiam a margine codem reliquorum hujus familiae generum nonnihil aberrans, scil. non crustaceus et opacus sed coriaceus aut submembranaceus pellucidus albus, perpendiculariter dependens, ad centrum ut in Omalo emarginatus.

Species tantum 3, 2 e promontorio bouac spei et 1 ex Aegypto, nobis cognitae, scil. 
196. Spintharis chrysonota Klug.

Subparva $2 \frac{1}{2}$ lin. decimal. long. confertim et fere subliliter punctata supra viridi-aurea subtus viridi-cyanea, mesopleuris inermibus, abdominis segmenti dorsalis 3 :tii margine anali uni-emarginato: emarginatura centrali distincta arcuala, utrinque lobo acute triangulari, venae radialis centro ramulum obliqum in discum emittente.

Spintharis clirysonata Kilug. Mus. Berolin. teste Spinola in litteris.

Habitat in Africa meridionali, ad promontorium bonae spei a Dom. Draege detecta, Mus. Dom. Spinola, qui unicum specimen communicavit.

Observ. Statura paullulum similis Hedychri rosei. - Caput transversum suborbiculatum, punctulatum; vertex aureus, pagina capitis inferior viridi-cyanea; cavitas facialis viridis tenuissime aureo, modice profunda superne transverse marginata: margine utrinque arcuato-deflexo; clypeus transversus brevis aureo-viridis confertim subtiliter punctulatus, apice area triangulari polita impunctata picea; antennae brevissimae sublineares aut brunneae aut rufo-testaceae, scapo viridi; mandibulae disco rufo-testaceae, basi apiceque piceae. Thorax ovato-subquadratus tenuiter punclatus (non punctulatus) dorso modice convexus; pronotum et mesonotum aurea plus minus viridi nitida; illius truncatura antica, mesopleura et metanotum viridia suturis cyaneis; sternum et femora viridicyanea; tibiae extus viridi-aenescentes ceterum unacum totis tarsis brunneo-testaceis; alae hyalinae aut subhyalinae, tegulis et mediocribus venis piceis, cellula radiali lanceolata incompleta; e centro venae radialis excurrit 


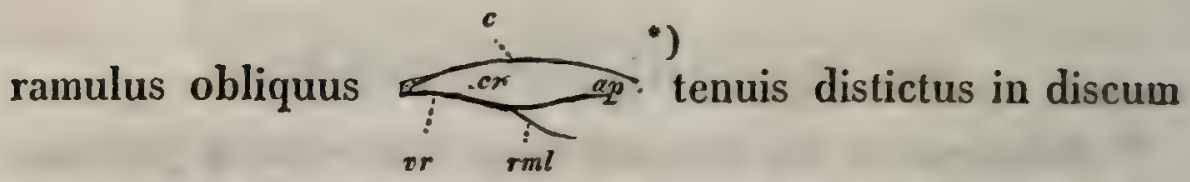
alae. Abdomen fere thorace brevius, rotundato-quadratum dorso subhemisphaerice convexum confertius tenuiter punctatum igneo-aureum; segmenti 3 : tii series ante-apicalis foveolis parvis rotundis numerosis, marginis analis emarginatura centralis subanguste arcuata profunda, angulus aut lobus utrinque acute triangularis. Venter fornicatus nigro-violascens maculis transversis arcuatis viridibus aut viridi-cyaneis.

197. Spintharis destituta Dlbm. $ఠ$.

Subparva $2 \frac{1}{2}$ lin. decimal. long. confertim tenuiter punclata supra viridi-aurea, subtus viridi-cyanea, mesopleuris inermibus, abdominis segmenti dorsalis 3:tii margine anali uni-emarginato: emarginatura centrali levi (non profunda) triangulari, utrinque lobo leniter arcuato-obtuso, vena radiali libera (ramulo destitula).

Spintharis Chrysonota Spinola in litteris.

Ilabilat in Africa meridionali, ad promontorium bonae spei a Dom. Draege, Mus. Dom. Spinola, qui specimen communicavit.

Observ. Praecedenti (Spinth. chrysonotae, no. 196) ita simillima, ut tamquam illius mas facillime existimari posset; at sequentes duae notac, me saltem judice, Spinth.

\footnotetext{
*) $\mathrm{cr}=$ cellula radialis, $a p=$ ejusdem apex apertus, $c=$ costa, $v r=$ vena radialis, $r m l=$ ramulus venae radialis; $-e=$ etuarginatura ccntralis, $l=$ lobus 1 . angulus ad emarginaturam utrinque situs.
} 
destitutam ut diversam speciem satis determinant, scil.

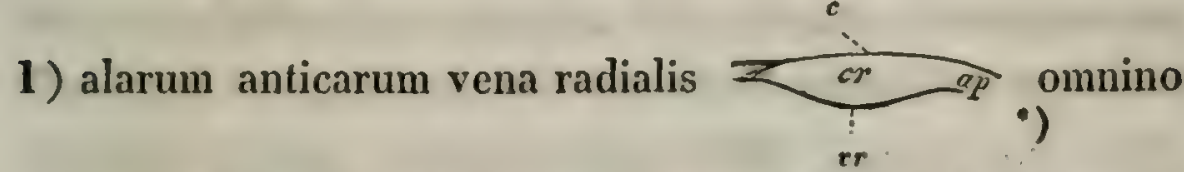
caret ramulo illo obliquo, quem apud Spinth. chrysonotam distinctum (licet tenuem) vidimus; 2 ) emarginatura marginis analis (abdominis segmenti dorsalis $3:$ tii) levis (nec profunda) et triangularis, atque utrinque instructa lobo arcuato-obtuso, fere hujus formae

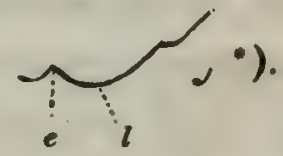

198. Spintharis singularis Spinola. ㅇ․

Subparva 2 lin. decimal. long. confertim modice punclata viridi-cuprea aut viridi-aenea, ventre igneo, femoribus posterioribus antice concoloribus; antennis ex parte, genubus tibiis tarsisque pallide testaceis; mesopleuris utroque apice bispinosis; abdominis segmenti dorsalis 3:tii margine anali tri-emarginato et 4-dentato.

Spintharis singularis Mus. Spinolae.

IIabilat in Aegypto, a Dom. Walll detecta, Mus. Dom. Spinola.

Observ. Corpus robustum, statura fere Hedychri luciduli parvi. Caput longiusculum rotundato-triangulum; vertex latus confertim punctatus viridi-cupreus; oculi rotundati valde prominuli; cavitas facialis angusta longiuscula rectangularis punctulata viridis albo - sericea, superne marginata: margine omnino ut in Chryside pul-

*) $c, c r, a p$, vr; $-e$ et $l=$ candem habent significationem quaın apud no. 196. 
chella (no. 168, cfr. figuram ibidem) constructo; antennae mediocres, scapus viridi-cupreus, pedicellus virescens, flagellum testaceum fusco-maculatum; clypeus longitudinalis subtrapezinus viridi-cupreus, carinula mediana levi, basi utrinque foveatus, disco convexus, superficic totus politissimus splendidus punctis paucis, margine apicali transverso nigro-aeneo; mandibulae albo-testaceae, basi viridi-aenescentes, margine cupreo-aeneo. Thorax robustus tetraëdro-cylindricus crasse punctato-reticulatus viridi-aeneus; pronotum utrinque, dorsuli area media, scutellum et metanoti anguli postico-laterales cuprata, hi (anguli) mediocres ad formam Chrysidis Wahlbergi (no. 178) nonnihil similes; mesopleurae scutello concolores utroque apice - fere hujus ${ }_{m a}$. for- $_{m p}$ mae - bispinosae, spinis proportionaliter robustis subconicis emarginatura mediocri separatis; sternum riride; femora viridi-cuprata, posteriora antice igneo-aureo splendidissima, genua tibiae tarsique testaceac; alae hyalinae, venis mediocribus aut subtenuibus brunnescentibus, cellula radiali ovato-lanceolata incompleta apice late aperta, vena radiali (libera, ut in no. 197 ramulo destituta) apice longe remota a costa. Abdomen mediocre rotundatosubrectangulum, longitudine capitis thoracisque simul sumtorum, dorso modice convexum et crebre modice punctatum, punclis maxime regularibus omnibus orbiculatis, riridi-aeneum, nonnihil vergens in cupreum, lineola me-

*) $m a=$ margo anticus, $m p=$ margo posticus. 
diana subelevata ad certum situm luminis in segmento 2:do observanda; segmentum dorsale 3 :lium mediocre, 2: do vix ad dimidium brevius, disco nonnihil transverse immersum, ante seriem paullo incrassatum, apice quasi auctum margine anali amplo cartilagineo 1 . submembranaceo lacteo; series ante-apicalis in basi hujus marginis posita pulcherrima foveolis mediocribus, orbiculatis, numerosis circ. 24, omnibus distinctis et pellucidis; margo analis (segm. 3:tii) latiusculus convexo-depressus, emarginaturis $3 \quad$ lo arcuatis, scil. unica apicali-centrali (cfr. hanc lateralibus el figuran ec) et duabus subbasali(unica utrinque, cfr. figuram $e l$ ), dentibus triangularibus subspinoideis 4) scil. 2 subbasalilateralibus (uno utrinque, cfr. figuram $d l$ ) et 2 apicalicentralibus (scil. ad emarginaturam centralem utrinque uno, cfr. figuram $d c$ ); summus finis hujus marginis subtilissime scariosus.

\section{Genus STILBUM Spinola.}

Ins. Lig. fasc. 1. pag. 9, et fasc. 2. pag. 3; Latr. gen. IV. 48:477.

Corpus robustum, aut magnum aut maximum $3-5$ lin. decinal. long. subovale convexissimum punctatum splendidissimum.

Caput subangustum longitudinale pendulum subovatum ore prominulo, in parte antica cavitatibus instructum duabus: superiore sive verlicina anticum superne includente, un margine apicali transverso trian to; cavitas inferior s. façialis elongata angusta dique marginata gulariter emargina- 
sublinearis profunda confertissime transverse strigosa medio canaliculata, superne inter cavitatem verticiuam et facialem adest spatium convexo-declive crebre punctatum; antennae mediocres aut subbreves normaliter constructae; clypeus a forma Chrysidum maxime normali aberrat, ut jam annis 1808 et 1838 sat demonstravit Ill. Spinola, scil. non transversus sed longitudinalis (et fere hujus formae), medio canaliculatus (non carinatus), utrinque convexo-depressus, apice arcuato-emarginatus lobo utrinque arcuato-obtuso; mandibulae elongatae falcatae acuminatae, margine interno pone apicem canicula obliqua instructae sed non inciso-denticulatae; labrum mediocre convexum rotundatum; labium subcordatum.

Thorax crassus gibbus globoso-subquadratus, antice posticeque truncatus, apice multo latior quam basi; pronotum antice posticeque late transverse arcuato-emarginatum; dorsuli suturae medianae crenatae et leniter arcuatae, areae laterales juxta tegulas, area media apice et totum scutellum crasse punctata; postscutellum a Chrysidum postscutello mucronatarum forma normali distincte aberrat (cfr. ex. gr. species no. 191 - 195), scil. ejusdem tota pars basalis sub scutello abscondita et solummodo pars apicalis instar mucronis validi exarati rel excavati procedens; mesopleurae fere ut in Chryside lyncen (no. 191, cfr. figuram ibidem) in areas oblique longitudinales divisae, apice autem aliter constructae et fere hoc modo tanoti an

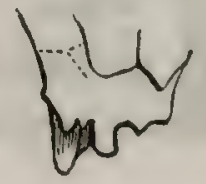
crenato irregulariter dentatae; meguli postico-laterales maximi sub- 
transversi fere ut in Chryside Wallbergi (no. 178, cfr. figuram ibidem) constructae; pedes et alae fere ut in Genere Chrysidis; alarum venae nigro-piceae et omnes, quae completae obveniunt, sunt firmae; - anticarum cellula costalis, medialis et I:ma discoidalium completae; cellula radialis lanceolata incompleta et late aperta. scil. venae radialis apex ab alae apice longe remota; cellula submedialis et cubitalis $\mathbf{1}: \mathrm{ma}$ subcompletae; haec $\mathbf{1}$. cubitalis 1 : ma apice gaudet venula transverso-cubitali coeca (ad certum situm luminis observanda) nonnihil angulata, ex angulo ramulum venae radiali parallelum emittente.

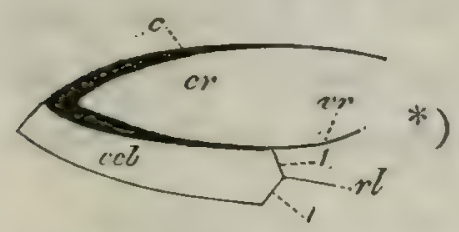

Abdomen validum capite thoraceque simul sumtis conspicue 1. $\frac{1}{4}$ l. $\frac{1}{3}$ longius, ovato-semiconicum, dorsum convexissimum splendidum politum punctatum; segmentum dorsale 1:mum breve; segmentum 2:dum maximum convexissimum 1:mo fere triplo longius, ad apicem marginis utriusque lateralis spinula triangulari munitum; segmentum 3:tium semper cyaneum, 2:do plerumque tantum duplo brevius: area basalis instar marginis ampli convexo-depressi transverso-arcuati supra seriem prominens, area analis humilis declivis convexo-depressa prominula subtrapezina dentibus 4 apicalibus robustis triangularibus armata; series ante-apicalis foveolis rotundatis numerosis; emarginaturae apicales arcuatac, centralis an-

*) $c=$ costa ; $c r=$ cellula radialis; $v r=$ vena radialis; $c c b=$ cellula cubitalis $1: \mathrm{ma} ; 1=$ venula transverso-cubitalis coeca, $r l=$ ramulus ex angulo ejusdem venulae excurrens.

II. 
gustior et lenior, externac duae latiores et nonnihil profundiores. Venter aut planiusculus aut fornicatus.

Species tantum 3 cognoscimus, scil. Stilbum splendidum Fabr., Stilb. Wesmaëli nob., et Stilbum calens Fabr.

199. Stilbum splendidum Fabr. $\sigma^{\top}$ ㅇ․

Magnum aut maximum 3-5 lin. decimal. long. pictura valde varians aut violaceum, aut viridi-cyaneum, aut viridi-auratum, verlice thoraceque plemumpe cyanescenti-viridibus rarius aureis, dorsulo (praeler puncturas laterales et apicales) aut subimpunctato aul levissime et parcissime punchulalo subpolito nitido, melanoti mucrone apice integerrimo.

var. $\boldsymbol{a}$. Corpus violaceum.

Stilbum splendidum Museis DD. Drewsen, Spinola et Wiestermann.

E Senegal, Java et Bengalia.

var. $b$. Dorsum thoracis abdominisque segmentormm $1: \mathrm{mi}$ et 2 : di aut viride aut cyaneo-viride l. vice versa.

Chrysis splendida Fabr. Ent. Syst. 2. 238:1, et Piez. 170:1 secundum specimina typica in Museis Havniensi et hiliensi anno 1847 a me examinata.

$\left.\begin{array}{l}\text { Stilbum calens Spinol. var. } \\ \text { Stilbum connectens Spinol. }\end{array}\right\}$ Mus. Spinolae.

E Gallia meridionali, Hispania, Italia, Graecia, insulis maris mediterranei, Africa Indiisque et occidentali et orientali nobis missa.

var. c. Thorax aut viridissimus aut viridi-cyaneus; abdominis segmenta dorsalia $1:$ mum et 2 : dum aurcoviridia. 
Stilbum calens var. Museis Imp. Viennensi, et Spinolac, - D. Mus. Westermanni.

Ex Italia, Graecia, Asia minori et Senegal nobis communicata.

var. d. Thoracis dorso (praesertim disco et antice) abdominisque segmentis dorsalibus 1 :mo et $2:$ do viridi-aureis.

Stilbum splendidum Mus. Drewoseni.

E Sicilia.

var. e. Corpus cupreo-aureum plus minus viridi splendens, excepto segmento dorsali - in hac ut in omnibus aliis varietatibus - cyaneo.

Stilbum calens var. Mus. Spinolae, et Viennensi.

Stilbum Westermanni Dlbm. Dispos. 16:4.

Ex Austria, Italia, Sicilia et Malta.

Habitat in plurimis orbis terrarum regionibus 1. calidis 1. calidissimis, ex. gr. Europa meridionali, Asia minori, Africa, Asia, Indiis etc. non rar.

200. Stilbum Wesmaëli Dlbm. $ర$.

Submaximum $4 \frac{1}{2}$ lin. decimal. viridissimum, abdominis dorso nomilil aureo nitido, (segmento 3:tio pure cyanco,) dorsulo (praeter puncluras laterales et apicales) levissime parcissime punclulato nilido, postscutelli mucrone ad centrum marginis apicalis inciso et utrinque arcuato-obtuso.

Stilbum Wesmaëli Dlbm. Dispos. 1845. 16:3.

IIabilat in insula Rhodo; specimen unicum e Mus. Reg. Acad. Scient. Stockholm. communicavit Dom. Boheman.

Observ. Simillimum praecedenti, praecipue hujus var. $c_{.}$; at primo intuitu dignoscitur: postscutelli mucrone 
paullo breviore, ad centrum marginis apicalis acute incisoemarginato, utrinque arcuato obtuso, fere hanc ${ } V^{\prime}$ formam referente.

201. Stilbum calens Fabr. $\delta$ 오.

Magnum aut maximum 4-5 lin. decimal. long. colore capilis thoracisque varians, abdominis segmentis dorsalibus 1:mo et 2:do aureis, 3:tio cyaneo, dorsulo toto confertim punctato- et punctulato-coriaceo (postsculelli mucrone apice integerrimo).

var. $a$. Caput et thorax ant cyanea levissime viridi-picta, aut viridi-cyanea aut viridia.

Chrysis calens Fabr. Ent. Syst. 2. 239:4, Piez. 171:4 secundum specimen typicum in Museo Havniensi anno $1817 \mathrm{cum}$ nostris speciminibus comparatum.

var. $b$. Caput et thorax aut aureo-viridia aut viridissime aurea.

Stilbum calens var. Mus. Spinolae.

var. c. Corpus igneo-aureum.

Stilbum calens var. Mus. Spinolae.

Habitat in Italia, Austria, Dalmatia, Graecia, Turcia, Sicilia et Rhodo, hoc loco jam mense Majo obserrata.

Observ. Stilbo splendido, praecipue hujus varictali $e$ simillimum, at dorsulo toto confertim punctatoet punctulato-coriaceo facillime dignoscitur. 


\section{Familia 5. EUCHROEIDAE Dlbm.}

Mandibulae margine interno intra apicem uni-denticulatae, l. apice etiam numerato bi-denticulatae.

Thorax subtetraëdro-cylindricus dorso depresso-convexus; pronotum transverso-subrectangulum margine postico emarginato-arcuato; postscutellum inerme.

Tarsorum unguiculi infra mutici et inermes.

Alae anticae cellula medialis triangularis, venula transverso-mediali obliqua: aut rectà currente aut leniter arcuato-inflexa.

Abdomen segmentis cataphractis conspicuis 3, supra convexum, subtus aut planiusculum aut fornicatum, segmenti 3:tii margo analis aut conspicue serratus aut subtilissime et fere microscopice serrulatus. 


\section{Genera hujus familiae ita dignoscuntur:}

A. Abdominis segmenti dorsalis 3 : tii margo analis denticulis minutis submicroscopicis aequalibus concinne serrulatus et spinulosissimus; mesopleurae apice inermes . . . . . . . . 10. Genus Spinolia nob.

B. Abdominis segmenti dorsalis 3 : tii margo analis dentibus conspicuis inaequalibus, et majoribus et minoribus lacero-serratus; mesopleurae utroque apice bispinosae . . . . . . . 11. Genus Euchroeus Latr. 


\section{Genus SPINOLIA Dlbm.}

Corpus ut in Chrysidibus normalibus constructum, a quibus facile et certe ut proprium Genus distinguitur margine anali segmenti (abdominis) dorsalis tertii, qui denticulis numerosissimis minutis subınicroscopicis, magniludine et forma invicem aequalibus munitus, concinne, subtilissime et confertissime serrulatus exslat. Species tantunmodo unica adhuc cognita, scil.

202. Spinolia magnifica Dlbm.

Magna 3 lin. decimal. long. robusta modice punctatucoriacea ryanea, maculis verlicis 2 , pronoto, viltis dorsuli 2 scutelloque viridi-aureis, abdominis dorso purpureo-aureo, ventre igneo.

IIabitat in Turcia, ad Constantinopolum a Dow. Friwaldsky detecta, Mus. Dom. Spinola, qui unicum specimen communicavit.

Observ. Species propter structuram marginis analis (segmenti abdominis 3 :tii) valde singularis; nam taleu formam, qualem hanc - per totum Hymenopterorum Chrysidiformium ordinem - antea non vidi; unde hanc structuram singularem ad Genus proprium pertinentem consideravi eandemque nomine viri illustris, Domini Marquisani Maximiliani Spinola, cui scientia entomologica per saeculum dimidium magna debet beneficia, conjungere ausus sum.

Corpus robustum crassum canopubescens. Caput latum rotundato-triangulum cyaneum, vertice brevi viridi utrinque macula viridi-aurea. Facies plana punctulatocoriacea viridi-cyanea medio canaliculata. Antennac ro- 
bustae, magnitudine mediocres fuscae, basi: subtus cyaneac et supra virescentes; clypeus magnus transversus inaequalis, regione apicali depressus nitidus nigro-aeneus et utrinque inter centrum et marginem foveolatus, ad centrum marginis apicalis leniter arcuato-emarginatus; mandibulae piceae, basi cyaneae et levissime viridi-pictae. Thorax crassus quadrato-subcylindricus punctato-coriaceus subreticulatus cyaneus, macula mesopleurarum viridi; pronoti pars dorsalis, scutellum et dorsuli vittae 2 parallelae viridi-aurea; metanotum striolis nonnullis transversis viridibus, angulis postico-lateralibus brevibus obtusis fere hujus $\_$formae; pedes viridi-cyanci tarsis brunneis; alae subhyalinae, disco et ad costam cellulae radialis nonnihil sordidae, venis mediocribus piceis, cellula radiali valde incompleta et fere tantummodo dimidiata propter venam radialem subrectam jam in disco alae desinentem. Abdomen fere ut in Hedichro lucidulo formatum, sed minus rotundatum et proportionaliter longius, longitudinem capitis thoracisque simul sumtorum vix superans, dorso confertissime punctatum purpureo-aureum, macula baseos semicirculari violacea viridi-limbata; lineola mediana subelevata ad initium segmenti dorsalis 2: di vix observanda, margo basalis hujus segmenti nigroaeneus; margo apicalis segmentorum $1:$ mi et 2 :di lacvissimus politus; series ante-apicalis foveolis mediocribus rotundatis numerosis; margo analis segmenti dorsalis

3: tii fere hanc figuram

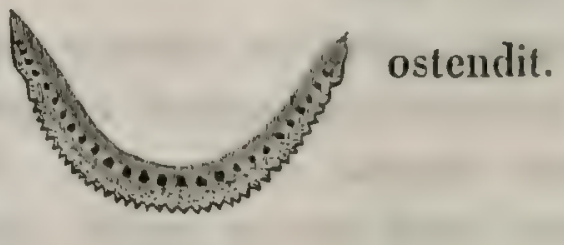




\section{Genus EUCHROEUS Latreille.}

Gen. Ins. 4. $49: 578$.

Corpus mediocre fere ut in Medychris constructum, aut mediocre aut submagnum, ovale, proportionaliter robustum. Caput robustum rotundato-triangulum; mandibulae jam in nota familiae descriptae; clypeus magnus depressus, aut transversus aut sublongitudinalis.

Mesopleurae thoracis omnino ut in Spinthari singulari (no. 198, cfr. figuram ibidem) utroque apice bispinosae.

Alae et pedes eandem habent constructionem quam apud Chrysidis species descripsimus, at cellula radialis ita valde incompleta ut in Spinolia.

Abdomen aut ovatum aut ovato- 1 . subquadratum 1 . subrectangulum, segmenti dorsalis 3:tii margo analis dentibus numerosis robustis aut mediocribus, invicem magnitudine inaequalibus lacerus et minus regulariter serratus.

Species Euchroei generis 7 cognoscimus, scil. 3 Africanas et 4 Europaeas. 


\section{Tabula Synoptica}

\section{Specierum nobis cognitarum Generis Eucleroei.}

\section{Sect. I. Postscutellum muticum (in-} erme).

Divis. 1. Abdominis segmenti dorsalis 3 : tii dentes anales angusti acuti, aut subtriangulares aut subspinoidei, numerosi $10-16$.

A. Abdominis dorsum aureum segmento 3:tio cyaneo . . . . . . 203. Euchroeus limbatus KI. Rossia meridinal.

B. Abdominis dorsum aut violascentipurpureum fasciis duabus vel viridibus vel aureis, aut viride fasciis duabus violaceo-purpurcis

. 204. Euclioeus purpuratus Fabr. Europa meridional.

C. Abdominis dorsum unicolor (non fasciatum ): aut purpureo - violascens, aut viridi-cyaneum aut viride.

1. Mlac brunneae violascentes.

$\times$ Abdominis dorsum purpureoviolaceum, scapi, tibiae tarsique testacei

. 205. Euchroeus candens Kl. Africa.

$X X$ Abdominis dorsum viridi-cyaneum, scapi et pedes aut concolores aut virides, tarsis brumneis

\section{. 206. Euchroeus cocrulans}

Kl. Mfrien.

2. Mlac albo-hyalinac disco plus minus sordidac. Abilominis dorsun aut cyaneum, aut viridi-cyancum 
Specierum nob. cognitar. Generis Euchroei.

aut viride; pedes concolores tarsis

testaceis

207. Luchroeus quadratus Klug. Europa nedia et meridional., Sibiria.

Divis. 2. Abdominis segmenti dorsalis 3 : tii dentes anales lati subquadrati crenulati, minus numerosi $6-9$.

Corpus viridi-cyaneum tarsis testaceis, alae albo-hyalinae disco nonnilil sordidae . . . 208, Euchroeus 6-dentatus Latr. Gallia.

Sect. II. Postscutellum mucronatum.

Corpus viride . . . 209. Eucliroeus festivus Fabr. Guinea. 
Sect. I. Postscutellum muticum (inerme, non mucronatum).

Divis. 1. Abdominis segmenti dorsalis 3:tii dentes anales angusti acuti, aut subtriangulares aut subspinoidei, numerosi $14-16$.

203. Euchroeus limbatus Klug.

Mediocris robustus via 3 lin. decimal. long. modice aut subcrasse et subconfertim pumctatus cyaneus, abdominis dorso aureo, segmento 3:tio cyaneo, margine anali summo testaceo, denticulis circ. 16 incequalibus.

Eucltoeus limbatus Klug Mus. Berolin. secundurn specimen ibidem anno 1847 a me descriptum.

Habilat in Rossia meridionali, Dom. Klug.

Observ. Quoad habitum Hedychro lucidulo masculo non multo dissinilis. - Caput cyaneum; clypeus magnus subquadratus disco convexiusculo viridis, apice nigro-aeneus; mandibulae basi nigrae, apice rufo-testaceae; maxillae palpique brumnescentes. Thorax cyaneus: pronotum utrinque, dorsuli area media, scutellum, postscutellum et mesopleurae viridi-irrorata; pedes virescenticyanei, genubus, tibiis 1. apice 1. uno latere, tarsisque totis brunneo-testaceis; tegulae concolores, basi cyaneae: alae sordide hyalinae, venis mediocribus brunneis, cellula radiali triangulariter lanceolata incompletissima scil. venac radialis apex a costa et ab alice apice longe lateque remota. Abdominis dorsum confertim subcrasse punctatum viridi-aureum, segmenta $1:$ mum et 2 :dum basi macula triangulari cyanea; segmentum 3:tium cyancum aut violascens, disco transverse subimmersum, tum - ante marginem analem - subincrassatum, margo analis brevis te- 
staceus centro emarginatus, utrinque irregulariter multidenticulatus, denticulis omnibus inaequalibus et parvis et minutis simul sumtis 16-20. Venter nigro-, cyaneo-et viridi-maculatus.

204. Euchroeus purpuratus Fabr.? ठㅇ.

Mediocris vix 3 lin. decimal. long. confertim modice punctatus subcoriaceus viridis, vertice ex parle, dorsuli vittis 3 abdominisque fasciis dorsalibus 2 ant cupreoaut violaceo-purpureis, tibiis tarsisque testaceis, denticulis analibus incequalibus $10-13$.

"Euchroeus purpureus Latr." Gen. Ins. 1. c. secundum specimen typicum Collectionis Latreilleanae a Dom. Spinola nobis communicatum.

Chrysis purpurata Fabr. Ent. Syst. 2. 240:9, et Piez. 172:12 (exclusis verbis ,ano quadridentato", quac ad Chrysidem nitidulam pertinent), secundum specimen e Museo Viennensi a Dom. Kollar nobis communicatum.

Habilat in Europa media et meridionali passim; „Parisiis, mense Augusto, in Eryngii campestris floribus; dum vivit cuprea, post mortem viridis". Latreille l. c.

Observ. Species sui generis pulcherrima. Corpus robustum confertim modice punctatum coriaceum subreticulatum. Caput aut viride aut viridi-aureum, verticis macula cupreo-purpurascens; cavitas facialis longitudinalis rectangularis parum profunda subtiliter punctulata, adpresse albopilosella aut sublanata, medio tenuiter carinulata, superne transverse subacute marginata; antennac robustae, magnitudine mediocres, fuscae basi l. virides 1 . cupreo- aut aureo, aut aeneo-virescentes; clypeus magnus sublongitudinalis, quadratus 1. potius subtrapezinus 
punctulatus, margine apicali subtruncatus, fere hujus

formae; mandibulae testaceae, summo apice nigro-piceae, macula basali viridi. Thorax laete viridis; pronoti truncatura antica polita purpurascenti-cuprea, pronoti pars dorsalis aut unicolore viridis aut punctis riridibus et interstitiis purpurascentibus; dorsuli vittae 3 parallelae (una in singula quaque arearum) etiam cupreae; metanoti anguli postico-laterales mediocres acute triangulares; femora aut viridia aut viridi-aenea, antica plerumque postice aurata; alae albo-hyalinae venis mediocribus aut subtenuibus nigro-piceis, tegulae cupratae. Abdomen ovale aut potius ovato-subrectangulum, longitudine capitis thoracisque simul sumtis, supra subtusque laete et submalachitico-viride, dorso modice converum; segmentum dorsale 1 :mum confertissime modice punctatum riride: macula basali amplissima et margine apicali tenuissime cupreis; segmentum dorsale 2:dum cupreum: maculis 2 lateralibus viridibus transversis oppositis suboralibus aut obtuse conicis fasciam medianam interruptam efformantibus, in colore cupreo magis nitidum et subremote punctatum, in colore viridi confertissime punctatum et subcoriaceum; segmentum dorsale 3:tium brere, 2:do fere triplo brevius, politum aut saltem valde nitidum punctulatum aut cupreum aut violascente-purpureum basi transverse subarcuato sulcatum, tum ante seriem margine convexo modice incrassatum; series ante-apicalis foreolis numerosis profundis rotundatis magnitudine mediocribus; margo analis mediocris aut potius subbrevis; denticuli in aliis et plurimis (forma normali) valde irregulares, atque interdum fere hanc

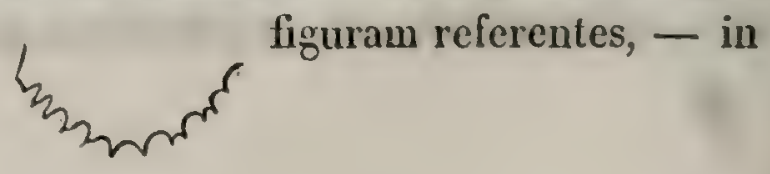


aliis autem (et forma optima) fere hoc modo constructi.

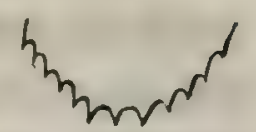

205. Euchroeus candens Klug.

MLediocris aut submagnus $3 \frac{1}{2}$ lin. decimal. long. robushus confertim punctatus cyaneus abdominis dorso saturate purpureo-violaceo, scapo et pedicello antennarum libiis tarsisque luteis; alis disco ample brunneis nonnihil violascentibus, segmenti dorsalis 3: lii denticulis analibus robustis acute triangularibus plurimis vegularibus, $11-12$.

Eucliroeus candens Klug. secundum Spinola in litt.

Wabitat in Africa ad promontorium bonae spei; Mus. Dom. Spinolr.

Observ. Corpus robustum. Caput punctulato-subreticulatum cyaneum; cavitas facialis tota planiuscula viridi-aurea subtiliter punctulato-coriacea albo-sericeo pilosella, medio canaliculata, superne transverse marginata: margine inaequali et cum verticis carinula arcuata cohaerente, fere ut in C\%ryside malachitica (no. 189, cfr. figuram ibidem) formato; antennae subbreves robustac, flagellum nigro-fuscum, scapus et pedicellus lutei, ille postice aureo-nitens; clypeus magnus quadratus confertissime punctulatus aureus, disco transverse convexus; mandibulae crassae, basi virides, infra medium lutescentes, apice nigro-piceae. Thorax cyaneus punctato-reticulatus, punctis pronoti amplioribus unde forma puncturae reticulata ibidem optima; dorsulum violascenti- aut nigricanti-aeneum; sternum viride, utrinque ad mesopleurarum spinas macula cuprea; femora viridi-aurea subtus 
viridi-cyanea, tibiae tarsique lutea, illae extus (ad certum situm luminis) nonnihil virescentes; alae disco brunnescentes et ad certum situm luminis violascentes, basi apiceque hyalinae, venis omnibus firmibus nigro-piceis, cellula radiali triangulariter lanceolata valde incompleta. Abdominis dorsum saturate purpureo-violaceum confertius et subtenuiter punctatum; segmentum dorsale 2: dum utrinque macula laterali subrectangulari cyanea viridilimbata; segmentum dorsale 3:tium fere ut in Euchroeo purpuralo constructum at paullo longius, basi lenius et amplius immersum, dentes anales plurimi regulares l. subaequales, punctura omnino eadem atque segmenti 2 :di. Venter laete viridis marginibus segmentorum aureis.

206. Euchroeus coerulans Klug.

Mediocris $2 \frac{3}{4}$ vix 3 lin. decimal. long. rabustus punctatus viridi-cyaneus tarsis fuscis, alis brunneis nonnihil violascentibus, abdominis segmenti dorsalis 3 : 1 ii denticulis analibus valde irregularibus et inaequalibus $11-15$.

Euchroeus coerulans Klug, secundum Spinola in litteris.

Iabitat in Africa ad promontorium bonae spei, Mus. Dom. Spinola.

Observ. Affinis et similis Euchroeo candenti (no. 205), at facillime dignoscitur corpore breviore viridi-cyaneo, antennis pedibusque nunquam lacteo-pictis, abdominis dorso immaculato unicolore viridi-cyaneo rage 1 . remote punctato etc. Caput cyaneum antice virescens, constructio cavitatis facialis et clypei omnino eadem ut in Euchroeo candente; antenuae breves nigro-fuscac, basi supra virides et subtus cyaneae; mandibulae piceo-brumneae, 
basi virescentes. Thorax cyaneus; pronotum et dorsuli maculae 2 (una in singula quaque arearum lateralium) virescentia; pectus et pedes viridia, plus minus cyanescentia; pronotum eximie reticulatum punctis nullo modo profundis sed fere planis amplis orbiculatis politis; mesonotum et postscutellum etiam punctato-reticulata sed confertius et profundius, punctis itaque non planis sed excavatis, scutelli et postscutelli crassissimis et profundissimis; metanoti anguli postico-laterales mediocres obtusi nonnihil curvi, fere hujus $\Omega$ formae. Abdomen robustum obtuse rectangulum, longitudine capitis thoracisque simul sumtorum, dorso sat convexum politum remote punctatum punctis mediocribus, viridi-cyaneum; segmenti 2: di discus medianus longitudinalis subimpunctatus; segmentum 3:tium mediocre, 2: do vix plus quam duplo brevius, transverso subrectangulum, margine anali leniter arcuatum, basi leniter transverso-immersum, ante seriem permodice convexo-incrassatum; series ante-apicalis foveolis numerosis parvis rotundatis; marginis analis denticuli fere ita inaequales et irregulares ut in $\boldsymbol{E u}$ chroei purpurali forma normali (no. 204, cfr. figuram ibidem). Venter abdominis dorso concolor.

207. Euchroeus quadratus Klug. $\delta$ Tㅇ.

Mediocris $3-3 \frac{1}{4}$ lin. decimal. long. robustus conferlim modice punctatus, aut viridis aut viridi-cyaneus gemubus tarsisque luteis 1 . testaceis, alis albo-lyyalinis, disco levissime sordidis, abdominis segmenti dorsalis 3:tii denticulis analibus valde irregularibus el inaequerlibus $11-15$.

II. 
var. a. Macula verticis, dorsulum - praeter villas tenues virescentes -, metanotum, margo basalis segmentorum abdom. 1:mi et 2: di, nec non segmentum 3:tium fere totum, cyanea; ceterum est corpus viride.

Eucliroeus quadratus Klug, secundum specimina quae in Musen Berolinensi anno 1847 descripsi.

Euchroeus polyodon Kollar, Mus. Viennens.

Chrysis 15-dentata Pallas, MIanuscript. teste Dom. Klug. var. $b$. Corpus dorso cyaneum unicolor.

Euclirocus festivus Mrss. Spinolae.

Habitat in Germania ad Berolinum, Dom. Klug; Italia, Dom. Zeller; - Hungaria, Dom. Kollar; - Aegrypto, Dom. Waltl, Mus. Dom. Spinola; - Sibiria, Dom. Pallas, Mus. Reg. Berolin.

Observ. Nonnihil similis Euchroeo coerulanti, at primo intuitu dignoscitur thorace undique confertius modice punctato subcoriaceo subopaco, pronoto non (ut in Euchroeo coerulanti) eximie et ample reticulato sed reliquis thoracis partibus sculptura simillimo, alis albo-hyalinis et tantum disco interdum leviter sordidis tarsis testaceis aut potius luteis etc. Capitis cavitas facialis mediocris subrectangularis modice profunda subtiliter punctulata transverse strigosa, lateribus cano-pilosella, superne fovea centrali et supra hanc transverse marginata: margine inaequali subangulato et fere hujus formae; clypeus ut in praecedenti constructus; antennac mediocres nigro-fuscae, basi cyaneo-virides l. vice versa; mandibulae basi aut virides ant cyaneae, disco fuscopiceae, ante apicem lutescentes, apice 1. rufo- I. brunneo- 
piceae. Pectus, pedes et venter aut viridia aut viridicyanea; pedum genua, apices tibiarum, tarsi toti et omnes spinulae lutea; reliquam corporis picturam jam in notis varietatum attulimus. Alarum venae mediocres aut brunneae aut nigro-piceae. Abdominis dorsum confertim modice punctatum, interstitiis subtilissime punctulatis, centrum segmenti dorsalis 2:di subtuberculato-gibbum; segmentum dorsale 3:tinu brevissimum, 2: do triplo brerius, basi sulco transverso continuo, ante seriem fortiter transverso-arcuato-incrassatum, series ante-apicalis et denticuli anales fere omnino ut in Euchroeo coerulanti.

Divis. 2. Abdominis segmenti dorsalis 3:tii dentes anales lati subquadrati crenulati, minus numerosi $6-9$.

208. Euchroeus 6-dentatus Latreille.

Mediocris vix 3 lin. decimal. long. robustus conferlim modice punctatus, supra viridi-cyaneus sublus vividis, gemubus tarsisque luteis, alis sordide hyalinis, abdominis segmenti dorsalis 3:tii dentibus 6-9: plurimis latis irregulariter subquadratis cremulatis, tantummodo $2-3$ acutis subtriaingularibus.

Euchroeus sexdentatus: Latreille, Mus. Spinolae.

Inabitat in Gallia, Parisiis a Dom. Latreille olim detectus; Mus. Dom. Spinola.

Observ. Speciebus proxime praecedentibus habitu et pictura similis et affinis, at armatura anali satis superque distinguitur. - Caput cyaneum, antice h. e. facie et clypeo virescens; cavitas facialis modice profunda subtiliter transverse strigosa, utrinque punctulata et cano-pi- 
losa, superne marginata: margine transwerso leniter crenulato; antennae breves nigro-fuscae, basi virescentes; clypeus ut in Eucluroeo quadrato magnus subquadratus punctulatus; mandibulae basi viridi-aenescentes, medio testaceae, apice brunneo-fuscae. Thoracis dorsum cyaneum; pronotum antice, dorsuli vittac 2 laterales, scutellum, angulique metanoti postico-laterales viridia; hi anguli ut in Euchroeo quadrato constructi; pectus, pedes et venter quoque viridia; genua tarsique lutea. Abdominis dorsum confertim irregulariter subcrasse punctatum cyaneum, segmentum 1:mum utrinque et 2:dum disco virescentia; segmenti 2 : di lineola mediana polita lanceolata et disco subgibbe elevata; segmentum 3:tium breve, 2: do duplo et ultra brevius, basi sulco transverso lato continuo, tum ante seriem fortiter convexo-incrassatum, series ante-apicalis foveolis mediocribus profundis rotundatis numerosis; margo analis (segmenti 3:tii) latiusculus cyaneus, sparse crenatus et emarginatus, hinc dentes irregulares $6-9$ fere hujus formae terunt.

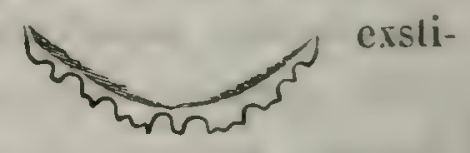

Sect. II. Postscutellum mucronatum.

209. Euchrocus festivus Fabr.

Mediocris viridissimus nitidus ,ano serrato coeruleo alis sordide hyalinis.

Chrysis festiva Fabr. Ent. Syst. 2. 239:3, Piez. 171:3, secundum specimen typicum, quod in Museo Reg. Havniensi anno 1847 examinavi et descripsi. detectus.

"Mabitat in Guinea" Fabr. I. c. a Dom. Isert olim 
Observ. Corpus viridissimum, nomunquam in aeneum vergens. Caput, pronotum et dorsulum modice crasse punctata; pleurae, scutellum et postscutellum crassissime punctata scabra; abdominis segmenta dorsalia medio modice punctata et ibidem maxime nitida subpolita, ad margines confertim punctulata; abdominis limbus 1. margo analis punctatissimus coeruleus lineola mediana convexa viridi. Antennae nigrae basi viridi-aenescentes. Cilypeus semilunatus margine apicali summo depresso testaceo. Mandibulae fulvac, apice piceae. Postscutelli mucro 1. processus discoidalis horizontalis orbiculatus margine 5-dentatus: dentibus utrinque lateralibus 2 parvis subconicis, dente postico-centrali maximo rotundato, angulato et crenulato. Pedes virides, genubus, apicibus tibiarum tarsisque totis luteis. Alae sordide hyalinae, scil. umbris fuscis minus determinatis. 


\section{Familia 6. PARNOPIDAE Dlbm.}

Maxillae et labium abnormia: ligula et maxillarum processus longissima, producta in rostrum tenue filiforme, promuscidem Apum simulans, in quiete sub pectore reflexum.

Mandibulae margine interno ante apicem acute unidentatae.

'Thorax subtetraëdro - cylindricus, dorso depresso convexus; pronotum transverso subrectangulum.

Unguiculi tarsorum infra mutici et inermes.

Abdomen segmentis cataphractis conspicuis $3-4$, supra depresso-convexum, subtus aut planiusculum aut subfornicatum, segmenti dorsalis 3:tii margo analis serrulatus. 
E Fanilia Parnopidarum adhuc tantummodo unicum Genus cognitum, scil.:

\section{Genus PARNOPES Fabr.}

Piez. $177: 27$. - Latr. Gen. Ins. 4. 47:476. - Dlbm. Dispos. 1845. pag. 1. Chrysis Fabr. Eunt. Syst. 2.

Corpus robustum elongatum $2 \frac{1}{2}-4$ lin. decimal. long. Caput rotundato - triangulum; clypeus submagnus transversus latus.

Mesopleurae thoracis subtiliter marginatae: margine aut subintegerrimo aut crenulato.

Scutelli discus elevatus planus trapezinus.

Postscutelli centrum aut tuberculatum, aut mucronatum: mucrone plano subhorizontali versus abdomen directo et fere instar sellae equestris formato, margine lacero.

Pedes fere ut in Chrysidibus constructae, at minus robustae et spinulae articulorum tarsorum apicales evidentiores et longiores.

Alarum tegulae maximae longitudinales ovato-scutatae et punctatae.

Nlae anticae tantummodo 2 cellulis completis, scil. costali et mediali; cellula submediali 1 :ma subcompleta; cellula radiali solum inchoata, propter venam radialem paullo post sligma desinentem.

Alae posticae duabus venis: costali et postcostali atque cellula costali gaudent. 
Abdomen ovato-subrectangulum, segmenta dorsalia: maris $4,-$ o 3 ; segmentum dorsale ultinum maximum serie ante-apicali - apud familias antecedentes normali - omnino caret, aut subsemicirculare aut conicosubtriangulare, parte basali et maxima modice convexum, ante marginem analem utrinque elliptico- aut ovato-immersum carinula mediana brevi minus determinata convexa intercedente. Limbus analis (segmenti dorsalis ultimi) crassus, aut depressus declivis aut convexus, duplici serie serrulatus: serie superiore marginem dorsalem sequente aut a tuberculis aut a spinulis microscopicis constituta, serie inferiore secundum marginem ventralem ducta et denticulis 1. subtriangularibus 1. spinoideis distinctis instructa.

Species Parnopis Generis 4 cognitae sunt: 3 Acgyptiacae et unica Europaea. 


\section{Tabula Synoptica Specierum adhuc cognitarum Generis Parnopis.}

Divis. 1. Postscutellum tuberculatum.

A. Corpus $2 \frac{1}{2}$ lin. decimal. long. viride, antennis tegulis pedibusque testaceis, abdominis dorso concolore aureo-micante 210. Parnopes elegans Kl. ․ Aegypto.

B. Corpus 3 lin. decimal. long. viridi-aureum, antennarum flagellis, pedibus, abdominisque segmentis dorsalibus 1 :no, 2 : do et 3 : tio rufo-testaceis, tegulis margineque anali pallidis . . . . 211. Parnopes denticulata Spin. ठ'. Aegypt.

Divis. 2. Postscutellum in centro armatum processu plano horizontali ovato aut rotundato, margine crenulato et veluti lacero.

A. Corpus vix 3 lin. decimal. long. politum subremote punctatum viride tegulis viridi-cyaneis, genubus, tibïs ex parte, tarsis totis et margine anali summo testaceis

212. Parnopes Fischeri Spin. ㅇ. Aegypt.

B. Corpus circ. 4 lin. decimal. long. subopacum confertim punctatum aeruginoso-viride, abdominis segmentis dorsalibus aut tribus aut duobus ultimis carneis

- 213. Parnopes carnea Fabr. ðㅇ‥ Europa meridional. 
Divis 1. Postscutellum tuberculatum.

210. Parnopes elegans Klug. ㅇ.

Mediocris $2 \frac{1}{2}$ lin. decimal. long. viridis aureo-nitida, ore, antennis, tegulis, pedibus margineque anali testaceis aut sublutescentibus, alis hyalinis venis tenuibus testaceis.

Parnopes elegans Klug. Symb. Phys. Dec. V. 1845. Tab. 45. fig. 1.

Habitat ,ad Ambukohl Aethiopiae mense Julio, semel lecta“. Klug. 1. c.

Observ. Parnopi carneae quoad picturam quodammodo similis; at facile dignoscitur corpore breviore magis nitido, ore, antennis, pedibus, alarum tegulis et renis abdomineque testaceis, hoc dorso viridi-aureo-micaute, postscutello vix tuberculato etc., segmentum dorsale 3 : tium ad apicem utrinque - ut in $\boldsymbol{P}$. carnea - ovato-impressum, impressione albo-sericea.

211. Parnopes denticulata Spinola. $\sigma$.

Mediocris 3 lin. decimal. long. viridi-aurea antennarum flagellis, pedibus, abdominisque segmentis dorsalibus 1:mo, 2:do et 3:tio rufo-testaceis, tegulis margineque anali pallidis, alis hyalinis venis mediocribus fuscis.

Parnopes denticulata Spinola Annal. Entomol. 1838. 455:6.

Inabitat in Aegypto (?), Dom. Géné, secundum Spinola l. c.

Observ. Insectum non vidimus, unde tantummodo notas a Dom. Spinola l. c. jam relatas referre possumus. Antennae, pedes abdominisque segmenta dorsalia tria an- 
tica rufo-testacea; caput, scapus antennarum, thorax, coxae pedum abdominisque segmentum dorsale 4 :tum metallice viridia ex aureo resplendentia. Alarum tegulae abdominisque margo analis pallescentes. Postscutellum tuberculatum: tuberculo parvo obtuso leniter cmarginato. Abdominis segmentum dorsale 4:tun convexum nec ante marginem utrinque - ut in reliquis hujus Generis speciebus - depressum; margo analis arcuatus nonnihil incrassatus - fortiter serrulatus: denticulis aculis oblique pendulis.

Divis. 2. Postscutellum in centro armatum processu plano horizontali ovato aut rotundato, margine crenulato et veluti lacero.

212. Parnopes Fischeri Spinola. 9.

Mediocris 3 lin. decimal. long. sparse aut subremote punctata polita viridissima tegulis viridi-cyaneis, $n$ ntennis genubus tibiisque ex parle tarsisque totis lutescentibus, margine anali triangulari subcarneo, alis hyalinis ad costam sordidis venis firmis piceis.

Parnopes Fischeri Spinola Annal. 1838. 455: XII. secundun specimen typicum mihi benevole communicatum.

IIabilat in Aegypto, a Dom. Fischer detecta; Mus. Dom. Spinola.

Observ. Pictura, punctura et politura corporis nec non forma segmenti abdom. dorsalis ultimi a reliquis speciebus hujus Generis valde diversa.

Corpus lineari-subellipticun, angustius et multo minus robustum quam in Parnope carner, viridissimum nitidum. Caput confertim punctato-rugulosum; Cavitas 
facialis mediocris subquadrata planiuscula punctulato-coriacea albo-sericea, superne libera nec marginata; antennae perbreves, scapus viridi-cyaneus, subtus castaneolineatus, pedicellus castaneus, flagellum subferrugineofuscum; clypeus magnus transversus subsemilunatus punctulatus, transversaliter subcarinato-convexus, margo apicalis (anne labrum clypeo arctissime conjunctum?) albus submembranaceus leniter arcuato-cmarginatus 1. potius arcuato-detrusus; mandibulae castaneae, basi apiceque nigro-piceae; maxillae nigro-piceae violascentes, palpi lutei, ligula his concolor. Thorax rectangulariter cyliudricus, dorso convexo-depressus; pronotum nitidum vage punctatum interstitiis non politis at subtilissime punctulatis, utroque margine laterali leniter arcuato-emarginatum, hinc omnes anguli laterales et antici et postici nonnihil prominuli; dorsulum politum nitidissimum vage I. remote sed irregulariter punctatum; scutellum crassissime punctato-reticulatum et scrobiculatum; metanotum coriaceum plus minus nitidum, postscutelli processus crasse punctato-rugosus, irregulariter subsemicircularis, margine inaequaliter crenulatus, fere hujus metanoti anguli postico-laterales me

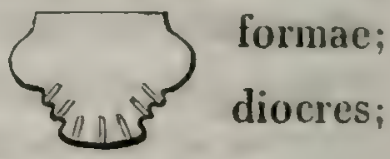
mesopleurae nitidae crasse punctato-reticulatac, margine apicali arcuatae et fere hoc modo denticulatae; tibiae virides; femora

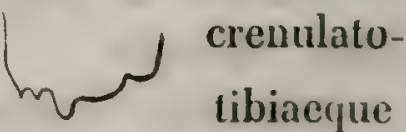
antica latere antico, femora posteriora postice, tibiac posteriores latere infero, genua et apices tibiarum ounia tarsique toti, lutea. Abdomen ovato-subconicum, dorso politum viridissimum punctis et mediocribus et minutis vage adspersum; seguientum dorsale 3:tium conico-sub- 
triangulare, obliqua sub irregulariter cimine com utraque impressio antemarginalis linearis; margo analis subcarneus multispinulosus. Venter (in spemunicato) piceus fornicatus, in speciminibus recentioribus forsitan metallice violascens, etenim Cel. Spinola 1. c. dixit: „Ventre violet metallique".

213. Parnopes carnea Rossi. $\delta$ ㅇ.

Magna circ. 4 lin. decimal. long. robusta subopaca confertim punctala aeruginoso-aeneoque viridis, abdominis segmentis dorsalibus ullimis $2-3$ carneis margine anali arcuato, tibiis tarsisque luteis, alis sordidis, venis firmis piceis.

Chrysis carnea Rossi Fn. Etr. Edit. Illig. 2. 120. 843. Tab. 8. fig. 5; - Fabr. Ent. Syst. 2. $240: 7$. - Coqueb. Icon. dec. 2. tab. 14. fig. 11.

Parnopes carnea Latr. Gen. Ins. 4. 47; - Fabr. Piez. 177. 27:1; - Dlbm. Dispos. 17 : 1.

Habitat in locis arenosis sylvaticis et fruticosis Europae meridionalis, praesertim Italiae, Galliae australis etc., mense Julio passim, interdum frequens.

De moribus hujus speciei jam Cel. Lepeletier de Saint-Fargeau in Encycl. Meth. Tom. X. pag. 8 et 497 observationes publicavit. Nobis autem opus citatum non possidentibus liceat sequentia verba e Cel. Westwoodi opere "Modern Classification of Insects" inscripto (pag. 179) referre: "Saint-Fargeau have detailed the economy of this genus (Parnopes), the type ( $\boldsymbol{P}$. carnea) of which is found in France, and which deposits its eggs in the nests of Bembex rostrata. According to the more re- 
cent observations of the last named Author, the femal Parnopes (carnea) enters backwards into the nest of the Bembex (thus evidently showing its object to be to deposit its eggs therein). It is probable that it only then deposits a single egg, as generally a single larva of the Parnopes is only found, and which is engaged at the beginning of the spring in sucking the larra of the Bembex, upon the back of which it has fastened itself."

Observ. Corpus robustum. Vertex, thorax et abdominis segmentum dorsale $\mathbf{1}$ : mun confertim crasse punctata subreticulata, punctura verticis tamen tenuior et confertior. Caput viride, vertice, fronte et cavitate faciali ant concoloribus, aut cupreo-irroratis aut unicolore cupreis; cavitas facialis brevis transverso-quadrata albosericea subtiliter punctulato-coriacea, medio superne plus minus distincte carinulata, utrinque modice profunda; antennae mediocres brunnescentes, scapo nigro-piceo aut nigro-aeneo; clypeus magnus transversus punctulatus aut viridis aut aureo-viridis aut aureus, disco conrerus, margine apicali amplo convexo-deflexo nigro-piceo polito, ad centrum arcuato-emarginato; mandibulae piceae, ante apicem castaneac; maxillae et labium nigro-piceae, marginibus ligulaque scarioso submembranaceis pallide testaceis. Thorax coriaceus crasse punctato-reticulatus, crassus subquadrato-cylindricus aut aeruginoso-viridis aut viridi-aeneus; pronotum raro totum viride, plerumque cupreo-irroratum, interdum totum cupreum, qui color tunc etiam partem dorsuli basalem obtegit; apud quaedam individua sunt dorsulum apice et totum scutellum cyanea atque posiscutelli processus nigro-aeneus; scutellum crassissime punctatum 1. scrobiculatum; postscu- 
telli processus fere eandem formam לv' habet, quam apud Parnopem Fischeri, at saepe proportionaliter longior et apice interdum truncato angustiori, crenaturis et denticulis lateralibus etiam distinctioribus; mesopleurarum margo apicalis irregulariter subarcuatus et fere hoc modo Le crenulato-denticulatus; pedum partes basales aut viridia, aut picea 1. nigro-aenea femoribus tunc testaceo-lineatis; interdum sunt femora latere externo aureo splendida; tibiae unacum tarsis luteae; alae sordidae, renis robustis piceis, tegulis remote modice punctatis aut testaceis, aut luteis, aut piceis aut nigroaeneis margine pallido. Abdomen robustum obtuse rectangulum, basi transversa, apice arcuato, in mare longius quam in femina, dorso modice convexum carneum; segmentum dorsale $\mathbf{1}$ :mum crasse punctatum aeruginosoviride, cupreo-irroratum, rarissime totum cupreum, interdum sunt maris segmenta dorsalia 2:dum et 3:tium fascia mediana violascente instructa, saepissime autem deprehenduntur reliqua segmenta (1:mo excepto) carnea; margo apicalis segmentorum 1 : mi et 2 : di in femina, $1: \mathrm{mi}$, 2: di et 3:tii in mare nonnihil impressus fulvus, post mortem plerumque testaceus; punctura segmentorum aut intermediorum aut intermedii disco crassa, ad margines basales et apicales confertissima et tenuis; segmentum dorsale 3: tium semicirculare convexum crebre punclatum subrugulosum, ante marginem utrinque ovato- aut subtriangulariter oblique depressum, depressionibus largiter albo-sericeis per carinulam medianam separatis; margo 
analis robustus arcuatus, denticulis numerosis distinctioribus acutis spinoideis. Venter aut fornicatus aut planiusculus, aliis testaceus, aliis luteus, aliis denique castaneus aut piceo-brunneus. 


\section{vi. Explicationes Icontum.}





\section{Tabula I.}

Figura 1. Cleptes fasciata.

2. Cleptes orientalis.

3. Caput antice risum; 4. Maxilla; 5. Labium; 6. Mandibula; 7. Clypeus; 8. Antenna; 9. Thoracis dorsum: $p r=$ pronotum, $d r s l=$ dorsulum, $s c=$ scutellum, $p s c=$ postscutellum, $m t=$ metanotum, $\operatorname{mapl}=$ metanoti anguli postico-laterales.

"10 a. Ala antica: $a=$ margo anticus, $b=$ margo apicalis, $c=$ margo posticus, $a=$ vena costalis, $d=$ vena postcostalis, $e=$ vena medialis, $f=$ vena analis, $u=$ venula transverso-medialis, $v=$ venula transverso-submedialis $1: \mathrm{ma}, w=$ venula transverso-subroedialis $2: \mathrm{da}$, $h=$ vena cubitalis rudimentaria, $i=$ vena discoidalis rudimentaria, $p=$ venula transverso-discoidalis (1: $\mathrm{ma}$ ); $1=$ stigma, $2=$ cellula radialis incompleta, $15=$ celIula costalis completa, $16=$ cellula medialis completa, $17=$ cellula submedialis $1: \mathrm{ma}$ completa, $18=$ cellula submedialis 2 :da rudimentaria, $19=$ cellula discoidalis plerumque rudimentaria, $20=$ regio cellulae analis.

" $10 \mathrm{~b}$. Nla postica: $a=$ vena costalis et margo anticus, $b=$ vena postcostalis, $c=$ vena medialis, $d=$ vena analis; $a b=$ spinulae recurrae quibuscum ala postica ad alam anticam sub rolatu affigitur, $1=$ regio stigmaticalis, $2=$ cellula costalis.

" 11. Abdominis dorsum 우 12. venter 우; 13. pes; 14. tarsi unguiculi.

"15. Heterocoelia nigriventris.

"16. Abdominis segmentum dorsale ultimum margine apicali 4 . dentatum in Heterocoelia nigriventri. 


\section{Tabula II.}

Fig. 17. Caput antice visum; 18. Mandibula; 19. Thorax supra visus; 20. Thorax e latere visus; 21. Ala antica; 22. Ala postica; 23 a. Unguiculi tarsorum; 23 b. Pes; 24 a. Abdominis dorsum, $24 \mathrm{~b}$. Venter; 25. Abdominis segmentum dorsale. 3 : tium, $e=$ emarginatura apicalis Omali aurati var. $\boldsymbol{a}$.

"26. Thorax e latere visus; 27. Abdominis segmentum dorsale 3 : tium Omali pusilli.

" 28. Omalus punctulatus.

" 29: Thorax e latere visus; 30. Abdominis segmentum dorsale 3 : tium Omali punctulati.

" 31 a. Thorax supra visus, $31 \mathrm{~b}$. Thorax e latere visus; 32 . Ahdominis segmentum dorsale 3 : tium Omali coerulei.

" 33 a. Thorax supra visus, $33 \mathrm{~b}$. Thornx e latere visus; 34 . Abdominis segmentum dorsale 3 :tium Omali aenei.

" 35 a. 'Thorax supra visus, $35 \mathrm{~b}$. Thorax e latere visus; 36 . Abdominis segmentum dorsale 3 :tium Omali coriacei.

\section{Tabula III.}

Fig. 37a. Postscutellum supra visum, 37 b. Postscutellum e latere visum; 38. Abdominis segmentum dorsale 3:tium Elampi bilentuli.

" 39 a. Postscutellum mucronatum supra visurn, 39 b. Postscutellum e latere visum; 40. Abdominis segmentum dorsale 3:tum Elampi ambigui.

" 41 a. Postscutellum mucronatum supra risum, 41 b. Postscutellum e latere visum; 42 a. Abdominis segmen- Elampi tum dorsale 3 : tium supra visum, 42 b. idem se- Spina.
gmentum e latere visum

" 43 a. Postscutellum obtuse conicum supra visum, 43 b. idem e latere visum; $44 \mathrm{a}$. Abdominis segmentum dorsale 3:tium supra visum, 44 b. idem segmentum e latere risum Elampi truneati.

"45. Elampus chrysonotus.

" 46 a. Postscutellum mucronatum supra visum, 46 b. idem e latere visum Elampi producti. 
Fig. 47. Caput antice visum; 48. Mandibula; 49 a. Postscutellum mucronatum supra visura, $49 \mathrm{~b}$. idem e latere visum; 50. Ala antica, 51. Ala postica; Elampi 52 a. Abdominis segmentum dorsale 3:tium su- Panzeri. pra et antice visum, $52 \mathrm{~b}$. idem segmentura e latere visum; 53 a. Pes; $\mathbf{5 3}$ b. Unguiculi tarsorum

"54. Postscutelli nucro semicircularis; $55 \mathrm{a}$. Abdominis segmentum dorsale 3:tium supra et antice visum, $55 \mathrm{~b}$. iden segmenturu e latere visum Elampi Gayi.

" 56 a. Holopyga Dolerni. 56 b. Abelominis segmentum dorsale 3 : tium ejusdem insecti.

"57. Caput antice visum; 58. Mandibula; 59. Ala antica, 60. Ala postica; $61 \mathrm{a}$. Unguiculi tarsorum; $61 \mathrm{~b}$. Abdominis segmentum dorsale 3:tium Holopygae ovatae.

\section{Tabula IV.}

[ïig. 62. Abdominis segmentum dorsale 3 : tium Hedychri brasiliani.

"63 ^. Hedychrum Zelleri.

" 63 b. Dorsum abdominis e latere visum, 63 c. Dorsum abdominis supra visum Hedychri incrassati.

" 64a. Caput antice visum; 64b. Vertex; 65. Mandibula; 66. Dorsum thoracis; 67. Ala antica, 68. Ala postica; 69. Abdominis-segnentum dorsale 3:tium; 70. Unguiculi tarsorum; 71. Pes Hedycleri luciduli var. $a$.

" 72. Dorsum thoracis; 73. Dorsum abdoninis Hedychri minuti.

"74. Dorsum thoracis; 75. Dorsum abdominis Hedychri cuprati.

" 76. Dorsum thoracis; 77. Dorsum abdominis Hedycliri integri.

"78. Dorsum thoracis; 79. Dorsum abdominis Hedychri fervidi

80. Structura corporis Hedychri fervidi e latere visa.

S1. Hedychrum femoratum.

, S2. Sculptura pronoti Hed. femorati in tabulis omissa est. 


\section{Tabula V. Familia CHRYSIDIDAE. \\ Genus CHRYSIS. Phalanx I.}

Fig. 83 a. Chrysis capitalis.

" $83 \mathrm{~b}$. Segmentum abdominis dorsale 3 :tium et sculptura ejusdem.

" 84 a. Chrysis incrassata.

" 84 b. Dorsum abdominis et sculptura ejusdem; 84c. Segmentum abdominis dorsale 3:tium e latere visum (= das 3te Segraent des Hinterleibsrückens von der Seite gesehen).

" 85 a. Chrysis Humboldti.

" $85 \mathrm{~b}$. Dorsum abdominis et sculptura ejusdem; $85 \mathrm{c}$. Segmeutum abdominis dorsale 3 :tium e latere visum.

\section{Tabula VI.}

Fig. 86 a. Chrysis aerata.

" $86 \mathrm{~b}$. Postscutellum a dorso visum (von oben gesehen); $86 \mathrm{c}$. Postscutellum e latere visum; $86 \mathrm{~d}$. Dorsum abdominis et sculptura ejusdem.

" 87. Chrysis socia.

" $87 \mathrm{~b}$. Segmentum abdominis dorsale $3:$ tium et sculptura ejusdem.

" 88a. Chrysis uniformis.

" 88 b. Dorsun thoracis et sculptura ejusdem; 86 c. Postscutellum c latere visum; 88d. Dorsum abdominis et sculptura ejusdem; 88c. Segmentum abdominis dorsale 3:tium e latere visum.

\section{Tabula VII.}

Genus CHRYSIS. Phalanx II.

Fig. 89a. Chrysis Dalmanni. 89b. Abdominis segmentum dorsale $3:$ tium et sculptura ejusdem, $89 \mathrm{c}$. hujus segmenti margo apicalis summus.

"90. Abdominis segmentum dorsale 3 :tium et sculptura ejusdem: Chrysidis sinuosae.

"91. Abdominis segmentum dorsale 3 :tium et sculptura cjusdem: Chrysidis erythromelas. 
Fig. 92 a. Abdominis segraentum dorsale 3:tium et sculptura ejusdem; $92 \mathrm{~b}$. Formae variantes marginis apicalis summi (in abdorainis segraento dorsali $3:$ tio) apud varictates $a, b, c, e$ et $f$ Chrysidis elegantis.

" 93 a. Chrysis mediocris. $93 \mathrm{~b}$. Abdominis segmentum dorsale 3 : turu et sculptura ejusden, $93 \mathrm{c}$. hujus segm. margo apicalis summus.

" $94 \mathrm{a}$. Dorsum abdorainis et sculptura ejusdem; $94 \mathrm{~b}$. Abdoninis segmenti dorsalis $3:$ tii margo apicalis suramus Chrysidis emarginatulae.

" 95 a. Dorsum abdominis et sculptura ejusdem; 95b. Abdoninis segmenti dorsalis $3:$ tii margo apicalis summus Chrysidis carinatae.

\section{Tabula VIII.}

Fig. 96a. Chrysis crassimargo. $96 \mathrm{~b}$. Abdominis segmentum dorsale 3 :tium et sculptura ejusdera, $96 \mathrm{c}$. hujus segmenti margo apicalis summus.

" 97 a. Clirysis foveata. 97b. Dorsum abdominis et sculptura ejusdem; 97 c. Abdominis segmenti dorsalis 3:tii margo apicalis summus.

" 98a. Dorsum abdominis et sculptura ejusdem; 98b. Abdom. segmenti dorsalis $3:$ tii margo apicalis summus Chrysidis versicoloris.

" 99 a. Chrysis albipennis. 99b. Dorsum abdominis et sculptura ejusdem; 99 c. Abdominis segmenti dorsalis 3 : tii margo apicalis summus.

\section{Tabula IX.}

Fig. 100 a. Chrysis unicolor. 100b. Dorsum abdominis et sculptura ejusdem; $100 \mathrm{c}$. Abdominis segmenti dorsalis $3:$ tii margo apicalis summus.

\section{Genus CHRYSIS. Phalanx III.}

Fig. 101 a. Chrysis succinclula. 101b. Dorsum abdominis et sculptura ejusdem; $101 \mathrm{c}$.Abdominis segmenti dorsalis $3:$ tii margo apicalis.

"102a. Clerysis Leachei. 102b. Dorsum abdoninis et sculptura ejusdem; 102 c. Abdom. segmenti dorsalis 3:tii snargo apicalis. 


\section{Genus CHRYSIS Phalanx IV.}

Fig. 103 a. Dorsum abdom. et ejustem sculptura; 103 b. Ab- Chr. bila dominis segmenti dorsalis 3 :tii margo apicalis $\left\{\right.$ matae $S_{p}$ iu.

\section{Tabula X.}

Fig. 104 a. Dorsum abdom. et sculptura ejusdem; 104b. Ab-) Chrysidis dominis segmenti dorsalis 3 :tii margo apicalis prasinae $\mathrm{Kl}$.

\section{Genus CHRYSIS. Phalanx V.}

Fig. 105a. Chrysis Bohemanni. 105b. Postscutellum muticum, e latere visum; $105 \mathrm{c}$. Abdominis dorsum et sculptura ejusdera; $105 \mathrm{~d}$. Abdominis segmenti dorsalis $3:$ tii margo apicalis.

" 106 a. Clerysis parvula. $106 \mathrm{~b}$. Postscutellum subconicum apice sublacerum, e latere visum; $106 \mathrm{c}$. Dorsum abdominis et scu!ptura ejusdem; $106 \mathrm{~d}$. Abdominis segmenti dorsalis 3 : tü marso apicalis.

"107a. Clerysis truncatella. 107b. Postscutellum e latere visun; 107 c. Dorsum abdominis et sculptura ejusdem; $107 \mathrm{~d}$. Abdominis segmenti dorsalis 3 : tii margo apicalis.

\section{'Tabula XI.}

Fig. 108. a. Chrysidis fasciatae Fbr. segmentum abdominis dorsale 3: tium ejusque sculptura,

b. idem segmentum e latere visum.

" 109. Chrysidis clitiensis Spin. dorsum abdominis ejusque sculptura.

"110. a. Clarysidis coerulantis Fbr. segmentum abdominis dursalt" 3: tiurn ejusque sculptura,

b. idem segmentum e latere visum.

"111. a. Chrysis Zetterstedti Dlbm.

b. segmentum abdominis dorsale 3 :tium ejusque sculptura.

"112. a. Chrysis Genbergi Dlbm.

b. segmentum abdominis dorsale 3 :tium ejusque sculptura, c. idem segmentum e latere visum. 


\section{Explicationes Icomum.}

\section{Tabula XII.}

1ig. 113. a. Chrysis Klugi.

b. segmentum abdominis dorsale 3 :tium ejusque sculptura,

c. idem segmentum e latere visum.

"114. Stilli splendidi Fbr. a. caput antice visum; b. thoracis dorsum ejusque sculptura; c. abdominis dorsura ejusque sculptura; d. segmentum abdominis dorsale 3:tium e latere visum; e. ala antica; f. ala postica.

"115. Stilbi calendis Fbr. dorsum thoracis ejusque sculptura. 


\section{Index systematicus}

Familiarum, Generum et Specierum in hoc libro adumbratorum.

Fam. 1. CLEPTIDAE

$$
\text { pag. 7, 8-22. }
$$

Genus 1. CLEPTES Auctor.

Species

$$
\text { pag. 9, 10-21. }
$$

1. fasciata pag.

2. nitidula . . . 13

3. semiaurata . . . 15

4. ignita . . . . 18

5. aurata . . . . 20

6. orientalis . . . 20

Genus 2. HE'TEROCOELIA

$$
\text { pag. 9, 21-23. }
$$

7. nigriventris . . . 22

Famil. 2. ELAMIPIDAE pag. 7, 24-56.

Genus 3. OMALUS pag. $25,26-38$.

8. auratus . . . 26

9. parvulus . . . . 31

10. pusillus . . . 31

11. punctulatus . . . 33

12. caerulescens . . . 34

13. aeneus . . . . 35

14. coriaceus . . . 37
Genus 4. ELAMPUS

pag. 25, 38-47.

Species (Omalús) pag.

15. bidentulus . . . 39

16. ambiguus . . . . 41

17. Spina . . . . 41

18. truncatus . . . 42

19. chrysonotus . . . 44

20. productus . . . . 44

21. Panzeri * . . 45

22. caeruleus . . . $46^{\circ}$

23. Gayi * . . . 47

Genus 5. HOLOPYG:

$$
\text { pag. 25, } 47-56 .
$$

24. Dohrni - . . . 48

25. luzulina . . . . 49

26. janthina . . . . 50

27. punctatissima . . 50

28. ovata . . . . 51

29. micans . . . . 55

Famil. 3. HEDYCHRIDAE

p. $7,57-94$.

Genus 6. HEDYCHIRUM

$$
\text { p. } 58-94 \text {. }
$$

30. brasilianum . . . 59

31. caclestinum . . . 60 
Index systematicus.

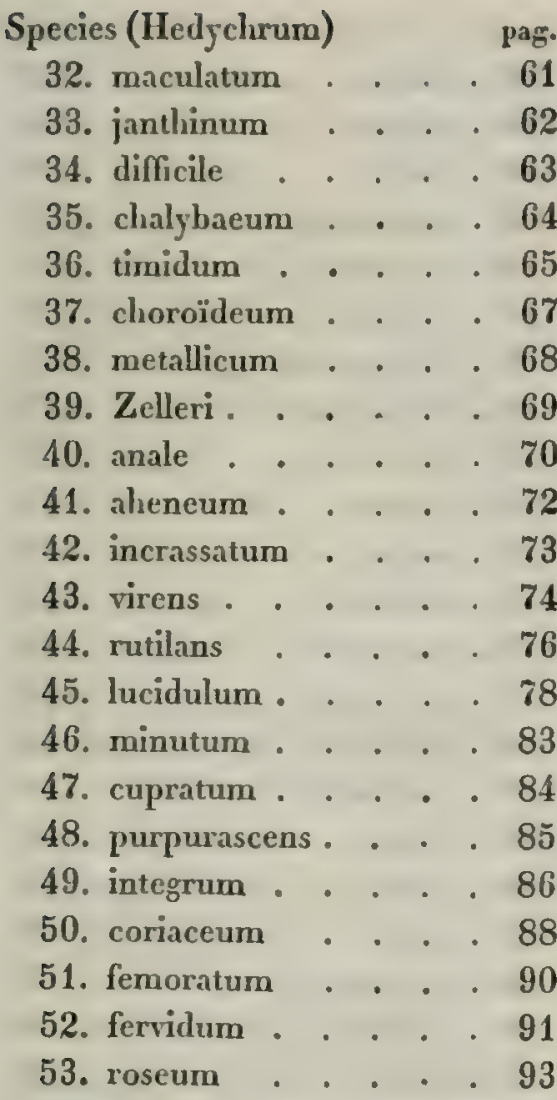

\section{Famil. 4. CHRYSIDIDAE} pag. 7, 95-360.

\section{Genus 7. CHRYSIS}

$$
\text { pag. 97, 98-349. }
$$

54. capitalis . . 100

55. Jugum 。 . . . 101

56. lilaris . . . 103

57. tarsata . . . 105

58. basalis . . . . 106

59. incrassata . . . 107

60. cyanura . . . 109

61. Humboldi . . . 110

62. transversa . . . 113

63. gastrica . . . 115

64. sulcata . . . . 116

65. varicornis . . . 118
Species (Clurysis) pag.

66. rufiventris . . . 119

67. austriaca . . . 120

68. aurifrons . . . 122

69. bicolor . . . . 123

70. simplex . . . 127

71. aerata . . . 129

72. refulgens . . . 131

73. neglecta . . . 133

74. cuprata . . . 135

75. viridana . . . 137

76. Germari . . . 137

77. candens . . 140

78. caerulipes . . 141

79. Gyllenhali . . 143

80. socia . . . . 145

81. dichroa . . . 146

82. uniformis . . . . 149

83. Dalmanni . . . 150

84. sinuosa . . . 153

85. erythromẹlas . . 155

86. integra . . . . 157

87. elegans . . . 158

88. mediocris . . . 162

89. emarginatula . . . 164

90. mixta . . . . 167

91. carinata . . . 167

92. crassimargo . . . 169

93. foveata . . . 171

94. versicolor . . . 173

95. albipennis . . . 175

96. unicolor . . . 177

97. succinctula . . . 179

98. Leachei . . . 181

99. bihamata . . . 182

100. prasina . . . . 184

101. Bohemanni . . 187

102. cyanea . . . 188

103. parvula . . . . 191

104. lunigera . . . 194 


\begin{tabular}{|c|c|c|c|c|c|c|c|c|}
\hline Species (Chrysis) & & & pag. & Species (Chrysis) & & & & pag. \\
\hline 105. truncatella & . & & .195 & 144. rutilans & & i & 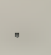 & 260 \\
\hline 106. fasciata & · & . & .197 & 145. terminata. & . & . & . & 261 \\
\hline 107. episcopalis & .. & . & .199 & 146. analis. & & - & . & 263 \\
\hline 108. assimilis & - & . & .201 & 147. scutellaris. & . & . & - & 265 \\
\hline 109. dissimilis . & . & 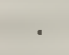 & .202 & 148. aeruginosa & . & . & . & 267 \\
\hline 110. Gayi . . & .. & . & .203 & 149. succincta . & . & . & . & 268 \\
\hline 111. chiliensis . & . & . & .204 & 150. Grohmanni & . & & . & 271 \\
\hline 112. Nisseri . & . $\cdot$ & . & . 206 & 151. Schousboei & . & . & & 272 \\
\hline 113. indigotea . & .. & . & .208 & 152. Drerseni. & . & 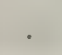 & . & 273 \\
\hline 114. chlorosoma & .. & . & .210 & 153. armena & . & . & . & 274 \\
\hline 115. distinctissima . & - & . & .211 & 154. manicata & 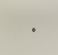 & . & . & 276 \\
\hline 116. caerulans . & 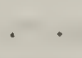 & . & . 212 & 155. angulata . & . & 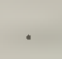 & . & 277 \\
\hline 117. nitidula & - & & .214 & 156. inaequalis. & . & & $\cdot$ & 278 \\
\hline 118, soluta . & . & . & .217 & 157. pyrophanis & . & . & . & 280 \\
\hline 119. Reichei & . & - & .218 & 158. distinguenda & . & - & . & 282 \\
\hline 120. Ehrenbergi & . & - & .220 & 159. comparata & . & 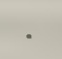 & . & 284 \\
\hline 121: megacephala & 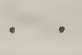 & . & . 222 & 160. verna & . & & . & 285 \\
\hline 122. laeta & 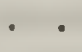 & 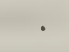 & .223 & 161. aestiva. & - & . & . & 286 \\
\hline 123. palliditarsis & 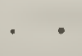 & 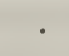 & . 225 & 162. impar. & . & 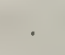 & . & 287 \\
\hline 124. orientalis . & - & $\cdot$ & . 225 & 163. curvidens. & . & . & . & 288 \\
\hline 125. diversa & . & - & . 226 & 164. auripes & . & . & . & 289 \\
\hline 126. delicatula . & - & - & .227 & 165. ignita . & . & $\cdot$ & . & 292 \\
\hline 127. amethystina . & 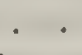 & - & - 229 & 166. lusca . & . & & . & 296 \\
\hline 128. Westermanni & $i$ & 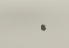 & . 232 & 167. Megerlei & 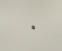 & - & . & 297 \\
\hline 129. spinigera . & . & & .233 & 168. pulchella & & & ${ }^{\circ}$ & 299 \\
\hline 130. alternans & . & . & . 236 & 169. dives & & 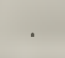 & . & 301 \\
\hline 131. Sehestedti & 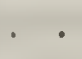 & 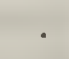 & .238 & 170. Vahli & ${ }^{\circ}$ & & . & 301 \\
\hline 132. aureo-maculat & ta. & . & . 239 & 171. micans. & 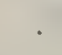 & . & . & 302 \\
\hline 133. soror . & - & - & . 240 & 172. Schönherri & 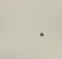 & . & . & 303 \\
\hline 134. semicincta & . & . & . 242 & 173. Zetterstedti & . & . & & 305 \\
\hline 135. zonata. & . & . & .244 & 174. equestris & . & . & . & 307 \\
\hline 136. fulgida . & • & . & .245 & 175. Schiödtei & . & . & - & 309 \\
\hline 137. exsulans . & . . & . & .247 & 176. oculata & . & . & & 310 \\
\hline 138. Ramburi . & 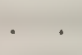 & 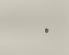 & .249 & 177. splendens. & ${ }^{\circ}$ & & & 312 \\
\hline 139. sinuata & . & & 251 & 178. Wahlbergi & & . & & 314 \\
\hline 140. cyanopyga & . . & & .253 & 179. violacea & . & . & & 316 \\
\hline 141. splendidula . & . . & . & 254 & 180. Smidti & . & . & & 317 \\
\hline 142. bidentata & & & 257 & 181. Genbergi & . & 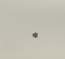 & . & 319 \\
\hline 143. pyrrhina & & & 259 & 182. Klugi & & & . & 21 \\
\hline
\end{tabular}


Index systematicus.

Species (Chrysis) pag.

183. sexdentata . . . . 324

184. modica . . . . 326

185. prominula . . . 328

186. aequinoctialis . . 330

187. insularis . . . . 332

188. inaequidens . . . 334

189. malachitica . . . 335

190. smaragdula : . . 337

191. lyncea . . . . . 339

192. Gueudei . . . . 343

193. mucronata . . . . 344

194. incerta . . . . 346

195. nobilis . . . . 347

Genus 8. SPINTHARIS

pag. $97,350-355$.

196. chrysonota ... . 351

197. destituta . . . . 352

198. singularis . . . . 353

\section{Genus 9. STILBUM} pag. $97,355-360$.

199. splendidum . . . 358

200. IVesmä̈li . . . . 359

201. calens . . . . 360
Famil. 5. EUCIIROEIDAE

pag. 7, $361-377$.

Genus 10. SPINOLIA

p. $362-364$.

Species

pag.

202. magnifica . . . . 363

\section{Genus 11. EUCHROEUS}

pag. $362,365-377$.

203. limbatus . . . . 368

204. purpuratus . . . 369

205. candens . . . . 371

206. caerulans . . . . 372

207. quadratus . . . . 373

208. 6 -dentatus . . . 375

209. festivus . . . . 376

\section{Famil. 6. PARNOPIDAE}

pag. 7, $377-388$.

Genus 12. PARNOPES

$$
\text { pag. } 379-388 \text {. }
$$

210. elegans . . . . 382

211. denticulata . . : : 382

212. Fischeri . . . . 383

213. carnea . . . . 385 


\section{Index alphabeticus.}

Nomina systematica literis rectis, Synonyma literis obliquis 1. s. d. cursivis excusa sunt. In quibus paginis nomina quacrenda sunt, mumeri post nomina positi significant.

CLEPTES Aut. Genus 1. p. 9-11. aurata Dlbm. 20.

auratus Megerl. 16.

fasciata Dlmn. 12.

ignita Fabr, 18.

nitidula Fabr. 13.

orientalis Dlbm. 20.

semiaurata Fabr. 16.

splendens Fabr. 16.

pallipes Lepel. 16.

CLEP'TIDAE Dlbm. Famil. 1.

pag. $7-22$.

CHRYSIDIDAE Dlbm. Famil. 4. pag. 7, 95 -360 .

CHRYSIS Lin. p. Genus \%. p. 97

-349 .

aenea Fabr. 36.

aequinoctialis Spin. 330 .

nerata Dlbm. 129.

aeruginosa Kl, 267.

aestiva Dlbm. 286.

ahenea Pallas. 72.

albipennis Dlbm. 175.
(Chrysis)

alternans Ḱl. 236.

amethystina Fabr. 229.

analis Megerl. 253.

analis Megerl. 297.

analis Zeller. 79.

analis $S_{\text {pin. }} 263$.

angulata Dlbm. 277.

armena Spin. 274.

assimilis Christoph. 180.

assimilis Spin. 201.

aurata Lin. 27.

aureo-maculata Dlbm. 239.

aurifrons K1. 122.

auripes Wesm. 289.

austriaca Dlbra. 134.

austriaca Fabr. 99, 120.

austriner Zett. 124.

basalis Dlbm. 106.

bicolor Dlbm. 123.

bicolor Megerl. 147.

bicolor Meg. 162.

bidentata Drews. 242. 


\section{Index alphabeticus.}

(Chrysis)

bidentata Lin. 257.

bidentata Spin. 249.

bihamata Spin. 99, 182.

Bohemanni Dlbm. 99, 186, 187.

caerulans Dlmn. 192.

caerulans Fabr. 99, 212.

caerulans Mleg. 215.

caerulea Dlbm. 36.

caerulea De Géer. 34.

cuerulea Pall. 46.

caeruleo-cephala Meg. 83.

caerulescens Fabr. 141.

caerulipes Fabr. 99, 141.

caerulipes Mus, Hav, 301.

caerulipes Spin. var. 147.

calens Fabr. 360.

candens Dlbm. 158.

candens Germar. 140.

capitalis Dlbm, 100.

carinata Spin. 167.

carnea Rossi. 385.

cellaris Christophor. 269.

chalybaea Meg. 189.

chalybaea Pall. 64.

chalybaea Ziegl. 34.

chalybaeata Kl. 189.

chiliensis Spin. 99, 204.

cliloroidea Ziegl. 67.

chlorosoma Dlbm. 210.

cliryso-cepluala Spin. 122.

comparata Lepel. 284.

confluens Dlbm. 159.

crassimargo Spin. 99, 169.

cuprata Hoffrmgg. 135.

curvidens Dlbm. 288.

cyanea Lin. 86, 99, 188.

cyanea Meg. 189.

cyanochrysa Foerster. 293.

cyanopyga Dlbra. 253.
(Chrysis)

cyanura Dlmn. 255.

cyanura Kl. 109.

Dalmanni Dlbm. 149.

delicatula Dlbm. 227.

dichroa Dlbm. 146.

dimidiata Oliv. 257.

discoidea Meg. 189.

dissimilis Dlbm. 200.

distinguenda Spin. 282.

distinctissima Dlbm. 211.

diversa Dlbm. 226.

dives K1. 299.

dives Kl. 301.

Drewseni Dlbm. 273.

Ehrenbergi Dlbm. 220.

elegans Pelet. 99, 158.

elegantula Spin. 236.

ernarginatula Spin. 164.

ephippium Ziegl. 140.

episcopalis Spin. 199.

equestris Dlman. 307.

erythromelas Dlmn. 155.

exsulans Dlbm. 247.

fasciata Fabr. 99, 212.

fasciata var. Mus. Havn, 211.

fasciata Spin. 211.

fasciata TVestermann. var. 319.

festiva Fabr. 376.

flammea Pall. 124.

foveata Dlbm. 171.

Friwaldskyi Spin. 260.

fulgida Lin. 99, 245.

fuscipennis Dlbm. 34.

gastrica Dlbm. 115.

Gayi Spin. 203.

Gentergi Dlbm. 319.

Germari Wesm. 137.

Germari Ziegl. 265.

gloriosa Dlbm. 272. 
(Chrysis)

gloriosa Fabr. 54.

gloriosa Mus. Havn. 272.

Grolımanni Spin. 271.

Gueudei Spin. 343.

haemorrlioea Meg. 246.

hilaris Dlbm. 103.

Humboldti Dlbm. 99, 110.

Tybrida Pelet. 181.

ignita Dlbm. var. 290.

ignita Fabr. var. 120.

ignita Lin. 99, 202.

ignita Spin. var. 279.

impar Dlbm. 287.

imperialis Dlmn. 242.

inaequalis Dlbm. 278.

inaequidens Dlbm. 334.

incerta Dlbm. 346.

incrassata Spin. 99, $10 \%$.

indigotea Duf. 208.

indigotea Duf. 316.

inermis Zett. 120.

insularis Gner. 332.

integra Dlbm. 83.

integra Fabr. 157.

integra Meg. 107.

integra Panz. 133.

integra Spin. var. 122.

integra Spin. var. 128.

intricans Spin. 211.

Iris Christophor. 215.

Jugum Dlbm. 101.

Klugi Dlbm. 321.

Kollari Ziegl. 293.

lacta Draege. 223.

Leachei Shuck. 99, 181.

lucidula Fabr. 78.

Iunigera Dlbm. 194.

lusca Fabr. 99, 296.

lyncea Fabr. 339.

\section{(Chrysis)}

maculata Fabr. 61.

malachitica Draeg. 335.

malachitica Mus. Berolin. 224.

manicata Dlbm. 275.

mediocris Dlbm. 162.

mediocris Dlbm. 326.

megacephala Bohern. 222.

Negerlei Dlbm. 297.

micans Meg. 175.

micans Ross. 302.

mixta Dlbm. 167.

modica Dlbm. 326.

mucronata Dlbm. 344 .

mutica Kl. 124.

neglecta Shuck. 99, 133.

nitidula Ahrens. 269.

nitidula Dlbm. ol. var. 217.

nitidula Fabr. 99, 214.

nitidula Germr. 138.

nobilis KI. 347.

obsoleta Dlbm. 293.

oculata Fabr. 310.

orientalis Dlbm. 225.

ovata Poll. 52.

palliditarsis Spin. 225.

Panzeri Fabr. 45.

parvula Fabr. 99, 186, 191.

prasina Kl. 99, 182, 184.

producta Spin. 347.

prominula Dlbm. 328.

pulchella Spin. 299.

pumila Kl. 201.

punctatissima Spin. 197.

purpurata Fabr. 215.

purpurata Fabr. 369.

pusilla Fabr. 31.

pyritosa Kl. 293.

pyrophana Kl. 280.

pyrrhina Dlnan. 259. 
(Chrysis)

4-dentata Meg. 215.

15-dentata Pall. 374.

Ramburi Spin. 249.

refulgens Fabr. 120.

refulgens Spin. 131.

regia Fall. 52.

regia Fabr. 72.

Reichei Spin. 218.

Rosue Dlbm. 93.

rosea Ross. 93.

rufa Panz. 93.

rufirentris Dlbm. 119.

rufiventris DImn. 158.

rutilans Oliv. 260.

Schiödtei Dlhm. 309.

Schoushoei Dlbm. 272.

Schönherri Dlbm. 303.

scutellata Panz. 131.

scutellaris Fabr. 265.

scutellaris Panz. 45.

Sehestedti Dlbm. 238.

segmentata Dlbm. 265.

semiaurata Lin. 16.

semicincta Lepel. 242.

separata Dlbm. 159.

sexdentata Fabr. 99, 324.

sexdentata Panz. 324.

sexdentata Panz. 316.

sicula Spin. 158.

similis Lepel. 302.

sinuata Dlbm. 153.

sinuata Dlmn. 251.

sinuosa Dlmn. 153.

smaragdula Fabr. 337.

smaragdula Mus. Ham. 309.

smaragdula Westerm. 309.

socia Dlbm. 142.

soluta Dlbm. 217.

soror Dlbm. 240.

II.

\section{(Chrysis)}

spinigera Spin. 233.

splendens Kl. 99, 312.

splendida Fabr. 358.

splendidula Ross. 254.

$\left.\begin{array}{ll}\text { stondera Jur. } \\ \text { stondera Panz. }\end{array}\right\} 246$.

succincta Lin. 99, 268.

succinctula Spin. 99, 179.

succinctula Spin. 269.

sulcata Dlbm. 116.

tarsata Kl. 105.

terminata Meg. 261.

torquata Dlmn. 151.

transversa Dlbm. 113.

3-dentata Dlbm. 192.

truncata Dlbm. 42.

truncatella Spin. 192.

Vahli Dlbna. 301.

varicornis Spin. 118.

verna Dlbm. 285.

versicolor Spin.99, 173.

violacea Panz. 316.

violacen Rioss. 34.

violacea Spin. 208.

viridana Dlbm, $13 \%$.

viridula Lin. 257.

Wahlbergi Dlbm. 99, 314.

Westermanni Spin. 229.

Westermanni Dlhm, 232.

Zetterstedti Dlbm. 305.

Zetterstedti Dlhm. 307.

zonata Dlbm. 244.

Chrysura Dlbm.

sulcata Dlbm. 116.

unicolor Dlbm. 177.

Cymura Dlbm.

splendida Dlbm. 60.

Diplolep is Fabr.

chrysis Fabr. 18. 
ELAMPID $\Lambda E$ Dlbm, Familia 2. pag. 7, 24-57.

ELAMPUS Spin. Gen. 4. pag. 7, $25,38-47$.

affinis Wesm. 36.

ahaeneus K1. 72.

? albipennis Kl.? 175.

ambiguus Dlbm. 41.

analis Kl. 70.

auratus Wesm. 27.

azureus Kl. 42.

bidentulus Kl. 39.

caerulescens Kl. 67.

caerulescens Pall. 34.

caeruleus Kl. 46 .

candens Kl. 91.

chrysonotus Dlbm, 44.

cupralus Kl. 84.

cyanocephalus Kl. 54.

excisus Kl. 36.

fervidus KI. 83.

fulgidicollis Spin. 55 .

Gayi Spin. 47.

gibbus KI. 34.

ignicollis $\mathbf{K} 1.54$.

micans Kl. 55.

minutus Dlbm. 42.

Panzeri Latr. 45.

parvulus Kl. 31.

productus Kl. 44.

punctatissimus Kl. 52.

pusillus Wesma. 40.

Ramburi Spiu. 45.

scintillum KI. 27.

truncatus Dlbm. 42.

Spina Spin. 41.

violaceus Kl. 36 .

violaceus Wesm. 34 .
EUCHROEIDAE Dlbm. Fanil. 5. pag. 7, 361-377.

EUCHROEUS Latr. Genus 11. pag. $362,365-377$.

caerulans Kl. $366,372$.

candens K1. $366,371$.

festivus Dlbm. 367, 376.

feslivus Spin. 374.

limbatus Kl. 366,368 .

polyodon Kollar. 374.

purpuratus Dlbm. 366, 369.

purpureus Latr. 369.

quadratus Kl. $367,373$.

6 -dentatus Latr. 367, 375.

Familia CLEPTIDAE Dlbm. 7, $8-23$.

" CHRYSIDID AE DHm, $95-360$.

) ELAMIPIDAE DIbr. ?, $24-57$.

》 EUCHROEID AE DIbn. 7, $361-37 \%$.

" HEDYCHRIDAE Dlhm. $7,57-94$.

" PARNOPIDAE Dlbm. 7, $377-388$.

Genus CLEPTES Aut. 9, 10-21.

»CHR YSIS Lin. 97, 98-349.

¿ELAMIPUS Spin.25,38-47.

" EUCHROEUS Spin. 362, $365-367$.

" HEDYCHRUMI Latr. 58 94.

" HE'TEROCOELIA Dlbm.9, 21-23.

" HOLOPYGA Dibm. 25, $47-56$. 
Genus OMILUS Panz. 25, 26 38.

" PARNOPES Fabr. 379388.

1) SPINOLIA Dlbm.362-364.

" SPINTHARIS KL. 97, 350 -355 .

1) STILBUM Fabr, 97, 355

-377 .

HEDYCHRIDAE Dlbm. Famil. 3. pag. 7, 57-94.

IIEDYCHRUM. Latr. Genus 6 .

pag. $58-94$.

ahaeneum Dlbru. 72.

alterum Duf. 79.

?amethystinum Kl.? 60.

anale Dlbm. 70.

ardens Latr. 79.

aulicum Cliristophor. 79.

auratum Drews. 52.

auratum Meg. 27.

aeneum Meg. 36.

bidentulum Pelet. 39 .

brasilianum Spin. 59.

caelestinum KI. 60 .

caerulans Kl. 64 .

caerulescens Pelet. 66.

caerulescens Sluck. 64.

candens Kl. 91.

chalybaeum KI. 64.

chalybaeum Meg. 175.

chloroideum Spin. 66.

congestum Kl. 79.

coriaceum Dlbm. 88.

cupratum Dlbm. 84.

cupreum Dlbm. 87.

cyanocephalum Meg. 54.

diffrcile Spin. 63.

Duponti Spin. 83.
(Hedychrum)

femoratum Meg. 90.

fervidum Drews. 78. fervidum Meg. 90, 91. fervidum Shuck. 76. imperiale Shuck. 34. incrassatunu Spin. 73. integrum Dlbm. 83. integrum Dlbm. 86. intermedium Spin. 77. janthinum Draege. 62. jantlinum Spin. 50. laetum Zeller 91. lucidulum Dlbm. 78. lucidulum Latr. 78. lucidulum Nyl. 52. lucidulum Pelet. 54. lucidulum Zell. 79. luzulinum Kl. 49. maculatum Dlbm. 61. raetallicum Dlbm. 68. minimum Duf, 33. minutum Pelet. 82, 83. nitidum Panz. 34. nitidum Pelet. 91. obtusicolle Meg. 54. pedatum KI. 79. purpurascens Dlbno. 85. pusillum Meg. 31. regium Mleg. 52. regium Pelet. $7 \%$. regium Wesm. 79. roseum Pelet. 93. rutilans Meg. 76. siculum Meg. 78. speciosum Drews. 76.

Spina Pelet. 41. limidum Dlbm. 65. virens KI. 74. viride Mus, Berol. 64 . 
(Hedyclinum)

viride Zell. 66.

viridicolle KI. 79.

viridissimum Meg. 66.

Zelleri Dlbm. 69.

Zimmermanni Dlbm. 61.

HETEROCOELIA Dlbm. Gen. 2. pag. 9, 21-23.

nigriventris Dlbm. 22.

HOLOPYGA Dlbm. Genus 5.

pag. $25,47-56$.

amoenula Dlbm. 53.

Dohrni Dlbm. 48.

janthina Dlbm. 50.

luzulina Dlbm. 49.

micans Dlbm. 55.

ovata Dlbm. 51.

punctatissima Dlbm. 50.

IIymenoptera Chrysidiformia pag. 1.

Ichneumon

auratus Panz. 16.

Chrysis Fabr. 18.

ignitus Fabr. 18.

nitidulus Fabr. 13.

semiauratus Panz. 16.

Nemophora

carinata Draeg. 168.

OMALUS Panz. Genus 3. pag. $26-36$.

anratus Dlbm. 26.

auratus Meg. 27.

deneus Meg. 36.

acneus Panz. 35.

caeruleus Dlbm. 34.

coriaceus Dlbm. 37.

Mavipennis MLg. 36 .
(Omalus)

nitidus Panz. 34.

parvulus Dlbm. 31.

punctulatus Dlbm. 33.

pusillus Dlbm. 31.

PARNOPES Fabr. Gen. 12. pag. $379-388$.

carnea Latr. 381, 385.

denticulata Spin. 381, 382.

elegans Kl. 381, 382.

Fischeri Spin. 381, 383.

Platycoelia

Ehrenbergi Dlbm. 220.

Poeciloechro:

alternans KI. 236.

bifasciata Kl. 236.

Pyria

Reiclıei Spin. 219.

Stilboides Spin. 347.

Splex

semiaurata Lin. 16.

violacea Scop. 34.

SPINOLIA Dlbn. Genus 10. pag. $362-364$.

magnifica Dlbm. 363.

SPINTHARIS Klug, Genus 8. pag.

$97,350-354$.

clirysonota Kl. 351.

clirysonota Spin. 352.

cuprata Kl. 136.

destituta Dlbm. 352.

dichroa Kl. 147.

elegans KI. 158.

emarginatula Kl. 167.

gastrica Kl. 115.

nitidula Kl. 181.

singularis Spiu. 353.

succinctula Ki. 180. 


\section{Index alphabeticus.}

STILBUM Fabr. p. Genus 9. pag.

97, $355-360$.

calens Dlbm. 360.

calens Spin. 358.

connectens Spin. 358.

Hedenlorgi Dlbm. 347.

lynceum Dlbm. 340.

sexdentatum Guer. 347.

splendidum Dlbm. 358.

Westermanni Dlbm. 359.

Vesnaäli Dlbm. 359.

\section{TABULA SYNOPTICA}

Familiarun pag. 7.
Gencrum Cleptidarum 9.

» Chrysidarum $9 \%$

» Elampidarum 25.

„Euchroeidarum 362.

Specierum Cleptis 10-11.

" Chrysidis 100-101, $150-151,186,197$, 298.

" Elampi $38-39$.

" Euchroei $366-367$.

" Hedychri 58-59.

Holopygae 47-48.

Omali $26-27$.

Parnopis 381. 


\section{Corrigenda.}

pag. 5. lin. ultima at caput et lege: ut caput et

"10. linea $10 \mathrm{ab}$ infr. Theracis lege: Thoracis

"10-11 (in Tabula Synoptica) lin. 17 viridicyanea 1.: viridicyanci

lin. 21 punctorem lege: punctorusu

"12. lin. 6 ab infr. abtusiusculo lege: obtusinsculo

"14. lin. $10 \mathrm{ab}$ infr, esiam lege: etiam

"15. lin. 11 ab infr, subvena lege: sub rena

"16. lin. $12 \mathrm{ab}$ infr. horte lege: horto

"21. lin. 10 opaca area lege: opaca, area

"23. lin. $16 \mathrm{ab}$ infr. armata lege: arcuata

lin. 4 ab infr. submediocra lege: submediocria,

" 33. lin. 10 delegit lege: detegit

"34. lin. 17 Men lege: Mou.

"37. lin. 3 adeque lege: adeoque

lin. 5 politissime lege: politissima

lin. 15. varius lege: rarius

38-39. (Tab. Synopt.) lin. 5 ab infr. segmentis lege: segmenti

42. lin. 13 ab infir. Chrysuris lege: Chrysidibus

Chrysura lege: Chryside

43. lin. 9 ab infr. tura aut late lege: emarginatura subarcuata 1. late

51. lin. 1 alarum Holop lege: alarum a Holop.

56. lin. 2 ab infr. micantia lege: micantibus

58-59. (Tab. Synopt.) lin. 17 angulis 3:tio lege: angulis tribus

lin. 26. chalibaeum lege: chalybaeum

in ejusden Tabulae pagina altera lin. 16 var. $a$ lege: var $d$

61. lin. 4 supra lege: superior

66. lin. 10 ab infr. Hedychr. chloroideum Ziegl. lege: Hedychr. cht.roideum Spin.

84. lin. 1 reticulatis lege: reticulatio

lin. 13 ab infr. aureo-nitidus lege: aureo-nitidis

85. lin. 14 ab infr. aurato- lege: arcuato-

100. lin. 7 ab infr. pedicellum lege: pedicellus

lin. $6 \mathrm{ab}$ infr, viridia lege: virides

lin. 5 ab infr. apice unde lege: apice, unde

100-101. (in Tab. Synopt. 59. Chr, incrassata Kl. 1.: 59. Chr. iucrassata Spin. in cjusd. Tab. 60. Chr. cyanura Pall. lege: 60. Clır. cyanura Kil.

76. Chr. Germari nob. 1.: 76. Chr. Germari Vesmaël

101. lin. 4 cyanea lege: cyaneae

lin. 11 sumtarum lege: sumtorusn

103. lin. 2 ab infr. quam lege:, quae

105. lin. 7 ab infr. constituto lege: constituta

112. lin. 13 laete lege: late

lin. 18 sumtarum lege: sumtorum

118. lin. 11 magni lege: magno

lin. 14 prominula lege: prominulo 


\section{Corrigenda.}

pag. 121. lin. 15 ab infr. viridi-aenco picta lege: viridi-aeneo pictae

"122. lin. 2 ab infr. auratus lege: auratum.

"128. lin. 4 ab infr. margine et lege: margine antico et

" 133. 73. Chrysis integrella Dlbm. lege: 73. Chrysis neglecta Shuck.

"134. lin. 3 (in Synonymia) Clerysis neglecta Shuck. secundum Don. Klug in Museo Berolin. anno 1847.

"135. lin. 8 integrellae lege: neglectae

74. Chr. cuprata Kl. lege: 74. Chr. cuprata Hoffrnannsegg.

142. ultim. anticae lege: antice

147. lin. 9 celluli lege: cellula

150. lin. 11 apices $1: \mathrm{mi}$ lege: apices segmentorum $1: \mathrm{mi}$

163. lin. 8 reticulatis lege: reticulatio

183. lin. 6 depressum nitidum lege: depressus nitidus

lin. 7 punctaturn lege: punctatus

184 lin. 12 ab infr. inter subrectangulari l. : inter spinas subrectangulari

192. lin. 10 parallelis lege: ramulis parallelis

193. lin. 4 modice ut lege: modice convexum ut

lin. 14 efformantes lege: efformantibus

201. lin. 6 ab infr. hiuc lege: hic

205. lin. 9 ab infr. proprius lege: propius

212. lin. 4 ab infr. caeruleans lege: caerulans

214. lin. 6 quum lege: quam

216. (in medio) quibusdam aut individuis $\mathrm{l}^{\cdot}$ quibusdam autem individuis

217. liu. 7 punctato et punctulato 1. : punctata et punctulata

lin. 17 var. 6 . lege: var. $b$.

lin. 2 ab infr. primis lege: primarius

230. lin. 11 vertex et sat lege: vertex sat

lin. $7 \mathrm{ab}$ infr. 2 :do et 3 : tio lege: 2 : dus et 3 : tius

231. lin. 2 ab infr. segm. cyaneo lege: segmento 3:tio cyaneo

232. lin. 12 quaque lege: quaeque

236. (. var. $a$. in Synonymia.) Mus. Spinolae 1.: Mus. Spinolae.

237. lin. 10 ab infr. subopacum in lege: subopacum, in

lin. 10 ab infr. fascia segmenti 1. : fascia segmenti aut $1:$ mi aut 2: di aut utriusque

238. lin. 14 ab infr. Schousbo lege: Schousboe

243. lin. $8 \mathrm{ab}$ infr. angulo centrali brevissinao acuto lege: angulum centralem brevissimum acutuma

" 247 lin. 12 stissima lege: tissima

"248. lin. 15 radialis nec lege: radialis nec non

250. lin. $7 \mathrm{ab}$ infr. proxima-utraque lege: proxima utraque

252. lin. 6 scutellum rubra lege: scutellum rubro-

255. lin. 6 Glosbo lege: Glösbo

lin. 14 dorsuli lege: dorsali

258. lin. 12 ab infr. firmibus lege: firmis

264. lin. 7 inaequales lege: inaequalis

265. lin. 11 paullum lege: paullulum

271. lin. 7 ab infr. complissimeque lege: amplissimeque

272. lin. 4 Schiodte lege: Schioedte

lin. $8 \mathrm{ab}$ infr. thoraceque abdomine lege: thorace abdomineque 
pag. 275. lin. 2 ab infr. quum lege: quam

"276. lin. 4 ab infr. firmibus lege: firmis

"280. lin: 15,16 nitidus punctulatus lege: nitida punctulata

"285. lin. 11 firmibus lege: firmis

"286. lin. 5 segmenti margo lege: segmenti 3 : tii margo

"288. lin. 17 mediocres lege: mediocris

"289. lin. 6 ab infr. Wasmaël lege: VVesmaël

"291. lin. 9 ab infr. inter medii lege: intermedii

"297. lin. 2 ab infr. furmibus lege: firmis

ad pag. 298. (in Tab. Synopt. V) lin. 13 ab infr. lateralis lege: laterali

pag. 300 lin. 11 apicalis $8-10$ lege: apicalis foreolis 8-10

" 301. lin. 1 Chrysis dives Lin. lege: Chrysis dives Klug.

lin. 7 at nullo lege: at nullo modo

"304. lin. 7 ab infr. scenti-aenea lege:' scenti-aenei; in ejusd. p. lin. 8 ab infr. crenulatus 1 . : crenulatum; lin. 2 ab infr firmibus l.: firmis

" 306. lin. $12 \mathrm{ab}$ infr. fasciolum $\mathrm{l}$. : fasciolam

"316. lin. 3 externe lege: externo.

"320. lin. 5 dorsale lege: dorsale $1:$ mum

" 322. lin. 3 emarginato-bidentalis lege: emarginato-bidentatis lin. $9 \mathrm{ab}$ infr. dorsulo intra marginem 1 . : et dorsulo intra marginem lin. $2 \mathrm{ab}$ infr. pellucidis lege: pellucidus

329. lin. 2 ab infr. femori lege: femoribus

" 336. lin. 12 ab infr. firmibus lege: firmis

" 337. lin. 1 paullum lege: paullulum

"339. lin. 6 uterque antelaterali-apicalis lege: uterque angulus lateraliapicalis

" 343. lin. 14 Gucidei lege: Gueudei

lin. 5 ab infr. dorsali multo lege: dorsali 3 : tio multo

"345. lin. 6 aenea purpurascentia opaca 1 . : aeneae purpurascentes opacae lin. 12 Gueidei lege: Gueudei

348. lin. 10 punctis aut scutelli postscutelli lege: punctis autem scutelli et postscutelli

"349. lin. 7 discoidalis lege: discoidalibus

"350. lin. 15 tibiae aut saltem lege: tibiac aut totae aut saltem

"351. lin. 8 chrysonata lege: chrysonota lin. 15 tenuissime aureo 1. : plus ninus aureo-micans, tenuissime albido-villosa

"352. lin. 1 distictus lege: distinctus

" 354. lin. 12 ab infr. bispinosae lege: bi-aut tri-spinosae

lin. $9 \mathrm{ab}$ infr. testaceae lege: testacea

"360. lin. 2. ab infr. tate simillimum lege: tate $e$ simillimum

"361. lin. 3 lata lege: latae

" 372. lin. 4 firmibus lege: firmis; in ejusd. p. lin. 6 ab infr. lateopictis lege: luteo-pictis; lin. 3 : ab infr. ctis lege: ctio

In Tab. acnea VII. Fig. 94. Chrysis imarginatula 1.: Chrysis emarginatula VIII. Fig. 97. Chrysis faveata lege: Chrysis foveata. 
Fan. I. litepticue. Jöb.

Yob. I.

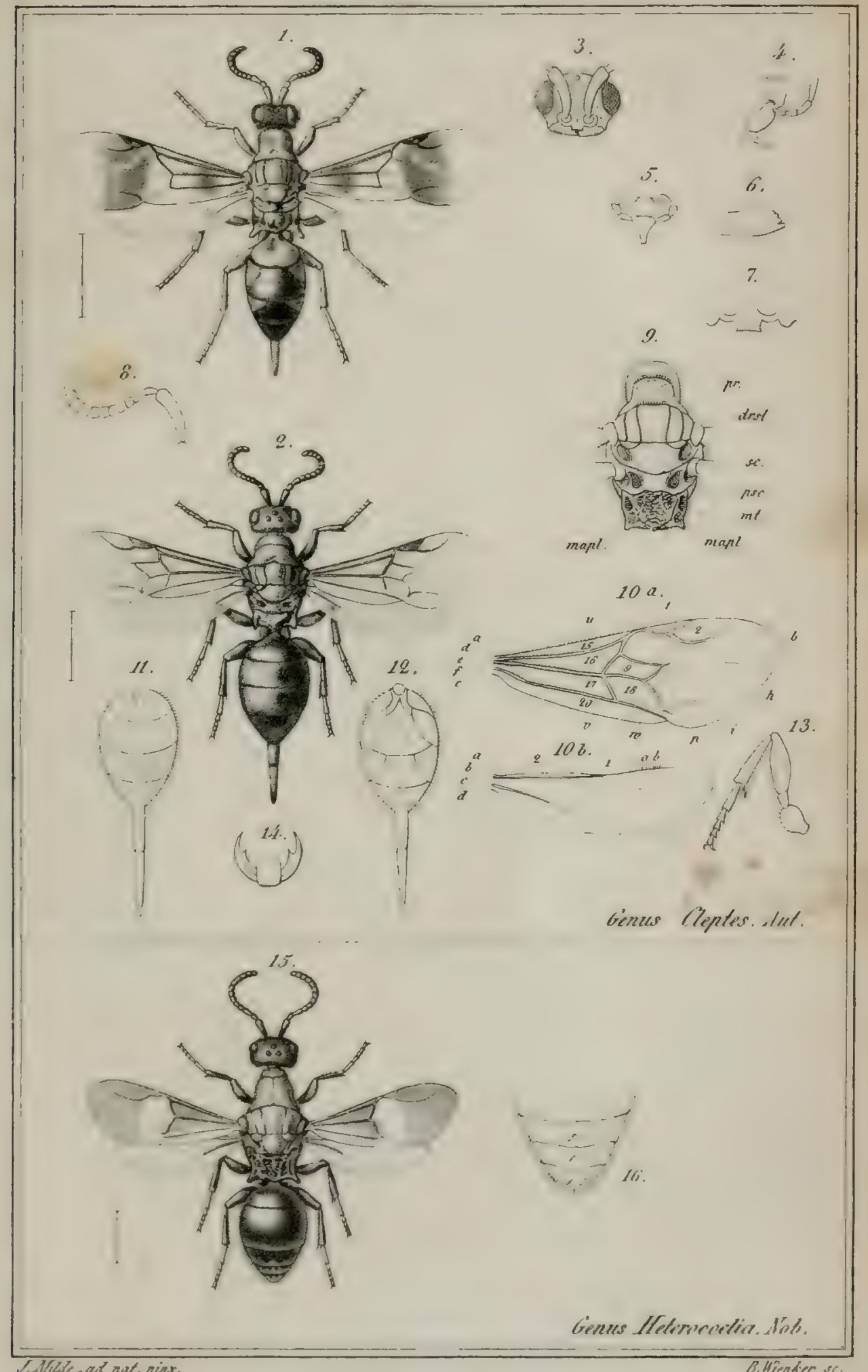

B. Mientier st. 


\section{Li3n1-ve \\ dF PUE}

UNIVERSITY OF I! LINOIE 


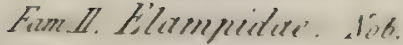

21
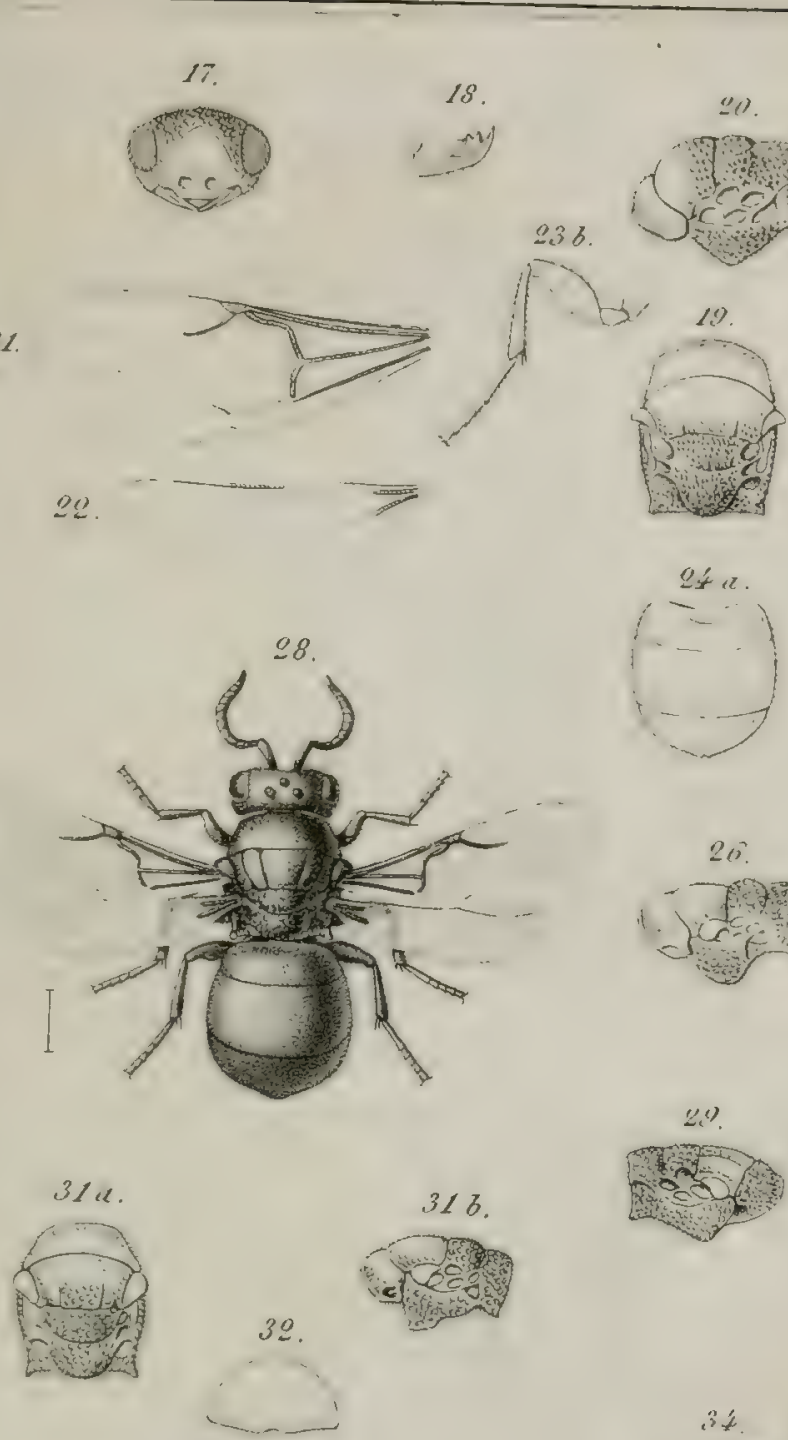

$33 \mathrm{~b}$.

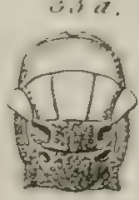

Q (1).

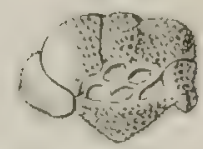

236
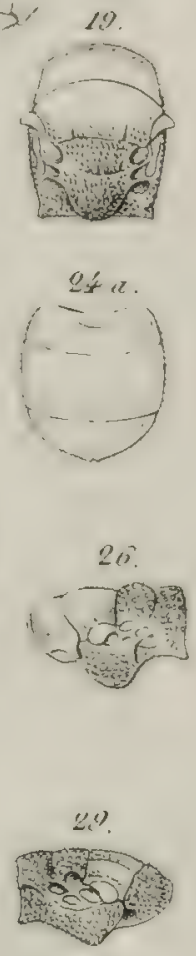

34

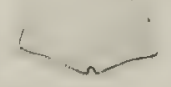

$3.3 \%$

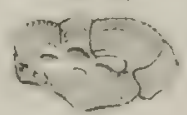

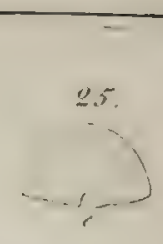

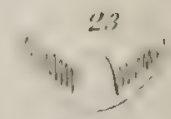

196.

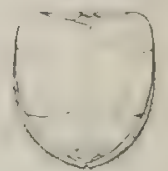

$a r$

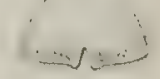

3.54.

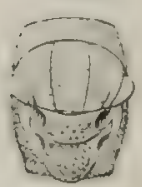

$38 i$

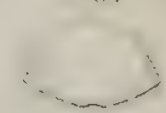

Gienus Tlmalus. Plunz

B. 


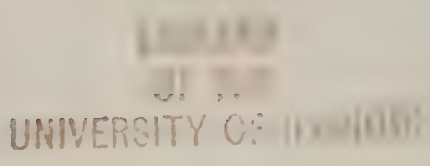

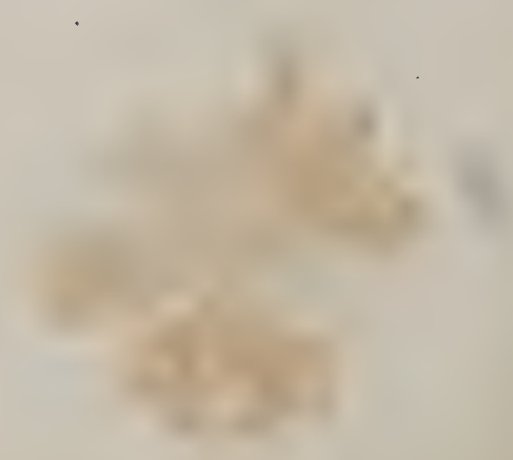


Fum II. Filampidne. Nob.

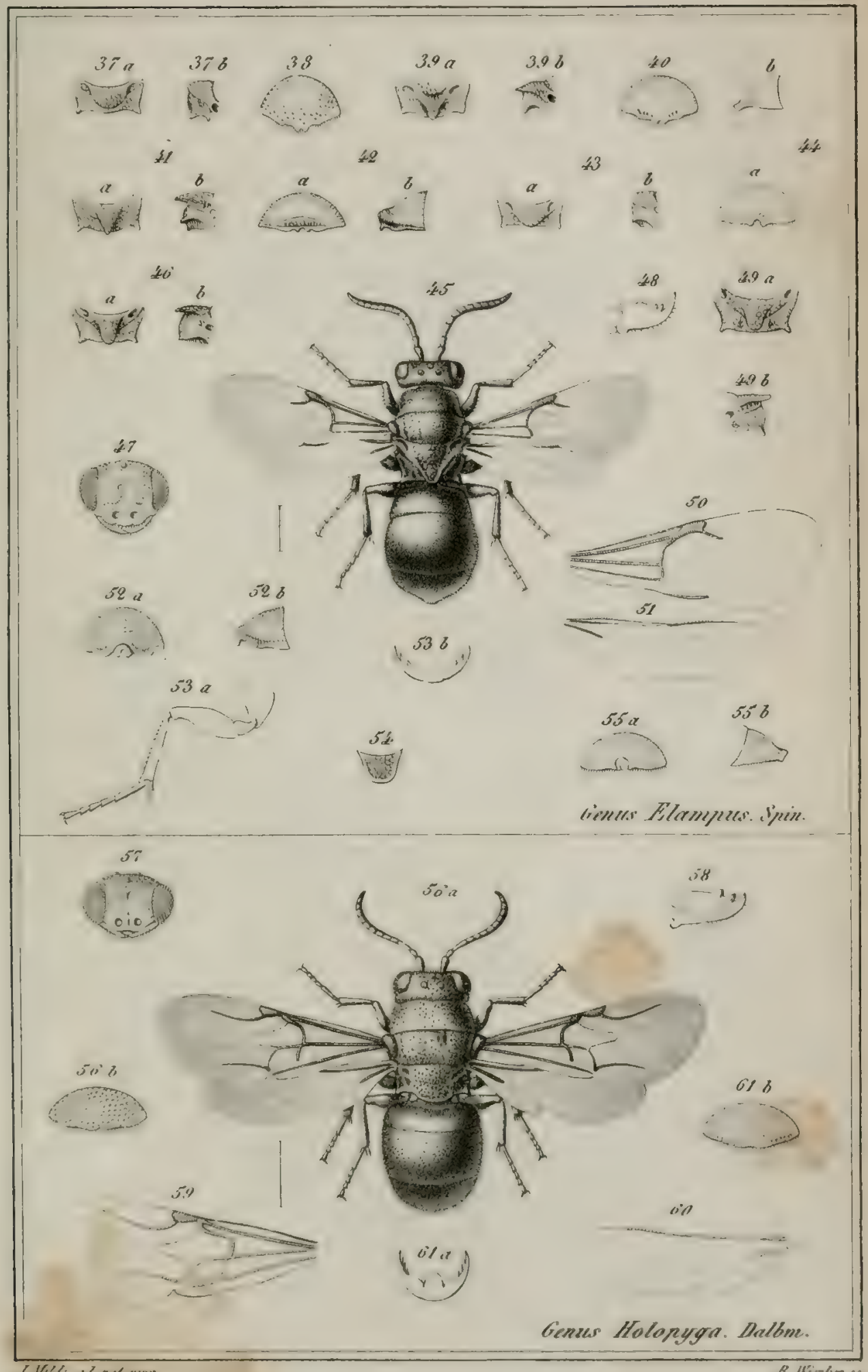

B. Ifintionse 


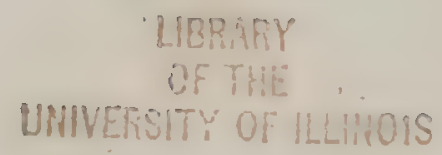

\section{.}




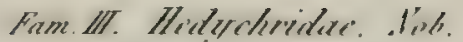

Iäl. $A$.

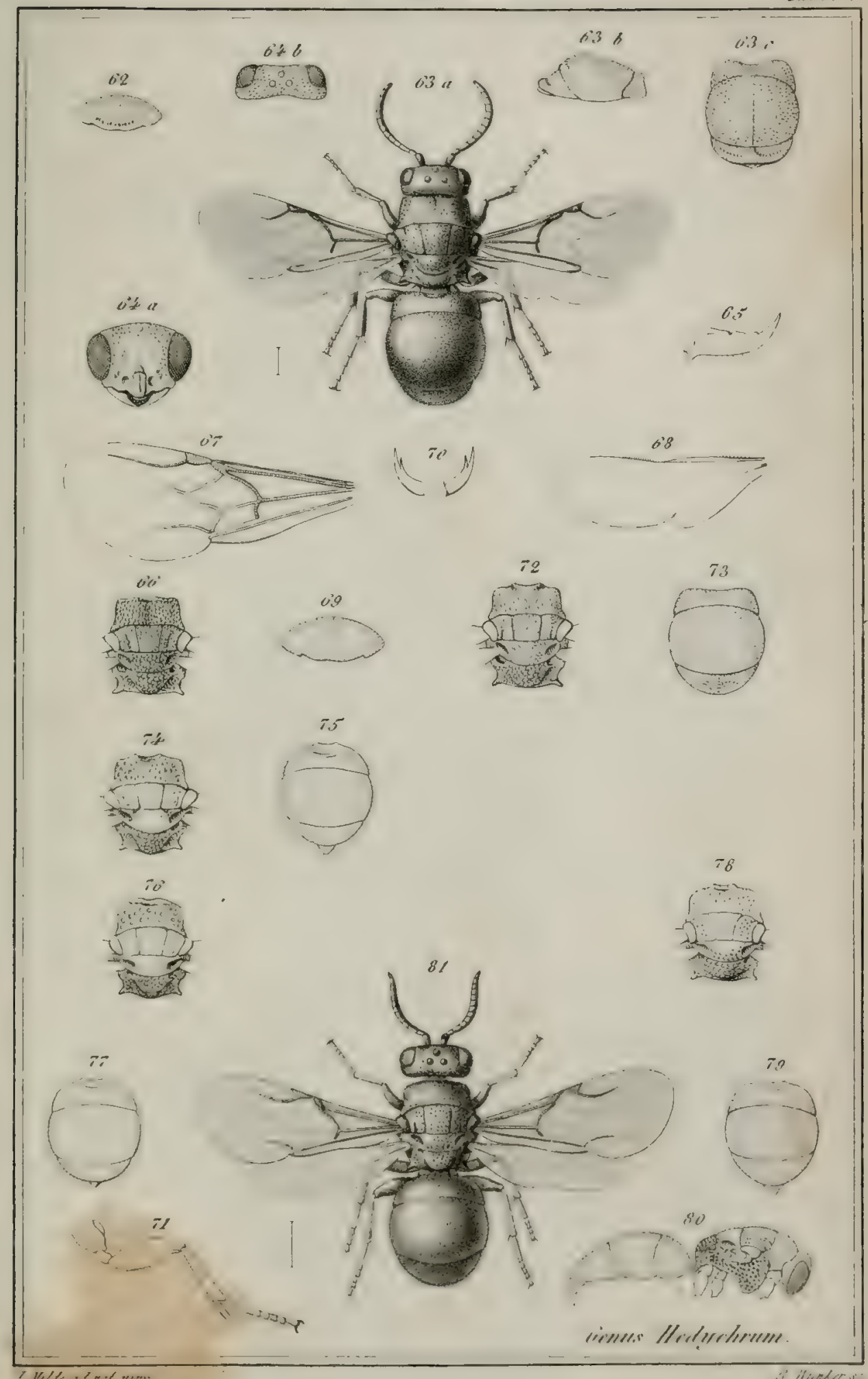


LIERAMY

of The

UNIVERSTI: OF HLLIIOIS 


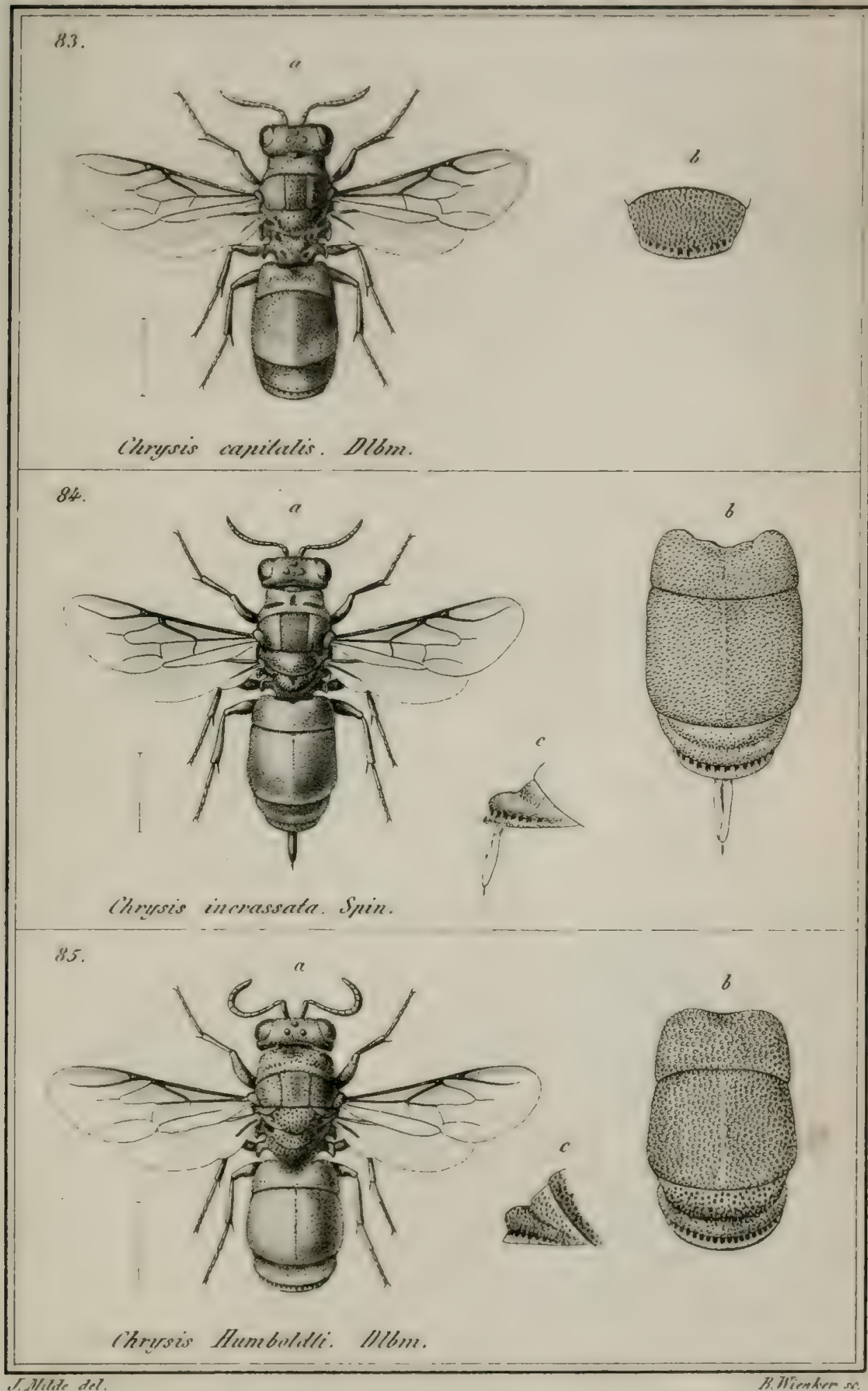




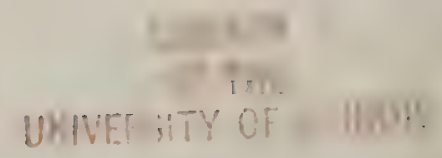


Tab. 17.

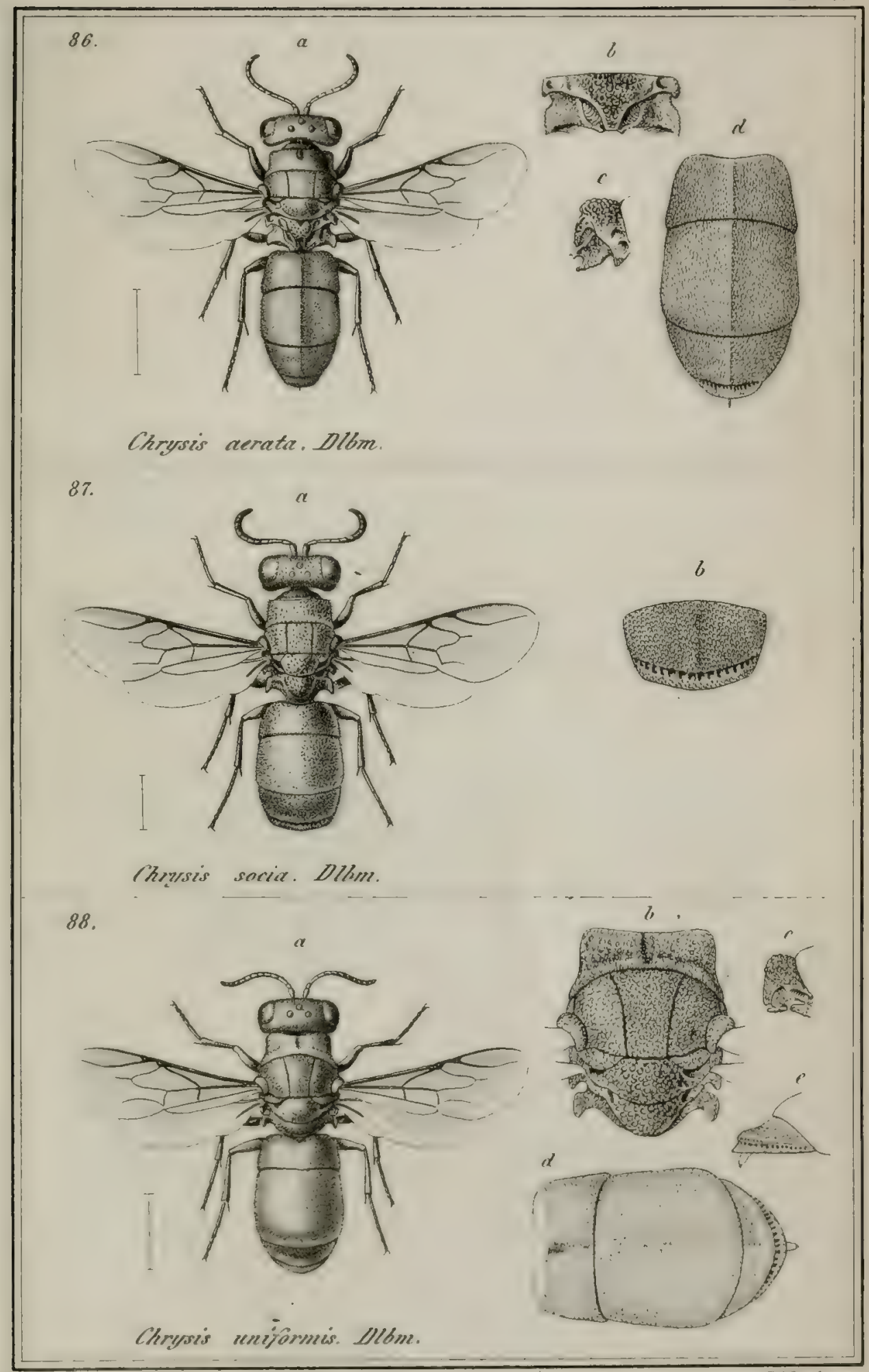

Thitile wel.

B. liestersen 
LIERARY

OF THE

UNIVERSITY OF ILLIHOIS 
Tul. IIT.

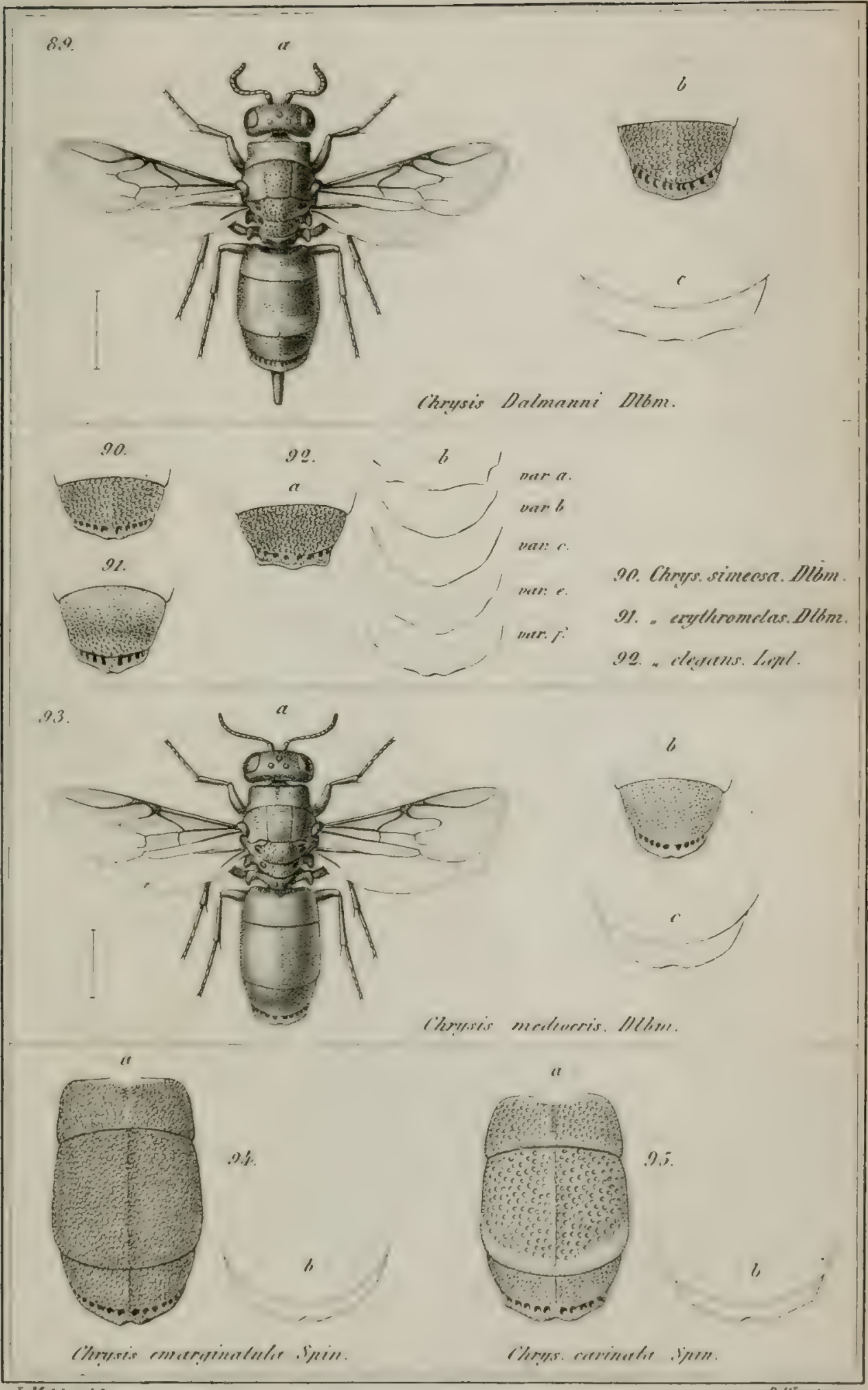

Jielde ath

Bllirnterm or. 

7in6. 7275.

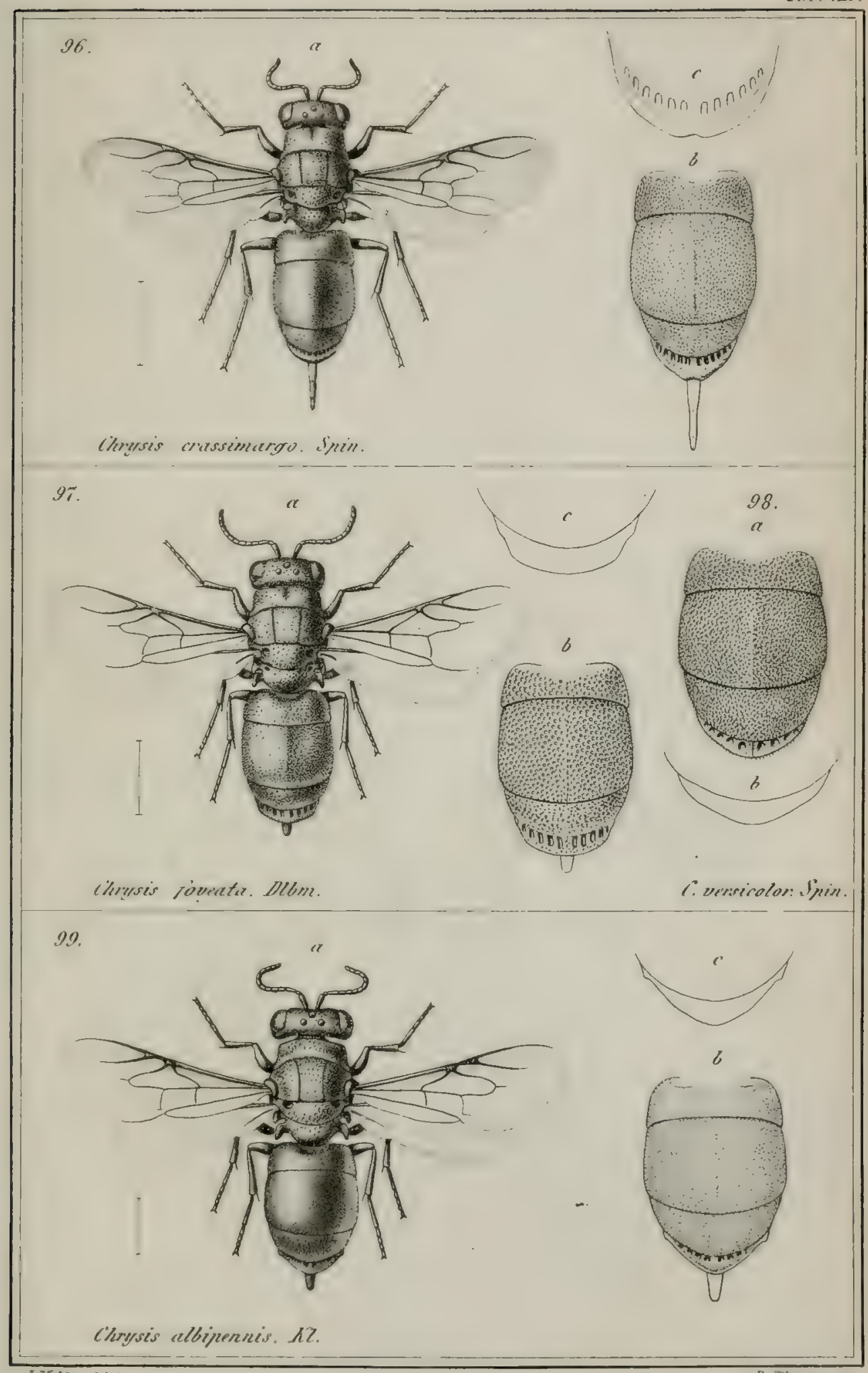

Thilde add.

B. Theenter se 


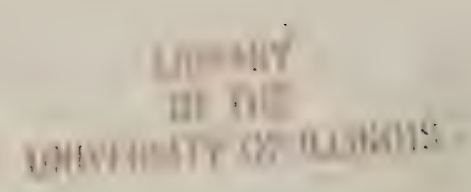


Tirs. A

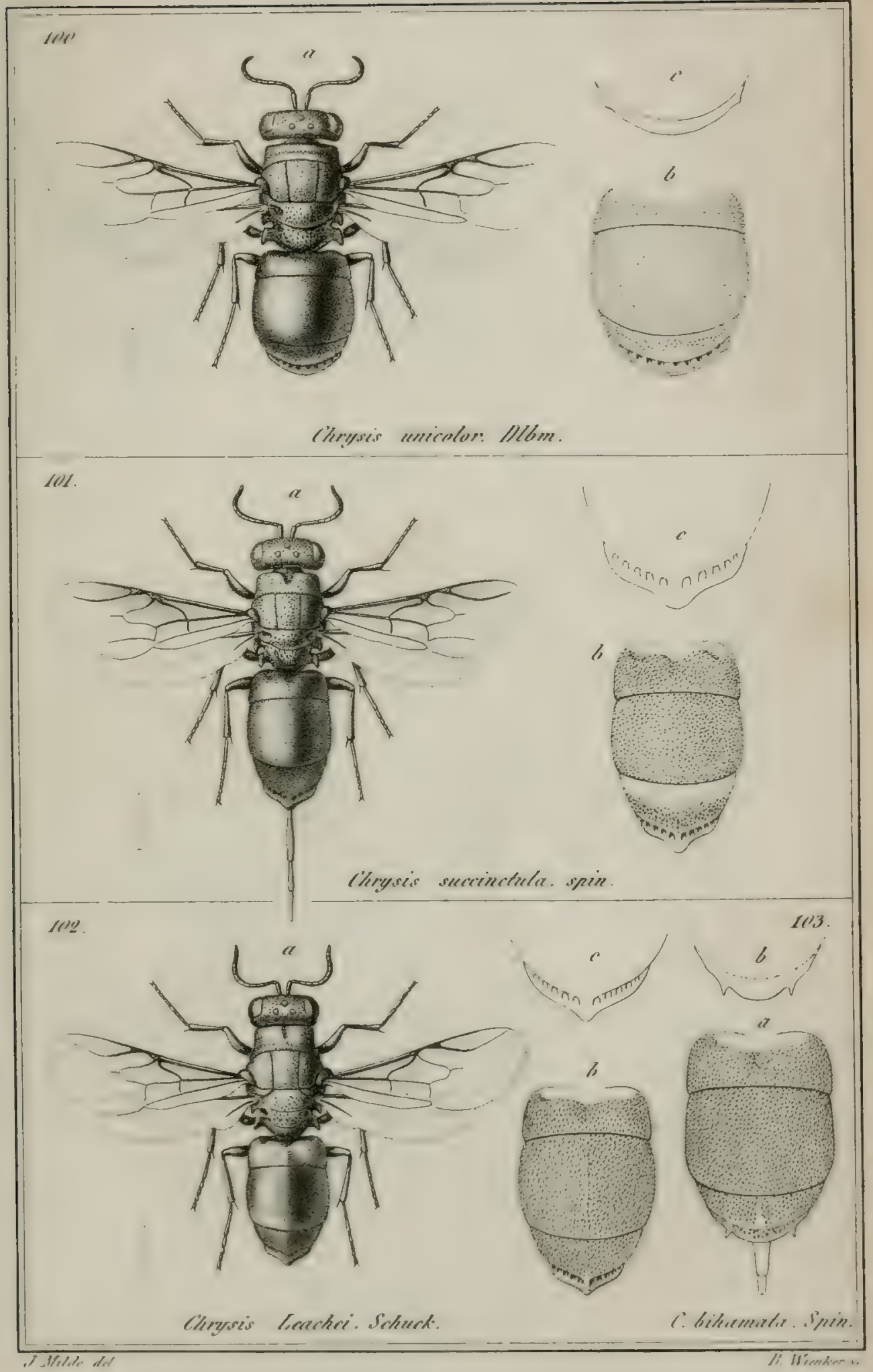




\section{zimesey}

uI $\mathrm{NW}$ Iikaver of of romols 
Tint. It

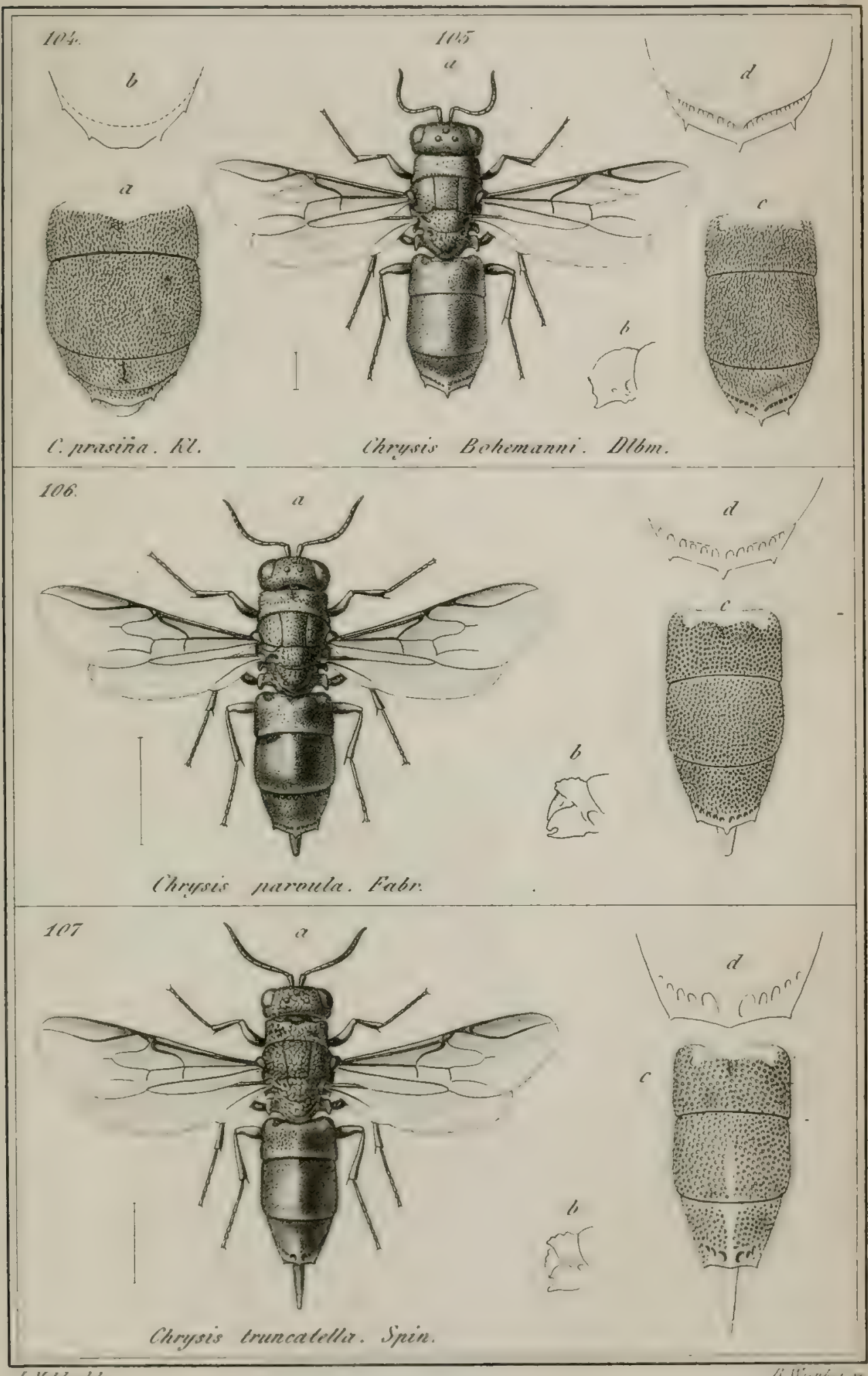




\section{nus iry \\ B. $7 \mathrm{~h}$

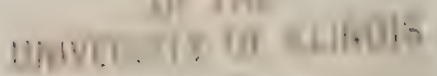




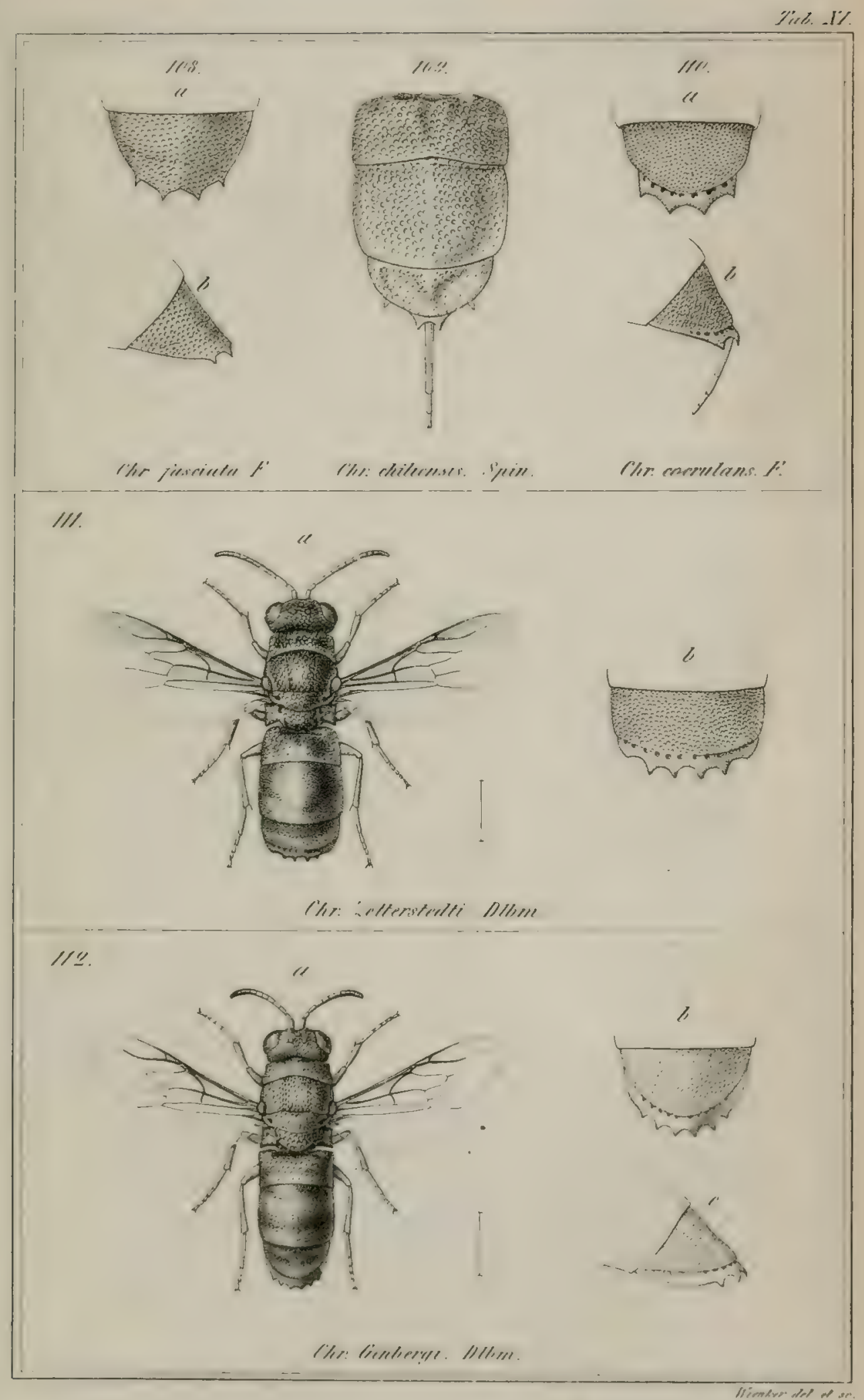




\section{1): 4 \\ OF THE \\ UNIVERSITY OF ILLINOIS}


Sins all.

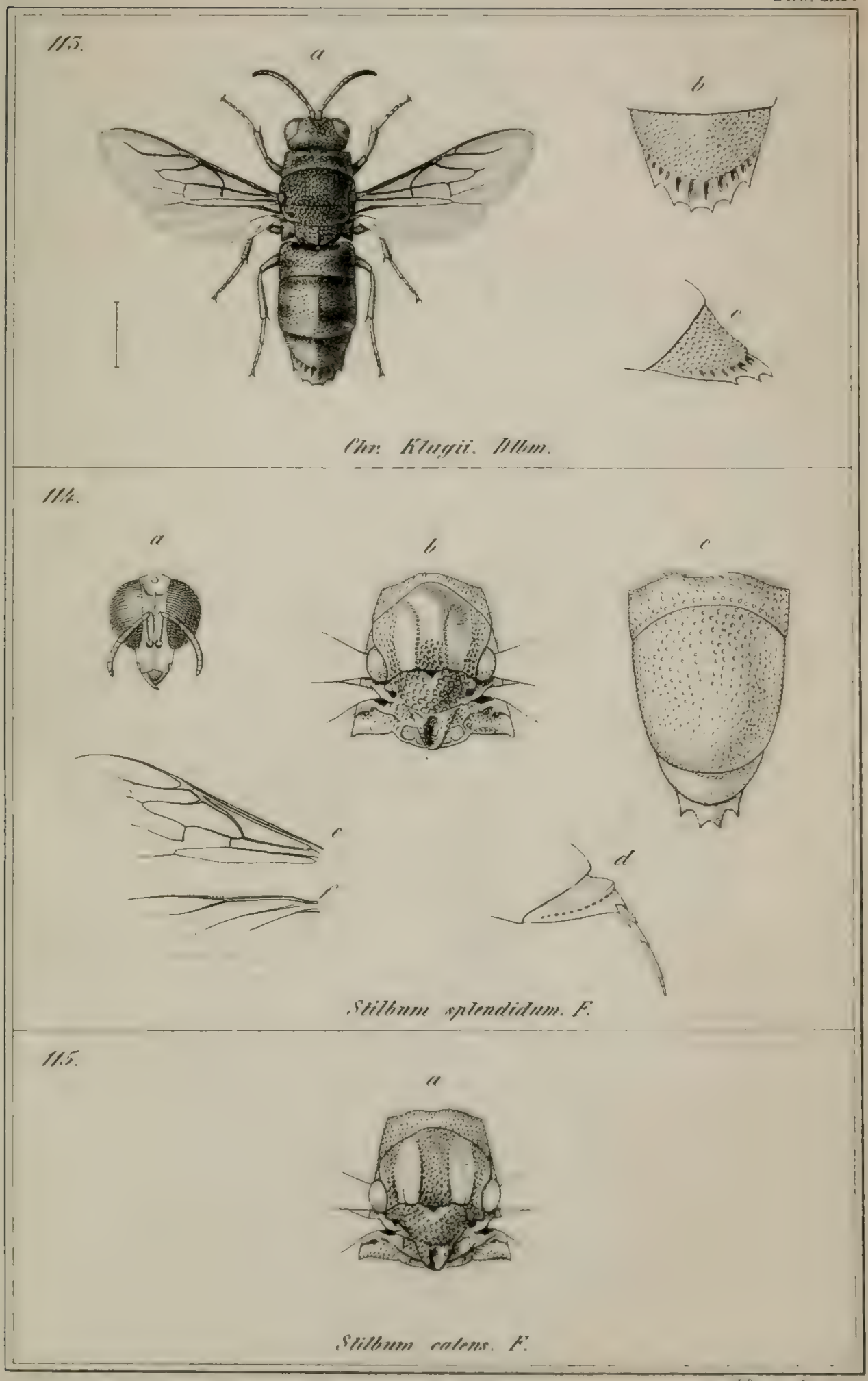

biendirn ded of on 


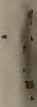

1

I"בxant

OF THE

UNIVEASTY OF ILEROIS 



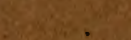

1 



\section{UNIVERSITY OF ILLINOIS-URBANA}

595.79013H

CO01 V002

HYMENOPTERA EUROPAEA PRAECIPUE BOREALIA,

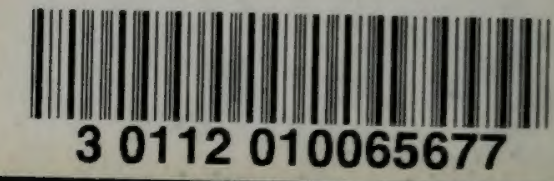

